\title{
Structural health assessment of bridges by long-term vibration monitoring and automated operational modal analysis
}

by

Serge L. Desjardins, P.Eng., B.A.Sc., M.A.Sc.

A thesis submitted to the

Faculty of Graduate and Postdoctoral Affairs

in partial fulfillment of the requirements for the degree of

Doctor of Philosophy in Civil Engineering

Ottawa-Carleton Institute for Civil Engineering

Department of Civil and Environmental Engineering

Carleton University

Ottawa, Ontario

April, 2019

(C)Copyright

Serge L. Desjardins, 2019 
The undersigned hereby recommends to the

Faculty of Graduate and Postdoctoral Affairs acceptance of the dissertation

\section{Structural health assessment of bridges by long-term vibration monitoring and automated operational modal analysis}

submitted by Serge L. Desjardins, P.Eng., B.A.Sc., M.A.Sc.

in partial fulfillment of the requirements for the degree of

Doctor of Philosophy in Civil Engineering

Professor Ashutosh Bagchi, External Examiner, Concordia University

Professor David T. Lau, Thesis Supervisor

Professor Yasser Hassan, Chair, Department of Civil and Environmental Engineering

Ottawa-Carleton Institute for Electrical and Computer Engineering Department of Civil and Environmental Engineering Carleton University

April, 2019 


\section{Abstract}

Vibration-based structural health monitoring (VBSHM) is a sub field of structural health monitoring (SHM) where field-measured vibration responses are used for condition assessment of civil engineering structures. The goal of VBSHM applications is reliable and consistent non-destructive condition assessments of structures (such as bridges, buildings, dam, towers, etc.). The main objective of this thesis is to contribute to, and to expand on, the current knowledge of VBSHM, with a focus on both the practical aspects such as instrumentation, data collection, data management and large scale data processing, and on the theoretical aspects such as advanced analysis and interpretation through improved system identification, automated operational modal analysis, long-term tracking of modal estimates and visualizations which will lead to proper condition assessment and damage detection. The Confederation Bridge's long-term remote vibration monitoring project in eastern Canada provides an important backdrop for the work described in this thesis.

The path to reliable and consistent condition assessments from vibration response measurements is through a thorough understanding of the causes of uncertainties and variability in the analysis results and where possible through the removal or the mitigation of these sources of variability. Several known sources of measurement noise inside the monitoring system have been identified and removed during the complete renewal of the monitoring system. The data processing engine has been completely 
overhauled in order to minimize computation noise and to streamline all data operations. The automation of these tools is required in order to batch process the large amounts of collected raw vibration responses. The well-established Stochastic Subspace Identification (SSI) technique was improved and automated to reduce the uncertainty associated with computation and human error. A new Automated Inline Full Space Identification (AI-FSI) technique is thus proposed and is presented in this thesis. This new technique integrates all aspects of automated modal parameter estimations (MPE) and modal tracking. With the new tools integrated in the third version of the signal processing platform for analysis of structural health (SPLASH), the processing and analysis of all the historical data collected by the Confederation Bridge monitoring project since 1998 was completed. This represents over 250000 logger files, 40000 hours of recording and 28TB of raw and processed data collected over 20 years. Several possible sources of environmental and operational variability are identified and quantified. Through multiple regression analysis it has been shown that depending on the operational vibration mode, these identified sources of variability can explain between $24 \%$ and $53 \%$ of the variations observed in the estimated modal frequencies. This is a significant finding that can further improve future condition or damage identification techniques that use modal features. A novel approach using a change point algorithm to detect mean shifts in the residual frequencies attributable to possible damage is successful in identifying small shifts in frequencies in the order of the standard deviation of residuals ( $0.68 \%$ to $0.95 \%$ of mean frequency).

Finally, practical examples of the successful applications of VBSHM for the evaluation of the health of structural components are described in this thesis. A fatigue analysis method is presented as a proof of concept for calculating the cumulative fatigue damage (cFD) from vibration response data and for estimating the remaining fatigue service life of in-operation bridge light poles. Such successful case studies are 
required to further advance research in the field of VBSHM for eventual large scale adoption and for improved infrastructure management. 
Véronik, Henri, Vincent et Thomas pour votre patience, amour et support. 


\section{Acknowledgments}

I would like to express my most sincere gratitude to Professor David T. Lau, for his time, expertise, friendship and enthusiasm throughout this research and for the past 17 years. It has been a great honor for me to work and learn from this great knowledgeable person and internationally renowned researcher.

Special thanks are owed to Donald McGinn of Strait Crossing Bridge Ltd. (SCBL) for his trust, suggestions and hospitality during the numerous visits to the bridge site. And to Mike Piskun of MAPScientific for his work and for the shared knowledge and expertise during the renewal of the vibration monitoring system.

Sincere gratitude to Gérard Poitras, friend and colleague for his valuable assistance on multiple trips to the bridge site during the light pole vibration monitoring study.

I would also like to acknowledge Gilles Roy, Dean of the Engineering Faculty at the Université de Moncton, and to all of the faculty members for their encouragement, understanding and continued support.

I wish to take this opportunity to express my deepest appreciations to my family and extended family for their love, support, patience and encouragements without which this work would not have been possible.

To Engineers Canada and Manulife Financial for their generous national scholarship, of which I am a grateful recipient.

The thesis research is part of the collaborative research project on monitoring 
of the Confederation Bridge funded by Natural Sciences and Engineering Research Council Canada, Public Works and Government Services Canada, and Strait Crossing Bridge Ltd. 


\section{Table of Contents}

Abstract iii

Acknowledgments vii

Table of Contents $\quad$ ix

List of Tables $\quad$ xvii

List of Figures $\quad$ xx

1 Introduction 1

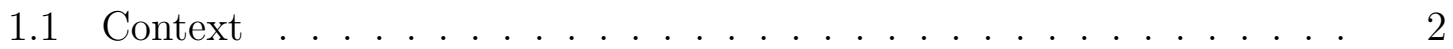

1.1.1 Public infrastructure challenges . . . . . . . . . . . . 2

1.1.2 Structural health monitoring - a global perspective . . . . . 6

1.2 Thesis Objectives . . . . . . . . . . . . . . . . . . . . . . 11

1.3 Thesis Outline . . . . . . . . . . . . . . . . . . . . . . . . 13

2 Advanced Operational Modal Analysis $\quad 15$

2.1 Stochastic Subspace Identification (SSI) . . . . . . . . . . . . 16

2.2 Automated In-line Full Space Identification (AI-FSI) $\ldots \ldots \ldots \ldots$

2.2 .1 Full Space Identification $\ldots \ldots \ldots$

2.2 .2 Improved parameter estimations . . . . . . . . . . . . 25 
2.2.3 Automated modal parameter estimations (MPE) . . . . . . 26

2.2.4 Mode tracking by recursive reduction of the MAC matrix . . . 29

\section{Confederation Bridge Vibration Monitoring Project 31}

3.1 Bridge Description . . . . . . . . . . . . . . . . . 32

3.2 Original Monitoring System . . . . . . . . . . . . . . . . . 34

3.2.1 Vibration monitoring . . . . . . . . . . . . . 35

3.3 Summary of Prior Studies . . . . . . . . . . . . . . . . . 38

3.3.1 Analytical modelling . . . . . . . . . . . . . . . . . . 39

3.3.1.1 Analytical model for seismic design (SAP-90 $\left.{ }^{\circledR}\right)$. . . 39

3.3.1.2 Updated analytical model for calibration (COSMOS $\left.{ }^{\circledR}\right) \quad 39$

3.3.1.3 Updated finite element model $\left(\right.$ Abaqus $\left.{ }^{\circledR}\right)$. . . . . . 42

3.3.2 Bridge behavior and early operational modal analysis . . . . . 43

3.3.2.1 Initial estimations of dynamic characteristics . . . . . 43

3.3.2.2 Pull test . . . . . . . . . . . . . . . 45

3.3.2.3 Controlled traffic load test . . . . . . . . . . . . . . 48

3.3.2.4 Local vibrations . . . . . . . . . . . . . . 49

3.3.2.5 Design verification . . . . . . . . . . 50

3.3.2.6 Baseline variability of modal properties . . . . . . . . 57

3.3.2.7 General variability of modal properties . . . . . . . 62

3.3.3 Signal processing platform for analysis of structural health $($ SPLASH v1.0) . . . . . . . . . . . . . . . 66

3.3.4 Advanced system identification techniques . . . . . . . . . 68

3.3.4.1 PolyMax - Polyreference Least Square Complex Frequency Estimator $(\mathrm{p}-\mathrm{LSCF}) \ldots \ldots 8$

3.3.4.2 Comparison study (FDD, ERA, SSI and PolyMax) . 68 
3.3.5 Damage detection . . . . . . . . . . . . . . . . . . 69

3.3.5.1 Finite element model updating . . . . . . . . . . 69

3.3.5.2 Statistical pattern recognition . . . . . . . . . 70

3.3.6 Research Platform for Smart Facilities Management (RP-

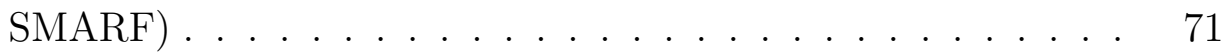

3.4 Summary . . . . . . . . . . . . . . . . . . . . . . . . 72

4 Implications of Instrumentation Renewals on Established Long$\begin{array}{ll}\text { term Vibration-based Monitoring Programs } & 75\end{array}$

4.1 Experience gained from a first generation long-term vibration monitoring system $\ldots \ldots \ldots \ldots \ldots \ldots \ldots \ldots$

4.1 .1 Instrumentation . . . . . . . . . . . . . . 76

4.1 .2 System diagnostics $\ldots \ldots \ldots \ldots \ldots$

4.1 .3 Collection parameters $\ldots \ldots \ldots \ldots \ldots$

4.1 .4 Data synchronization . . . . . . . . . . . . . . . . 79

4.1.5 Data management . . . . . . . . . . . . . . 81

4.1.6 Automation of processing and analysis . . . . . . . . 82

4.2 Instrumentation Renewal . . . . . . . . . . . . . . . . . . . . 82

5 Redevelopment of the Specialized Application Platform for VB$\begin{array}{ll}\text { SHM } & 87\end{array}$

5.1 Practical Considerations for SPLASH Update . . . . . . . . . 87

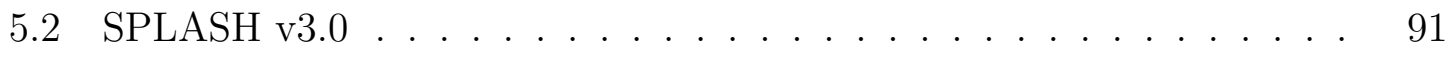

5.2 .1 Database management module . . . . . . . . . . . . . 91

5.2.1.1 Database creation . . . . . . . . . . . . 91

$5.2 .1 .2 \quad$ Database viewer . . . . . . . . . . . . . . 94

5.2.1.3 Data acquisition diagnostic . . . . . . . . . 96 
5.2 .1 .4 Other functions . . . . . . . . . . . . . 96

5.2 .2 Data synchronization . . . . . . . . . . . . . . . . 96

$5.2 .3 \quad$ Streamlined preprocessing . . . . . . . . . . . . 98

5.2 .4 Metadata . . . . . . . . . . . . . . . . . . . . . . 104

5.2 .5 Sensor diagnostic . . . . . . . . . . . . . . . . . 104

5.2.6 Multiple system identification techniques . . . . . . . . . 107

5.2.7 Automated operational modal analysis . . . . . . . . . 108

$5.3 \quad$ Summary . . . . . . . . . . . . . . . . . . . . . . 108

6 Historical Database of Processed Data 109

6.1 Data Collection Statistics _. . . . . . . . . . . . . 110

6.1.1 Vibration data collection . . . . . . . . . . . . . . 110

6.1.2 Environmental data collection . . . . . . . . . . . . . 111

6.1 .3 Collection statistics . . . . . . . . . . . . . . . . . . . 112

6.1.3.1 From February 1998 to February 2014 . . . . . . . 114

6.1.3.2 From March 2014 to May 2018 . . . . . . . . . . . 115

6.2 Processed Data . . . . . . . . . . . . . . . . 116

6.2 .1 Data collection per sensor . . . . . . . . . . . 116

6.2 .2 Maximum acceleration . . . . . . . . . . . . . . . 118

6.2 .3 Strength of signal (RMS) . . . . . . . . . . . . . . . 121

6.2.4 Operational modal estimates . . . . . . . . . . . . . . 123

6.2.4.1 OMA results from February 1998 to February 2014 . 124

6.2.4.2 OMA results from March 2014 to May 2018 . . . . . 126

6.2.4.3 Variability of OMA results . . . . . . . . . . 128

\section{Condition Assessment by Statistical Analysis of Operational Modal}

$\begin{array}{ll}\text { Estimates } & 131\end{array}$ 
7.1 OMA Results . . . . . . . . . . . . . . . . . . . . . . 132

7.2 Smoothing of OMA Estimates . . . . . . . . . . . . 135

7.3 Extended Kalman Filtering of OMA estimates . . . . . . . . . . . 142

7.4 Regression Analysis of OMA estimates . . . . . . . . . . . 150

$7.4 .1 \quad 0.47 \mathrm{~Hz}$ mode $\ldots \ldots \ldots \ldots \ldots \ldots \ldots$

$7.4 .2 \quad 0.67 \mathrm{~Hz}$ mode $\ldots \ldots \ldots \ldots \ldots \ldots \ldots$

$7.4 .3 \quad 0.96 \mathrm{~Hz}$ mode $\ldots \ldots \ldots \ldots \ldots$

$7.4 .41 .62 \mathrm{~Hz}$ mode $\ldots \ldots \ldots \ldots \ldots \ldots$

$7.4 .5 \quad 1.85 \mathrm{~Hz}$ mode $\ldots \ldots \ldots \ldots \ldots$

7.4 .6 Summary . . . . . . . . . . . . . . . . . . . 172

7.5 Residual Analysis and Change Point Detection for Damage Identification174

8 Use of Vibrations for Health Monitoring of Structural Components179

8.1 Modal Analysis and Vibration Mitigation of the Confederation Bridge

Light Poles . . . . . . . . . . . . . . . . . . . 180

8.1 .1 Summary . . . . . . . . . . . . . . . 180

8.1 .2 Introduction . . . . . . . . . . . . . . . 181

8.1 .3 Pole dynamics . . . . . . . . . . . . . . . . . . . . . . . 181

8.1.4 Analytical modelling . . . . . . . . . . . . . . . . 184

8.1.4.1 Structural component models (SCM) . . . . . . . 185

8.1.4.2 Finite element model (FEM) _ . . . . . . . . 187

8.1.4.3 Frequency analysis . . . . . . . . . . . . . 188

8.1.4.4 Static analysis . . . . . . . . . . . . . 189

8.1.4.5 Superstructure frequency . . . . . . . . . . . . 190

8.1.5 Experimental methodology . . . . . . . . . . . . . . . . 190

8.1.5.1 Subject light poles . . . . . . . . . . . . . . . . 191 
8.1.5.2 Vibration mitigation options . . . . . . . . . . . 192

8.1.5.3 Instrumentation and data acquisition . . . . . . . 196

8.1.6 Manipulations . . . . . . . . . . . . . . . . . . 197

8.1.6.1 Field setup . . . . . . . . . . . . . . . . 197

8.1.6.2 Laboratory setup and verifications . . . . . . . . 198

8.1 .7 Analysis . . . . . . . . . . . . . . . . . . . 199

8.1.7.1 Data processing ............... 199

8.1.7.2 Free vibration - $\log$ decrement . . . . . . . . . . 199

8.1.7.3 Free vibration - frequency domain . . . . . . . . . 200

8.1.7.4 Stochastic subspace identification (SSI) - SPLASH . 201

8.1.8 Results and interpretation . . . . . . . . . . . . . . 202

8.1.8.1 Modal properties of installed light poles . . . . . . . 202

8.1.8.2 Damper comparison .............. . 204

8.1.9 Conclusion and recommendations . . . . . . . . . . . . . . 210

8.2 Vibration Monitoring and Damper Performance of the Confederation

Bridge Light Poles . . . . . . . . . . . . . . . . . . . . . . . . . . . . 212

8.2.1 Summary . . . . . . . . . . . . . . . . . 212

8.2 .2 Introduction . . . . . . . . . . . . . . . . . 214

8.2 .3 Methodology . . . . . . . . . . . . . . . 214

8.2.3.1 Light poles . . . . . . . . . . . . . . 215

8.2.3.2 Pole top impact damper . . . . . . . . . . . . . 215

8.2.3.3 Instrumentation . . . . . . . . . . . . . 215

8.2.3.4 Data acquisition . . . . . . . . . . 216

8.2.3.5 Damping configurations . . . . . . . . . . 218

8.2.4 Datasets and environmental conditions . . . . . . . . . . . 219

8.2.5 Data analysis . . . . . . . . . . . . . . . . 220 
8.2.5.1 Data processing . . . . . . . . . . . . . . 220

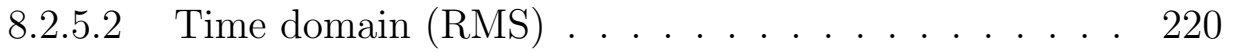

8.2.5.3 Frequency domain . . . . . . . . . . . . . . 221

8.2.5.4 Pole base stress estimation . . . . . . . . . . . . 222

8.2.5.5 Rainflow count for fatigue analysis . . . . . . . . 222

8.2.5.6 Cumulative fatigue damage (cFD) . . . . . . . 225

8.2.5.7 Fatigue life estimations . . . . . . . . . . . 226

8.2 .6 Results . . . . . . . . . . . . . . . . . . . . . . 227

8.2.6.1 Extended monitoring results . . . . . . . . . . . 228

8.2.6.2 Damping performance . . . . . . . . . . . . 228

8.2.6.3 Estimated fatigue service life . . . . . . . . . . 228

8.2.7 Interpretation and discussions . . . . . . . . . . . . . 231

8.2.8 Conclusions and recommendations . . . . . . . . . . . . 236

8.3 Estimating the Remaining Service Life of Bridge Bearings Using Dynamic Measurements . . . . . . . . . . . . . . . . 238

8.4 Use of Vibration Monitoring for Estimating Prestress Loss of In-Service External Post Tensioning Cables . . . . . . . . . . . . . . . . . 240

9 Conclusions 242

9.1 Discussion . . . . . . . . . . . . . . . . . . . . . . . . . . . . . . . 242

9.2 Future work . . . . . . . . . . . . . . . . . . . . . 244

$\begin{array}{ll}\text { List of References } & 247\end{array}$

Appendix A Operational Mode Shapes 255

$\begin{array}{lll}\text { Appendix B Linear regression parameter estimations } & 261\end{array}$ 
Appendix D Light pole monitoring - complete list of collected datasets and associated results 


\section{List of Tables}

3.1 Sensor location with make and model of accelerometers. . . . . . . . . 36

3.2 Specifications for the original accelerometers located on the Confederation Bridge. . . . . . . . . . . . . . . . . . . . 37

3.3 Modal properties extracted by different authors from the vibration monitoring data of the Confederation Bridge. . . . . . . . . . . . . 46

3.4 Verification of experimental modal properties with respect to the analytical results from the beam element model spanning two portal frames $[28] . \ldots \ldots \ldots \ldots \ldots$

3.5 Summary of in-depth verification study by Londono et al [29]. . . . . 55

3.6 Variability of results from analysis of 42 data sets for environmental effects study [30]. . . . . . . . . . . . . . . . . . . . . . 64

3.7 Results of regression analysis in environmental variability study [30]. . 65

4.1 On-line dates for the replacement high speed data loggers. . . . . . . 83

4.2 Sensor location and channel numbering with make and model of original and new accelerometers. . . . . . . . . . . . . . . . . . . 84

4.3 Specifications for accelerometers located on the Confederation Bridge. 85

6.1 Vibration data collection statistics. . . . . . . . . . . . . 113

6.2 Identification and variability of operational modal estimates. . . . . . 129

7.1 Variability of operational modal estimates - November 2017. . . . . . 134 
7.2 Description of smoothing methods considered in this study. . . . . . . 136

7.3 Response and predictor variables considered for linear regression. . . . 151

7.4 Linear regression statistics for modal frequencies of $0.47 \mathrm{~Hz}$ mode. . . 155

7.5 Linear regression statistics for modal frequencies of $0.67 \mathrm{~Hz}$ mode. . . 159

7.6 Linear regression statistics for modal frequencies of $0.96 \mathrm{~Hz}$ mode. . . 163

7.7 Linear regression statistics for modal frequencies of $1.62 \mathrm{~Hz}$ mode. . . 167

7.8 Linear regression statistics for modal frequencies of $1.85 \mathrm{~Hz}$ mode. . . 171

7.9 Linear regression statistics for the five dominant modal frequencies. . 173

7.10 Results of the change point sensitivity analysis on the residuals of five dominant operational modes. . . . . . . . . . . . . . . . . 177

8.1 Light pole structural properties considered for analytical modeling. 186

8.2 Analytical modal frequencies $(\mathrm{Hz})$ for the first four vibration modes of the light poles. . . . . . . . . . . . . . . . . . . . . . . . . . . . . . . 188

8.3 Sensitivity of modal frequencies $(\mathrm{Hz})$ of light pole with varying light fixture weights. . . . . . . . . . . . . . . . . . . . . 189

8.4 Superstructure frequencies near light pole's fundamental frequencies [29].190

8.5 Damping configurations for site testing. . . . . . . . . . . . . . . 194

8.6 Modal frequencies $(\mathrm{Hz})$ of the original undamped light pole structure with LED light fixture. . . . . . . . . . . . . . . . . . . . 203

8.7 Modal frequencies $(\mathrm{Hz})$ of the replacement undamped light pole structure with LED light fixture. . . . . . . . . . . . . . . . 203

8.8 First mode properties (frequencies and damping ratios) of the tested damping configurations. . . . . . . . . . . . . . . 209

8.9 Collected datasets during wind events with corresponding environmental conditions. . . . . . . . . . . . . . . . . . . . 219

8.10 Analysis results averaged over wind events. . . . . . . . . . . . . 229 
8.11 Estimate of cumulative fatigue damage $(\mathrm{cFD})$ in percentage of total fatigue life (cumulated from November 1997 to March 2018). . . . . . 234

8.12 Longitudinal pier sweep estimates $(\mathrm{mm} / \mathrm{min})$ obtained from vibration measurements of pier 30. . . . . . . . . . . . . . . . 239

B.1 Linear regression coefficients for modal frequencies of $0.47 \mathrm{~Hz}$ mode, ordered according to significance. . . . . . . . . . . . . . . . . 261

B.2 Analysis of variance of the linear regression model for modal frequencies of the $0.47 \mathrm{~Hz}$ mode. . . . . . . . . . . . . . . . . . . 262

B.3 Linear regression coefficients for modal frequencies of $0.67 \mathrm{~Hz}$ mode, ordered according to significance. . . . . . . . . . . . . . . . 262

B.4 Analysis of variance of the linear regression model for modal frequencies of the $0.67 \mathrm{~Hz}$ mode. . . . . . . . . . . . . . . . . 263

B.5 Linear regression coefficients for modal frequencies of $0.96 \mathrm{~Hz}$ mode, ordered according to significance. . . . . . . . . . . . . . . . . 264

B.6 Analysis of variance of the linear regression model for modal frequencies of the $0.96 \mathrm{~Hz}$ mode. . . . . . . . . . . . . . . . . . . . . 264

B.7 Linear regression coefficients for modal frequencies of $1.62 \mathrm{~Hz}$ mode, ordered according to significance. . . . . . . . . . . . . . . 265

B.8 Analysis of variance of the linear regression model for modal frequencies of the $1.62 \mathrm{~Hz}$ mode. . . . . . . . . . . . . . . . . . 265

B.9 Linear regression coefficients for modal frequencies of $1.85 \mathrm{~Hz}$ mode, ordered according to significance. . . . . . . . . . . . . . . 266

B.10 Analysis of variance of the linear regression model for modal frequencies of the $1.85 \mathrm{~Hz}$ mode. . . . . . . . . . . . . . . . . 267

D.1 List of collected datasets with analysis results for the Confederation Bridge light pole study of Section 8.2. . . . . . . . . . . . . . . . 272 


\section{List of Figures}

1.1 Research context. . . . . . . . . . . . . . . . . 5

2.1 Typical stabilization plot showing stability of poles. . . . . . . . . 21

3.1 Geographical location of the Confederation Bridge [38] . . . . . . . 33

3.2 Confederation Bridge elevation. . . . . . . . . . . . . 33

3.3 Confederation Bridge components. . . . . . . . . . . . . 34

3.4 Accelerometer locations on the Confederation Bridge. . . . . . . . 35

3.5 3D shell element model of one typical portal frame of the Confederation Bridge $\left(\operatorname{COSMOS}^{\circledR}\right)[41] . \ldots \ldots \ldots$

3.6 Finite element model using 3D beam elements of one typical portal frame of the Confederation Bridge $\left(\operatorname{COSMOS}^{\circledR}\right)[41]$. . . . . . . . 41

3.7 Finite element model using 3D beam elements spanning two portal frames of the Confederation Bridge (COSMOS $\left.{ }^{\circledR}\right)[41] \ldots \ldots$. . . . . 42

3.8 3D model of one typical portal frame of the Confederation Bridge

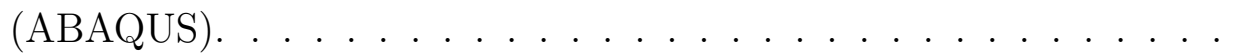

3.9 Comparison of experimental and analytical mode shapes. Vibration modes from the beam finite element model are shown in green with doted lines and the modes identified from the monitoring data are shown in gray with full lines. Modes from 0 to $1 \mathrm{~Hz}[24] \ldots$. . . . . 58 
3.10 Comparison of experimental and analytical mode shapes. Vibration modes from the beam finite element model are shown in green with doted lines and the modes identified from the monitoring data are shown in grey with full lines. Modes above $1 \mathrm{~Hz}[24] \ldots$. . . . . . . . 59

3.11 Operational modal frequency variation with respect to temperature [30]. 66

3.12 Screen-capture of SPLASH modules for Confederation Bridge VBSHM

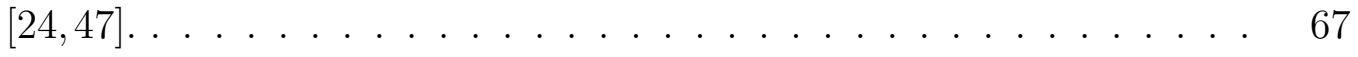

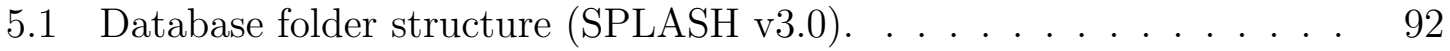

5.2 Screen capture of new Database Management module of SPLASH v3.0. 95

5.3 Screen capture of database management module showing data captured over a few hours on November 4th 2014 and associated metadata. . . 95

5.4 Example of a log file created while creating a new database in SPLASH

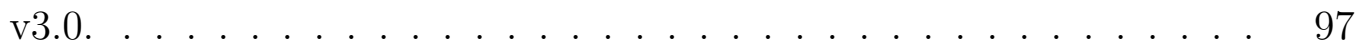

5.5 Integration of acceleration and velocity data to obtain displacement data. . . . . . . . . . . . . . . . . . . 103

5.6 Metadata tagged to the translated logger data files (SPLASH v3.0). . 104

5.7 Metadata tagged to the processed event files (SPLASH v3.0). . . . . 105

5.8 Metadata tagged to the individual processed channels (SPLASH v3.0). 105

5.9 Three dimensional plot of maximum normalized cross correlations between each sensor signal. . . . . . . . . . . . . . . . . 106

5.10 Averaged maximum normalized cross-correlations for each sensor signal.107

6.1 Historical environmental data from November 1997 to March 2018. . 112

6.2 Data collection per data logger from February 1998 to February 2014. 114

6.3 Data collection per data logger from March 2014 to May 2018. . . . . 115

6.4 Data collection per sensor from February 1998 to May 2018. . . . . . 117 
6.5 Maximum accelerations per event with corresponding mean wind speed and temperature data for all events collected between February 1998 and May 2018. . . . . . . . . . . . . . . . . . . . . . . . . . 119

6.6 Maximum accelerations per event with corresponding mean wind speed and temperature data for all events collected in November 2017. . . . 120

6.7 Average signal strength (RMS) per event with corresponding mean wind speed and temperature data for all events collected between February 1998 and May 2018. . . . . . . . . . . . . . . . . . . . . 121

6.8 Average signal strength (RMS) per event with corresponding mean wind speed and temperature data for all events collected in November

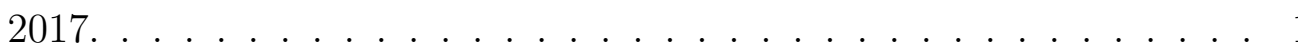

6.9 Histogram of extracted modal frequencies from full events collected between February 1998 and February 2014. . . . . . . . . . . . . . . . 124

6.10 Extracted modal frequencies from full events collected between February 1998 and February 2014. . . . . . . . . . . . . . . 125

6.11 Histogram of extracted modal frequencies from full events collected between March 2014 and May 2018. . . . . . . . . . . . . . . 126

6.12 Extracted modal frequencies from full events collected between March 2014 and May 2018. . . . . . . . . . . . . . . . . . . 127

6.13 Box plot of normalized operational modal frequencies from full events collected between February 1998 and February 2014. . . . . . . . . . 130

6.14 Box plot of normalized operational modal frequencies from full events collected between March 2014 and May 2018. . . . . . . . . . . . . . 130

7.1 Extracted frequencies from full events collected in November 2017. . . 133

7.2 Box plot of normalized operational modal frequencies - November 2017. 135

7.3 Data smoothing of the operational modal frequencies - 0.47Hz mode. 137 
7.4 Data smoothing of the operational modal frequencies $-0.67 \mathrm{~Hz}$ mode. 138

7.5 Data smoothing of the operational modal frequencies $-0.96 \mathrm{~Hz}$ mode. $\quad 139$

7.6 Data smoothing of the operational modal frequencies $-1.62 \mathrm{~Hz}$ mode. $\quad 140$

7.7 Data smoothing of the operational modal frequencies $-1.85 \mathrm{~Hz}$ mode. 141

7.8 Extended Kalman filtering of the modal frequencies (0.47Hz mode). . 147

7.9 Extended Kalman filtering of the modal frequencies (0.67Hz mode). . 147

7.10 Extended Kalman filtering of the modal frequencies (0.96Hz mode). . 148

7.11 Extended Kalman filtering of the modal frequencies (1.62Hz mode). . 148

7.12 Extended Kalman filtering of the modal frequencies (1.85Hz mode). . 149

7.13 Modal frequencies vs continuous predictors $-0.47 \mathrm{~Hz}$ mode. . . . . . 153

7.14 Modal frequencies vs categorical predictors - 0.47Hz mode. . . . . . . 154

7.15 Regression model for modal frequencies of $0.47 \mathrm{~Hz}$ mode. . . . . . . . 156

7.16 Modal frequencies vs continuous predictors $-0.67 \mathrm{~Hz}$ mode. . . . . . . 157

7.17 Modal frequencies vs categorical predictors - 0.67Hz mode. . . . . . 158

7.18 Regression model for modal frequencies of $0.67 \mathrm{~Hz}$ mode. . . . . . . . 160

7.19 Modal frequencies vs continuous predictors $-0.96 \mathrm{~Hz}$ mode. . . . . . . 161

7.20 Modal frequencies vs categorical predictors $-0.96 \mathrm{~Hz}$ mode. . . . . . . 162

7.21 Regression model for modal frequencies of $0.96 \mathrm{~Hz}$ mode. . . . . . . . 164

7.22 Modal frequencies vs continuous predictors $-1.62 \mathrm{~Hz}$ mode. . . . . . . 165

7.23 Modal frequencies vs categorical predictors $-1.62 \mathrm{~Hz}$ mode. . . . . . . 166

7.24 Regression model for modal frequencies of $1.62 \mathrm{~Hz}$ mode. . . . . . . . 168

7.25 Modal frequencies vs continuous predictors - $1.85 \mathrm{~Hz}$ mode. . . . . . . 169

7.26 Modal frequencies vs categorical predictors $-1.85 \mathrm{~Hz}$ mode. . . . . . . 170

7.27 Regression model for modal frequencies of $1.85 \mathrm{~Hz}$ mode. . . . . . . . 172

7.28 Change point detection example of mean shift in residuals of $0.47 \mathrm{~Hz}$ mode. . . . . . . . . . . . . . . . . 176 
8.1 Effects of vibration dampers [63]. . . . . . . . . . . . . . . 184

8.2 Rubbing pipe chain type damper [63] . . . . . . . . . . . . . 185

8.3 Structural component model of the Confederation Bridge light poles. 186

8.4 Mode-shape comparison of transverse $1.32 \mathrm{~Hz}$ mode. Grey model with full lines represents extracted mode-shapes while green model with dashed lines represents the mode-shape result from the analytical 3D

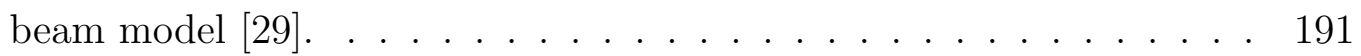

8.5 Approximate location of subject poles on the Confederation Bridge. . 191

8.6 Accelerometer stations and pull location for on-site testing. . . . . . . 192

8.7 Pole top impact dampers installed during testing. . . . . . . . . . . 195

8.8 Installed wireless sensors. GLink2-LXRS tri-axial MEMS accelerometer.196

8.9 On-site setup for vibration testing. . . . . . . . . . . . . . . 197

8.10 Laboratory setup for the testing of the light poles. . . . . . . . . . . 198

8.11 Free vibration of a lightly damped system. . . . . . . . . . . . 200

8.12 Analytical and experimental mode shapes of the original light pole. . 204

8.13 Free vibration displacement time histories for the damping configurations of the original pole. . . . . . . . . . . . . . . 205

8.14 Free vibration displacement time histories for the damping configurations of the replacement pole. . . . . . . . . . . . . . 206

8.15 Free vibration displacement time histories for the damping configurations of the laboratory pole (without chain damper). . . . . . . . . 207

8.16 Prototype of first mode damper Two balls on top of pole with obstructions. . . . . . . . . . . . . . . . . 216

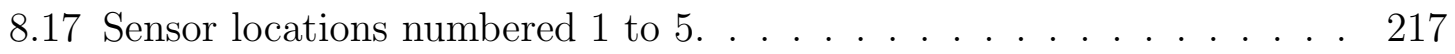

8.18 Pole top displacement time history with RMS. . . . . . . . . . . 221

8.19 Frequency content of pole top displacement signal. . . . . . . . . . . . 222 
8.20 Rainflow counts for a sample five minutes dataset. . . . . . . . . . . . 224

8.21 Pole to base plate connection of fatigue category E1 [71] . . . . . . . 226

8.22 Fatigue damage versus measured ten minute mean wind speed, v, with best fit exponential model for both the undamped and the damped poles.227

8.23 Estimated cumulative fatigue damage (cFD) of Confederation Bridge light poles (damped vs undamped). . . . . . . . . . . . . . . 230

8.24 Fatigue damage versus wind speed with corresponding exponential fatigue model. . . . . . . . . . . . . . . . . . . . . . . 233

A.1 Mode-shape comparison of transverse $0.47 \mathrm{~Hz}$ mode. Grey model with full lines represents extracted mode-shapes while green model with dashed lines represents the mode-shape result from the analytical 3D

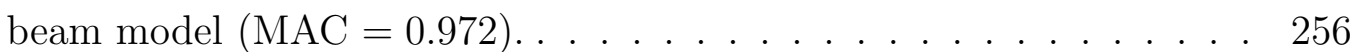

A.2 Mode-shape comparison of transverse $0.67 \mathrm{~Hz}$ mode. Grey model with full lines represents extracted mode-shapes while green model with dashed lines represents the mode-shape result from the analytical 3D

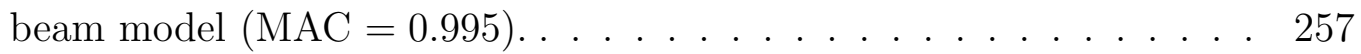

A.3 Mode-shape comparison of transverse $0.96 \mathrm{~Hz}$ mode. Grey model with full lines represents extracted mode-shapes while green model with dashed lines represents the mode-shape result from the analytical 3D beam model $(\mathrm{MAC}=0.993) \ldots \ldots . . . . . . . . . . .258$

A.4 Mode-shape comparison of transverse $1.62 \mathrm{~Hz}$ mode. Grey model with full lines represents extracted mode-shapes while green model with dashed lines represents the mode-shape result from the analytical 3D

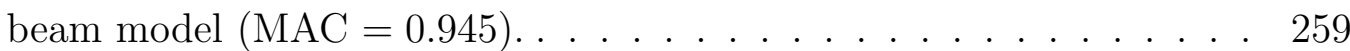


A.5 Mode-shape comparison of transverse $1.85 \mathrm{~Hz}$ mode. Grey model with full lines represents extracted mode-shapes while green model with dashed lines represents the mode-shape result from the analytical 3D

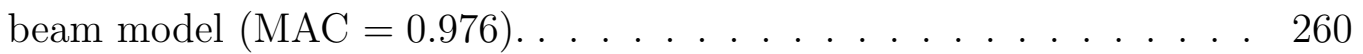

C.1 Data-sheet for the wireless sensors used in the light pole studies of

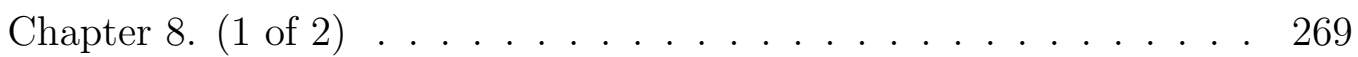

C.2 Data-sheet for the wireless sensors used in the light pole studies of

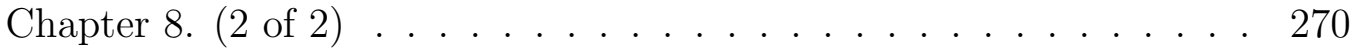




\section{Chapter 1}

\section{Introduction}

The work described in this thesis expands on the practical applications of vibrationbased structural health monitoring (VBSHM) of civil engineering structures. VBSHM is a sub field of structural health monitoring (SHM) where field-measured vibration responses are used for condition assessment. VBSHM techniques have expanded greatly in the past decades and have become an important, and sometimes essential, component of a complete structural health monitoring program. Advances in information technology with respect to instrumentation and computer processing have allowed a significant blossoming of this field in recent years.

The ultimate objective of VBSHM applications is reliable and consistent nondestructive condition assessments of structures (such as bridges, buildings, dam, towers, etc.). The main objective of this thesis is to contribute to, and to expand on, the current knowledge of VBSHM, with a focus on both the practical aspects such as instrumentation, data collection, data management and large scale data processing, and on the theoretical aspects such as advanced analysis and interpretation through improved system identification, automated operational modal analysis, long-term modal tracking and visualizations which will lead to proper condition assessment and damage detection. Section 1.2 expands on the main objectives stated here. 
The Confederation Bridge's long-term remote monitoring project in eastern Canada provides an important backdrop for the work described in this thesis. Sections of this thesis will highlight recent developments with respect to its vibration monitoring program. Much has been written about this project in the past, however this thesis will expand on the long-term aspects of this significant program such as (but not limited to) the recent instrumentation renewal and its implication for ongoing research and applications and the automation of all processing and analysis steps required for the operational modal analysis of all vibration responses collected in the past 20 years.

Context for the current work is provided in Section 1.1. The practical implications of this research for public (and private) infrastructure management are stated and a brief description of structural health monitoring in its global context is provided. The detailed objectives and the outline of the thesis document are presented in Sections 1.2 and 1.3 respectively.

\subsection{Context}

\subsubsection{Public infrastructure challenges}

According to the U.S. National Science Foundation, "A civilization's rise and fall is linked to its ability to feed and shelter its people and to defend itself. These capabilities depend on infrastructure; the underlying, often hidden, foundation of a society's wealth and quality of life. A society that neglects its infrastructure loses the ability to transport people and food, provide clean air and water, control disease and conduct commerce." 1

\footnotetext{
${ }^{1}$ Quoted in library of Parliement's report by Haggart : "Canada's Infrastructure Debt Part I: assessing the infrastructure shortfall" [1].
} 
It is well understood that public civil infrastructure is the foundation for a country's economic well-being and its citizens' quality of life. In Canada, infrastructure is central to every aspect of life. It is a driver of growth and productivity in the national economy. It is a contributor to the health and well-being of Canadian citizens and a critical component for the transportation of goods and services across the country. It is a means for providing essential services such as water, waste and wastewater management, and electricity and is a predictor of how our communities grow and contribute to our collective social fabric [2].

According to Haggart [1], over the past decade, a number of groups - including the Federation of Canadian Municipalities, the Conference Board of Canada, and the various associations of Canadian engineers - have concluded that years of underfunding have left Canada with about $\$ 60$ billion in outstanding infrastructure repair and replacement costs. These challenges are not unique to Canada. In the United States, the American Society of Civil Engineers (ASCE) estimated in 2003 that the U.S. needed to invest $\$ 1.6$ trillion (USD) over the following five years to bring its infrastructure to acceptable levels.

In more recent years, an increasing number of publications and reports have been issued drawing attention to Canada's growing infrastructure needs [3-7]. Canadian municipalities currently spend $\$ 12$ to $\$ 15$ billion annually on infrastructure [8] but it never seems to be enough. Existing infrastructure is ageing while demand grows for more and better roads and bridges, and improved water and sewer systems responding both to higher standards of safety, health and environmental protection as well as population growth. According to the Federation of Canadian Municipalities [8], the solution is to change the way infrastructures are planned, designed and, most importantly, managed. Only by doing so can the core public infrastructures meet new demands within a fiscally-responsible and sustainable framework, while preserving 
the expected quality of life of citizens.

In Canada, investments on bridges have been under the level required to hold their age constant. Hence, the average age of bridges has increased since the mid-1980s; from 21.3 years in 1985 to 24.5 years in 2007, an increase of 3.2 years, which means that Canada's bridges have passed $57 \%$ of their useful life [9]. Many bridges have already exceeded their estimated service life and many more are approaching this limit. Sustainable development urges to better protect these built assets. While, for example, transportation infrastructures are steadily aging and traffic volume is increasing, assessment and damage detection dictate the choice of maintenance policies for these types of infrastructures. Since maintenance is very costly, reliable and sensitive early damage detection capabilities could pay off rapidly.

The continued safe and economical operation of bridge infrastructure for the benefit of society depends on their proper management, which includes timely assessments of their condition for public safety and for efficient maintenance. Today, these tasks are still largely carried out by visual inspection and by other localized measurement techniques. However, increased public awareness of the economic and social effects of aging, deterioration and extreme events on these core civil infrastructures has been accompanied by recognition of the need for more advanced structural health monitoring and condition assessment.

Structural health monitoring (SHM) is a relatively new concept for civil infrastructure stakeholders (such as transportation authorities, infrastructure managers, operators, engineers, consultants, contractors, etc.) that has received much attention in the last three decades as witnessed by an acceleration of academic and applied research in areas such as sensing and data acquisition technologies, data mining, system identification, and condition assessment

Policy makers and decision makers at all levels of government are now striving 
towards smart infrastructure systems (SIS) $[8,10]$. SIS are a combination of smart structures (structures that monitors themselves and their environment in order to respond to changes in their conditions/health) and the management systems that operate them including design, installation, operation, use of the data generated and the decision-making $[11,12]$. Such a system, with integrated structural health monitoring, will be able to remotely monitor a large number of structures simultaneously. This ability to continuously and non-destructively evaluate many civil engineering structures is particularly enticing to bridge infrastructure managers and stakeholders. With such a bridge infrastructure management tool, decision makers will be able to identify high-risk structures and plan for more efficient retrofitting. These tools will reduce and focus maintenance costs where they are needed and potentially greatly reduce public infrastructure risks. In this goal, structural health monitoring will play a key role. SIS and SHM technologies will allow the current time-based maintenance practices to evolve into potentially more cost effective condition-based maintenance practices [13]. As costs for instrumentation and hardware for monitoring of structures are diminishing and as the knowledge-base for structural health monitoring is increasing, it is very likely that policy makers will incorporate SIS and SHM in future infrastructure management strategies $[8,10]$. There is therefore a strong potential for a new industry in SHM, from hardware (sensors, loggers, network equipment etc.) to software and eventual operation of the monitoring system [11,12].

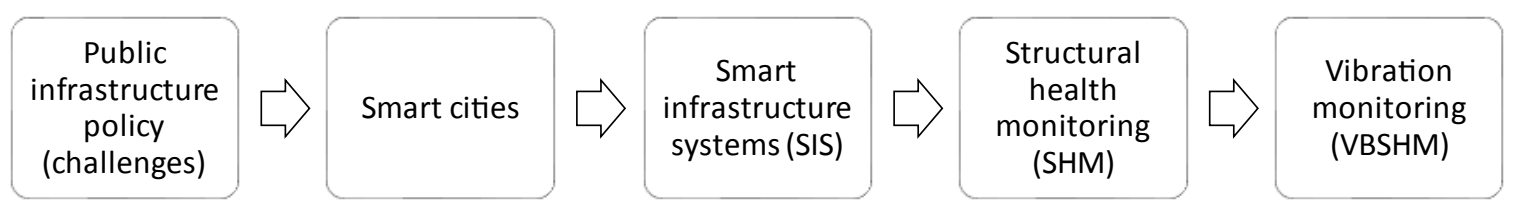

Figure 1.1: Research context.

Before making the case to include SHM in national public policies on infrastructure, clear proof of concepts must be presented with successful case studies. What 
policy makers and stakeholders require from SHM research are practical applications that answer basic and pragmatic questions about in-service performance, maintenance and security. Questions like: What is the health of the structure? Is there presence of significant deterioration or damage? What is the severity of the deterioration/damage? Where is the deterioration/damage located? What is the remaining service life of the structure or of its components? It is clear that research in SHM technologies must strive to answer these fundamental questions.

\subsubsection{Structural health monitoring - a global perspective}

What is Structural Health Monitoring? Reproduced here is one the many definitions of SHM offered by Charles Farrar [13]:

"The process of implementing a damage detection strategy for aerospace, civil and mechanical engineering infrastructure is referred to as Structural Health Monitoring (SHM). The SHM process involves the observation of a system over time using periodically-sampled response measurements from an array of sensors. The extraction of damage-sensitive features from these measurements and the statistical analysis of these features is then used to determine the current state of system health. For longterm SHM, the output of this process is periodically updated information regarding the ability of the structure to perform its intended function in light of the inevitable aging and degradation resulting from operational environments. After extreme events, such as earthquakes or blast loading, SHM is used for rapid condition screening and aims to provide, in near real time, reliable information regarding the integrity of the structure."

Structural health monitoring (SHM) can also be loosely defined as the observation 
and interpretation of performance of civil infrastructure. At its core, SHM is the continuous identification of a physical or parametric model of the structure using time-dependant data.

As in other fields, new information and communication technologies (ICT) are permitting the evolution of this very specialized field. Over the last decades, developments in sensing and information technology have made it possible (even easy) to monitor various structural responses and obtain data regarding the operations of the structures with a high level of detail and accuracy. The monitored data may include short and long-term deformations, strains, temperature profiles, corrosion rate, environmental data (wind and weather), and vibration responses to name a few. The low cost and the relative ease with which such monitoring system can now be implemented greatly increased the availability of such information. This has opened whole new avenues for investigation. Considerable research efforts have been, and continue to be, directed towards finding how to use the collected response data for structural condition assessment.

One type of data that has received plenty of attention lately is the vibration response data. Vibration data is relatively economical to record; the measurements are non-intrusive, they do not interrupt services or operations, and they are well suited for continuous monitoring. Also, most importantly, the vibration responses carry the essential information about the structural health: changes in the vibration frequencies reflect changes in stiffness and changes in the vibration mode shapes reflect changes in the distribution of the stiffness. This very important statement is the basis for all vibration-based monitoring programs.

For the above reasons, and with continued advances, vibration-based structural health monitoring (VBSHM), a sub field of SHM, has been attracting interest as a promising method for non-destructive evaluation of civil engineering structures. From 
the vibration data, modal properties, such as vibration frequencies, mode shapes and damping ratios, of the structure can be extracted. By treating the estimated modal parameters of the structure as features and by monitoring these extracted features in time, any observed changes in the modal frequencies, mode-shapes or damping ratios are considered as manifestations of possible damage or deterioration of the structure. It is well understood that changes in modal parameters reflect changes in physical properties (such as stiffness and mass) and therefore in the physical conditions of the structure. Difficulties occur in the implementation of VBSHM in the field, where monitoring data are affected by noise and other uncertainties of the environment. The solution to alleviate this problem and facilitate correct interpretation of the engineering information embedded in the data is to establish the trend and detailed behaviour and characteristics of the monitoring data by long-term collection of the information, even over the life span of the facility. For long-term monitoring, specialized instrumentation systems are permanently installed on the structure to capture operational responses and responses from extreme events such as earthquakes, hurricanes, ice floes or impacts. The captured monitoring data during and after extreme events can be used to rapidly evaluate the structure's safety, its performance and condition. Vibration-based monitoring systems are generally non-intrusive and they do not interfere with the normal function and operation of the monitored structure. To extract the needed information from the data acquired from these monitoring systems, complex numerical operations for signal processing purposes need to be performed. System identification of modal properties of the structure from the response monitoring data is a central component of most of the VBSHM techniques. It is the basic initial step in many of the current algorithms used for identifying damage in the structure or assessing the structural condition from response vibrations. It should be noted, however, that some new condition assessment techniques are completely data 
driven (meaning they do not rely on identification of a physical model) [14].

Structural responses caused by normal operating loads, such as moderate wind or traffic (in the case of bridges), are referred to as ambient responses. These ambient responses are very important for continuous long-term vibration-based structural evaluations. The estimation of modal parameters from these ambient responses has been shown to be very complicated due to the high level of uncertainties of the input excitations, the relatively low amplitude of the responses, the high noise contaminations of the data, the non-stationarity of the signals, the non-linearity of the structural behavior resulting in high uncertainty of the signals. Special system identification algorithms that can effectively reduce the effects of noise contaminations by means of signal correlations have been proven to be the most robust output-only identification techniques. It is only after the successful and consistent identification of modal properties, that condition assessment algorithms can be developed which deal with the variability of the estimated modal parameters. At the time of writing, the author has found no documented field monitoring project that has successfully detected the occurrence of actual structural damage after implementing these aspects in a complete continuous vibration-based condition evaluation of a large-scale operating civil engineering structure. The goal of VBSHM research is to provide continuous remote online evaluation of structural conditions for safety and/or maintenance purposes. It should be stated that vibration monitoring constitutes only a part of an integrated SHM program; the future of successful SHM is integration of all aspects of SHM, which includes but is not limited to vibration monitoring, strain monitoring, corrosion monitoring, temperature and wind monitoring and structural condition assessment (SCA). In this document, the term SCA is used to define the classical methods of evaluating structural condition which consists of activities of visual observation, measuring, 
photographing, probing and sampling, field and laboratory testing, engineering analyses, record keeping, documentation, and report preparation. The reliability of SCA lies in the interpretation of the collected and generated data and in the judgments converting them to accurate conclusions and recommendations [15].

Very promising at first, some believe the recent advances in VBSHM applications have been slow to deliver real-world results. According to Brownjohn [16], VBSHM's effectiveness in automatically detecting classical damage still remains to be proven for operational civil structures. The very small signal to noise ratio is a major obstacle for these techniques due to the effect of varying environmental conditions on the modal parameters, masking the effects of structural changes. However, Brownjohn notes that one significant technological advance that improves the potential of VBSHM is the use of automated operational modal analysis (OMA) [16]. More recent developments in OMA have addressed the statistical properties of the OMAderived parameters (frequencies, mode shapes and damping ratios) with filtering of environmental effects to provide effective structural identification, potentially even of deterioration or damage. This represents the basis for most of the work presented in this thesis.

It is well understood that through long-term continuous monitoring of civil structures, a thorough grasp of the structural behavior can be achieved. Establishing a detailed baseline of "healthy" vibration signatures of a civil structure early in its service life will aid in developing robust condition assessment techniques. While these advantages are well understood, the realities of establishing and maintaining a longterm monitoring program are not without difficulties. Not the least of which is the fact that the life cycle of a civil infrastructure is typically much longer than the life cycle of the monitoring instrumentation. In a long-term monitoring program, the instrumentation may undergo several cycles of renewal. While designing the renewed 
instrumentation systems, careful attention must be accorded to assure data quality and integrity. In fact, data quality, integrity and continuity must be considered as criteria of the highest importance during the decision making process.

\subsection{Thesis Objectives}

The ultimate objective of VBSHM applications is reliable and consistent nondestructive condition assessments of structures. The main objectives for the work described in this thesis, as stated in this section, are aligned with this ultimate objective.

It is now well understood that the path to reliable and consistent condition assessments from vibration response measurements is through a thorough understanding of the causes of noise and variability in the analysis results. The objectives for the work described in this thesis are therefore stated as follows:

- The known sources of uncertainties shall be mitigated by judicious choices in monitoring instrumentation;

- The sources of computational uncertainty and noise shall be reduced by streamlining all of the data processing operations;

- The existing operational modal analysis technique shall be improved to reduce the uncertainty due to computation and human error;

- All data processing and analysis operations shall be automated to allow for the large scale data analysis required for proper statistical analyses;

- All available information on sources that may contribute to the variability of the analysis results shall be gathered (i.e. environmental and loading conditions);

- The identified sources of variability shall be correlated with the operational modal analysis results in order to understand their contributions; 
- The effects from known sources of variability shall be filtered or removed from the operational modal analysis results;

- Temporal changes in the filtered or residual modal parameters shall be detected;

- The sensitivity of change detection shall be evaluated to provide an indication of the possible severity of damage to be detected.

The stated objectives have significant practical implications:

- There is an opportunity during the renewal of the instrumentation, to make judicious choices to reduce the measurement noise. These include the use of sensors with better range and resolution and requiring minimal signal treatments prior to recording.

- For proper analysis of variability, large amounts of vibration response data (on a much larger scale that was previously realized) needs to processed and analyzed. If possible, back calculation of all historical data is envisaged. There is approximately 20 years of vibration response data that is available for this work.

- New automated operational modal analysis techniques are therefore required, as the obligatory user interactions of the previous identification methods are inadequate for the scale of the analysis required to attain the stated objectives.

- There is now a need for new automated mode tracking algorithms to track the evolution of modal parameters on large time scales.

- New tools and methods are required to investigate the relationship between the modal parameters and the possible correlated environmental and operational processes.

- The existing processing and analysis tools developed more than 15 years ago need to be significantly updated to streamline the data processing and to incorporate the new automated OMA and mode tracking algorithms. 
An additional objective of this thesis, is to provide concrete practical examples of the successful applications of vibration-based monitoring for the evaluation of the health of structures. Such case studies are required to further advance research in this field for eventual large scale adoption and improved infrastructure management.

The following section outlines how the stated objectives are to be addressed in this document.

\subsection{Thesis Outline}

In Chapter 2, the operational modal analysis (OMA) techniques are presented. Based on previous research and comparison studies, a method that has demonstrated its abilities to successfully and robustly identify the modal properties of in-operation civil engineering structures was improved to align with the stated objectives of automation and reduction of computational uncertainties. Two significant contributions related to the automation of OMA and to the temporal tracking of modes are also described in this chapter.

Chapter 3 describes the Confederation monitoring project. The original monitoring system is presented with a focus on the vibration instrumentation. The previous works by the various research teams on this project since the bridge opening in 1997 are discussed in Section 3.3. These discussions help to situate the contributions presented in this thesis with respect to previous work on this important monitoring project.

A discussion on the implications of the recent vibration instrumentation renewal undertaken on the Confederation Bridge is included in Chapter 4. To accomplish the goals previously stated, much of the computational processes must be automated, efficient, robust and reliable. It was found that the existing tools developed more 
than 15 years ago for research and monitoring required significant updating to accommodate (and to better manage) the large amounts of data and to make use of the advances in instrumentation and processing capabilities. The redevelopment of the specialized platform for VBSHM is described in Chapter 5. This extensive software renewal constitutes one of the author's original contributions to the Confederation Bridge monitoring project.

With over 20 years of historical vibration measurements, the Confederation Bridge's vibration monitoring project represents a unique opportunity to study large quantities of real vibration data with all its uncertainties and noise. Chapter 6 presents the compiling of a historical database of processed vibration data that contributes to furthering the understanding of the vibration characteristics and their use in the condition assessment of this important structure. This represents the processing and analysis of over 250000 logger files, 40000 hours of recording and $28 \mathrm{~TB}$ of raw and processed data collected since 1998.

In Chapter 7, a statistical approach to the analysis of operational modal estimates is presented. Successful regression and filtering techniques are applied to the OMA results from approximately 4000 datasets. A novel technique using residual analysis and change detection is presented and is another of the author's original contribution.

Chapter 8 presents the use of vibration responses for the health monitoring of bridge components such as light poles, bridge bearings and external post-tensioning cables. Successful practical applications such as those presented in Chapter 8 are required to showcase the potential of these advanced VBSHM techniques in providing actionable engineering information to bridge operators and infrastructure managers. 


\section{Chapter 2}

\section{Advanced Operational Modal Analysis}

Despite being a relatively new field, operational modal analysis (OMA) has attracted significant attention in the research community and has become more viable especially where traditional experimental modal analysis (EMA) techniques cannot be used. For large-scale civil structures like bridges, it is impractical to carry out forced vibration measurements which have been the common practices for EMA and considered as the most reliable means for vibration-based evaluation of mechanical systems. Consequently, operational loadings are relied upon as the input excitations in the dynamic response measurements of bridges. Since it is practically unfeasible to measure the operational forces, output-only system identification (or operational modal analysis) techniques are required in the analysis of such vibration response data. Recent advances in the field of OMA include automation and mode tracking [17-19].

The reader is directed to the following references for a critical review and comparison study of different state-of-the art system identification techniques: [20-23].

Among the different system identification techniques proposed for civil engineering monitoring applications, the stochastic subspace identification (SSI) method has been found to be a reliable OMA technique which compares favorably to other available 
methodologies. A summary of this well documented method is provided in the following section $[20,22,24]$.

\subsection{Stochastic Subspace Identification (SSI)}

The SSI method, also known as Covariance Block Hankel Matrix method (CBHM) [25], is a robust output-only identification technique that is similar to the Eigensystem Realization Algorithm with Data Correlations (ERA-DC) [26] except that the ERA-DC method uses data correlations of the system impulse responses, which are estimated from both input and output measurements, instead of using data correlations of the output-only responses as in the SSI method. The SSI method described in this section, and its variant described in Section 2.2, are the OMA techniques used in the studies presented in this thesis.

The dynamic behaviour of physical systems, such as civil engineering structures, is traditionally modeled through a discrete-time approximation, which is represented by the following matrix equation of motion:

$$
\mathbf{m} \ddot{\mathbf{u}}(t)+\mathbf{c} \dot{\mathbf{u}}(t)+\mathbf{k u}(t)=\mathbf{f}(t)=\mathbf{r p}(t)
$$

where $\ddot{\mathbf{u}}(t), \dot{\mathbf{u}}(t)$ and $\mathbf{u}(t)$ are the $n \times 1$ acceleration, velocity and displacement vectors of the $n$ degree of freedom (DOF) model, and where $\mathbf{m}, \mathbf{c}$, and $\mathbf{k}$ are the $n \times n$ mass, viscous damping, and stiffness matrices, respectively, $\mathbf{f}$ is an $n \times 1$ load vector, $\mathbf{r}$ is an $n \times m$ location coefficient that relates the locations of the $m$ inputs with the response degrees-of-freedom of the system model, and $\mathbf{p}$ is an $m \times 1$ vector describing the $m$ inputs or excitations. For system identification of discretely-sampled responses, it is often more convenient to reformulate the model into a discrete-time state-space model. The structural dynamics and the data observed from it are represented by 
the following discrete-time stochastic state-space modeling:

$$
\begin{gathered}
\mathbf{x}_{k+1}=\mathbf{A} \mathbf{x}_{k}+\mathbf{w}_{k} \\
\mathbf{y}_{k}=\mathbf{C} \mathbf{x}_{k}+\mathbf{v}_{k}
\end{gathered}
$$

where $\mathbf{x}_{k}$ is an $n \times 1$ vector describing the system state (displacement and velocity) at the instant $k \Delta t, \Delta t$ is the sampling interval, $\mathbf{y}_{k}$ represents the measurements or outputs, $\mathbf{A}$ is the discrete state matrix containing the system eigenvalues and describing the dynamics of the system, $\mathbf{C}$ is the discrete output matrix that relates the system state to the measured outputs, while $\mathbf{w}_{k}$ and $\mathbf{v}_{k}$ account for the immeasurable inputs, which are assumed to be white, zero-mean stochastic processes, independent of the state vector. Measured outputs are typically accelerations, but may also be displacements or velocities. For typical bridge monitoring projects the measured outputs are the acceleration responses obtained from accelerometers.

Under the assumptions of stationarity of the state vector and whiteness of the stochastic input terms it can be shown that output correlation matrices $\mathbf{R}_{i}$, defined as $\mathbf{R}_{i}=\mathbf{E}\left[\mathbf{y}_{k+i} \mathbf{y}_{k}^{T}\right]$, where $\mathbf{E}$ is the expected value operator, can be factorized into a triplet containing the system matrices as follows,

$$
\mathbf{R}_{i}=\mathbf{C A}^{i-1} \mathbf{G}
$$

where $\mathbf{G}$ is defined as $\mathbf{G}=\mathbf{E}\left[\mathbf{x}_{k+1} \mathbf{y}_{k}^{T}\right]$. Equation 2.3, which is analogous to the factorization of impulse response functions in input-output modal analysis, is the cornerstone of the stochastic subspace method. By taking advantage of this factorization property, correlation matrices are first estimated from the data and then decomposed to obtain the system matrices, ultimately yielding the eigenvalues and eigenvectors, as is briefly summarized below. 
Based on an ergodicity assumption, estimates of the output correlation matrices can be obtained as

$$
\hat{\mathbf{R}}_{i}=\frac{1}{N} \sum_{k=0}^{N-1} \mathbf{y}_{k+i} \mathbf{y}_{k}^{T}
$$

where $N$ is the number of samples. Data correlations offer the advantages of significantly compressing the data while preserving the modal information and eliminating uncorrelated noise.

In experimental modal analysis applications, typically many sensors are used to measure the response of the structural system in order to obtain the mode shapes with a reasonable spatial resolution. As a result, there is usually some redundancy in the data because all sensors carry essentially the same information regarding the frequency and damping ratios, except if they are located at modal nodes. From this observation, computations are substantially reduced by computing data correlations with respect to just a small subset of reference sensor channels. Hence the "subspace" in "stochastic subspace identification".

Reference output correlation matrices computed at different time lags are gathered into a block Toeplitz matrix as follows

$$
\mathbf{T}_{1 \mid i}^{r e f}=\left[\begin{array}{cccc}
\mathbf{R}_{i}^{r e f} & \mathbf{R}_{i-1}^{r e f} & \cdots & \mathbf{R}_{1}^{r e f} \\
\mathbf{R}_{i+1}^{r e f} & \mathbf{R}_{i}^{r e f} & \cdots & \mathbf{R}_{2}^{r e f} \\
\vdots & \vdots & & \vdots \\
\mathbf{R}_{2 i-1}^{r e f} & \mathbf{R}_{2 i-2}^{r e f} & \cdots & \mathbf{R}_{i}^{r e f}
\end{array}\right]
$$

where $\mathbf{R}_{i}^{r e f}=\mathbf{E}\left[\mathbf{y}_{k+i}\left(\mathbf{y}_{k}^{r e f}\right)^{T}\right]$, and $\mathbf{y}_{k}^{r e f}$ is the $r \times 1$ vector of sampled reference responses at time instant $k, r$ is the number of reference channels, and $i$ is a parameter that defines the portion of the data correlations (i.e. the number of time lags) to be 
used for system identification. Mathematically, this parameter must satisfy the relation $r \times i>n$, where $n$ is the order of the state-space model. Based on Equation (2.3), the block Toeplitz matrix can be expressed as follows :

$$
\mathbf{T}_{1 \mid i}^{r e f}=\left[\begin{array}{c}
\mathbf{C} \\
\mathbf{C A} \\
\vdots \\
\mathbf{C A}^{i-1}
\end{array}\right]\left[\begin{array}{llll}
\mathbf{A}^{i-1} \mathbf{G}^{r e f} & \cdots & \mathbf{A G}^{r e f} & \mathbf{G}^{r e f}
\end{array}\right]=\mathbf{O}_{i} \Gamma_{i}^{r e f}
$$

These two factors $\mathbf{O}_{i}$ and $\boldsymbol{\Gamma}_{i}^{r e f}$ are known as the extended observability and reversed extended stochastic controllability matrices, respectively. Estimates of these two factors are obtained via the Singular Value Decomposition (SVD) of the block Toeplitz matrix:

$$
\mathbf{T}_{1 \mid i}^{r e f}=\mathbf{U S V}^{T}=\left[\begin{array}{ll}
\mathbf{U}_{1} & \mathbf{U}_{2}
\end{array}\right]\left[\begin{array}{ll}
\mathbf{S}_{1} & \mathbf{0} \\
\mathbf{0} & \mathbf{0}
\end{array}\right]\left[\begin{array}{c}
\mathbf{V}_{1}^{T} \\
\mathbf{V}_{2}^{T}
\end{array}\right]=\mathbf{U}_{1} \mathbf{S}_{1} \mathbf{V}_{1}^{T}
$$

where $\mathbf{S}_{1}$ is a diagonal matrix containing singular values in descending order. Using equations 2.6 and 2.7 the following internally-balanced realization may be obtained:

$$
\begin{gathered}
\mathbf{O}_{i}=\mathbf{U}_{1} \mathbf{S}_{1}^{\frac{1}{2}} \\
\Gamma_{i}^{r e f}=\mathbf{S}_{1}^{\frac{1}{2}} \mathbf{V}_{1}^{T}
\end{gathered}
$$

Since the true model order of the system is typically unknown and difficult to 
determine, solutions of increasing model orders, 1 to $n_{\max }$, are obtained by taking an increasing number of singular values from the SVD to construct the estimates of $\mathbf{O}_{i}$ and $\boldsymbol{\Gamma}_{i}^{r e f}$. At each model order, system matrices $\mathbf{A}$ and $\mathbf{C}$ are extracted from $\mathbf{O}_{i}$ and $\boldsymbol{\Gamma}_{i}^{\text {ref }}$. Then, an eigenvalue decomposition of $\mathbf{A}$ yields the system's complex eigenvalues and vectors as follows:

$$
\mathrm{A}=\boldsymbol{\Psi} \Lambda \boldsymbol{\Psi}^{-1}
$$

where $\boldsymbol{\Psi}$ is a matrix of the complex eigenvectors and $\boldsymbol{\Lambda}$ is a diagonal matrix containing the discrete-time eigenvalues $\mu_{j}$, which are directly related to the continuous time eigenvalues $\lambda_{j}$ that contain the modal frequencies $\omega_{j}$ and damping ratios $\xi_{j}$ :

$$
\lambda_{j}=\frac{\log _{e}\left(\mu_{j}\right)}{\Delta t}=-\xi_{j} \omega_{j}+i \sqrt{1-\xi_{j}^{2}} \omega_{j}
$$

It should be noted that the frequency resolution of the modal frequencies identified by the SSI method is not limited by the duration of the data recordings as in the traditional frequency-domain techniques. Finally, the observed part of the mode shapes is obtained from the eigenvectors as follows:

$$
\mathrm{V}=\mathbf{C} \Psi
$$

Although theoretically the order of the system $n$ can be determined by looking at the number of non-zero singular values of the SVD, when analyzing the data from real structures the model order is typically masked by the noise in the data. The noise may arise from a combination of the following sources :

- modeling approximations;

- measurement inaccuracies; 
- the use of finite datasets to compute estimates of the correlations;

- non-stationarity and non-linearity behavior in the data.

To overcome the problem of the unknown and hidden system order, a technique known as stabilization diagrams is typically employed. In this technique, the set of models of different order $1<n<n_{\max }$ identified by taking a different number of singular values and vectors from the SVD are presented in a diagram where the distinction between "true" or stable system solutions and "spurious" solutions caused by the noise becomes apparent to the analyst. A typical stabilization diagram is shown in Figure 2.1. In this figure, "4" stands for a stable solution pole, "3" stands for

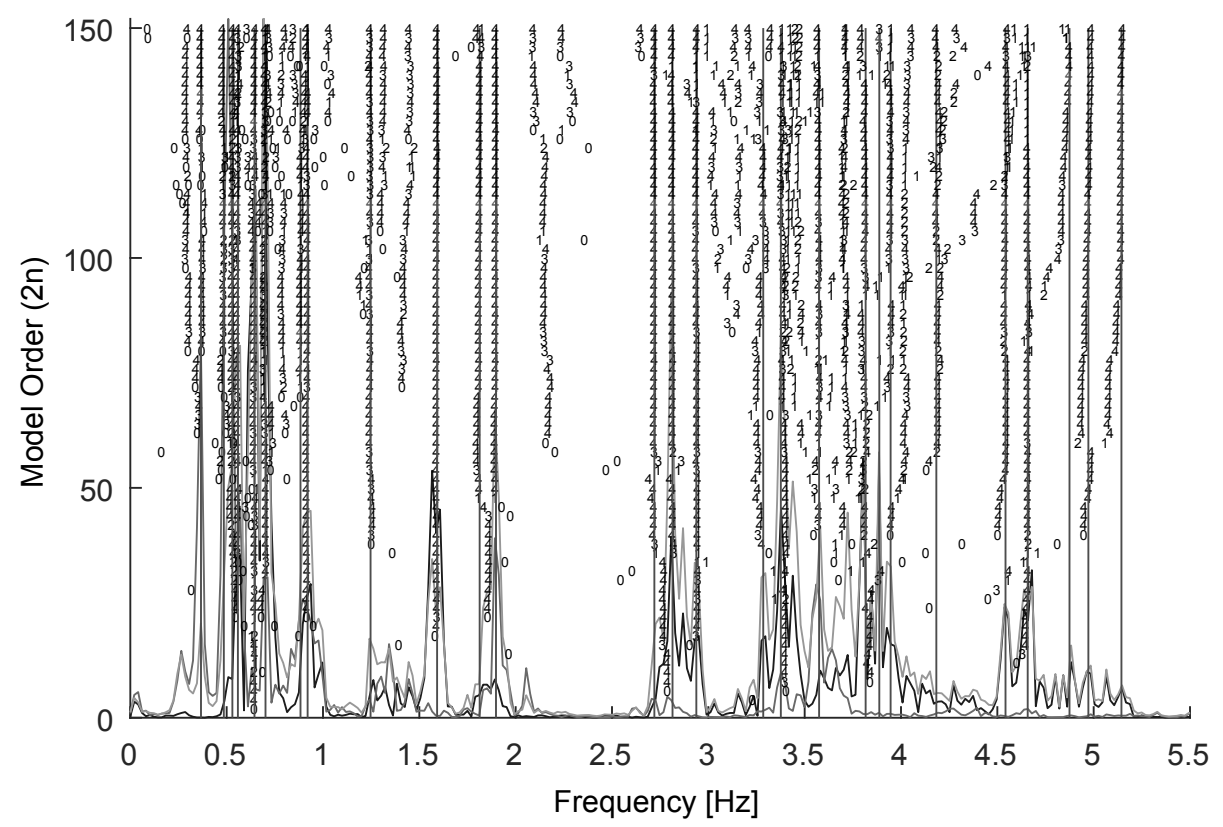

Figure 2.1: Typical stabilization plot showing stability of poles.

frequency and mode stable, "2" stands for frequency and damping stable, "1" stands for frequency only stable and "0" stands for new frequency. For a more in-depth look at the SSI method the reader may refer to the following references : [20,27]. 


\subsection{Automated In-line Full Space Identification (AI-FSI)}

Significant modifications and improvements to the Stochastic subspace identification method (described in Section 2.1) are proposed in this section and are implemented in the processing and analysis platform described in Chapter 5 with the corresponding OMA results presented in Chapters 6 and 7. A new Automated In-line Full Space Identification (AI-FSI) method is described in this section to address the needs associated with long-term continuous vibration-based structural health monitoring, such as the automation of all data processing and analysis operations (including modal parameter estimations and mode tracking), and the need for high quality OMA results, with minimal measurement and computational uncertainties and variability.

The original SSI algorithm, developed more than 25 years ago, had to deal with a major constraint, that of computer processing limits. Many operations were in fact limited by the computer processing capabilities. Some data operations were originally implemented with the only objective of reducing computational requirements all the while introducing possible sources of uncertainties and variabilities in the results. With the proposed improvements to the SSI technique described below and with the improved parameter estimates described in Section 2.2.2, it is believed that the quality of the operational modal parameters thus extracted will be of high quality with minimal computational noise.

The automation of the modal parameter estimations from the stabilization diagrams is described in Section 2.2.3 and is an integral component of the proposed AI-FSI technique along with the novel mode tracking algorithm described in Section 2.2.4. 


\subsubsection{Full Space Identification}

The SSI technique described in Section 2.1, used only a small subset of reference sensors (instead of all available sensors) for the computation of data correlations to reduce computing time. This can have significant impact on the quality and completeness of the identification results. In the proposed method, the complete block Toeplitz matrix is calculated with the output correlation matrices using all available sensors. Equation (2.13) shows the full output correlation matrices.

$$
\mathbf{R}_{i}=\mathbf{E}\left[\mathbf{y}_{k+i} \mathbf{y}_{k}^{T}\right]
$$

where $\mathbf{y}_{k}$ and $\mathbf{y}_{k+i}$ are the $s \times 1$ vectors of all sampled responses at time instants $k$ and $k+i$ respectively; where $s$ is the total number of available channels, and $i$ defines the portion of the data correlations (i.e. the number of time lags) used for system identification. This parameter must satisfy the relation $s \times i>n$, where $n$ is the order of the state-space model. By using all available sensors, this relation is easier to satisfy and permits a wider variety of parameter estimates to be considered. The improved parameter estimates are described in Section 2.2.2.

The full output correlation matrices computed at different time lags are then gathered into the full block Toeplitz matrix as in Equation (2.14).

$$
\mathbf{T}_{1 \mid i}=\left[\begin{array}{cccc}
\mathbf{R}_{i} & \mathbf{R}_{i-1} & \cdots & \mathbf{R}_{1} \\
\mathbf{R}_{i+1} & \mathbf{R}_{i} & \cdots & \mathbf{R}_{2} \\
\vdots & \vdots & & \vdots \\
\mathbf{R}_{2 i-1} & \mathbf{R}_{2 i-2} & \cdots & \mathbf{R}_{i}
\end{array}\right]
$$


Based on Equation (2.3), the block Toeplitz matrix can be expressed as in Equation $(2.15)$ :

$$
\mathbf{T}_{1 \mid i}=\left[\begin{array}{c}
\mathbf{C} \\
\mathbf{C A} \\
\vdots \\
\mathbf{C A}^{i-1}
\end{array}\right]\left[\begin{array}{llll}
\mathbf{A}^{i-1} \mathbf{G} & \cdots & \mathbf{A G} & \mathbf{G}
\end{array}\right]=\mathbf{O}_{i} \boldsymbol{\Gamma}_{i}
$$

Estimates of the extended observability and reversed extended stochastic controllability matrices are then obtained via the Singular Value Decomposition (SVD) of the block Toeplitz matrix as described in Section 2.1. The remaining data operations (the extraction of system matrices $\mathbf{A}$ and $\mathbf{C}$, and the eigenvalue decomposition of $\mathbf{A}$ ) are similar to those described in Section 2.1.

In operational modal analysis applications, typically many sensors are used to measure the response of the structural system in order to obtain the mode shapes with a reasonable spatial resolution. By using all available information, the quality of modal identifications (especially in regards to mode shapes) is maximized, and the computational noise is minimized. As a result, computation loads are substantially increased since many more correlation calculations are performed. However, with proper code implementation of the AI-FSI algorithm to fully exploit the parallel computing capabilities of modern computers, the computing time can be kept at a level that permits in-line analysis of the monitoring data for eventual real-time applications. 


\subsubsection{Improved parameter estimations}

In previous operational modal analysis studies using the SSI technique a maximum model order $n_{\max }=150$ to 200 is used in the construction of the stabilization diagrams to allow for a clear visualization of stabilized trends [22,27-30]. The actual

model order of the physical system considered is typically lower than this, typically around 40 for the considered bridge structure, but may vary between different datasets depending on the number of modes being excited. The number of time lags of the data correlations that was typically used in the referenced studies for the construction of the Toeplitz matrix (Eq. 2.5), which forms the basis of the system identification, was taken as $i=200$ for all datasets analyzed. This value of $i$ not only satisfied the mathematical requirement of $r \times i>n_{\max }$ but also ensured that the data correlations of the Toeplitz matrix span more than a full vibration cycle of the lowest mode. This criterion is important as it ensures there is enough information from the lowest modes in the data correlations to ensure enough accuracy in their identification. Also, in these studies, the data correlations were computed with respect to a subset of reference sensors in order to reduce the computational burden. Typically, six sensors (out of the available 58 sensors installed on the referenced structure) were used. The reference sensors selected were deemed suitable because of their relatively high response amplitudes and because their locations do not coincide with modal nodes of any of the important vibration modes. However the relatively low number of chosen reference channels could explain the less accurate estimation of mode shapes in the previous studies.

According to theory any value of $i$ represented by an integer larger than the ratio between the model order $n$ and the number of measured outputs $s$ can be chosen. Engineering practice, however, has shown that if the system has a low fundamental frequency with respect to the sampling frequency and the number of time lags $i$ is set 
too low, only a few samples of that modal response are included in the block matrix and, as a consequence, the corresponding modal parameters are not well identified [31]. In [32] it is suggested to set the value of the number of block rows according to Equation (2.16).

$$
i \geq \frac{f_{s}}{2 f_{0}}
$$

where $f_{s}$ is the sampling frequency and $f_{0}$ is the lowest frequency of interest. In the operational modal analysis described in this thesis a time lag $i=200$ is used since the lowest identified mode of vibration for the monitored structure is $0.34 \mathrm{~Hz}$.

The maximum order of the model $n_{\max }$ should equal, according to theory, twice the number of modes that are needed to accurately describe the structural response. However, it is often necessary to consider much larger values in order to detect weakly excited modes [18]. For the OMA results presented in this thesis a value of $n_{\max }=400$ was considered. Again, with parallel computing, processing time with these relatively high parameter estimates was kept to a reasonable level.

\subsubsection{Automated modal parameter estimations (MPE)}

A custom clustering algorithm is developed and integrated in the AI-FSI algorithm for the automation of the modal parameter estimations carried out in this thesis. Reynders et al. [17] identify five target criteria that should be respected when considering a fully-automated approach for the interpretation of stabilization diagrams:

- The method should not rely on more than one dataset, or on prior estimates;

- The method should be as physically intuitive as possible and be similar to the manual analysis;

- The method should produce similar results as in a manual analysis;

- The method should work in an OMA framework and with any parametric system 
identification algorithm that produces a stabilization diagram.

- The method should not contain parameters that need to be specified or tuned by the user.

Several automated OMA techniques employ clustering algorithms that satisfy all (or most) of these criterias $[18,19,33]$. The clustering algorithm described in this section is similar to these methods but has been developed independently and satisfies all the target criteria imposed by Reynders et al. [17]. It should be made clear that the algorithm described below is used for modal parameter estimations (MPE) on a single dataset of measured signals. A distinction needs to be made between MPE and mode tracking, which is the tracking of a single mode through time by repeated MPE [17]. A separate algorithm for mode tracking has been developed and implemented in this thesis and is described in Section 2.2.4.

The clustering algorithm for MPE works recursively on the solutions of the system identification algorithm described in Section 2.2.1. Starting at the highest model order $n_{\max }$ all solutions modes are used to initially seed the clusters. If $n_{\max }$ was set to 400, then there are 400 different solution poles available for initial seeding. For each subsequent model order in descending order the solution poles are added into the seeded cluster bins according to modal parameter match (mode shape, frequency and damping ratios). Mode shape comparisons are calculated using the modal assurance criterion shown in Equation (2.17) [34]:

$$
\operatorname{MAC}\left(\boldsymbol{\Psi}_{i}, \boldsymbol{\Psi}_{j}\right)=\frac{\left|\boldsymbol{\Psi}_{i}^{*} \boldsymbol{\Psi}_{j}\right|^{2}}{\left(\boldsymbol{\Psi}_{i}^{*} \boldsymbol{\Psi}_{i}\right)\left(\boldsymbol{\Psi}_{j}^{*} \boldsymbol{\Psi}_{j}\right)}
$$

where $\Psi_{i}$ and $\Psi_{j}$ are two different eigen-vectors (mode-shapes), the symbol "**" indicates complex conjugate transpose. An MAC of 1 indicates perfect correlation of the mode-shapes while an MAC of 0 indicates no correlation. Relatively low tolerances 
are used for clustering in order to ensure proper mode identification, and to ensure that each cluster represents a single true structural mode. Frequency tolerances of 0.01 ; mode shape tolerance of 0.05 and damping tolerance of 0.15 are used for proper mode identifications. If a modal match is not found at the current model order, the algorithm seeds a new cluster with in the solution pole which is then used for mode comparisons in the subsequent iterations.

By setting the maximum order of the model $n_{\max }$ relatively high (as indicated in Section 2.2.2), many spurious, non-physical solutions are generated by the identification algorithm. With clustering, the spurious numerical solutions (which occur randomly, are not properly sorted and are distributed uniformly over the frequency spectrum) are essentially filtered out by the algorithm. Only clusters that contain consistently-identified solution poles through the model orders are kept as true mode solutions. At set intervals, the algorithm flushes out the spurious solutions which are represented by small cluster sizes. A large cluster size indicates good mode consistency through the model orders. The threshold on cluster size specified to identify true modes from spurious modes is arbitrary and depends on the requirements of mode tracking (Section 2.2.4) but must not be set too low to avoid including non-physical solutions. A threshold of $n_{\max } / 8$ is used here to validate consistently-identified modes. The size of each cluster provides a measure of the strength of identification and is stored as an operational parameter by the algorithm. This parameter, which also serves as an indication of mode quality, is considered as a linear regression predictor in Chapter 7. 


\subsubsection{Mode tracking by recursive reduction of the MAC ma- trix}

Although similar principles are present, tracking the evolution of a single mode through time from multiple MPE is much more complex than modal parameter estimations of a single dataset [17]. Difficulties lie in the fact that all modal parameters vary due to environmental and operational influences. Literature suggests to use hard-validation criteria to sort out complex modes, which is not always applicable in the cases of closely-spaced modes, as the mode-shape may lie in a subspace that is spanned by two corresponding modes shapes of closely-spaced modes [19,35]. Reynders suggests using baseline modal parameters to start an automated modal tracking algorithm [17].

Described here is a novel two-stage approach to the grouping of modes proposed for long-term modal tracking. In the first stage, the dominant modal frequencies are identified based on their frequency of identification as determined from histogram calculations of multiple MPE. These dominant modes are used to start the modal tracking algorithm. In order to confirm proper mode pairing of modal frequency and mode shape, a clustering algorithm is employed to group modes around the identified dominant modal frequencies. A relatively high frequency range $( \pm 20 \%)$ is considered in this first step so as not to hide potential frequency variations due to damage or deterioration. It is possible and not uncommon that the identified clusters may overlap during this first clustering step. Also, by grouping modes according to frequency only, there is also a high likelihood that several closely-spaced modes are grouped together in a single cluster. These are typical scenarios that are properly handled by the algorithm.

In the second stage of the mode tracking algorithm, inside each cluster, the modal 
assurance criteria of Equation (2.17) is calculated to ensure that compared modes are indeed the same mode and not separate closely-spaced modes [36]. Each mode shape is compared to all the other mode shapes within each cluster by calculating the MAC matrix. By averaging across a single dimension of the MAC matrix we obtain average MAC values for each of the identified modes. The algorithm then recursively reduces the MAC matrix by excluding the modes with average MAC values below a minimum threshold. For each subsequent iterations, the threshold is incrementally increased until the minimum MAC value for a single mode inside the sub-cluster is greater than 0.75 . A true mode sub-cluster is therefore confirmed when the smallest average MAC value of a mode shape with all other mode shapes inside the subcluster is greater than 0.75 . This threshold is chosen so as not to exclude modes with variations of mode shapes due to environmental or operation influences or due to damage or deterioration. The excluded modes are gathered and passed through the recursive MAC matrix reduction algorithm again in order to find other true mode groups (in the case of closely-spaced modes). This step is repeated until there are no more modes to group. The result of this second stage are sub-clusters of true modes inside each dominant frequency clusters. Theses sub-clusters are assumed to be true grouped modes.

It should be noted that a relatively high computational load is required for the repeated calculation of the MAC matrix. Optimization of computation steps is possible, however with proper code implementation, parallel processing kept computing times reasonable, even without code optimization.

OMA results using the AI-FSI algorithm are presented in Chapters 6 and 7 


\section{Chapter 3}

\section{Confederation Bridge Vibration Monitoring Project}

The Confederation Bridge monitoring project is one of only a few documented longterm vibration-based monitoring projects in the world. The project provides a unique opportunity to study real bridge responses from data with presence of real world uncertainties and noise. With over 20 years of captured vibration data, representing one fifth of the bridge's design service life, the Confederation Bridge monitoring project is a tremendous resource for testing and implementing various state-of-the art system identification and condition assessment techniques.

Less documented are long-term monitoring projects that have undergone a significant instrumentation renewal process. It is well understood that the service life of a monitoring system is significantly less than that of the monitored civil structure. This is due to various factors, the most significant are failures: failures of sensors, failures of data loggers, communication failures, etc. Other factors include technological advances which make the installed technology obsolete. In some cases, to monitor the structure throughout its service life, a monitoring system may need to be renewed multiple times. This produces multi-state and multi-scaled vibration response data, 
where the number of sensors and the sampling parameters (sampling rate, signal resolution, dataset duration, etc.) used for analysis are rarely constant in time. What are the implications of instrumentation renewals on an established monitoring program? Chapter 4 will address this question.

In this chapter, a description of the physical characteristics of the bridge is given, and the main structural components of the Confederation Bridge are presented. The original monitoring system itself is also described with details on the original vibration instrumentation configuration. For details on the new instrumentation configuration, the reader is directed to Chapter 4. An overview of past research at the Ottawa Carleton Bridge Research Institue (OCBRI) is presented in Section 3.3. This should provide context for the work presented in this thesis.

\subsection{Bridge Description}

With 43 main spans of 250 meters each and a 100-year design life, the $12.9 \mathrm{~km}$ long confederation Bridge is the world's longest prestressed concrete box girder bridge built over salt water [37]. Upon completion in the summer of 1997, the Confederation Bridge provides a fixed link between the provinces of New-Brunswick and PrinceEdward Island in eastern Canada as shown in Figure 3.1. Spanning the Northumberland strait between Cape Tormentine NB and Borden PEI, the crossing consists of 21 approach spans, 2 transition spans and 43 main spans at a typical height of $40 \mathrm{~m}$ above the mean sea level (60 $\mathrm{m}$ at the navigation spans) as shown in Figure 3.2.

The main-spans portion of the bridge is comprised of 22 repetitive structural frame modules of $500 \mathrm{~m}$ length each. Each frame module is a $440 \mathrm{~m}$ portal frame made up of a $250 \mathrm{~m}$ center span and two $95 \mathrm{~m}$ overhangs, one on each side of the center span, plus a $60 \mathrm{~m}$ simply supported drop-in expansion span, as shown in Figure 3.3. The 


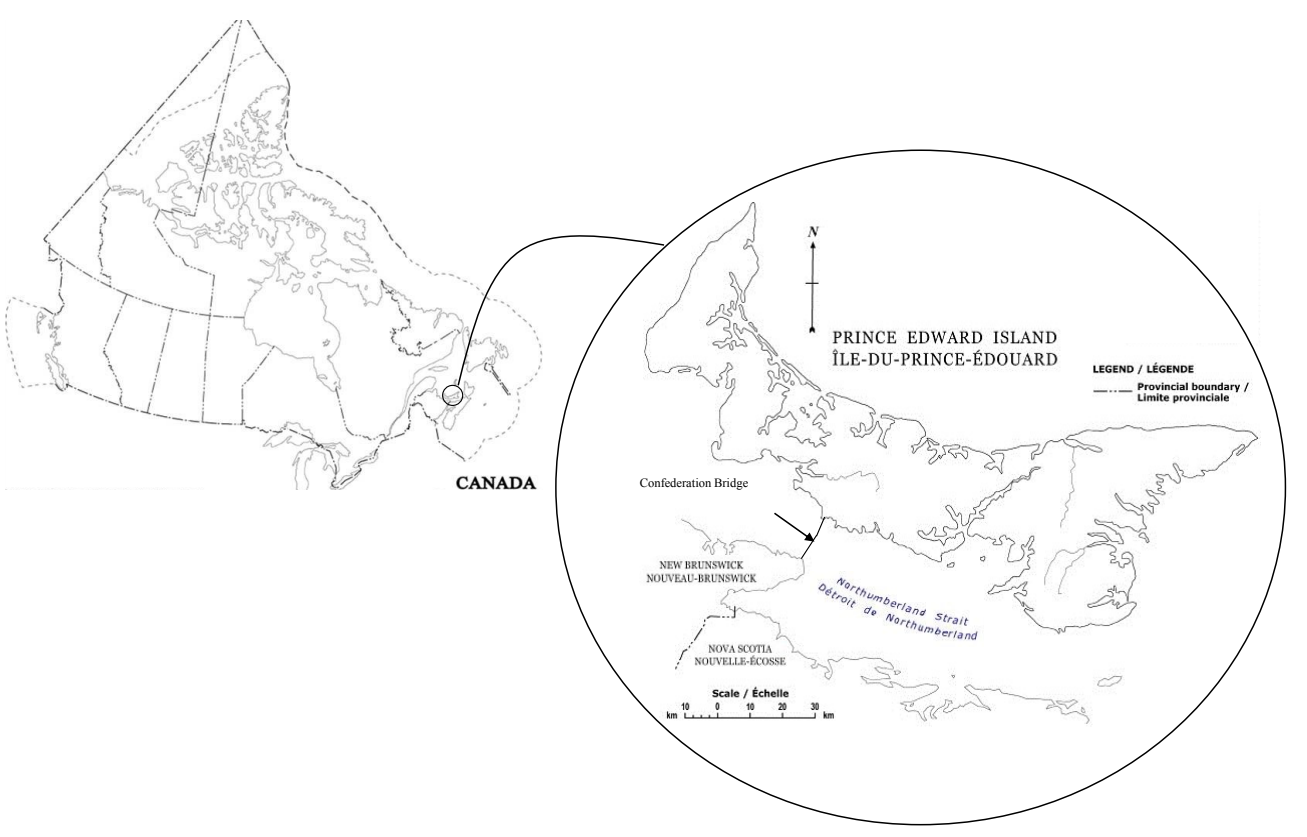

Figure 3.1: Geographical location of the Confederation Bridge [38].

portal frame is constructed of four types of prefabricated component units, pier base, pier shaft, main girder and fixed drop-in span girder. The connections between the different units of the portal frame are made continuous by post tensioning prestressing and grouting. The bridge girders are single trapezoidal cell box girders with section depth varying from $14 \mathrm{~m}$ above the piers to $4.5 \mathrm{~m}$ at mid-span of the drop-in girder, and a cross-section width varying from $5.0 \mathrm{~m}$ at the bottom to $7.0 \mathrm{~m}$ at the top, with $2.5 \mathrm{~m}$ deck overhangs.

The prestressing is assured by both internally-bonded and external post-tensioned

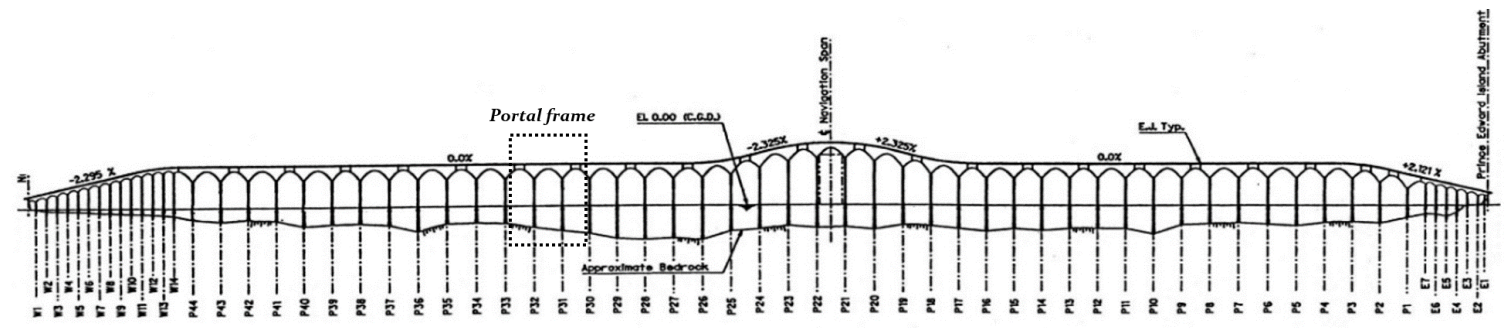

Figure 3.2: Confederation Bridge elevation. 


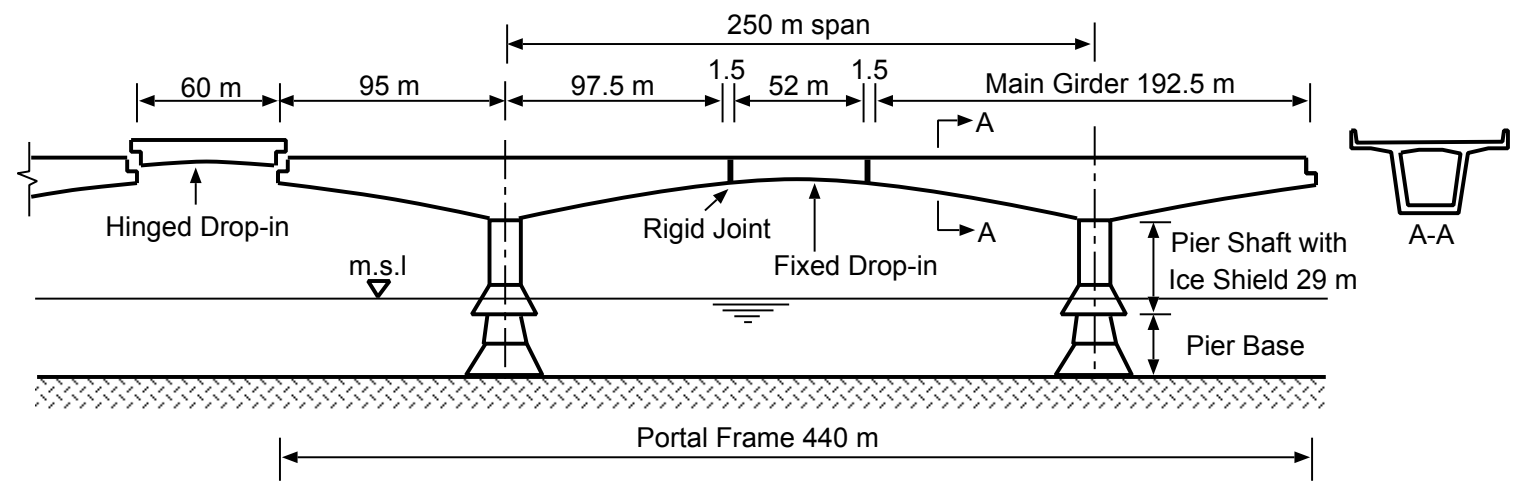

Figure 3.3: Confederation Bridge components.

prestressing tendons. Each pier consists of a hollow shaft with an octagonal shape in the upper portion, a conical ice shield at mean sea level, and a conical shell with a ring-shaped footing on the sea bed. With its numerous long spans and a design life of 100 years (which was twice the code required design life for typical bridges at the time), the design of the Confederation Bridge was unique and was not covered by any code or standard in the world [37]. It was for these reasons, and the fact that the bridge was expected to operate under very severe environmental conditions over its very long service life, that it was essential to obtain a comprehensive and detailed understanding of the bridge behavior in operation.

\subsection{Original Monitoring System}

A comprehensive long-term remote monitoring system on the Confederation Bridge has been in operation since the bridge opening to collect data and information about its behaviour and performance. The monitoring system which was commissioned in December 1997 measures and records both environmental and bridge response data related to wind, ice forces, short and long-term deflections, thermal effects, traffic loading, corrosion and vibration responses. All instruments incorporated in 
the bridge structure are located in two adjacent spans, a rigid frame and a drop-in simply supported span covering a one kilometer stretch of the bridge in the deep-sea part of the Northumberland Strait with some additional wind measurements being also taken at the highest elevation of the navigation span.

\subsubsection{Vibration monitoring}

The dynamic monitoring system is dedicated to the measurement of the ambient vibration responses of the bridge caused by sources of dynamic excitations, including wind, traffic, ice loads and earthquakes. The vibration instrumentation comprises 76 accelerometers distributed around a typical structural frame module, as shown in Figure 3.4. The response behavior observed in the instrumented segment of the bridge is considered representative of the behavior of the main span portion of the structure. Vibration responses of the bridge girders are measured in the vertical and transverse directions as shown in Figure 3.4. This setup facilitates the recovery of vertical bending, transverse bending and torsional vibration modes of the bridge superstructure. Detailed descriptions of the original installed system can be found in $[37,39]$.

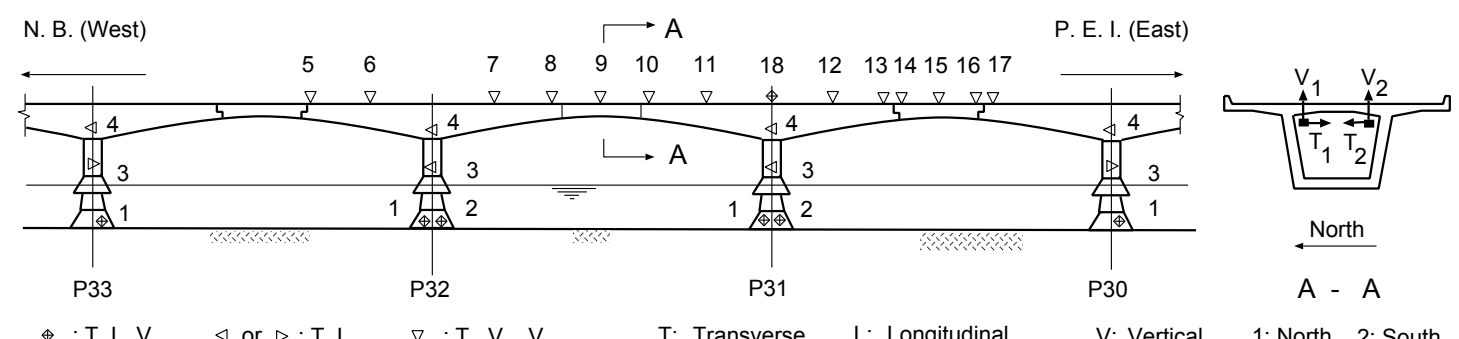

Figure 3.4: Accelerometer locations on the Confederation Bridge.

From December 2012 to March 2014, the monitoring system underwent an instrumentation renewal. Having been in operation for approximately 15 years, the 
monitoring system was showing signs of reaching the end of its service life. Sensors were failing at greater rate and data acquisition and transmission issues were more frequent. During this monitoring system renewal on the instrumented portion of the Confederation Bridge, all accelerometers were replaced and new data loggers were installed. A detailed description of the renewal and its implication on data integrity is provided in Chapter 4. The vibration sensors used in the original monitoring system included both piezo-electric accelerometers and servo accelerometers. Locations of the sensors are listed in Table 3.1 and shown in Figure 3.4.

Table 3.1: Sensor location with make and model of accelerometers.

\begin{tabular}{|c|c|c|c|c|c|c|c|c|}
\hline \multirow{2}{*}{$\begin{array}{l}\text { Pier (Data } \\
\text { Logger } \\
\text { Number) }\end{array}$} & \multirow{2}{*}{$\begin{array}{l}\text { Loca- } \\
\text { tion }\end{array}$} & \multicolumn{5}{|c|}{ Original channel numbering inside data logger tables } & \multicolumn{2}{|c|}{ Original sensors } \\
\hline & & $\mathrm{T} 1$ & $\mathrm{~T} 2$ & $\mathrm{~L}$ & V1 & $\mathrm{V} 2$ & $\begin{array}{l}\text { Accelerometer } \\
\text { model }\end{array}$ & Scale $\mathrm{V} / \mathrm{g}$ \\
\hline \multirow{5}{*}{ P.30 (No.1) } & 1 & 7 & & 8 & 9 & & PCB 326 A03 & 1.00 \\
\hline & 3 & 12 & & 13 & & & QLC 400 & 2.66 \\
\hline & 4 & 10 & & 11 & & & QLC 400 & 2.66 \\
\hline & 16 & & 3 & & 1 & 2 & PCB 393C & 1.00 \\
\hline & 17 & & 6 & & 4 & 5 & PCB 393C & 1.00 \\
\hline \multirow{11}{*}{ P.31 (No.4) } & 1 & 1 & & 2 & 3 & & PCB 326 A03 & 1.00 \\
\hline & 2 & 4 & & 5 & 6 & & PCB 326 A03 & 1.00 \\
\hline & 3 & 7 & & 8 & & & QA 1200 & 99.0 \\
\hline & 4 & 9 & & 10 & & & QA 1200 & 99.0 \\
\hline & 10 & & 30 & & 29 & 31 & PCB 393C & 1.00 \\
\hline & 11 & & 26 & & 28 & 27 & QLC 400 & 2.66 \\
\hline & 12 & & 15 & & 16 & 14 & PCB 393C & 1.00 \\
\hline & 13 & & 17 & & 19 & 18 & QLC 400 & 2.66 \\
\hline & 14 & & 20 & & 21 & 22 & PCB 393C & 1.00 \\
\hline & 15 & & 23 & & 25 & 24 & QLC 400 & 2.66 \\
\hline & 18 & 11 & & 12 & 13 & & QA 1200 & 99.0 \\
\hline \multirow{9}{*}{ P.32 (No.5) } & 1 & 1 & & 2 & 3 & & PCB 326 A03 & 1.00 \\
\hline & 2 & 4 & & 5 & 6 & & PCB 326 A03 & 1.00 \\
\hline & 3 & 10 & & 11 & & & QA 1200 & 99.0 \\
\hline & 4 & 16 & & 15 & & & QA 1200 & 99.0 \\
\hline & 5 & 24 & & & 23 & 25 & PCB 393C & 1.00 \\
\hline & 6 & 28 & & & 26 & 27 & PCB 393C & 1.00 \\
\hline & 7 & 30 & & & 29 & 31 & PCB 393C & 1.00 \\
\hline & 8 & 32 & & & 34 & 33 & PCB 393C & 1.00 \\
\hline & 9 & 20 & & & 21 & 22 & QLC 400 & 2.66 \\
\hline \multirow{3}{*}{ P.33 (No.6) } & 1 & 1 & & 2 & 3 & & PCB 326 A03 & 1.00 \\
\hline & 3 & 4 & & 5 & & & QLC 400 & 2.66 \\
\hline & 4 & 6 & & 7 & & & QLC 400 & 2.66 \\
\hline
\end{tabular}

\footnotetext{
* Location numbers in this table correspond to the location numbers in Figure 3.4.
} 
The types and specifications of accelerometer sensors used in the original system are listed in Table 3.2. The original piezo-electric accelerometers were PCB Model 393C, and the original servo accelerometers were Allied Signal QLC 400 and QA 1200.

Table 3.2: Specifications for the original accelerometers located on the Confederation Bridge.

\begin{tabular}{|c|c|c|c|}
\hline & $\begin{array}{c}\text { PCB 393C } \\
\text { (piezo-electric) }\end{array}$ & QLC 400 (servo) & QA 1200 (servo) \\
\hline $\begin{array}{l}\text { Principal } \\
\text { location }\end{array}$ & along girder & along girder & $\begin{array}{l}\text { in piers (initially for } \\
\text { ice floe monitoring) }\end{array}$ \\
\hline Sensitivity & $1 \mathrm{~V} / \mathrm{g}$ & $2.66 \mathrm{~V} / \mathrm{g}$ & $99.0 \mathrm{~V} / \mathrm{g}$ \\
\hline Frequency range & $\begin{array}{l}0.01-1200 H z \\
\quad( \pm 10 \%)\end{array}$ & $0-300 \mathrm{~Hz}( \pm 10 \%)$ & $0-300 \mathrm{~Hz}( \pm 10 \%)$ \\
\hline Amplitude range & $\pm 1.5 \mathrm{~g}$ & $\pm 10 g$ & $\pm 25 g$ \\
\hline Resolution & $0.1 m g$ & $0.01 \mathrm{mg}$ & $0.005 \mathrm{mg}$ \\
\hline $\begin{array}{l}\text { Operational } \\
\text { temperature } \\
\text { range }\end{array}$ & $-54^{\circ} \mathrm{C}$ to $93^{\circ} \mathrm{C}$ & $-20^{\circ} \mathrm{C}$ to $71^{\circ} \mathrm{C}$ & $-54^{\circ} \mathrm{C}$ to $107^{\circ} \mathrm{C}$ \\
\hline
\end{tabular}

In the first generation of the vibration monitoring system, the measured analog accelerometer signals were conditioned and filtered for anti-aliasing by 8 -pole $50 \mathrm{~Hz}$ low-pass Bessel filters. Signals were then sampled and digitized by a network of distributed high-speed data loggers before being sent to on-site computers and transmitted back to the research team at Carleton University in Ottawa for data archival and research. The data loggers were CR9000 with 16 Bit 100kHz A/D conversion from Campbell Scientific. The vibration data acquisition system operates in continuous buffered data collection mode, which upon triggering by detection of specific dynamic events, such as heavy traffic signals or high winds, or simply upon user request, store time history responses in data tables in memory. Otherwise, only statistic information determined from the time history data, such as mean, maximum, minimum and standard deviation etc., are stored to establish long-term trends 
in the behavior of the bridge. Triggered data, typically sampled at $125 \mathrm{~Hz}$, are stored temporarily in logger memory until retrieval at specified scheduled intervals from a remote network computer assigned to the control and operation of each logger. The logger computer retrieves the data from the data logger and sends them to a centralized platform where they are made accessible over the internet to researchers at OCBRI located at Carleton University in Ottawa, approximately 1300 kilometers away, for data processing and analysis. Data acquisition software is installed in each logger control computer and can be remotely accessed for system control or maintenance. This software controls the acquisition of data from the sensors connected to the data-loggers and collection of data from the loggers to the control computers. It also permits editing of logger programming code to modify the functioning of the logger. Details of the original monitoring system setup have been described in the references [37,39]. All prior studies summarized in Section 3.3 were carried out using captured vibration response data from the original monitoring system described in this section.

\subsection{Summary of Prior Studies}

This section will provide research context for the current work on the Confederation Bridge monitoring project. It is quite evident that since the bridge opening, much work has been carried out by various members of the Ottawa Carleton Bridge Research Institute (OCBRI) on the vibration monitoring project. As many as three doctoral theses and five master's theses and many more published articles have been written with the Confederation Bridge monitoring project as a background. This section will present, as briefly as possible, the important work which was previously accomplished. This section will provide context and will situate the current work 
presented in this thesis with respect to past work on this vital project.

\subsubsection{Analytical modelling}

A series of Finite Element (FE) bridge models for analytical modal analysis have been developed by various researchers at OCBRI. The FE models help to understand the analytical behavior and response of a representative portion of the Confederation Bridge. The behavior of the complete portal frame containing the instrumented portion of the structure between Piers 31 and 32, as shown in Figure 3.4, is considered representative of the rest of the structure. Several FE models of the bridge have been developed to analyze the dynamic behavior of the Confederation Bridge. Some of these models are described here because of their relevance and importance in subsequent correlations of the analytical results with the field-obtained dynamic properties of the bridge such as those presented in Section 3.3.2.5.

\subsubsection{Analytical model for seismic design (SAP-90 ${ }^{\circledR}$ )}

Jaeger [40] presents a finite element model of the Confederation Bridge for its seismic design analysis. Two and a half portal frames including two drop-in spans are modeled with 821 concentrated mass, 452 beam elements and 395 nodes. Fifty-six separate modes were obtained using the SAP-90 ${ }^{\circledR}$ structural analysis program. The first nine modes range from $0.297 \mathrm{~Hz}$ to $0.443 \mathrm{~Hz}$ (Table 3.3).

\subsubsection{Updated analytical model for calibration (COSMOS $\left.{ }^{\circledR}\right)$}

Lau et al. [41] present three more analytical models: a 3D shell elements model for one complete portal frame of the structure as shown in Figure 3.5, a 3D beam elements model for one complete portal frame in Figure 3.6, and 3D beam element model spanning two complete portal frames including a drop-in span in Figure 3.7. 
The 3D shell element model of a complete portal frame in 30 meters of water depth was developed using the finite element code $\operatorname{COSMOS}^{\circledR}$ (Figure 3.5). The girders and piers are modeled with 8-node quadrilateral thin shell elements and 6node triangular shell elements. The modeling of the interaction of the portal frame with adjacent drop-in spans is considered by adding half the mass of each drop-in span to the supporting overhang in both the transverse and vertical direction and the full mass or no mass in the longitudinal direction, depending if actual connection with drop-in span is hinged or is sliding plate. The nominal value for the modulus of elasticity of concrete is specified for the model as $E_{c}=35 \mathrm{GPa}$. A total of 1292 nodes and 444 elements are included in this FE model. The resulting fundamental frequency is found to be $0.299 \mathrm{~Hz}$ and the first twelve modes range in frequency from $0.299 \mathrm{~Hz}$ to $2.268 \mathrm{~Hz}$ as shown in Table 3.3. The mode-shapes of the first 6 modes are presented in [41].

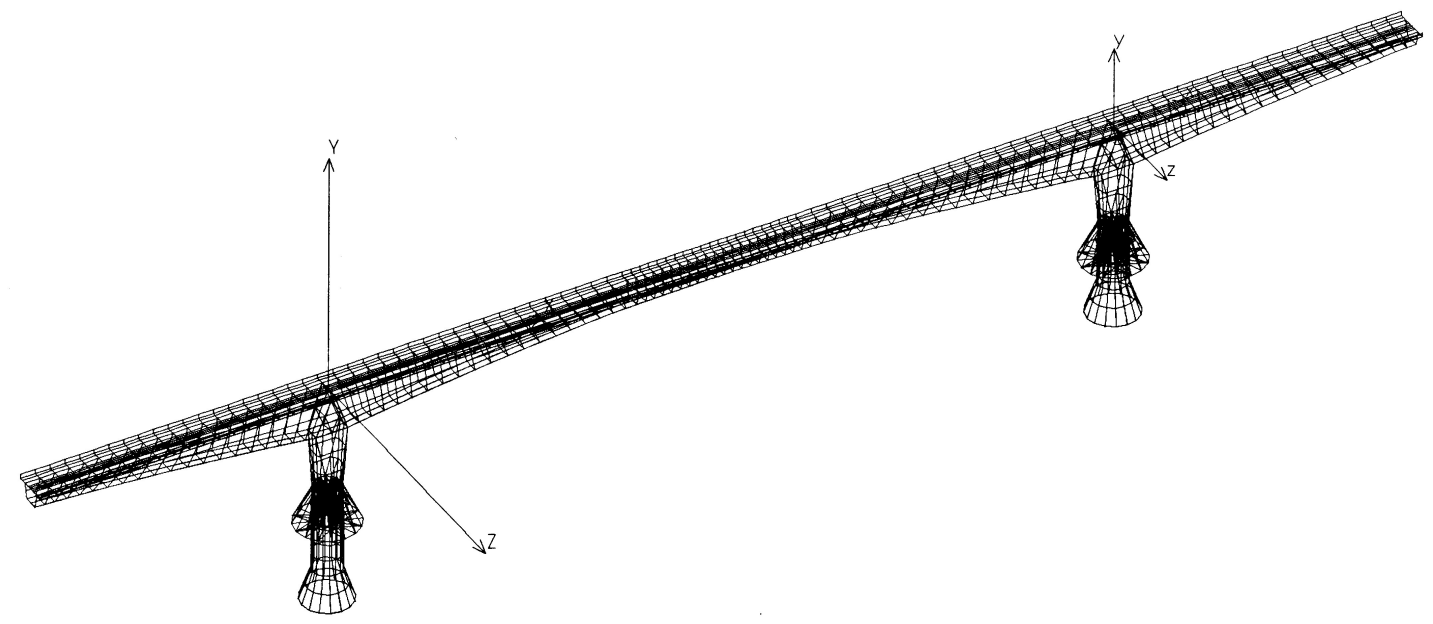

Figure 3.5: 3D shell element model of one typical portal frame of the Confederation Bridge $\left(\operatorname{COSMOS}^{\circledR}\right)[41]$.

This same portal frame is modeled using 3D beam elements in Figure 3.6. The geometrical properties of the variable cross-section beam elements are determined 
from design drawings and calibrated with deformations of the actual member. The interactions with adjacent drop-in spans are modeled by means of lumped mass elements at the two ends of the girder. For each structural component, such as girder, pier shaft, pier base and drop-in span, the mass density is calculated from the weight of the component and its volume in the model. The mass of significant non-structural components, such as road barriers and pavement are included as additional masses to the beam elements. However, the stiffness contribution of these components is neglected. The 3D beam model of one portal frame contains a total of 52 elements; 40 elements comprising the girder and 6 elements comprising each of the two piers. The beam model, with $E_{c}=35 \mathrm{GPa}$, is verified and calibrated using the shell model results. The first twelve natural frequencies obtained from this model range from $0.299 \mathrm{~Hz}$ to $2.306 \mathrm{~Hz}$ as summarized Table 3.3 and have less than $2 \%$ discrepancy with the results from the 3D shell model [41].

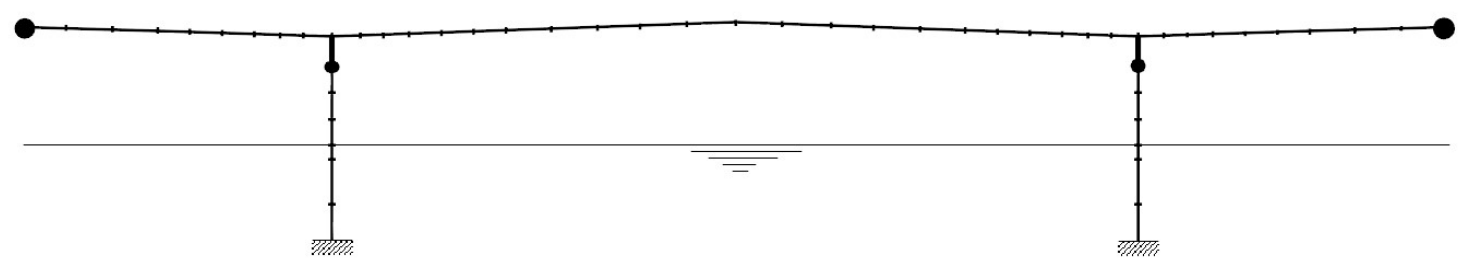

Figure 3.6: Finite element model using 3D beam elements of one typical portal frame of the Confederation Bridge (COSMOS $\left.{ }^{\circledR}\right)[41]$.

To better understand the overall dynamic behavior of the Confederation Bridge two consecutive portal frames plus a drop-in span between piers 29 and 32, of the instrumented portion of the bridge, are modeled using 3D beam elements by the finite element code COSMOS ${ }^{\circledR}$, as shown in Figure 3.7.

The objective of the investigation of two portal frames of the bridge is to study the interaction between adjacent repetitive frame units. The two portal-frame beam model is also verified and calibrated using the shell model results. Eighteen beam 


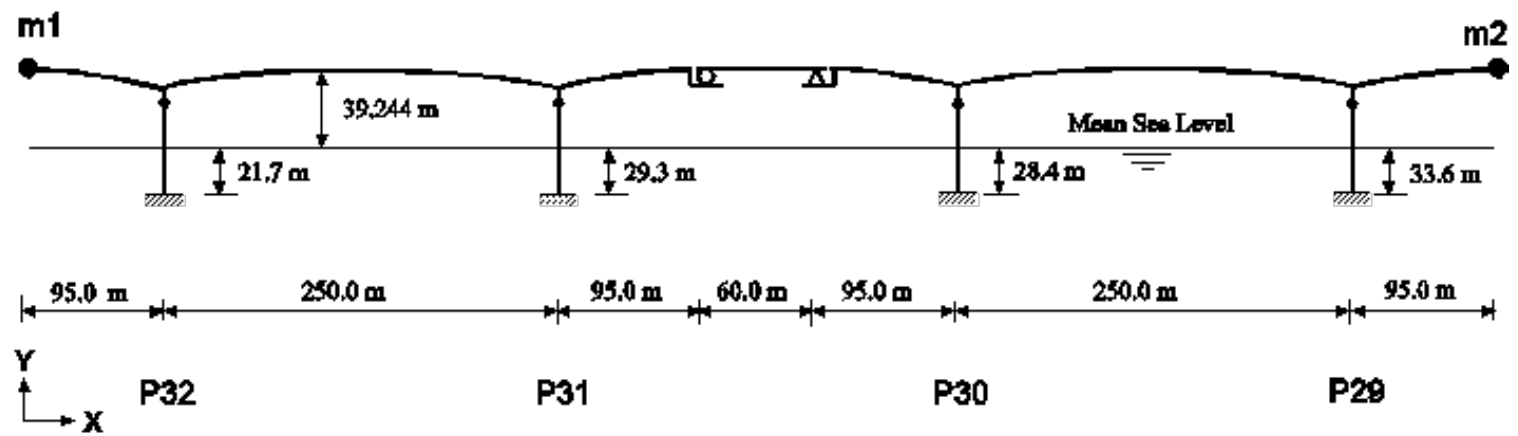

Figure 3.7: Finite element model using 3D beam elements spanning two portal frames of the Confederation Bridge (COSMOS $\left.{ }^{\circledR}\right)$ [41].

elements are used in the modeling of the main girder, four for each drop-in span and seven for each pier. In total, the beam model comprises 112 beam elements, 6 mass elements and 113 nodes. Appropriate end release conditions of the beam elements are adopted to model the hinge and sliding plate connections between the drop-in span and the portal frames. Half of the mass of the drop-in span is added at the boundary node in the transverse and vertical directions for the case of sliding plate bearings, while the entire mass of the drop-in is added to the boundary node in the longitudinal direction in the case of a fixed hinge bearing. The results of analysis presented in the reference by [41] include the 10 first modes ranging in frequency from $0.3 \mathrm{~Hz}$ to $0.67 \mathrm{~Hz}$ summarized in Table 3.3. These models provide the basis for the verification of experimentally-obtained dynamic properties of the Confederation Bridge. They have been and are continually being calibrated with research results obtained from monitoring data analysis.

\subsubsection{Updated finite element model (Abaqus ${ }^{\circledR}$ )}

In 2014, a new FEM model of a complete portal frame of the bridge was created by researchers at OCBRI using the Abaqus ${ }^{\circledR}$ suite of commercial software. The model, described in an unpublished report and shown in Figure 3.8, is composed of 28048 
solid elements of which 27848 are quadratic hexahedrals, 104 are quadratic wedges and 96 quadratic lines with 193676 nodes. A concrete modulus of $E_{c}=43 \mathrm{GPa}$ was specified for this model. The first ten modes obtained are : $0.315 \mathrm{~Hz}, 0.366 \mathrm{~Hz}$, $0.435 \mathrm{~Hz}, 0.491 \mathrm{~Hz}, 0.639 \mathrm{~Hz}, 0.776 \mathrm{~Hz}, 0.911 \mathrm{~Hz}, 1.081 \mathrm{~Hz}, 1.130 \mathrm{~Hz}$ and $1.655 \mathrm{~Hz}$.

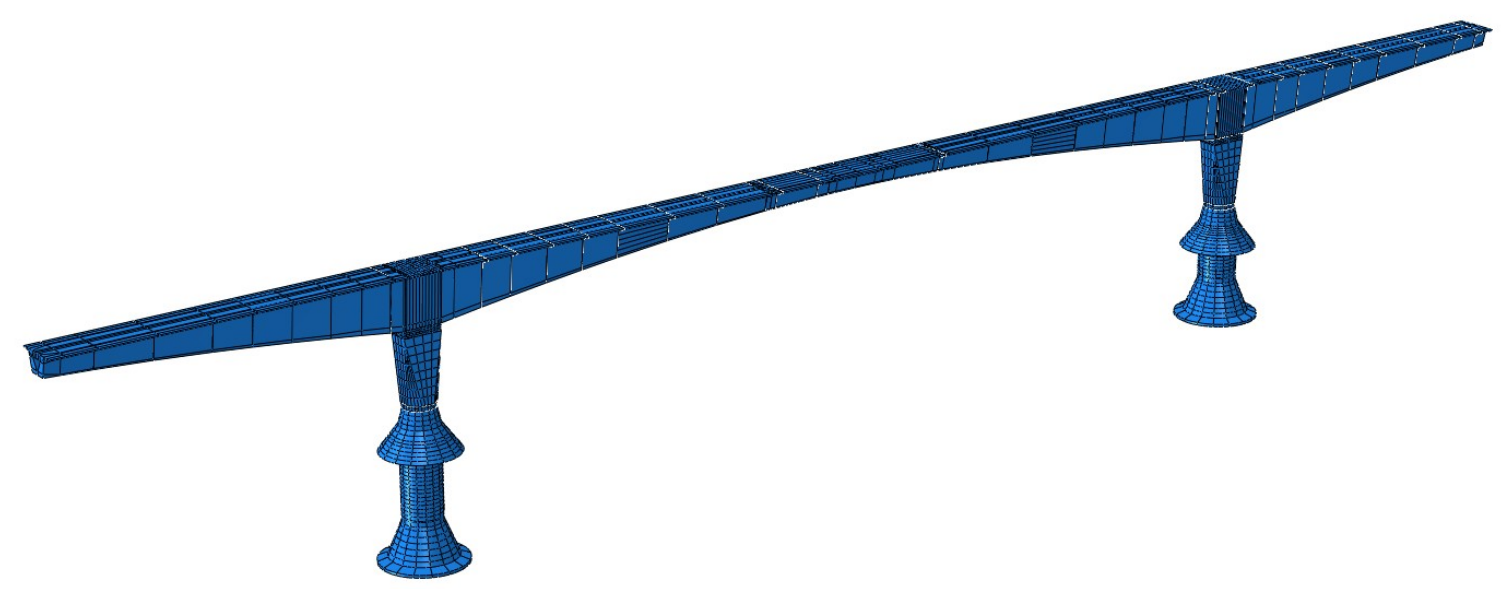

Figure 3.8: 3D model of one typical portal frame of the Confederation Bridge (ABAQUS).

\subsubsection{Bridge behavior and early operational modal analysis}

The first studies carried out with the vibration response data of the Confederation Bridge helped understand the complexities of the bridge's behavior under different types of environmental and controlled loading scenarios. This section will describe some of these studies.

\subsubsection{Initial estimations of dynamic characteristics}

Naumoski et al. [42] studied vibration responses from ambient conditions from the Confederation Bridge to determine the dynamic characteristics of the structure. By ambient data it is meant data sets collected under moderate wind and traffic conditions. Using a total of 8 ambient wind- and traffic-triggered data sets from January 
and February 2000 they have identified several modes. The methodology for obtaining the dynamic properties (frequencies and mode-shapes only) consists in computing the average Fourier spectra of each signal and picking peaks for the identification of natural frequencies. Band-pass filtering around these identified frequencies isolates the modal acceleration time histories. From the acceleration time histories, they obtain the modal displacement time histories for the vertical or transverse channels, depending on which mode they intend to shape. Once each displacement time history from all proper measurement locations are obtained and properly normalized they are animated to show the shapes. The identified vertical modes include $0.7 \mathrm{~Hz}$, $1.7 \mathrm{~Hz}, 2.9 \mathrm{~Hz}$, and $4.8 \mathrm{~Hz}$ and the identified transverse modes include $0.5 \mathrm{~Hz}, 0.9 \mathrm{~Hz}$, $1.4 \mathrm{~Hz}, 1.95 \mathrm{~Hz}$, and $2.8 \mathrm{~Hz}$. The results are shown in Table 3.3. The mode-shapes for these modes can be found in the reference [42]. The torsion modes are identified by computing the Fourier spectra of the record obtained from subtracting the measurement records at each location V1 from the records at each location V2. Two torsion modes were identified, in this manner, at $3.3 \mathrm{~Hz}$ and $4.2 \mathrm{~Hz}$ as shown in Table 3.3 . An important observation of the dynamic characteristics is the identification of local vibration modes in the frequency range of 10 to $16 \mathrm{~Hz}$ which is covered exhaustively by a subsequent study [43] which is discussed briefly in Section 3.3.2.4.

The extracted dynamic characteristics from [42] were compared with those obtained by Jaeger's FE model presented in Section 3.3.1.1. The correlation of modes seems to have been done qualitatively by observing figures of analytical versus experimental mode-shapes. Naumoski et al. [42] conclude their analytical comparison by stating that the experimentally-extracted frequencies are significantly higher than those obtained by Jaeger [40] and that more research is needed to fully understand the dynamic response of the structure.

Zhang [44] also extracted dynamic properties of the Confederation Bridge from 
ambient response data. For the first time in this project an advanced system identification technique, called the stochastic subspace identification (SSI) method is employed [44]. This approach has its advantages since it considers the noise contamination problem in the monitoring data and tries to minimize its influence on the resulting modal properties. The commercially-available software CADA-X ${ }^{\circledR}$ (in which the SSI technique is implemented) is used in the data analysis [44]. The data signals are low-pass filtered at $15 \mathrm{~Hz}$ prior to detailed system identification analysis. The two vertical records from the sensors at a single measurement location were combined by averaging. A total of 22 modes were identified in the frequency range of 0 to $5.33 \mathrm{~Hz}$. Table 3.3 lists the identified modes. Mode-shapes are illustrated using a wire geometric model of the bridge in the reference [44]. Damping ratios are also experimentally-obtained and shown in Table 3.3. The system identification technique employed permits identification of many modes, some of which are closely spaced. Section 2.1 of this thesis describes the stochastic subspace identification method.

\subsubsection{Pull test}

In a 2004 study, Lau et al. [41] present the calibration of the two portal frame beam models, shown in Figure 3.7, with experimental data acquired through a dynamic pulltest performed on the Confederation Bridge. The pull-test was conducted in April 1997, two months prior to the official opening of the bridge. A cable was attached above the ice shield of pier 31 at one end and pulled by a test boat at the other. The horizontal load was applied slowly up to a maximum of $1.40 \mathrm{MN}$ and suddenly released. 
Table 3.3: Modal properties extracted by different authors from the vibration monitoring data of the Confederation Bridge.

\begin{tabular}{|c|c|c|c|c|c|c|c|c|c|c|c|c|c|c|c|c|c|c|c|c|c|c|c|c|}
\hline \multicolumn{4}{|c|}{ Analytical } & \multicolumn{21}{|c|}{ Experimental } \\
\hline Beam & Shell & Beam & $\begin{array}{l}\text { Beam } \\
\text { dou- }\end{array}$ & \multicolumn{3}{|c|}{ Pull test } & \multicolumn{5}{|c|}{ Ambient wind \& traffic } & \multicolumn{5}{|c|}{ Controlled traffic test } & \multicolumn{4}{|c|}{ Design verification } & \multicolumn{4}{|c|}{ Baseline variability } \\
\hline 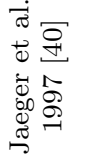 & 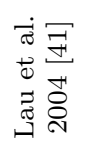 & 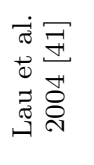 & 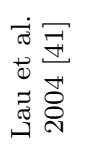 & & 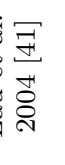 & & 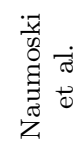 & 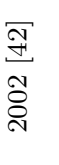 & & 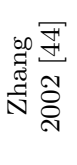 & & 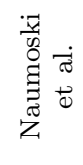 & 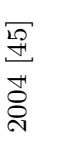 & & 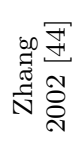 & & & & 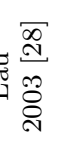 & & & 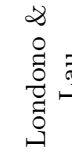 & 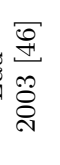 & \\
\hline 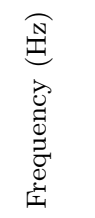 & 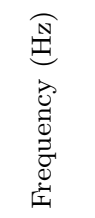 & 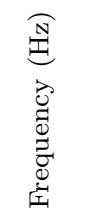 & 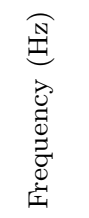 & 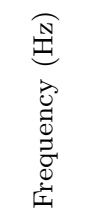 & 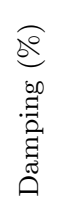 & \begin{tabular}{l}
0 \\
\multirow{2}{0}{} \\
0 \\
0 \\
0 \\
$\dot{\Sigma}$
\end{tabular} & 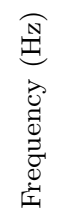 & 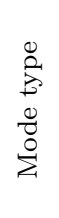 & 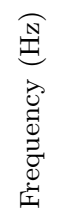 & 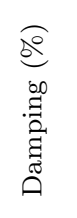 & 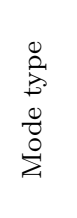 & 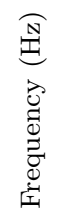 & 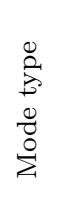 & 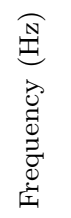 & 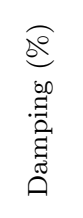 & 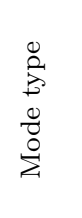 & 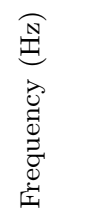 & 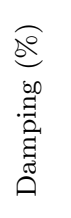 & 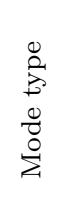 & 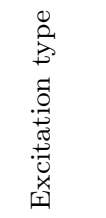 & 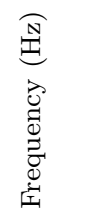 & 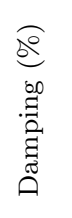 & 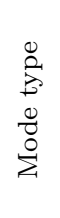 & 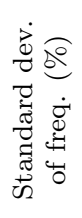 \\
\hline$\overline{0.297}$ & $\overline{0.299}$ & $\overline{0.299}$ & $\overline{0.299}$ & 0.477 & 3.1 & $\mathrm{~T}$ & 0.50 & $\mathrm{~T}$ & 0.33 & 3.85 & $\mathrm{~T}$ & 0.50 & $\mathrm{~T}$ & 0.33 & 3.85 & $\mathrm{~T}$ & 0.36 & 0.79 & $\mathrm{~T}$ & Wind & 0.474 & 1.53 & $\mathrm{~T}$ & 0.61 \\
\hline 0.304 & 0.354 & 0.354 & 0.312 & 1.304 & 3.7 & $\mathrm{~T}$ & 0.70 & $\mathrm{~V}$ & 0.47 & 3.92 & $\mathrm{~T}$ & 0.70 & $\mathrm{~V}$ & 0.47 & 3.92 & $\mathrm{~T}$ & 0.40 & 0.35 & $\mathrm{~T}$ & Wind & 1.641 & 1.34 & V & 0.56 \\
\hline 0.313 & 0.453 & 0.459 & 0.355 & & & & 0.90 & $\mathrm{~T}$ & 0.61 & 6.97 & $\mathrm{~V}$ & 0.90 & $\mathrm{~T}$ & 0.65 & 1.81 & $\mathrm{~V}$ & 0.65 & 0.1 & $\mathrm{~V}$ & Wind & 1.828 & 1.56 & V & 0.53 \\
\hline 0.322 & 0.476 & 0.473 & 0.387 & & & & 1.40 & $\mathrm{~T}$ & 0.65 & 1.81 & $\mathrm{~V}$ & 1.00 & $\mathrm{~V}$ & 0.96 & 1.88 & $\mathrm{~T} / \mathrm{V}$ & 0.71 & 1.48 & $\mathrm{~V}$ & Storm & 2.774 & 0.91 & $\mathrm{~V}$ & 0.50 \\
\hline 0.350 & 0.620 & 0.623 & 0.460 & & & & 1.70 & $\mathrm{~V}$ & 0.79 & 8.15 & $\mathrm{~V}$ & 1.40 & $\mathrm{~T}$ & 1.47 & 4.08 & $\mathrm{~T} / \mathrm{V}$ & 0.91 & 0.07 & $\mathrm{~V}$ & Wind & & & & \\
\hline 0.375 & 0.793 & 0.815 & 0.475 & & & & 1.95 & $\mathrm{~T}$ & 0.85 & 1.66 & $\mathrm{~T} / \mathrm{V}$ & 1.70 & $\mathrm{~V}$ & 1.54 & 1.35 & $\mathrm{~T} / \mathrm{V}$ & 1.00 & 0.47 & $\mathrm{~V}$ & Traffic & & & & \\
\hline 0.429 & 0.878 & 0.856 & 0.493 & & & & 2.80 & $\mathrm{~T}$ & 0.96 & 1.88 & $\mathrm{~T} / \mathrm{V}$ & 1.95 & $\mathrm{~T}$ & 1.68 & 4.15 & $\mathrm{~V}$ & 1.21 & 0.40 & $\mathrm{~V} / \mathrm{L}$ & Traffic & & & & \\
\hline 0.434 & 1.045 & 1.034 & 0.504 & & & & 2.90 & $\mathrm{~V}$ & 1.23 & 6.13 & $\mathrm{~T} / \mathrm{V}$ & 2.80 & $\mathrm{~T}$ & 1.81 & 1.01 & $\mathrm{~V}$ & 1.62 & 0.04 & $\mathrm{~V} / \mathrm{L}$ & Wind & & & & \\
\hline \multirow[t]{12}{*}{0.443} & 1.134 & 1.163 & 0.622 & & & & 3.30 & $\mathrm{~T}$ & 1.34 & 2.33 & $\mathrm{~T} / \mathrm{V}$ & 2.90 & $\mathrm{~V}$ & 2.77 & 1.47 & $\mathrm{~V}$ & 2.75 & 0.11 & $\mathrm{~V}$ & Traffic & & & & \\
\hline & 1.594 & 1.604 & 0.669 & & & & 4.20 & $\mathrm{~T}$ & 1.54 & 1.35 & $\mathrm{~T} / \mathrm{V}$ & 3.30 & $\mathrm{~T}$ & 2.84 & 2.43 & $\mathrm{~V}$ & 3.44 & 0.22 & $\mathrm{~V}$ & Traffic & & & & \\
\hline & 1.639 & 1.634 & & & & & 4.80 & $\mathrm{~V}$ & 1.81 & 1.01 & $\mathrm{~V}$ & 4.20 & $\mathrm{~T}$ & 3.00 & 2.22 & $\mathrm{~V}$ & 3.38 & 0.10 & $\mathrm{~T}$ & Traffic & & & & \\
\hline & 2.268 & 2.306 & & & & & & & 2.77 & 1.47 & $\mathrm{~V}$ & 4.80 & $\mathrm{~V}$ & 3.05 & 1.32 & $\mathrm{~V}$ & 4.71 & 0.38 & $\mathrm{~V}$ & Wind & & & & \\
\hline & & & & & & & & & 3.00 & 2.22 & $\mathrm{~V}$ & & & 3.42 & 1.18 & $\mathrm{~V}$ & 10.73 & 0.02 & $\mathrm{~V}$ & Traffic & & & & \\
\hline & & & & & & & & & 3.21 & 1.24 & $\mathrm{~V}$ & & & 3.68 & 1.80 & $\mathrm{~V}$ & 12.7 & 0.05 & $\mathrm{~V}$ & Traffic & & & & \\
\hline & & & & & & & & & 3.42 & 1.18 & $\mathrm{~V}$ & & & 3.88 & 1.32 & $\mathrm{~V}$ & & & & & & & & \\
\hline & & & & & & & & & 3.88 & 1.32 & $\mathrm{~V}$ & & & 4.50 & 1.21 & $\mathrm{~V}$ & & & & & & & & \\
\hline & & & & & & & & & 4.65 & 1.82 & $\mathrm{~V}$ & & & 5.12 & 2.27 & $\mathrm{~V}$ & & & & & & & & \\
\hline & & & & & & & & & 4.95 & 1.62 & $\mathrm{~V}$ & & & 5.33 & 0.44 & $\mathrm{~V}$ & & & & & & & & \\
\hline & & & & & & & & & 5.12 & 2.27 & $\mathrm{~V}$ & & & & & & & & & & & & & \\
\hline & & & & & & & & & 5.33 & 0.44 & $\mathrm{~V}$ & & & & & & & & & & & & & \\
\hline
\end{tabular}


The dynamic monitoring system captured the subsequent "free-vibration" response of the structure. The power spectrum of the response measurements from sensor location 9T1 is computed (see Figure 3.4 for sensor locations). The FFT of the 20s data set sampled at $294 \mathrm{~Hz}$ was computed with 217 points for the FFT. The dominant frequencies were then determined from Lagrangian interpolation through several points around the corresponding peak. The dominant frequency is found to be $0.4769 \mathrm{~Hz}$ and the second dominant frequency is found to be $1.3042 \mathrm{~Hz}$ (Table 3.3). The modal free-vibration acceleration time histories of the two identified modes are obtained by digital band-pass filtering the results of the FFT around the identified frequency values and then applying the inverse FFT. The pass-band for the digital filters was set wide enough to avoid distortion of the time-histories but narrow enough not to include adjacent vibration modes. The pass-band for the first dominant mode was 0.41 to $0.69 \mathrm{~Hz}$ while the pass-band for the second mode was 1.0 to $1.53 \mathrm{~Hz}$. The damping ratios were subsequently obtained from the modal free-vibration time histories and were found to be $3.1 \%$ for the first mode and $3.7 \%$ for the second. See Table 3.3 for results.

With the resulting values of the dynamic properties obtained from the analysis of the pull-test measurement data, the two-portal beam model presented in Section 3.3.1.2 and detailed in the reference [41] is calibrated. The material properties of $E_{c}$ and $K_{r f}$, representing the elastic modulus of the structure and the rotational stiffness of the foundation respectively, are updated by trial and error analysis. Different $E_{c}$ and $K_{r f}$ properties of the $\mathrm{FE}$ model are specified for each analysis and the resulting dominant frequency is compared with the experimentally-obtained dominant frequency $(0.4769 \mathrm{~Hz})$. The values of $E_{c}=34 \mathrm{GPa}$ and $K_{r f}=7.06 \mathrm{MN} \mathrm{m} / \mu \mathrm{rad}$ produce a resulting dominant frequency matching the experimental results and are therefore taken as the model's material properties. The average damping ratio (3.4\%) 
from the two extracted modes is also adopted in the FE beam model of two portal frames.

\subsubsection{Controlled traffic load test}

The traffic tests conducted overnight of the $13^{\text {th }}$ to $14^{\text {th }}$ of December 2000 consisted of loaded trucks traveling over the instrumented portion of the bridge at different speeds and different configurations under low wind conditions. The first set of test cases consisted of a single loaded truck traveling at the constant speeds of 40 and $80 \mathrm{~km} / \mathrm{h}$ in the right-hand lane over the instrumented portion. The second set of test cases included two trucks tailgating in a single lane at constant speeds of 40 and $60 \mathrm{~km} / \mathrm{h}$ while the third set of cases involved both trucks traveling side-by-side at speeds of 40 and $60 \mathrm{~km} / \mathrm{h}$. Dynamic data were recorded by the high-speed data acquisition system during these tests.

Zhang [44] presents details of the traffic tests and provides a detailed example of the analysis procedures undertaken with the response data from the three test cases. The stochastic subspace identification method, integrated into the software CADA-X ${ }^{\circledR}$, is employed to analyze the traffic test data which produces the dynamic properties listed in Table 3.3. It should be noted that the damping ratios obtained from the monitoring data of ambient wind and traffic excitations and those obtained from the controlled traffic test data as reported by Zhang are similar. This is contrary to the observations in a later study [46] which show that the complexity of the damping phenomenon makes it difficult to determine accurately and consistently damping ratio results from vibration monitoring data. The observations on variability of damping ratios are discussed in more detail in Section 3.3.2.6.

Following the same analysis procedure as described in Section 3.3.2.1 on the controlled traffic test data, Naumoski et al. [45] identify a new vertical mode at $1.0 \mathrm{~Hz}$ 
(Table 3.3) caused by slower moving vehicles which is not identified in their earlier study [42].

\subsubsection{Local vibrations}

The size and dimensions of the box girders and the wide spacing of the webs permit some local vibrations of the box girders under heavy traffic loadings. Local vibrations have been observed in the frequency range of 10 to $16 \mathrm{~Hz}$ [42]. In a separate unpublished study, Cheung et al. [43] have placed seven extra accelerometers at location 15 during the controlled traffic test described in the previous section. The response measurement of the sensor placed at the center of the deck slab clearly identifies some important local vibration modes. The Fourier amplitude spectrum of the time history responses of the extra sensors during the test case of a single truck traveling at $80 \mathrm{~km} / \mathrm{h}$ clearly shows a dominant mode at $13.5 \mathrm{~Hz}$ and some other less dominant modes at $11.7 \mathrm{~Hz}, 12.7 \mathrm{~Hz}$, and $16.4 \mathrm{~Hz}$. The study further states that these girder box vibrations occurring under traffic excitations are more quickly dissipated than the global vibrations.

An updated 3D shell model with a finer mesh than the one described in Section 3.3.1.2 has been developed for the study on local vibrations of the box girder cross-sections [43]. The new 3D shell model is developed using finite element code COSMOS ${ }^{\circledR}$ and represents one typical bridge portal frame corresponding to the instrumented portion of the structure between Piers 31 and 32 (Figure 3.4). Main girders and piers are modeled using 4-node quadrilateral thin shell elements and 3node triangular shell elements. The ice shield component of the pier is modeled by 4-node tetrahedron solid elements which have compatible nodal degrees of freedom with the adjacent shell elements. The interaction of the portal frame with the connecting drop-in spans, which are not included in the model, is represented by lumped 
mass elements. The mass of the drop-in span is equally divided and added to each bearing in the transverse and vertical directions, since all four bearings of a drop-in span prevent motion in these directions. The entire mass of the drop-in span is added in the longitudinal direction of the bearing which prevents longitudinal motion since only one of the four bearings on the drop-in span prevents relative motion in this direction. The dimensions and material properties of this model are based on the design drawing dimensions and specifications. The model comprises a total of 2381 elements and 2206 nodes, resulting in a reasonably fine mesh. The 3D shell model described here in detail is also the subject of comparison in the design verification studies presented in Section 3.3.2.5.

The finite element structural model of the bridge was subjected to load simulations to observe the effects of local vibrations. The finer mesh of the 3D shell model permitted observation of the local deformations which occurred at different points along the structure correlating with the location of the moving load. The box-girder contour mode-shapes were identified and were found to be varying in amplitude in a longitudinal manner.

\subsubsection{Design verification}

A detailed design verification study was undertaken in 2003 [28]. Data acquired from the dynamic monitoring system were processed and analyzed to obtain the modal properties of the Confederation Bridge. Three types of data sets which correspond to different excitation scenarios were chosen for analysis:

- data sets corresponding to bridge responses due to moderate wind with average speeds of approximately $60 \mathrm{~km} / \mathrm{h}$;

- data sets corresponding to responses from the controlled traffic tests;

- data sets corresponding to responses of the bridge due to a major wind storm 
in November 2001.

As an aside, one vibration data set corresponding to the windstorm was processed and analyzed in a separate study by Naumoski [45]. Maximum deflections were presented. The largest measured transverse deflection from the data set studied was $13.5 \mathrm{~mm}$ while the maximum vertical deflection was $9.0 \mathrm{~mm}$. It is worth noting here that only one of the four data sets recorded during the windstorm was studied, while the other unused three data sets did include higher bridge responses recorded during the windstorm.

The complete data sets mentioned above were processed and analyzed by Londono et al. [28] using tools developed in Matlab ${ }^{\circledR}$. This early processing engine is the basis for part of the computer platform presented in Section 3.3.3 and in the references $[24,47]$. The processed signals were analyzed using the operational modal analysis software CADA-X ${ }^{\circledR}$ which integrates the stochastic subspace identification method. The identification method incorporated in CADA-X ${ }^{\circledR}$ includes a stabilization diagram to differentiate stable solutions from spurious ones. The extracted modal properties from these data sets are included in Table 3.3. A simple geometric wire model was built in Matlab ${ }^{\circledR}$ for visualization of the identified mode-shapes.

The extracted modal properties were used in a verification study of the design parameters and assumptions of the bridge. The FE model used for comparison is the 3D beam element model spanning two portal frames including a drop-in span detailed in Section 3.3.1.2 and shown in Figure 3.7. This FE model was also calibrated with data captured from the pull-test detailed in Section 3.3.2.2. A comprehensive comparison study of experimentally-identified and analytical mode-shapes was completed [28]. A modal assurance criterion (MAC) was also calculated between the experimental and analytical mode-shapes [20,28,34] as per Equation (2.17). An MAC of 1 indicates perfect correlation of the mode-shapes while an MAC of 0 indicates no correlation. 
Table 3.4 shows the analytical and experimental modes and their corresponding MAC values. The experimental modes show a consistent higher frequency than the design value, which implies that the structure is generally stiffer than the model.

Table 3.4: Verification of experimental modal properties with respect to the analytical results from the beam element model spanning two portal frames [28].

\begin{tabular}{cccc}
\hline $\begin{array}{c}\text { Analytical } \\
\text { frequency }(\mathrm{Hz})\end{array}$ & $\begin{array}{c}\text { Experimental } \\
\text { frequency }(\mathrm{Hz})\end{array}$ & $\begin{array}{c}\text { Modal assurance } \\
\text { criterion (MAC) }\end{array}$ & Mode type \\
\hline 0.28 & 0.36 & 0.94 & Transverse \\
0.33 & 0.40 & 0.93 & Transverse \\
0.58 & 0.65 & 0.86 & Vertical \\
0.62 & 0.71 & 0.96 & Vertical \\
0.79 & 0.91 & 0.94 & Vertical \\
0.82 & 1.00 & 0.72 & Vertical \\
1.08 & 1.21 & 0.78 & Vertical/Longitudinal \\
1.60 & 1.62 & 0.83 & Vertical/Longitudinal \\
2.34 & 2.75 & 0.94 & Vertical \\
2.73 & 3.44 & 0.89 & Vertical \\
\hline
\end{tabular}

This study [28] successfully demonstrated the effectiveness of the stochastic subspace identification method in dealing with noisy output only structural response data by successfully identifying modes which correlate well with those previously obtained by FE modeling. An important finding is the observed variability in the identified modal parameters. Modal properties extracted from different data sets tend to give slightly different results in identified frequency and damping ratios. The observed variability in the modal parameters complicates the practical use of ambient vibration response data for condition assessment which rely on changes in dynamic characteristics to detect and locate damage. Potential damage may be masked by this observed variability. This is a very important finding that needs further study. 
A second in-depth design verification study was performed using the signal processing platform for analysis of structural health (SPLASH), which is a combination of custom computer tools and modules developed for the project $[24,47]$. The SPLASH application is also described in Section 3.3.3. Ten different data sets were chosen for analysis in a more in-depth study into design verification [29]. The chosen data sets correspond to the following loading scenarios:

- high winds;

- controlled traffic test cases of two trucks following each other and traveling side by side;

- ambient operational conditions with ice cover;

- ambient operational conditions with no ice cover.

The stochastic subspace identification (SSI) technique, developed in Matlab ${ }^{\circledR}$ and fully implemented in the SPLASH platform, was used to estimate the modal parameters from the chosen data sets.

Two different finite element models, the fine-meshed 3D shell element model presented in Section 3.3.1.2 and shown in Figure 3.5 and the equivalent 3D beam element model spanning two portal frames presented in the same section and shown in Figure 3.7, were used to obtain the theoretical dynamic behavior of the bridge and are used in this verification study [29]. The sensitivity of the dynamic properties of the bridge to long-term changes in material properties is examined by varying the material properties of the concrete of the analytical bridge models. Computer analyses of the bridge models have been carried out using two different values of the Young's modulus "E" of concrete. The first value of $E_{c}$ is the nominal design value, and the second value of $E_{c}$ is based on the monitoring data and research by the material monitoring group of the project from tests on concrete samples used in the Confederation Bridge [48].

The elastic modulus of the concrete determines the stiffness of the bridge and has a 
direct effect on its static and dynamic behavior. Therefore, this material parameter is an important design assumption that must be verified after construction. The target 28-day strength of concrete in the design specifications was $f_{c 28}^{\prime}=55 \mathrm{MPa}$ and the target elastic modulus was $E_{c 28}=35 \mathrm{GPa}$. as determined from tests of 24 concrete cylinders taken from two Confederation Bridge batches [48]. The cylinders were tested at the ages of $2,7,14,28,90$, and 180 days. The average measured value of $f_{c 28}^{\prime}$ was $63 \mathrm{MPa}$ and $E_{c 28}$ was approximately $38.7 \mathrm{GPa}$. Based on the measurements, a best fit equation for the time-dependent variation of $E_{c}$ was proposed as follows [48]:

$$
E_{c}(t)=E_{c 28} \sqrt{\exp \left[0.25\left(1-\sqrt{\frac{28}{t}}\right)\right]}
$$

in which $E_{c}(t)$ is the elastic modulus of concrete at age $t$, in days, and $E_{c 28}$ is $38.7 \mathrm{GPa}$, approximately the average measured value. The constant of 0.25 in the equation is for normal hardening cement, assumed for the Confederation Bridge concrete [48]. The 1000-day value of $E_{c}$ given by this expression is $42.9 \mathrm{GPa}$. In the referenced study [29], two values of $E_{c}$ are used in the bridge $\mathrm{FE}$ models for comparison purpose: the target 28 -day value of $35 \mathrm{GPa}$ and a 3 -year value of $43 \mathrm{MPa}$, which is expected to more realistically reflect the actual properties of the Confederation Bridge concrete.

Table 3.5 presents analysis results from the monitoring data sets and results from both the beam and shell finite element models using the two different values for $E_{c}$. The first 23 identified modes with mean frequency values ranging from $0.34 \mathrm{~Hz}$ to $5.13 \mathrm{~Hz}$ are shown with the corresponding modes from the analytical models. The differences, in percentage value, between the experimentally-obtained frequencies and those from the two analytical models with the two different modulus values are also shown in Table 3.5. By examining the average differences of all modes, it is shown that there is a better correlation of the system identification results from field monitoring 
Table 3.5: Summary of in-depth verification study by Londono et al [29].

\begin{tabular}{|c|c|c|c|c|c|c|c|c|c|c|c|c|c|c|c|c|c|c|c|c|c|c|}
\hline \multirow[b]{2}{*}{ 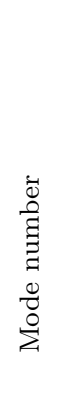 } & \multirow[b]{2}{*}{$\stackrel{\infty}{\rightleftharpoons}$} & \multicolumn{6}{|c|}{ Experimental frequency } & \multicolumn{4}{|c|}{ Theoretical frequency } & \multicolumn{4}{|c|}{$\begin{array}{l}\text { Experimental vs. } \\
\text { theoretical frequency }\end{array}$} & \multicolumn{3}{|c|}{$\begin{array}{l}\text { Modal damping } \\
\text { ratio } \xi \%\end{array}$} & \multicolumn{4}{|c|}{$\begin{array}{l}\text { Mode-shapes modal } \\
\text { assurance criterion } \\
\text { (MAC) }\end{array}$} \\
\hline & & 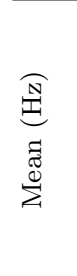 & $\begin{array}{l}\underset{\mathrm{N}}{\Xi} \\
\underset{\Sigma}{\Xi}\end{array}$ & 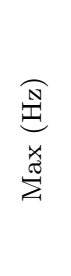 & 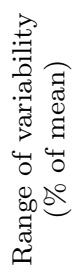 & 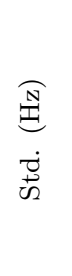 & 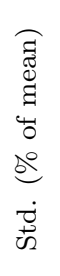 & 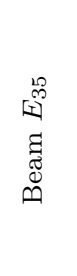 & 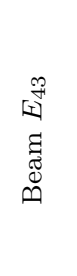 & 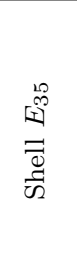 & 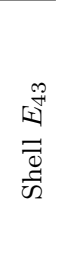 & 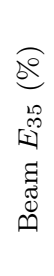 & 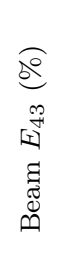 & 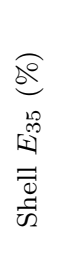 & 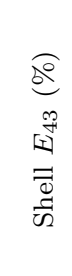 & 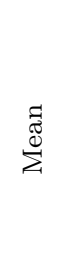 & 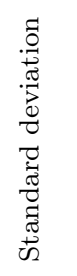 & 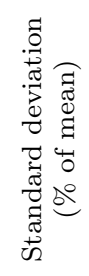 & 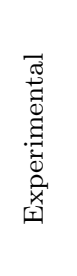 & 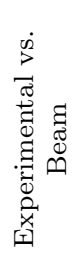 & 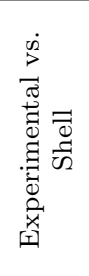 & 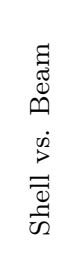 \\
\hline 1 & $\mathrm{~T}$ & 0.34 & 0.32 & 0.37 & 13 & 0.02 & 5.2 & 0.28 & 0.31 & 0.32 & 0.35 & 19 & 10 & 7 & -3 & 0.019 & 0.011 & 57 & 0.88 & 0.85 & 0.78 & 0.998 \\
\hline 2 & $\mathrm{~T}$ & 0.37 & 0.35 & 0.39 & 12 & 0.01 & 4.0 & 0.33 & 0.37 & 0.38 & 0.42 & 10 & 0 & -2 & -13 & 0.048 & 0.015 & 32 & 0.54 & 0.64 & 0.60 & 0.98 \\
\hline 3 & $\mathrm{~T}$ & 0.47 & 0.46 & 0.49 & 6 & 0.01 & 1.7 & 0.46 & 0.51 & 0.49 & 0.54 & 2 & -8 & -2 & -13 & 0.016 & 0.008 & 49 & 0.69 & 0.57 & 0.74 & 0.96 \\
\hline 4 & $\mathrm{~V}$ & 0.57 & 0.52 & 0.62 & 16 & 0.03 & 4.9 & 0.47 & 0.52 & 0.49 & 0.55 & 17 & 8 & 13 & 4 & 0.026 & 0.016 & 59 & 0.59 & 0.59 & 0.74 & 0.98 \\
\hline 5 & $\mathrm{~V}$ & 0.65 & 0.61 & 0.68 & 10 & 0.02 & 2.5 & 0.58 & 0.64 & 0.63 & 0.69 & 11 & 1 & 3 & -8 & 0.020 & 0.010 & 52 & 0.70 & 0.72 & 0.80 & 0.98 \\
\hline 6 & $\mathrm{~V}$ & 0.68 & 0.66 & 0.70 & 6 & 0.01 & 2.0 & 0.62 & 0.69 & 0.63 & 0.69 & 9 & -1 & 8 & -1 & 0.022 & 0.017 & 78 & 0.54 & 0.64 & 0.79 & 0.98 \\
\hline 7 & $\mathrm{~T}$ & 0.90 & 0.87 & 0.95 & 9 & 0.02 & 2.6 & 0.84 & 0.93 & 0.88 & 0.98 & 7 & -3 & 2 & -8 & 0.017 & 0.009 & 55 & 0.35 & 0.35 & 0.44 & 0.98 \\
\hline 8 & $\mathrm{~V}$ & 0.92 & 0.90 & 0.94 & 5 & 0.01 & 1.3 & 0.79 & 0.88 & 0.86 & 0.95 & 14 & 5 & 7 & -3 & 0.016 & 0.005 & 32 & 0.80 & 0.83 & 0.91 & 0.98 \\
\hline 9 & $\mathrm{~V}$ & 0.97 & 0.92 & 1.00 & 8 & 0.03 & 2.7 & 0.82 & 0.91 & 0.86 & 0.95 & 15 & 6 & 11 & 1 & 0.019 & 0.010 & 50 & 0.78 & 0.78 & 0.89 & 0.98 \\
\hline 10 & $\mathrm{~V}$ & 1.25 & 1.23 & 1.27 & 3 & 0.01 & 1.0 & 1.08 & 1.20 & 1.11 & 1.22 & 13 & 4 & 12 & 2 & 0.018 & 0.010 & 53 & 0.68 & 0.59 & 0.52 & 0.97 \\
\hline 11 & $\mathrm{~T}$ & 1.32 & 1.31 & 1.36 & 4 & 0.02 & 1.8 & 1.20 & 1.33 & 1.28 & 1.42 & 10 & 0 & 3 & -7 & 0.014 & 0.004 & 31 & 0.53 & 0.42 & 0.47 & 0.99 \\
\hline 12 & $\mathrm{~V}$ & 1.61 & 1.58 & 1.64 & 4 & 0.02 & 1.3 & 1.60 & 1.78 & 1.65 & 1.82 & 1 & -10 & -2 & -13 & 0.018 & 0.013 & 72 & 0.75 & 0.73 & 0.88 & 0.995 \\
\hline 13 & $\mathrm{~V}$ & 1.81 & 1.78 & 1.83 & 3 & 0.02 & 1.0 & 1.60 & 1.78 & 1.65 & 1.82 & 11 & 2 & 9 & -1 & 0.014 & 0.007 & 50 & 0.89 & 0.86 & 0.93 & 0.995 \\
\hline 14 & $\mathrm{~V}$ & 2.75 & 2.69 & 2.77 & 3 & 0.03 & 1.0 & 2.34 & 2.59 & 2.64 & 2.92 & 15 & 6 & 4 & -6 & 0.008 & 0.004 & 53 & 0.84 & 0.83 & 0.78 & 0.85 \\
\hline 15 & $\mathrm{~V}$ & 2.88 & 2.81 & 2.96 & 5 & 0.05 & 1.8 & 2.46 & 2.72 & 2.64 & 2.92 & 15 & 5 & 8 & -1 & 0.010 & 0.006 & 60 & 0.60 & 0.73 & 0.82 & 0.99 \\
\hline 16 & $\mathrm{~V}$ & 3.08 & 3.02 & 3.11 & 3 & 0.03 & 0.8 & 2.73 & 3.03 & - & - & 11 & 2 & - & - & 0.009 & 0.007 & 84 & 0.55 & 0.52 & - & - \\
\hline 17 & To & 3.31 & 3.27 & 3.35 & 2 & 0.03 & 0.9 & 2.91 & 3.22 & 3.17 & 3.51 & 12 & 3 & 4 & -6 & 0.005 & 0.003 & 63 & 0.37 & 0.07 & 0.33 & 0.46 \\
\hline 18 & $\mathrm{~V}$ & 3.76 & 3.70 & 3.82 & 3 & 0.05 & 1.2 & 3.08 & 3.42 & 3.25 & 3.60 & 18 & 9 & 13 & 4 & 0.008 & 0.004 & $\begin{array}{l}49 \\
+\quad 4\end{array}$ & 0.62 & 0.60 & 0.57 & 0.78 \\
\hline 19 & $\mathrm{~V}$ & 3.45 & 3.42 & 3.47 & 1 & 0.01 & 0.4 & 3.15 & 3.50 & 3.45 & 3.83 & 9 & -1 & 0 & -11 & 0.009 & 0.005 & 55 & 0.83 & 0.42 & 0.29 & 0.70 \\
\hline 20 & $\mathrm{~V}$ & 4.03 & 3.89 & 4.16 & 7 & 0.12 & 3.1 & 3.49 & 3.87 & 3.62 & 4.01 & 13 & 4 & 10 & 0 & 0.006 & 0.003 & 41 & 0.57 & 0.59 & 0.65 & 0.69 \\
\hline 21 & $\mathrm{~V}$ & 4.53 & 4.46 & 4.63 & 4 & 0.05 & 1.2 & 3.95 & 4.38 & - & - & 13 & 3 & - & - & 0.006 & 0.006 & 96 & 0.70 & 0.62 & - & \\
\hline 22 & $\mathrm{~V}$ & 4.92 & 4.83 & 5.01 & 4 & 0.07 & 1.4 & 4.34 & 4.81 & 4.52 & 5.01 & 12 & 2 & 8 & -2 & 0.007 & 0.003 & 44 & 0.54 & 0.66 & 0.74 & 0.99 \\
\hline 23 & $\mathrm{~V}$ & 5.13 & 4.99 & 5.22 & 4 & 0.07 & 1.3 & 4.43 & 4.91 & 4.52 & 5.01 & 14 & 4 & 12 & 2 & 0.006 & 0.004 & 60 & 0.72 & 0.63 & 0.60 & 0.86 \\
\hline Ave & & & & & 5.9 & & 2.0 & & & & & 12 & 2 & 6 & -4 & 0.015 & & 55 & 0.66 & 0.62 & 0.68 & 0.91 \\
\hline
\end{tabular}


data with the computer predictions using the updated parameter of $E_{c}=43 \mathrm{GPa}(2 \%$ and $-4 \%$ versus $12 \%$ and $6 \%$ ). This confirms the materials monitoring results and the previous observations of consistently-higher experimental frequencies which are due to the fact that the structure is actually stiffer than the analytical model based on nominal material design specifications. Some information regarding the variability of the extracted modal parameters from data sets collected under different loading scenarios and environmental conditions is also presented in Table 3.5. Averages over all 23 modes indicate a frequency range of variability of $5.9 \%$ and a standard deviation of $2 \%$ of the mean frequency from the 10 data sets. Lower modes also tend to be less consistently identified than higher frequency modes, which is a very important observation because the lower modes tend to represent the dominant vibration behavior of the structure. The variability of modal frequency poses a major challenge for identification of damage from vibration data because change in frequency due to stiffness degradation or damage may be masked by the normal variability of the data. However, a $2 \%$ standard deviation from the mean of an extracted modal property from highly-uncertain, complex and noisy output-only monitoring data collected at different times under different loading and environmental conditions is a promising result.

The analytical and experimental modes were correlated by comparing both their frequency values and their mode-shape's MAC values. In the comparison of the mode-shapes obtained from FE models with those identified from the monitoring data, modal coefficients at sensor locations are obtained by interpolating the modal coefficients at the adjacent beam model nodes. This permits the MAC calculation between experimental and analytical modes and more importantly the visual comparison of mode-shapes using the animation modules of SPLASH. Two mode-shapes 
can be simultaneously animated to visualize any discrepancies. Figure 3.9 and Figure 3.10 show examples of using the animation module for comparisons between experimentally-obtained and analytical mode-shapes (from beam FE model) of identified modes listed in Table 3.5. Some local discrepancies may be observed at the drop-in span where FE modeling assumptions for the bearing and hinged connections may not accurately represent the actual behavior of the connections and at the piers, where reference channels chosen for SSI analysis did not include pier channels. However, the overall close correlation demonstrates the validity of the SSI algorithm to successfully extract mode-shape information from the monitoring data. With the new techniques and greater processing power it is believed that greater accuracies can be achieved with mode shape estimation. Damping estimates were also successfully extracted from the monitoring data but with a higher variability than the other modal properties (Table 3.5). The complicated nature of the damping phenomenon and the viscous damping assumption in the state-space model are most likely the chief contributions to the $55 \%$ average standard deviation of damping ratios from all modes extracted from the 10 data sets.

\subsubsection{Baseline variability of modal properties}

After observing the significant variability of identified parameters in their verification studies, a series of data sets with similar loading and environmental conditions were analyzed to determine the baseline variability of extracted dynamic properties [46]. The level of variability of the structure during the healthy early stage of the bridge is determined in order to be able to use future monitoring data for vibration-based structural condition assessment. By analyzing data sets of similar loading and environmental conditions, the identified variability in modal properties represents the baseline, or lower bound, level of variability, assuming no damage occurred during the 

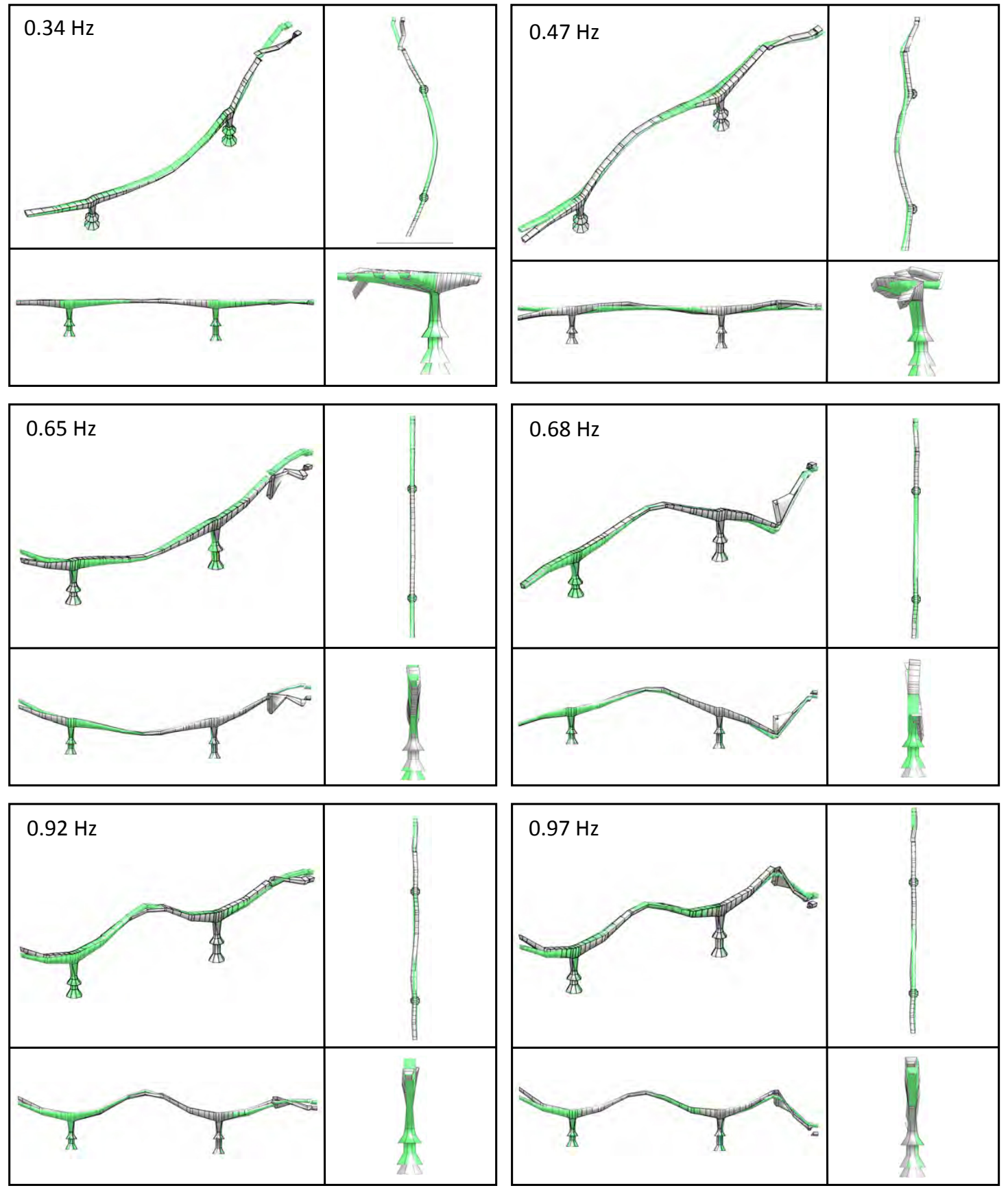

Figure 3.9: Comparison of experimental and analytical mode shapes. Vibration modes from the beam finite element model are shown in green with doted lines and the modes identified from the monitoring data are shown in gray with full lines. Modes from 0 to $1 \mathrm{~Hz}[24]$. 

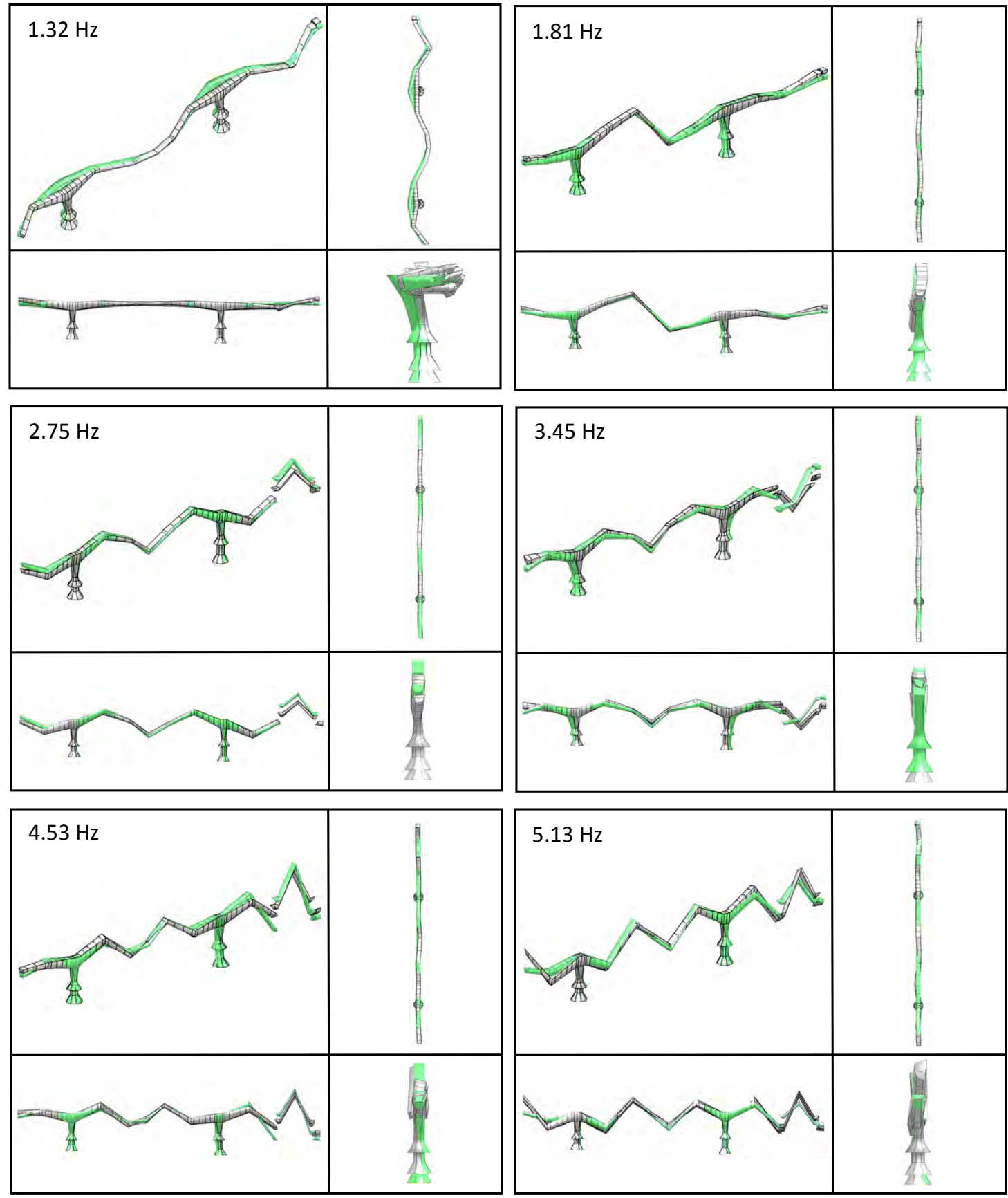

Figure 3.10: Comparison of experimental and analytical mode shapes. Vibration modes from the beam finite element model are shown in green with doted lines and the modes identified from the monitoring data are shown in grey with full lines. Modes above $1 \mathrm{~Hz}[24]$. 
collection of the data sets which spanned 4 days in this study. The objective of this and future studies is to determine if the identified baseline variability is significant enough to mask any changes in frequency, or other modal properties to prevent their use in condition assessment algorithms for the identification of damage or deterioration.

A total of ten data sets representing bridge behavior during typical ambient operational conditions were collected over four days in March of 2003 [46]. Temperature and wind speeds were similar in all the data sets. The system identification method used in this study is the same as in the verification study which is a variant of the SSI method incorporated in CADA-X ${ }^{\circledR}$ software. The duration of data sets is 10 minutes in this study, which gives good information of the lower frequency modes and consistent identification. The analysis results of this study are shown in Table 3.3. The four modes shown are identified from all ten data sets. The extracted frequencies are more consistently obtained than the damping ratio estimates. The four eigenfrequencies were estimated from the ten data sets with standard deviations at or below $0.6 \%$ of the mean as opposed to the damping estimates which have standard deviations between $34 \%$ and $55 \%$ of the mean. The $0.6 \%$ standard deviation of identified frequencies from data sets of similar loading scenarios and environmental conditions shows that it is possible to retrieve highly consistent modal frequencies. Two reasons are presented in this study [46] as to why the damping ratios are not as consistently identified. Firstly, the physical damping phenomenon is very complex and may not be accurately represented by the structural model. Secondly, the viscous damping modeling assumption is only a practical approximation of the actual damping behavior. The mode-shapes from a single mode acquired from all ten data sets are compared to each other by averaging the MAC value of all mode-shapes with respect to all other mode-shapes of the identified mode. Average MAC values of the 
four identified modes of the study are $0.987,0.984,0.990$ and 0.974 respectively. This shows very promising results of consistent identification of mode-shape properties. There are some localized discrepancies in mode-shape amplitude observed while correlating the individual amplitudes from all data sets with the amplitudes from the first data set. This may limit the use of monitoring data for use in damage localizing algorithms which rely on localized changes of mode-shapes.

Average normalized power spectral density estimates are computed in the vertical and transverse directions for the different loading scenarios studied [46]. The obtained spectral plots show very clear the behavior of the bridge under different loading scenarios. Some typical bridge frequencies are more strongly excited under different loading scenarios. It is very important here to distinguish the frequency behavior from variability of modal parameters. While different loading scenarios excite different modes, the frequency values of these modes should, in theory, be the same with no discrepancy in the case of a healthy structure. Therefore, it is assumed that loading scenarios do not account for the observed variability of the modal parameters but that they do change the frequency behavior of the response in that they more strongly excite certain modes. The four following loading scenarios were studied [46]:

- high winds;

- controlled heavy traffic test;

- ambient operational conditions with ice cover;

- ambient operational conditions with no ice cover.

Loading scenarios involving high winds typically excite lower frequencies in both the vertical and transverse directions; the dominant modes (identified by peak picking of power density plots) being $0.68 \mathrm{~Hz}, 0.64 \mathrm{~Hz}$ and $0.95 \mathrm{~Hz}$ in the vertical and $0.48 \mathrm{~Hz}$, $0.33 \mathrm{~Hz}$ and $0.36 \mathrm{~Hz}$ in the transverse directions. The traffic scenarios seem to excite more the frequencies in the range of $2.5 \mathrm{~Hz}$ to $3 \mathrm{~Hz}$ (and local vibration range above 
$10 \mathrm{~Hz}$ ) with dominant modes (identified by peak picking from PSD plots) being $3.43 \mathrm{~Hz}$, $3.28 \mathrm{~Hz}$ and $3.47 \mathrm{~Hz}$ in the vertical direction and $3.35 \mathrm{~Hz}, 3.39 \mathrm{~Hz}$, and $3.31 \mathrm{~Hz}$ in the transverse direction. Ambient operational conditions involving moderate to calm winds and normal traffic with ice cover tend to excite evenly all the frequency ranges with dominant modes identified by peak picking of PSD at $3.82 \mathrm{~Hz}, 2.86 \mathrm{~Hz}$ and $0.66 \mathrm{~Hz}$ in the vertical direction and $0.47 \mathrm{~Hz}, 0.36 \mathrm{~Hz}$ and $3.62 \mathrm{~Hz}$ in the transverse direction. Typical operational conditions with no ice cover represents the most frequent loading scenario experienced by the bridge. The dominant modes picked from peaks are $0.64 \mathrm{~Hz}, 3.27 \mathrm{~Hz}$, and $0.68 \mathrm{~Hz}$ in the vertical direction and $0.50 \mathrm{~Hz}, 0.34 \mathrm{~Hz}$, and $0.47 \mathrm{~Hz}$ in the transverse direction. Although an important peak at $4.16 \mathrm{~Hz}$ in the vertical direction is found only in scenarios involving ice cover, more research needs to be carried out to more fully understand the dynamic behavior of the bridge during significant ice cover and ice floes.

\subsubsection{General variability of modal properties}

Previous studies by Londono et al. $[28,29,46]$ discussed in earlier sections have clearly identified variability in the extracted modal parameters from dynamic vibration monitoring data of the Confederation Bridge. This variability is a serious obstacle for the use of ambient field vibration monitoring data for structural condition assessment that relies upon the detection of changes in the dynamic properties. Changes caused by damage or deterioration of the structure may be masked by the normal variability of the data. The potential causes of variation in the dynamic properties can be grouped as follows:

- environmental effects (e.g. temperature, wind, moisture);

- measurement, modeling, and computational inaccuracies;

- non-linearity of the structure; 
- non-stationarity in the measured data;

- stiffness degradation reflecting deterioration or damage of the structure.

The lower bound level of variability of extracted frequencies has been studied and quantified at a baseline level of $0.6 \%$ standard deviation from the mean [46]. The baseline variability shows that it is possible to retrieve highly consistent modal frequencies and mode-shapes from monitoring data sets of similar loading scenarios and environmental conditions.

Forty-two data sets collected over a period of ten months of bridge operation under varying environmental conditions and loading scenarios have been analyzed to evaluate the potential effect of temperature, cross-section temperature differentials, amplitude of response signal, and wind speed on the identified vibration properties of the Confederation Bridge. The data sets used in a co-authored study [30], are selected from the dynamic monitoring database based on the average concrete temperatures at the time of acquisition. It is assumed here that during the elapsed period between data sets the structural condition of the bridge remained unchanged. To avoid bias and give equal weight to all temperatures, one data set is selected per degree wide temperature range between $-18^{\circ} \mathrm{C}$ to $25^{\circ} \mathrm{C}$ of average concrete temperatures. Each of the $10-$ minute-duration data sets used in the study consists of the sampled acceleration responses from 19 sensors at locations 3 to 9 of Figure 3.4. The analysis of 42 data sets would have been a daunting task if it were not for the efficiency of the developed modules of SPLASH for processing, analysis and visualization of the data and results. The identification of eight dominant modes and statistical information on their variability is shown in Table 3.6.

A multiple linear regression analysis is carried out to investigate the correlation of the natural modal vibration frequencies with the environmental and amplitude 
Table 3.6: Variability of results from analysis of 42 data sets for environmental effects study [30].

\begin{tabular}{|c|c|c|c|c|c|c|c|c|}
\hline & \multicolumn{8}{|c|}{ Mode } \\
\hline & 1 & 2 & 3 & 4 & 5 & 6 & 7 & 8 \\
\hline Frequency average $(\mathrm{Hz})$ & 0.474 & 0.665 & 1.634 & 1.820 & 2.769 & 3.387 & 4.641 & 5.143 \\
\hline Standard deviation $(\mathrm{Hz})$ & 0.007 & 0.010 & 0.018 & 0.031 & 0.033 & 0.074 & 0.093 & 0.057 \\
\hline Standard deviation (\% of mean) & 1.5 & 1.5 & 1.1 & 1.7 & 1.2 & 2.2 & 2.0 & 1.1 \\
\hline Damping average $(\xi \%)$ & 2.641 & 2.945 & 2.103 & 2.637 & 1.583 & 2.257 & 2.672 & 2.283 \\
\hline Standard deviation $(\xi \%)$ & 3.177 & 1.321 & 1.344 & 1.900 & 0.684 & 1.529 & 1.449 & 0.931 \\
\hline Standard deviation ( $\xi \%$ of mean) & 120 & 45 & 64 & 72 & 43 & 68 & 54 & 41 \\
\hline MAC average & 0.948 & 0.944 & 0.982 & 0.957 & 0.954 & 0.878 & 0.899 & 0.925 \\
\hline
\end{tabular}

variables. The linear regression model used in this study is the following :

$$
\mathbf{y}=\beta_{0}+\beta_{1} \mathbf{x}_{1}+\ldots+\beta_{k} \mathbf{x}_{k}+E
$$

where $\mathbf{x}_{k}$ are the predictor variables, $\beta_{k}$ the regression coefficients, $\mathbf{y}$ is the dependent variable and $E$ is the error. In the present study the vibration modal frequency is the dependent variable, and the average concrete temperature, cross-section temperature differentials, average signal amplitude (RMS), and wind speed are the predictor variables. The results of the regression analysis are presented in Table 3.7. The 95\% confidence intervals for the regression coefficients are calculated. These confidence intervals allow the identification of the variables most significantly correlated with the variations in the frequencies. The variables found to have a high correlation with frequency are shown in bold in Table 3.7.

The frequencies of the eight different modes considered in this case study are plotted against temperature in Figure 3.11. For the first time, as can be observed from the plots of Figure 3.11, there is identification of a clear trend of reduction in the vibration frequencies with increase in temperature. This is likely a reflection of the 
Table 3.7: Results of regression analysis in environmental variability study [30].

\begin{tabular}{|c|c|c|c|c|c|c|c|c|}
\hline & & $\beta_{0}$ & $\beta_{R M S v}$ & $\beta_{R M S t}$ & $\beta_{T}$ & $\beta_{\delta T x}$ & $\beta_{\delta T y}$ & $\beta_{W i n d s p}$ \\
\hline \multirow{3}{*}{$\frac{N}{\stackrel{N}{I}}$} & $\beta$ & 0.473 & 0.487 & -0.544 & -0.000810 & 0.00250 & 0.00142 & 0.00042 \\
\hline & $95 \%$ c.i. for $\beta$ & 0.009 & 1.407 & 2.634 & 0.000403 & 0.00542 & 0.00125 & 0.000317 \\
\hline & $95 \%$ c.i. for $\beta(\%)$ & 2.0 & 289 & 484 & 50 & 216 & 88 & 76 \\
\hline \multirow{3}{*}{$\stackrel{N}{\stackrel{N}{N}}$} & $\beta$ & 0.677 & 0.867 & -3.897 & 0.000139 & -0.00336 & -0.00224 & 0.000096 \\
\hline & $95 \%$ c.i. for $\beta$ & 0.012 & 1.752 & 3.163 & 0.000559 & 0.00545 & 0.00165 & 0.000425 \\
\hline & $95 \%$ c.i. for $\beta(\%)$ & 1.7 & 202 & 81 & 402 & 162 & 73 & 445 \\
\hline \multirow{3}{*}{ 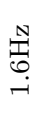 } & $\beta$ & 1.633 & 0.642 & 0.277 & -0.001167 & -0.00991 & 0.00018 & -0.00052 \\
\hline & $95 \%$ c.i. for $\beta$ & 0.043 & 6.720 & 10.007 & 0.001462 & 0.02354 & 0.00425 & 0.00148 \\
\hline & $95 \%$ c.i. for $\beta(\%)$ & 2.6 & 1047 & 3608 & 125 & 238 & 2409 & 284 \\
\hline \multirow{3}{*}{ 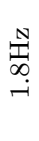 } & $\beta$ & 1.809 & 2.409 & 1.088 & -0.001623 & -0.00358 & -0.00161 & -0.00087 \\
\hline & $95 \%$ c.i. for $\beta$ & 0.030 & 4.680 & 8.100 & 0.001541 & 0.01334 & 0.00426 & 0.00119 \\
\hline & $95 \%$ c.i. for $\beta(\%)$ & 1.6 & 194 & 744 & 95 & 373 & 265 & 137 \\
\hline \multirow{3}{*}{$\begin{array}{l}\stackrel{N}{\mathbb{N}} \\
\infty \\
\stackrel{i}{N}\end{array}$} & $\beta$ & 2.776 & -3.63 & 3.996 & -0.001522 & 0.01133 & -0.00068 & 0.00045 \\
\hline & $95 \%$ c.i. for $\beta$ & 0.034 & 4.937 & 7.981 & 0.001600 & 0.01254 & 0.00443 & 0.00111 \\
\hline & $95 \%$ c.i. for $\beta(\%)$ & 1.2 & 136 & 200 & 105 & 111 & 655 & 244 \\
\hline \multirow{3}{*}{$\underset{\stackrel{N}{F}}{\stackrel{N}{F}}$} & $\beta$ & 3.484 & -4.80 & -4.114 & -0.005784 & 0.02017 & 0.00162 & -0.00176 \\
\hline & $95 \%$ c.i. for $\beta$ & 0.162 & 19.288 & 34.297 & 0.005883 & 0.07341 & 0.01705 & 0.00481 \\
\hline & $95 \%$ c.i. for $\beta(\%)$ & 4.6 & 402 & 834 & 102 & 364 & 1053 & 274 \\
\hline \multirow{3}{*}{$\begin{array}{l}\stackrel{N}{U} \\
\stackrel{+}{+}\end{array}$} & $\beta$ & 4.716 & -21.1 & 28.288 & -0.008383 & 0.01449 & 0.00836 & -0.00142 \\
\hline & $95 \%$ c.i. for $\beta$ & 0.125 & 19.150 & 37.231 & 0.005989 & 0.05114 & 0.01856 & 0.00404 \\
\hline & $95 \%$ c.i. for $\beta(\%)$ & 2.7 & 91 & 132 & 71 & 353 & 222 & 284 \\
\hline \multirow{3}{*}{$\underset{1}{\stackrel{N}{Z}}$} & $\beta$ & 5.165 & 7.829 & -11.649 & -0.004924 & 0.01885 & 0.00253 & -0.00057 \\
\hline & $95 \%$ c.i. for $\beta$ & 0.073 & 11.697 & 21.009 & 0.003836 & 0.03287 & 0.01093 & 0.00265 \\
\hline & $95 \%$ c.i. for $\beta(\%)$ & 1.4 & 149 & 180 & 78 & 174 & 433 & 469 \\
\hline
\end{tabular}

changes in the material properties with temperature, in particular the elastic modulus of concrete. This significant correlation demonstrates the importance of temperature effects on modal properties and that temperature should be considered or filtered in any subsequent damage detection algorithm. 

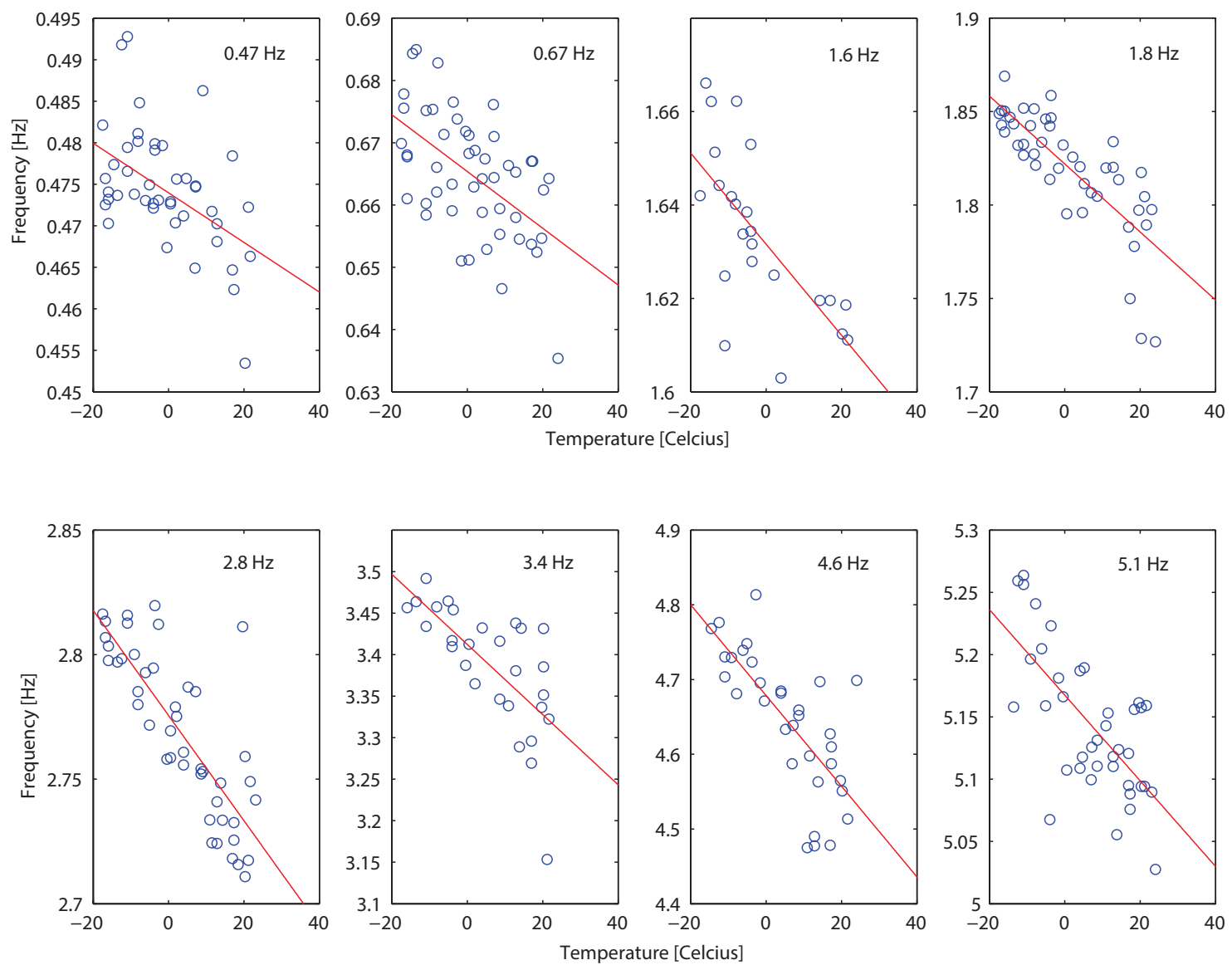

Figure 3.11: Operational modal frequency variation with respect to temperature [30].

\subsubsection{Signal processing platform for analysis of structural health (SPLASH v1.0)}

To properly capture motions of a physical system such as a bridge or building, sampling of information needs to be performed at relatively-high frequencies; in the order of $100 \mathrm{~Hz}$ to $250 \mathrm{~Hz}$. This range of sampling frequency is typically required to prevent aliasing and to permit proper capture of all significant vibration modes of civil structures. These relatively-high sampling rates combined with long-term monitoring 
operations produces large amounts of structural response data that needs to be efficiently managed, processed, analyzed and evaluated. The great amount of collected vibration data shows there is evident need for a sophisticated computer platform for rapid and efficient data management, signal processing, and system identification operations. The development of a Signal processing PLatform for Analysis of Structural Health (SPLASH) is presented in the references $[24,47]$. SPLASH is a centralized network computer platform for all VBSHM operations. What distinguishes this computer platform from others are the sophisticated easy-to-use custom graphical user interfaces (GUI) created for all vibration-based data operations and interactions as shown in Figure 3.12. The visualization modules integrated in the platform are prac-

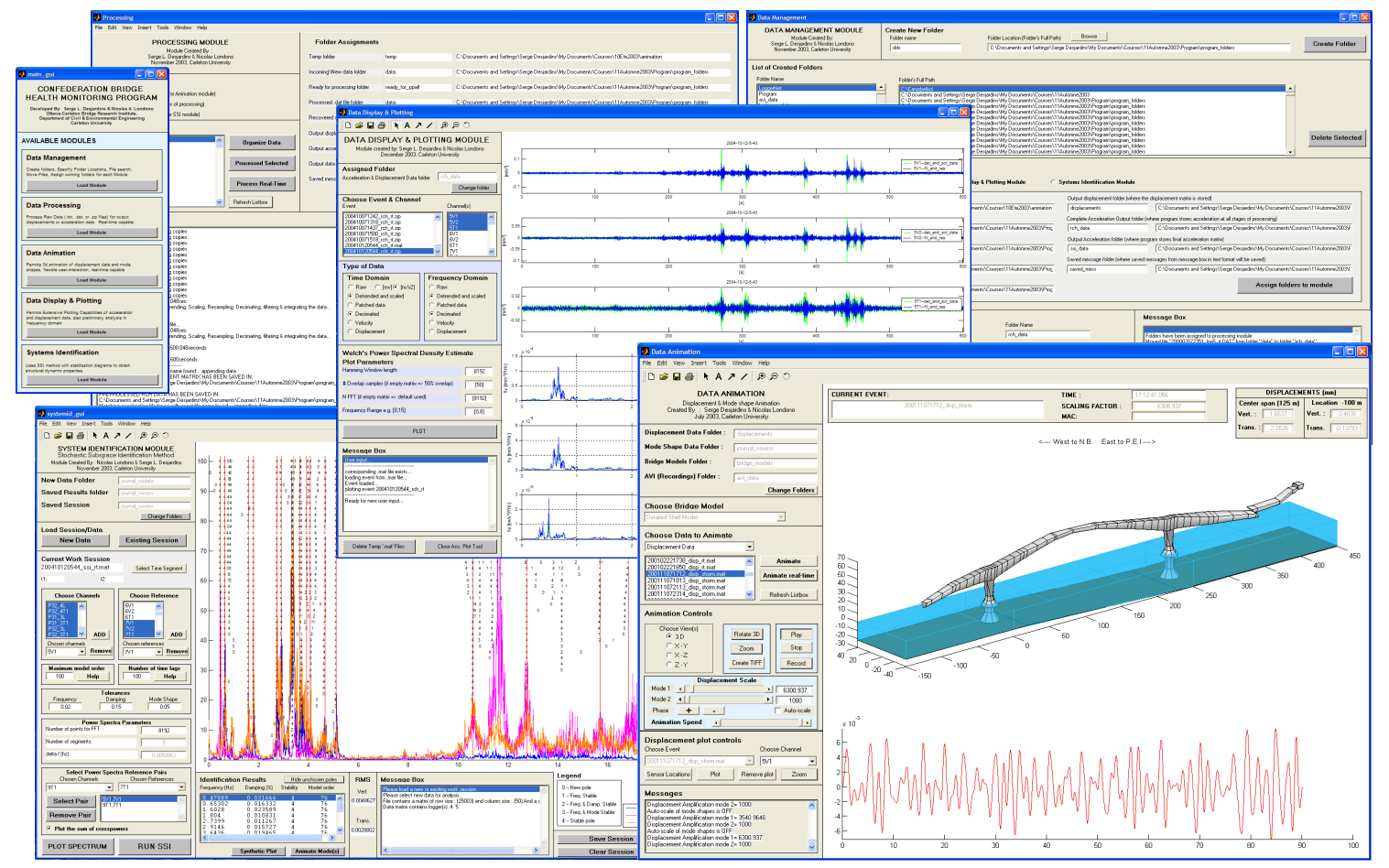

Figure 3.12: Screen-capture of SPLASH modules for Confederation Bridge VBSHM $[24,47]$.

tical and provide useful engineering information. The ability to visualize animation of structural responses (deformations and mode shapes; both extracted and numerical) 
is an asset for any structure's engineer and/or operator. Detailed description of the data processing and of the operational modal analysis modules are included in [24].

\subsubsection{Advanced system identification techniques}

\subsubsection{PolyMax - Polyreference Least Square Complex Frequency Esti- mator (p-LSCF)}

A new system identification module was developed for the application of the newlydeveloped polyreference least square complex frequency algorithm to enhance the real-time automatic data processing and analysis functionality of the monitoring software platform [49]. The new technique, called PolyMax, has the advantage of generating clean stabilization diagrams which facilitates the development of an automated pole selection algorithm. This new algorithm, which reduces the subjectivity of the results, is an important contribution in the goal to achieve a reliable continuous online monitoring due to its automation [49]. It should be stated that in its application, the newly-developed system identification module using PolyMax developed by O'Connell, does not provide the user with proper mode shape estimates [49]. This represents a significant shortcoming for an advanced system identification application module and limits the capabilities of the developed automated pole picking algorithm.

\subsubsection{Comparison study (FDD, ERA, SSI and PolyMax)}

In a comparison study by Rahman [21], four state-of-the-art stochastic system identification methods (the Frequency-Domain Decomposition (FDD), the Eigensystem Realization Algorithm (ERA), the Stochastic Subspace Identification (SSI) and the newly developped PolyMAX method) were compared with a simulated 8-DOF example and with the Confederation Bridge monitoring data. From the comparison of the 
estimated modal parameters, Rahman concluded that the stochastic subspace identification method is the preferred method, since it is more consistent in estimating modal parameter among the four studied methods. However, Rahman notes that none of the methods are robust against less-excited modes and that the mode shape estimation by the PolyMAX method is the victim of comparatively high bias and variance due to biased correlogram spectrum estimation which makes it less than ideal for system identification purposes. Rahman has also created analysis modules for three of the four studied methods (ERA, FDD and PolyMax) the fourth (SSI) having already been implemented in SPLASH.

\subsubsection{Damage detection}

This section resumes the work by Londono [22] and Tehranian [14] on damage detection techniques applied to the Confederation Bridge monitoring data.

\subsubsection{Finite element model updating}

A recently-introduced variant of the finite element model updating method, using socalled "damage functions", has been used as an algorithm for the damage detection simulations presented in [22]. Results from three localized and distributed damage scenarios, show that the variability in the data inevitably leads to some significant errors in the identified damage patterns. However, despite the significant variability in the modal parameters, especially in the mode shapes, Londono [22] states that it is still possible to obtain some useful engineering information regarding the presence and location of damage from the output-only monitoring data using the FE updating approach. To counter the effects of random numerical and environmental noises, the use of multiple independent datasets is necessary, which requires the collection and analysis of relatively-large amounts of monitoring data collected at different times 
and under different conditions for both the "undamaged" and "damaged" structures. This requirement can only be met through continuous long-term monitoring.

\subsubsection{Statistical pattern recognition}

In [14], a recently-proposed approach using time series analysis for statistical pattern recognition is improved to evaluate the validity and effectiveness of using fieldcaptured vibration monitoring data for assessing the performance of bridge structures in the field. The methodology is employed to evaluate the structural conditions of the Confederation Bridge. In this study by Tehranian et al. [14], field-measured data of monitored vibration responses of the bridge under operational conditions are analyzed by the proposed methodology. The applied methodology is a combination of the random decrement averaging method, autoregressive modelling, and Mahalanobis squared distance measure for outlier detection. First, the measured vibration response data are filtered through a random decrement function to obtain pseudofree vibration responses. An autoregressive model is then used to extract a cluster of features for each pseudo-free vibration response data record. To ensure the accuracy of the autoregressive curve fitting technique, a confidence level criterion is enforced to pass only high-quality extracted features. This process is repeated for a group of sensors in each measured dataset triggered by different loading scenarios such as ambient wind, traffic, or ice floe impact. Finally, a database of extracted features is considered as a reference for healthy states, and a Mahalanobis distance function is used to quantify the deviation of each observation from the reference. Although no significant structural changes have been detected, it is shown that this condition assessment method may be used to assure performance of the structure. It is also shown that the method may be used as a complementary tool for health and function monitoring of the sensors and instrumentation of the vibration monitoring system. 


\subsubsection{Research Platform for Smart Facilities Management (RP-SMARF)}

The Research Platform for Smart Facilities Management (RP-SMARF) is a middleware platform whose main object is to facilitate resource and data sharing among researchers within a smart facility domain. The cloud-based research software platform, is the result of a multi-disciplinary collaboration between Carleton University researchers, in the departments of systems and computer engineering, civil and environmental engineering, mechanical and aerospace engineering, and their industrial partner, Solana Networks [50]. It allows researchers to share data-analysis tools, sensor data, and expertise to manage smart facilities. Two projects are currently using RP-SMARF, the first is the Confederation Bridge vibration monitoring project, the second is the industrial and Aerospace Machine Monitoring program at Carleton University's mechanical and aerospace engineering department. Funding for the development of RP-SMARF was provided through CANARIE's Research Software Program. Within the context of RP-SMARF a resource refers to a computing resource (a physical or a virtual computer), a data storage resource refers to files or folders located on a computer and a software application is called a tool. By facilitating resource sharing, researchers are able to access a large and diverse set of resources and data through a network which leads to higher productivity and effectiveness for the various collaborative efforts in the research of smart facilities management.

In order to improve productivity, the platform allows users the ability to attach and detach resources to the platform, to search for the availability of specific types of resources, to reserve resources for a specific period of time, and to transfer data between resources. By connecting resources to the platform these resources become network enabled and can be accessed by anyone with access to the network and 
appropriate authorization. Data collected within such a domain can be considered sensitive; the system therefore guarantees that access to the data is given to those who are authorized to access the data. A similar requirement is handled in the case of resources; as resource access may be limited to certain individuals. Furthermore, only certain subsets (files/folders/software) of resources may be available for access; this platform provides mechanisms for allowing resource owners to specify who can access data/resources and which parts can be accessed. The Confederation Bridge vibration monitoring project can produce data in real-time hence the platform is responsible for handling both static and real-time data. The platform is also able to monitor the health and status of its own services which can be viewed by a system administrator for finding and dealing with system errors.

\subsection{Summary}

The current and the future work on the Confederation Bridge vibration monitoring project will build on the work of others, it is therefore critical that the past work on this project is properly understood and as such this work has been summarized in this section. Early work focused on the bridge's behaviour under various environmental and controlled loading scenarios. The dynamic characteristics obtained at this time were extracted using preliminary system identification techniques to understand the bridge behaviour and to verify its design parameters. The first identification techniques used peak picking of the averaged Fourier spectra to determine modal frequencies and band pass filtering around peaks for modal displacement time histories (mode shapes) [42]. A limited number of datasets of varying durations were used to determine the initial dynamic behaviour of the bridge. A more advanced identification technique, the stochastic subspace identification algorithm, was then used to 
obtain more accurate and reliable modal parameters from the response data, first in a commercially-available software [44], then in an improved custom Matlab ${ }^{\circledR}$ application specifically created for the project [28]. The subsequent studies used datasets of longer durations (10 minutes) and were instrumental in confirming design parameter. Table 3.3 shows results of these preliminary studies.

The variability of the estimated modal properties, which was observed during the previous studies, was then quantified by Londono et al. [30,46]. The first of these studies established a baseline variability of $0.6 \%$ standard deviation of identified frequencies from ten data sets of similar loading scenarios and environmental conditions which shows that it is possible to retrieve highly consistent modal frequencies. The second study established a general variability of 1.1 to $2.2 \%$ standard deviation of identified frequencies using 42 datasets of varying loading and environmental conditions (see Table 3.6).

Subsequent studies by O'Connell [49] and Rahman [21] implemented newer OMA techniques in an attempt to improve the accuracy of the modal parameter estimations. In a comparison study, Rahman demonstrated that the stochastic subspace identification technique was the preferred method as compared with three other established methods, since it is more consistent in estimating modal parameters. Having demonstrated that consistent and accurate identification of modal parameters was possible with SSI, subsequent studies focused on damage detection. Among the many existing damage detection techniques, Londono [22] focused on finite element model updating while Tehranian [14] focused on statistical pattern recognition. Both studies showed promising results while clearly highlighting the challenges of using real in-operation response data due to variability and uncertainty of captured responses.

Throughout these studies, analytical models of a representative portion of the bridge were created and used for design verification and for model calibration and 
updating; the latest of which is a fine meshed model created in Abaqus ${ }^{\circledR}$ in 2014 (Figure 3.8). Many of the described studies (specifically those undertaken after 2003) were carried out using the tools and the modules included in the SPLASH platform which was originally developed for the Confederation Bridge vibration monitoring project in $2003[24,47]$.

Natural progression requires that all current and future work on the Confederation Bridge vibration monitoring project consider the following points:

- Having been proven as a robust and reliable identification technique, the stochastic subspace identification shall be used with improvements and refined parameters as described in Chapter 2;

- Having been used since 2003, the SPLASH platform has received plenty of constructive feedback. The SPLASH platform has therefore undergone a significant upgrade for its third version as described in Chapter 5;

- With updated tools and automation, results can be obtained on a much larger scale. Many more datasets shall be processed and analyzed, with all results consolidated in a complete historical database (Chapter 6);

- Since a proven damage detection technique has yet to be implemented in this project, new damage detection techniques based on quantifying and filtering uncertainties and variabilities are developed as described in Chapter 7. 


\section{Chapter 4}

\section{Implications of Instrumentation Renewals on Established Long-term Vibration-based Monitoring Programs}

With the completion of the Bridge and the experiences of its successful monitoring operation since 1997, there is the opportunity to not only assess and verify the behaviour and performance of the structure but also of the monitoring system itself. Since the original monitoring system underwent a full life cycle, there is an opportunity to assess its performance and propose improvements for its second generation. In this chapter, a brief description of the renewal of the instrumentation system is presented. Details on the requirements for the renewed system and its implications on data integrity and continuation are discussed.

For example, one of the most pressing issues arising from this first generation of the monitoring system is the need for rapid and on-line self diagnostic of the monitoring system especially as the system approaches the end of its service life. New techniques have been developed to monitor the state of the installed instruments. These tools,

which will aid in proper and timely maintenance of the monitoring system to avoid disruption of continuity of data collection, are detailed in Chapter 5. 
In Section 4.1 the observations and the gained experience on the functioning of the first generation of the vibration monitoring system is discussed. The renewal of the vibration monitoring instrumentation is presented in Section 4.2 and the redevelopment of the data management, processing and analysis tools are detailed in Chapter 5.

\subsection{Experience gained from a first generation long- term vibration monitoring system}

In this section several key lessons learned from the operation of the first generation of the vibration-based monitoring system installed on the Confederation Bridge are described.

\subsubsection{Instrumentation}

With a better understanding of the bridge behavior and of the current and future research needs, some improvements to the instrumentations were noted during the operation of the first generation of the monitoring system. It should be noted that this first generation performed well over the 15 years of operation, generated large amounts of useful data and led to important insightful research results. Some issues with sensor and logger malfunctions were noted, but these are not uncommon for systems of this size and are generally- expected especially near the end of service life.

With regard to sensor locations, the only issue of note was the positioning of the underwater sensors at the base of piers 30 to 32 (i.e. locations 1 and 2 in Figure 3.4). These sensors located below the water line malfunctioned after a few years of operation and were omitted from all subsequent vibration responses. 
Three different types of sensors were included in the original configuration of the vibration monitoring system with detailed specifications shown in Table 3.2. A combination of servo and piezo electric accelerometers were used. This setup while adequate, created some complications, especially in post-processing (e.g. extra filtering was required for the QA 1200 servo accelerometers). Also, the servo accelerometers required extra filtering prior to the analog to digital (A/D) conversion. In these cases a low-pass Bessel filter was used prior to sending the analog signal to the data logger. To reduce extra computations and to avoid introducing extra variability and noise in the vibration responses, future monitoring systems should employ a single type of sensor and avoid extra filtering steps prior to A/D conversion. This should improve data consistency and avoid measurement bias in the vibration responses. With a better idea of the range of accelerations expected on the bridge deck, the choice of accelerometers can be optimized. With maximum accelerations below $0.5 \mathrm{~g}$ and with ambient vibrations at much lower levels, sensors with adequate range and better resolution should be selected. This would permit higher quality analysis results from low level vibration responses.

\subsubsection{System diagnostics}

The most pressing issues arising from the first generation of the monitoring system is the need for rapid and on-line self diagnostics of the monitoring system. As the first generation of the system approached the end of its service life, sensors were failing at greater rate and data acquisition and transmission issues were more frequent. Typically these issues were identified manually through visual inspections of processing results. Experienced analysts familiar with the bridge's behavior could identify problematic sensors by observing their time history signals and their associated frequency-content. If erratic behavior of a signal was observed over several 
datasets, the information was relayed to the bridge operator who could then physically inspect and hopefully fix the issues at the bridge site in a timely manner. These faulty signals were then manually removed from the processing results prior to further analysis. This method proved to be very time consuming and prone to human errors. Sometimes, faulty signals were not properly identified and therefore contaminated analysis results. Data logger issues were also identified manually by the analyst and corrected in the same manner. It is clear that automated sensor and logger diagnostic tools are required to improve data collection and processing efficiency, especially if these operations were to be scaled with continuous monitoring and/or higher sampling rates. The development of new automated tools to monitor the state of the installed instruments will improve the timely maintenance of the monitoring system to avoid disruptions in data collection. These tools are detailed in Chapter 5 .

\subsubsection{Collection parameters}

The data collection schemes have varied greatly during the first generation of the monitoring system. Data collection started shortly after bridge opening in February of 1998. The collection of the vibration responses has been managed by the Ottawa Carleton Bridge Research Institute (OCBRI) housed at Carleton University in Ottawa, Canada. For the past 20 years, graduate students and post-doctoral fellows within OCBRI had the responsibility of managing all aspects of vibration data collection, from sampling rates, length of recordings, collection triggers, collection frequency, data storage and backup. Vibration data are mainly collected via three high speed data loggers numbered 1, 4 and 5 located in the bridge piers as shown in Figure 3.4. A fourth logger, logger number 6, collected vibration data briefly from February to June of 1998. Data collection parameters have varied throughout the years; while a sampling rate of $125 \mathrm{~Hz}$ is most common, sampling rates of $100 \mathrm{~Hz}, 166 \mathrm{~Hz}$ and $200 \mathrm{~Hz}$ 
have been used in the past. The length of recordings has also varied greatly, from 30 seconds to 10 minutes. Shorter duration datasets were mainly collected in the first 10 years of the vibration monitoring program. While data collection can be triggered manually (and has been in the past), most of the data collection operates under automated trigger scenarios. Trigger routines are programmed in the logger codes by researchers at OCBRI. The specified thresholds for triggering have varied throughout the past 20 years, according to the needs of on-going research. Consequently, the quantity of data collected has also varied as discussed later in Section 6.1.3. Also, data collection has not always been synchronized across loggers due to a variety of factors, which include faulty or unspecified trigger routines, erratic trigger signals, and choices made by various researchers according to their research needs (where only a subset of sensor signals were required).

These highly variable collection parameters (recording durations, sampling rates, collection frequency, trigger thresholds, combinations of loggers and sensors, etc.) lead to multi-state and multi-scale response data that need to be properly processed prior to any analysis operations. There is therefore an evident need to automate processing to recognize and deal with these multi-state and multi-scale response data to ensure data integrity and continuity for proper interpretation of results. Also, future data collection paradigms should not only consider the needs of current research but also the needs of future works. In this sense, continuous collection with higher sampling rates is considered for the design of the second generation of the vibration monitoring system, with all that it implies in regards to data management and storage.

\subsubsection{Data synchronization}

Due to the relatively high number of accelerometers (58) included in the monitoring system of the Confederation Bridge, the vibration response data are recorded by 
several high speed data loggers. For this reason data synchronization becomes a very critical and sometimes complex post-processing operation. For most operational modal analysis techniques, it is critically important that the responses across all sensors are synchronized. Although each logger attaches a time stamp to the acquired signals, it is not prudent to rely on these values for synchronization.

During the early stages of the monitoring program, the individual sensor responses, when collected across several loggers, were synchronized based on the triggering signal (when present). This trigger signal, which is sent from a single logger to the other loggers when a set condition is met (usually when a response amplitude threshold is surpassed), is assumed to be instantaneous. By ignoring the individual logger's timestamps and aligning the trigger signals from each of the loggers, a complete response matrix representing a synchronized dataset is assembled by the processing engine. While this method performed reasonably well, issues did arise when the logger clocks deviated significantly from one another (in some cases by several hours). It should be noted that logger tables are stored in text files named according the logger's internal time clocks. Using the internal clock times in the data tables makes it very difficult to properly synchronize logger files from different loggers during processing. Also, as stated in Section 4.1.3, trigger routines and trigger signals were not consistently present during the collection schemes of the first generation of the monitoring system. When the trigger signal between loggers is absent, due to erroneous triggering routines or due to faulty trigger signals, it becomes very difficult to accurately synchronize the response data.

From these observations, it is evident that there is a need for a proper automated robust synchronization algorithm to properly assemble full datasets of response data. Combined with proper triggering (or by including a separate repeating synchronization signal between loggers) and by resetting the logger clocks in a timely manner, the 
synchronization algorithm described in Section 5.2.2 creates complete synchronized datasets.

\subsubsection{Data management}

During the operation of the original monitoring system, it was clear that data management was a very important component of the vibration monitoring program. It is now well understood that for vibration response measurements, where the sampling rates are relatively high $(>100 \mathrm{~Hz})$ and the recordings are relatively long $(\approx 10 \mathrm{~min})$, raw data accumulates quickly, and depending on collection frequency, this data can become unmanageable without the proper tools. Also, depending on the processing protocols, this data can multiply very quickly during the processing and analysis phases, where copies of data at different stages are stored for future troubleshooting purposes. Section 6.1 provides some data collection statistics for the first and second generation of the Confederation Bridge vibration monitoring program.

With a growing database of raw and processed data, it becomes very difficult to search for specific datasets. Depending on the research needs, datasets corresponding to certain environmental or operational scenarios may have been required for further analysis. Significant effort was required to manually search for and to identify these datasets inside the database. For this reason, a systematic approach of collecting and storing metadata at all stages of processing and analysis was determined to be of paramount importance. Combined with a relational database structure and a large volume networked or cloud storage solution, the monitoring program will be better equipped to manage the ever increasing data volume. Especially if higher sampling rates and continuous monitoring conditions are envisaged. 


\subsubsection{Automation of processing and analysis}

Automation of all processing and analysis steps is a near necessity for a long-term or permanent vibration monitoring system, as the large amount of response data generated cannot be manually-processed and analyzed in a timely manner. During the first generation of the monitoring system, significant effort was focused on the development of efficient and robust processing and analysis tools as described in Section 3.3.3. While these tools were necessary and very useful, they were not developed with automation in mind. Many of the steps required user interaction, especially during the analysis phase. For the second generation of the monitoring system, all processing and analysis steps shall be automated.

\subsection{Instrumentation Renewal}

Having been in operation for approximately 15 years, the first generation of the monitoring system was showing signs of reaching the end of its service life. Sensors were failing at greater rate and data acquisition and transmission issues were more frequent. Recent advances in sensor and data acquisition technologies allow a more streamlined approach to the data acquisition process as compared to the original system. Also, the technological advancements with regards to processing, storage and data transmission permit increases to the collection and sampling rates to accommodate current and future analysis techniques.

From December 2012 to March 2014, the vibration monitoring system of the Confederation Bridge underwent an instrumentation renewal. During this renewal, all accelerometers were replaced and new data loggers were installed. The replacement of the data loggers and their associated accelerometers occurred on the dates indicated in Table 4.1. 
Table 4.1: On-line dates for the replacement high speed data loggers.

\begin{tabular}{ccc}
\hline Logger identification & & On-line date \\
\hline HSL \# 1 & & $2013-07-08$ \\
HSL \# 4 & $2012-12-15$ \\
HSL \# 5 & $2014-03-16$ \\
\hline
\end{tabular}

The vibration sensors used in the original monitoring system included both piezoelectric accelerometers and servo accelerometers. These sensors have been completely replaced with new piezo-electric accelerometers. The location of the sensors on the bridge remain unchanged and are listed in Table 4.2 and shown in Figure 3.4. However the channel numbering for each channel inside the logger tables has changed and is well documented in Table 4.2. It should be noted that during renewal, sensors located inside the piers below the water line were not replaced. These locations are marked as "decommissioned" in Table 4.2. Also of note is the removal of the high-speed data logger at pier 33 (data logger No. 6). Sensors at location 3 and 4 on pier 33 have been connected to the new logger at pier 32 (data logger No. 5).

The types and specifications of accelerometer sensors used in the original and in the new monitoring systems are listed in Table 4.3. The original piezo-electric accelerometers were PCB Model 393C, and the original servo accelerometers were Allied Signal QLC 400 and QA 1200. The new sensors are all Dytran model 3191-A1.

In the original vibration monitoring system, the measured analog accelerometer signals were conditioned and filtered for anti-aliasing by 8-pole $50 \mathrm{~Hz}$ low-pass Bessel filters. Signals were then sampled and digitized by a network of distributed highspeed data loggers before being sent to on-site computers and transmitted back to the research team at OCBRI for data archival and research. The data loggers were CR9000 with 16 Bit 100kHz A/D conversion from Campbell Scientific. 
Table 4.2: Sensor location and channel numbering with make and model of original and new accelerometers.

\begin{tabular}{|c|c|c|c|c|c|c|c|c|c|c|}
\hline \multirow{2}{*}{$\begin{array}{l}\text { Pier } \\
\text { (Data } \\
\text { Logger } \\
\text { Number) }\end{array}$} & \multirow{2}{*}{$\begin{array}{l}\text { Loca- } \\
\text { tion }^{*}\end{array}$} & \multicolumn{5}{|c|}{$\begin{array}{l}\text { Channel numbering inside data logger tables } \\
\text { (original) }\end{array}$} & \multicolumn{2}{|c|}{ Original sensors } & \multicolumn{2}{|c|}{ New sensors } \\
\hline & & $\mathrm{T} 1$ & $\mathrm{~T} 2$ & $\mathrm{~L}$ & V1 & $\mathrm{V} 2$ & $\begin{array}{l}\text { Accelerometer } \\
\text { model }\end{array}$ & Scale V/g & $\begin{array}{l}\text { Accelerometer } \\
\text { model }\end{array}$ & Scale V/g \\
\hline \multirow{5}{*}{$\begin{array}{l}\text { P.30 } \\
(\text { No.1) }\end{array}$} & 1 & $-(7)$ & & $-(8)$ & $-(9)$ & & PCB 326 A03 & 1.00 & \multicolumn{2}{|c|}{ Decommissioned } \\
\hline & 3 & $9(12)$ & & $10(13)$ & & & QLC 400 & 2.66 & Dytran 3191A1 & 10.0 \\
\hline & 4 & $7(10)$ & & $8(11)$ & & & QLC 400 & 2.66 & Dytran 3191A1 & 10.0 \\
\hline & 16 & & $3(3)$ & & $1(1)$ & $2(2)$ & PCB 393C & 1.00 & Dytran 3191A1 & 10.0 \\
\hline & 17 & & $6(6)$ & & $4(4)$ & $5(5)$ & PCB 393C & 1.00 & Dytran 3191A1 & 10.0 \\
\hline \multirow{11}{*}{$\begin{array}{l}\text { P.31 } \\
(\text { No.4) }\end{array}$} & 1 & $-(1)$ & & $-(2)$ & $-(3)$ & & PCB 326 A03 & 1.00 & \multicolumn{2}{|c|}{ Decommissioned } \\
\hline & 2 & $-(4)$ & & $-(5)$ & $-(6)$ & & PCB 326 A03 & 1.00 & \multicolumn{2}{|c|}{ Decommissioned } \\
\hline & 3 & $1(7)$ & & $2(8)$ & & & QA 1200 & 99.0 & Dytran 3191A1 & 10.0 \\
\hline & 4 & $3(9)$ & & $4(10)$ & & & QA 1200 & 99.0 & Dytran 3191A1 & 10.0 \\
\hline & 10 & & $23(30)$ & & $22(29)$ & $24(31)$ & PCB 393C & 1.00 & Dytran 3191A1 & 10.0 \\
\hline & 11 & & $19(26)$ & & $21(28)$ & $20(27)$ & QLC 400 & 2.66 & Dytran 3191A1 & 10.0 \\
\hline & 12 & & $8(15)$ & & $9(16)$ & $7(14)$ & PCB 393C & 1.00 & Dytran 3191A1 & 10.0 \\
\hline & 13 & & $10(17)$ & & $12(19)$ & $11(18)$ & QLC 400 & 2.66 & Dytran 3191A1 & 10.0 \\
\hline & 14 & & $13(20)$ & & $14(21)$ & $15(22)$ & PCB 393C & 1.00 & Dytran 3191A1 & 10.0 \\
\hline & 15 & & $-(23)$ & & $18(25)$ & $17(24)$ & QLC 400 & 2.66 & Dytran 3191A1 & 10.0 \\
\hline & 18 & $5(11)$ & & $6(12)$ & $16(13)$ & & QA 1200 & 99.0 & Dytran 3191A1 & 10.0 \\
\hline \multirow{11}{*}{$\begin{array}{c}\text { P.32 } \\
(\text { No.5) }\end{array}$} & 1 & $-(1)$ & & $-(2)$ & $-(3)$ & & PCB 326 A03 & 1.00 & \multicolumn{2}{|c|}{ Decommissioned } \\
\hline & 2 & $-(4)$ & & $-(5)$ & $-(6)$ & & PCB 326 A03 & 1.00 & \multicolumn{2}{|c|}{ Decommissioned } \\
\hline & 3 & $10(10)$ & & $11(11)$ & & & QA 1200 & 99.0 & Dytran 3191A1 & 10.0 \\
\hline & 4 & $16(16)$ & & $15(15)$ & & & QA 1200 & 99.0 & Dytran 3191A1 & 10.0 \\
\hline & 5 & $24(24)$ & & & $23(23)$ & $25(25)$ & PCB 393C & 1.00 & Dytran 3191A1 & 10.0 \\
\hline & 6 & $28(28)$ & & & $26(26)$ & $27(27)$ & PCB 393C & 1.00 & Dytran 3191A1 & 10.0 \\
\hline & 7 & $30(30)$ & & & $29(29)$ & $31(31)$ & PCB 393C & 1.00 & Dytran 3191A1 & 10.0 \\
\hline & 8 & $32(32)$ & & & $34(34)$ & $33(33)$ & PCB 393C & 1.00 & Dytran 3191A1 & 10.0 \\
\hline & 9 & $20(20)$ & & & $21(21)$ & $22(22)$ & QLC 400 & 2.66 & Dytran 3191A1 & 10.0 \\
\hline & P33_3 & 37 & & 38 & & & & & Dytran 3191A1 & 10.0 \\
\hline & P33_4 & 35 & & 36 & & & & & Dytran 3191A1 & 10.0 \\
\hline \multirow{3}{*}{$\begin{array}{c}\text { P.33 } \\
\text { (No.6) }\end{array}$} & 1 & $-(1)$ & & $-(2)$ & $-(3)$ & & PCB 326 A03 & 1.00 & \multirow{3}{*}{\multicolumn{2}{|c|}{$\begin{array}{c}\text { Decommissioned } \\
\text { Moved to logger No. } 5 \\
\text { Moved to logger No. } 5\end{array}$}} \\
\hline & 3 & $-(4)$ & & $-(5)$ & & & QLC 400 & 2.66 & & \\
\hline & 4 & $-(6)$ & & $-(7)$ & & & QLC 400 & 2.66 & & \\
\hline
\end{tabular}


To accommodate the new Dytran sensors, the three high-speed data loggers are replaced with the newer Campbell Scientific CR9000X. The Dytran sensors are directly connected (via coaxial cables) to new CR9052IEPE input modules on the CR9000X high-speed data loggers. The A/D conversion (16 Bit 100kHz), the signal conditioning and the anti-alias filtering is carried out by the data loggers and its input modules. By removing the low-pass Bessel filters, the acquisition scheme was streamlined and an additional possible source of measurement noise was eliminated.

Table 4.3: Specifications for accelerometers located on the Confederation Bridge.

\begin{tabular}{|c|c|c|c|c|}
\hline & \multicolumn{3}{|c|}{ Original sensors } & \multirow{2}{*}{$\begin{array}{c}\text { New sensors } \\
\begin{array}{c}\text { Dytran 3191A1 } \\
\text { (piezo-electric) }\end{array}\end{array}$} \\
\hline & $\begin{array}{c}\text { PCB 393C } \\
\text { (piezo-electric) }\end{array}$ & QLC 400 (servo) & QA 1200 (servo) & \\
\hline $\begin{array}{l}\text { Principal } \\
\text { location }\end{array}$ & along girder & along girder & $\begin{array}{l}\text { in piers (initially } \\
\text { for ice floe } \\
\text { monitoring) }\end{array}$ & $\begin{array}{c}\text { at all locations (see } \\
\text { Table } 3.1 \text { ) }\end{array}$ \\
\hline Sensitivity & $1 \mathrm{~V} / \mathrm{g}$ & $2.66 \mathrm{~V} / \mathrm{g}$ & $99.0 \mathrm{~V} / \mathrm{g}$ & $10 \mathrm{~V} / \mathrm{g}$ \\
\hline $\begin{array}{l}\text { Frequency } \\
\text { range }\end{array}$ & $\begin{array}{c}0.01-1200 \mathrm{~Hz} \\
( \pm 10 \%)\end{array}$ & $\begin{array}{c}0-300 H z \\
( \pm 10 \%)\end{array}$ & $\begin{array}{c}0-300 H z \\
( \pm 10 \%)\end{array}$ & $\begin{array}{c}0.08-1000 H z \\
( \pm 5 \%)\end{array}$ \\
\hline $\begin{array}{l}\text { Amplitude } \\
\text { range }\end{array}$ & $\pm 1.5 g$ & $\pm 10 g$ & $\pm 25 g$ & $\pm 0.5 g$ \\
\hline Resolution & $0.1 m g$ & $0.01 \mathrm{mg}$ & $0.005 \mathrm{mg}$ & $0.003 \mathrm{mg}$ \\
\hline $\begin{array}{l}\text { Operational } \\
\text { temperature } \\
\text { range }\end{array}$ & $-54^{\circ} \mathrm{C}$ to $93^{\circ} \mathrm{C}$ & $-20^{\circ} \mathrm{C}$ to $71^{\circ} \mathrm{C}$ & $-54^{\circ} \mathrm{C}$ to $107^{\circ} \mathrm{C}$ & $-51^{\circ} \mathrm{C}$ to $121^{\circ} \mathrm{C}$ \\
\hline
\end{tabular}

The vibration data acquisition system operates in continuous buffered data collection mode, which upon triggering by detection of specific dynamic events, such as heavy traffic signals, high winds, ice floe impacts or earthquakes, or simply upon user request, store time history response data in data tables in memory. Otherwise, only statistic information determined from the time history data, such as mean, maximum, minimum and standard deviation etc., are stored to establish long-term trends in the behavior of the bridge. 
The triggered data tables, typically sampled at $125 \mathrm{~Hz}$, are stored temporarily in logger memory until retrieval at specified scheduled intervals from a remote network computer assigned to the control and operation of each logger. The logger computer retrieves the data from the data logger and sends them to a centralized platform where they are made accessible over the Internet to researchers at OCBRI, for data processing and analysis. Data acquisition software is installed in each logger control computer and can be remotely accessed for system control or maintenance. This software controls the acquisition of data from the sensors connected to the dataloggers and the collection of data from the loggers to the control computers. It also permits editing of the logger programming code to modify the functioning of the data acquisition. Details of the original monitoring system setup have been described in the references $[37,39]$.

With the completion of the Bridge and the experiences of its successful vibration monitoring since 1997, there is the opportunity to not only assess and verify the behavior and the performance of the structure but also of the monitoring system itself. Important lessons have been learned, and the experiences described in this section lead to significant improvements to the monitoring, the collection, the management, the processing and the analysis of the response data of this important long-term vibration-based structural health monitoring project. These improvements are further discussed in Chapter 5. 


\section{Chapter 5}

\section{Redevelopment of the Specialized Application Platform for VBSHM}

\subsection{Practical Considerations for SPLASH Update}

Since the monitoring system underwent a recent instrumentation renewal, a reflection on the current SPLASH application was required. Just as a monitoring system requires renewal, so do the tools built around them. New advances in computing, in storage capacities, and in communications render some tools inefficient or obsolete. Moreover, having been utilized by a multitude of researchers, much feedback has been received over the past decade in order to improve or to build upon this application.

Significant effort was put on the redevelopment of the SPLASH application and is one of the author's significant contributions to the Confederation Bridge monitoring project. The third version of the SPLASH application is complete. A software requirement specification (SRS) has been drafted to detail the requirements for the development for this new (and for future versions) of the software. The requirements, as defined by the author and based on recommendations of previous users of the application, are stated below. 
The technical requirements are:

- The application should run on all platforms (i.e. write once run anywhere WORA). For the current Matlab based application, this implies compiling the program.

- The eventual integration in a web and/or cloud based application (with a front end web browser or a stand alone client application with back end computing on host server) must be anticipated and considered while coding the current version.

- The application should make use of parallel computing. This requires the updating of the code to make full use of new powerful machines with multiple processing cores.

The functional requirements are:

- For eventual continuous on-line monitoring, all of the processing and analysis operations should be automated, with minimal user input or interactions. This implies significant improvements to the current OMA techniques and integration of the new AI-FSI algorithm described in Section 2.2.

- In order to analyze large amounts of historical data, the operations must be able to run automatically and in batch mode. This means that the application must also be able to background process large amounts of off-line data.

- To ensure data continuity and integrity any changes made to the application must be backward compatible. Meaning that the third version of the SPLASH application must maintain the capabilities of managing, processing and visualizing multi-state and multi-scale data captured by the previous and the current generation of the monitoring system.

- For the large amounts of raw and processed data, the platform must create, update and maintain an organized and intuitive database of all vibration response 
data.

- To properly organize and query this database, metadata must be extracted from the data at all stages of operations.

- New capabilities must be integrated in the third version of the application in order to monitor the proper functioning of the monitoring system itself.

- The processing modules must be adaptive with respect to the sensor conditions, meaning faulty sensors or loggers must be identified and excluded from the analysis results automatically.

- The redevelopment of this application must ensure that all operations are implemented for general use. This means minimizing static built-in definitions of parameters so that the applications can be used for other vibration monitoring projects with minimal setup procedures.

- The integration of data other than vibrations (i.e. environmental data such as temperatures and wind speeds) into the platform's database should be considered.

The implementation of the above requirements in the third version of the SPLASH platform has great implications for the studies presented in this thesis.

- The ability to background process off-line data permits the creation of an historical database of raw and processed data. Queries of this database are now possible using the metadata, for targeted research purposes. The ability to filter data depending on the type of research is a powerful tool for ongoing and future studies. For example, there may be a need to find all the available datasets of minimum 10 minute durations containing signals from specified sensors with ambient air temperatures between $0^{\circ} \mathrm{C}$ and $10^{\circ} \mathrm{C}$ in the past 10 years. Previously, this relatively simple task required significant human effort.

- To understand the variability of the identified signal features or of the estimated 
modal parameters, previous studies have focused on environmental conditions and have found significant correlations. The integration of this environmental data in the platform greatly facilitates studies of this kind.

- Faster and more efficient computing permits a drastic increase in the quantity of processed datasets to better understand and quantify uncertainties and variability in the estimated modal properties. The proposed approach for condition assessment presented in Chapter 7 uses regression and filtering of the estimated modal parameters in time with dependent variables such as signal amplitude, temperature, wind, traffic, etc. For some regression and filtering techniques, more data means better understanding of variability and uncertainty.

- It should be noted that many of the processing algorithms were developed more than 15 years ago and had to deal with a major constraint: computer processing limits. Many operations were limited by computer processing capabilities. Some data operations were originally implemented with the only objective of reducing computational requirements all the while introducing possible sources of uncertainties and variability in the results. For example, while preprocessing the data, some filtering and decimation of the signals was carried out to reduce computational load. Also, in the previous implementation of the system identification algorithm, a small subset of reference sensors is used (instead of all available sensors) to reduce computing time. This simplification can have significant impact on identification results. The effect of using a reference subset of sensors for identification is most evident on the mode shape of the $1.32 \mathrm{~Hz}$ mode, where the measurements from the pier sensors were not chosen as reference channels (Figure 3.10). 


\subsection{SPLASH v3.0}

In this section the development of the third version of the SPLASH platform is described.

\subsubsection{Database management module}

A significant shortcoming of the previous versions of SPLASH was its manual folder based data management paradigm. This methodology which consisted of manually specifying locations for data storage was not intuitive and was not as robust as desired. One of the first significant changes to the application is the implementation of a database management module that effectively replaces two modules of the previous version of SPLASH: the data management module and the processing module.

\subsubsection{Database creation}

The main function of the new database module is the creation of databases. The steps for creating a new database, which are described below, can also function in batch mode when the user selects a directory containing raw data. After selection, all the preprocessing and processing operations are automatically carried out in the background by the engine. The following operations are completed, in order:

- In a first step, a database structure is created. It should be noted that many database solutions have been considered for storing data at all stages of processing, including relational database management systems (RDMS) such as SQL. It is found that one important limitation of RDMS is the size of the data files that can be included inside the database; it has been shown that for large data files, file systems perform better than RDMS [51]. The referenced study indicates that if objects are larger than one megabyte on average, NTFS file 
systems have a clear performance advantage over relational databases like SQL. Based on these findings, the database structure consists of a series of statically named folders under a single directory of the user's choosing as shown in Figure 5.1 .

\begin{tabular}{|c|c|c|c|}
\hline \multicolumn{4}{|c|}{$\begin{array}{l}\text { Documents library } \\
\text { DB LoadTest }\end{array}$} \\
\hline Name & Date modified & Type & Size \\
\hline 1.3. 01-identified raw & 11/05/2018 10:07 AM & File folder & \\
\hline D. 02-organised raw & 11/05/2018 10:07 AM & File folder & \\
\hline 13. 03-translated & 11/05/201810:07 AM & File folder & \\
\hline D. 04-processed & 26/06/2016 8:35 PM & File folder & \\
\hline 05-systemid & 26/06/2016 8:35 PM & File folder & \\
\hline 13. 97-log files & 26/06/2016 8:35 PM & File folder & \\
\hline 138-backup & 11/05/201810:07 AM & File folder & \\
\hline 1. 99-temp & 11/05/2018 10:07 AM & File folder & \\
\hline
\end{tabular}

Figure 5.1: Database folder structure (SPLASH v3.0).

- A subroutine recursively searches through all the folders and subfolders under the user specified directory of raw data and gathers all the found files. Each file is verified in order to identify files of proper format which correspond to text files containing the required data acquisition information in the header. All properly identified files are then saved in the corresponding folder in the database structure (01-identified raw).

- A subroutine written in java language then organizes all properly identified data files into ASCII files of contiguous time segments and renames the files based on the start time (according to first time stamp of the logger tables) and the originating logger number (for example: 201606221818_log1.dat). When gaps in data are found, the data files are separated in distinct data files and renamed accordingly. These organised files are then stored in the corresponding folder 
of the database structure (02-organised raw).

- The next subroutine translates the data files into proprietary . mat files for faster importing into the Matlab environment [52]. This task, which was historically computationally intensive in Matlab, used to rely on an old Fortran function which has since become obsolete. With improved performance and more efficient coding, this function is now completed inside the Matlab environment. This is noted here because of its implication for future compiling of the application (previous attempts were unsuccessful because of the presence of the Fortran code). While translating, the subroutine also stores some preliminary metadata as described in Section 5.2.4.

- After translation, integrity checks are completed on the data files. Separate subroutines complete the following tasks: duplicate files are removed, duplicate records are truncated from data files and data files are merged if their time stamps are contiguous. Translated data files are then stored in their assigned folder in the database structure (03-translated).

- It should be noted that up to this point in the database creation, no data operations is carried out on the data itself. Once the data translation is complete, the pre-processing of the data commences. A subroutine is called which identifies synchronized data files from separate loggers in order to build complete data events of all sensor measurements. The synchronization is based on trigger signals or on synchronization signals sent and received by the loggers and stored in the data tables. Section 5.2.2 provides additional details on the data synchronization. Unsynchronized files are kept and can be further processed if desired.

- Once the synchronized files are identified they are sent to the main data processing engine. This important subroutine is significantly streamlined compared 
to its previous version (Section 5.2.3 provides additional details on the processing operations). Processed files are stored in the corresponding folder of the database structure (04-processed). These processed files contain all synchronized sensor signals from a combination of loggers as a result of the synchronization process (when the proper trigger or synchronized signals are found).

- The next subroutine identifies all the full processed events (i.e. containing synchronized signals from all data loggers) with a minimum 10-minute recording length.

- These identified full events are then analyzed automatically using the AI-FSI method presented in Section 2.2. The preset parameters having been specified by the user before hand. The AI-FSI algorithm includes a new automatic pole picking algorithm created to select the physical poles from the stabilization diagram of the modified SSI technique. The estimated modal parameter structures and the associated metadata are then stored in the 05-systemid folder of the database.

\subsubsection{Database viewer}

The database module includes a database viewer (Figure 5.2). The database viewer permits visualization of the data files captured by the monitoring system. Shown in Figure 5.2 are all the translated logger data files captured over eleven days in November 2014. It is also possible to view the processed files for the same time frame. The module also has zoom and pan capabilities to permit quick searches for data in the chosen time frame. Figure 5.3 includes a zoomed view of the timeline plot showing data captured over 7 hours on November 4th 2014. Further capabilities

of the module include clicking on an element in the timeline plot to gather metadata information on the selected data file. 


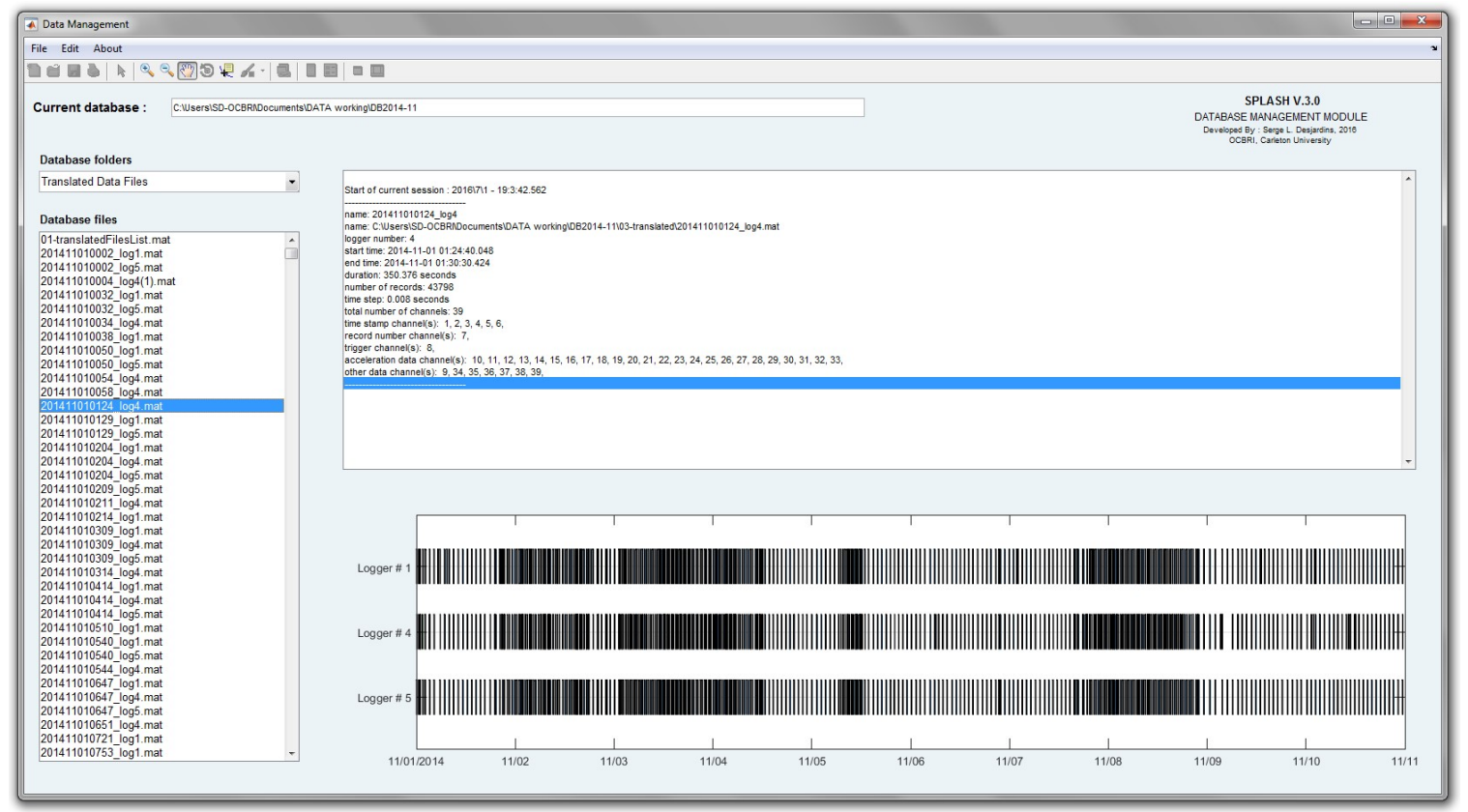

Figure 5.2: Screen capture of new Database Management module of SPLASH v3.0.

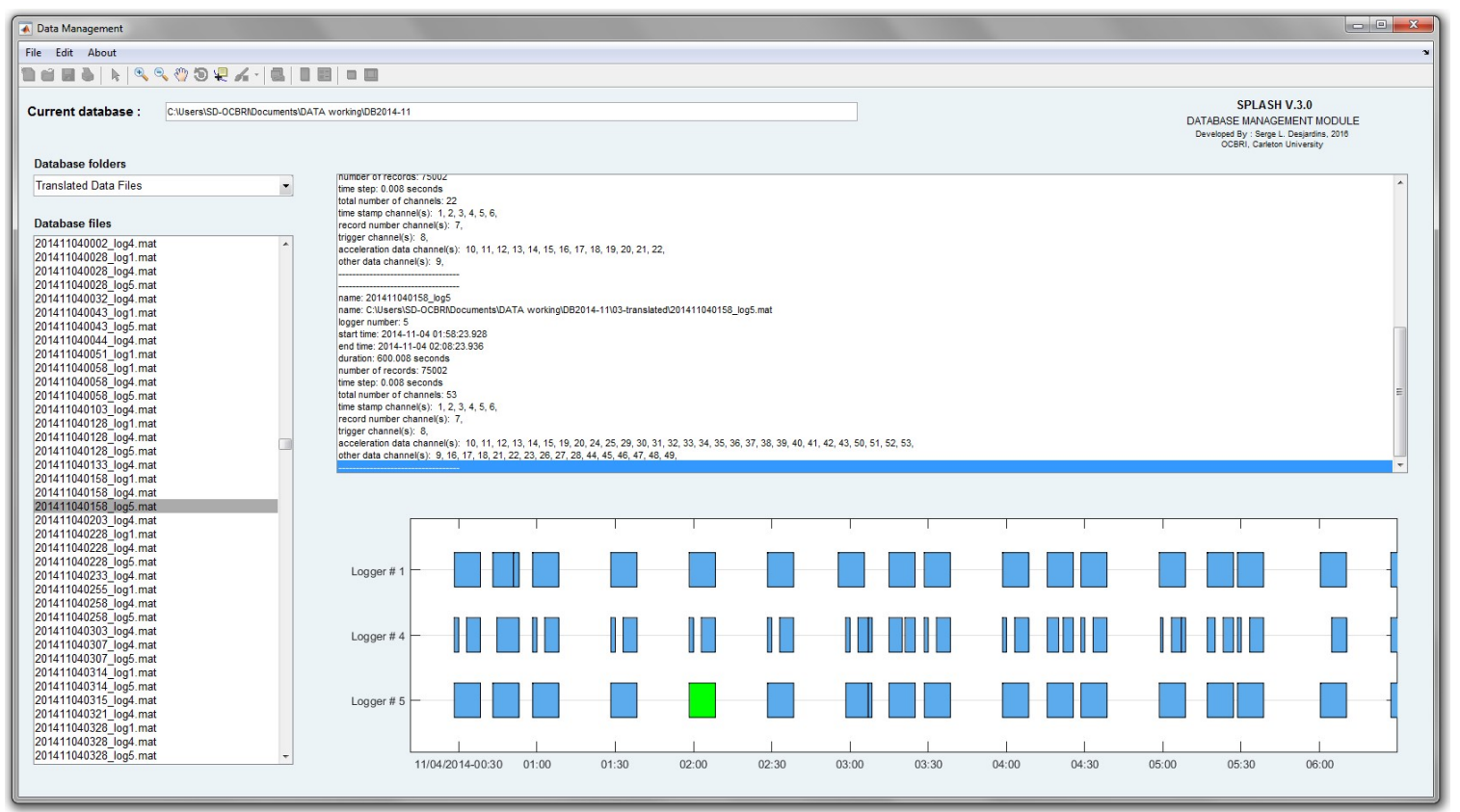

Figure 5.3: Screen capture of database management module showing data captured over a few hours on November 4th 2014 and associated metadata. 


\subsubsection{Data acquisition diagnostic}

As an added feature, the database viewing capabilities now permit qualitative determinations of data acquisition issues. As can be observed in Figure 5.3, there are data collection issues with logger no. 4 that create fragmented data files which should be investigated and corrected. This is an example of how the timeline plots included in the database viewer permit quick interpretations of data collection operations. This feature also serves to visualize the synchronization issues between the collected logger files. The collection times of the logger files are plotted in this figure according to the time stamps related to the internal logger clocks. Collection lags can be quickly identified. Section 5.2.2 covers data synchronization in detail.

\subsubsection{Other functions}

Other functions of the database management module include: opening and searching existing databases, adding data to an existing database, checking for database integrity, fixing or refreshing databases. The last two capabilities are automated and required if manual modifications are made to the databases.

For all interactions in the database module, the program operations are displayed in a scrolling display window of the database viewer and a log file is created (shown in Figure 5.4) and saved inside the database and can be opened from the database viewer for future reference.

\subsubsection{Data synchronization}

Data synchronization of vibration data collected at relatively high (and sometimes different) sampling rates from multiple data loggers represented a significant programming challenge. It is evident, that for the system identification techniques to 


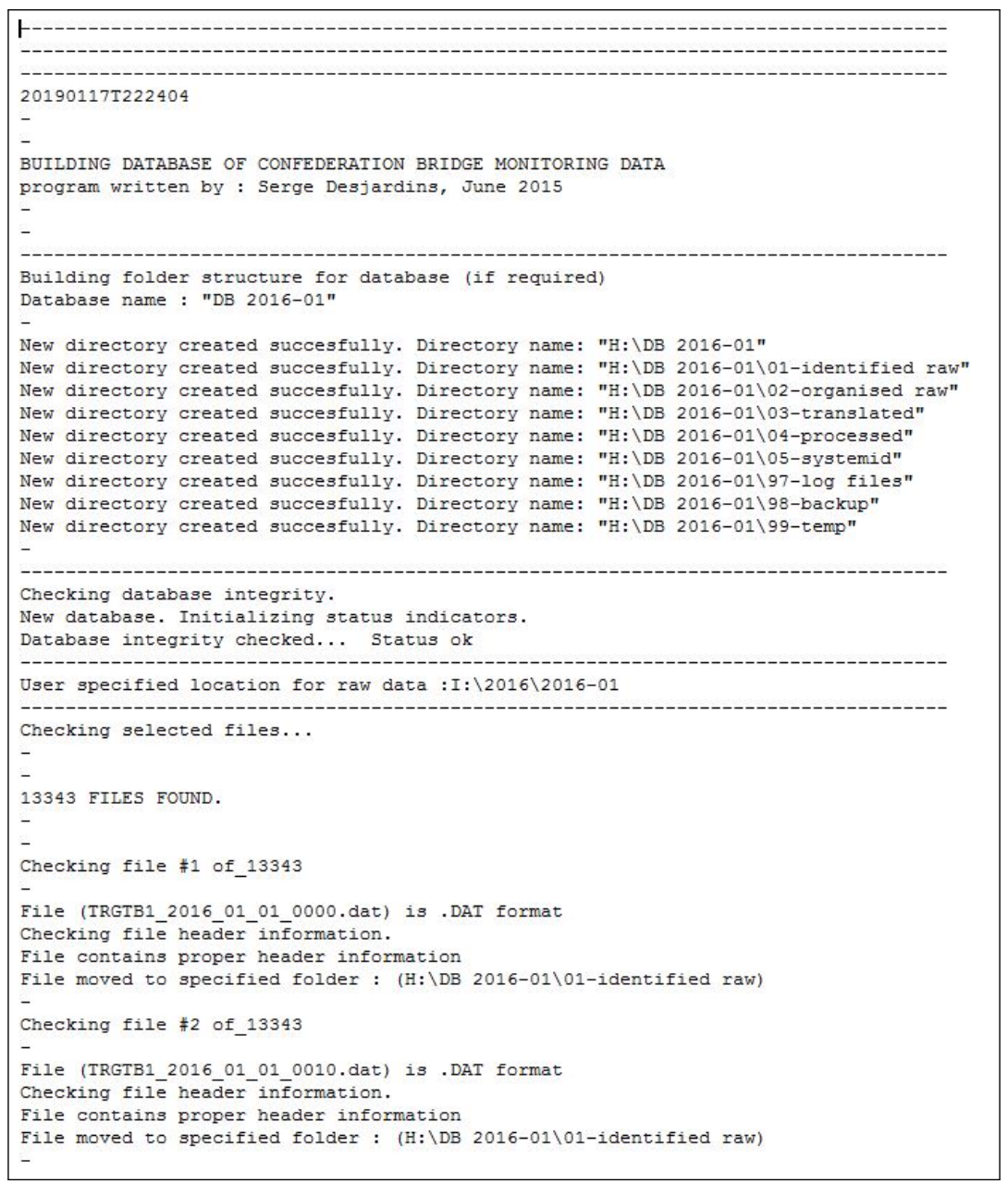

Figure 5.4: Example of a log file created while creating a new database in SPLASH v3.0.

work and give meaningful results, data from multiple data loggers must be synchronized precisely. It is not sufficient to simply rely on the time-stamps stored in the logger data tables as these are based on the logger's internal clock which is not necessarily synchronized with the other loggers to the precision required (time differences of minutes and even hours have been observed in the time stamps of different loggers). Figure 5.3 shows the imprecise alignment of logger files based on internal logger clocks. In the past, the triggered data events from multiple loggers were assembled, as is, to form a complete event assuming they were synchronized. While this may 
work for most of the captured data files when the data collection is running perfectly, we have seen that this is not always the case. A more robust method to assure data synchronicity was required. An algorithm was developed that searches through the trigger channels (as identified by the file header) of the logger data files to find trigger instances. A trigger instance occurs when a low voltage signal is sent from one logger to the other loggers when a specific response threshold is surpassed (a strong vertical acceleration measured at a specified sensor location due to the passage of heavy truck, for example). This trigger signal is assumed to occur simultaneously across all loggers. The algorithm can identify and align the synchronized data files according to this trigger signal and send them to the processing engine which then combines them to form a complete synchronized processed event. Additionally, since 2014, a data synchronicity signal is being sent from one logger to the others via the triggering channels at every 5 minutes to confirm data synchronicity under a multitude of data collection scenarios (such as continuous monitoring or under manual data collection).

As an added verification, a correlation function is used to temporally align the logger files based on the collection patterns of each logger. In this manner it was discovered that the logger clocks had differed by as much as twelve hours during the fall of 2001.

\subsubsection{Streamlined preprocessing}

All the processing operations performed by SPLASH have been streamlined in order to minimize the effects on the data and to avoid introducing additional computational noise. The less data manipulations the better. Some operations are done on data sets as a whole, while others are performed on individual channels. The different data processing operations and procedures described below are carried out by the processing engine as required: 
- Channel identification: Although individual channels within the logger files are mapped according to Table 4.2, the processing engine confirms the channel numbering with the information contained in the header of each logger file.

- Purging of duplicate records: The subroutine loops through row vectors of the data matrix, starting with the earliest in chronological order, to find potential duplicating records. A potential duplicate record occurs when a sampling interval, calculated from the time-stamps, is found to be less than zero. The algorithm will then compare the potential duplicate records with earlier records to find a match. When a match is found the duplicate record is purged from the data set.

- Patching small data gaps: Small data gaps, where data samples are missing, are patched by a cubic polynomial interpolation algorithm using Piecewise Cubic Hermit Interpolating Polynomial (PCHIP). It operates on the column vectors of the signal matrix by taking a set number of samples, four in the present case, before and after the missing samples for interpolation.

- De-trending of data (baseline correction): The mean is calculated and removed from each signal. Theoretically, this has no effect on acceleration signals since a residual acceleration signal has no physical meaning (it would mean that the structure is in continuous unidirectional motion).

- Scaling of data using calibration factors: Calibration factors are channel specific and are used to convert the acceleration readings from voltage into engineering units of $\mathrm{m} / \mathrm{s}^{2}$. A sensor table which contains the accelerometer information, such as sensitivity and calibration factors, is used in the algorithm and throughout the processing engine. For multi-scale and multi-state data, this data sensor table is historically accurate and is queryable according to timestamps. This is critical in a long term monitoring setup where sensor replacements are common. 
- Re-sampling of data to a common sampling rate: The data sets of a dynamic event can be collected by multiple data loggers. The data-loggers of the acquisition system typically run at the same sampling rates but they may sample at different rates (in fact, they have run with different sampling rates in the past). To synchronize the multiple signal matrices for output compatibility, the minimum sampling rate is determined and the other data sets are subjected to a re-sampling operation. This re-sampling operation lowers the sampling rate of the signal at a ratio of integers $a / b$ established by the data set sampling intervals $\Delta t / \max (\Delta t)$ using an interpolation algorithm to upsample the signal by the integer factor $b$ and then down-sampled by the integer factor $a$. This operation is carried out on each individual column vector of the signal matrix of a given data set. Down-sampling operations can result in aliasing of the signal and reduce the useful frequency range of the otherwise properly captured signal. Therefore, signal filtering of the down-sampled data is needed. In this case a Finite Impulse Response (FIR) filter is used to low-pass filter the original signal vector to prevent aliasing. The filter design characteristics vary depending on the ratio of integers $a / b$ and are automatically chosen based on operation requirements of the data. A new FIR filter is designed by the algorithm whenever a re-sampling operation is performed. In order to avoid these data manipulations, sampling rates are now kept constant across all data loggers.

- Decimation of data: The processing and output computations of the data can be economized by reducing the amount of samples contained in the signal matrices. Decimation is very similar to re-sampling in that it includes both low-pass filtering and down-sampling operations. The signal filter used in this case is an Infinite Impulse Response (IIR) filter (8th order Chebyshev type 1). By 
decimating with an integer factor $n$, the cutoff frequency $\left(F_{\text {pass }}\right)$ for the filter is determined by dividing the Nyquist frequency $\left(F_{s} / 2\right)$ by the chosen decimation factor $n$. A factor of 0.8 is added to obtain a conservative cut-off frequency. This filter has a phase response function that is near-linear. Since off-line data are considered, signal distortions caused by the near-linear phase response function can be canceled out by filtering the signal in both directions in the time-domain. Once data is filtered, the down-sampled data is obtained by taking every $n^{\text {th }}$ sample which significantly reduces the amount of data in the matrices. This function has been kept for backward compatibility and is described here. However, in order to streamline the processing operations and to minimize data manipulations (and more importantly, to avoid adding computational noise to the signals), the decimation is not performed on data described in the following chapters.

- Un-filtering of channels: The QA-1200 servo-accelerometers at locations 3, 4 and 18 of pier 31 and at locations 3 and 4 of pier 32 were initially installed for the ice floe monitoring in the original monitoring system. It was decided that the measurements from these locations would be valuable for the vibration monitoring project. A simple one-pole resistor/capacitor filter was fabricated with a $75 \mathrm{k} \Omega$ resistor and $0.68 \mu \mathrm{F}$ capacitor and installed on-site at the above locations in order to reduce the output voltage signal from $99 \mathrm{~V} / \mathrm{g}$ to $10 \mathrm{~V} / \mathrm{g}$. A discrete time transfer function is created in the algorithm with the same properties of the on-site $\mathrm{R} / \mathrm{C}$ filter; the inverse of this transfer function is applied to data from these pier transducers. It should be noted that these sensors have been replaced by Dytran piezo-electric accelerometers during the instrumentation renewal operation as shown in Table 4.2. However, this function has been kept in the new processing engine for backwards compatibility. 
- Double Integration: The signal is doubly integrated to obtain the displacement response of the structure using the cumulative trapezoidal numerical integration (CTNI) method. Precautions with low frequency content need to be taken when integrating a signal. Any DC component or near-zero frequency content are amplified during an integration operation. Therefore, a high-pass filter is designed for use prior to the first and after each integration operation (Chebyshev type 2 IIR filter). The algorithm operations for double-integration are listed as follows:

- High-pass filtering of the acceleration data using the Chebyshev filter.

- Integrating the filtered acceleration data with the CTNI method yielding velocity signal.

- High-pass filtering the velocity with the Chebyshev filter to remove any constant offset from integration.

- Integrating the filtered velocity yielding using the CTNI method yielding displacement signal.

- High-pass filtering the displacement signal with the Chebyshev filter.

- Displacements are created mainly for the animation module; therefore, to increase animation speed the data can be further down-sampled without loss of visible response information. Figure 5.5 shows a typical signal subjected to the double integration algorithm.

- Signal statistics: Once all data operations are completed, a subroutine calculates the signal statistics of each channel and saves it as metadata for future querying purposes. The calculated signal statistics include the maximum acceleration, the strength of the signal by root mean square (RMS), the standard deviation, the variance, the FFT peaks and the $90 \%$ occupied bandwidth of the signal.

- Sensor diagnostics: A new sensor diagnostic tool has been developed and is run 

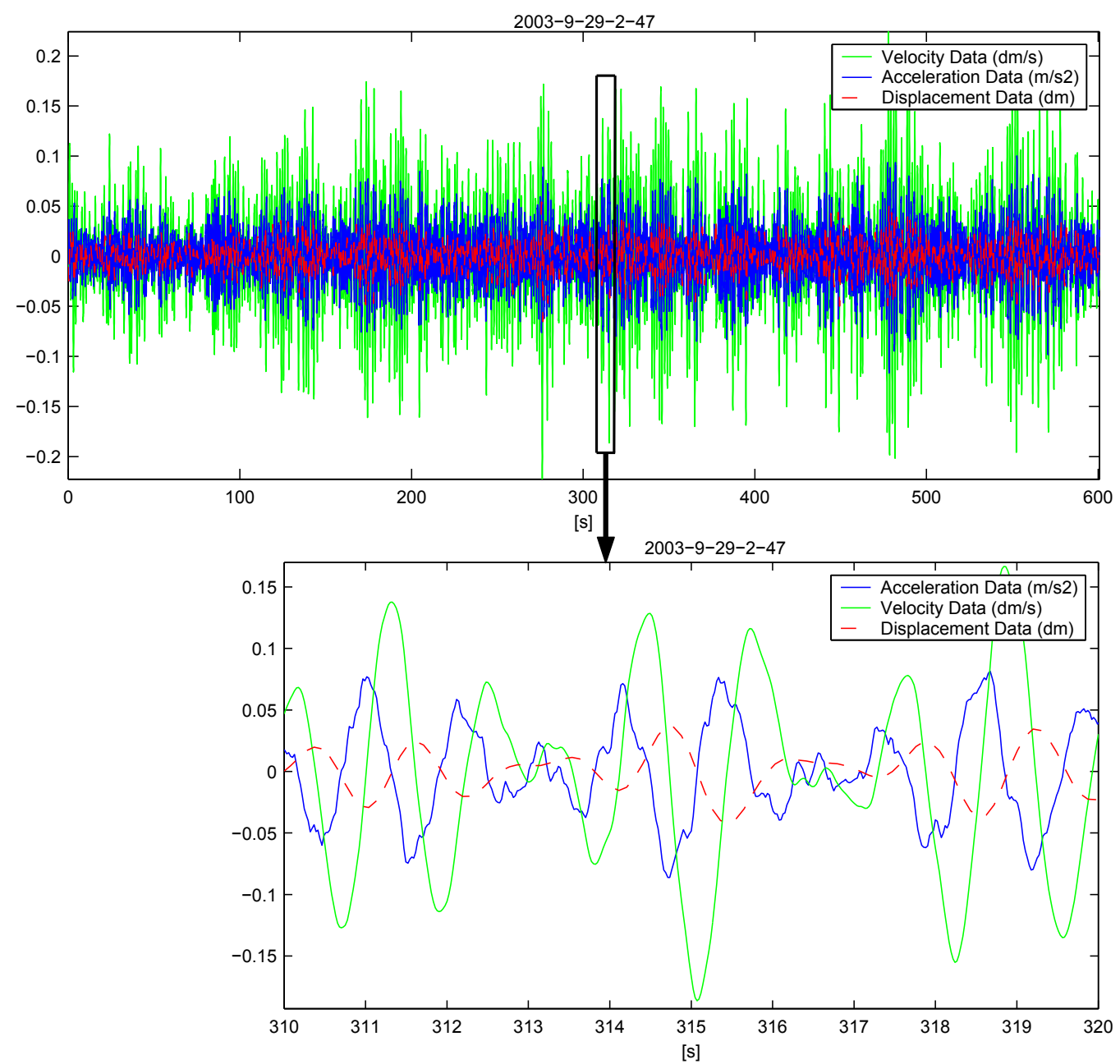

Figure 5.5: Integration of acceleration and velocity data to obtain displacement data.

at each processing cycle. Section 5.2.5 describes this tool in detail. If faulty sensors are identified they are removed from the output matrices.

- Processing outputs: The output of the processing engine is a data structure containing data at all stages of processing and two output matrices containing processed acceleration and displacements of synchronized sensors. The saved outputs are used in the other modules of SPLASH: the data visualization/plotting module, the animation module and the operational modal analysis module. 


\subsubsection{Metadata}

An important improvement to the SPLASH application has been the gathering of metadata at all stages of operations. This metadata is useful in querying the database for targeted research. During the translation of data, the metadata shown in Figure 5.6 is saved and tagged to the translated logger data files. Metadata is also generated and stored during the processing of events. Figure 5.7 shows the typical event metadata while Figure 5.8 shows the metadata tagged to the individual processed sensor signal.

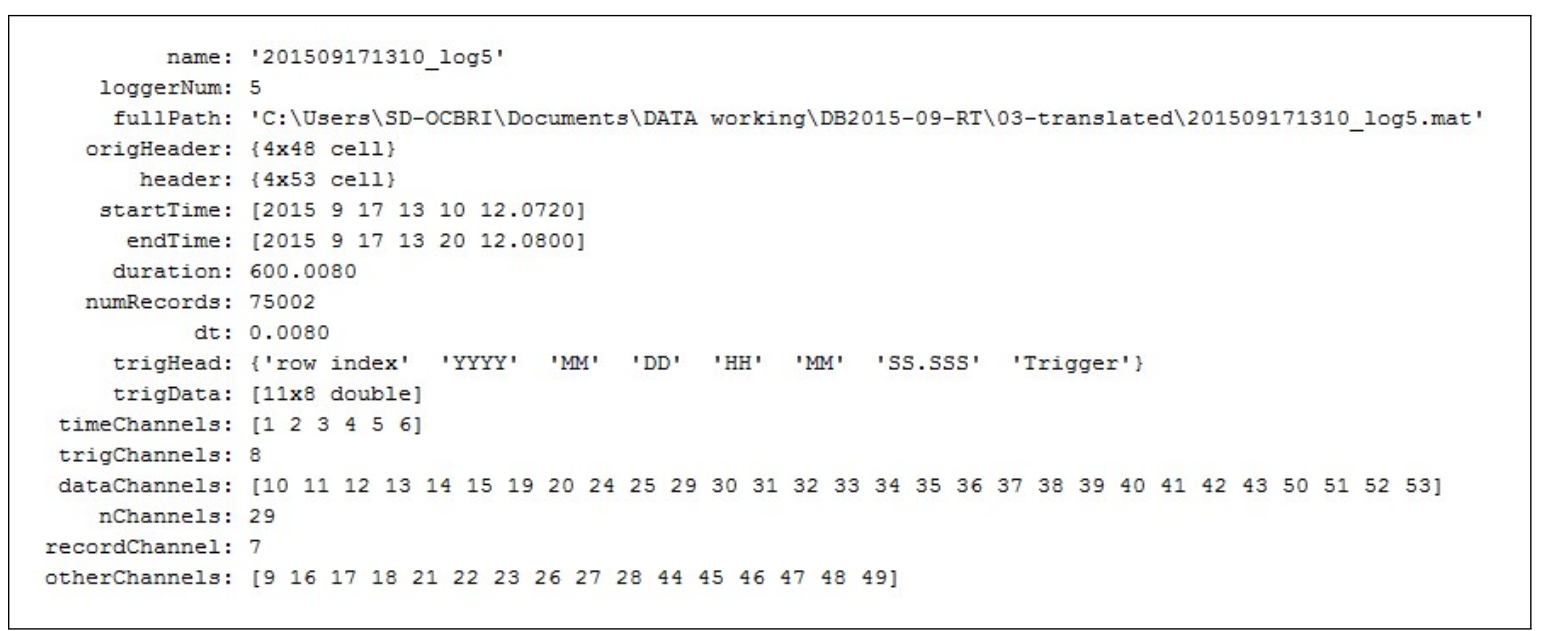

Figure 5.6: Metadata tagged to the translated logger data files (SPLASH v3.0).

\subsubsection{Sensor diagnostic}

Another significant contribution to the SPLASH application is the development of the sensor diagnostic algorithm used to identify the faulty or empty signal channels corresponding to problematic or missing sensors. Once properly identified these faulty signals are removed from the processed output displacement and output acceleration matrices. 


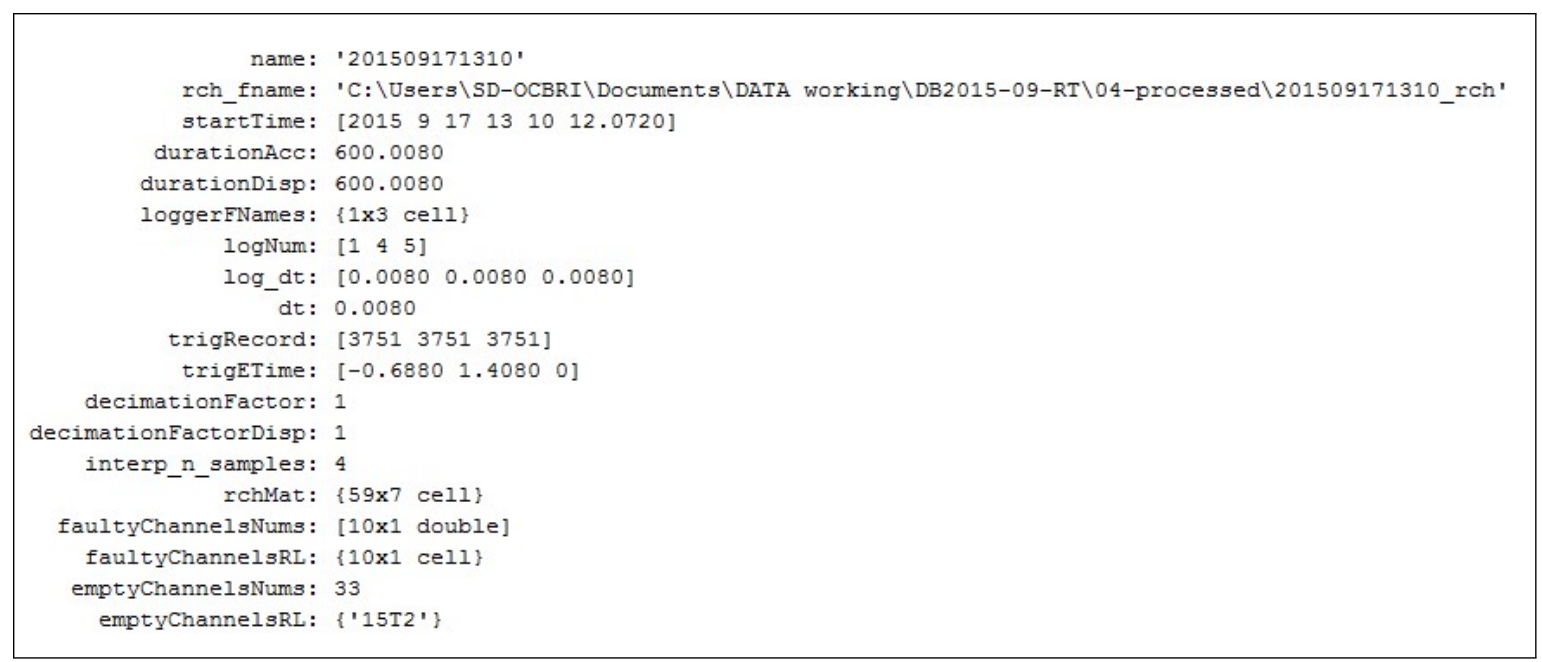

Figure 5.7: Metadata tagged to the processed event files (SPLASH v3.0).

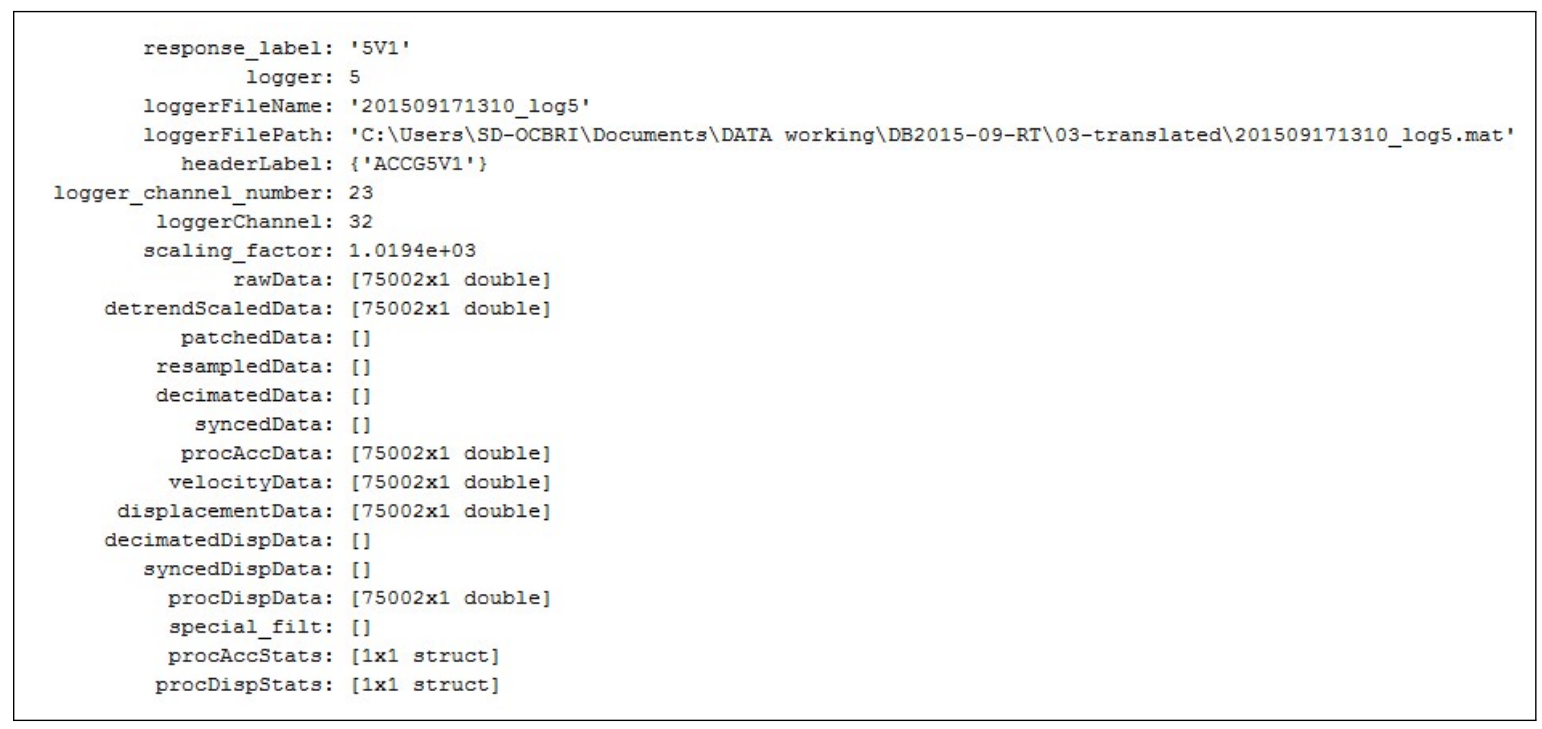

Figure 5.8: Metadata tagged to the individual processed channels (SPLASH v3.0).

The algorithm functions in two stages. The first stage considers each signals occupied bandwidth. If the lower bound of the occupied bandwidth is below a threshold value (taken as $0.01 \mathrm{~Hz}$ ) the signal is considered faulty and is removed from the processing results. The second stage is carried out as follows: the normalized cross correlations are calculated between each signal and every other signal. The normalization is done with respect to the maximum value of the-auto correlations with a 
value of one. The maximum absolute value of each correlation is stored in a symmetric matrix of size equivalent to the number of channels. Figure 5.9 shows a 3D representation of the maximum normalized cross correlations between each sensor signal. Valleys represent possibly faulty or empty channels.

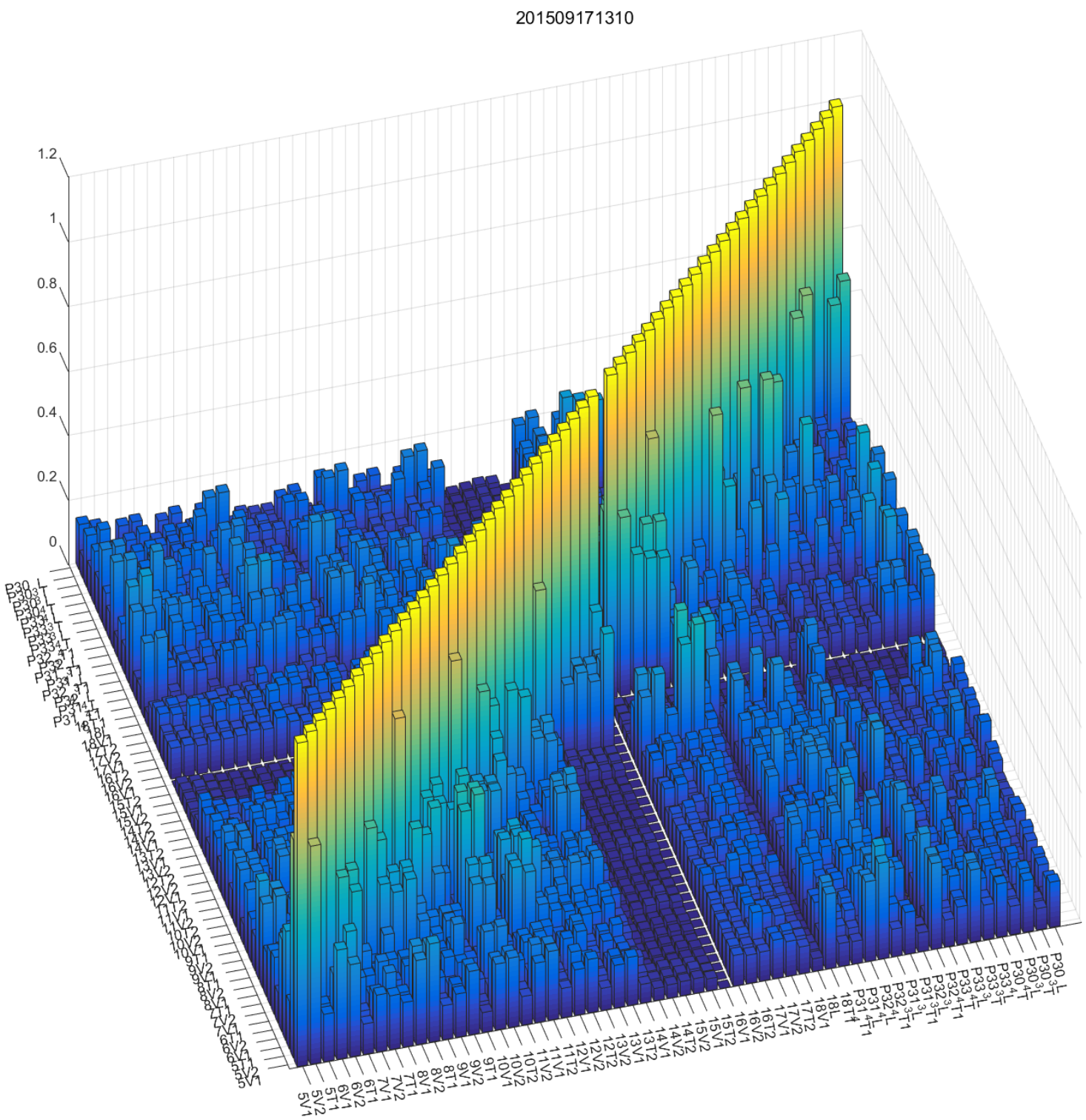

Figure 5.9: Three dimensional plot of maximum normalized cross correlations between each sensor signal.

To confirm that a signal is faulty or empty, an average of the maximum cross 
correlations values for each sensor is calculated across a single dimension of the correlation matrix and plotted as illustrated in Figure 5.10. The algorithm identifies faulty or empty sensors when the calculated average falls below one standard deviation.

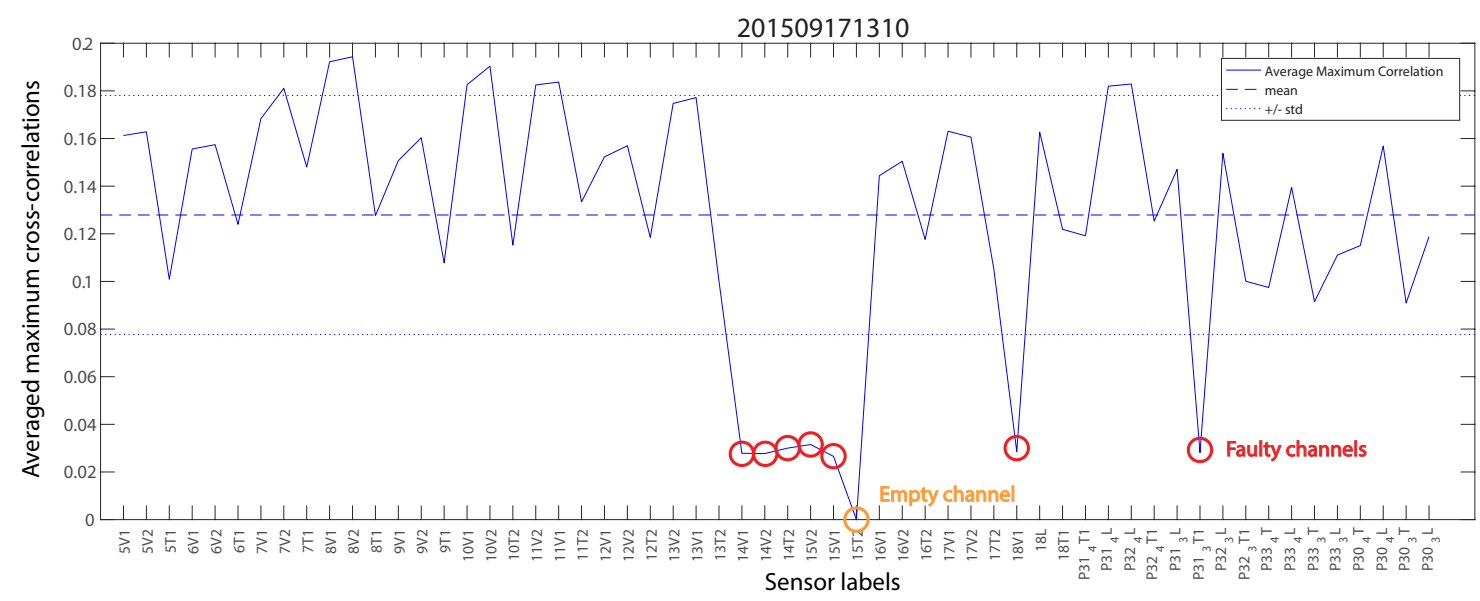

Figure 5.10: Averaged maximum normalized cross-correlations for each sensor signal.

\subsubsection{Multiple system identification techniques}

Several modules have been added to the SPLASH application. These include the two system identification modules created by Rahman [21] the first using the Eigensystem Realization Algorithm and the second using the improved PolyMax method [49]. Minor code adjustments were required to integrate these modules within the new version of SPLASH. It should be noted that these modules, combined with the existing SSI module provide easy to use, graphical user interfaces for user interactions and for viewing identification results, and as such do not provide automatic system identification which is described in the next section. 


\subsubsection{Automated operational modal analysis}

The AI-FSI algorithm described in Section 2.2 is fully implemented in the third version of SPLASH. After the processing operations, a subroutine identifies all the full processed events (i.e. datasets containing the synchronized data from all data loggers) with a minimum 10 minute duration. These identified full events are then analyzed automatically with the FSI technique described in Section 2.2.1 using the improved parameter estimates presented in Section 2.2.2. The automatic pole selection algorithm described in Section 2.2.3 is fully implemented in the third version of the SPLASH application. The obtained identification results (i.e. the estimated modal parameters) are stored for future visualization and evaluation and are used as inputs to the mode tracking algorithm described in Section 2.2.4.

\section{$5.3 \quad$ Summary}

The third version of the SPLASH application platform is presented in this section. The renewal of the computer tools used for the monitoring of the Confederation Bridge vibration responses coincided with the renewal of the installed instrumentation. Most of the improvements and additions to SPLASH were a result of the needs arising from the multi-state and multi-scale data created by the two generations of vibration monitoring system as described in Chapter 4 and from the practical considerations arising from over 10 years of use as described in Section 5.1. With these tools, an unprecedented feat was realized with the processing and analysis of all the historical data collected by the Confederation Bridge monitoring project since 1998. This represents over 250000 logger files, 40000 hours of recording and $28 \mathrm{~TB}$ of raw and processed data collected over 20 years. The historical database of raw and processed data is presented in Chapter 6. 


\section{Chapter 6}

\section{Historical Database of Processed Data}

The Confederation Bridge vibration monitoring project has been in operation since shortly after the bridge opening in 1997 which means that a significant database of raw vibration data now exists. This database includes data from two different data acquisition configurations due to the replacement of all vibration sensing equipment in 2013-2014. The tools required to manage, query and process the data need to be robust, reliable, adaptive and simple of use. New data processing and mining techniques are continuously being developed and perfected, which often require back processing of historical data. Some damage detection techniques may also require data from a "healthy" structure or new structure. For all these reasons, the new techniques and advances in processing capabilities can be put to good use in processing and analyzing the historical data in order to build a comprehensive database of processed data (which includes the estimated modal parameters). To accomplish this task, all of the processing and analysis operations are automated in the SPLASH application (as described in Chapter 5) in order to permit batch processing of significant amounts of raw data.

In this Chapter, the collection of vibration data is described and the processing results of all historical vibration data is presented. 


\subsection{Data Collection Statistics}

Most of the instruments incorporated in the bridge structure are located in two adjacent spans, a rigid frame and a drop-in simply supported span covering a one kilometer stretch of the bridge in the deep-sea part of the Northumberland Strait. The vibration instrumentation comprises 76 accelerometers distributed around a typical structural frame module, as shown in Figure 3.4. The response behavior observed in the instrumented segment of the bridge is considered representative of the behavior of the main span portion of the structure.

\subsubsection{Vibration data collection}

Data collection has been ongoing since shortly after bridge opening. The earliest recorded vibration data dates from February of 1998. High speed data collection (vibration) has been managed by the Ottawa Carleton Bridge Research Institute (OCBRI) housed at Carleton University in Ottawa, Canada. For the past 20 years, graduate students and post-doctoral fellows within OCBRI had the responsibility of managing all aspects of data collection, from sampling rates, data set durations, collection triggers, collection frequency, data storage and backup.

Vibration data are mainly collected via three data loggers numbered 1, 4 and 5 located in the bridge piers as shown in Figure 3.4. A fourth logger, logger number 6, collected vibration data briefly from February to June of 1998. Raw data are stored in logger files containing data tables of continuously recorded vibration data from a subset of accelerometers. The accelerometer channel mappings inside the logger tables are shown in Table 3.1. Data collection parameters have varied throughout the years; while a sampling rate of $125 \mathrm{~Hz}$ is most common, sampling rates of $100 \mathrm{~Hz}$, $166 \mathrm{~Hz}$ and $200 \mathrm{~Hz}$ have been used in the past. Dataset duration has also varied greatly, 
from 30 seconds to 10 minutes. Shorter duration datasets were mainly collected in the first 10 years of the vibration monitoring program. While data collection can be triggered manually (and has been in the past), most of the data collection has been under automated trigger scenarios. Trigger routines are programmed in the logger codes by researchers at OCBRI. The specified thresholds for triggering have varied greatly throughout the past 20 years, according to the needs of on-going research. Consequently, the quantity of data collected has also varied greatly as discussed later in Section 6.1.3. It should be noted that since October 2015, the collection mode has been set to continuous. Meaning that data is collected continuously, independent of trigger thresholds. This results in substantial amounts of collected raw vibration data and a unique opportunity to study bridge behaviour under actual ambient conditions.

\subsubsection{Environmental data collection}

Environmental data in the form of wind speed, wind direction and ambient air temperature have also been collected since bridge opening. The wind and ambient air temperature measurements are obtained from existing weather stations installed near pier 20 above the deck of the Confederation Bridge (see Figure 3.2). The sustained wind measurements are 10-minute mean, while gusts are based on peak measurement (one-second mean). Historical environmental data have been collected since November 1997 (date of first wind records). Wind and temperature data have been collected almost continually since. On average, a measurement is taken every 6 to 7 minutes. Using the available 1.7 million data samples, correlations between environmental conditions and processing and analysis results are made possible.

It should also be noted that the bridge operators limit vehicular traffic on the bridge during strong wind events. Travel restrictions may be enforced when steady winds recorded at the Bridge exceed $70 \mathrm{~km} / \mathrm{h}$ with gusts consistently exceeding 85 


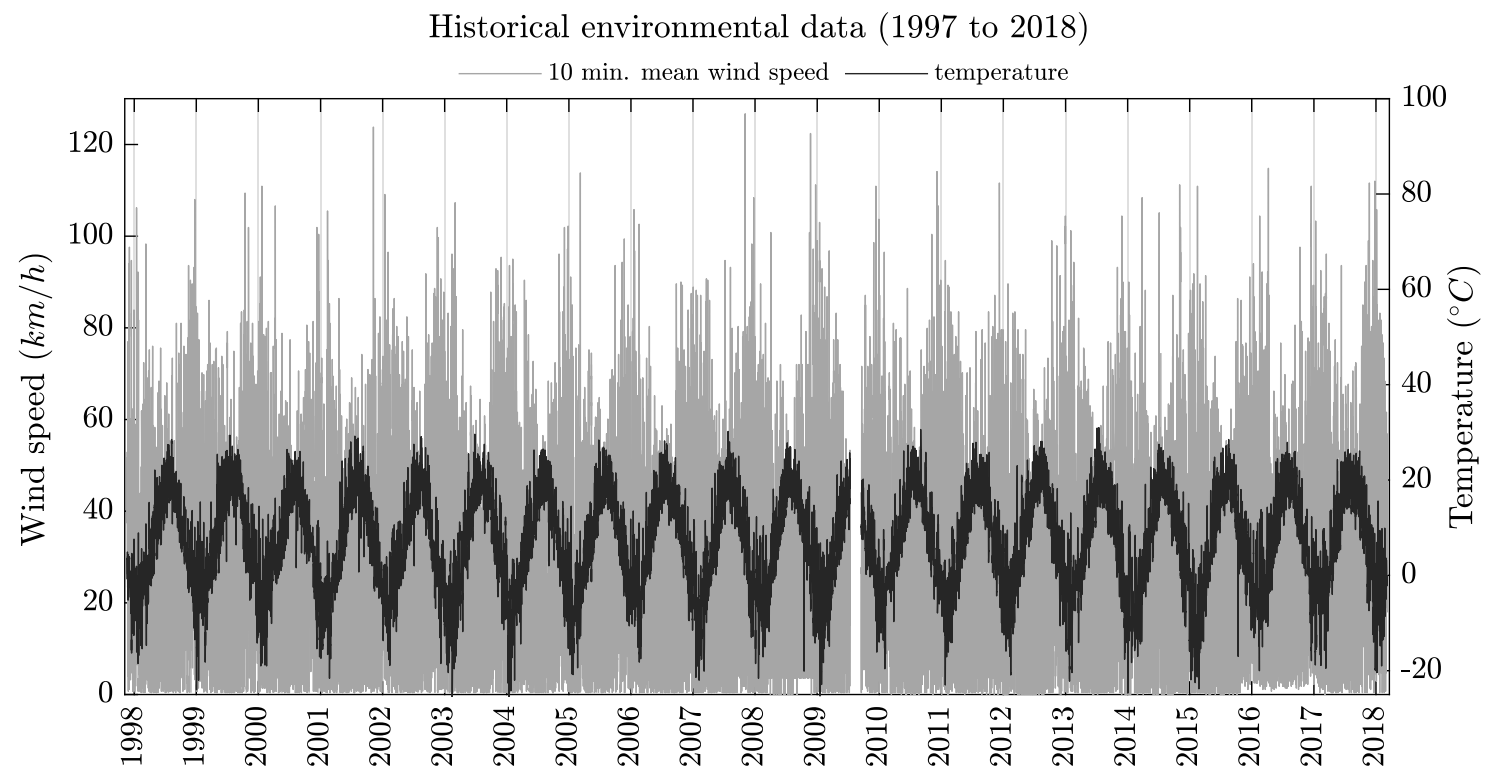

Figure 6.1: Historical environmental data from November 1997 to March 2018.

$\mathrm{km} / \mathrm{h}$. Restrictions during these strong wind events usually only apply to high-sided vehicles, motorcycles, and vehicles with objects in tow. On rare occasions, when extreme wind conditions occur with sustained winds greater than $100 \mathrm{~km} / \mathrm{h}$, the bridge is closed to all traffic.

\subsubsection{Collection statistics}

Data collection statistics are shown in the following sections for the periods of February 1998 to February 2014 and for the period of March 2014 to May 2018. These periods correspond to the first and second generations of the vibration monitoring system. Table 6.1 provides some data collection statistics. It is evident that collection schemes have varied in the past according to research needs. It should be noted that raw data are stored in data tables inside logger files of temporally-continuous vibration recordings of varying lengths. The number of data files collected is a metric provided in Table 6.1 and in Figures 6.2 and 6.3. It provides a good measure of the 
amount of datasets collected from each logger. A full event is described as a combination of datasets from the logger files of logger 1, 4 and 5, synchronized as described in Section 5.2.2. Partial events are composed of synchronized data from a subset of loggers. As explained, the length of recording for individual triggered events (either partial or full) is a preset parameter that can be modified by the user and as such is not constant in the data collection scheme. The total duration of events shown in Table 6.1 was calculated from a cumulative sum of all events for the periods specified. The file sizes of Table 6.1 were obtained from the size on disk occupied by the raw and processed data and serve as an indication of the amount of data required to be managed under a long-term vibration monitoring program.

Table 6.1: Vibration data collection statistics.

\begin{tabular}{|c|c|c|c|}
\hline & $\begin{array}{l}\text { February } 1998 \text { to } \\
\text { February } 2014\end{array}$ & $\begin{array}{c}\text { March } 2014 \text { to May } \\
2018\end{array}$ & Total \\
\hline Number of logger files & 72846 & 455079 & 527925 \\
\hline Number of partial events & 55530 & 97812 & 153342 \\
\hline Number of full events & 1579 & 101339 & 102918 \\
\hline Total Number of events & 57109 & 199151 & 256260 \\
\hline $\begin{array}{l}\text { Recorded duration of partial } \\
\text { events (hours) }\end{array}$ & 7226 & 16302 & 23528 \\
\hline $\begin{array}{l}\text { Recorded duration of full } \\
\text { events (hours) }\end{array}$ & 76 & 16890 & 16966 \\
\hline $\begin{array}{l}\text { Total recorded duration - all } \\
\text { events (hours) }\end{array}$ & 7302 & 33192 & 40494 \\
\hline Size on disk of raw data (MB) & 846871 & 6100197 & 6947068 \\
\hline $\begin{array}{l}\text { Size on disk of processed data } \\
\text { (MB) }\end{array}$ & 5067643 & 14383402 & 19451045 \\
\hline
\end{tabular}




\subsubsection{From February 1998 to February 2014}

Figure 6.2 shows the quantity of data (number of logger files) collected between February 1998 and February 2014. This represents the first 17 years of Confederation Bridge vibration monitoring data collected by the first generation of the vibration monitoring system. Data collection during the first ten years was relatively modest as compared to later periods. Data collection varied from zero datasets per month to a maximum of 800 datasets per month in the period between February 1998 and November 2012. While the current research focuses on operational modal analysis, which requires full events for better modal estimates, the previous research and hence previous collection schemes did not require full synchronized events to obtain meaningful results. This explains why very few full events were collected in the first 15 years of data collection. The period from December 2012 to February 2014 corresponds to the transition period from the first to the second generation of the monitoring system.

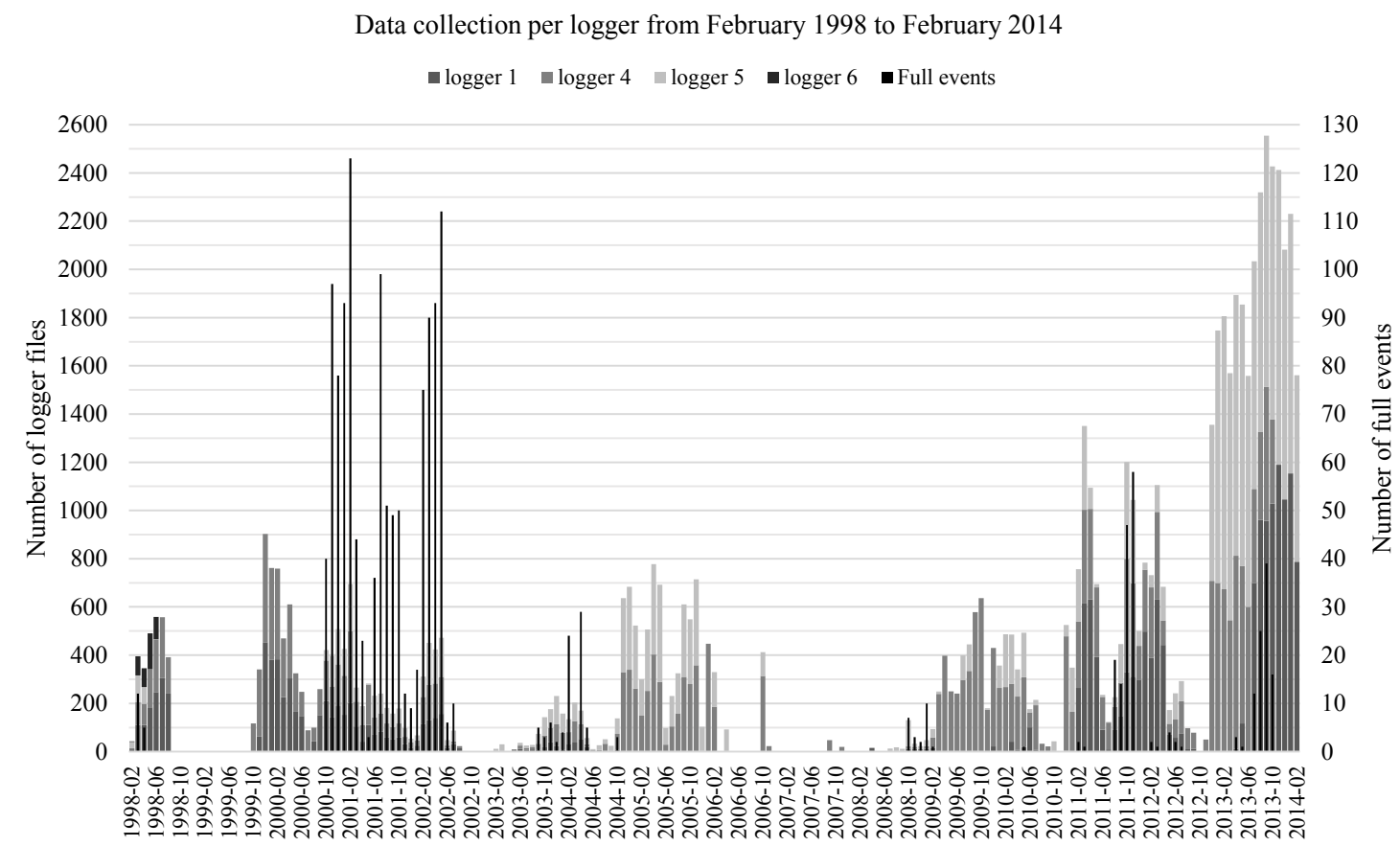

Figure 6.2: Data collection per data logger from February 1998 to February 2014. 
During this period a combination of old and new sensors and loggers were in place and the collection schemes were in flux. The three high speed data loggers and their associated sensors were replaced sequentially over a period of 15 months (logger 5 in December 2012, logger 1 in June 2013 and logger 4 in March 2014). Trigger thresholds were also modified at this time and hence more data were collected with the new instrumentation. Details on the instrumentation renewal are presented in Chapter 4.

\subsubsection{From March 2014 to May 2018}

Figure 6.3 shows the quantity of datasets (number of logger files) collected between March 2014 and May 2018. This represents the last 4 years of Confederation Bridge vibration monitoring data collected by the second generation of the vibration monitoring system. The last 4 years of data collection can be split in two periods, the first of which, from March 2014 to September 2015, corresponds to a relative increase in

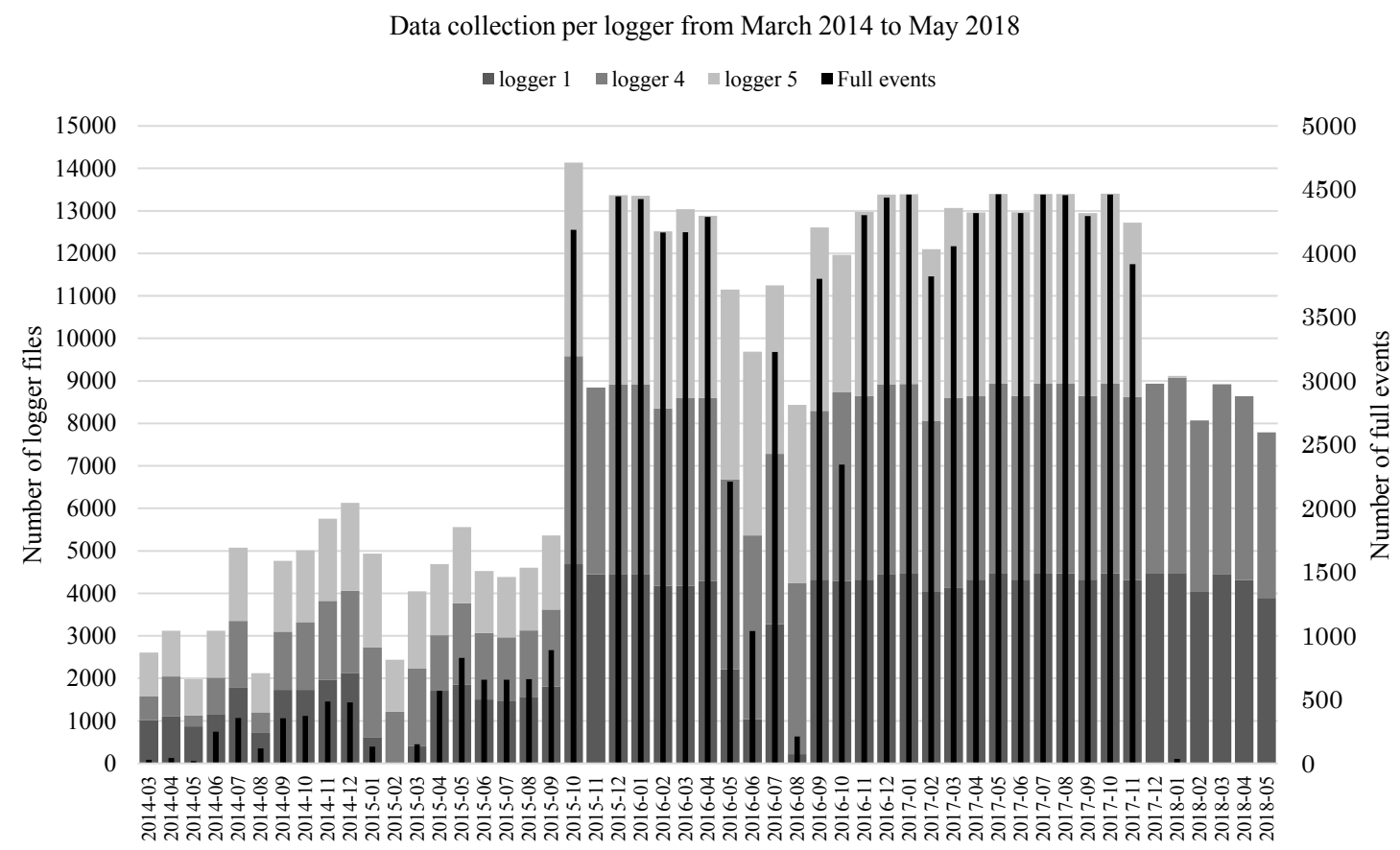

Figure 6.3: Data collection per data logger from March 2014 to May 2018. 
data collection under a new monitoring system. The number of datasets collected in this period varied from 2000 to 5000 a month. A marked increase in the number of full events is also noted, due to better triggering routines and the addition of a synchronized signal communicated to all loggers. The period from October 2015 to May 2018 corresponds to a period of continuous data collection. The maximum number of datasets were collected in this period, which sums to, on average, close to 13000 datasets per month.

\subsection{Processed Data}

Using the tools presented in Chapter 5, all the historical vibration data were processed and analyzed. With the third version of SPLASH, which is now automated, streamlined and optimized for use with powerful parallel processing machines, the processing and analysis of 20 years of data took approximately 8 months to complete under continuous operation (24 hours a day, seven days a week). Details on the data processing and analysis operations are provided in Section 5.2.3.

\subsubsection{Data collection per sensor}

Problems with sensors are inevitable in a long-term structural health monitoring system. A variety of factors can lead to problematic sensors, such as sensor malfunction, deterioration, faulty data acquisition electronics or unit, etc. With a new automated sensor diagnostic tool in post-processing, the empty and/or faulty signals are identified and removed from the data events. This new algorithm is integrated in the third version of SPLASH and is detailed in Section 5.2.5.

Plotted in Figure 6.4 are the data collection points per sensor after removal of problematic signals for all historical data (from February 1998 to May 2018). 


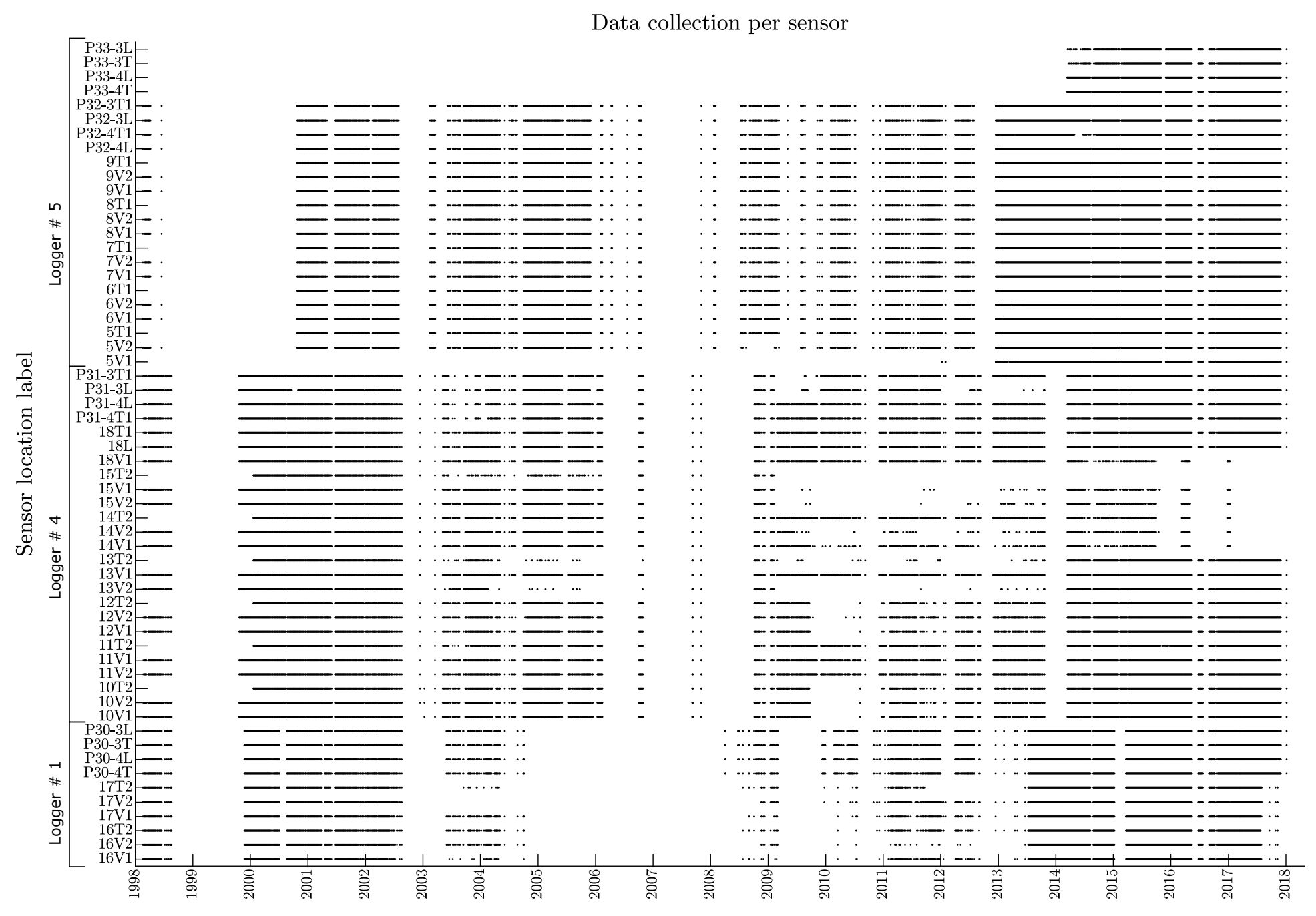

Figure 6.4: Data collection per sensor from February 1998 to May 2018. 
A data collection point in this figure represents a continuous signal recording for a specific sensor. Duration of recordings can vary from 30 seconds to 600 seconds. In this figure, sensors are grouped according to their data logger. Location labels correspond to those shown in Table 3.1 and in Figure 3.4. The long-term perspective provided by Figure 6.4 permits a rapid interpretation on the functioning of the monitoring system. From this figure it can be observed that without a full-time staff on call to fix instrumentation issues, certain problems can remain and affect data integrity for long durations. For example, at location 13, the erratic data collection from the transverse sensor (13T2) and from one of the vertical sensors (13V2) can be observed starting in mid 2003. This behavior continued until 2014 when the sensors and the logger were replaced. In other occasions, disruption to data collection may be due to issues of the data loggers. For example, missing data from all sensors connected to a particular logger does not necessarily relate to malfunction of the sensors, but may indicate the absence of a data collection trigger signal sent to the logger for the logger to start data collection in the data collection process.

\subsubsection{Maximum acceleration}

The maximum recorded acceleration of a signal is an important indicator used in operation modal analysis and structural health monitoring. Maximum accelerations provide an indication of significant transient events occurring on the structure, such as impacts (from vehicles, boats or ice floes), earthquakes or other structural issues causing higher than normal bridge vibrations (bearing failures, tendon ruptures, pole failures, etc.).

Figure 6.5 shows the averaged maximum acceleration across the vertical and the transverse signals along with the maximum values from two sensors at location 9 (mid-span) in the vertical and in the transverse direction. For each single event, the 
averaged values shown in Figure 6.5 are obtained by averaging the maximum values from each signal measured in the indicated direction (either vertical or transverse).

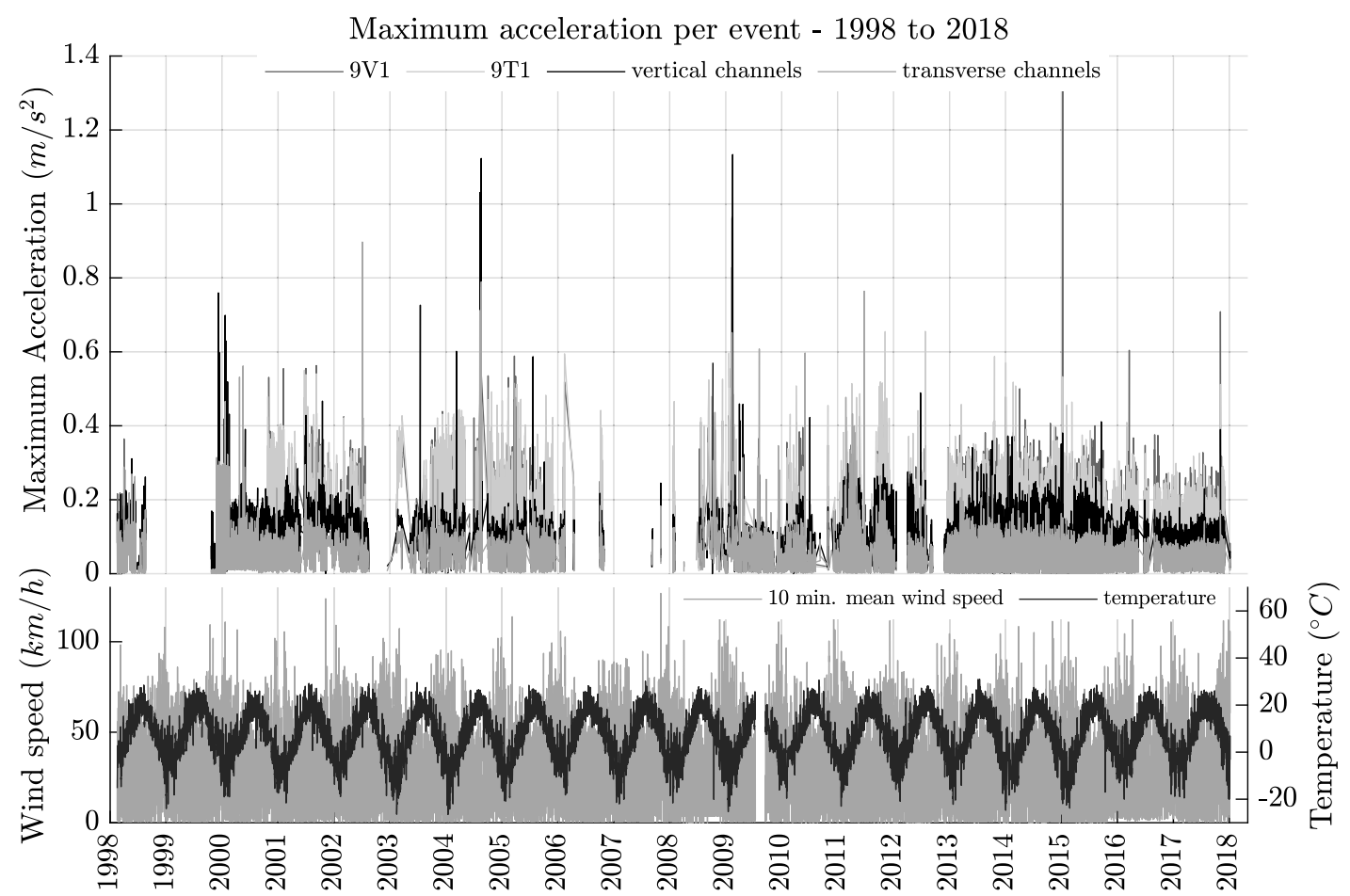

Figure 6.5: Maximum accelerations per event with corresponding mean wind speed and temperature data for all events collected between February 1998 and May 2018.

We can observe, from Figure 6.5, that the signals measuring in the vertical directions generally record higher maximum values than those in the transverse direction on average. The peaks observed in this figure are outliers that would require further investigation to identify the cause. Typically, the cause of very high maximum accelerations are due to exceptional loading conditions (such as an overweight or permit load vehicle crossing the bridge). Faulty instrumentation (sensor or data logger) must also be ruled out by investigation. Even with a sensor diagnostic algorithm, it is possible, albeit rare, that some misbehaving signals are not properly filtered from the processing results. 


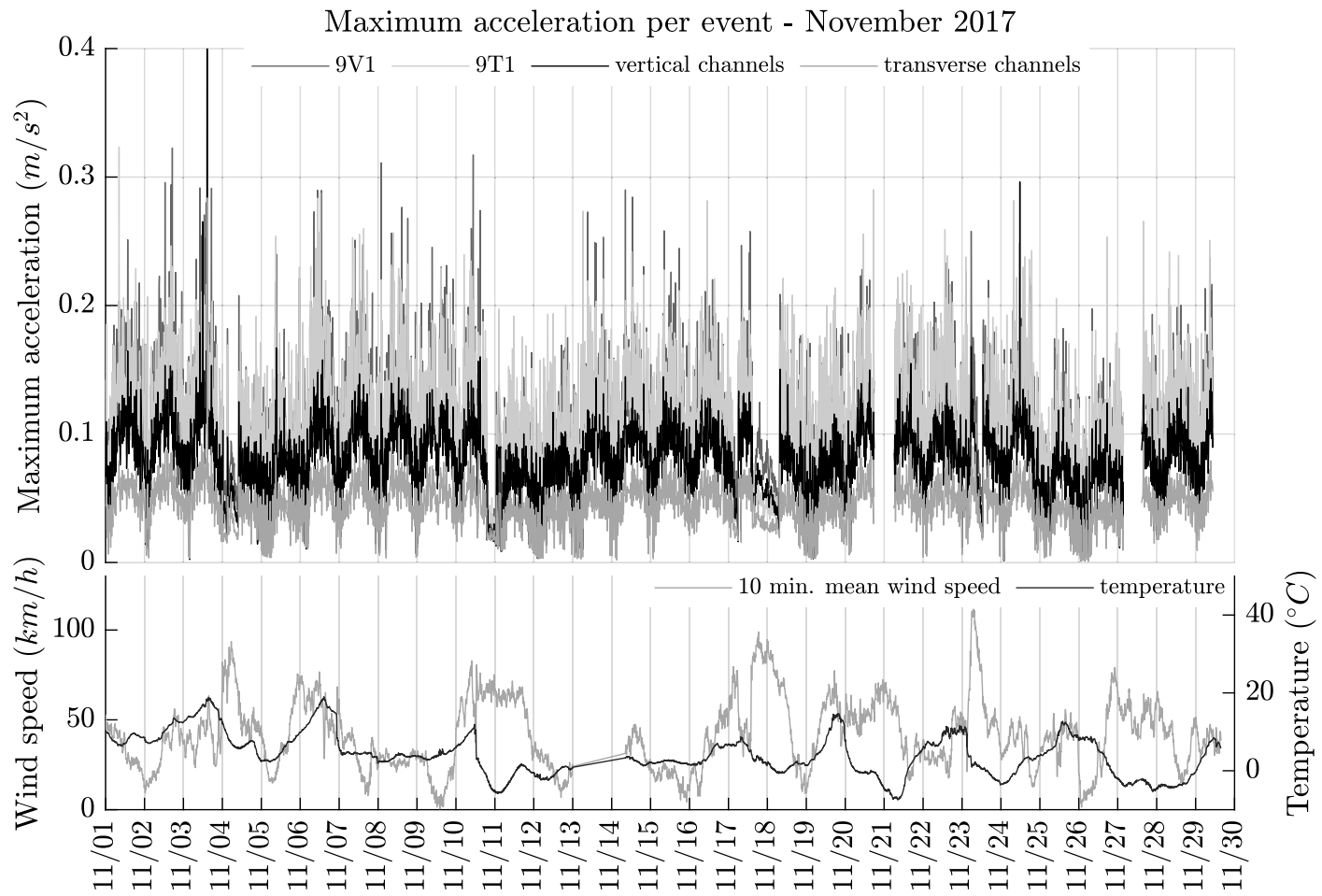

Figure 6.6: Maximum accelerations per event with corresponding mean wind speed and temperature data for all events collected in November 2017.

Figure 6.6 shows maximum accelerations for all events collected in November 2017. As peak accelerations occur under vehicular traffic, daily variations of maximum accelerations are clearly observed. Interestingly, during strong wind events, the maximum recorded accelerations are comparatively lower than the corresponding observed data on normal days. This is due to travel restrictions on the bridge which are in place when sustained winds are greater than $70 \mathrm{~km} / \mathrm{h}$. We can therefore deduce that maximum accelerations are mainly due to vehicular traffic whereas signal strength (RMS) are more correlated with wind speed. These correlations are explored in detail with a regression analysis presented in Chapter 7. 


\subsubsection{Strength of signal (RMS)}

In operational modal analysis, the amplitude or strength of a signal is an important indicator in the reliability of the extracted modal estimates. The calculation of the root-mean-squared (RMS) value is an effective way to evaluate the strength of a given signal. The RMS value of a discrete signal $\mathbf{x}_{n}$ is calculated as the square root of the average of squared values of the signal, mathematically represented in Equation (6.1):

$$
x_{R M S}=\sqrt{\frac{1}{N} \sum_{n=1}^{N}\left|\mathbf{x}_{n}\right|^{2}}
$$

where $\mathbf{x}_{n}$ is a vector of $N$ acceleration samples. Figure 6.7 shows the averaged RMS values of signals for all processed events between February 1998 and May 2018.

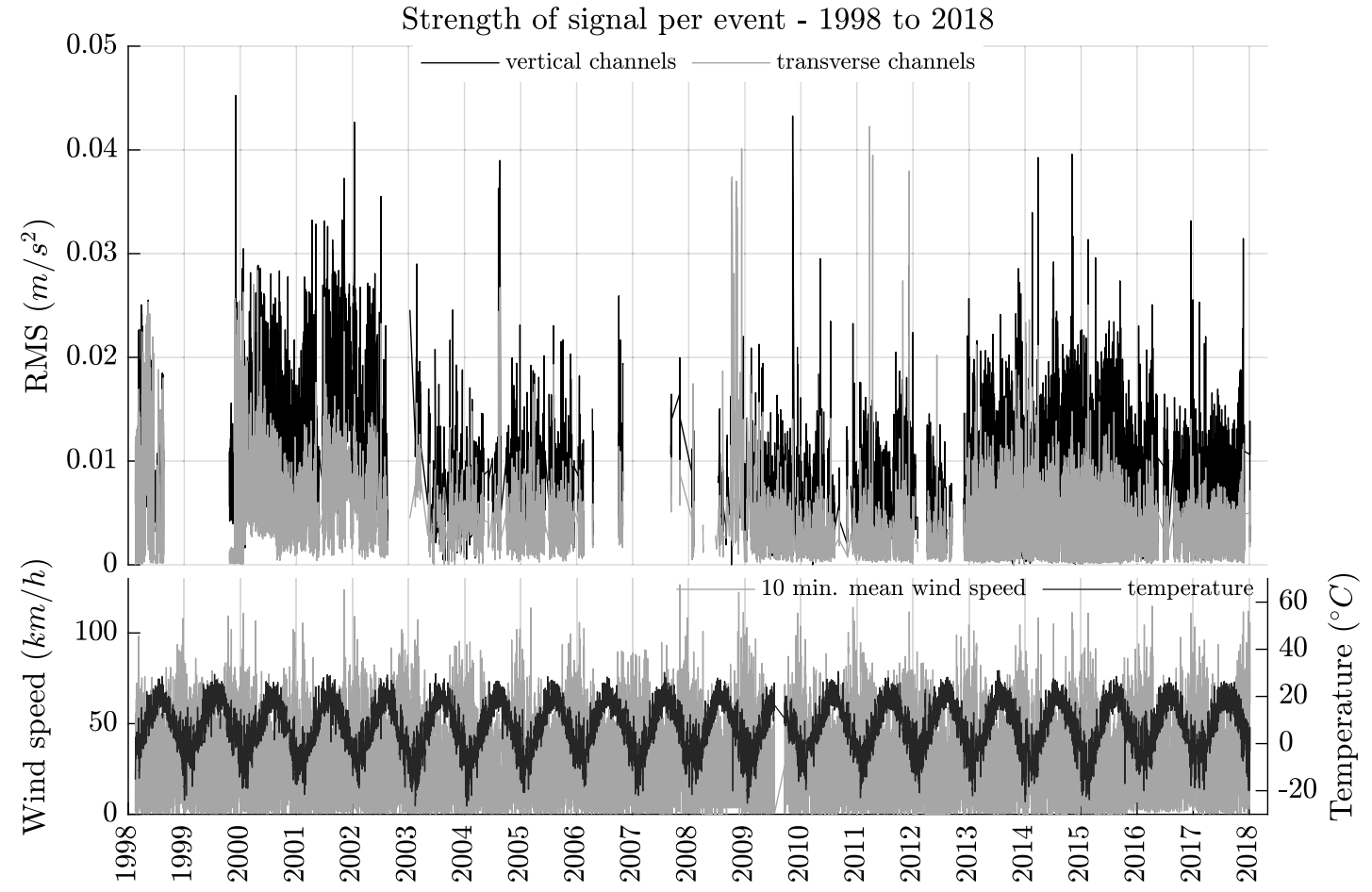

Figure 6.7: Average signal strength (RMS) per event with corresponding mean wind speed and temperature data for all events collected between February 1998 and May 2018. 
The RMS values are calculated on the de-trended and scaled acceleration signals with units of $\mathrm{m} / \mathrm{s}^{2}$. The averaged values are calculated over all vertical and transverse sensors for a given data collection event. A contributing factor to the variations observed in the averaged RMS values of individual data collection events is the sensors which are included in the determination of the RMS value. It is discussed in Section 6.2.1 that the processed events are comprised of signals from various sensors collected by different data loggers. Faulty or missing signals are removed from the processed results of data collection events. The removed faulty signals affect the calculated average RMS values.

Figure 6.8 provides a closer look of RMS values for all events collected in November 2017. In this figure we can clearly see a daily cycle in the amplitudes of the RMS

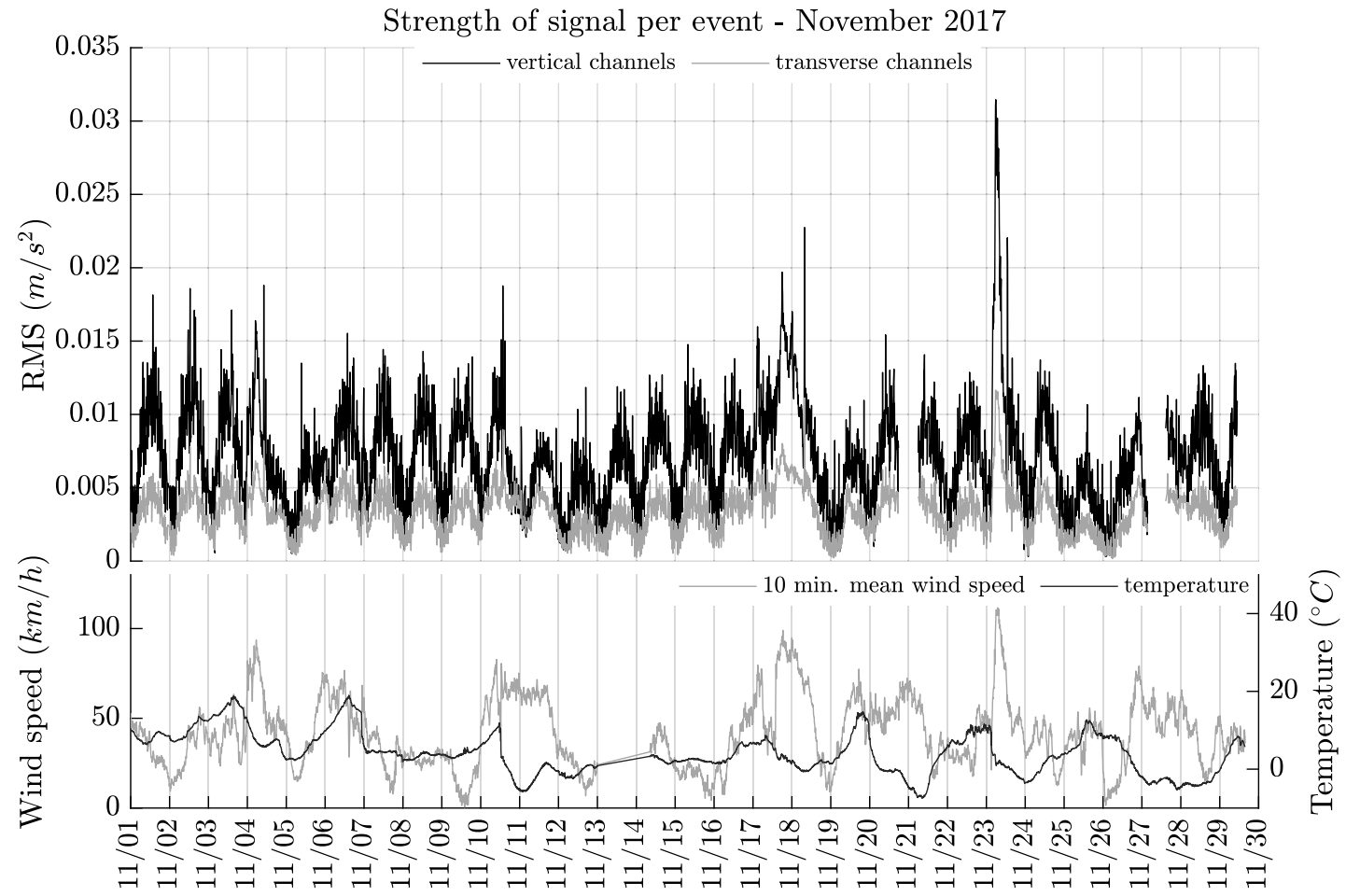

Figure 6.8: Average signal strength (RMS) per event with corresponding mean wind speed and temperature data for all events collected in November 2017. 
values. The most likely cause of these cycles are traffic patterns which peak during mid-day and are relatively low late at night and in the early morning.

Wind also has an effect on the strength of signal, in fact the highest RMS values are observed from events collected during strong wind conditions. In general, the sensors measuring in the vertical directions are subjected to stronger vibrations than those in the transverse direction. The RMS values are used as a dependent variable in the statistical analyses of the operational modal estimates described in Chapter 7.

\subsubsection{Operational modal estimates}

The analysis of historical data includes the extraction of modal properties from full processed events. The estimated modal properties obtained (modal frequencies, mode shapes and to a lesser extent damping ratios) are primordial for the damage detection approach proposed in Chapter 7. Full events are data collection events comprised of data collected by the three main data loggers located on the Confederation Bridge. Potentially, data from all sensors are included in full events; however, due to faulty instrumentation, some sensors are removed from the processed results. This removal is carried out automatically by the sensor diagnostic algorithm incorporated inside the processing engine of the third version of the SPLASH platform as described in Section 5.2.5. As is evident in this section and in the next chapter, the OMA results are highly dependent on the sensors included in the processed events. Great care is taken to ensure the maximum number of sensors are included in each processed event, however, due to sensor and logger issues, this is not always possible. While investigating the results in this section, the reader is directed to Section 6.2.1 and Figure 6.4 for a complete overview of the available sensors for each collection event.

In the following sections, the extracted modal frequencies of the Confederation Bridge are presented for both generations of the monitoring system. For the first 
generation, this corresponds to the period from February 1998 to February 2014 and for the second generation, the period is from March 2014 to May 2018.

\subsubsection{OMA results from February 1998 to February 2014}

In this data collection period, a total of 1569 full events were collected, processed and analyzed. The extracted modal frequencies from these events are plotted in histogram form in Figure 6.9 with bin edges specified at $0.0034 \mathrm{~Hz}$.

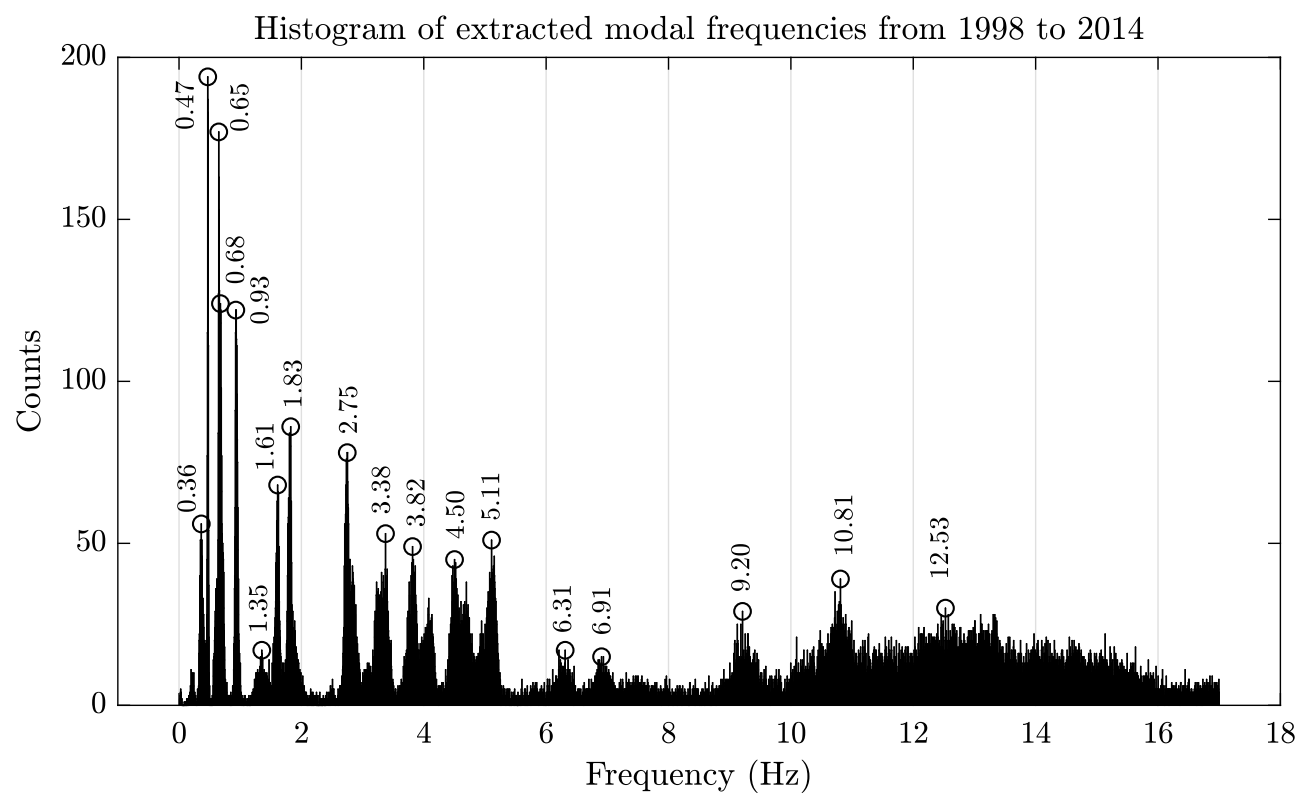

Figure 6.9: Histogram of extracted modal frequencies from full events collected between February 1998 and February 2014.

Section 2.2.3 describes the algorithm developed for automated selection of poles containing the estimated modal properties. The peak frequencies are labeled directly on the plot of Figure 6.9 with units in Hz. The peaks correspond to the most frequently extracted modal frequencies, and provide a good indication of the dominant modes of the bridge superstructure.

Figure 6.10 shows the ten top modes from each full event plotted temporally with 
corresponding environmental (wind and temperature) measurements. From this figure we can observe interesting temporal trends in the extracted modal properties. In this long-term perspective, even with sporadic collection schemes, certain trends in OMA results emerge. Clearly, some modes vary according to temperature. The quantification of the correlation of the extracted frequencies with environmental conditions is presented in Chapter 7 . While the scatter of plotted results for certain frequencies is relatively high for this period, the main modes of vibration of the structure are identifiable.

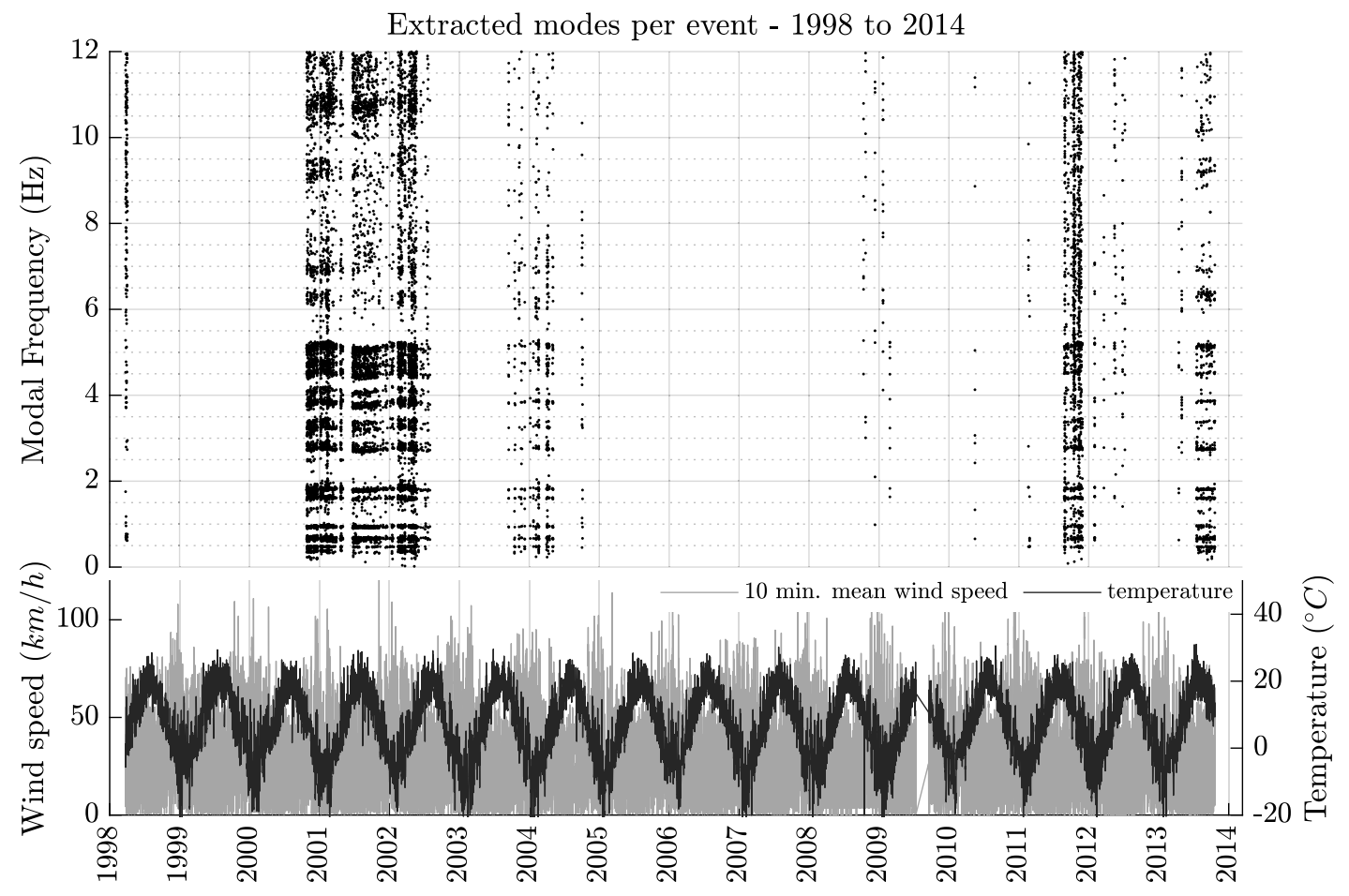

Figure 6.10: Extracted modal frequencies from full events collected between February 1998 and February 2014. 


\subsubsection{OMA results from March 2014 to May 2018}

From March 2014 to May 2018, data collection was relatively more uniform, especially after September 2015 when data collection was set to continuous. A total of 101339 full events were collected and processed; a subset of which was analyzed with the methods described in Section 2.2. The extracted modal properties from these 12750 events are plotted in histogram form in Figure 6.11.

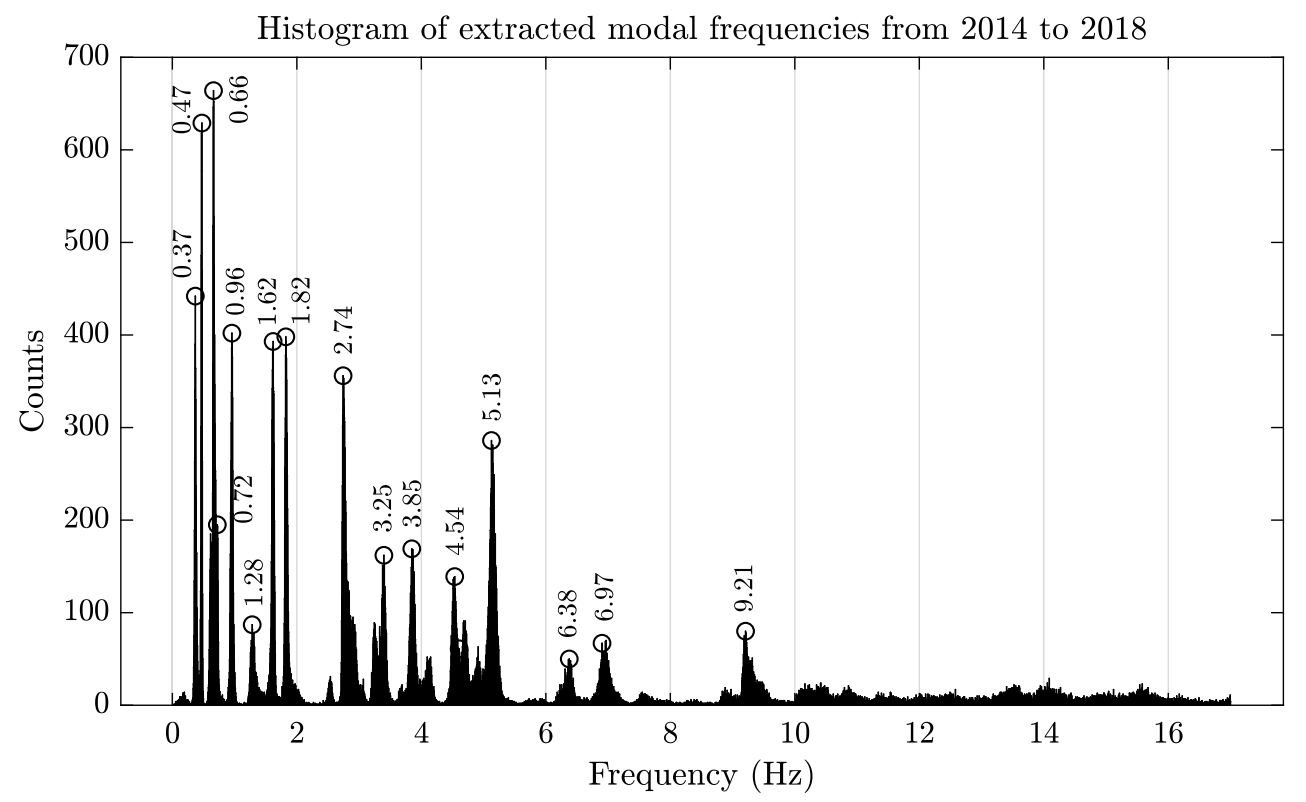

Figure 6.11: Histogram of extracted modal frequencies from full events collected between March 2014 and May 2018.

The bin edges specified for the histogram of Figure 6.11 are $0.0034 \mathrm{~Hz}$; the same as those specified in Figure 6.9. While the count numbers are comparatively higher, the peaks identified in Figure 6.11 are similar to those identified in Figure 6.9 albeit with very minor variations in frequency values. The histogram for this period is cleaner, meaning the the peaks are more pronounced with less distribution, which indicates less variability in the extracted modal properties. Also of note is the relatively low identification rate of modes in the 10 to $16 \mathrm{~Hz}$ range. 


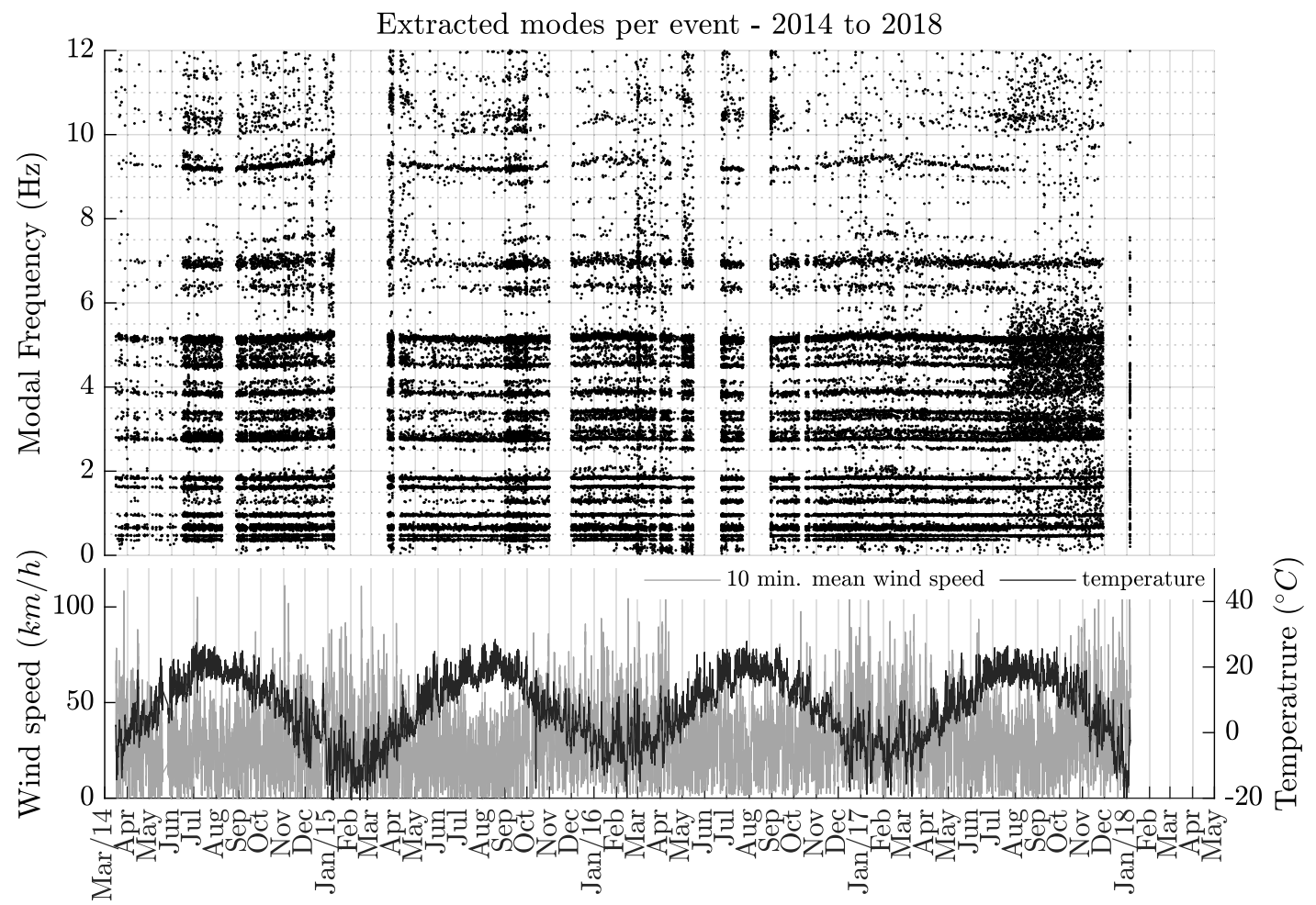

Figure 6.12: Extracted modal frequencies from full events collected between March 2014 and May 2018.

Figure 6.12 shows the ten top modes from each of the 12750 analyzed full events plotted temporally with corresponding environmental (wind and temperature) measurements. From Figure 6.12 the dominant modes and their temporal variations can be observed. The vertical gaps in the scatter plot correspond to periods where full events were not collected. Qualitatively, the correlation between the modal frequencies and temperature can be observed. This correlation is more evident for higher modes such as the $9.21 \mathrm{~Hz}$ and the $6.97 \mathrm{~Hz}$ modes. Also of note is the sudden increase in scatter observed in the OMA results of the data collection events occurring after late July 2017. This corresponds to the sudden malfunction of several sensors of data logger \# 1 on or near July 23rd 2017, as can be observed in Figure 6.4. It is now evident that contributing sensors greatly affect the results of operation modal analysis. 
Further statistical analysis using OMA results must consider the contributing sensors for comparisons. OMA results obtained with the maximum number of sensors seem to be more consistent with lower variability.

\subsubsection{Variability of OMA results}

The identification of dominant modes and the statistical information on their variability is shown in Table 6.2. The very complex task of tracking each mode through time was completed by the newly developed mode tracking algorithm described in Section 2.2.4. This mode tracking algorithm is an integral part of the new AI-FSI algorithm detailed in Section 2.2. In the first stage of the mode tracking algorithm, the dominant modes are clustered based on their frequency of identification as determined from the histogram calculations of Figure 6.9 and Figure 6.11. In a second stage, in order to confirm proper mode pairing of modal frequency and mode shape, the mode tracking algorithm recursively reduces the MAC matrix to ensure that the grouped modes are indeed the same mode and not a separate closely spaced mode. With this algorithm, the grouped modal parameter estimates (frequencies, mode-shapes and damping ratios) are assumed to be of the same mode.

The results shown in Table 6.2 correspond those obtained by the AI-FSI algorithm for each generation of the vibration monitoring system. The interquartile range shown in Table 6.2 is equal to the difference between the $75^{\text {th }}$ and $25^{\text {th }}$ percentiles of the distribution of modal frequencies and provides a good indication of variability along with the calculated standard deviation. The MAC average shown in this table is the calculated average in both dimensions of the MAC matrix obtained for a true mode group identified by the mode tracking algorithm. In the last column of Table 6.2 , the

number of identifications represents the number of times the mode was identified in the time period specified. 
Table 6.2: Identification and variability of operational modal estimates.

\begin{tabular}{|c|c|c|c|c|c|c|c|c|c|c|c|c|c|}
\hline \multirow[b]{2}{*}{ 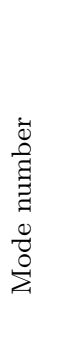 } & \multirow[b]{2}{*}{ 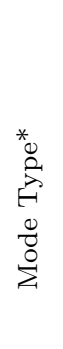 } & \multicolumn{6}{|c|}{ February 1998 to February 2014} & \multicolumn{6}{|c|}{ March 2014 to May 2018} \\
\hline & & 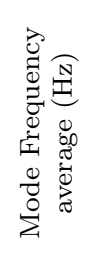 & 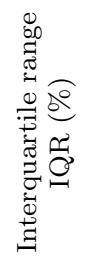 & 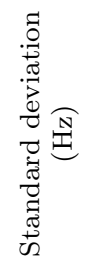 & 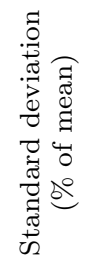 & 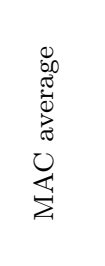 & 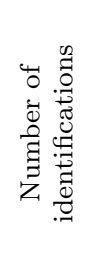 & 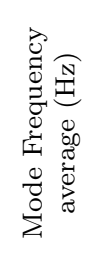 & 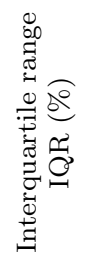 & 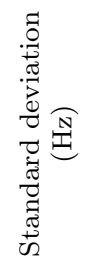 & 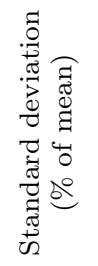 & 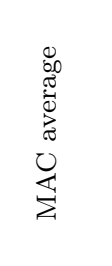 & 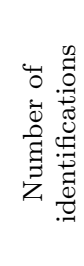 \\
\hline 1 & $T$ & 369 & 8.19 & 0.025 & 6.75 & 0.826 & 312 & 0.374 & 4.46 & 0.016 & 4.33 & 0.840 & 2411 \\
\hline 2 & $T$ & 0.474 & 1.91 & 0.007 & 1.45 & 0.863 & 544 & 0.470 & 1.91 & 0.007 & 1.48 & 0.966 & 4203 \\
\hline 3 & $V$ & 0.655 & 1.83 & 0.009 & 1.36 & 0.873 & 444 & 0.663 & 1.89 & 0.010 & 1.46 & 0.844 & 1662 \\
\hline 4 & $V$ & 677 & 2.11 & 0.011 & 1.60 & 0.831 & 295 & .674 & 1.58 & 0.011 & 1.56 & 0.937 & 4030 \\
\hline 5 & $V$ & 0.940 & 2.44 & 0.017 & 1.80 & 0.864 & 868 & 0.958 & 1.37 & 0.010 & 1.06 & 0.919 & 3156 \\
\hline 6 & $V$ & 269 & 4.31 & 0.043 & 3.36 & 0.811 & 56 & 06 & 3.22 & 0.036 & 2.76 & 0.890 & 2718 \\
\hline 7 & $V$ & 1.609 & 2.29 & 0.026 & 1.62 & 0.868 & 650 & 1.618 & 1.07 & 0.013 & 0.83 & 0.944 & 3649 \\
\hline 8 & $V$ & 1.814 & 2.00 & 0 & 1.45 & 0 & 0 & 2 & 0.98 & 15 & 0.78 & 5 & 2896 \\
\hline 9 & $V$ & 2.735 & 1.44 & 0.029 & 1.06 & 0.848 & 560 & 2.819 & 2.97 & 0.058 & 2.07 & 0.976 & 4042 \\
\hline 10 & $V$ & 848 & 2.07 & 0.046 & 1.61 & 0.828 & 290 & 2.860 & 4.14 & 0.072 & 2.50 & 0.935 & 2861 \\
\hline 11 & $V$ & 3.240 & 1.67 & 0.043 & 1.32 & 0.849 & 517 & 3.269 & 1.56 & 0.055 & 1.67 & 0.949 & 3777 \\
\hline 12 & $V$ & 3.825 & 1.61 & 0.043 & 1.12 & 0.881 & 635 & 3.844 & 1.06 & 0.032 & 0.82 & 0.898 & 1311 \\
\hline 13 & $V$ & 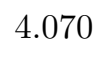 & 2.42 & 0. & 1.57 & 0.852 & 014 & 2 & 2.53 & 7 & 1.63 & 0.935 & 3020 \\
\hline 14 & $T_{o}$ & 4.640 & 3.70 & 0.105 & 2.26 & 0.883 & 1050 & 4.637 & 3.84 & 0.111 & 2.39 & 0.961 & 3930 \\
\hline 15 & $V$ & 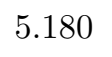 & 1.5 & 0. & 1. & 0. & 77 & 4 & 1.23 & 5 & 1.07 & 0.936 & 4080 \\
\hline 16 & $V$ & 6.351 & 1.84 & 0.077 & 1.22 & 0.943 & 303 & 6.348 & 0.95 & 0.046 & 0.72 & 0.914 & 3293 \\
\hline 17 & $T_{o}$ & 6.956 & 2.06 & 0.102 & 1.47 & 0.942 & 411 & 6.975 & 1.32 & 0.074 & 1.06 & 0.965 & 3935 \\
\hline 18 & $T_{o}$ & 9.350 & 2.84 & 0.189 & 2.02 & 0.812 & 231 & 9.725 & 0.63 & 0.058 & 0.60 & 0.924 & 1688 \\
\hline 19 & $T_{o}$ & 10.94 & 3.19 & 0.400 & 3.65 & 0.904 & 948 & 10.93 & 8.16 & 0.489 & 4.48 & 0.896 & 3129 \\
\hline 20 & $T_{o}$ & 12.85 & 6.23 & 0.484 & 3.77 & 0.916 & 967 & 12.57 & 3.93 & 0.473 & 3.59 & 0.919 & 2252 \\
\hline
\end{tabular}

${ }^{*} T$ : transverse mode; $V$ : vertical mode; $T_{o}:$ torsional mode.

Figure 6.13 and Figure 6.14 show box plots of the dominant modes extracted in each of the identified collection period. Results from Table 6.2 and from Figures 6.13 and 6.14 indicate that operational modal estimates can be reliably, consistently and automatically-extracted from highly uncertain and noisy data on a much larger 


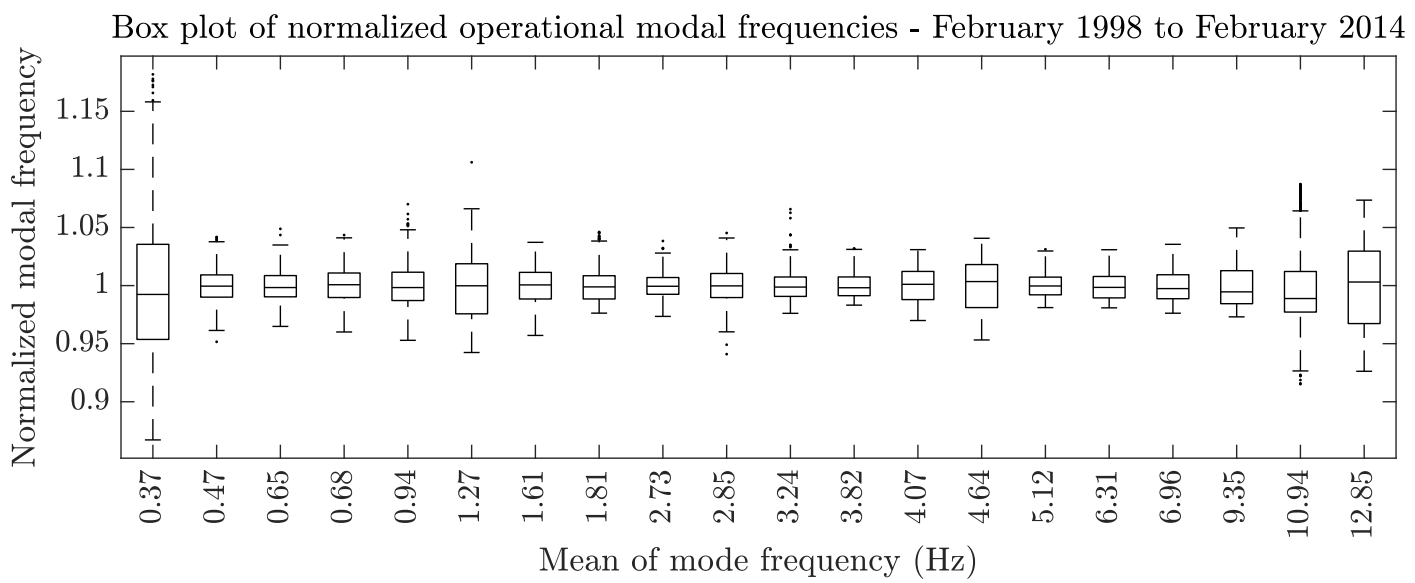

Figure 6.13: Box plot of normalized operational modal frequencies from full events collected between February 1998 and February 2014.

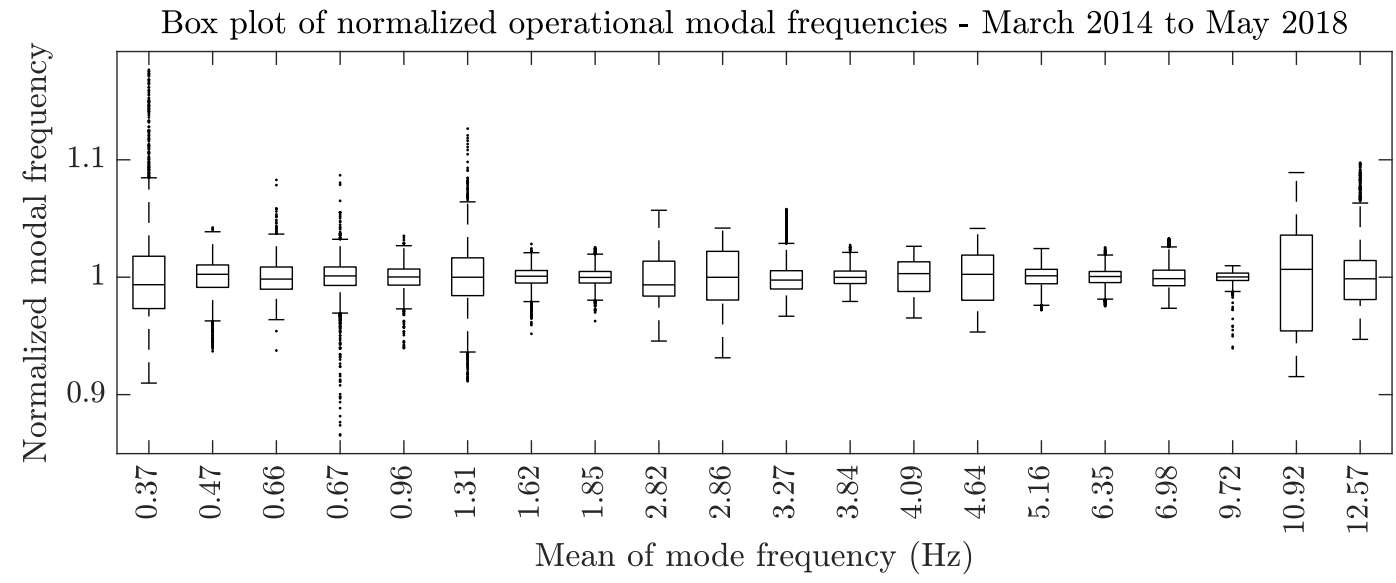

Figure 6.14: Box plot of normalized operational modal frequencies from full events collected between March 2014 and May 2018.

scale than was previously undertaken. A general improvement in the quality of OMA results can also be observed from the first to the second generation of the monitoring system, especially regarding mode shape identification. Improvements are attributable to better data acquisition system (sensors, loggers, etc.), more streamlined processing and improved parameter estimations in the OMA. 


\section{Chapter 7}

\section{Condition Assessment by Statistical Analysis of Operational Modal Estimates}

The promising perspective of vibration-based structural damage detection has inspired many researchers internationally. Doebling et al. surveyed and classified the literature [53]. The most promising of the recent damage detection techniques are data driven; such as statistical pattern recognition $[14,54]$ and data mining of statistical properties of vibration responses $[55,56]$. Most of these data driven techniques use vibration responses directly for system identification or to either identify patterns or features in the acceleration signals or to observe trends in signal properties such as maximums, signal amplitudes or standard deviations of the response signals. The approach proposed in this chapter, considers the operational modal properties extracted with the AI-FSI method described in Section 2.2 as the features for statistical analysis. By exponentially increasing the collection and the operational modal analyses of response data, and by tracking the modal parameter estimates through time, trends and features can be observed. Only through a good understanding of the variability of the estimates and the correlations with environmental effects and other operational parameters, can reliable proper damage identification be considered. 
In this Chapter, in-depth data analysis of the data collected over a one month period is carried out. The vibration response data of a total of 3910 full events were collected in November 2017 and these data have been processed and analyzed with the SPLASH application described in Chapter 5. The operational modal estimates are detailed in Section 7.1, and their statistical analysis is described in Sections 7.2 to 7.4. Using the results of filtering and regression, a sensitive change point detection algorithm is implemented in Section 7.5 and proposed for detection of possible structural damage or deterioration scenarios.

\subsection{OMA Results}

The extracted modal frequencies and mode shapes from data events collected in November 2017 are presented in this section. Figure 7.1 shows the most dominant extracted modal frequencies in the range of 0 to $12 \mathrm{~Hz}$ for each full event.

Only the ten most strongly identified modes in the 0 to $12 \mathrm{~Hz}$ for each single event were plotted in Figure 7.1. The selection of the top ten extracted modal frequencies are based on the consistency of identification in the stabilization plots of the improved system identification technique described in Section 2.2.3. The number of times the mode was identified through the model orders was recorded by the AI-FSI algorithm and was used here to sort the extracted modes and plot the top ten modes within the frequency range of interest for each single data event. This parameter is closely related to modal participation, where strongly excited modes are more consistently identified through the solution model orders. Wind and temperature measurements are also presented in Figure 7.1 for qualitative observations of correlation with the extracted modal frequency information. While the scatter of the extracted vibration modes is relatively high, certain modes are well identified and interesting patterns 


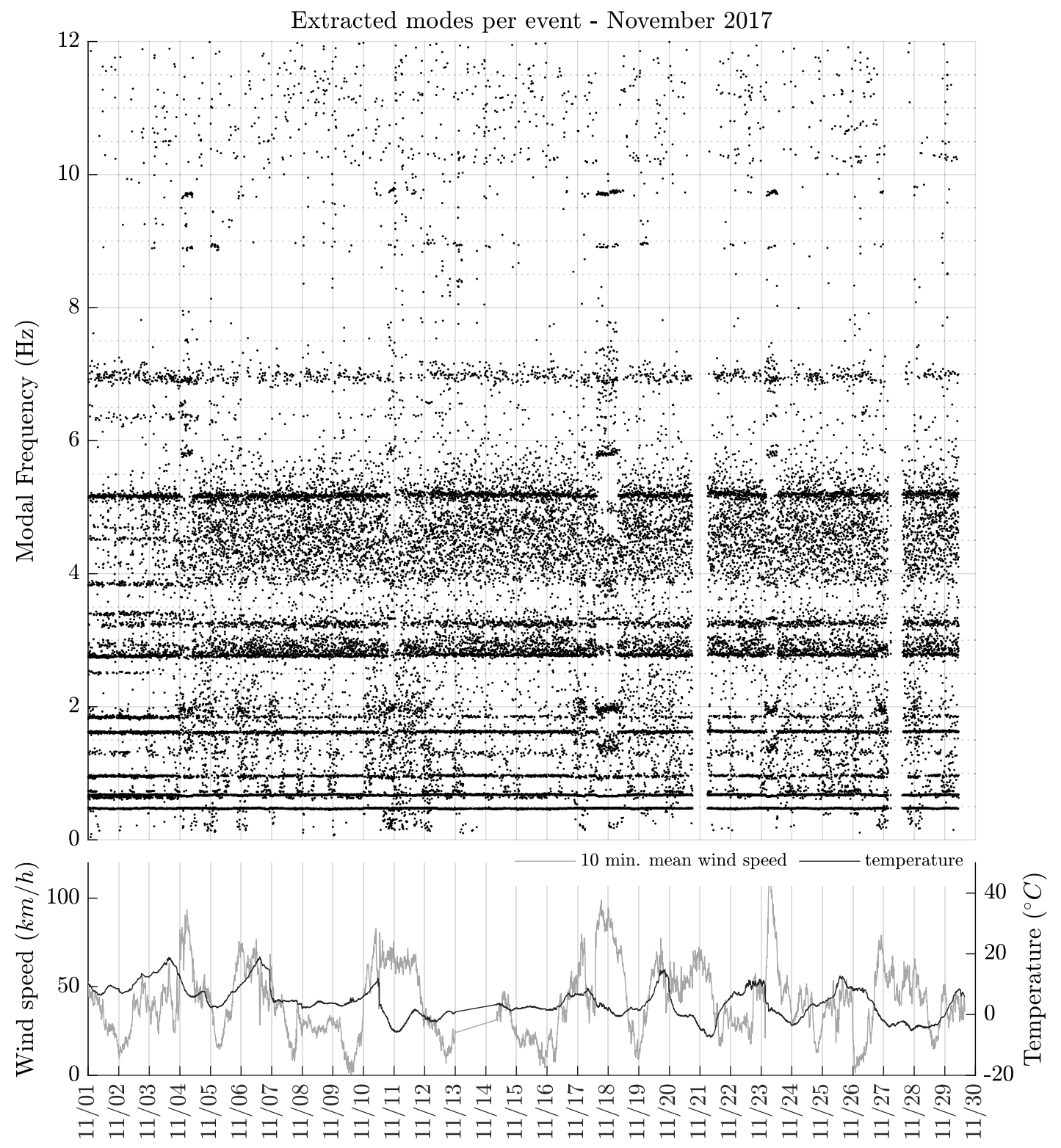

Figure 7.1: Extracted frequencies from full events collected in November 2017.

can be observed during stronger wind conditions. The month of November 2017 was a particularly windy month with several strong wind events as can be observed in Figure 7.1.

Table 7.1 shows the variability of the operational modal estimates for the first 20 consistently identified modes from the full events collected in November 2017. 
The grouping of each dominant mode was completed by the newly developed mode tracking algorithm described in Section 2.2.4. The interquartile range of the operational modal frequencies shown in Table 7.1 is equal to the difference between the $75^{\text {th }}$ and $25^{\text {th }}$ percentiles of the distribution of modal frequencies and provides a good indication of variability along with the standard deviation.

Table 7.1: Variability of operational modal estimates - November 2017.

\begin{tabular}{|c|c|c|c|c|c|c|c|}
\hline $\begin{array}{c}\text { Mode } \\
\text { number }\end{array}$ & $\begin{array}{l}\text { Mode } \\
\text { Type* }\end{array}$ & $\begin{array}{l}\text { Mode } \\
\text { Frequency } \\
\text { average } \\
(\mathrm{Hz})\end{array}$ & $\begin{array}{l}\text { Interquartile } \\
\text { range IQR } \\
\quad(\%)\end{array}$ & $\begin{array}{c}\text { Standard } \\
\text { deviation } \\
(\mathrm{Hz})\end{array}$ & $\begin{array}{l}\text { Standard } \\
\text { deviation } \\
(\% \text { of } \\
\text { mean })\end{array}$ & $\begin{array}{c}\text { MAC } \\
\text { average }\end{array}$ & $\begin{array}{l}\text { Number of } \\
\text { identifica- } \\
\text { tions }\end{array}$ \\
\hline 1 & $T$ & 0.389 & 5.77 & 0.017 & 4.26 & 0.856 & 878 \\
\hline 2 & $T$ & 0.471 & 1.68 & 0.006 & 1.29 & 0.983 & 3518 \\
\hline 3 & $V$ & 0.650 & 3.97 & 0.017 & 2.60 & 0.908 & 2243 \\
\hline 4 & $V$ & 0.674 & 1.54 & 0.008 & 1.21 & 0.964 & 3444 \\
\hline 5 & $V$ & 0.958 & 1.36 & 0.009 & 0.98 & 0.926 & 2907 \\
\hline 6 & $V$ & 1.308 & 3.15 & 0.035 & 2.68 & 0.902 & 2340 \\
\hline 7 & $V$ & 1.619 & 0.99 & 0.013 & 0.80 & 0.951 & 3305 \\
\hline 8 & $V$ & 1.853 & 0.96 & 0.014 & 0.78 & 0.904 & 2809 \\
\hline 9 & $V$ & 2.820 & 2.82 & 0.056 & 1.98 & 0.983 & 3463 \\
\hline 10 & $V$ & 2.862 & 4.08 & 0.072 & 2.50 & 0.939 & 2551 \\
\hline 11 & $V$ & 3.268 & 1.43 & 0.052 & 1.58 & 0.954 & 3176 \\
\hline 12 & $V$ & 3.871 & 2.18 & 0.050 & 1.30 & 0.960 & 1068 \\
\hline 13 & $V$ & 4.097 & 2.43 & 0.066 & 1.60 & 0.937 & 2566 \\
\hline 14 & $T_{o}$ & 4.639 & 3.84 & 0.111 & 2.40 & 0.963 & 3288 \\
\hline 15 & $V$ & 5.170 & 1.07 & 0.052 & 1.00 & 0.940 & 3421 \\
\hline 16 & $V$ & 6.351 & 0.90 & 0.044 & 0.69 & 0.930 & 2832 \\
\hline 17 & $T_{o}$ & 6.979 & 1.31 & 0.073 & 1.04 & 0.978 & 3301 \\
\hline 18 & $T_{o}$ & 9.729 & 0.58 & 0.054 & 0.55 & 0.928 & 1471 \\
\hline 19 & $T_{o}$ & 10.30 & 0.84 & 0.235 & 2.28 & 0.914 & 2076 \\
\hline 20 & $T_{o}$ & 12.59 & 3.03 & 0.354 & 2.81 & 0.919 & 1932 \\
\hline
\end{tabular}

${ }^{*} T$ : transverse mode; $V$ : vertical mode; $T_{o}:$ torsional mode.

The last column in Table 7.1 indicates the number of times the mode was identified during the month of November 2017. The MAC average values for each mode are obtained by averaging the MAC matrix along a single dimension and then calculating the mean of the resulting vector. It should be noted that the MAC is a statistical indicator that is most sensitive to large differences and relatively insensitive to small 
differences in the mode shapes [36].

Figure 7.2 shows the box plots of the dominant normalized operational modal frequencies of Table 7.1. It is clear from this figure that some modes are more consistently identified and are inherently less variable than others.

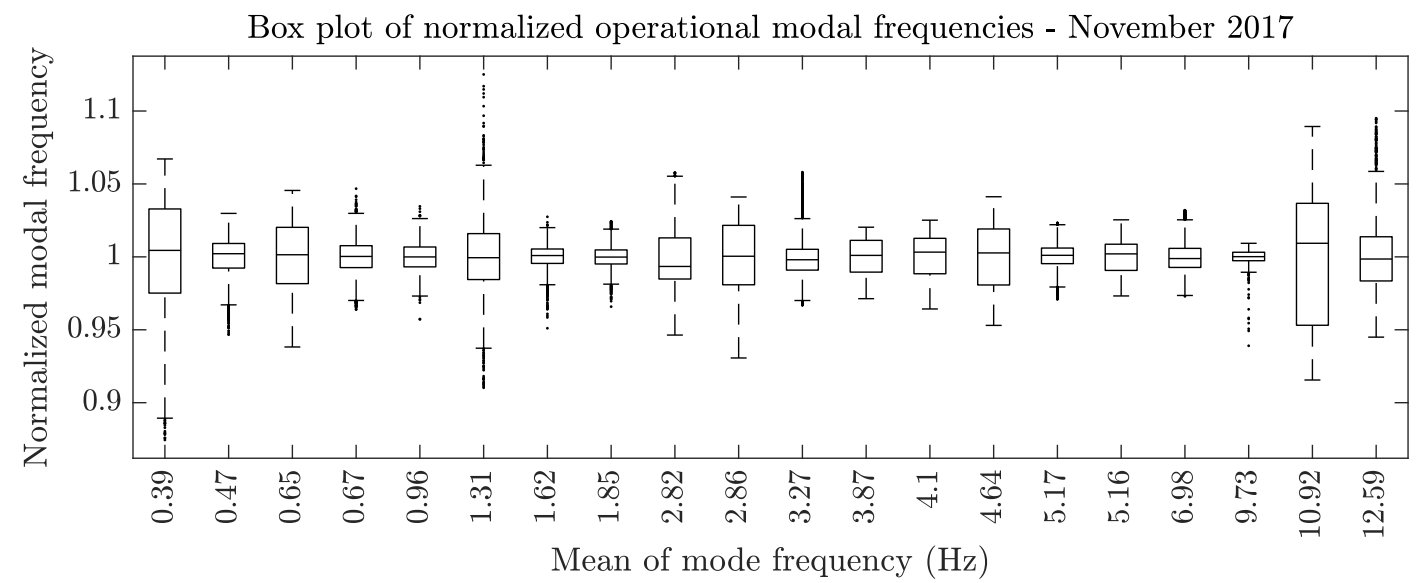

Figure 7.2: Box plot of normalized operational modal frequencies - November 2017.

The modes at $0.47 \mathrm{~Hz}, 0.67 \mathrm{~Hz}, 0.96 \mathrm{~Hz}, 1.62 \mathrm{~Hz}$, and $1.85 \mathrm{~Hz}$ are identified consistently with standard deviations between 0.78 and 1.3 percent of mean. These well tracked and well identified modes are further analyzed in the following sections. The estimated operational mode shapes for these modes are shown in Appendix A.

\subsection{Smoothing of OMA Estimates}

In this section, the modal frequencies of the $0.47 \mathrm{~Hz}, 0.67 \mathrm{~Hz}, 0.96 \mathrm{~Hz}, 1.62 \mathrm{~Hz}$ and $1.85 \mathrm{~Hz}$ modes extracted from the data collected in November 2017 are subjected to several data smoothing operations in order to observe any possible temporal trends which may be related to environmental or operational processes.

By treating the extracted modal frequencies of a single mode as a time series signal, the data smoothing techniques detailed in Table 7.2 are applied. It should be 
noted that the modal frequencies included in each time series are for tracked modes obtained from the automated mode tracking algorithm incorporated in the AI-FSI technique described in Section 2.2. This means that the mode shape associated with each modal frequency matches all other mode shapes to within MAC tolerance of 0.75 (see Table 7.1 for MAC averages).

Table 7.2: Description of smoothing methods considered in this study.

\begin{tabular}{|c|c|}
\hline $\begin{array}{l}\text { Smoothing } \\
\text { method }\end{array}$ & Description \\
\hline $\begin{array}{l}\text { Moving } \\
\text { average }\end{array}$ & $\begin{array}{l}\text { Moving average over each window of the operational modal frequency time series. } \\
\text { This method is useful for reducing some periodic trends in data, such as measure- } \\
\text { ment and computational noise. The average is calculated with equal weight to } \\
\text { each frequency sample. Not only is the moving average filter suitable for many } \\
\text { applications, it is optimal for the common problem of reducing random noise while } \\
\text { keeping a sharp step response. }\end{array}$ \\
\hline $\begin{array}{l}\text { Gaussian- } \\
\text { weighted } \\
\text { moving } \\
\text { average }\end{array}$ & $\begin{array}{l}\text { Gaussian-weighted moving average over each window of the operational modal } \\
\text { frequency time series. A variable weight is applied to each sample inside the } \\
\text { moving window. The weighting follows a Gaussian distribution around the center } \\
\text { of the moving window. }\end{array}$ \\
\hline $\begin{array}{l}\text { Linear } \\
\text { regression }\end{array}$ & $\begin{array}{l}\text { Linear regression over each window of the operational modal frequency time series. } \\
\text { This method can be computationally expensive, but results in fewer discontinuities. } \\
\text { The advantage of the linear regression method over a normal moving average is } \\
\text { that it has less lag than the moving average, responding quicker to changes in } \\
\text { modal frequencies. }\end{array}$ \\
\hline $\begin{array}{l}\text { Quadratic } \\
\text { regression }\end{array}$ & $\begin{array}{l}\text { Quadratic regression over each window of the operational modal frequency vector. } \\
\text { This method is slightly more computationally expensive than linear regression with } \\
\text { better results on signals whose variations can be described by a second degree } \\
\text { polynomial (parabola). }\end{array}$ \\
\hline
\end{tabular}

Each smoothing method described in Table 7.2 is applied to the modal frequency time series of the dominant modes $(0.47 \mathrm{~Hz}, 0.67 \mathrm{~Hz}, 0.96 \mathrm{~Hz}, 1.62 \mathrm{~Hz}$ and $1.85 \mathrm{~Hz})$ and plotted in Figures 7.3 to 7.7. Each method was applied with centered window lengths of 5, 10, 20 and 50 samples. Also plotted at the bottom of each figure are two environmental parameters (wind and temperature) which are added for qualitative observations of possible correlations. The right side of the figures provides a closer perspective of variations and smoothing of data collected on a time scale of 2 days. 

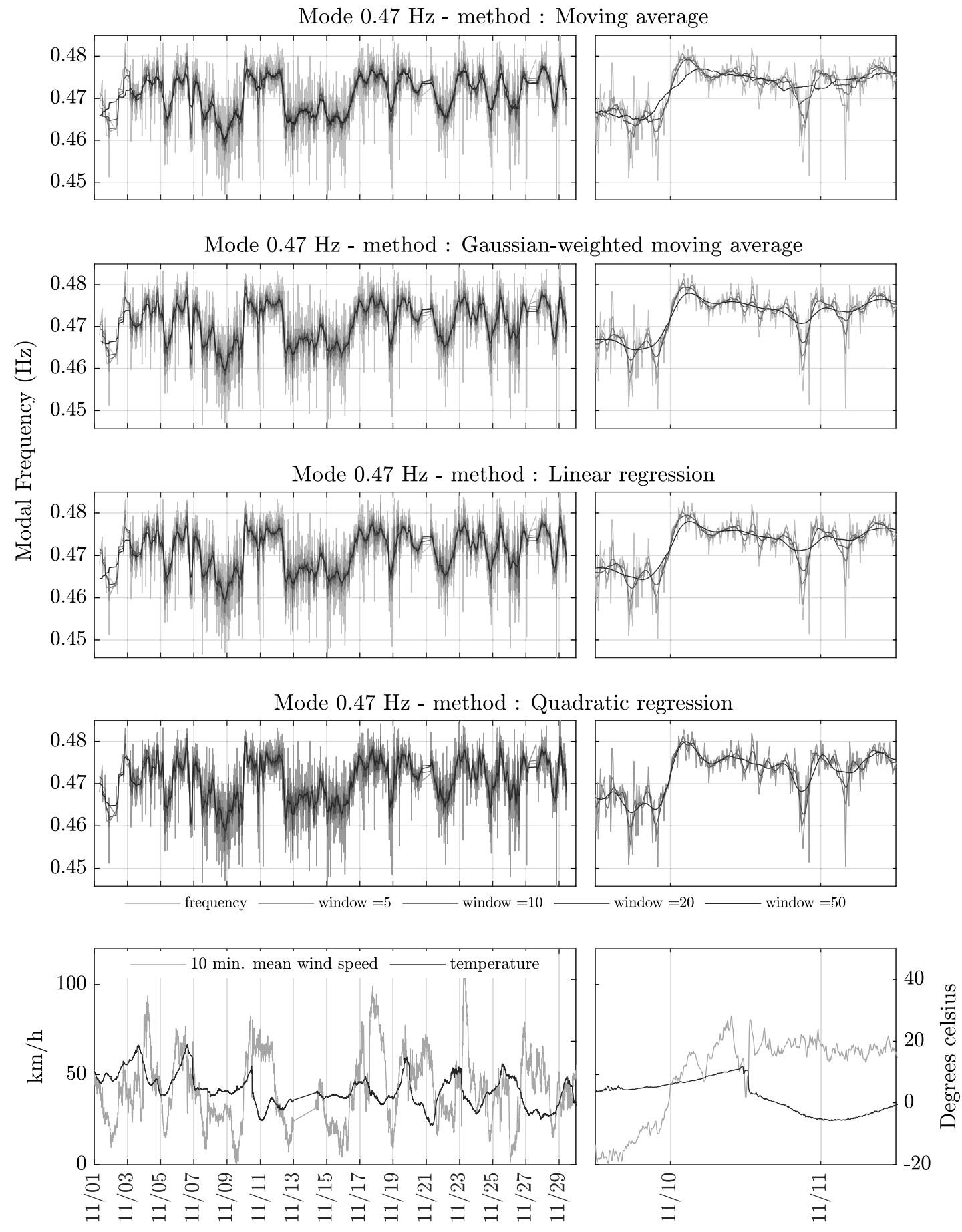

Figure 7.3: Data smoothing of the operational modal frequencies $-0.47 \mathrm{~Hz}$ mode. 

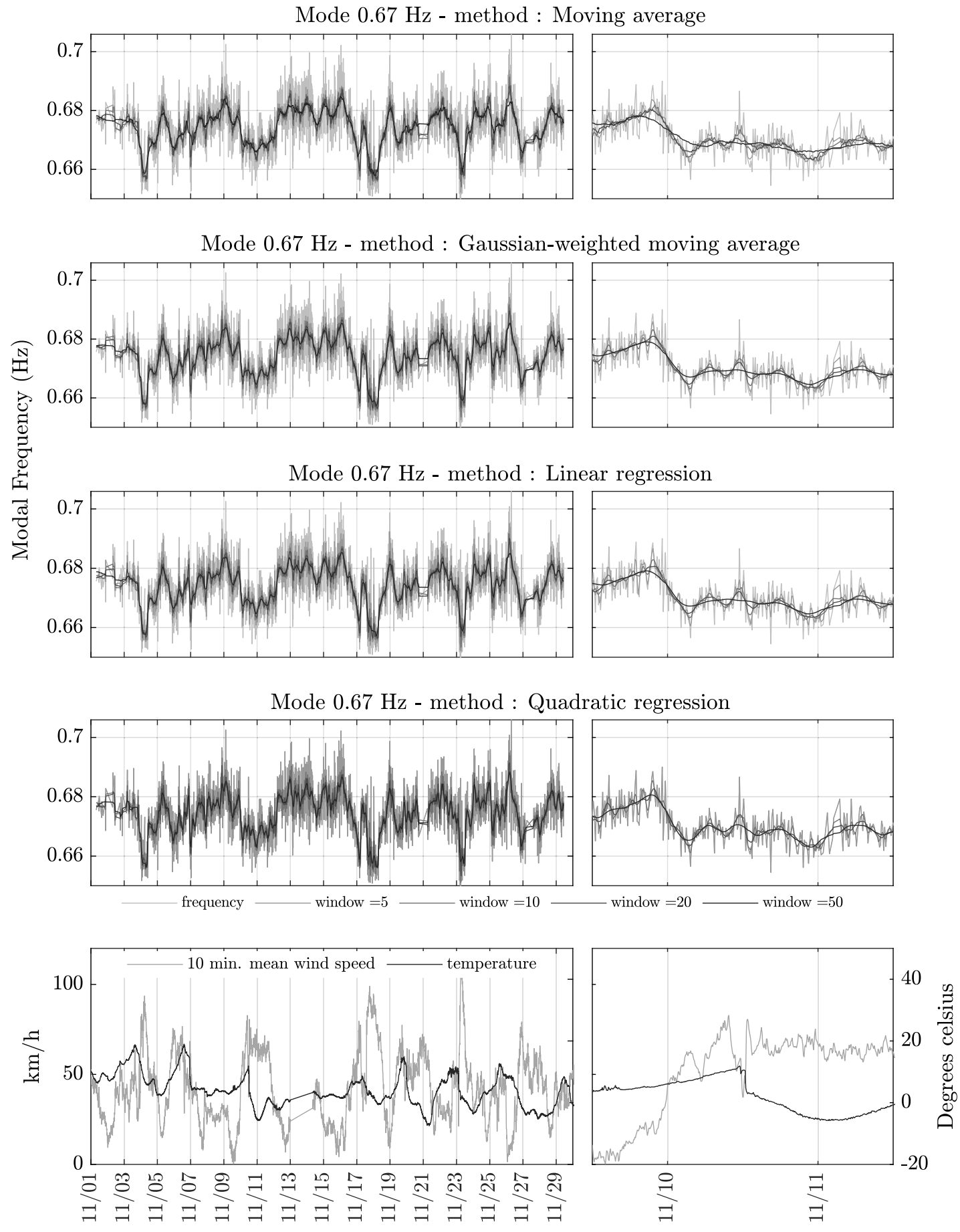

Figure 7.4: Data smoothing of the operational modal frequencies $-0.67 \mathrm{~Hz}$ mode. 

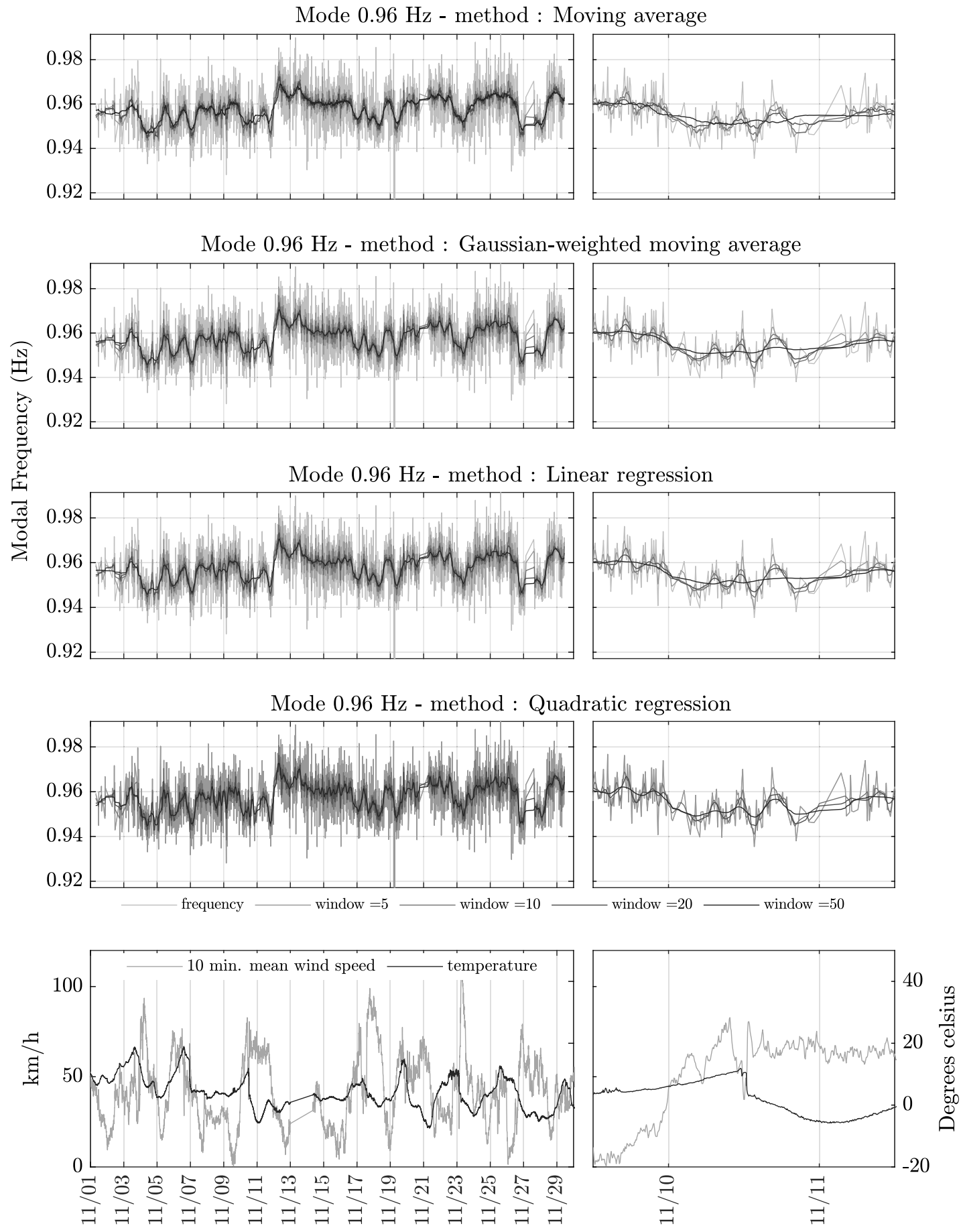

Figure 7.5: Data smoothing of the operational modal frequencies $-0.96 \mathrm{~Hz}$ mode. 

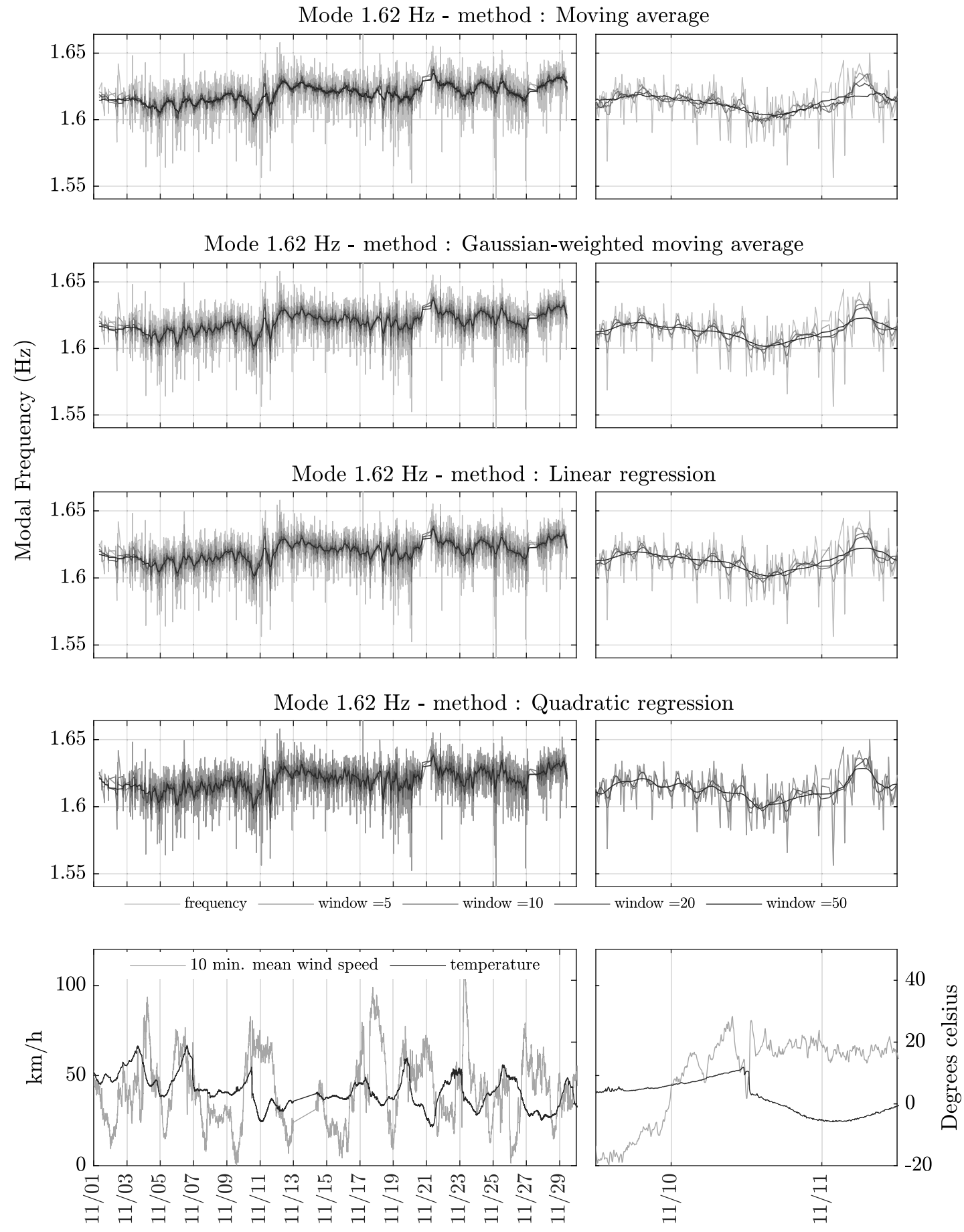

Figure 7.6: Data smoothing of the operational modal frequencies $-1.62 \mathrm{~Hz}$ mode. 

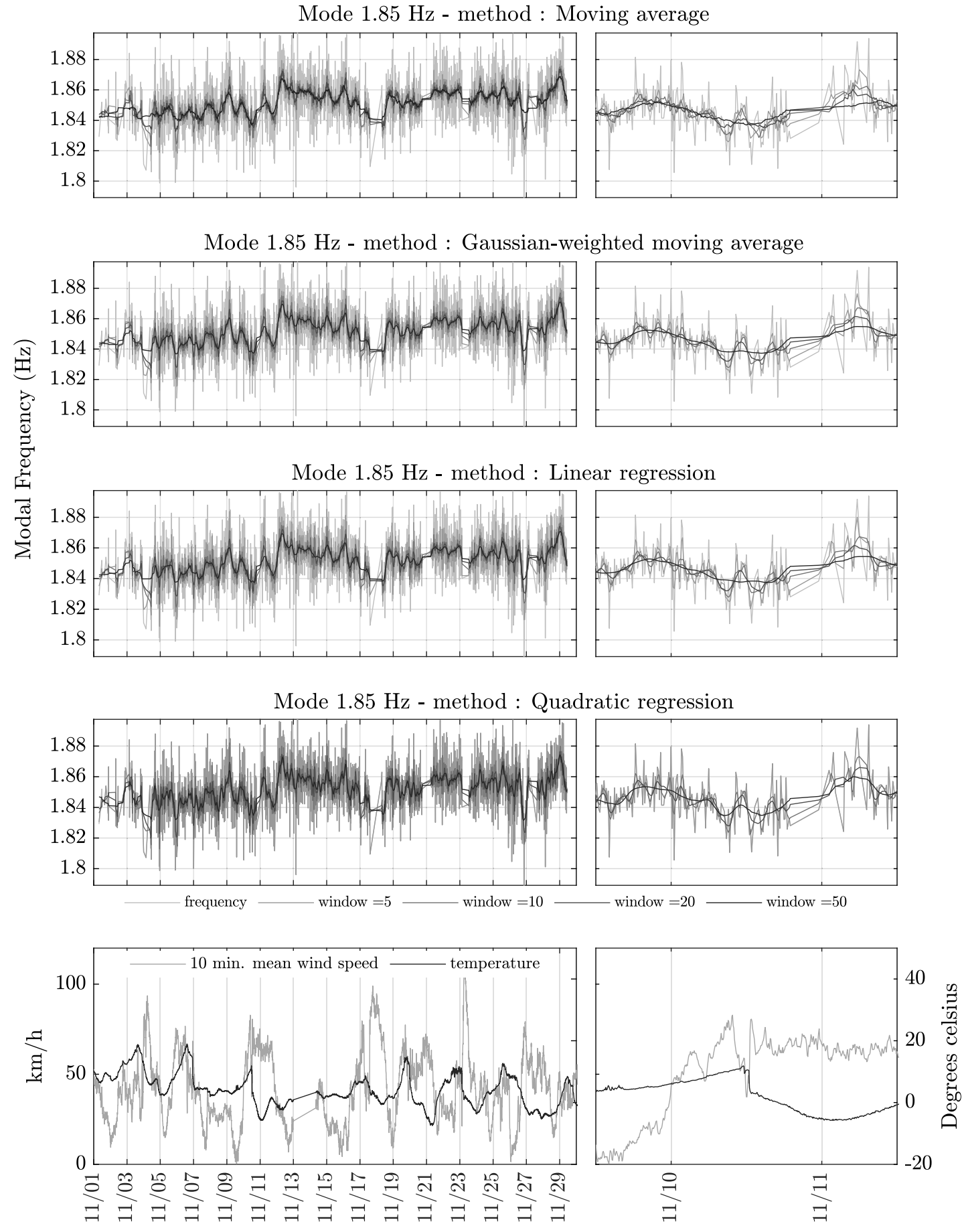

Figure 7.7: Data smoothing of the operational modal frequencies $-1.85 \mathrm{~Hz}$ mode. 
Definite correlations between the estimated modal frequencies and the wind are observed; especially for the lower modes. The transverse mode at $0.47 \mathrm{~Hz}$ seems to be directly related to wind while the modes at $0.67 \mathrm{~Hz}$ and $0.96 \mathrm{~Hz}$ seem to be inversely proportional to wind speed, meaning the amplitude of their estimated frequencies diminish with increases in wind speed. Correlations with the ambient air temperatures are less evident in these figures. However, some temperature effects on modal frequencies are observable on longer time scales. The reader is referred to Figures 6.10 and 6.12 for evidence of temperature trends in modal frequencies.

The mode at $1.85 \mathrm{~Hz}$ plotted in Figure 7.7 seem to show daily cycles of variations which are also observed in the variations of RMS (Figure 6.8). This may indicate some correlation with traffic patterns. Also of note is the lack of proper identification of this mode during strong wind events. Which further corroborates the hypothesis that this mode is related to traffic excitations as the bridge operators limits vehicular travel during strong wind events.

To quantify these observations, linear regression models are created and evaluated in Section 7.4.

\subsection{Extended Kalman Filtering of OMA estimates}

In order to account for the inherent variability of OMA results due to modeling error, noise, non-stationarity of the actual ambient vibrations, and non-linearity of the structure, the application of an Extended Kalman Filter (EKF) on the system identification results is proposed. The Kalman filter uses a series of measurements observed over time, containing noise, random variations and other inaccuracies. The Kalman filter algorithm estimates the state of unknown variables more precisely than the estimates that would be based on measurements alone. Specifically, the Kalman 
filter operates recursively on time series of noisy input data to produce an optimal estimate of the underlying state. The noisy input data considered in this study are the estimated modal parameters of the system, specifically: modal frequencies. Any statistically significant deviations from the Kalman estimates could indicate damage or deterioration of the system.

In this section, the estimated modal parameters obtained from the 3910 full datasets collected in November 2017 are processed through an Extended Kalman Filter $(\mathrm{EKF})$, which shows promising results in obtaining meaningful estimation in the presence of noise and significant outliers [57-59]. The first-order discrete-time Kalman filter algorithm is summarily described here. The general formulation of the state transition and measurement functions are shown in Equation (7.1).

$$
\begin{gathered}
\mathbf{x}_{k}=f\left(\mathbf{x}_{k-1}, \mathbf{u}_{k}\right)+\mathbf{w}_{k} \\
\mathbf{y}_{k}=h\left(\mathbf{x}_{k}\right)+\mathbf{v}_{k}
\end{gathered}
$$

In the general formulation of Equation (7.1), the function $f$ is a nonlinear transition function that describes the evolution of the states $\mathbf{x}$ and can be used to compute the predicted state from the previous estimate. Similarly the function $h$ which relates $\mathbf{x}$ to the measurement $\mathbf{y}$ at time step $k$, can be used to compute the predicted measurement from the predicted state. The vector $\mathbf{u}_{k}$ is an optional control vector passed on to the non linear transition function, while $\mathbf{w}_{k}$ and $\mathbf{v}_{k}$ are the process and observation noises which are both assumed to be zero mean Gaussian noises with covariance $\mathbf{Q}_{k}$ and $\mathbf{R}_{k}$ respectively as shown in Equation (7.2).

$$
\begin{aligned}
\mathbf{w}_{k} & \sim \mathcal{N}\left(0, \mathbf{Q}_{k}\right) \\
\mathbf{v}_{k} & \sim \mathcal{N}\left(0, \mathbf{R}_{k}\right)
\end{aligned}
$$

The state transition function $f$ and the measurement function $h$ are not applied 
to the covariance directly. Instead a matrix of partial derivatives (the Jacobian) is computed. The Jacobians of the state transition and measurement matrices with respect to the state are shown in Equation (7.3)

$$
\begin{gathered}
\mathbf{F}_{k}=\left.\frac{\partial f}{\partial \mathbf{x}}\right|_{\hat{\mathbf{x}}_{k-1 \mid k-1}, \mathbf{u}_{k}} \\
\mathbf{H}_{k}=\left.\frac{\partial h}{\partial \mathbf{x}}\right|_{\hat{\mathbf{x}}_{k \mid k-1}}
\end{gathered}
$$

At each time step $k$, the Jacobian is evaluated with current predicted states. These matrices are used in the Kalman filter equations. This process essentially linearizes the non-linear function around the current estimate. The predicted state estimates and covariance estimates are shown in Equation (7.4).

$$
\begin{gathered}
\hat{\mathbf{x}}_{k \mid k-1}=f\left(\hat{\mathbf{x}}_{k-1 \mid k-1}, \mathbf{u}_{k}\right) \\
\mathbf{P}_{k \mid k-1}=\mathbf{F}_{k} \mathbf{P}_{k-1 \mid k-1} \mathbf{F}_{k}^{T}+\mathbf{Q}_{k}
\end{gathered}
$$

The update or a posteriori equations are then calculated using the predicted state and covariance estimates. First, the measurement and covariance residuals (innovation) are calculated in Equation (7.5)

$$
\begin{gathered}
\overline{\mathbf{z}}_{k}=\mathbf{y}_{k}-h\left(\hat{\mathbf{x}}_{k \mid k-1}\right) \\
\mathbf{S}_{k}=\mathbf{H}_{k} \mathbf{P}_{k \mid k-1} \mathbf{H}_{k}^{T}+\mathbf{R}_{k}
\end{gathered}
$$

Then, the Kalman gain is calculated in Equation (7.6)

$$
\mathbf{K}_{k}=\mathbf{P}_{k \mid k-1} \mathbf{H}_{k}^{T} \mathbf{S}_{k}^{-1}
$$


With the Kalman gain, the updated state and covariance estimates can be calculated according to Equation (7.7)

$$
\begin{gathered}
\hat{\mathbf{x}}_{k \mid k}=\hat{\mathbf{x}}_{k \mid k-1} \mathbf{K}_{k} \overline{\mathbf{z}}_{k} \\
\mathbf{P}_{k \mid k}=\mathbf{P}_{k \mid k-1}-\mathbf{K}_{k} \mathbf{H}_{k} \mathbf{P}_{k \mid k-1}
\end{gathered}
$$

In Figures 7.8 to 7.12 , the updated state estimates of modal frequencies are plotted over the actual operational modal frequencies. For each of the identified dominant modes $(0.47 \mathrm{~Hz}, 0.67 \mathrm{~Hz}, 0.96 \mathrm{~Hz}, 1.62 \mathrm{~Hz}$ and $1.85 \mathrm{~Hz})$ the state estimates are the extracted modal frequencies. Since the underlying assumption is that the modal frequencies are invariable in time for a healthy structure, any observed variations are attributed to measurement and process noise. Therefore, the state transition function and the measurement functions considered are shown in Equation (7.8).

$$
\begin{gathered}
x_{k}=x_{k-1}+w_{k} \\
y_{k}=x_{k}+v_{k}
\end{gathered}
$$

Once the state and measurement functions are set, the remaining user defined parameters for the extended Kalman filter include the initial state $x_{0}$, the initial state covariance $P_{0}$, the variance of process noise $Q$ and the measurement noise $R$. The initial state is obtained from measurements prior to time step $k=1$; a mean of prior measurements in this case. The initial covariance $P_{0}$ is defined based on the initialization error. Typically, if there is high confidence in the initial state estimate, meaning that the states are very close to the actual values, a small $P_{0}$ is used (for an exact match $P_{0}$ is taken as 0 ). Alternatively if the confidence in the initial state is low, the value of $P_{0}$ should be large. A value corresponding to the variance of measurements prior to time step $k=1$ was chosen here with good results as shown 
in Figures 7.8 to 7.12 . The variance of process noise $Q$ can vary from zero when assuming perfect process model (i.e. frequency is a constant steady state value) to a value greater than zero (meaning there is a probability that the modal frequencies vary in time). For each mode several values of $Q$ were considered to observe the effect on filtered results as shown in Figures 7.8 to 7.12 .

The measurement noise variance $R$, is determined from the regression results of Section 7.4 and is kept constant. For each mode, the variance of the residuals are used as the estimated variance of the measurement noise in the extended Kalman filter. This means that any variations of modal frequency due to known environmental and operational parameters are considered as process by the Kalman filter and can be filtered out by lowering the variance of the process noise. Essentially, the relative magnitude between process and measurement noise determines the importance of the measurements. In one extreme, if the process noise is zero the Kalman filter will effectively ignore new measurements because the process model is considered exact (i.e. zero noise). At the other extreme, if the process noise is very large and the measurement noise is very small, the state essentially resets to match the measurements at each time step. For the modes at $0.47 \mathrm{~Hz}, 0.67 \mathrm{~Hz}, 0.96 \mathrm{~Hz}, 1.62 \mathrm{~Hz}$ and $1.85 \mathrm{~Hz}$ the respective measurement noise variances considered are $R=1.44 \times 10^{-5} \mathrm{~Hz}^{2}$, $R=2.40 \times 10^{-5} \mathrm{~Hz}^{2}, R=6.63 \times 10^{-5} \mathrm{~Hz}^{2}, R=9.22 \times 10^{-5} \mathrm{~Hz}^{2}, R=1.19 \times 10^{-4}$ $\mathrm{Hz}^{2}$.

By reducing the process noise, the short term variations of modal frequencies due to environmental and operational effects are filtered out and an estimate of the actual modal frequency can be made. Estimated frequencies of $0.471 \mathrm{~Hz}, 0.674 \mathrm{~Hz}, 0.958 \mathrm{~Hz}$, $1.620 \mathrm{~Hz}$ and $1.852 \mathrm{~Hz}$ are thus obtained. After an initial training phase, the filtered estimates with non-zero process noise vary according to trends due to environmental and or operational processes and confirm the trends observed from data smoothing 


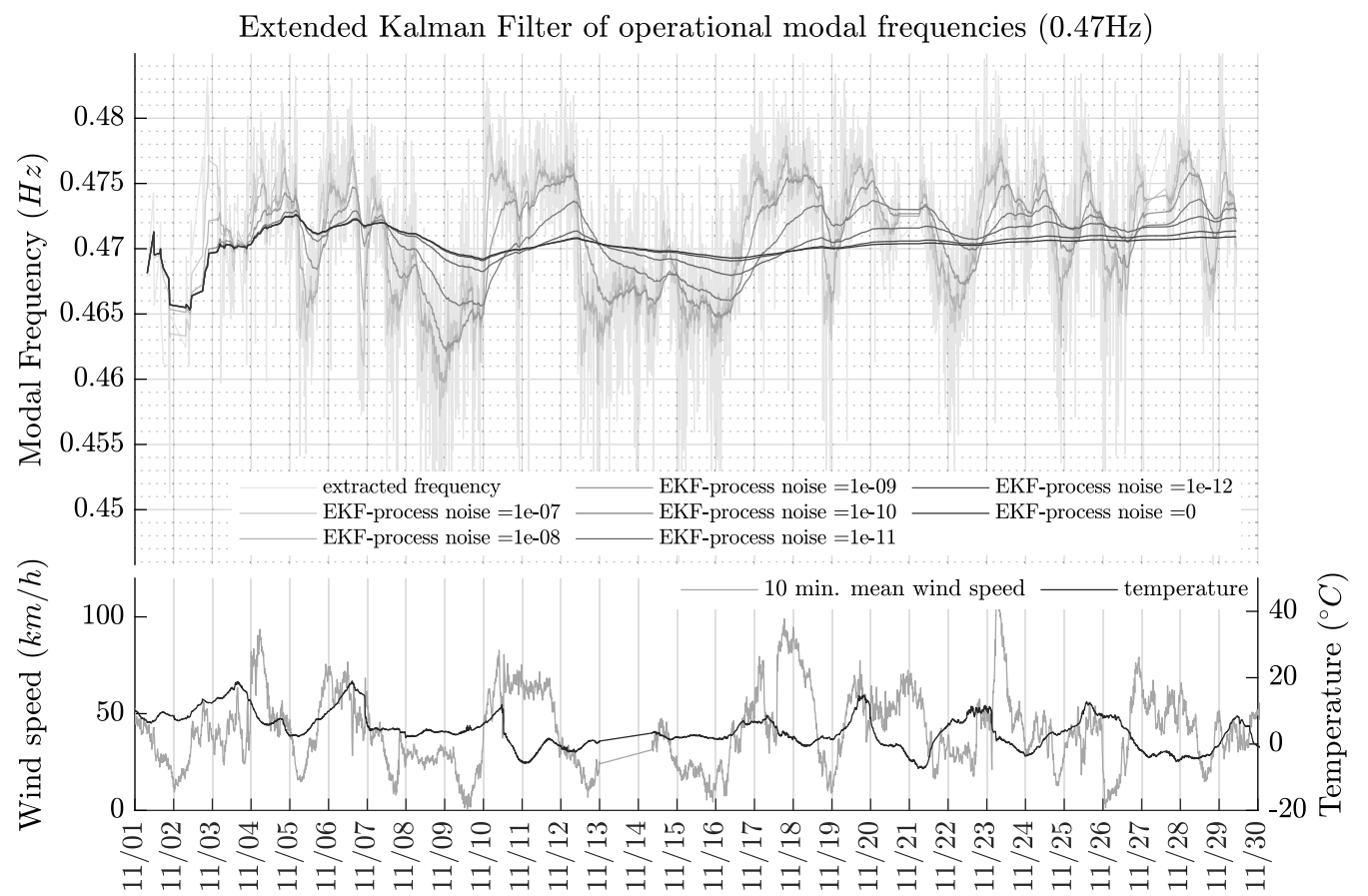

Figure 7.8: Extended Kalman filtering of the modal frequencies (0.47Hz mode).

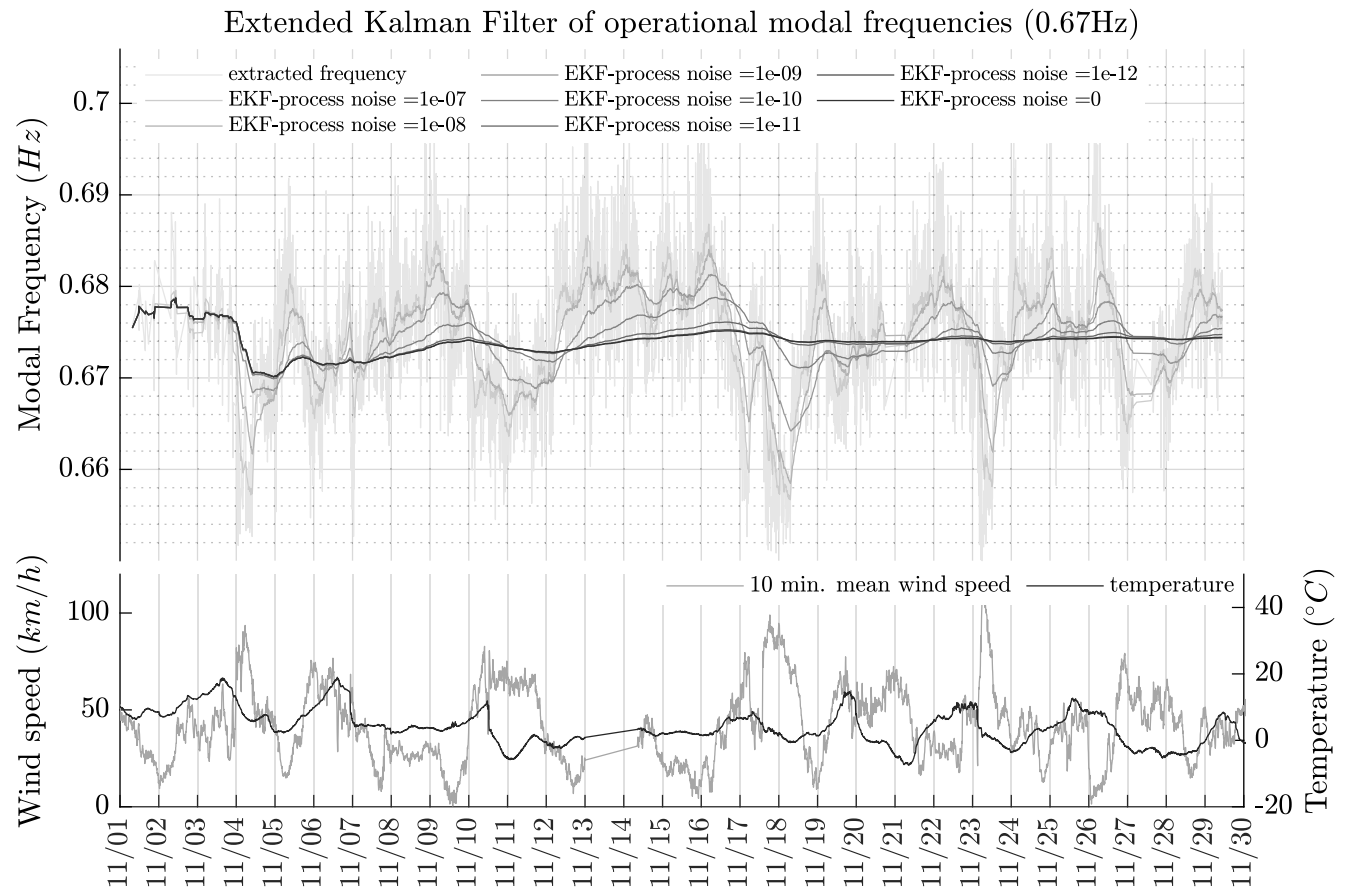

Figure 7.9: Extended Kalman filtering of the modal frequencies (0.67Hz mode). 


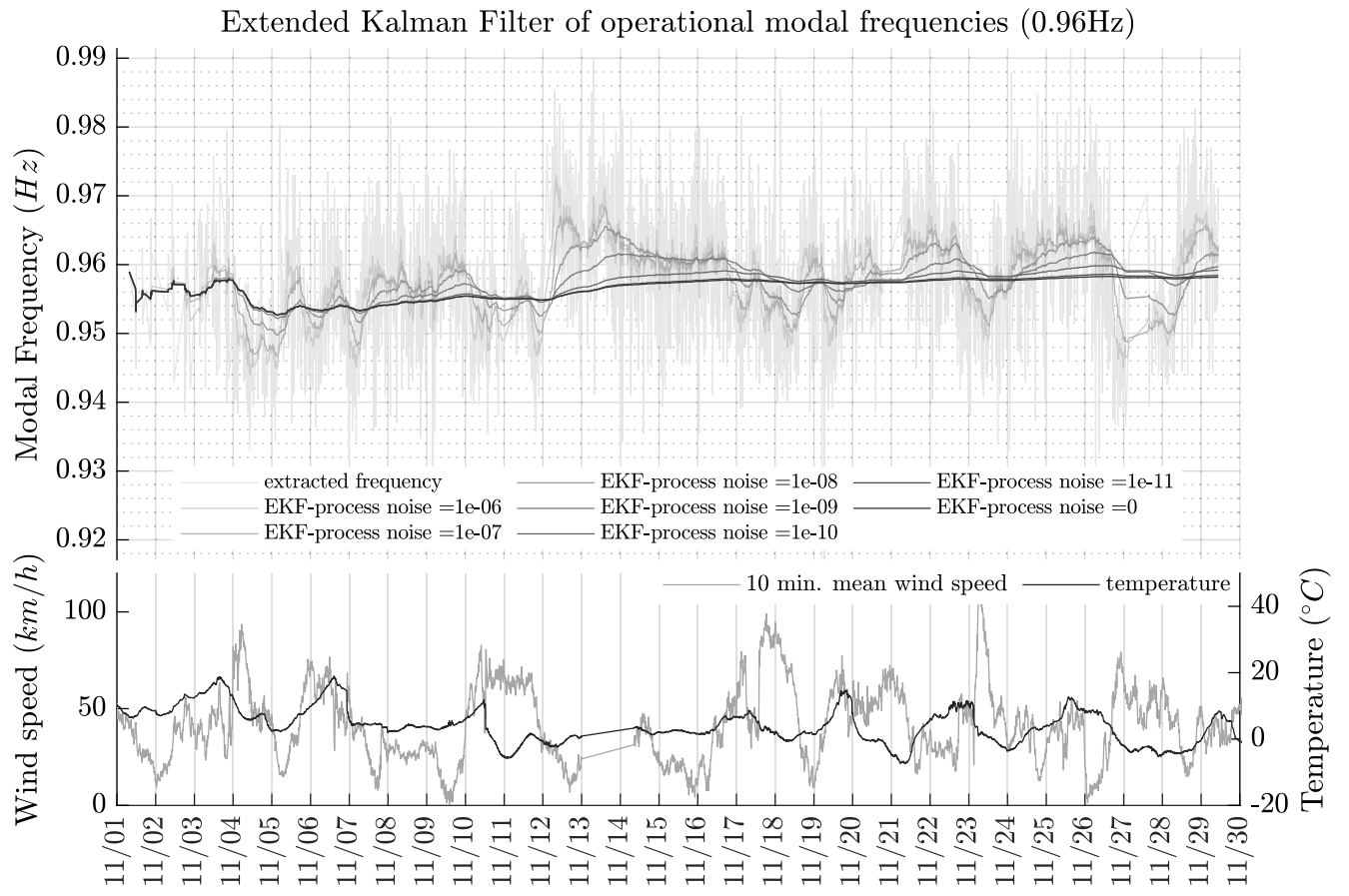

Figure 7.10: Extended Kalman filtering of the modal frequencies (0.96Hz mode).

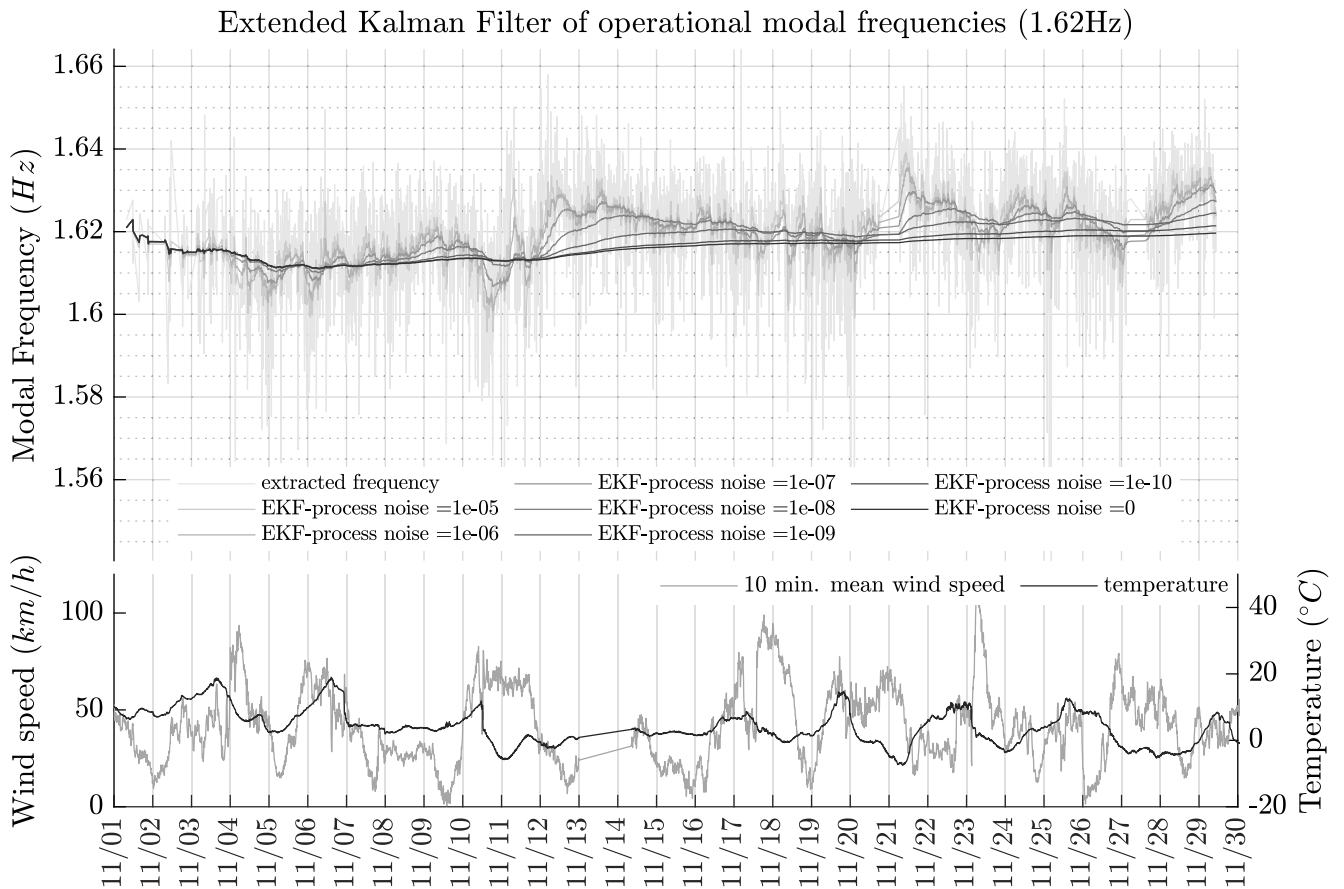

Figure 7.11: Extended Kalman filtering of the modal frequencies (1.62Hz mode). 


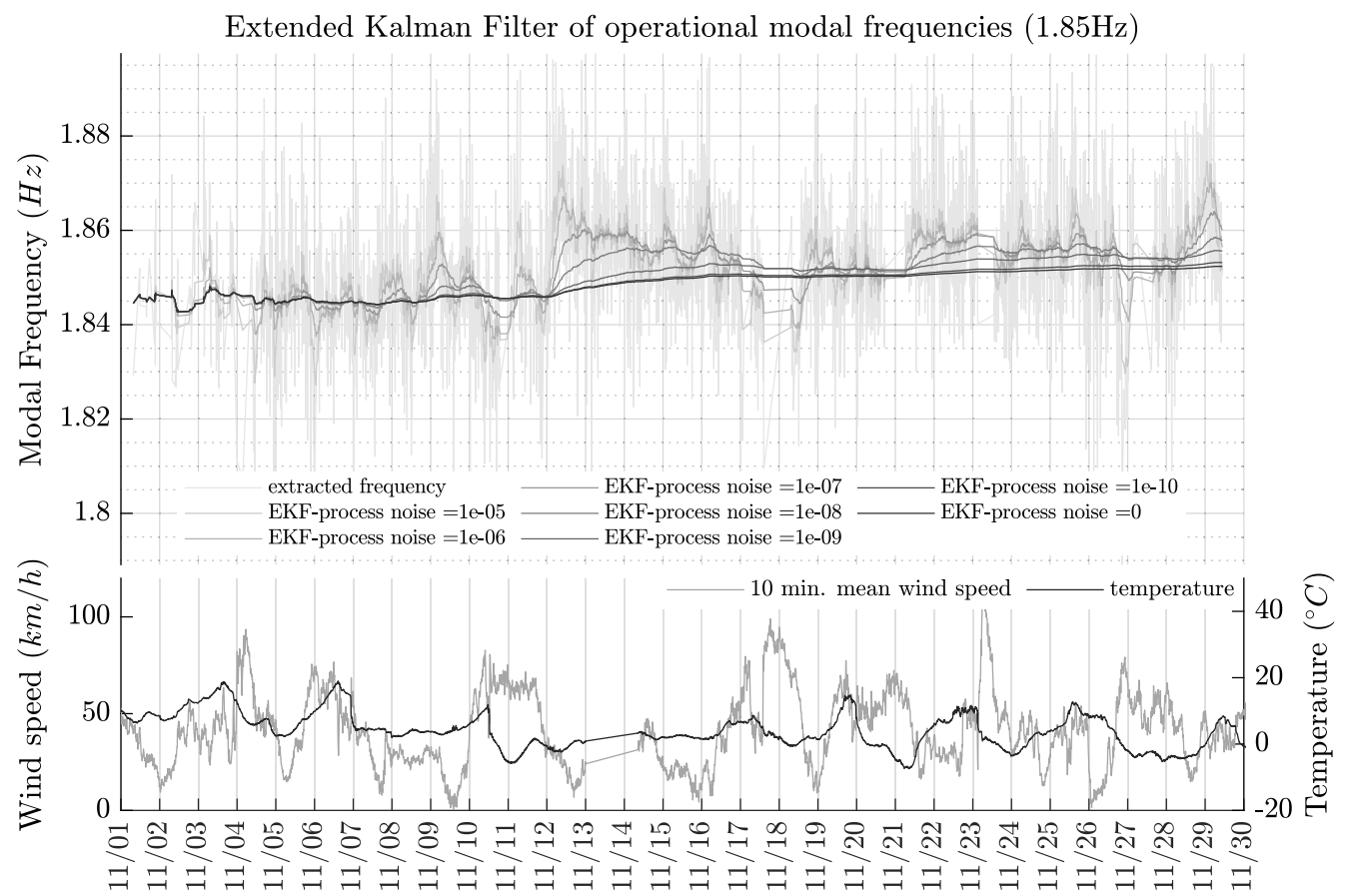

Figure 7.12: Extended Kalman filtering of the modal frequencies (1.85Hz mode).

presented in Section 7.2.

With the specified state transition function and a process noise value of $Q=0$ the filter estimates are essentially a cumulative mean of the estimated frequency time series. Therefore to gather any meaningful interpretation, the estimates obtained with non-zero process noise must be considered. While the specified parameters for the current implementation of the extended Kalman filter are relatively simple, the application validates a proof of concept that can be later scaled to include several parameters in the state vector, including, but not limited to the mode shapes, wind, temperature, and other predictors. Future state transition functions should utilize regression coefficients to better estimate actual state. 


\subsection{Regression Analysis of OMA estimates}

In this section, a multiple linear regression analysis was carried out on each of the modal frequency time series of the dominant modes extracted from the data collected in November $2017(0.47 \mathrm{~Hz}, 0.67 \mathrm{~Hz}, 0.96 \mathrm{~Hz}, 1.62 \mathrm{~Hz}$ and $1.85 \mathrm{~Hz}$ modes $)$. Linear regressions were also carried out on the smoothed data of Section 7.2.

The objective in this study is to observe and quantify any possible temporal trends which may be related to environmental or operational processes. Several predictor variables are considered in this comprehensive study of modal frequency variability. The dependent variable in the regression analyses described in this section is the operational modal frequency time series. Nine predictors, or independent variables are considered due to their apparent qualitative correlations with modal frequencies. The predictor variables are described in Table 7.3. A combination of continuous and categorical predictors are used to identify any statistically significant correlations. Continuous predictors are numeric variables that have an infinite number of values between any two values while categorical predictor variables contain a finite number of categories or distinct groups.

The linear regression model used in this study is shown in Equation (7.9)

$$
\mathbf{y}=\beta_{0}+\beta_{1} \mathbf{x}_{1}+\ldots+\beta_{k} \mathbf{x}_{k}+\mathbf{e}
$$

where $\mathbf{x}_{k}$ are the predictor variable vectors, $\beta_{k}$ the regression coefficients, $\mathbf{y}$ is the dependent variable and $\mathbf{e}$ is the error. $\mathbf{y}, \mathbf{x}$ and $\mathbf{e}$ are vectors of length $n$, where $n$ is the number of data points. For categorical predictors, a regression coefficient is obtained for each of the categories contained in the variable. The original categorical variable is separated into logical vectors of zeros and ones for each of the categories contained in the original vector. 
Table 7.3: Response and predictor variables considered for linear regression.

\begin{tabular}{|c|c|}
\hline Variable name & Description \\
\hline ModalFrequency & $\begin{array}{l}\text { The extracted modal frequencies are stored in a time series vector and is taken } \\
\text { as the response variable in the multiple linear regressions performed in this } \\
\text { section. }\end{array}$ \\
\hline ModalMAC & $\begin{array}{l}\text { The modal assurance criterion is used as a continuous predictor in order to } \\
\text { detect any correlation with modal frequency. A weakly identified mode may } \\
\text { have a divergent identified modal frequency and a corresponding low MAC } \\
\text { value. It should be noted that the MAC values stored in the prediction } \\
\text { variable for each mode is an average of the MAC values obtained with each } \\
\text { of the other modes in the vector. }\end{array}$ \\
\hline AirTemperature & $\begin{array}{l}\text { The ambient air temperature is measured on the bridge deck. It is used as } \\
\text { a continuous predictor and is thought to be related to longer term trends } \\
\text { observed in the estimated modal frequencies. }\end{array}$ \\
\hline WindSpeed & $\begin{array}{l}\text { The } 10 \text { minute mean wind speed is a continuous predictor and is thought to } \\
\text { be closely related to lower modes. Evident trends with several modes were } \\
\text { observed in Section } 7.2\end{array}$ \\
\hline Dotw & $\begin{array}{l}\text { The day of the week (dotw) is used a categorical predictor, with seven cate- } \\
\text { gories. Possible trends relating to day of the week may be detected. These } \\
\text { are likely to be traffic related. }\end{array}$ \\
\hline Hour & $\begin{array}{l}\text { The hour of the day is a categorical predictor with } 24 \text { categories which are } \\
\text { thought to be closely related to traffic excitations. }\end{array}$ \\
\hline SensorsComb & $\begin{array}{l}\text { The sensor configuration predictor is a categorical variable which accounts for } \\
\text { the number and location of sensors included in the analyzed datasets. Some } \\
\text { sensor signals are either absent or are removed from the analysis results by the } \\
\text { sensor diagnostic algorithm during processing due to faulty or erratic signal } \\
\text { behavior. Many categories are created based on the combinations of sensors } \\
\text { available at the time of collection. It is thought that the sensor configuration } \\
\text { affects the quality of the extracted modal properties (mode shapes especially, } \\
\text { and possibly modal frequencies). }\end{array}$ \\
\hline ModeIdent. & $\begin{array}{l}\text { This continuous predictor is an amplitude variable related to the strength and } \\
\text { consistency of the mode identification. It is a processing parameter stored by } \\
\text { the automated OMA method described in Section } 2.2 .3 \text {. Strongly identified } \\
\text { modes with higher mode identifications may be an indicator of trustworthiness } \\
\text { of the operational modal properties. }\end{array}$ \\
\hline rmsVertical & $\begin{array}{l}\text { The average strength of the acceleration signals in the vertical direction is } \\
\text { used as continuous amplitude predictor variable. This amplitude variable is } \\
\text { thought to be closely related to traffic patterns as described in Section } 6.2 .3\end{array}$ \\
\hline rmsTransverse & $\begin{array}{l}\text { The average strength of the acceleration signals in the transverse direction } \\
\text { is used as continuous amplitude predictor variable. This amplitude variable } \\
\text { is thought to be closely related to traffic and wind patterns as described in } \\
\text { Section } 6.2 .3\end{array}$ \\
\hline
\end{tabular}


Multiple linear regressions were performed on each of the five dominant modes identified from the November 2017 data. The performance of each linear model can be determined from the model statistics which include, R-squared, adjusted R-squared, F-statistic, global p-value, root mean squared error (RMSE) and standard deviation of residuals. Regression analyses were also performed on the smoothed data. Results are included in Tables 7.4 to 7.8 for the $0.47 \mathrm{~Hz}, 0.67 \mathrm{~Hz}, 0.96 \mathrm{~Hz}, 1.62 \mathrm{~Hz}$ and $1.85 \mathrm{~Hz}$ modes.

Results for each linear regression model include the estimates, or regression coefficients $\left(\beta_{k}\right)$, the standard error (SE), the t-statistic, the p-value and the $95 \%$ confidence interval for each $\beta_{k}$. The results of the regression analyses on the original (unsmoothed) data are described Tables B.1, B.3, B.5, B.7 and B.9 for the $0.47 \mathrm{~Hz}$, $0.67 \mathrm{~Hz}, 0.96 \mathrm{~Hz}, 1.62 \mathrm{~Hz}$ and $1.85 \mathrm{~Hz}$ modes respectively.

\subsection{1 $\quad 0.47 \mathrm{~Hz}$ mode}

The $0.47 \mathrm{~Hz}$ mode is a dominant transverse mode. Figures 7.13 and 7.14 show the plots of the extracted modal frequencies for this mode (the dependant variable) versus each predictor (independent variables). Figure 7.13 shows the relations to the continuous variables, while Figure 7.14 shows the relation to the categorical variables in box plot format.

Qualitatively, from Figure 7.13, we can observe possible significant correlations of modal frequencies with wind speeds, modal identifications (strength of identification) and to a lesser extent with transverse RMS and temperature. From the MAC plot we can see a tendency towards better mode shape assurance with higher modal frequencies. Interestingly, from Figure 7.14 we can observe some statistical variations between the days of the week. However, this categorical predictor would be better used on longer term data (over several months or years), as the number of Fridays 
(for example) in a month is no more than 5 , and depending on weather conditions on these days, the results could be skewed. The hour of the day, however, are more suited for data collected during a single month as approximately 30 different sampling opportunities are present. However, for the $0.47 \mathrm{~Hz}$ it is difficult to observe trends qualitatively from Figure 7.14.

Frequency vs Continuous Predictors - Mode $0.47 \mathrm{~Hz}$
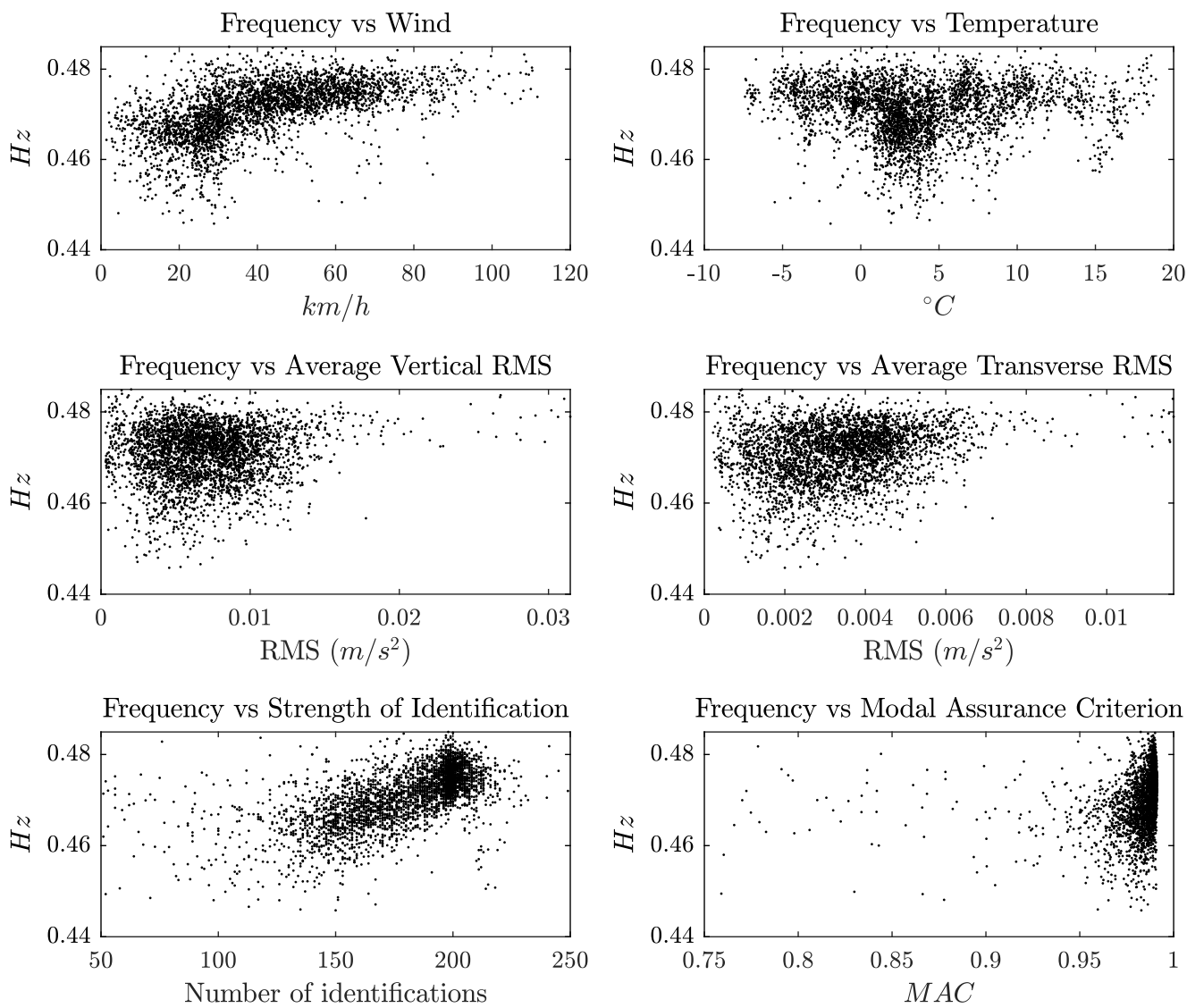

Figure 7.13: Modal frequencies vs continuous predictors $-0.47 \mathrm{~Hz}$ mode.

Table 7.4 shows the results of multiple regression analyses for the modal frequencies of the $0.47 \mathrm{~Hz}$ mode. Included in the top section of this table are the model statistics for the original data and in the bottom section are the model statistics for each multiple linear regression analyses performed on the smoothed data. 
Frequency vs Categorical Predictors - Mode $0.47 \mathrm{~Hz}$
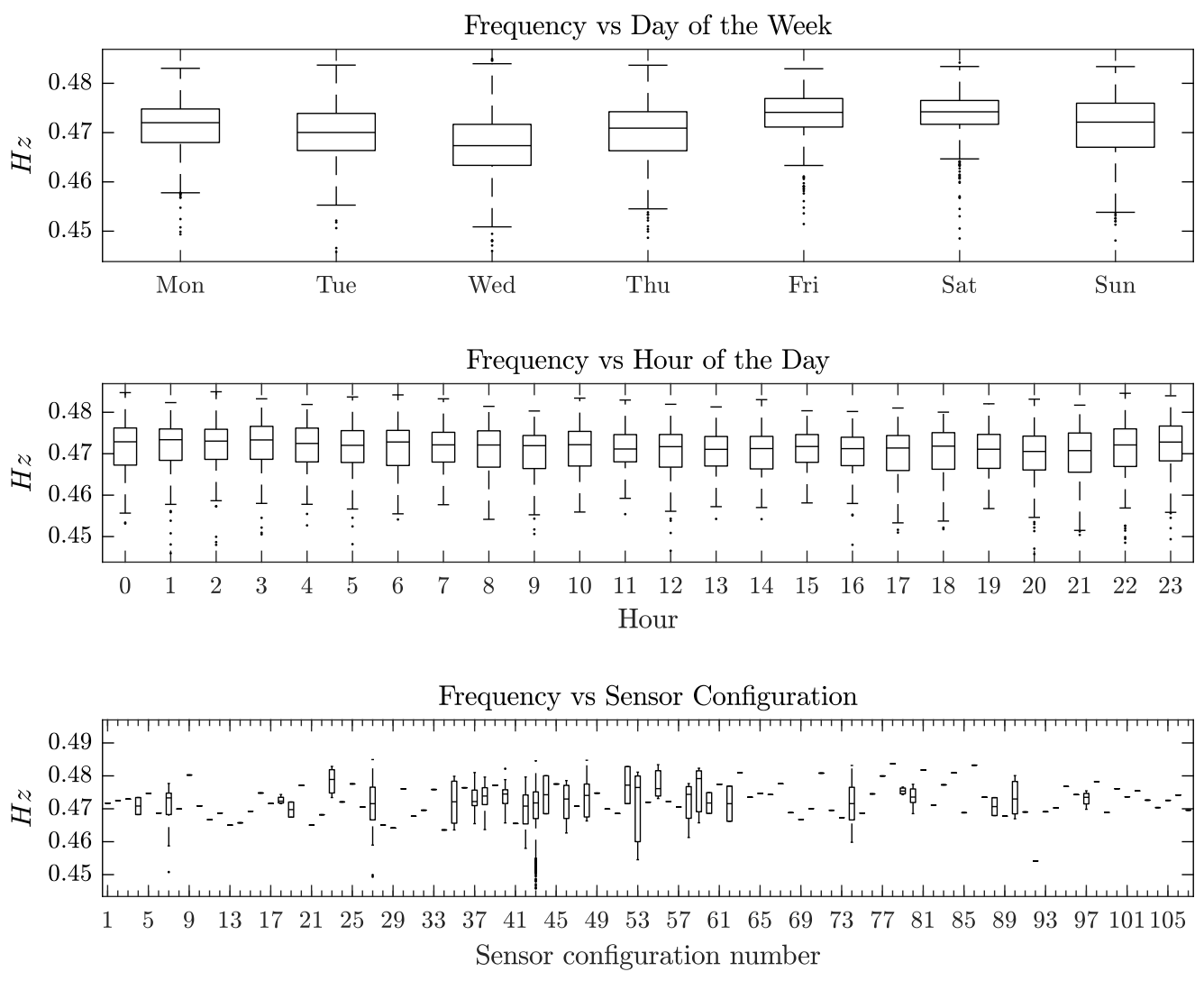

Figure 7.14: Modal frequencies vs categorical predictors - $0.47 \mathrm{~Hz}$ mode.

An R-squared value of 0.468 is obtained for the linear model of the original (unsmoothed) data. This essentially means that $46.8 \%$ of the variations of the modal frequencies for this mode can be explained by the variations of the included predictor variables. A relatively high F-statistic of 21.1 with a corresponding very low p-value (essentially zero) means that the model is statistically significant and the null hypothesis can be rejected. In statistical hypothesis testing, the null hypothesis represents a model where all the predictor coefficients are null. A p-value (or probability value) less than the significance level (typically taken as 0.05) indicates that the null hypothesis can be rejected since it is very unlikely that the dependent variable varies by chance alone. It is therefore likely, with a very low p-value, that changes 
Table 7.4: Linear regression statistics for modal frequencies of $0.47 \mathrm{~Hz}$ mode.

\begin{tabular}{|c|c|c|c|c|c|c|c|}
\hline \multicolumn{8}{|c|}{$0.47 \mathrm{~Hz}$ mode } \\
\hline \multicolumn{8}{|c|}{$\begin{array}{l}\text { R-squared: } 0.468, \text { Adjusted R-Squared } 0.446 \\
\text { F-statistic vs. constant model: } 21.1, \mathrm{p} \text {-value }=0 \\
=0.00454, \text { Standard deviation of residuals }=0.00445 \\
\text { Top coefficients : ModeIdent., WindSpeed }\end{array}$} \\
\hline \multicolumn{8}{|c|}{ Smoothing models } \\
\hline Method & $\begin{array}{l}\text { Window } \\
\text { size }\end{array}$ & $R^{2}$ & $\begin{array}{c}\text { Adjusted } \\
R^{2}\end{array}$ & $\begin{array}{c}\text { F- } \\
\text { Statistic }\end{array}$ & pVal. & $\begin{array}{l}\text { Standard } \\
\text { deviation of } \\
\text { residuals }\end{array}$ & Top coefficients \\
\hline \multirow{4}{*}{$\begin{array}{l}\text { Moving } \\
\text { average }\end{array}$} & 5 & 0.622 & 0.606 & 39.4 & 0 & $3.048 \times 10^{-3}$ & ModeIdent., WindSpeed \\
\hline & 10 & 0.653 & 0.638 & 45.0 & 0 & $2.784 \times 10^{-3}$ & ModeIdent., WindSpeed \\
\hline & 20 & 0.677 & 0.664 & 50.2 & 0 & $2.562 \times 10^{-3}$ & WindSpeed, ModeIdent. \\
\hline & 50 & 0.697 & 0.684 & 55.0 & 0 & $2.279 \times 10^{-3}$ & WindSpeed, ModeIdent. \\
\hline \multirow{4}{*}{$\begin{array}{l}\text { Gaussian } \\
\text { weighted } \\
\text { moving } \\
\text { average }\end{array}$} & 5 & 0.605 & 0.588 & 36.6 & 0 & $3.219 \times 10^{-3}$ & ModeIdent., WindSpeed \\
\hline & 10 & 0.643 & 0.628 & 43.1 & 0 & $2.896 \times 10^{-3}$ & ModeIdent., WindSpeed \\
\hline & 20 & 0.670 & 0.656 & 48.5 & 0 & $2.665 \times 10^{-3}$ & ModeIdent., WindSpeed \\
\hline & 50 & 0.697 & 0.684 & 55.0 & 0 & $2.387 \times 10^{-3}$ & WindSpeed, ModeIdent. \\
\hline \multirow{4}{*}{$\begin{array}{l}\text { Linear } \\
\text { regression }\end{array}$} & 5 & 0.588 & 0.571 & 34.1 & 0 & $3.348 \times 10^{-3}$ & ModeIdent., WindSpeed \\
\hline & 10 & 0.642 & 0.627 & 43.0 & 0 & $2.896 \times 10^{-3}$ & ModeIdent., WindSpeed \\
\hline & 20 & 0.668 & 0.654 & 48.2 & 0 & $2.669 \times 10^{-3}$ & ModeIdent., WindSpeed \\
\hline & 50 & 0.695 & 0.682 & 54.6 & 0 & $2.391 \times 10^{-3}$ & WindSpeed, ModeIdent. \\
\hline \multirow{4}{*}{$\begin{array}{l}\text { Quadratic } \\
\text { regression }\end{array}$} & 5 & 0.468 & 0.446 & 21.1 & 0 & $4.451 \times 10^{-3}$ & ModeIdent., WindSpeed \\
\hline & 10 & 0.600 & 0.583 & 35.9 & 0 & $3.244 \times 10^{-3}$ & ModeIdent., WindSpeed \\
\hline & 20 & 0.637 & 0.622 & 42.0 & 0 & $2.930 \times 10^{-3}$ & ModeIdent., WindSpeed \\
\hline & 50 & 0.671 & 0.658 & 48.9 & 0 & $2.638 \times 10^{-3}$ & ModeIdent., WindSpeed \\
\hline
\end{tabular}

in the predictors are related to changes in the dependent variable. The F-statistic is inversely proportional to the p-value, therefore for very low (i.e. negligible) pvalues, the F-statistics provides a metric for comparison between regression models. A low RMSE of $0.00454 \mathrm{~Hz}(0.96 \%$ of mean) indicates a relatively low variability of modal frequencies after removing the modeled effects of the predictor variables. The standard deviation of residuals is closely related to RMSE, but where the RMSE calculates the mean over the degrees of freedom of the linear model, the standard 
deviation calculates the mean over all samples. Residuals from the linear model are a key parameter for the proposed damage detection algorithm that will be presented in Section 7.5. The two most statistically significant regression variables for this mode are the strength of identification (as represented by the model identification variable) and the wind speeds. The statistical parameters of all the regression coefficients for the $0.47 \mathrm{~Hz}$ mode are shown in Table B.1 and the analysis of variance results are shown in Table B.2. It should be noted that only statistically significant coefficients (with a p-value lower than 0.05) are include in Table B.1. The linear regression models for the smoothed data seem to perform better overall. Section 7.2 describes the smoothing methods. Interestingly, for most of the methods, as the window length increases, the wind speed becomes the most statistically significant regression coefficient.

Figure 7.15 shows the fitted modal frequencies obtained from the linear regression model of the original (unsmoothed) data plotted over the original modal frequencies.
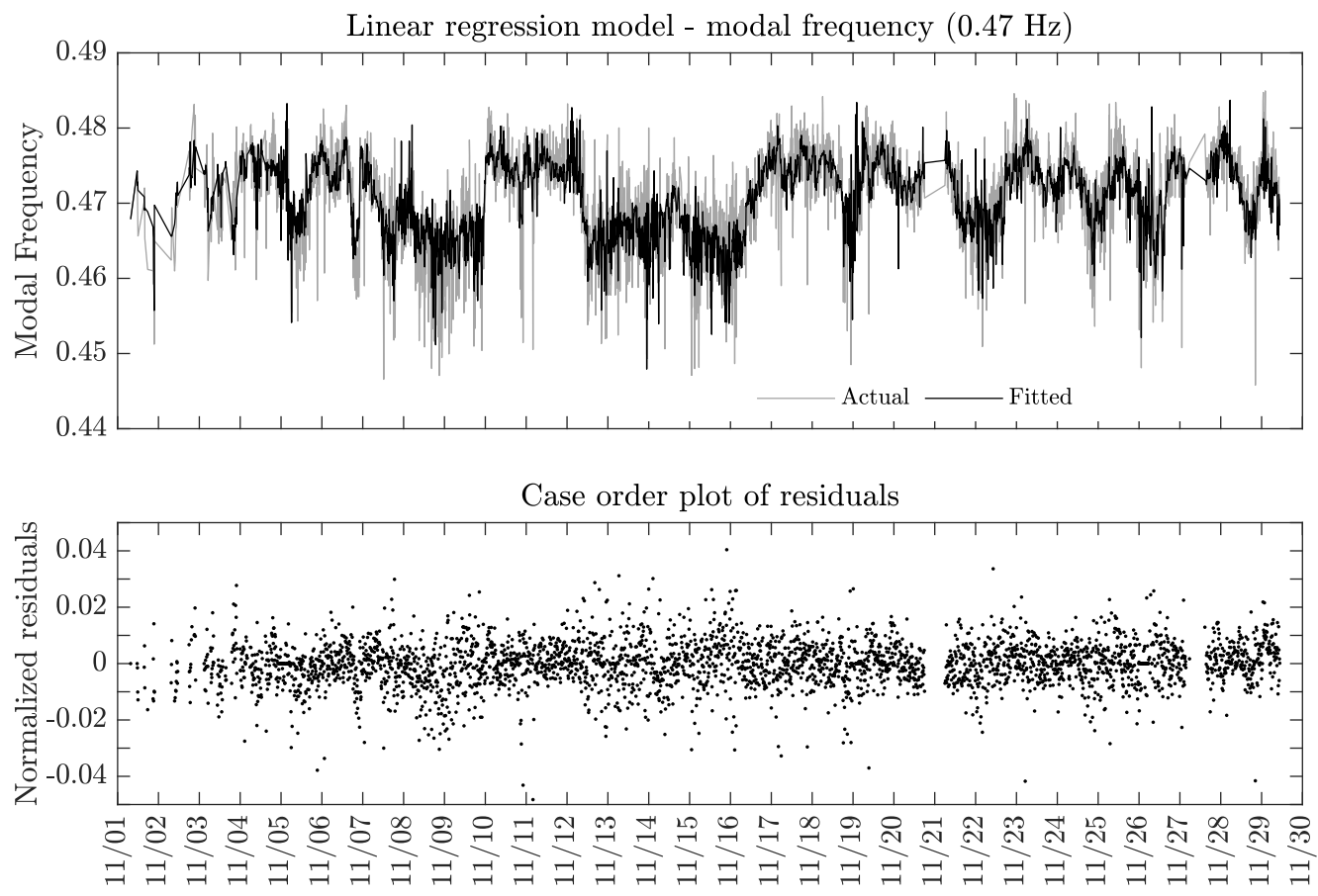

Figure 7.15: Regression model for modal frequencies of $0.47 \mathrm{~Hz}$ mode. 
Normalized raw residuals are plotted in the bottom half of the figure. A relatively good fit is observed from this figure. The distribution of residuals is fairly uniform which confirms the suitability of the model to effectively remove the contributions from the predictor variable from the modal frequency time series signal.

\subsection{2 $\quad 0.67 \mathrm{~Hz}$ mode}

The $0.67 \mathrm{~Hz}$ mode is a dominant vertical mode. Figure 7.16 shows the plots of the extracted modal frequencies for this mode versus each continuous predictor variable.
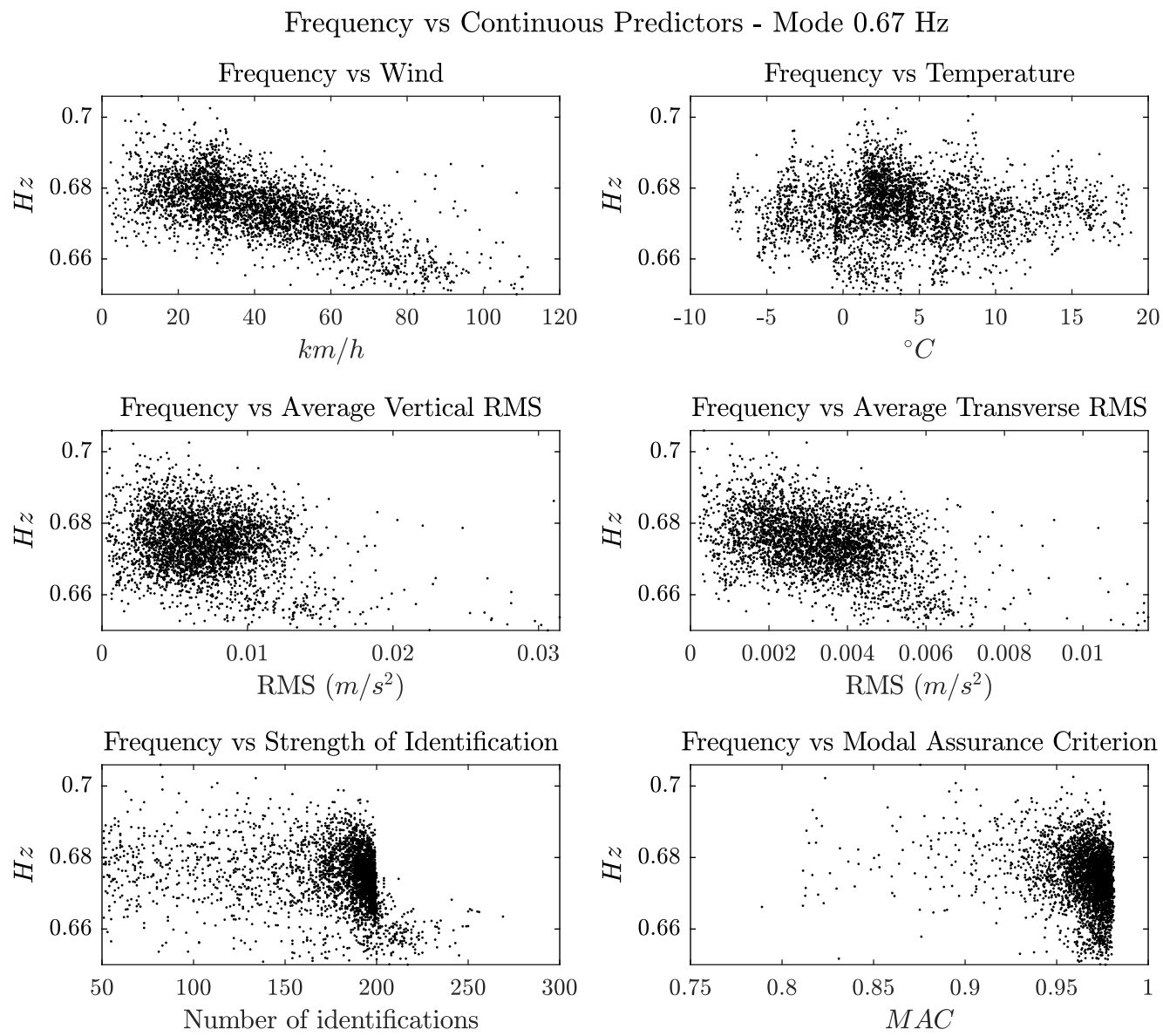

Figure 7.16: Modal frequencies vs continuous predictors - $0.67 \mathrm{~Hz}$ mode.

From Figure 7.16, we can observe correlations of modal frequencies with wind 
speeds, transverse RMS, and MAC. The observed trends for this mode are the inverse of the trends observed for the $0.47 \mathrm{~Hz}$. There is a decrease in modal frequencies with an increase in wind speeds and from the MAC plot we can see a tendency towards better mode shape assurance with lower modal frequencies. Figure 7.17 shows the relations between the modal frequencies and the categorical variables in box plot format. From Figure 7.17 we can observe some significant statistical variations with the hours of the day, which indicates possible correlations with traffic excitations.

Frequency vs Categorical Predictors - Mode $0.67 \mathrm{~Hz}$
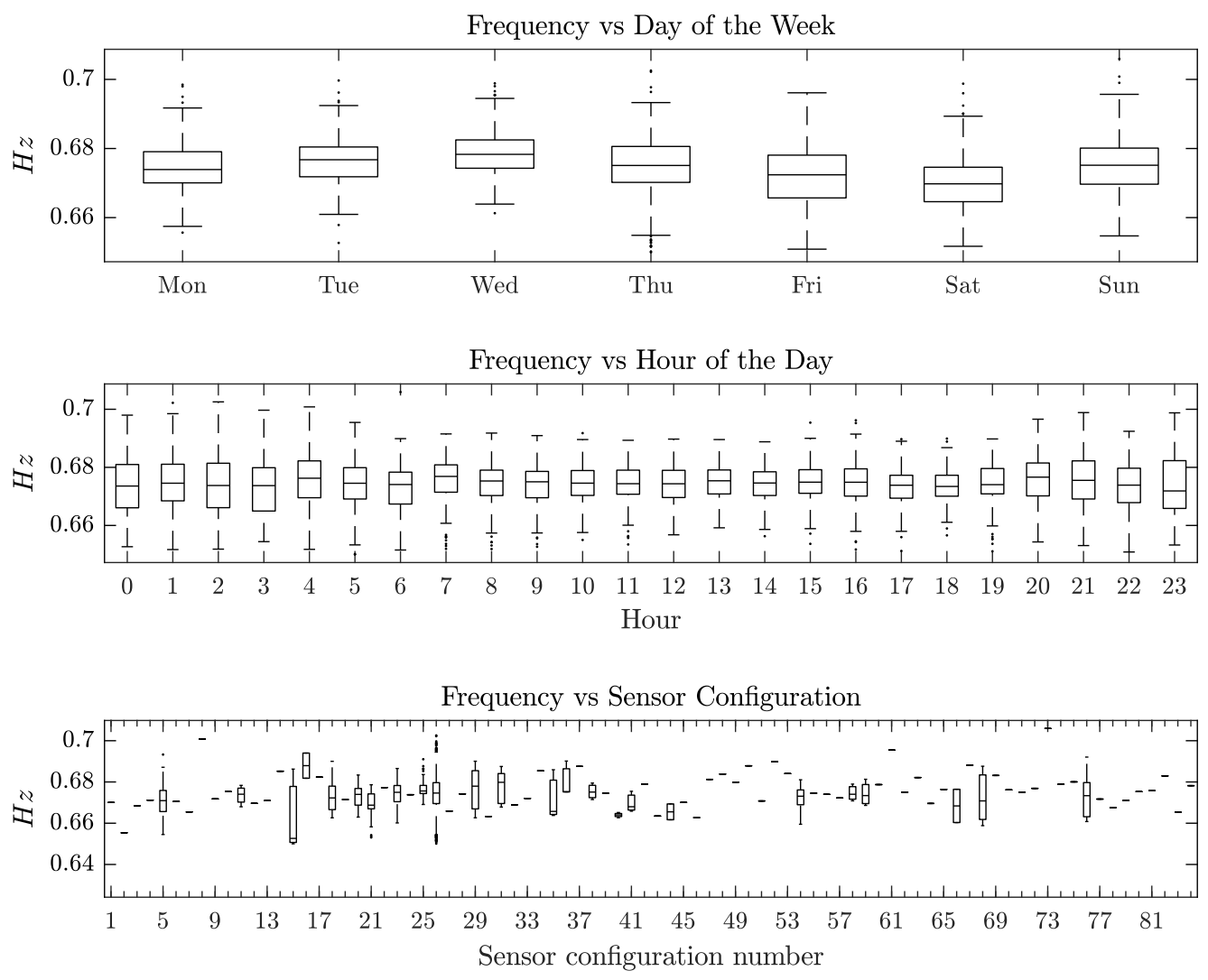

Figure 7.17: Modal frequencies vs categorical predictors - $0.67 \mathrm{~Hz}$ mode.

The results of multiple regression analyses for the modal frequencies of the $0.67 \mathrm{~Hz}$ mode are shown in Table 7.5.

An R-squared value of 0.532 is obtained for the linear model which essentially 
Table 7.5: Linear regression statistics for modal frequencies of $0.67 \mathrm{~Hz}$ mode.

\begin{tabular}{|c|c|c|c|c|c|c|c|}
\hline \multicolumn{8}{|c|}{$0.67 \mathrm{~Hz}$ mode } \\
\hline \multicolumn{8}{|c|}{$\begin{array}{l}\text { Model statistics : } \\
.532, \text { Adjusted R-Squared } 0.515 \\
\text { constant model: } 31.9, \text { p-value }=0 \\
\text { andard deviation of residuals }=0.0056 \\
\text { ents : WindSpeed, ModeIdent. }\end{array}$} \\
\hline \multicolumn{8}{|c|}{ Smoothing models } \\
\hline Method & $\begin{array}{l}\text { Window } \\
\text { size }\end{array}$ & $R^{2}$ & $\begin{array}{c}\text { Adjusted } \\
R^{2}\end{array}$ & $\begin{array}{c}\text { F- } \\
\text { Statistic }\end{array}$ & pValue & $\begin{array}{l}\text { Standard } \\
\text { deviation of } \\
\text { residuals }\end{array}$ & Top coefficients \\
\hline \multirow{4}{*}{$\begin{array}{l}\text { Moving } \\
\text { average }\end{array}$} & 5 & 0.726 & 0.716 & 74.5 & 0 & $3.506 \times 10^{-3}$ & WindSpeed, rmsTransverse \\
\hline & 10 & 0.771 & 0.763 & 94.7 & 0 & $3.075 \times 10^{-3}$ & WindSpeed, rmsTransverse \\
\hline & 20 & 0.804 & 0.797 & 115.0 & 0 & $2.719 \times 10^{-3}$ & WindSpeed, rmsTransverse \\
\hline & 50 & 0.803 & 0.796 & 115.0 & 0 & $2.481 \times 10^{-3}$ & WindSpeed, rmsTransverse \\
\hline \multirow{4}{*}{$\begin{array}{l}\text { Gaussian } \\
\text { weighted } \\
\text { moving } \\
\text { average }\end{array}$} & 5 & 0.696 & 0.686 & 64.6 & 0 & $3.792 \times 10^{-3}$ & WindSpeed, rmsTransverse \\
\hline & 10 & 0.750 & 0.741 & 84.4 & 0 & $3.284 \times 10^{-3}$ & WindSpeed, rmsTransverse \\
\hline & 20 & 0.788 & 0.780 & 104.0 & 0 & $2.909 \times 10^{-3}$ & WindSpeed, rmsTransverse \\
\hline & 50 & 0.818 & 0.811 & 126.0 & 0 & $2.510 \times 10^{-3}$ & WindSpeed, rmsTransverse \\
\hline \multirow{4}{*}{$\begin{array}{l}\text { Linear } \\
\text { regression }\end{array}$} & 5 & 0.676 & 0.665 & 58.5 & 0 & $3.986 \times 10^{-3}$ & WindSpeed, rmsTransverse \\
\hline & 10 & 0.751 & 0.742 & 84.8 & 0 & $3.274 \times 10^{-3}$ & WindSpeed, rmsTransverse \\
\hline & 20 & 0.788 & 0.781 & 105.0 & 0 & $2.903 \times 10^{-3}$ & WindSpeed, rmsTransverse \\
\hline & 50 & 0.816 & 0.810 & 125.0 & 0 & $2.519 \times 10^{-3}$ & WindSpeed, rmsTransverse \\
\hline \multirow{4}{*}{$\begin{array}{l}\text { Quadratic } \\
\text { regression }\end{array}$} & 5 & 0.531 & 0.515 & 31.9 & 0 & $5.583 \times 10^{-3}$ & WindSpeed, ModeIdent. \\
\hline & 10 & 0.695 & 0.684 & 64.2 & 0 & $3.800 \times 10^{-3}$ & WindSpeed, rmsTransverse \\
\hline & 20 & 0.748 & 0.739 & 83.5 & 0 & $3.310 \times 10^{-3}$ & WindSpeed, rmsTransverse \\
\hline & 50 & 0.795 & 0.787 & 109.0 & 0 & $2.846 \times 10^{-3}$ & WindSpeed, rmsTransverse \\
\hline
\end{tabular}

means that $53.2 \%$ of the variations of the modal frequencies for this mode can be explained by the variations of the included predictor variables. A relatively high F-statistic of 31.9 and very low p-value (essentially zero) means that the model is statistically significant versus the null hypothesis. A low RMSE of $0.0057 \mathrm{~Hz}(0.85 \%$ of mean) indicates a relatively low variability of modal frequencies after removing the modeled effects of the predictor variables. The two most statistically significant regression variables for this mode are the wind speed and the strength of identification. 
The statistical parameters of all the regression coefficients for the $0.67 \mathrm{~Hz}$ mode are shown in Table B.3 and the analysis of variance results are shown in Table B.4. The linear regression model for the Gaussian weighted smoothing over a window of 50 samples has a high $\mathrm{R}$-square values of 0.818 , which means that $82 \%$ of the variation of the smooth data with this method is explained by the variance of the predictor variables. For most of the linear models of smoothed data, the average RMS in the transverse direction becomes the second most statistically significant regression coefficient. While data smoothing increases model performance, there is a risk that statistically significant short period correlations are lost.

Figure 7.18 shows the fitted modal frequencies obtained from the linear regression model of the unsmoothed data plotted over the original modal frequencies.
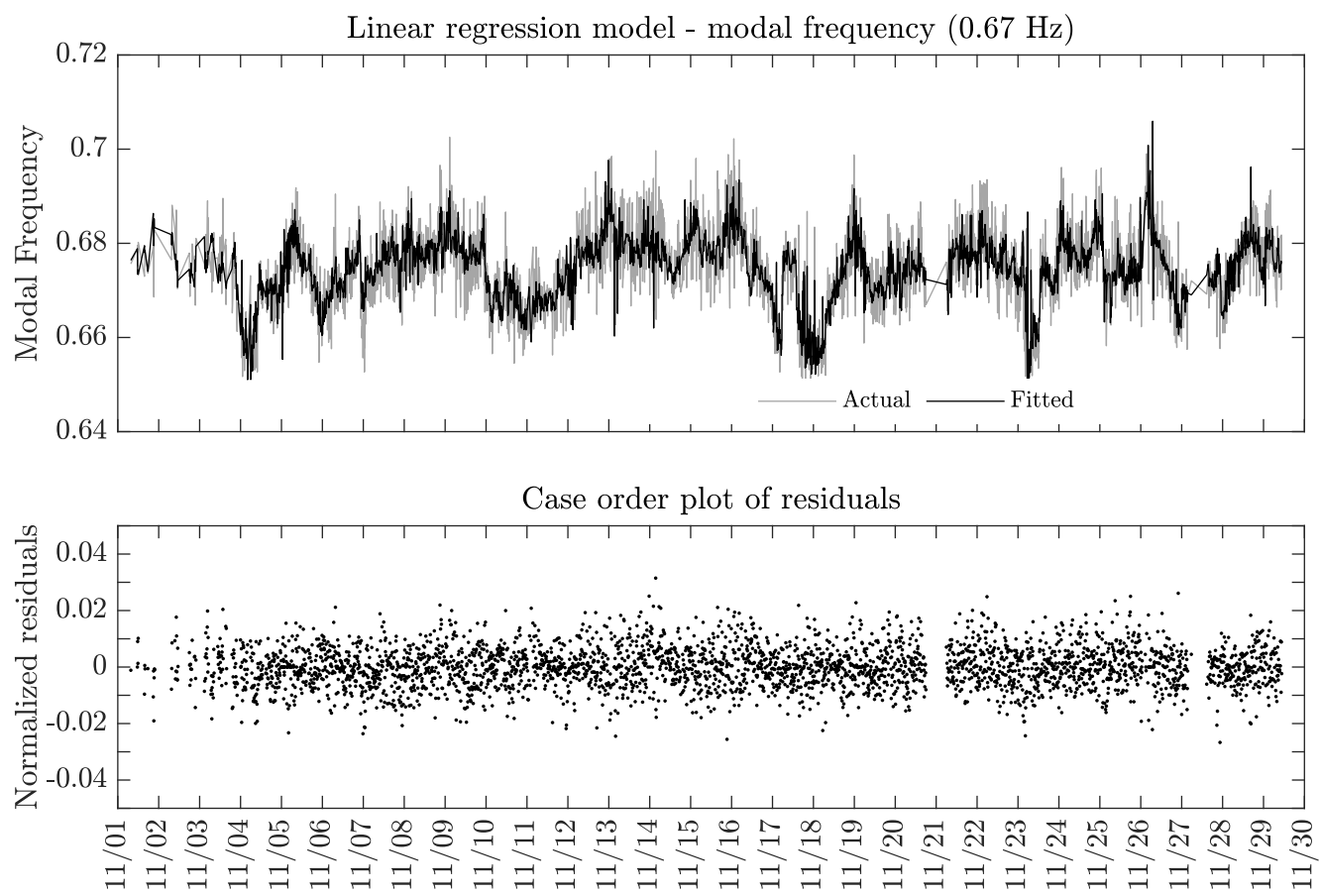

Figure 7.18: Regression model for modal frequencies of $0.67 \mathrm{~Hz}$ mode.

The normalized raw residuals are plotted in the bottom half of Figure 7.18. An indicator of proper modeling is the distribution of residuals, which is fairly uniform 
for this mode. This confirms the suitability of the model to effectively remove the contributions from the predictor variable from the modal frequency time series signal.

\subsection{3 $0.96 \mathrm{~Hz}$ mode}

The $0.96 \mathrm{~Hz}$ mode is a dominant vertical mode. Figures 7.19 and 7.20 show the plots of the operational modal frequencies for this mode versus each predictor variable.

Frequency vs Continuous Predictors - Mode $0.96 \mathrm{~Hz}$
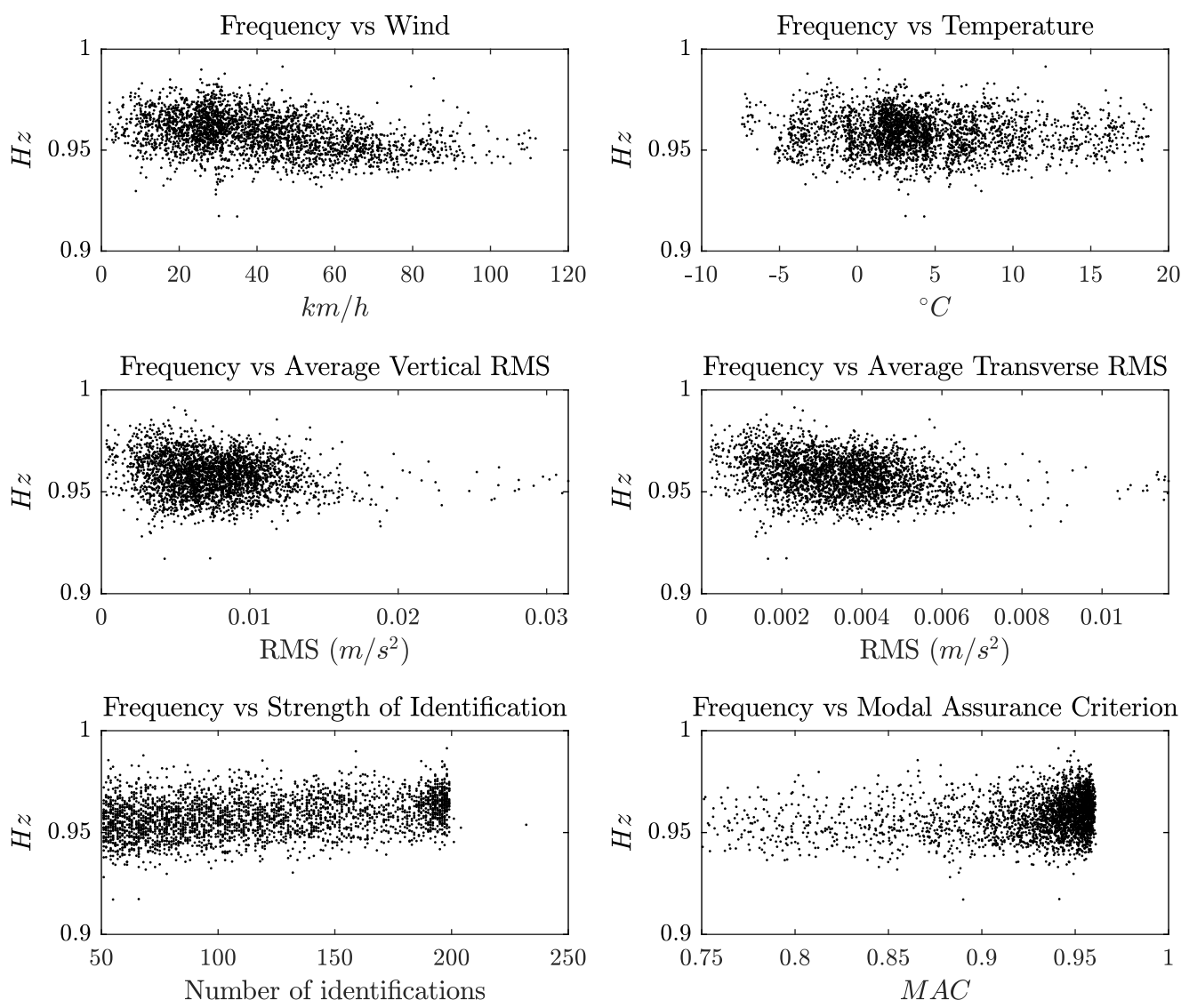

Figure 7.19: Modal frequencies vs continuous predictors $-0.96 \mathrm{~Hz}$ mode.

In Figure 7.19, we can observe possible significant correlations of modal frequencies with wind speeds and RMS and to a lesser extent with temperature, MAC and strength of identification. Figure 7.14 shows there are some statistical variations with 
the hours of the day.

Frequency vs Categorical Predictors - Mode $0.96 \mathrm{~Hz}$
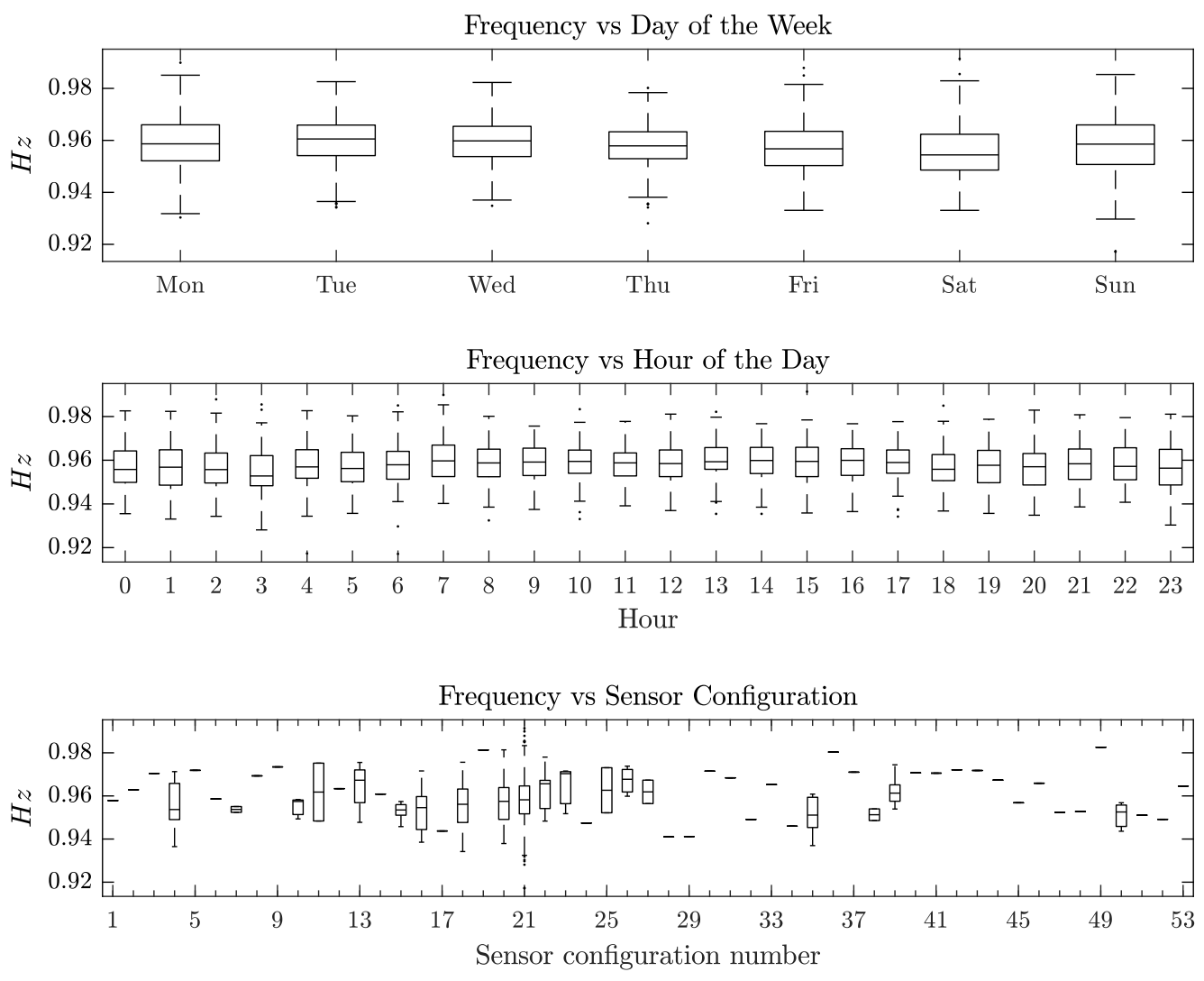

Figure 7.20: Modal frequencies vs categorical predictors - $0.96 \mathrm{~Hz}$ mode.

The multiple regression results for the modal frequencies of the $0.96 \mathrm{~Hz}$ mode are shown in Table 7.6. The top section of this table present the model statistics for the original data while the bottom section summarizes the model statistics for each multiple linear regression analyses performed on the smoothed data.

A comparatively low R-squared value of 0.272 is obtained for the linear model of the data. Theoretically, this indicates that only $27.2 \%$ of the variations of the modal frequencies for this mode can be explained by the variations of the included predictor variables. A modest F-statistic of 12.1 with a corresponding very low p-value (essentially zero) confirms that the model is still statistically significant versus the null 
Table 7.6: Linear regression statistics for modal frequencies of $0.96 \mathrm{~Hz}$ mode.

\begin{tabular}{|c|c|c|c|c|c|c|c|}
\hline \multicolumn{8}{|c|}{$0.96 \mathrm{~Hz}$ mode } \\
\hline \multicolumn{8}{|c|}{$\begin{array}{l}\text { R-squared: } 0.272 \text {, Adjusted R-Squared } 0.250 \\
\text { F-statistic vs. constant model: } 12.1, \mathrm{p} \text {-value }=5.81 \times 10^{-137} \\
\text { RMSE }=0.0081, \text { Standard deviation of residuals }=0.0080 \\
\text { Top coefficients : ModeIdent., rmsTransverse }\end{array}$} \\
\hline \multicolumn{8}{|c|}{ Smoothing models } \\
\hline Method & $\begin{array}{l}\text { Window } \\
\text { size }\end{array}$ & $R^{2}$ & $\begin{array}{l}\text { Adjusted } \\
R^{2}\end{array}$ & $\frac{\text { F- }}{\text { Statistic }}$ & pValue & $\begin{array}{l}\text { Standard } \\
\text { deviation of } \\
\text { residuals }\end{array}$ & Top coefficients \\
\hline \multirow{4}{*}{$\begin{array}{l}\text { Moving } \\
\text { average }\end{array}$} & 5 & 0.438 & 0.421 & 25.3 & 0 & $4.674 \times 10^{-3}$ & rmsTransverse, rmsVertical \\
\hline & 10 & 0.501 & 0.486 & 32.5 & 0 & $4.025 \times 10^{-3}$ & rmsTransverse, rmsVertical \\
\hline & 20 & 0.535 & 0.521 & 37.3 & 0 & $3.639 \times 10^{-3}$ & rmsTransverse, rmsVertical \\
\hline & 50 & 0.527 & 0.513 & 36.2 & 0 & $3.291 \times 10^{-3}$ & rmsTransverse, WindSpeed \\
\hline \multirow{4}{*}{$\begin{array}{l}\text { Gaussian } \\
\text { weighted } \\
\text { moving } \\
\text { average }\end{array}$} & 5 & 0.415 & 0.397 & 23.0 & 0 & $5.087 \times 10^{-3}$ & rmsTransverse, rmsVertical \\
\hline & 10 & 0.478 & 0.462 & 29.7 & 0 & $4.301 \times 10^{-3}$ & rmsTransverse, rmsVertical \\
\hline & 20 & 0.524 & 0.510 & 35.7 & 0 & $3.810 \times 10^{-3}$ & rmsTransverse, rmsVertical \\
\hline & 50 & 0.543 & 0.529 & 38.5 & 0 & $3.427 \times 10^{-3}$ & rmsTransverse, WindSpeed \\
\hline \multirow{4}{*}{$\begin{array}{l}\text { Linear } \\
\text { regression }\end{array}$} & 5 & 0.392 & 0.373 & 20.9 & 0 & $5.391 \times 10^{-3}$ & rmsTransverse, ModeIdent. \\
\hline & 10 & 0.478 & 0.462 & 29.6 & 0 & $4.290 \times 10^{-3}$ & rmsTransverse, rmsVertical \\
\hline & 20 & 0.524 & 0.509 & 35.6 & 0 & $3.808 \times 10^{-3}$ & rmsTransverse, rmsVertical \\
\hline & 50 & 0.540 & 0.526 & 38.1 & 0 & $3.435 \times 10^{-3}$ & rmsTransverse, WindSpeed \\
\hline \multirow{4}{*}{$\begin{array}{l}\text { Quadratic } \\
\text { regression }\end{array}$} & 5 & 0.272 & 0.250 & 12.1 & 0 & $8.013 \times 10^{-3}$ & ModeIdent., rmsTransverse \\
\hline & 10 & 0.406 & 0.388 & 22.2 & 0 & $5.119 \times 10^{-3}$ & rmsTransverse, rmsVertical \\
\hline & 20 & 0.477 & 0.461 & 29.5 & 0 & $4.306 \times 10^{-3}$ & rmsTransverse, rmsVertical \\
\hline & 50 & 0.534 & 0.520 & 37.1 & 0 & $3.731 \times 10^{-3}$ & rmsTransverse, rmsVertical \\
\hline
\end{tabular}

hypothesis. A low RMSE of $0.0081 \mathrm{~Hz}$ (0.86\% of mean) indicates a relatively low variability of modal frequencies after removing the modeled effects of the predictor variables. It is worth noting, that although the model's R-squared and F-statistic are lower for this mode than for previous modes, the RMSE (as a percentage of mean) is comparable. The operational modal frequency of this mode is therefore more resistant (i.e. less sensitive) to the variations caused by the chosen predictor variables. As the residuals from the linear model are the key parameter for the proposed damage 
detection algorithm (presented in Section 7.5), The RMSE value is much more significant than the R-squared and F-statistic as a predictor of performance of the linear models. The two most statistically significant regression variables for this mode are the strength of identification and the average signal strength (RMS) in the transverse direction.

In Appendix B the statistical parameters of all the regression coefficients and the analysis of variance results for the $0.96 \mathrm{~Hz}$ mode are presented (Table B.5 and Table B.6). Only statistically significant coefficients (with a p-value lower than 0.05) are include in these tables. It is observed from Table 7.6, that with the data smoothing, the RMS values in the transverse and in the vertical direction are the most statistically significant regression coefficient.

The performance of the obtained linear regression model can be observed in Figure 7.21 .
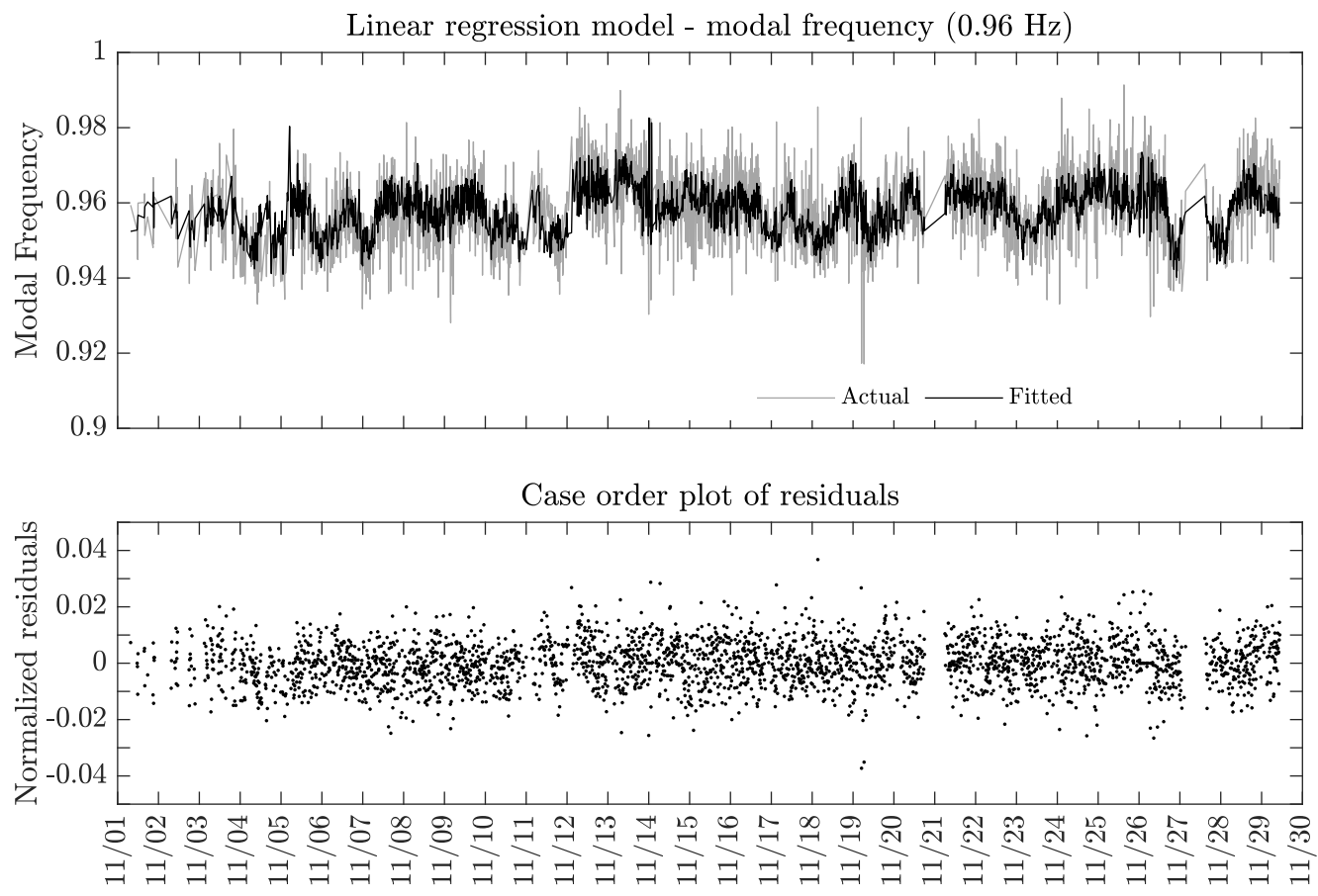

Figure 7.21: Regression model for modal frequencies of $0.96 \mathrm{~Hz}$ mode. 
The fitted modal frequencies obtained from the linear regression model of the original data is plotted over the original modal frequencies. A relatively good fit is observed from this figure. Also, the distribution of residuals is fairly uniform as can be observed in the bottom half of the figure, which confirms the suitability of the model to effectively remove the contributions from the predictor variables.

\subsection{4 $1.62 \mathrm{~Hz}$ mode}

Plotted in Figure 7.22 are the extracted modal frequencies for the $1.62 \mathrm{~Hz}$ mode relative to each predictor variable.

Frequency vs Continuous Predictors - Mode $1.62 \mathrm{~Hz}$
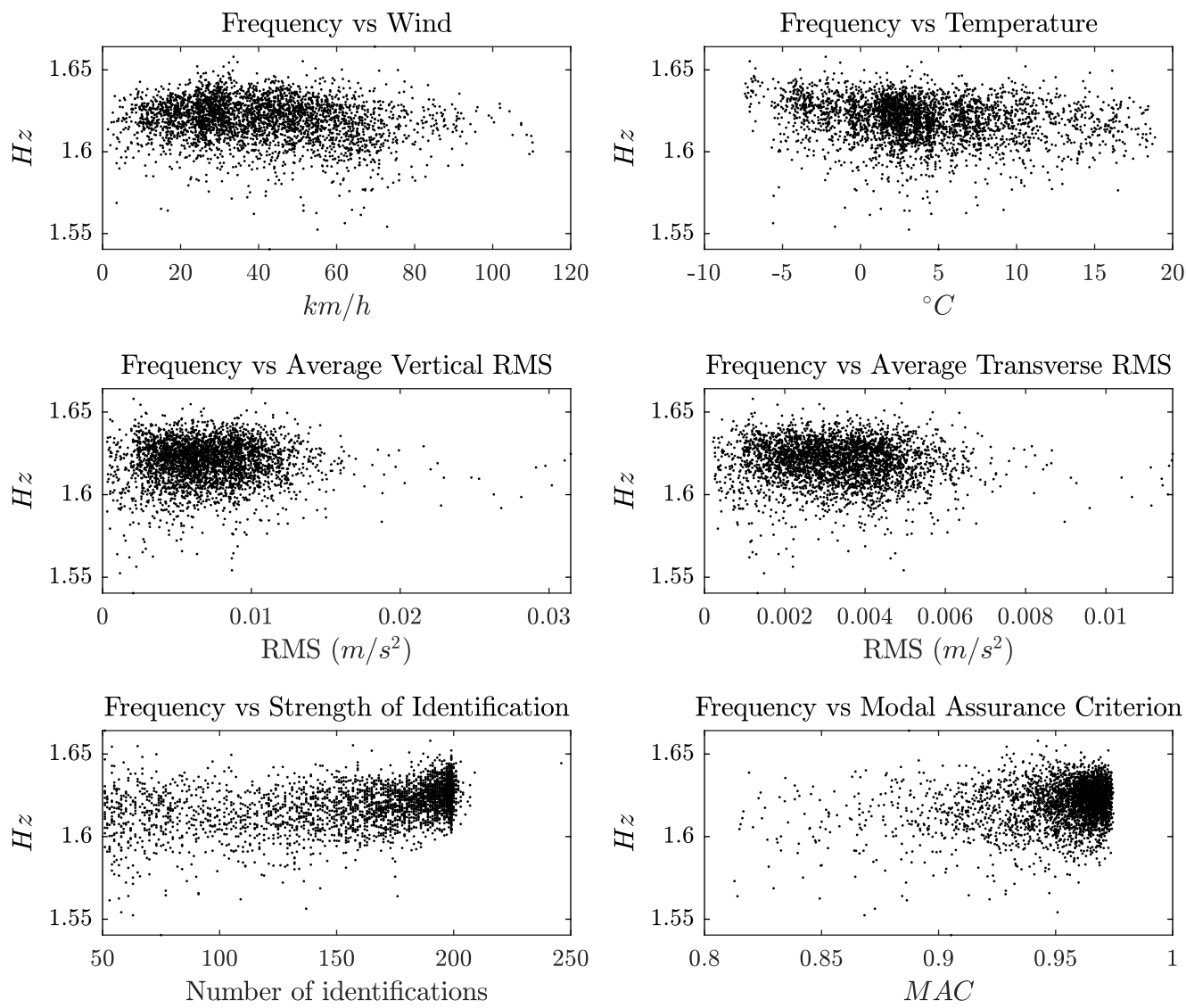

Figure 7.22: Modal frequencies vs continuous predictors - $1.62 \mathrm{~Hz}$ mode. 
The $1.62 \mathrm{~Hz}$ mode is a dominant vertical mode. From Figure 7.22, possible significant correlations can be observed between the modal frequencies and several continuous variables such as wind speed, temperatures and to a lesser extent, vertical and transverse RMS and MAC.

Figure 7.23 shows the relation to the categorical variables in box plot format. From this figure, statistical variations related to the hours of the day can be observed. Again, this indicates possible correlations with traffic excitations.

Frequency vs Categorical Predictors - Mode $1.62 \mathrm{~Hz}$
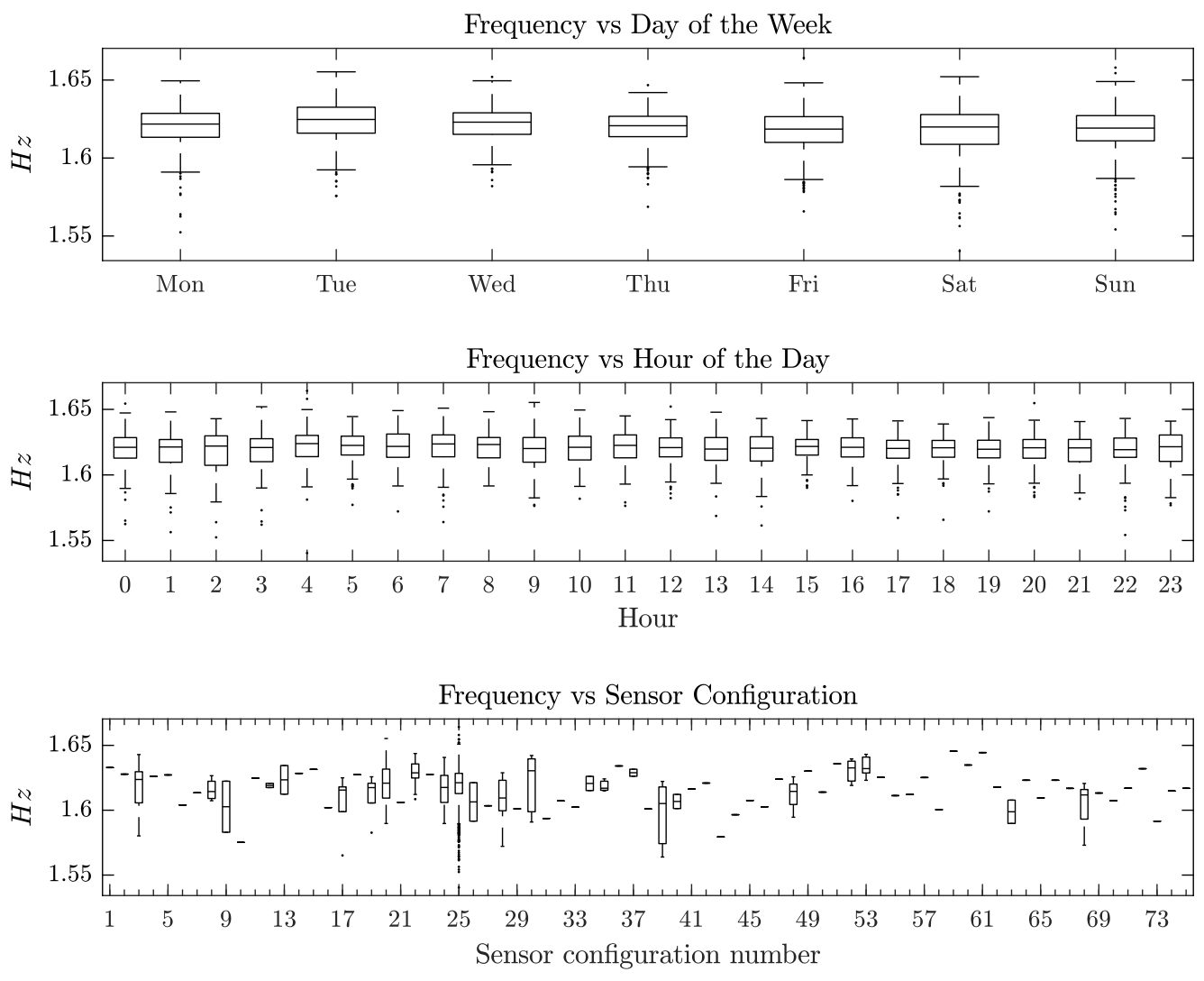

Figure 7.23: Modal frequencies vs categorical predictors $-1.62 \mathrm{~Hz}$ mode.

Multiple regression analyses were also carried out for the $1.62 \mathrm{~Hz}$ mode; Table 7.7 shows the results. In the first part of the table the model statistics for the original data are presented. In the bottom section are the model statistics for each multiple linear 
regression analyses performed on the smoothed data using the methods presented in

\section{Section 7.2 .}

Table 7.7: Linear regression statistics for modal frequencies of $1.62 \mathrm{~Hz}$ mode.

\begin{tabular}{|c|c|c|c|c|c|c|c|}
\hline \multicolumn{8}{|c|}{$1.62 \mathrm{~Hz}$ mode } \\
\hline \multicolumn{8}{|c|}{$\begin{array}{l}\text { R-squared: } 0.278, \text { Adjusted R-Squared } 0.253 \\
\text { F-statistic vs. constant model: } 11.3, \text { p-value }=8.39 \times 10^{-155} \\
\text { RMSE }=0.0113, \text { Standard deviation of residuals }=0.0111 \\
\text { Top coefficients : ModeIdent., AirTemperature }\end{array}$} \\
\hline \multicolumn{8}{|c|}{ Smoothing models } \\
\hline Method & $\begin{array}{l}\text { Window } \\
\text { size }\end{array}$ & $R^{2}$ & $\begin{array}{c}\text { Adjusted } \\
R^{2}\end{array}$ & $\begin{array}{c}\text { F- } \\
\text { Statistic }\end{array}$ & pValue & $\begin{array}{l}\text { Standard } \\
\text { deviation of } \\
\text { residuals }\end{array}$ & Top coefficients \\
\hline \multirow{4}{*}{$\begin{array}{l}\text { Moving } \\
\text { average }\end{array}$} & 5 & 0.317 & 0.294 & 13.6 & 0 & $6.813 \times 10^{-3}$ & AirTemperature, rmsTransverse \\
\hline & 10 & 0.364 & 0.342 & 16.8 & 0 & $5.882 \times 10^{-3}$ & AirTemperature, rmsTransverse \\
\hline & 20 & 0.396 & 0.376 & 19.3 & 0 & $5.291 \times 10^{-3}$ & AirTemperature, rmsTransverse \\
\hline & 50 & 0.422 & 0.402 & 21.4 & 0 & $4.776 \times 10^{-3}$ & AirTemperature, rmsTransverse \\
\hline \multirow{4}{*}{$\begin{array}{l}\text { Gaussian } \\
\text { weighted } \\
\text { moving } \\
\text { average }\end{array}$} & 5 & 0.318 & 0.295 & 13.7 & 0 & $7.379 \times 10^{-3}$ & AirTemperature, ModeIdent. \\
\hline & 10 & 0.352 & 0.330 & 15.9 & 0 & $6.272 \times 10^{-3}$ & AirTemperature, rmsTransverse \\
\hline & 20 & 0.388 & 0.367 & 18.5 & 0 & $5.548 \times 10^{-3}$ & AirTemperature, rmsTransverse \\
\hline & 50 & 0.420 & 0.400 & 21.2 & 0 & $4.953 \times 10^{-3}$ & AirTemperature, rmsTransverse \\
\hline \multirow{4}{*}{$\begin{array}{l}\text { Linear } \\
\text { regression }\end{array}$} & 5 & 0.301 & 0.277 & 12.6 & 0 & $7.835 \times 10^{-3}$ & AirTemperature, ModeIdent. \\
\hline & 10 & 0.349 & 0.327 & 15.7 & 0 & $6.263 \times 10^{-3}$ & AirTemperature, rmsTransverse \\
\hline & 20 & 0.386 & 0.365 & 18.4 & 0 & $5.541 \times 10^{-3}$ & AirTemperature, rmsTransverse \\
\hline & 50 & 0.420 & 0.400 & 21.2 & 0 & $4.938 \times 10^{-3}$ & AirTemperature, rmsTransverse \\
\hline \multirow{4}{*}{$\begin{array}{l}\text { Quadratic } \\
\text { regression }\end{array}$} & 5 & 0.278 & 0.253 & 11.3 & 0 & $1.107 \times 10^{-2}$ & ModeIdent., AirTemperature \\
\hline & 10 & 0.300 & 0.276 & 12.5 & 0 & $7.460 \times 10^{-3}$ & AirTemperature, rmsTransverse \\
\hline & 20 & 0.346 & 0.324 & 15.5 & 0 & $6.304 \times 10^{-3}$ & AirTemperature, rmsTransverse \\
\hline & 50 & 0.393 & 0.373 & 19.0 & 0 & $5.408 \times 10^{-3}$ & AirTemperature, rmsTransverse \\
\hline
\end{tabular}

With the presented R-squared value of the original (unsmoothed) data, $27.8 \%$ of the variations of the modal frequencies for this mode can be explained by the variations of the included predictor variables. The F-statistic is comparitively lower than those obtained with other modes (11.3). However, the model is still statistically significant versus the null hypothesis. The most significant metric for the purposes of 
this study, the RMSE, is relatively low $(0.0113 \mathrm{~Hz}$ or $0.70 \%$ of mean) which indicates a low variability of modal frequencies after removing the modeled effects of the predictor variables. The strength of identification and the ambient air temperature are the two most statistically significant regression variables for this mode, as shown in Table 7.7. In Appendix B, the parameters of all the statistically significant regression coefficients and the analysis of variance results are shown for the $1.62 \mathrm{~Hz}$ mode (Table B.7 and Table B.8).

Figure 7.24 shows the fitted modal frequencies obtained from the linear regression model of the original data plotted over the original modal frequencies. Normalized raw residuals are plotted in the bottom half of the figure. A relatively good fit is observed from this figure.
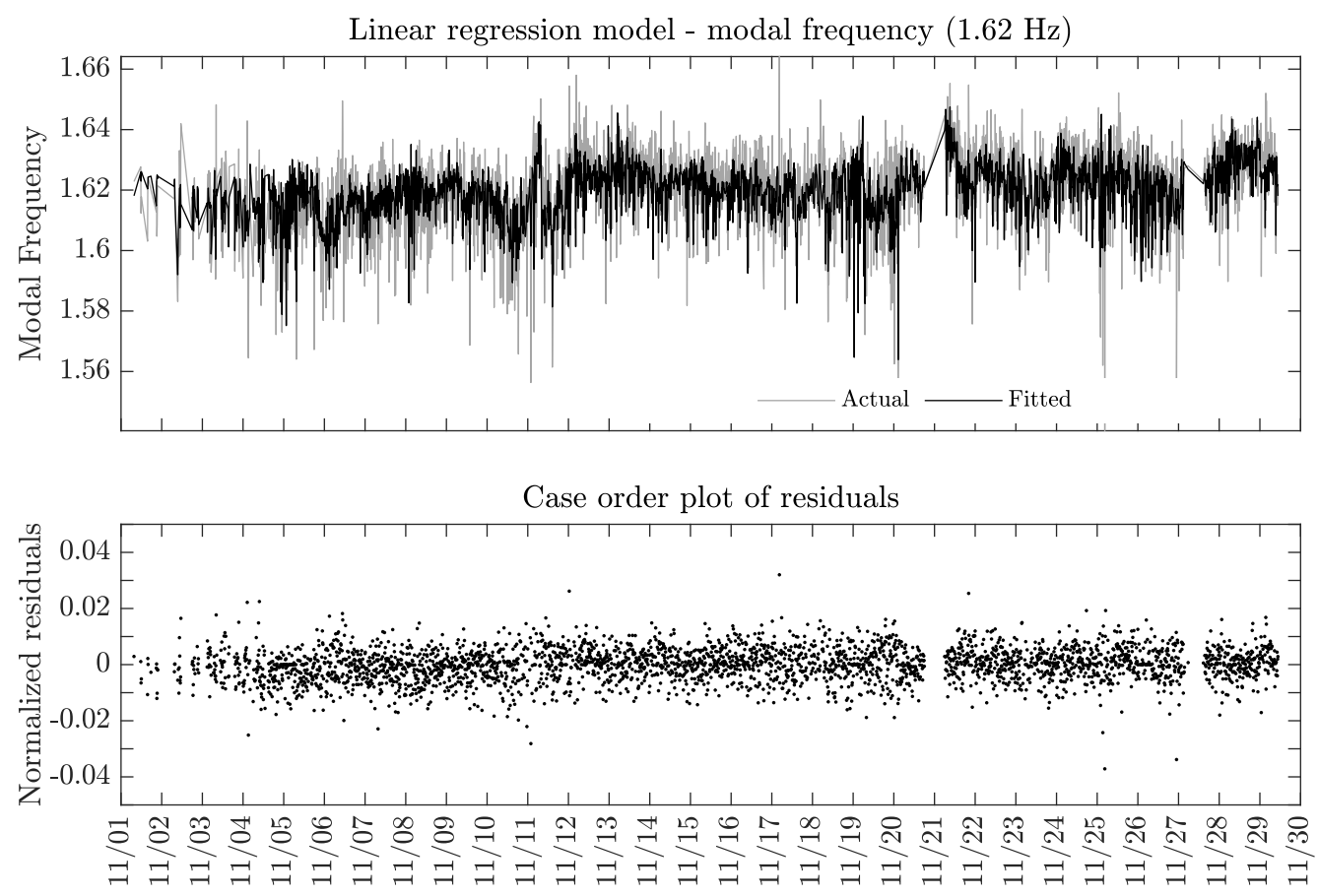

Figure 7.24: Regression model for modal frequencies of $1.62 \mathrm{~Hz}$ mode.

The distribution of residuals is also fairly uniform for this mode which confirms the suitability of the model of the $1.62 \mathrm{~Hz}$ mode to effectively remove the contributions 
from the predictor variable from the modal frequency time series signal.

\subsection{5 $1.85 \mathrm{~Hz}$ mode}

The $1.85 \mathrm{~Hz}$ mode is a dominant vertical mode. Figures 7.25 show the plots of the extracted modal frequencies for this mode versus each continuous predictor. Qualitatively, from Figure 7.25, we can observe possible significant correlations of modal frequencies with vertical and transverse RMS and to a lesser extent with wind and temperature.

Frequency vs Continuous Predictors - Mode $1.85 \mathrm{~Hz}$
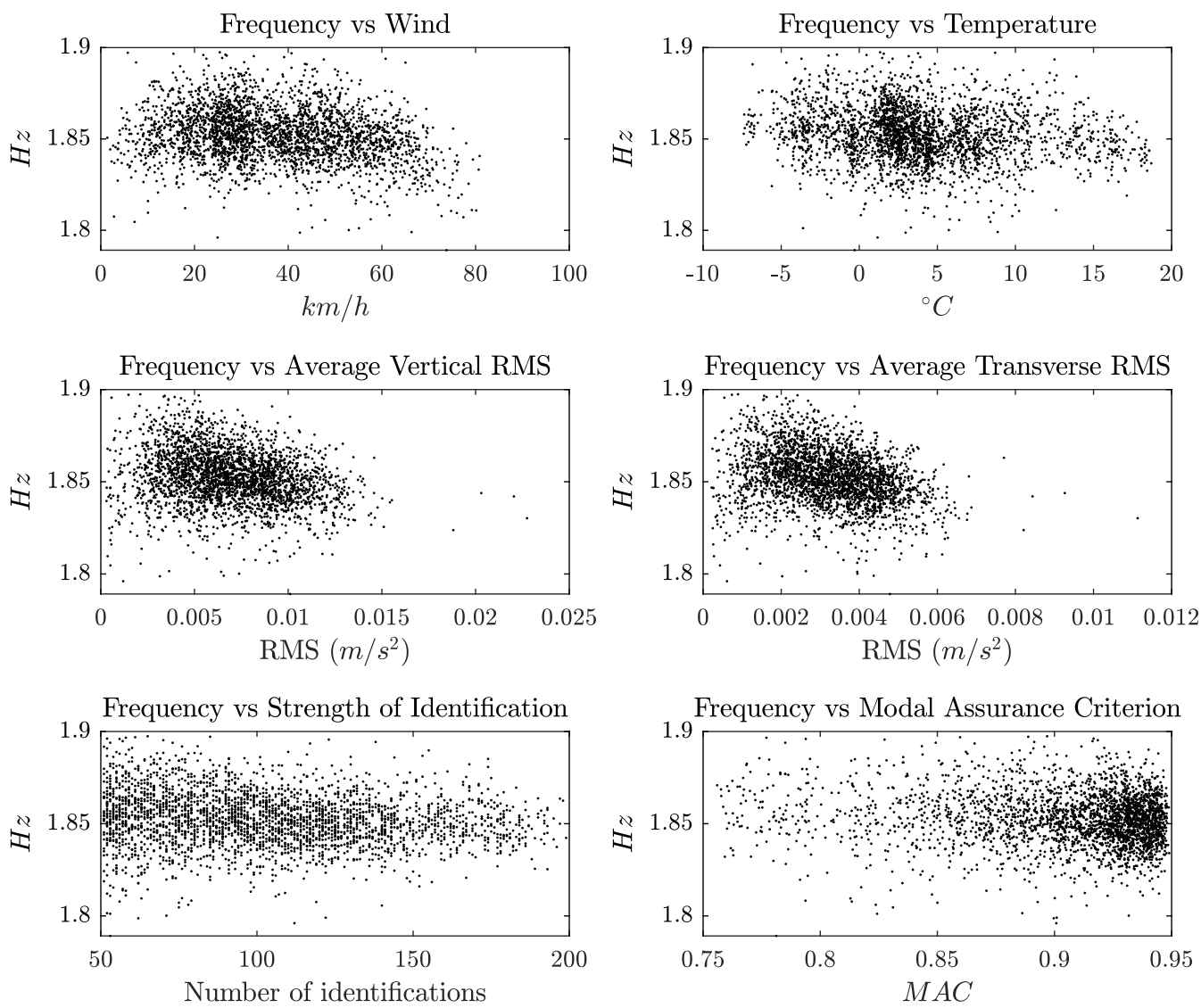

Figure 7.25: Modal frequencies vs continuous predictors $-1.85 \mathrm{~Hz}$ mode.

Figure 7.26 shows the relation to the categorical predictor variables. From this 
figure significant statistical variations of modal frequencies is observed with the hours of the day. The $1.85 \mathrm{~Hz}$ mode is a known traffic mode which could explain this observation.

\section{Frequency vs Categorical Predictors - Mode $1.85 \mathrm{~Hz}$}
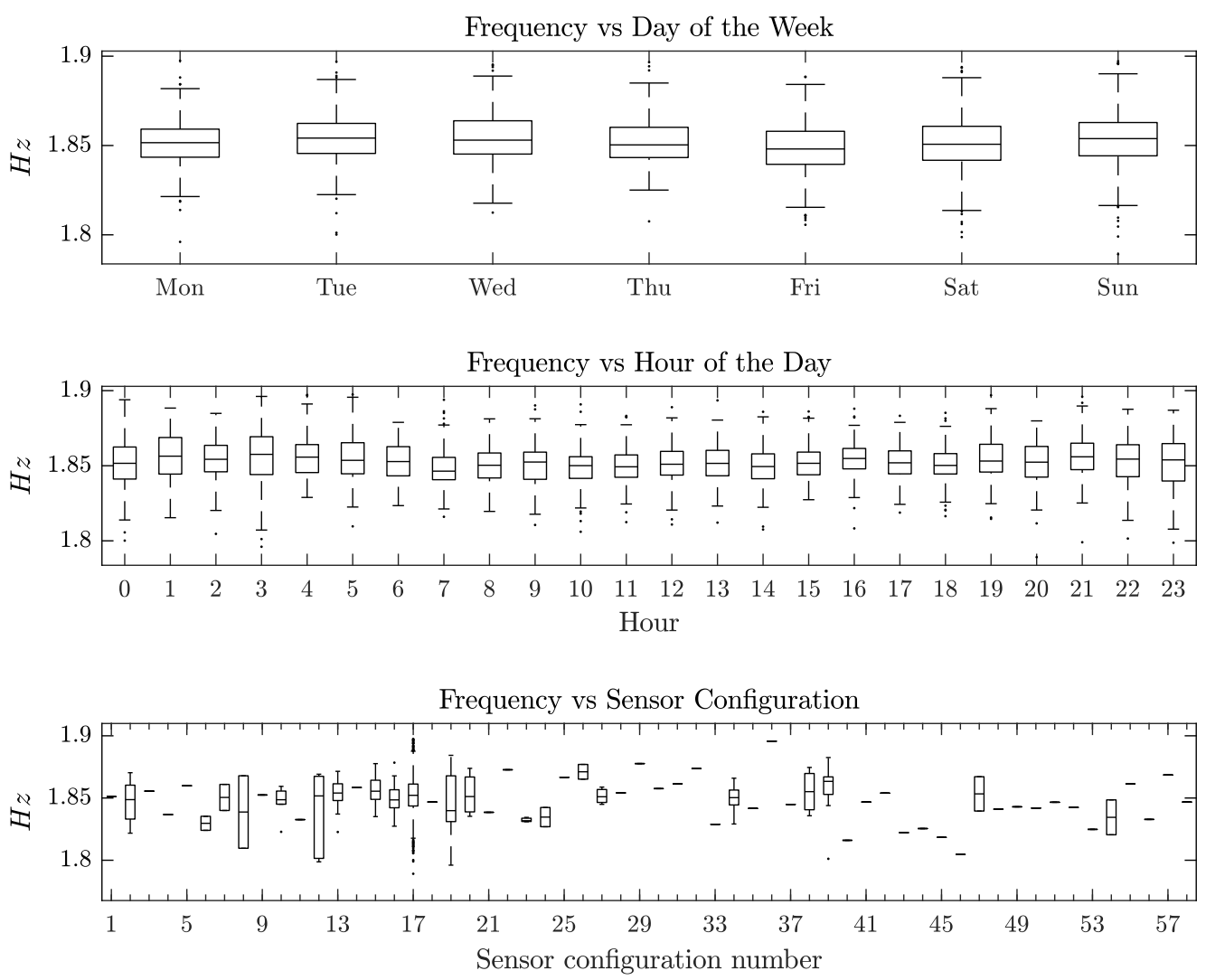

Figure 7.26: Modal frequencies vs categorical predictors $-1.85 \mathrm{~Hz}$ mode.

Table 7.8 shows the results of multiple regression analyses for the modal frequencies of the $1.85 \mathrm{~Hz}$ mode with model statistics for the original data in the top section of the table and the model statistics for each multiple linear regression analyses performed on smoothed data in the bottom portion of the Table. As much as $23.6 \%$ of the variations of the modal frequencies for this mode can be explained by the variations of the included predictor variables. With a comparatively low F-statistic of 9.1, the model is still statistically significant versus the null hypothesis. The RMSE of 0.0128 
Table 7.8: Linear regression statistics for modal frequencies of $1.85 \mathrm{~Hz}$ mode.

\begin{tabular}{|c|c|c|c|c|c|c|c|}
\hline \multicolumn{8}{|c|}{$1.85 \mathrm{~Hz}$ mode } \\
\hline \multicolumn{8}{|c|}{$\begin{array}{l}\text { Model statistics : } \\
0.236, \text { Adjusted R-Squared } 0.210 \\
\text { ant model: } 9.11, \mathrm{p} \text {-value }=2.79 \times 10^{-102} \\
\text { tandard deviation of residuals }=0.0126 \\
\text { ents : ModalMAC, rmsTransverse }\end{array}$} \\
\hline \multicolumn{8}{|c|}{ Smoothing models } \\
\hline Method & $\begin{array}{l}\text { Window } \\
\text { size }\end{array}$ & $R^{2}$ & $\begin{array}{l}\text { Adjusted } \\
R^{2}\end{array}$ & $\begin{array}{c}\text { F- } \\
\text { Statistic }\end{array}$ & pValue & $\begin{array}{l}\text { Standard } \\
\text { deviation of } \\
\text { residuals }\end{array}$ & Top coefficients \\
\hline \multirow{4}{*}{$\begin{array}{l}\text { Moving } \\
\text { average }\end{array}$} & 5 & 0.352 & 0.331 & 16.1 & 0 & $7.432 \times 10^{-3}$ & rmsTransverse, AirTemperature \\
\hline & 10 & 0.394 & 0.374 & 19.2 & 0 & $6.434 \times 10^{-3}$ & rmsTransverse, AirTemperature \\
\hline & 20 & 0.416 & 0.396 & 21.0 & 0 & $5.754 \times 10^{-3}$ & rmsTransverse, AirTemperature \\
\hline & 50 & 0.421 & 0.401 & 21.4 & 0 & $5.002 \times 10^{-3}$ & AirTemperature ,rmsTransverse \\
\hline \multirow{4}{*}{$\begin{array}{l}\text { Gaussian } \\
\text { weighted } \\
\text { moving } \\
\text { average }\end{array}$} & 5 & 0.319 & 0.296 & 13.8 & 0 & $8.216 \times 10^{-3}$ & rmsTransverse, AirTemperature \\
\hline & 10 & 0.377 & 0.356 & 17.9 & 0 & $6.907 \times 10^{-3}$ & rmsTransverse, AirTemperature \\
\hline & 20 & 0.414 & 0.394 & 20.8 & 0 & $6.074 \times 10^{-3}$ & rmsTransverse, AirTemperature \\
\hline & 50 & 0.430 & 0.410 & 22.2 & 0 & $5.301 \times 10^{-3}$ & AirTemperature, rmsTransverse \\
\hline \multirow{4}{*}{$\begin{array}{l}\text { Linear } \\
\text { regression }\end{array}$} & 5 & 0.297 & 0.273 & 12.5 & 0 & $8.724 \times 10^{-3}$ & rmsTransverse, AirTemperature \\
\hline & 10 & 0.380 & 0.359 & 18.1 & 0 & $6.869 \times 10^{-3}$ & rmsTransverse, AirTemperature \\
\hline & 20 & 0.413 & 0.393 & 20.8 & 0 & $6.069 \times 10^{-3}$ & rmsTransverse, AirTemperature \\
\hline & 50 & 0.426 & 0.406 & 21.9 & 0 & $5.311 \times 10^{-3}$ & AirTemperature, rmsTransverse \\
\hline \multirow{4}{*}{$\begin{array}{l}\text { Quadratic } \\
\text { regression }\end{array}$} & 5 & 0.236 & 0.210 & 9.1 & 0 & $1.256 \times 10^{-2}$ & ModalMAC, rmsTransverse \\
\hline & 10 & 0.318 & 0.295 & 13.8 & 0 & $8.224 \times 10^{-3}$ & rmsTransverse, AirTemperature \\
\hline & 20 & 0.379 & 0.358 & 18.0 & 0 & $6.911 \times 10^{-3}$ & rmsTransverse, AirTemperature \\
\hline & 50 & 0.414 & 0.394 & 20.9 & 0 & $5.957 \times 10^{-3}$ & rmsTransverse, AirTemperature \\
\hline
\end{tabular}

$\mathrm{Hz}$ or $0.70 \%$ of mean indicates a relatively low variability of the modal frequencies. The statistical parameters of all the regression coefficients for the $1.85 \mathrm{~Hz}$ mode are shown in Table B.9 and the analysis of variance results are shown in Table B.10.

Again, the linear regression models for the smoothed data for this mode seem to perform better overall. Section 7.2 describes the smoothing methods. With data smoothing, the top two significant regression coefficients are RMS (transverse) and air temperature. 
Model performance can be observed in Figure 7.27 which shows the fitted modal frequencies obtained from the linear regression model plotted over the original modal frequencies.
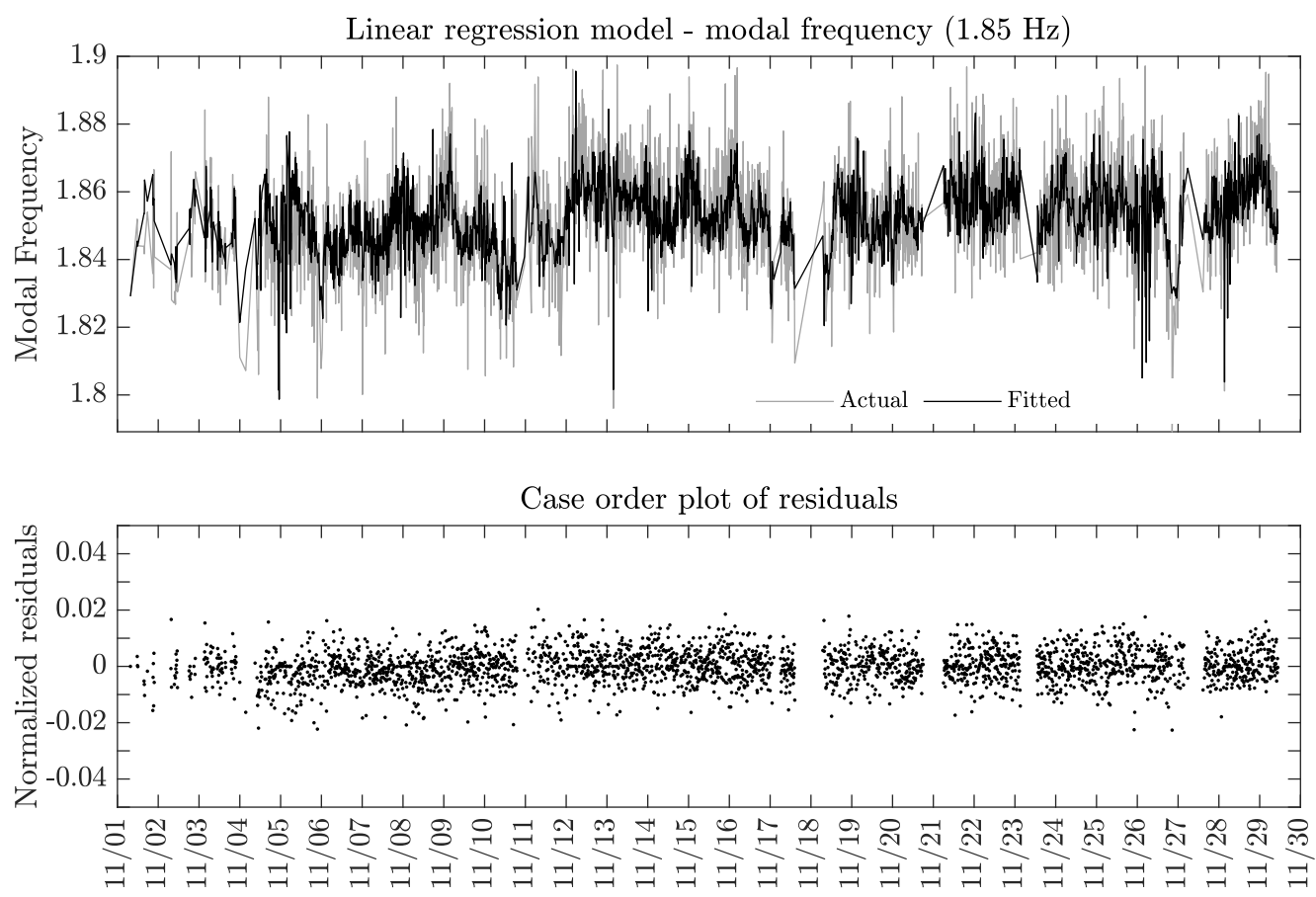

Figure 7.27: Regression model for modal frequencies of $1.85 \mathrm{~Hz}$ mode.

From the normalized raw residuals plotted in the bottom half of the figure, a uniform distribution is observed which confirms the suitability of the model to effectively remove the statistically significant contributions from the predictor variables.

\subsubsection{Summary}

In this section, the results from a series of multiple linear regressions performed on the five dominant operational modes of the Confederation Bridge are presented. The results, summarized in Table 7.9, indicate that the operational modes of the superstructure are differently affected by the environmental and operational processes. 
It should be noted that the month of November 2017 was a particularly windy month with several strong wind events (as can be observed in Figure 7.1). The five dominant modes considered in this section are therefore principally excited by wind loads. A distinction needs to be made in regards to excitation versus correlation. For example, a mode can be strongly excited by a certain environmental or operational load, but the variation of its modal parameters may not be strongly correlated to the variations of its excitation load.

It is also observed, from the summary table, that certain modes are much more sensitive to the variations in the predictor variables considered in this study. For example the operational modal frequencies of the $0.47 \mathrm{~Hz}$ and $0.67 \mathrm{~Hz}$ modes, which exhibited higher variability according to Table 7.1 than those associated with the modes at $0.96 \mathrm{~Hz}, 1.62 \mathrm{~Hz}$ and $1.85 \mathrm{~Hz}$, have linear models with comparatively higher $R^{2}$ values. A higher percentage of the variability from these modes can therefore be explained by the variations of the predictor variables. The higher modes, already less variable, were less sensitive to the variations of the predictor variables.

Table 7.9: Linear regression statistics for the five dominant modal frequencies.

\begin{tabular}{|c|c|c|c|c|c|c|}
\hline Mode & Data & $R^{2}$ & Adj. $R^{2}$ & F-Stat. & $\sigma_{\text {Resid. }} \%^{* *}$ & Top coefficients \\
\hline \multirow{2}{*}{$0.47 \mathrm{~Hz}$} & Original data & 0.468 & 0.446 & 21.1 & 0.95 & ModeIdent., WindSpeed \\
\hline & Smoothed data* & 0.697 & 0.684 & 55.0 & 0.51 & WindSpeed, ModeIdent. \\
\hline \multirow{2}{*}{$0.67 \mathrm{~Hz}$} & Original data & 0.532 & 0.515 & 31.9 & 0.84 & WindSpeed, ModeIdent. \\
\hline & Smoothed data* & 0.818 & 0.811 & 126.0 & 0.37 & WindSpeed, rmsTransverse \\
\hline \multirow{2}{*}{$0.96 \mathrm{~Hz}$} & Original data & 0.272 & 0.250 & 12.1 & 0.83 & ModeIdent., rmsTransverse \\
\hline & Smoothed data* & 0.543 & 0.529 & 38.5 & 0.36 & rmsTransverse, WindSpeed \\
\hline \multirow{2}{*}{$1.62 \mathrm{~Hz}$} & Original data & 0.278 & 0.253 & 11.3 & 0.69 & ModeIdent., AirTemperature \\
\hline & Smoothed data* & 0.420 & 0.400 & 21.2 & 0.31 & AirTemperature, rmsTransverse \\
\hline \multirow{2}{*}{$1.85 \mathrm{~Hz}$} & Original data & 0.236 & 0.210 & 9.11 & 0.68 & ModalMAC, rmsTransverse \\
\hline & Smoothed data* & 0.430 & 0.410 & 22.2 & 0.29 & AirTemperature, rmsTransverse \\
\hline
\end{tabular}

\footnotetext{
* Smoothing method : Gaussian-weighted moving average with a window length of 50 .

** Standard deviation of residuals (percentage of mean).
} 
The proposed approach to damage detection described in the next section (Section 7.5) focuses on the residuals obtained from the multiple linear regressions described in this section. The standard deviation of the residuals noted in Table 7.9 are therefore important predictors of the performance of the proposed damage detection technique. The residuals represent the unexplained variation of the operational modal frequencies. These variations are most likely due to measurement, computational and un-modeled process noise which potentially include variations due to damage and deterioration. By removing the variability due to known processes, the remaining variability is relatively low with standard deviations in percentage of mean of $0.68 \%$ to $0.95 \%$. These values approach the baseline variability $(0.50 \%$ to $0.61 \%)$ as determined by [28] and shown in Table 3.3. It should be noted that these promising results are obtained through large scale automated OMA using newly developed tools described in Chapter 2. Since the variability of the modal parameters can mask the changes due to damage or deterioration, the overriding objective of this study is to reduce the variability as much as possible in order to detect the lowest possible level of damage.

\subsection{Residual Analysis and Change Point Detection for Damage Identification}

In this section, the residuals obtained from the multiple linear regression analysis of the five chosen modal frequencies of Section 7.4 are used to model a change point detection algorithm. It is important to note that the residuals contain the variations of modal frequencies after removing the effects of all other known correlated parameters. For a properly modeled system, the residuals should be uniformly distributed and random. It is believed, from structural analysis theory, that damage or deterioration 
will result in a change in the stiffness matrix of a structure which is reflected in a change in its eigenvalues (modal frequencies). If we observe a statistically significant change in the modal frequency residuals (loss of randomness, shift in mean, change of slope or variance), this may indicate occurrence of damage or deterioration of the structure.

In this section a change point algorithm is applied to detect any shift in residual frequencies attributable to possible damage. By definition a change point is a sample or time instant at which some statistical property, such as the mean, the slope or the variance, of a signal changes abruptly. To find a change point, the algorithm chooses a point and divides the signal into two sections and computes an empirical estimate of the chosen statistical property for each section. At each point within a section, the algorithm measures how much the property deviates from the empirical estimate and then adds the deviations for all points. The deviations from section-to-section are then added to find the total residual error. Finally, the algorithm varies the location of the division point until the total residual error attains a minimum [52,60,61].

To detect a shift in the mean of the residual frequencies, the algorithm minimizes the total residual error from the best horizontal level for each section. Given a signal $\mathbf{x}_{i}$ where $i=1$ to $N$, the function finds $k$ such that Equation (7.10) is minimized.

$$
J=\sum_{i=1}^{k-1}\left(x_{i}-\langle x\rangle_{1}^{k-1}\right)^{2}+\sum_{i=k}^{N}\left(x_{i}-\langle x\rangle_{k}^{N}\right)^{2}
$$

Theoretically, a shift in mean equal to or greater than the standard deviation of the residuals should be detected by the change point detection algorithm. For illustrative purposes, the vector of residual modal frequencies from the multiple linear regression model of the $0.47 \mathrm{~Hz}$ mode is artificially shifted by an amount equal to its standard deviation of $0.96 \%$ of mean. This represents the variation attributed to a fictitious 
damage scenario occurring on November 15th 2017 (or at sample number 2060 of the residuals vector). Figure 7.28 shows the results of the algorithm in successfully identifying the change point in the residuals vector. The change point accuracy is calculated as the difference in samples between the actual change point (which is a known variable for this test case) and the detected change point. The detected mean shift is the difference in mean of the residuals before and after the change point and the detection lag is measured in number of samples required for detection after the actual change point. The detection lag represents the amount of samples that was required in order to successfully identify the change point. This value is important as it effectively limits the instantaneous identification of damage, since a certain number of samples is required after actual change occurrence to properly detect a change point.

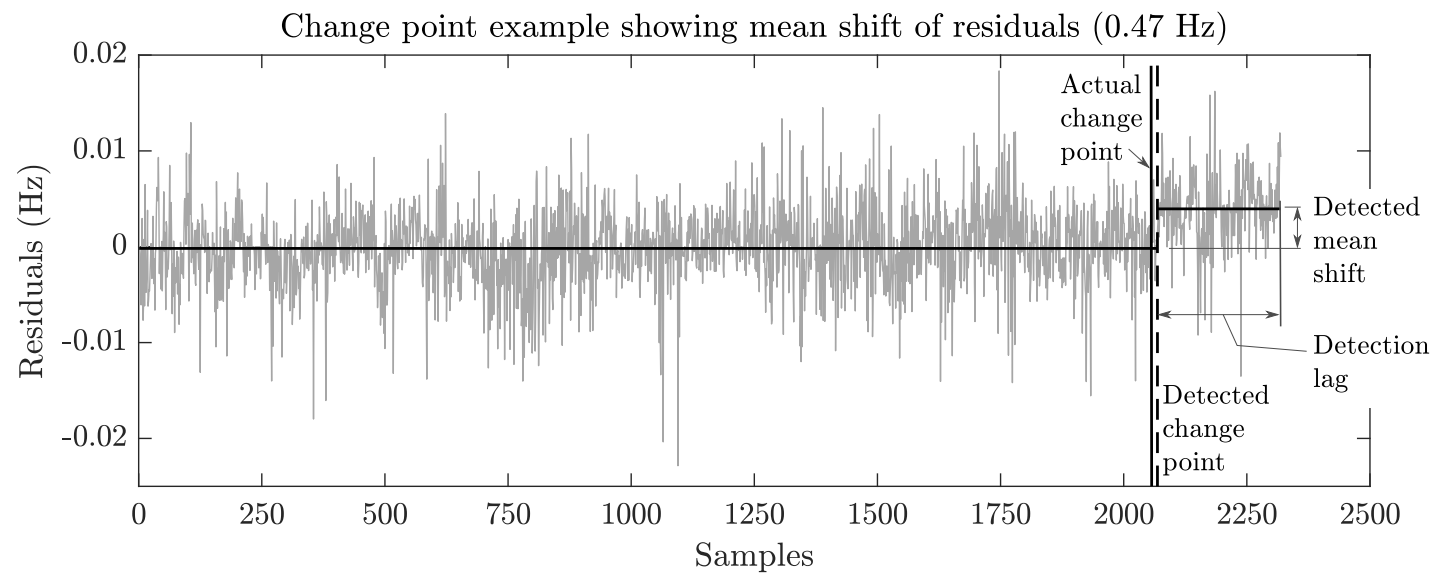

Figure 7.28: Change point detection example of mean shift in residuals of $0.47 \mathrm{~Hz}$ mode.

Table 7.10 shows a change point sensitivity analysis performed on the residuals from the multiple linear regression analysis of the five dominant modes $(0.47 \mathrm{~Hz}$, $0.67 \mathrm{~Hz}, 0.96 \mathrm{~Hz}, 1.62 \mathrm{~Hz}$ and $1.85 \mathrm{~Hz})$. For the sensitivity analysis, five hundred runs of the change point detection algorithm was performed on each residuals vector from the 
five dominant modes. Several amplitudes of the imposed mean shift were considered.

The location of the actual shift in mean was chosen randomly. The performance of the change point algorithm is evaluated using three metrics : the change point accuracy, the detection lag and the detected mean difference.

Table 7.10 shows the algorithm performed especially well for the lower modes. An imposed shift in mean of approximately $0.4 \%$ was successfully detected by the

Table 7.10: Results of the change point sensitivity analysis on the residuals of five dominant operational modes.

\begin{tabular}{|c|c|c|c|c|c|c|c|}
\hline \multirow[t]{2}{*}{ Mode } & \multirow{2}{*}{$\begin{array}{l}\text { Imposed } \\
\text { shift in } \\
\text { mean }(\%)\end{array}$} & \multicolumn{2}{|c|}{$\begin{array}{c}\text { Change point } \\
\text { accuracy (number } \\
\text { of samples) }\end{array}$} & \multicolumn{2}{|c|}{$\begin{array}{l}\text { Detection lag } \\
\text { (number of } \\
\text { samples) }\end{array}$} & \multicolumn{2}{|c|}{$\begin{array}{l}\text { Detected mean } \\
\text { shift }(\%)\end{array}$} \\
\hline & & Avg. & $\sigma$ & Avg. & $\sigma$ & Avg. & $\sigma$ \\
\hline \multirow{7}{*}{$0.47 \mathrm{~Hz}$} & 0.4 & 60.0 & 87.6 & 358.8 & 112.8 & 0.55 & $4.03 \times 10^{-2}$ \\
\hline & 0.6 & 16.6 & 29.3 & 276.6 & 77.0 & 0.75 & $4.40 \times 10^{-2}$ \\
\hline & 0.8 & 10.3 & 20.0 & 224.0 & 46.0 & 0.95 & $4.31 \times 10^{-2}$ \\
\hline & 1.0 & 5.3 & 14.3 & 152.7 & 32.4 & 1.15 & $4.95 \times 10^{-2}$ \\
\hline & 1.2 & 2.7 & 7.7 & 105.9 & 22.9 & 1.36 & $7.18 \times 10^{-2}$ \\
\hline & 1.4 & 1.7 & 2.7 & 80.0 & 16.7 & 1.55 & $6.55 \times 10^{-2}$ \\
\hline & 1.6 & 1.4 & 1.9 & 61.3 & 12.2 & 1.75 & $5.86 \times 10^{-2}$ \\
\hline \multirow{7}{*}{$0.67 \mathrm{~Hz}$} & 0.4 & 28.4 & 36.1 & 298.6 & 101.2 & 0.45 & $3.07 \times 10^{-2}$ \\
\hline & 0.6 & 12.3 & 19.9 & 220.8 & 64.0 & 0.64 & $3.51 \times 10^{-2}$ \\
\hline & 0.8 & 8.5 & 17.9 & 161.5 & 42.1 & 0.83 & $4.42 \times 10^{-2}$ \\
\hline & 1.0 & 4.3 & 9.4 & 102.4 & 27.0 & 1.04 & $4.47 \times 10^{-2}$ \\
\hline & 1.2 & 2.4 & 5.3 & 74.1 & 16.8 & 1.23 & $4.23 \times 10^{-2}$ \\
\hline & 1.4 & 1.5 & 1.9 & 53.4 & 11.8 & 1.43 & $4.22 \times 10^{-2}$ \\
\hline & 1.6 & 1.3 & 1.2 & 41.4 & 8.9 & 1.63 & $4.14 \times 10^{-2}$ \\
\hline \multirow{7}{*}{$0.96 \mathrm{~Hz}$} & 0.6 & 33.9 & 80.3 & 135.0 & 38.5 & 0.69 & $5.90 \times 10^{-2}$ \\
\hline & 0.8 & 10.2 & 20.1 & 108.2 & 32.4 & 0.89 & $5.64 \times 10^{-2}$ \\
\hline & 1.0 & 5.9 & 14.5 & 72.9 & 21.7 & 1.09 & $8.01 \times 10^{-2}$ \\
\hline & 1.2 & 6.0 & 16.3 & 51.9 & 14.3 & 1.29 & $5.91 \times 10^{-2}$ \\
\hline & 1.4 & 3.7 & 11.4 & 39.3 & 11.0 & 1.50 & $7.52 \times 10^{-2}$ \\
\hline & 1.6 & 3.1 & 11.0 & 30.5 & 7.9 & 1.70 & $7.56 \times 10^{-2}$ \\
\hline & 1.8 & 1.6 & 5.6 & 24.6 & 6.2 & 1.89 & $7.88 \times 10^{-2}$ \\
\hline \multirow{7}{*}{$1.62 \mathrm{~Hz}$} & 2.4 & 33.6 & 255.7 & 6.4 & 1.2 & 2.48 & $2.60 \times 10^{-1}$ \\
\hline & 2.6 & 39.8 & 271.7 & 5.5 & 1.0 & 2.67 & $2.99 \times 10^{-1}$ \\
\hline & 2.8 & 24.3 & 210.8 & 4.9 & 0.9 & 2.89 & $2.52 \times 10^{-1}$ \\
\hline & 3.0 & 1.0 & 0.1 & 4.3 & 0.8 & 3.11 & $4.74 \times 10^{-2}$ \\
\hline & 3.2 & 1.0 & 0.1 & 3.9 & 0.7 & 3.31 & $4.39 \times 10^{-2}$ \\
\hline & 3.4 & 1.0 & 0.1 & 3.5 & 0.6 & 3.51 & $4.74 \times 10^{-2}$ \\
\hline & 3.6 & 1.0 & 0.1 & 3.1 & 0.6 & 3.71 & $4.65 \times 10^{-2}$ \\
\hline \multirow{7}{*}{$1.85 \mathrm{~Hz}$} & 0.8 & 230.3 & 509.1 & 41.6 & 9.8 & 0.85 & $1.46 \times 10^{-1}$ \\
\hline & 1.0 & 22.2 & 130.8 & 28.3 & 6.3 & 1.09 & $5.43 \times 10^{-2}$ \\
\hline & 1.2 & 1.1 & 0.9 & 20.1 & 4.3 & 1.30 & $3.38 \times 10^{-2}$ \\
\hline & 1.4 & 1.1 & 0.6 & 14.9 & 3.0 & 1.50 & $3.56 \times 10^{-2}$ \\
\hline & 1.6 & 1.0 & 0.5 & 12.0 & 2.3 & 1.70 & $3.20 \times 10^{-2}$ \\
\hline & 1.8 & 1.0 & 0.4 & 9.6 & 1.9 & 1.90 & $3.31 \times 10^{-2}$ \\
\hline & 2.0 & 1.0 & 0.3 & 7.8 & 1.4 & 2.10 & $3.59 \times 10^{-2}$ \\
\hline
\end{tabular}


algorithm on the residuals of the $0.47 \mathrm{~Hz}$ and $0.67 \mathrm{~Hz}$ modes; albeit with lower accuracy and longer detection lags than those obtained for higher amplitude shifts. As the amplitude of shift is increased, the change point accuracy is significantly improved and more importantly the detection lags are greatly reduced. The results of the higher modes $(1.62 \mathrm{~Hz}$ and $1.85 \mathrm{~Hz})$ are more variable. Further investigation revealed a statistical feature in the residuals of these modes that skewed the results for the lower amplitude shifts, but at higher amplitudes the change detection algorithm was successful in identifying the imposed shift. This serves as an example of how damage can be hidden by unmodeled correlated parameters.

In this Chapter, it has been shown that the variability can be significantly reduced by filtering out the effects of known parameters on the extracted modal properties. Any statistical variations in the residuals can be attributed to unknown (unmodeled) parameters which include possible damage and deterioration. A change point algorithm has been implemented with promising results in identifying shifts in residual means with amplitudes in the order of the standard deviation of residuals $(0.68 \%$ to $0.95 \%$ of mean as shown in Table 7.9 ). It should be noted that the change point algorithm can also be applied on residuals obtained from the smoothing models or from the Kalman filter results. Future work will focus on this subject. 


\section{Chapter 8}

\section{Use of Vibrations for Health Monitoring of Structural Components}

Three separate studies were proposed by Strait Crossing Bridge Limited (SCBL are the current operators of the Confederation Bridge) to demonstrate the practical application of vibration-based monitoring for the health evaluation of structural components. There is a need, in the field of VBSHM for successful case studies demonstrating the practical applications and actionable results obtained from vibration response monitoring. The first study focused on the steel light poles located on the deck of the Confederation Bridge and was completed in two phases presented in Sections 8.1 and 8.2. The second proposed study is a preliminary proof of concept study on using vibration measurements for the estimation of remaining service life of bridge bearings and is presented in Section 8.3. The third is a proposed study on the use of vibration measurements to estimate the remaining stress in external post tensioning cables and is presented in Section 8.4. 


\subsection{Modal Analysis and Vibration Mitigation of the Confederation Bridge Light Poles}

\subsubsection{Summary}

The goals of this study are to provide a better understanding of the dynamic behavior of the light poles installed on the deck of the Confederation Bridge and to propose a damping solution to attenuate the large amplitude vibrations observed during significant wind events. To attain these objectives, the full-scale testing of two installed light poles (an original light pole and a replacement light pole) was carried out on the deck of the Confederation Bridge. The testing of a light pole was also carried out in a laboratory setting to validate the testing methodology. Four wireless tri-axial accelerometers installed on the light poles captured the free vibration response of the poles subjected to pull and release excitations.

Results were obtained via two modal analysis techniques (log decrement and stochastic subspace identification) and were compared to the results obtained from an analytical structural component model. The experimentally obtained modal properties (frequency and mode shapes of the first 4 modes) correlate well with those obtained analytically and with those obtained from a previous internal study [62].

To attenuate the large amplitude vibrations, the second part of the study focused on pole-top impact dampers by comparing the damping performance of five custom prototypes and a commercial option from the Hapco Company. A prototype using two lead balls within a pipe cylinder added to the top of the pole was found to offer comparable or better damping under large displacements than the commercial damper. This configuration has added advantages of being more easily fitted to the existing

poles and more durable for the harsh Atlantic environment. Recommendations are 
included in Section 8.1.9.

\subsubsection{Introduction}

This study is undertaken to better understand the dynamic behavior of the light poles installed on the deck of the Confederation Bridge and to propose a damping solution to attenuate the large amplitude vibrations observed during significant wind events.

During a strong wind event in December 2016, the bridge maintenance crews observed strong swaying motions of the light poles, generally in the direction of the wind. During these strong wind events, a few poles experienced structural failures and following these events, during special inspections, more poles were replaced as a precautionary measure as they showed signs of structural weakness. The new replacement poles have an increased wall thickness and an improved weld detail at the base plate interface. The objectives of the current study are limited to:

- properly identifying the modal properties of the light poles currently installed on the Confederation Bridge,

- comparing the identified modal properties of the light poles with the operational modal properties of the bridge superstructure,

- and to propose an effective solution to attenuate the pole vibrations during strong wind events, and thus extend the remaining service life of these important bridge components.

\subsubsection{Pole dynamics}

Light poles are vertical cantilever structures that vibrate under certain conditions. They vibrate in different modes and at different frequencies. Natural wind is the most common excitation force for these pole structures. Once the excitation force is 
removed, the vibration decays and the pole returns to rest. The rate of this decay is used to determine the damping properties. Very tall and slender structures such as light poles have inherently low damping coefficients. When certain conditions exist, these light poles may vibrate strongly for long periods of time.

It has been shown that under one or more of the following conditions, poles are more prone to vibrate [63]. When severe enough, pole failures can occur.

- Open terrain with little or no trees or buildings to break up the wind;

- Steady prevailing winds in the 12 to $40 \mathrm{~km} / \mathrm{h}$ range; however, under very high gusting conditions, more severe oscillation may result. When gusts occur at very high wind speeds, (80 to $100+\mathrm{km} / \mathrm{h}$ range) violent "whipping" and "pulsing" may occur;

- Elevated and exposed areas such as parking decks, bridges and overpasses, mountain passes;

- Poles lengths of 6 meters and over;

- Poles with very light weight luminaires (or no luminaires);

- Luminaire of low profile, or low EPA.

The light poles installed on the deck of the Confederation Bridge are subjected to each of these conditions and are therefore prone to large vibrations under wind loadings. In December 2011, the luminaires were changed from high pressure sodium (HPS) to LED style with a resulting reduction in weight and EPA.

The most significant effect of these vibrations is the inducement of stresses at the pole base. The greater the vibration amplitude (or displacement), the greater the stress. When these stresses are continually repeated over time, they are called fatigue stress and may lead to stress cracks and premature failures of the light poles. It is therefore advantageous, in the case of fatigue stress, to either limit the amplitude of vibrations or to limit the number of high amplitude cycles or both. 
For this study, it is important to distinguish between first mode vibrations and second mode vibrations of light poles. First mode vibrations, more commonly referred to as "pole sway", starts at moderate wind speeds, but when gusts occur at very high wind speeds, more violent behavior such as whipping and pulsing may occur resulting in very high repetitive stresses at the base. It is important to note that first mode vibration movement occurs generally in the direction of the wind.

Second mode vibrations is caused by vortex shedding, which is a phenomenon where small eddies are alternately spinning off the sides of the pole under moderate wind (12 to $32 \mathrm{~km} / \mathrm{h}$ ). When the vortex spins off the pole, it creates a pressure collapse that drives the pole in the direction of the vortex. This happens continually and alternately on both sides of the pole normal to the wind direction. Second mode vibration movement therefore occurs at a $90^{\circ}$ angle to the direction of the wind.

It is also important to state that the service life of light poles installed in wind prone areas is not typically measured temporally, but rather by the number of stress cycles it experiences while in service. According to fatigue theory, the higher the stress amplitude of the cycles of oscillations, the lower the number of cycles until the end of life. The service life of poles can be increased (sometimes significantly) by limiting the high amplitude stress cycles. Conversely, light poles can suffer failure during a single event if extreme oscillations occur.

From anecdotal evidence, pole movements observed during very strong wind events on the Confederation Bridge, were generally in the direction of the wind and corresponded with first mode vibration (sway) with the maximum displacements occurring at the top of the pole (and maximum stresses at the pole base). From this anecdotal evidence, the second objective of the current study therefore focuses on mitigating the first mode vibrations of the Confederation Bridge light poles. The proposed solutions to attenuate these vibrations focus on pole top impact dampers which, according to 
literature, is very effective against these types of vibrations [64,65]. Pole top impact dampers have shown to reduce displacement amplitudes and are very effective in reducing the number of high amplitude vibrations as illustrated in Figure 8.1. Section 8.1.5.2 describes the mitigation options considered in this study.

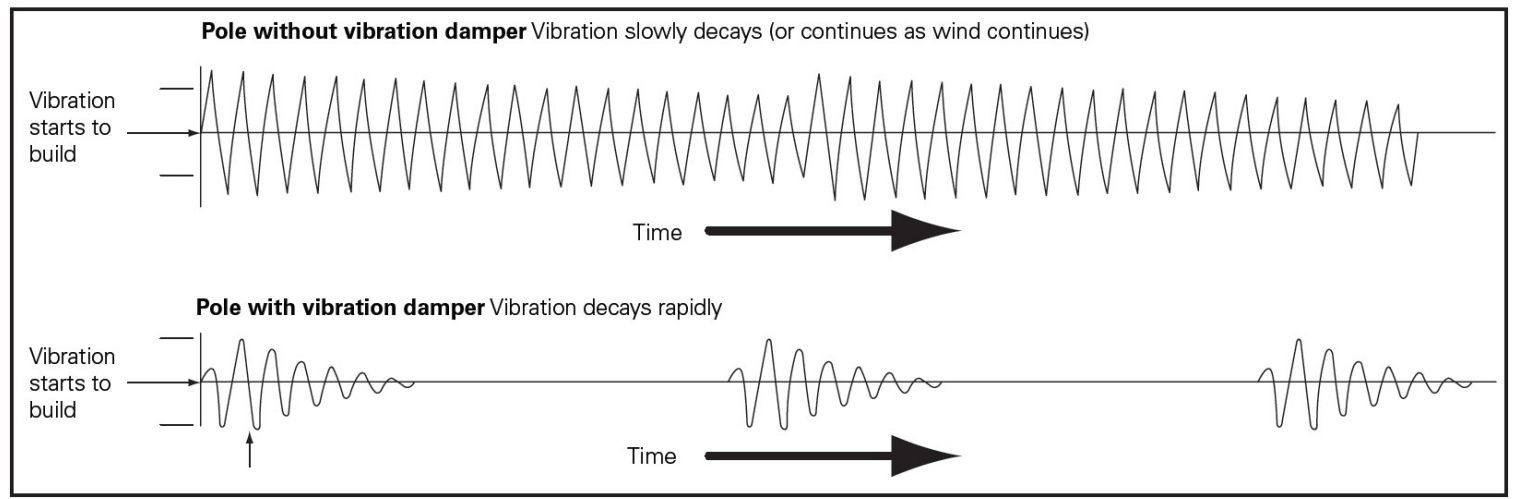

Figure 8.1: Effects of vibration dampers [63].

It should be noted that a previous study carried out in 2002 [62] of the Confederation Bridge light poles focused on the second mode vibrations. This study was mandated after repeated occurrences of premature light bulb and fixture failures (incandescent) were thought to be due to pole vibrations of the 2nd mode. The experimental results from the 2002 study [62] showed that the rubbing-pipe "chain" configuration is the most effective in increasing the damping ratio for the 2nd mode vibrations. Following this study, all light poles on the Confederation Bridge were outfitted with a rubbing pipe type chain damper as shown schematically in Figure 8.2, and since, the rate of light failures has drastically diminished.

\subsubsection{Analytical modelling}

An analytical frequency analysis was carried out to confirm the vibration characteristics of the original and the replacement light poles (each mounted with either the 


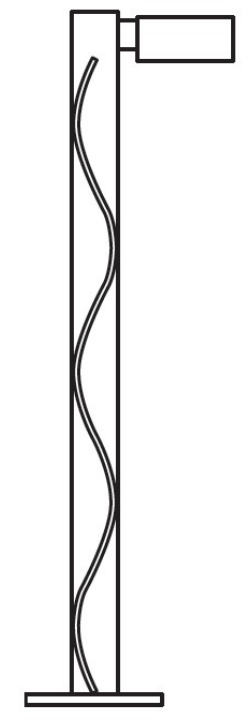

Figure 8.2: Rubbing pipe chain type damper [63].

original or the new LED fixtures) and to compare these values with the bridge superstructure's vibration behavior during strong wind events. Two structural component models of the light poles were created based on the manufacturer's shop drawings and on information provided by SCBL. The light poles are manufactured by NOVA POLE international Inc.

\subsubsection{Structural component models (SCM)}

The software program SAFI ${ }^{\circledR}$ was used to create the structural component models [66]. The light poles were modeled as a variable section cantilever beam with a fixed end support as shown in Figure 8.3a. The octagonal cross section was idealized with a circular cross section as indicated in Figure $8.3 \mathrm{~b}$ with a diameter equal to the across flats (A/F) dimension of the light poles as specified by the shop drawings. The 9.1 $\mathrm{m}$ length was subdivided into 200 elements of linearly varying diameters for added precision. Table 8.1 summarizes the input parameters used in the frequency analysis.

The principal difference between the new and the original light pole structures is 


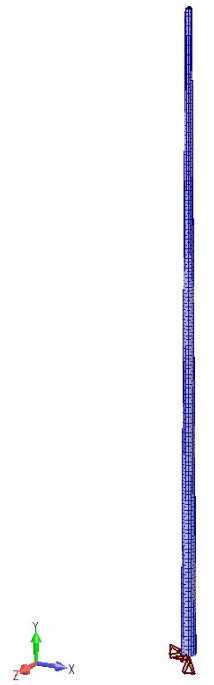

(a) 3D model of the light pole.

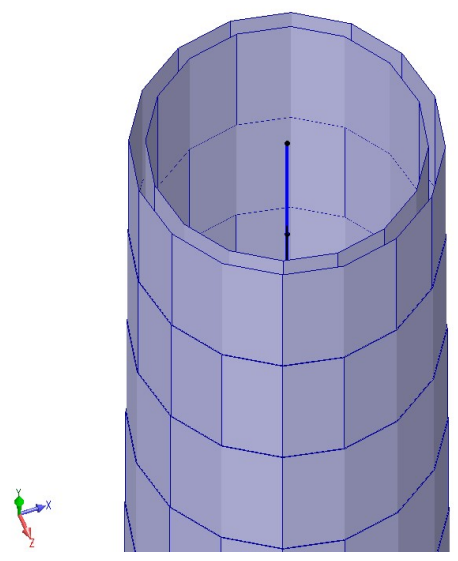

(b) Isometric view of the idealized cross section.

Figure 8.3: Structural component model of the Confederation Bridge light poles.

the wall thickness $(4.8 \mathrm{~mm}$ vs. $3.0 \mathrm{~mm})$. The light fixture is modeled as an eccentric load at the top of the light post. It should be noted that the tenon $(2.8 \mathrm{~kg})$, as detailed on the shop drawings at the top of the light poles, was considered in the analysis as being of same shape and dimension as the light pole to simplify the analysis. In

Table 8.1: Light pole structural properties considered for analytical modeling.

\begin{tabular}{lcc}
\hline & Original pole & Replacement pole \\
\hline Height (mm) & 9100 & 9100 \\
Wall thickness (mm) & 3.04 & 4.76 \\
A/F base (mm) & 203 & 200 \\
A/F top (mm) & 83 & 89 \\
Steel & $300 \mathrm{~W}$ & $300 \mathrm{~W}$ \\
Original HPS fixture weight $(\mathrm{kg})^{*}$ & 17.4 & $\mathrm{~N} / \mathrm{A}$ \\
Original HPS fixture c.g. (lever arm from & 520 & $\mathrm{~N} / \mathrm{A}$ \\
face of pole) (mm) & 13.23 & 13.23 \\
LED fixture weight $(\mathrm{kg})^{* *}$ & 290 & 290 \\
LED fixture c.g. $(\mathrm{mm})$ & \\
\hline
\end{tabular}

\footnotetext{
* The high pressure sodium light fixture was weighed and measured by SCBL.

** The LED light fixture was weighed and measured in the laboratory.
} 
December 2011, the light fixtures were changed from high pressure sodium (HPS) to LED style with a resulting reduction in weight and effective projected area (EPA). The original light fixtures were weighed by SCBL on-site in January $2017(17.4 \mathrm{~kg})$. Also of note are the weights of the original fixtures as indicated on the original shop drawings and on the original design calculations (as provided by SCBL). The original shop drawings indicate a fixture mass of $27 \mathrm{~kg}$ and the design calculations indicate a design weight of $30 \mathrm{~kg}$ ). However, both measured values of $17.4 \mathrm{~kg}$ and $13.23 \mathrm{~kg}$ were used for the analytical frequency analyses of the original light poles. The weight of the light fixture has a significant effect on vibration frequencies of the pole structure, and as such, is further investigated in Section 8.1.4.3.

\subsubsection{Finite element model (FEM)}

A three-dimensional model of the light pole was also created, and a finite element analysis was carried out using Solidwork ${ }^{\circledR}$ commercial software [67]. To ensure convergence of the results, the mesh control option was used. The runs were realized with increasingly fine mesh sizes until constant results were obtained (43064 solid mesh elements, 4 Jacobians points). The final mesh control size used was set at $30 \mathrm{~mm}$ for the pole and $10 \mathrm{~mm}$ for the light fixture $(60 \mathrm{~mm}$ diameter tubing, $3 \mathrm{~mm}$ thick, 331.5 $\mathrm{mm}$ in length from the center of the pole and weighs $1.25 \mathrm{~kg}$ ). A load of $11.98 \mathrm{~kg}$ was placed at the end of the light fixture tubing (center of gravity of the light) for a total mass of the light and light fixture of $13.23 \mathrm{~kg}$. An elastic support was used at the pole base in the vertical direction $(740 \mathrm{MN} / \mathrm{m})$ calibrated to match the experimental results obtained on site since perfect fixity at the pole base is not always achievable. In fact, when using a perfectly fixed base, the finite element analysis results in a first mode frequency of $2.04 \mathrm{~Hz}$ compared to $1.92 \mathrm{~Hz}$ obtained experimentally. This type of behaviour was also found in the laboratory setting when varying the base fixity of 
the pole to the strong floor. Modal results of the finite element analysis are presented in Table 8.2.

\subsubsection{Frequency analysis}

The SCM are used for the analytical analysis that determines modal frequencies and mode shapes (modal damping is assumed and therefore is not calculated in a frequency analysis). Modal frequencies obtained from the frequency analysis of the two light pole structures are compared with previous theoretical and experimental results obtained by Lau et al. [62].

Table 8.2 presents the first four modes obtained from the frequency analysis of the two light pole configurations detailed in Table 8.1. Also, included in Table 8.2 are the first four modes obtained from the theoretical finite element model (FEM) and the experimental analysis of the original light poles conducted by Lau et al. in $2002[62]$.

Table 8.2: Analytical modal frequencies $(\mathrm{Hz})$ for the first four vibration modes of the light poles.

\begin{tabular}{|c|c|c|c|c|c|c|}
\hline \multirow[t]{2}{*}{ Mode } & \multirow{2}{*}{$\begin{array}{c}\text { Mass } \\
\text { partic- } \\
\text { ipation } \\
(\%)\end{array}$} & \multicolumn{3}{|c|}{$\begin{array}{l}\text { Original light pole with original HPS light } \\
\text { fixture }\end{array}$} & \multirow{2}{*}{$\begin{array}{c}\text { Original } \\
\text { light pole } \\
\text { with LED } \\
\text { light fixture } \\
\text { Analytical }^{* *}\end{array}$} & \multirow{2}{*}{$\begin{array}{l}\text { Replacement } \\
\text { light pole } \\
\text { with new } \\
\text { LED light } \\
\text { fixture } \\
\\
\text { Analytical }\end{array}$} \\
\hline & & $\begin{array}{l}\text { Analytical }^{*} \\
{[62]}\end{array}$ & $\begin{array}{l}\text { Experimental } \\
{[62]}\end{array}$ & Analytical $^{* *}$ & & \\
\hline 1 & $48-56$ & 1.89 & 1.85 & 1.82 & 1.95 & 2.09 \\
\hline 2 & $19-20$ & 9.39 & 9.06 & 9.13 & 9.38 & 9.83 \\
\hline 3 & 8-9 & 24.94 & 25.18 & 24.88 & 25.18 & 25.92 \\
\hline 4 & $4-5$ & 46.36 & 48.45 & 49.35 & 49.67 & 50.63 \\
\hline
\end{tabular}

The results obtained from the frequency analysis of the structural component 
models described in Section 8.1.4.1 show a good correlation with the 2002 experimental study by Lau et al. [62]. These results confirm the validity of the structural component models. The new models are therefore validated and can be used for future dynamic studies and/or verifications. The results also indicate that the replacement light pole configuration has consistently higher frequencies as compared to the original light pole configuration. It is found that the difference in frequencies is mainly due to the reduced weight of the new LED light fixtures. Further analyses show that the vibration frequencies are very sensitive to the light fixture weight. A quick sensitivity analysis shows that increasing the weight of the components at the top of the light poles significantly reduces the fundamental frequency.

The results of the sensitivity analysis are detailed in Table 8.3. The results show the effect of varying the weight at the top of the light pole structures on modal frequencies. Results show a significant reduction of modal frequencies in the lower modes with increasing weight.

Table 8.3: Sensitivity of modal frequencies $(\mathrm{Hz})$ of light pole with varying light fixture weights.

\begin{tabular}{cccccccc}
\hline \multirow{2}{*}{ Mode } & \multicolumn{3}{c}{ Original light pole - SCM } & \multicolumn{3}{c}{ Replacement light pole - SCM } \\
& $0 \mathrm{~kg}$ & $11.4 \mathrm{~kg}$ & $17.4 \mathrm{~kg}$ & $34.8 \mathrm{~kg}$ & $0 \mathrm{~kg}$ & $11.4 \mathrm{~kg}$ & $22.8 \mathrm{~kg}$ \\
\hline \multirow{2}{*}{1} & 2.70 & 2.02 & 1.82 & 1.46 & 2.61 & 2.15 & 1.86 \\
2 & 11.96 & 9.52 & 9.13 & 8.62 & 11.86 & 9.99 & 9.29 \\
3 & 30.01 & 25.37 & 24.88 & 24.33 & 30.00 & 26.16 & 25.19 \\
4 & 56.67 & 49.86 & 49.35 & 48.79 & 56.83 & 50.92 & 49.83 \\
\hline
\end{tabular}

\subsubsection{Static analysis}

A static analysis of the analytical models shows that displacements under a pull force of $222 \mathrm{~N}(50 \mathrm{lb})$ for the original pole is $51 \mathrm{~mm}$ and for the replacement pole is 35 $\mathrm{mm}$. These values validate the experimental results described later in Section 8.1.8. 


\subsubsection{Superstructure frequency}

Table 8.4 represents a partial summary of an in-depth bridge vibration study [29]. The study investigated in detail the extracted modal frequencies of the Confederation Bridge. In this table, the vibration modes of the superstructure within the frequency range of interest corresponding to the fundamental frequency of the light pole structure as identified in Table 8.2 are shown. Figure 8.4 shows the only transverse mode in the frequency range of interest.

Table 8.4: Superstructure frequencies near light pole's fundamental frequencies [29].

\begin{tabular}{|c|c|c|c|c|c|c|c|c|c|c|}
\hline \multirow[b]{2}{*}{ 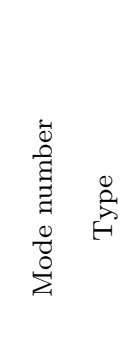 } & \multicolumn{6}{|c|}{ Experimental frequency } & \multicolumn{4}{|c|}{ Analytical frequency } \\
\hline & 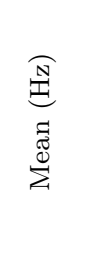 & $\begin{array}{l}\underset{\Theta}{\mathbb{B}} \\
\underset{\Sigma}{\Xi}\end{array}$ & 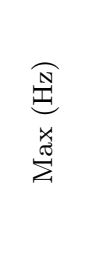 & 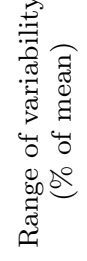 & 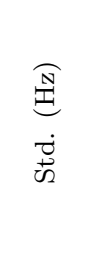 & 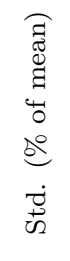 & 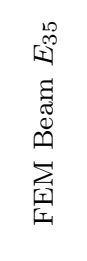 & 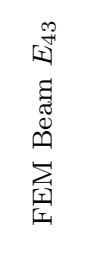 & 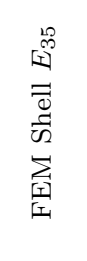 & 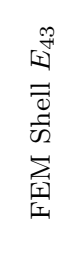 \\
\hline $11 \mathrm{~T}$ & 1.32 & 1.31 & 1.36 & 4 & 0.02 & 1.8 & 1.20 & 1.33 & 1.28 & 1.42 \\
\hline $12 \mathrm{~V}$ & 1.61 & 1.58 & 1.64 & 4 & 0.02 & 1.3 & 1.60 & 1.78 & 1.65 & 1.82 \\
\hline $13 \mathrm{~V}$ & 1.81 & 1.78 & 1.83 & 3 & 0.02 & 1.0 & 1.60 & 1.78 & 1.65 & 1.82 \\
\hline $14 \mathrm{~V}$ & 2.75 & 2.69 & 2.77 & 3 & 0.03 & 1.0 & 2.34 & 2.59 & 2.64 & 2.92 \\
\hline $15 \mathrm{~V}$ & 2.88 & 2.81 & 2.96 & 5 & 0.05 & 1.8 & 2.46 & 2.72 & 2.64 & 2.92 \\
\hline
\end{tabular}

\subsubsection{Experimental methodology}

To attain the objectives stated in Section 8.1.2, the full-scale testing of two installed light poles (an original light pole and a replacement light pole) was carried out on the deck of the Confederation Bridge. This section describes the site setup, the instrumentation and the manipulations. Details on the laboratory verifications of the equipment and manipulations are included in Section 8.1.6.2. 

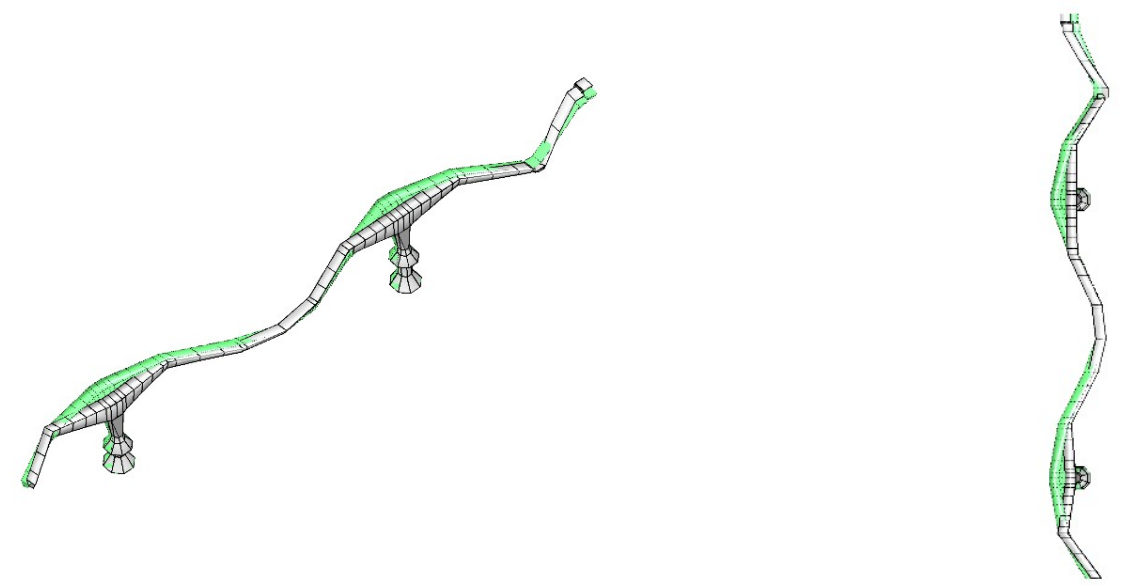

(a) Isometric view.

(b) Top view.

Figure 8.4: Mode-shape comparison of transverse $1.32 \mathrm{~Hz}$ mode. Grey model with full lines represents extracted mode-shapes while green model with dashed lines represents the mode-shape result from the analytical 3D beam model [29].

\subsubsection{Subject light poles}

Two adjacent light poles on the north side of the bridge deck near span 30 were identified for testing, one original light pole (N096) and one replacement light pole (N097) as shown in Figure 8.5.

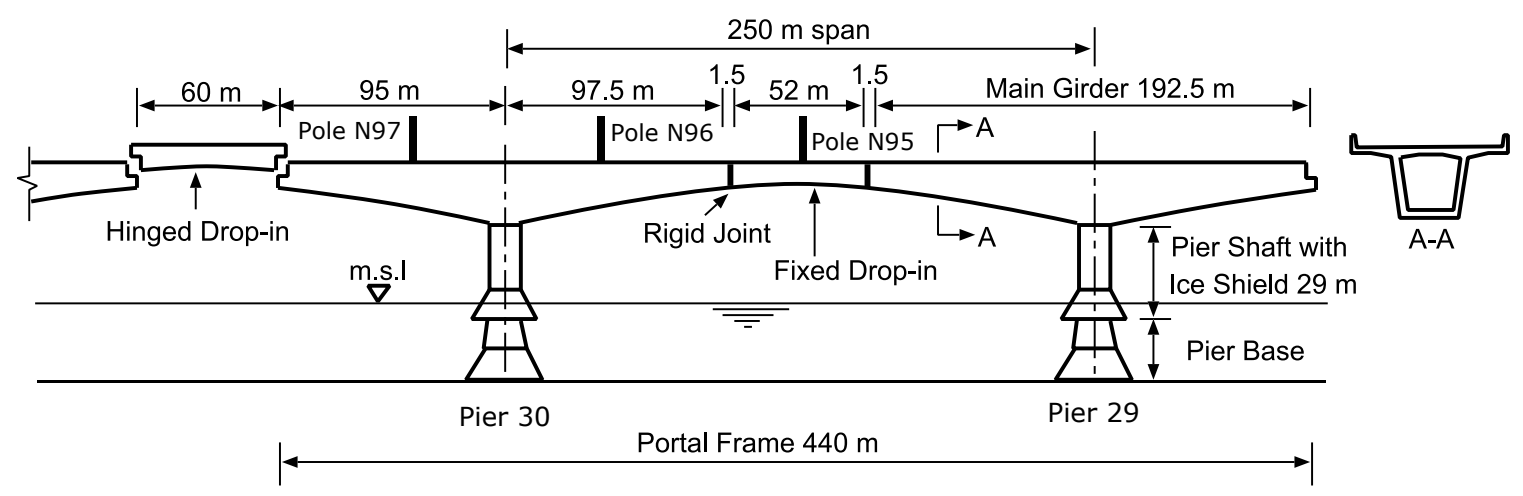

Figure 8.5: Approximate location of subject poles on the Confederation Bridge.

Each pole is outfitted with the LED light fixture which was weighed and measured in the laboratory. For this study, each light pole was instrumented with four tri-axial 
accelerometers as shown in Figure 8.6.

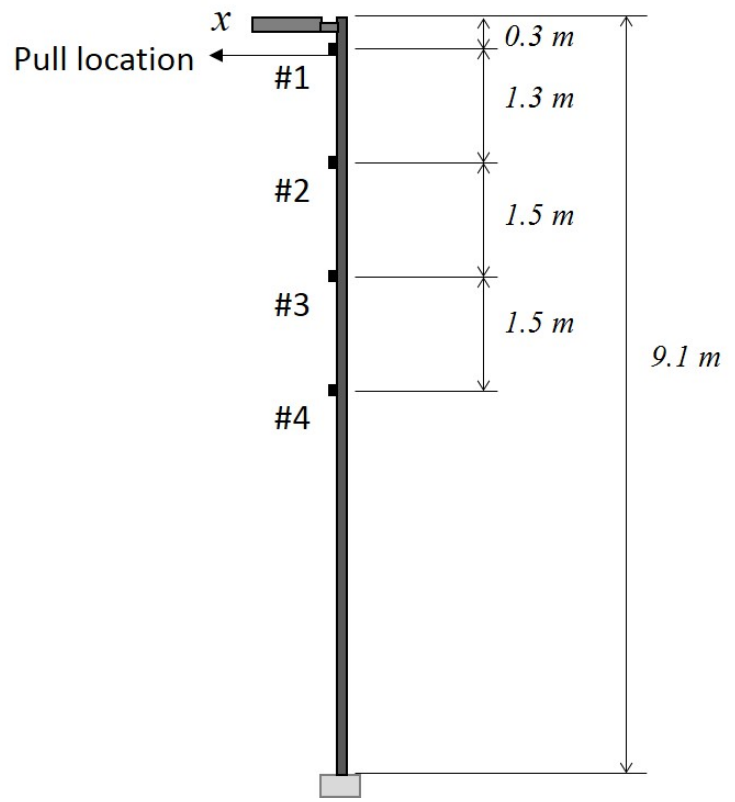

Figure 8.6: Accelerometer stations and pull location for on-site testing.

While the first mode is of primary interests, the accelerometers are positioned to properly capture the first four modes of vibration while avoiding modal node positions. Sensors are numbered 1 to 4 starting at the top. The measured $\mathrm{x}$-direction is in-line with the luminaire and follows the pull direction. The z-direction is horizontal and normal to the $\mathrm{x}$-direction, $\mathrm{y}$-direction is upwards.

\subsubsection{Vibration mitigation options}

The light poles were tested in nine different damping configurations summarized in Table 8.5. As noted, all light poles installed on the Confederation Bridge are currently outfitted with rubbing pipe type chain dampers as shown in Figure 8.2.

For configuration 1 this chain damper was removed, and the light poles were completely undamped. The remaining configurations include the chain damper; either by itself in configuration 2 , or in combination with a pole top impact damper in 
configurations 3 through 8 . Configuration 9 , consisting of a dead weight, was only tested in the lab to confirm the effect of the added weight of the impact dampers without the beneficial effects of the energy dissipating "impacts".

A custom pole top impact damper was developed and fabricated with the help and input of the bridge's operations and maintenance personnel. Five iterations of the prototype were fabricated and tested. The design parameters considered for the development of the new impact damper include cost, ease of fabrication, ease of installation, durability and performance. To evaluate the performance, the various iterations of the prototype contained variations on the physical parameters such as the number of weighted balls, the ball travel distance, the overall weight and the inclusion of obstructions. Obstructions are included in the final prototype to prevent "lock-in" of the weighted balls. "Lock-in" describes the behavior where the ball travels in a circular motion around the inside of the circular pole or cap instead of dissipating energy by impacting the sides of the cylinder. The included obstructions force the ball out of its circular path and increase the number of energy dissipating impacts which improves performance.

The performance was evaluated through free vibration testing as described in Section 8.1.6. The prototype of configuration 8 shown in Figure 8.7f represents the final iteration considered. It utilizes readily available stock materials, can be easily fabricated by the bridge crew, fits snugly on the pole tops for quick installation and is sealed to prevent water infiltration. The free vibration displacement time histories of the top sensors in the direction of the pull force for each damping configuration of Table 8.5 are shown in Figures 8.13, 8.14 and 8.15. 
Table 8.5: Damping configurations for site testing.

\begin{tabular}{|c|c|c|}
\hline Configuration & Description & Illustration \\
\hline 1 & $\begin{array}{l}\text { Undamped: In this configuration, the light poles are completely } \\
\text { undamped. The previously installed chain dampers are removed. }\end{array}$ & - \\
\hline 2 & $\begin{array}{l}\text { Chain only: In this configuration, a rubbing pipe type damper com- } \\
\text { posed of a chain inside a flexible PVC pipe is installed inside the } \\
\text { pole. Since 2002, all the light poles on the bridge are outfitted with } \\
\text { this damper following the recommendations of an internal technical } \\
\text { report [62]. }\end{array}$ & Figure 8.2 \\
\hline 3 & $\begin{array}{l}\text { Chain with commercial damper: In addition to the chain damper } \\
\text { of configuration } 2 \text {, the light pole is outfitted with a commercial pole } \\
\text { top impact damper from Hapco Inc. }\end{array}$ & Figure 8.7a \\
\hline 4 & $\begin{array}{l}\text { Chain with prototype damper } \# 1-\text { ball in pole top: In ad- } \\
\text { dition to the chain damper of configuration } 2 \text {, the light pole is out- } \\
\text { fitted with a custom pole-top impact damper prototype consisting of } \\
\text { a weighted ball installed inside the pole on a fixed plate. A cap is } \\
\text { added for protection. }\end{array}$ & Figure $8.7 \mathrm{~b}$ \\
\hline 5 & $\begin{array}{l}\text { Chain with prototype damper } \# 2-\text { ball on top of pole: In } \\
\text { this configuration, the custom pole top impact damper consists of a } \\
\text { weighted ball sitting on top of the pipe on an installed plate. The } \\
\text { protective cap with a larger inside diameter increases the ball travel. }\end{array}$ & Figure $8.7 \mathrm{c}$ \\
\hline 6 & $\begin{array}{l}\text { Chain with prototype damper \#3 - two balls (inside and } \\
\text { on top of pole): The pole top impact damper in this configuration } \\
\text { consists of two weighted balls, one inside the pole diameter, and one on } \\
\text { top of the pole. The chain damper of configuration } 2 \text { is also installed } \\
\text { in this configuration. }\end{array}$ & Figure $8.7 \mathrm{~d}$ \\
\hline 7 & $\begin{array}{l}\text { Chain with prototype damper \#4-two balls (on top of pole): } \\
\text { This first mode impact damper prototype encapsulates two weighted } \\
\text { balls separated by plates welded on the inside of a schedule pipe. The } \\
\text { damper slides on the top of pole for a quick field installation. The } \\
\text { chain damper of configuration } 2 \text { is also present in this configuration. }\end{array}$ & Figure $8.7 \mathrm{e}$ \\
\hline 8 & $\begin{array}{l}\text { Chain with prototype damper } \# 5 \text { - two balls on top with ob- } \\
\text { structions: This configuration corresponds to the pole top damper } \\
\text { described in configuration } 7 \text { except that obstructions are added in the } \\
\text { form of welded rebar to prevent the weighted ball from traveling in } \\
\text { a circular motion around the pipe diameter which is thought to limit } \\
\text { the damping effects. This configuration is added since the results } \\
\text { from a presumed failed test (where a screw was protruding inside the } \\
\text { pole diameter, limiting the ball travel) showed good damping results. }\end{array}$ & Figure $8.7 \mathrm{f}$ \\
\hline 9 & $\begin{array}{l}\text { Dead weight }(4.7 \mathrm{~kg}) \text { : In this configuration, a dead weight of } 4.7 \mathrm{~kg} \\
\text { (corresponding to the weight of the pole top damper of configuration } \\
\text { 8) is installed on top of the pole. This configuration was studied in } \\
\text { the laboratory setting only. }\end{array}$ & - \\
\hline
\end{tabular}




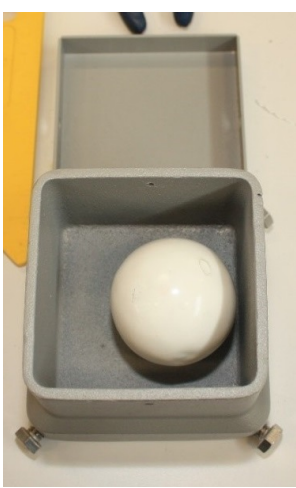

(a) Commercial first mode impact damper.
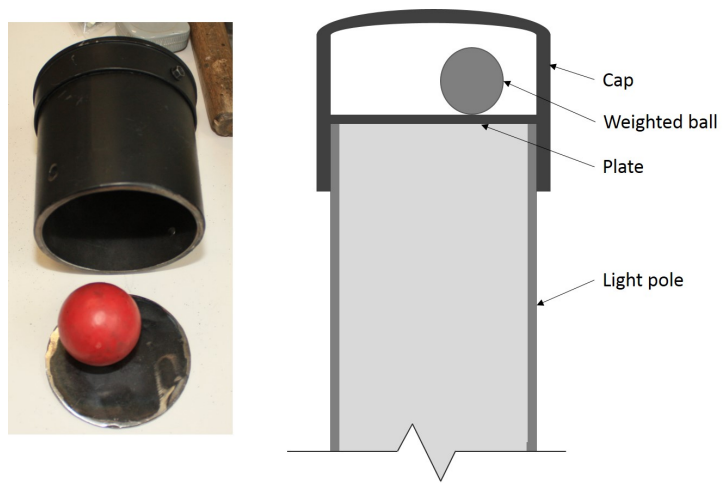

(c) Prototype of first mode impact damper - Ball on top of pole.
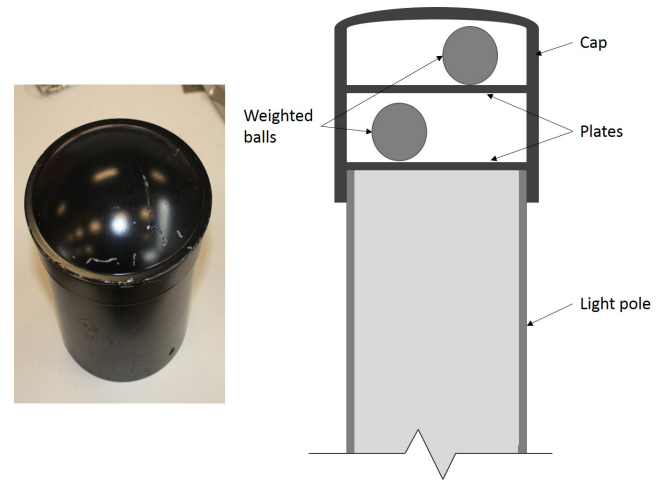

(e) Prototype of first mode impact damper - Two balls on top of pole.
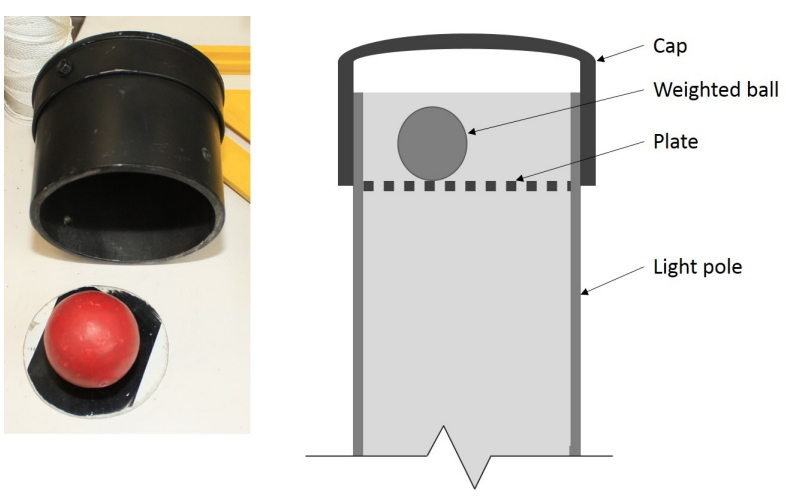

(b) Prototype of first mode impact damper - Ball in pole top.

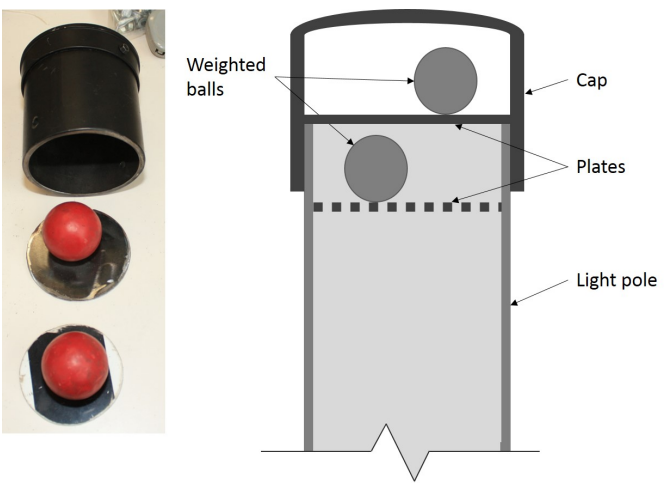

(d) Prototype of first mode impact damper - One ball inside and one ball on top of pole.
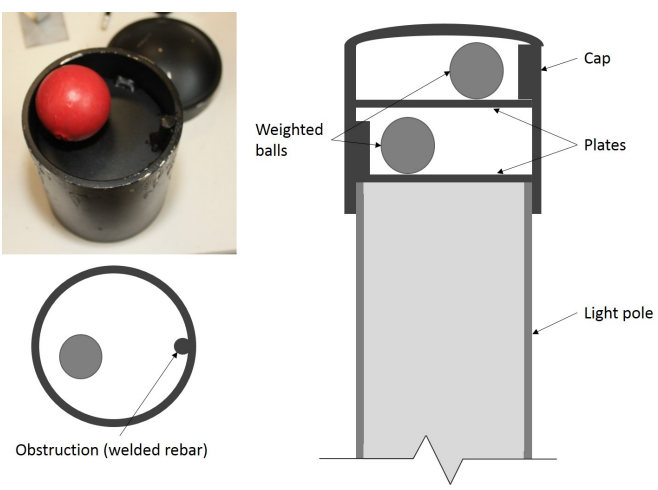

(f) Prototype of first mode damper Two balls on top of pole with obstructions.

Figure 8.7: Pole top impact dampers installed during testing. 


\subsubsection{Instrumentation and data acquisition}

The installed sensors were GLINK2-LXRS wireless tri-axial MEMS accelerometers with \pm 2 g measurement range and 16-bit resolution by LORD Microstrain (Figure 8.8 and Appendix C). This wireless setup permitted quick and efficient installation of the sensors on the light poles on site and in the lab. Custom removable mounting brackets were created to facilitate the installation of the accelerometers to the light poles using band clamps as shown in Figure 8.8.

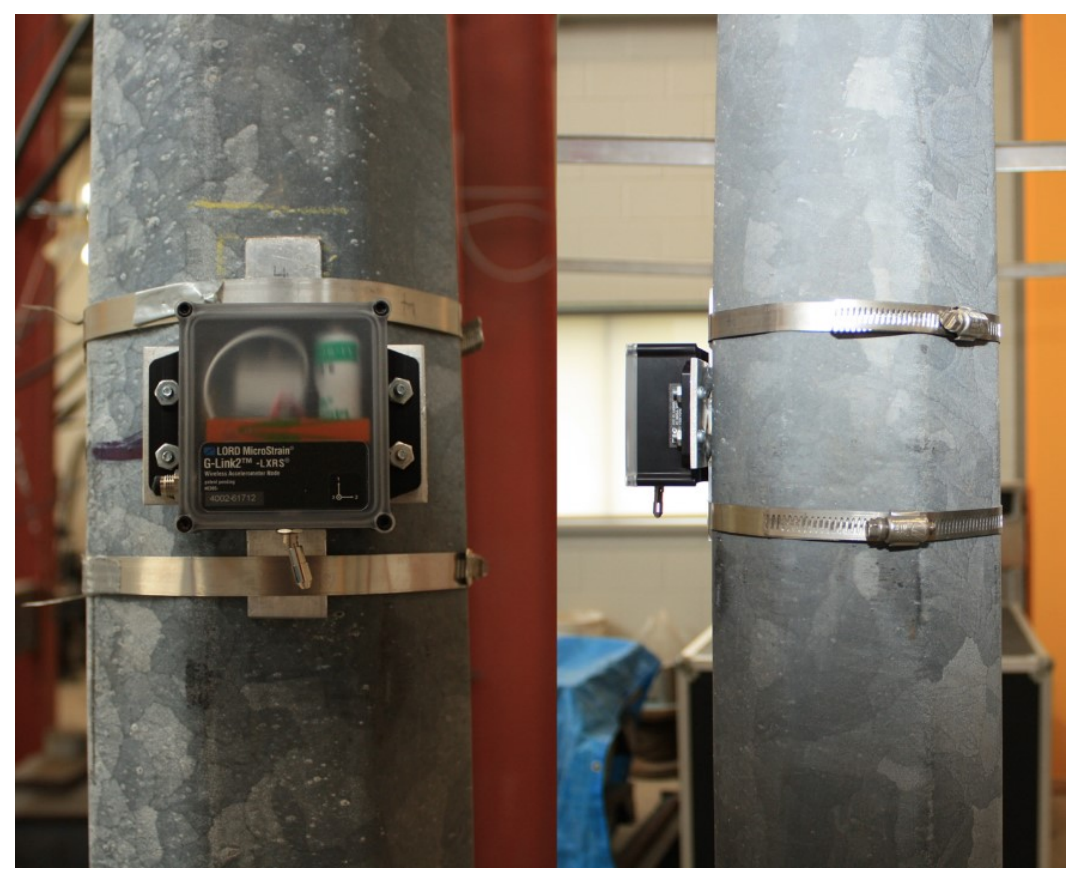

Figure 8.8: Installed wireless sensors. GLink2-LXRS tri-axial MEMS accelerometer.

The data acquisition was controlled by the specialized software, Node Commander, by LORD Microstrain. Data capture is synchronous across sensors at a sampling rate of $128 \mathrm{~Hz}$. Data were captured in datasets of 60 second duration and stored in a comma separated ASCII file for further data processing and analysis. 


\subsubsection{Manipulations}

Since first mode vibrations are of primary importance, the light poles are subjected to free vibrations via a pull and release load applied at the top of the pole. See Figure 8.6 for the pull location. The pull and release is carried out manually with a force gage and a pull string. At the proper force level of $222 \mathrm{~N}$ (50 lb), the string is cut and the pole is permitted to oscillate freely until its return to rest.

\subsubsection{Field setup}

Most tests were completed on April 20th, 2017. Site conditions on this day was cloudy with low wind (10 to $20 \mathrm{~km} / \mathrm{h}$ ), with temperatures near freezing $\left(-1\right.$ to $\left.2{ }^{\circ} \mathrm{C}\right)$. Figure 8.9 shows the site conditions and the setup on pole N096 (original light pole).

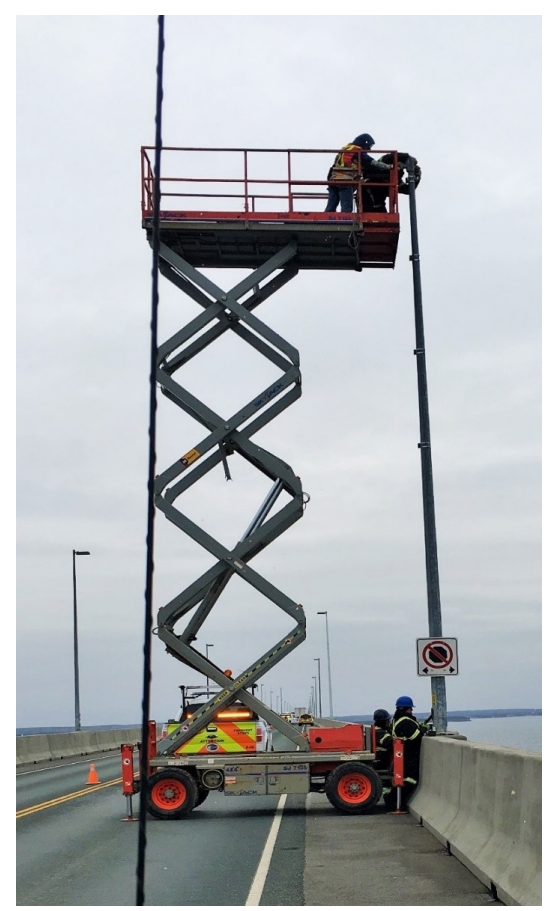

Figure 8.9: On-site setup for vibration testing.

Replacement pole N097 can be seen in the background (on the same side of the bridge deck). Also in Figure 8.9, we can see the installation of the chain damper 
through the hand hole at the bottom of the pole and the installation of a pole top impact damper.

With one exception, the first eight configurations identified in Table 8.5 were tested on-site on both poles, the original light pole (N096) and the replacement light pole (N097). The single exception was due to an installation problem on-site where the clearance between plates of the sixth configuration did not permit the free travel of the weighted ball inside the replacement light pole.

\subsubsection{Laboratory setup and verifications}

A truncated pole of $6.1 \mathrm{~m}$ in height was mounted in a laboratory environment for verification and calibration purposes. Figure 8.10 shows the test pole in the laboratory environment.

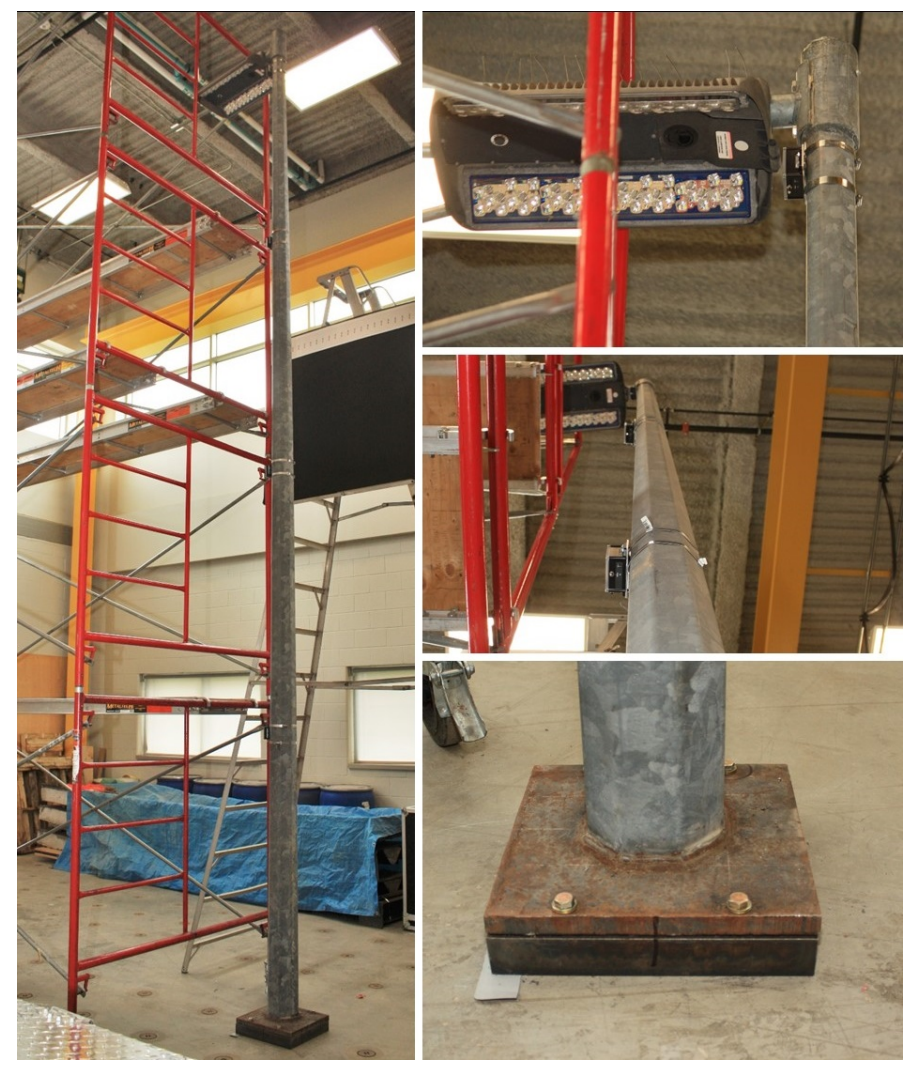

Figure 8.10: Laboratory setup for the testing of the light poles. 
The lab pole is fixed to the strong floor with a single $50 \mathrm{~mm}$ diameter anchor bolt threaded directly into a $50 \mathrm{~mm}$ thick anchor plate. The base plate welded to the bottom of the pole is bolted to the anchor plate with four $13 \mathrm{~mm}$ diameter grade 8 bolts.

The laboratory setup allows for proper verification of instrumentations and methodology prior to site testing. With this setup, every pole top damping configurations mentioned in Table 8.5 was also tested in the laboratory, with the exception that the rubbing pipe chain damper was not included in the lab configurations. Laboratory testing was carried out over four weeks from April 10th to May 4th 2017.

\subsubsection{Analysis}

This section describes the data processing and the analysis operations carried out on the raw data.

\subsubsection{Data processing}

Data processing techniques include removal of duplicate records (if present), detrending by mean removal, scaling of data, double integration of acceleration data with filtering to obtain displacement data. The filters are high-pass sixth order Chebyshev type 2 filter $(0.1 \mathrm{~Hz}$ cut-off for filtering of acceleration data and $0.5 \mathrm{~Hz}$ cut-off for filtering of velocity and displacement data).

\subsubsection{Free vibration - log decrement}

Since it is not possible to determine the damping ratio analytically, this elusive property is typically determined experimentally by testing on practical structures. Free vibration tests provide an efficient means with which to determine damping. Figure 8.11 shows the free vibration displacement time history of a lightly damped structure. 


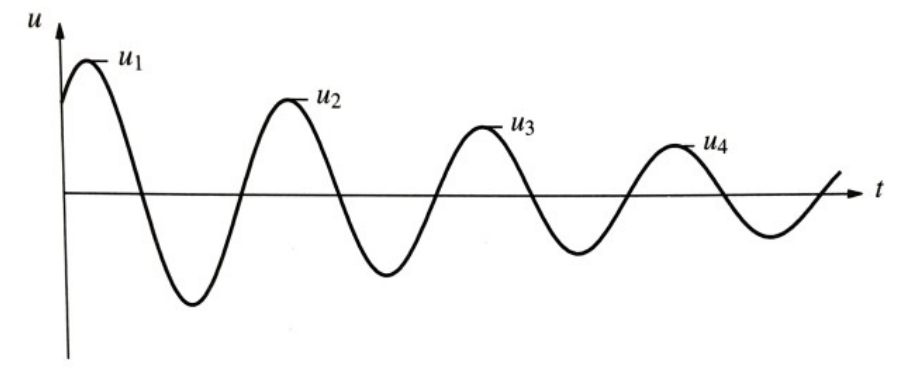

Figure 8.11: Free vibration of a lightly damped system.

The natural logarithmic of the ratio of two peaks $\left(u_{i}\right.$ and $\left.u_{i+j}\right)$ of a damped free vibration system is called the logarithmic decrement, $\delta$. For lightly damped systems $(\zeta<20 \%)$, we can state: $\delta=2 \pi \zeta$. Therefore, the damping ratio, $\zeta$, can be determined from Equation (8.1):

$$
\zeta=\frac{1}{2 \pi j} \ln \frac{u_{i}}{u_{i+j}}
$$

where $u_{i}$ is the displacement amplitude of the $i^{\text {th }}$ peak. All data operations were carried out by a custom script written in Matlab using the signal processing toolboxes.

\subsubsection{Free vibration - frequency domain}

Frequency estimates of the first four modes of vibrations were obtained by peak picking in the frequency domain. The power spectral density (PSD) function represents the power content of a signal in an infinitesimal frequency band. An improved estimator of the PSD by Welch is employed in the analysis of the light pole acceleration data [68]. The method consists of dividing the time series data into windowed overlapping segments, computing a modified periodogram of each segment, and then averaging the PSD estimates. These segments are subjected to a windowing operation using a Hamming window to prevent leakage in the Fast Fourier Transform (FFT) from time domain to frequency domain of the data. An $n$-point FFT is applied to 
the windowed data where $n$ is approximately equal to the number of samples in the segment (8192 in this case). The periodogram of each windowed segment is then computed to obtain the segment's power spectrum. The set of periodograms is averaged to form the spectrum of all segments which is then scaled with the sampling frequency to compute the power spectral density. Results of the free-vibration frequency domain analysis for the undamped pole are shown in Table 8.6 and in Table 8.7.

\subsubsection{Stochastic subspace identification (SSI) - SPLASH}

Among the different system identification techniques proposed for civil engineering monitoring applications, the stochastic subspace identification (SSI) method has been found to be a reliable output-only identification technique which compares favourably to other available methodologies [21]. The technique is derived from the work of B. Peeters [20] and is the preferred operational modal analysis method for the Confederation Bridge superstructure $[22,47,49]$.

As with all the system identification methods, a model of the underlying system is needed, in the SSI method a stochastic state-space model is used. Since it is generally impossible to measure the input forces of in-operation structure, these terms are modeled as stochastic white noise. The SSI method uses data correlations which compresses the data while still preserving vibration information. The data correlations also eliminate the uncorrelated noise and can be factorized into state space matrices. Taking advantage of these correlations, data at different time lags are assembled into a matrix, that is then decomposed into factors using single value decomposition. From these factors, the state space matrices can be extracted and the modal parameters such as modal frequency, modal damping and mode shapes can be directly identified. For a detailed description of the method, the reader is directed to Chapter 2

The Signal processing PLatform for Analysis of Structural Health (SPLASH) is 
a sophisticated computer platform for rapid and efficient vibration monitoring data management, signal processing, and system identification operations. The initial development of SPLASH is presented in the references [24,47]. SPLASH has been in continued use since its initial development in 2003 for the Confederation Bridge vibration monitoring program. The platform underwent a significant update (version 3.0), as described in Chapter 5, and among other notable improvements, it now has the capability to monitor any type of structure. The light pole vibration data of the current study is therefore processed, analysed and visualized using the SPLASH platform to obtain the dynamic properties of the first four modes and to confirm the first mode results obtained from the log decrement method.

\subsubsection{Results and interpretation}

Aligning with the objectives of the study, this section shows the results of the modal analysis (Section 8.1.8.1) and the results of the damping tests (Section 8.1.8.2).

\subsubsection{Modal properties of installed light poles}

The modal properties of the undamped light pole were determined analytically and experimentally. The results are compared in Tables 8.6 and 8.7. Table 8.6 shows the modal frequencies of the original light pole structure while Table 8.7 shows the modal frequencies of the replacement light pole structure; both poles being completely undamped.

The analytical results were obtained from the structural component models and the finite element models described in Section 8.1.4.1 and Section 8.1.4.2 respectively. The experimental determination of the modal properties was carried out by picking peaks from the free vibration signals (in the time domain and in the frequency domain) and by the stochastic subspace identification method. While only data from sensor 
Table 8.6: Modal frequencies $(\mathrm{Hz})$ of the original undamped light pole structure with LED light fixture.

\begin{tabular}{|c|c|c|c|c|c|}
\hline \multirow{3}{*}{ Mode } & \multicolumn{2}{|c|}{ Analytical } & \multicolumn{3}{|c|}{ Experimental } \\
\hline & \multirow{2}{*}{$\begin{array}{c}\text { Structural } \\
\text { component } \\
\text { model (SCM) }\end{array}$} & \multirow{2}{*}{$\begin{array}{c}\text { Finite } \\
\text { element } \\
\text { model }(\mathrm{FEM})\end{array}$} & \multicolumn{2}{|c|}{ Free vibration analysis } & \multirow{2}{*}{$\begin{array}{c}\text { Stochastic } \\
\text { subspace } \\
\text { identification } \\
\text { (SSI) }\end{array}$} \\
\hline & & & Time domain & $\begin{array}{c}\text { Frequency } \\
\text { domain }\end{array}$ & \\
\hline 1 & 1.95 & 1.92 & 1.92 & 1.92 & 1.92 \\
\hline 2 & 9.38 & 9.13 & - & 9.27 & 9.27 \\
\hline 3 & 25.2 & 24.0 & - & 23.6 & 23.7 \\
\hline 4 & 49.7 & 42.6 & - & 51.9 & 51.9 \\
\hline
\end{tabular}

no. 1 (in the $\mathrm{x}$-direction) were used for the free vibration analysis, data from four sensors (12 channels) were used for the stochastic subspace identification which allows proper identification of the first 4 modes as shown in Tables 8.6 and 8.7.

Experimental results correlate well with the analytical results, especially for the lower modes with an average relative error of $0.8 \%$ and $1.4 \%$ for mode 1 and 2 respectively. Although the manual pull and release method strongly excites the first mode of vibration, the SSI technique identifies the higher vibration modes, albeit with greater uncertainty. While the frequency domain analysis provides modal frequencies,

Table 8.7: Modal frequencies $(\mathrm{Hz})$ of the replacement undamped light pole structure with LED light fixture.

\begin{tabular}{|c|c|c|c|c|c|}
\hline \multirow{3}{*}{ Mode } & \multicolumn{2}{|c|}{ Analytical } & \multicolumn{3}{|c|}{ Experimental } \\
\hline & \multirow{2}{*}{$\begin{array}{c}\text { Structural } \\
\text { component } \\
\text { model }(\mathrm{SCM})\end{array}$} & \multirow{2}{*}{$\begin{array}{c}\text { Finite } \\
\text { element } \\
\text { model }(\mathrm{FEM})\end{array}$} & \multicolumn{2}{|c|}{ Free vibration analysis } & \multirow{2}{*}{$\begin{array}{c}\text { Stochastic } \\
\text { subspace } \\
\text { identification } \\
\text { (SSI) }\end{array}$} \\
\hline & & & Time domain & $\begin{array}{c}\text { Frequency } \\
\text { domain }\end{array}$ & \\
\hline 1 & 2.09 & & 2.12 & 2.13 & 2.13 \\
\hline 2 & 9.83 & & - & 9.89 & 9.90 \\
\hline 3 & 25.9 & & - & 25.5 & 25.5 \\
\hline 4 & 50.6 & & - & 56.8 & 56.8 \\
\hline
\end{tabular}


the SSI technique identifies all modal properties; including frequency, damping and mode shapes.

Figure 8.12 shows a qualitative comparison between the analytical mode shapes from the frequency analysis of the structural component model and the experimental mode shapes extracted using the SPLASH application with its integrated SSI module.

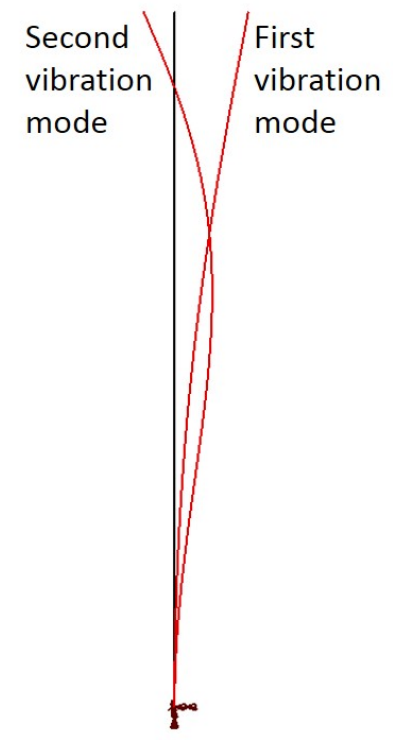

a) Analytical mode shapes - SCM

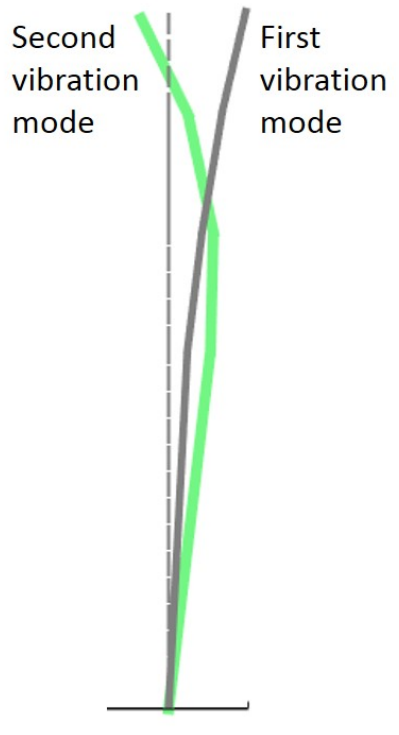

b) Experimental mode shapes - SSI

Figure 8.12: Analytical and experimental mode shapes of the original light pole.

\subsubsection{Damper comparison}

The displacement time histories for each damping configuration of the poles tested on-site are shown in Figures 8.13 and 8.14 and the free-vibrations of the pole tested in the laboratory are shown in Figure 8.15. The displacement time histories are from sensor no. 1 (at the top of the pole) in the x-direction (parallel to pull force).

Qualitatively, from Figures 8.13, 8.14 and 8.15, we can clearly see the effects of the installed dampers on the duration of vibration. While the reduction of maximum 

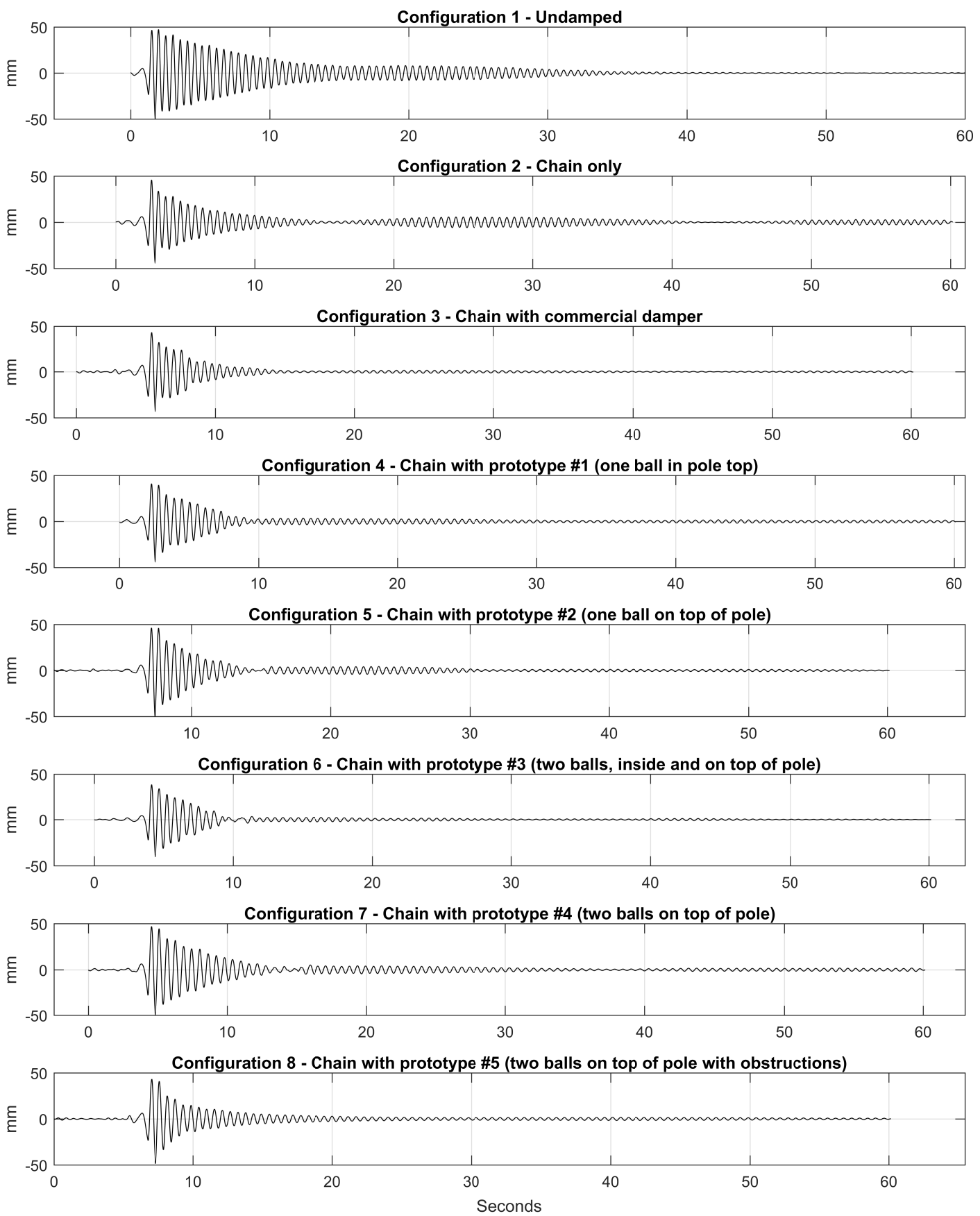

Figure 8.13: Free vibration displacement time histories for the damping configurations of the original pole. 

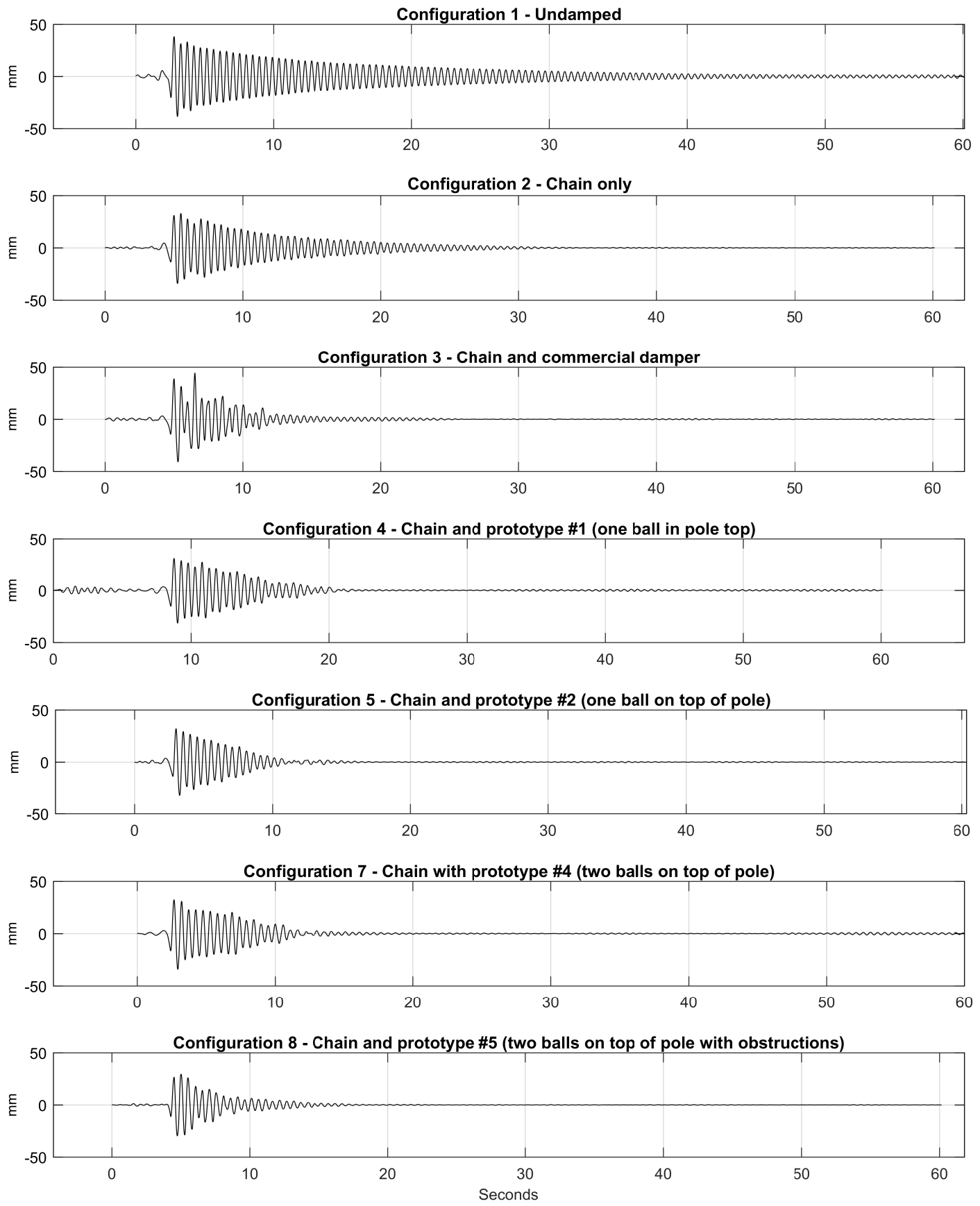

Figure 8.14: Free vibration displacement time histories for the damping configurations of the replacement pole. 

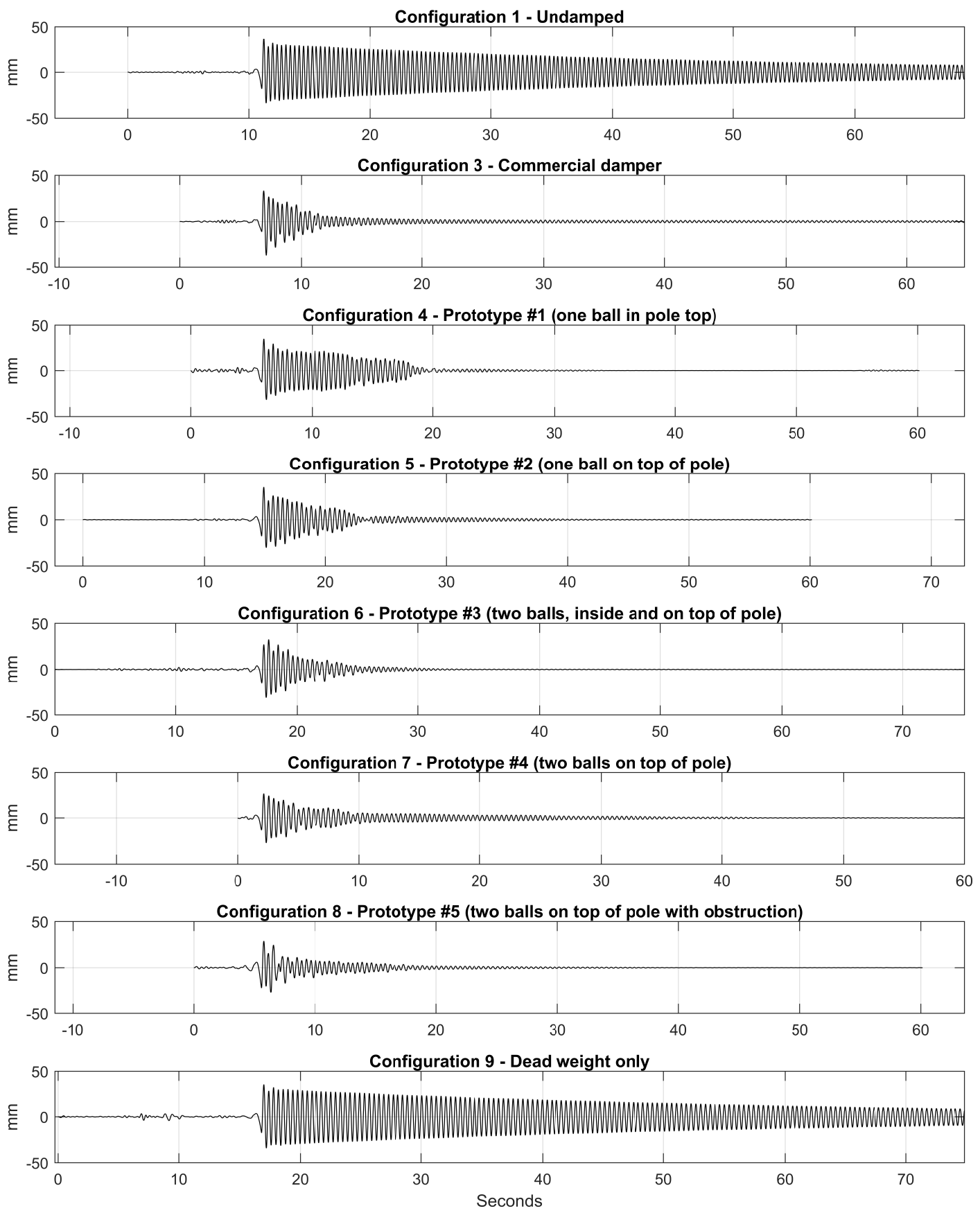

Figure 8.15: Free vibration displacement time histories for the damping configurations of the laboratory pole (without chain damper). 
displacements amplitude is minimal in some cases, the number of high amplitude cycles is generally greatly reduced with respect to the undamped configuration meaning the light poles come to rest at a much faster rate with the added pole top dampers.

Table 8.8 quantitatively summarizes the damping results for all the configurations. Results are averaged for each pull load (three pull tests per load level). The damping results were obtained by the log decrement method described in Section 8.1.7.2. For this type of analysis, the displacements time histories of the top most sensors were considered (i.e., plots from Figures 8.13, 8.14 and 8.15). The maximum peak displacement from each signal is identified and all subsequent peaks until a decay of $75 \%$ are considered for the calculation of the damping ratio, $\zeta(\%)$. The number of oscillation cycles until $75 \%$ decay gives a good indication of the attenuating effects of various dampers.

Some observations on the results from Table 8.8:

- The damping ratio of the original pole is significantly higher than the replacement pole for the same undamped configurations. This may be due to a difference in pole base fixity, differing wind conditions affecting external damping, or other aspect associated with the structural configuration.

- The calculated maximum displacements for the original and replacement light poles in their undamped configuration ( $47.5 \mathrm{~mm}$ and $34.3 \mathrm{~mm}$ respectively) are very similar to the displacements from the static analysis of the analytical models (51.0 $\mathrm{mm}$ and $35.0 \mathrm{~mm}$ respectively). Section 8.1 .4 describes the analytical models.

- The dead weight configuration tested in the laboratory clearly shows that adding weight to the top of the pole (in this case a dead weight of $4.7 \mathrm{~kg}$ corresponding to the weight of the impact damper of configuration 8) does not significantly affect the damping properties (in fact, a very slight reduction is noted). 
Table 8.8: First mode properties (frequencies and damping ratios) of the tested damping configurations.

\begin{tabular}{|c|c|c|c|c|c|c|c|c|c|c|c|c|}
\hline \multirow[b]{2}{*}{ Damping config. } & \multicolumn{4}{|c|}{ Original light pole } & \multicolumn{4}{|c|}{ Replacement light pole } & \multicolumn{4}{|c|}{ Laboratory pole $^{*}$} \\
\hline & $\begin{array}{l}\text { Freq. } \\
(\mathrm{Hz})\end{array}$ & $\begin{array}{c}75 \% \\
\text { decay } \\
\text { cycles } \\
(\#)\end{array}$ & $\begin{array}{l}\text { Max. } \\
\text { disp. } \\
(\mathrm{mm})\end{array}$ & $\begin{array}{c}\text { Damp. } \\
\text { ratio } \\
(\zeta)\end{array}$ & $\begin{array}{l}\text { Freq. } \\
(\mathrm{Hz})\end{array}$ & $\begin{array}{c}75 \% \\
\text { decay } \\
\text { cycles } \\
(\#)\end{array}$ & $\begin{array}{l}\text { Max. } \\
\text { disp. } \\
\text { (mm) }\end{array}$ & $\begin{array}{c}\text { Damp. } \\
\text { ratio } \\
(\zeta)\end{array}$ & $\begin{array}{l}\text { Freq. } \\
(\mathrm{Hz})\end{array}$ & $\begin{array}{c}75 \% \\
\text { decay } \\
\text { cycles } \\
(\#)\end{array}$ & $\begin{array}{l}\text { Max. } \\
\text { disp. } \\
(\mathrm{mm})\end{array}$ & $\begin{array}{c}\text { Damp. } \\
\text { ratio } \\
(\zeta)\end{array}$ \\
\hline 1 - Undamped & 1.92 & 16 & 47.5 & $1.38 \%$ & 2.13 & 35 & 34.3 & $0.61 \%$ & 2.77 & 139 & 38.8 & $0.16 \%$ \\
\hline 2 - Chain only & 1.89 & 9 & 45.6 & $2.23 \%$ & 2.07 & 22 & 31.6 & $0.96 \%$ & \multicolumn{4}{|c|}{$\begin{array}{l}\text { Chain damper not available for } \\
\text { laboratory testing }\end{array}$} \\
\hline $\begin{array}{l}3 \text { - Chain with } \\
\text { comm. damper }\end{array}$ & 1.84 & 7 & 41.1 & $3.00 \%$ & 2.03 & 10 & 39.7 & $1.90 \%$ & 2.68 & 13 & 34.0 & $1.52 \%$ \\
\hline $\begin{array}{l}4-\text { Chain with } \\
\text { prototype \#1 }\end{array}$ & 1.83 & 8 & 44.2 & $2.59 \%$ & 1.97 & 13 & 30.0 & $1.53 \%$ & 2.59 & 33 & 33.2 & $0.64 \%$ \\
\hline $\begin{array}{l}5 \text { - Chain with } \\
\text { prototype } \# 2\end{array}$ & 1.82 & 7 & 45.4 & $2.82 \%$ & 1.99 & 14 & 30.5 & $1.57 \%$ & 2.56 & 20 & 34.7 & $0.97 \%$ \\
\hline $\begin{array}{l}6 \text { - Chain with } \\
\text { prototype \#3 }\end{array}$ & 1.79 & 7 & 36.7 & $2.63 \%$ & \multicolumn{4}{|c|}{$\begin{array}{l}\text { Installation problems due to } \\
\text { geometric constraints on-site }\end{array}$} & 2.52 & 13 & 32.6 & $1.39 \%$ \\
\hline $\begin{array}{l}7 \text { - Chain with } \\
\text { prototype \#4 }\end{array}$ & 1.72 & 9 & 43.0 & $2.20 \%$ & 1.93 & 13 & 32.1 & $1.68 \%$ & 2.47 & 20 & 29.1 & $1.20 \%$ \\
\hline $\begin{array}{l}8 \text { - Chain with } \\
\text { prototype } \# 5\end{array}$ & 1.77 & 7 & 42.5 & $3.01 \%$ & 1.97 & 6 & 33.7 & $3.21 \%$ & 2.59 & 13 & 34.4 & $1.72 \%$ \\
\hline $\begin{array}{l}9-\text { Dead weight } \\
\text { only }\end{array}$ & \multicolumn{8}{|c|}{ Dead weight configuration tested in laboratory setting only } & 2.52 & 155 & 34.4 & $0.14 \%$ \\
\hline
\end{tabular}

${ }^{*}$ Laboratory pole configurations do not include a chain damper. 
- The final prototype impact damper (prototype no. 5) using two lead balls within a pipe cylinder added to the top of the pole was found to offer comparable damping properties (damping ratio, decay cycles and maximum pole top displacements) to the commercial damper..

The SSI technique described in Section 2.1 was also used to extract the first mode properties from the measured data; modal damping ratios of $0.68 \%, 0.79 \%, 2.14 \%$ and $3.06 \%$ were obtained for configurations 1 to 4 respectively. Modal damping is an inherently highly uncertain physical phenomenon. Nevertheless, a relatively marked improvement of damping performance is noted between the undamped configuration and the configurations with a pole-top impact damper. These output only identification results, while not exactly corroborating the results from the free vibration analysis (more work is required here), do confirm the suitability of the pole top impact dampers to reduce the amplitude and the quantity of fatigue stress cycles under free vibration scenarios.

\subsubsection{Conclusion and recommendations}

The modal properties of the original and replacement light poles installed on the Confederation Bridge were successfully determined following on-site testing carried out in the months of April and August 2017. The extracted modal properties (frequencies and mode shapes) correlate well with the analytical models (within $0.8 \%$ and $1.4 \%$ for estimated frequencies of modes 1 and 2 respectively). This confirms the suitability of the experimental modal analysis techniques employed in this study. While picking peaks in the frequency domain properly identifies the modal frequencies of the first four vibration modes, the variant of the stochastic subspace identification (SSI) technique described in this study identifies all modal properties (frequencies, damping and mode shapes) for the same four modes of vibration. Furthermore, the SSI algorithm 
is an output only identification technique, meaning that the modal properties can be extracted regardless of the excitation mechanism (pull-release, impact, or ambient wind conditions).

In the second part of this study, four different light pole damping configurations were tested on-site. These configurations allowed the comparison of six different pole top impact dampers; a commercially available damper and five prototype dampers developed and fabricated in collaboration with the Confederation Bridge operations and maintenance personnel. The displacement time history plots (Figures 8.13, 8.14 and 8.15) show the effectiveness of the impact dampers in reducing the number of high amplitude cycles. After the pull and release, the poles come to rest at a much quicker rate in the configurations with an impact damper than in the other configurations. According to fatigue theory, this attenuating effect can increase the remaining service life of the light poles. To properly quantify the comparison between each damping configurations, a free-vibration analysis in the time-domain was carried out to quantify the damping properties of each pole configuration. Under the $222 \mathrm{~N}$ pull load, the fifth and final prototype using two lead balls within a pipe cylinder added to the top of the pole was found to offer comparable damping properties to the commercial damper. Also, the developed prototype has the following advantages: it can be efficiently fabricated from stock material, it can be installed rapidly by snuggly fitting on the top of the pole and it can be readily sealed to prevent water infiltration and therefore increase durability. It should be noted that if optimal damping performance is desired from a custom-designed pole top impact damper, further testing and development is required to determine the optimal properties of the physical parameters of the weighted balls (weight, size, quantity, coating, etc.) and the container (size, shape, travel distance, partial obstructions, etc.).

The following recommendation was therefore made to the bridge operator: 
- A pole top impact damper has the potential to reduce harmful sway (first mode vibration) in the steel roadway light poles on the Confederation Bridge. This may reduce stress in the poles and extend their useful life. A long-term monitoring program to evaluate the performance of the chosen damper is recommended. The selected pole-top impact damper (shown in configuration 8 of Table 8.8) should be installed on-site for a duration spanning several months (ideally in windy season) to determine the actual damping performance. It is believed that results from such a study would better indicate the suitability of the proposed solution in extending the service life of the installed light poles.

\subsection{Vibration Monitoring and Damper Perfor- mance of the Confederation Bridge Light Poles}

Following the recommendations of the previous study described in Section 8.1, all light poles located on the deck of the Confederation Bridge were outfitted with custom pole-top impact dampers. According the recommendations, an extended full-scale vibration monitoring program of two bridge light poles under actual wind loading conditions was carried out over a period of several months (from August 2017 to January 2018) to evaluate the in-operation performance of the custom prototype pole-top impact damper.

\subsubsection{Summary}

275 Datasets were collected by a custom wireless remote data acquisition system primarily during medium to strong wind events (12 events total). The performance of the custom prototype damper was evaluated based on several metrics, the most 
significant of which are "signal power" expressed as RMS and the cumulative fatigue damage (cFD) expressed in fractions of total fatigue life. Global reductions of $7 \%$ RMS and 13\% cFD were observed over all collected datasets which indicates a good overall performance of the pole-top impact damper.

A fatigue analysis method is also presented in this report as a proof of concept for calculating the cumulative fatigue damage $(\mathrm{cFD})$ from vibration data and for estimating the remaining fatigue service life of in-operation bridge light poles. It was found that the dampers installed in the fall of 2017 could increase the remaining fatigue service life of the light poles by approximately 2.5 years. And if the dampers had originally been installed with the light poles, the cumulative fatigue damage could have been reduced by an average of $25 \%$ with a projected service life increase of $48 \%$ (which represents an additional 12 years of service above the actual estimated life of 25 years).

The subject poles are located 83 meters apart on the north side of the Confederation Bridge deck. Despite their physical proximity, several factors can influence the vibration and damping behavior of individual poles, the most important of which may be inherent damping. In fact during the free vibration testing in august 2017, one pole was found to have a damping ratio $41 \%$ greater than the other pole. Results from the fatigue analysis seem to confirm this finding; one pole has relatively high fatigue damage ( $87 \%$ of total fatigue life) with good damping performance provided by the installed pole-top damper $(40 \% \mathrm{cFD}$ reduction achieved over the life of the hypothetical damped pole), while the other pole has relatively low fatigue damage ( $29 \%$ of total fatigue life) with negligible damping performance provided by the damper. From this very limited sample set of two light poles, it is forwarded (with cautious reservations) that the dampers seem to have a positive effect on poles that have relatively high fatigue damage and no effect (or very little negative effect) on poles that 
have relatively low fatigue damage.

\subsubsection{Introduction}

A pole top impact damper has the potential to reduce harmful sway (first mode vibration) in the steel roadway light poles on the Confederation Bridge. These devices are considered as means to reduce stress in the poles and extend their useful life. A long-term monitoring program to evaluate the performance of a pole-top impact damper in place on the Confederation Bridge was recommended in the previous study described in Section 8.1. It was recommended to install the selected prototype poletop impact damper on-site for a duration spanning several months (ideally in windy season) to determine the actual damping performance of the custom pole top impact damper. It is believed that results from this study will better indicate the suitability of the proposed solution in extending the service life of the installed light poles.

Therefore, based on the recommendations of the previous report and as mandated, the objectives of this second study are:

- The implementation of an extended full-scale vibration monitoring of two bridge light poles under actual wind loading conditions over a period of several months;

- The in-operation performance evaluation of a custom prototype pole top impact damper.

\subsubsection{Methodology}

To attain the stated objectives, the instrumentation of two original light poles (one outfitted with the pole-top impact damper and the second serving as control without a pole-top damper) was carried out on the deck of the Confederation Bridge. This section describes the physical parameters of the study: the light poles, the pole-top 
impact damper, the instrumentation and the wireless data acquisition system.

\subsubsection{Light poles}

Two original adjacent light poles, separated by 83 meters, on the north side of the bridge deck near pier 30 were identified for testing : light pole N95 and light pole N96 (see Figure 3.2 for pier location and Figure 8.5 for approximate pole locations). Table 8.1 shows the structural properties of the original poles. The current LED light fixture was weighed and measured in the laboratory. It should be noted that all current light poles installed on the Confederation Bridge are outfitted with a rubbing pipe type chain damper, following the recommendations of a 2002 study [62] to better control the second mode vibrations.

\subsubsection{Pole top impact damper}

The damper considered in this study is a pole-top impact damper, custom fabricated by the maintenance personnel of SCBL and tested under controlled conditions as detailed in Section 8.1. The prototype damper contains two weighted lead balls inside a schedule pipe, capped and placed on top of the light pole, see Figure 8.16.

As detailed in Section 8.1, obstructions are added inside the schedule pipe in the form of welded rebar to prevent the lock-in phenomena where the weighted balls travel in a circular motion around the pipe diameter which limits the damping effects.

\subsubsection{Instrumentation}

For this study, a total of five wireless tri-axial accelerometers were used. Light pole N96 was instrumented with three accelerometers and light pole N95 was instrumented with 2 accelerometers as illustrated in Figure 8.17. While the first mode vibration of the poles is of primary interests, the accelerometers are positioned to properly capture 

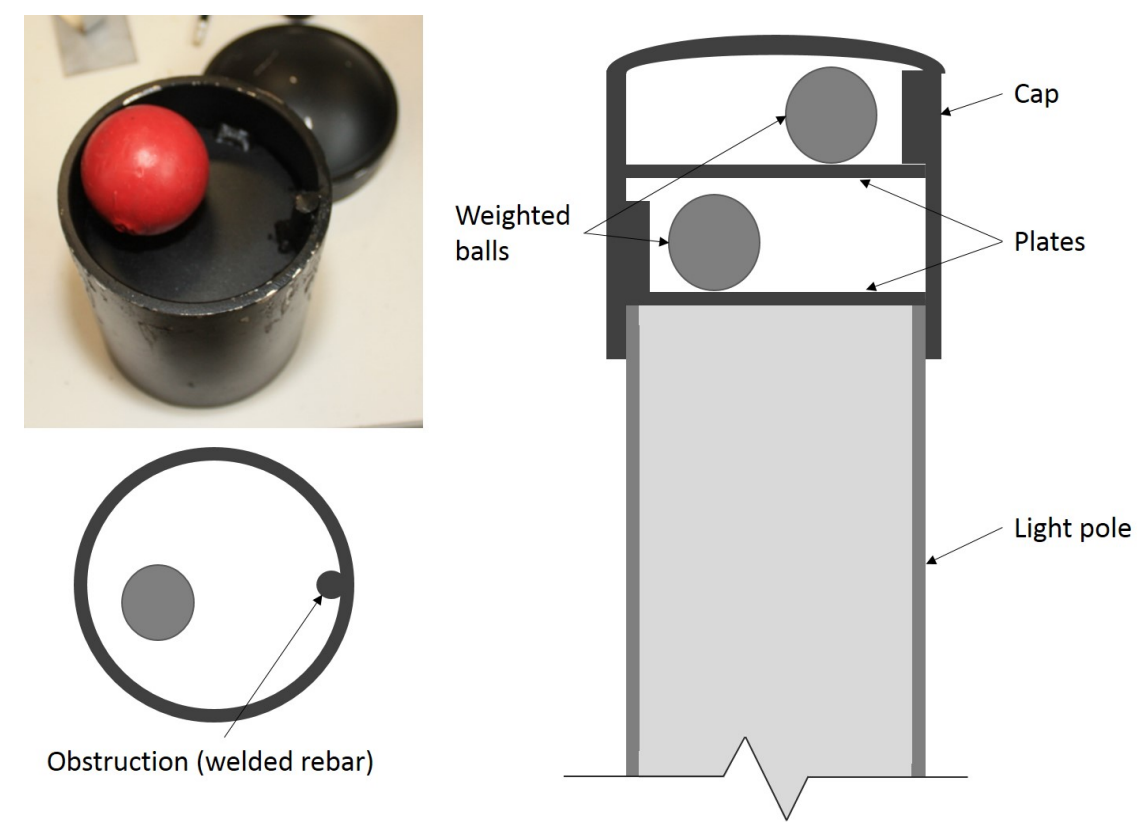

Figure 8.16: Prototype of first mode damper Two balls on top of pole with obstructions.

the first two or three modes of vibration while avoiding modal node positions. Sensors are numbered from the top. The measured $\mathrm{x}$-direction is in-line with the luminaire fixture. The z-direction is horizontal and normal to the $\mathrm{x}$-direction, $\mathrm{y}$-direction is upwards.

The installed sensors were GLINK2-LXRS wireless tri-axial MEMS accelerometers with \pm 2 g measurement range and 16-bit resolution by LORD Microstrain (Appendix C). This wireless setup permitted quick and efficient installation of the sensors on the light poles on-site. Custom removable mounting brackets were created to facilitate the installation of the accelerometers to the light poles using band clamps (Figure 8.8 shows an example of an installed sensor in a laboratory setting).

\subsubsection{Data acquisition}

A ruggedized PC loaded with the data acquisition software, Node Commander, by LORD Microstrain was temporarily installed at the base of light pole N95 on the 


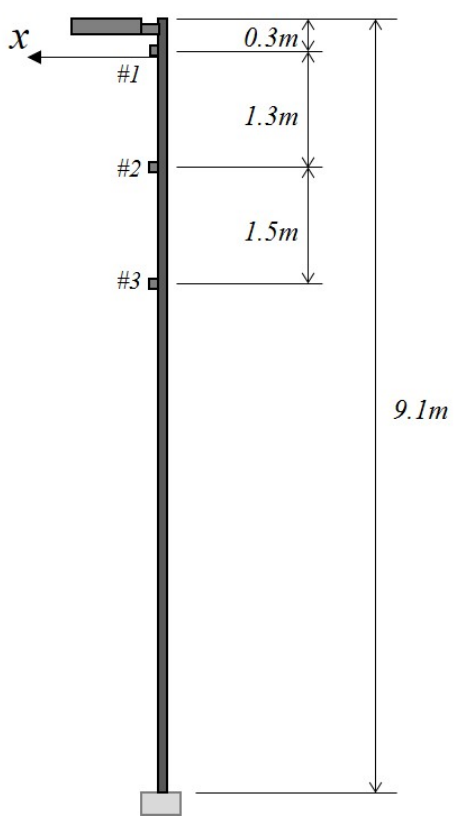

(a) Light pole N96.

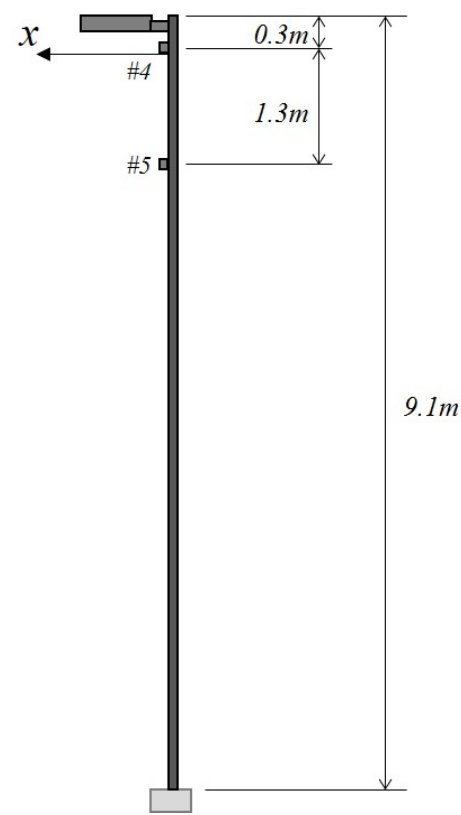

(b) Light pole N95.

Figure 8.17: Sensor locations numbered 1 to 5 .

bridge and powered directly from a callbox at this location. A custom insulated enclosure was fabricated to contain the PC, the WSBA base station by LORD Microstrain and a wireless communications modem. The remote operation of this PC was possible via a connection to the internet. The various data collection operations were carried out remotely from the research facility $100 \mathrm{~km}$ away and data sampling was triggered manually based on the local wind conditions. Data capture was synchronous across sensors at a sampling rate of $128 \mathrm{~Hz}$. Data were generally captured in datasets of 300 seconds duration and stored in a comma separated ASCII file for further data processing and analysis. During wind events the datasets were typically captured in burst sampling mode where, once triggered, datasets of 300 seconds were automatically collected at a preset interval of time (i.e. every 15, 20 or $30 \mathrm{~min}$, depending wind conditions and estimated duration of event). 


\subsubsection{Damping configurations}

The subject poles are located 83 meters apart on the north side of the bridge deck (Figure 8.5). Despite their physical proximity, several factors can influence the vibration and damping behavior of individual poles:

- Localized wind effects: Different wind profiles (turbulence, buffeting, etc.) may be present at each pole locations due to the difference in superstructure depth (see Figure 8.5).

- Superstructure vibrations: The amplitudes of superstructure vibration may differ between pole locations.

- Local inherent damping effects: Structural damping is a complex phenomenon. The inherent damping of poles may differ due to installed signs or call boxes on the poles or due to differences in base fixity (loose nuts, cracked grouting below base plate, etc.), or to differences in EPA of light fixtures, etc. In fact during the free vibration testing in august 2017, average damping ratios of $3.11 \%$ for pole N95 and 2.2\% for pole N96 were obtained. This implies that pole N95 has greater natural damping ( $41 \%$ greater damping than pole N96).

To observe the possible effects of these localized differences in vibration and damping behavior between poles, two measurement scenarios were considered in this study. In the first configuration, the pole-top impact damper was installed on pole N96, while pole N95 acted as the undamped control. In the second configuration the pole top impact damper was positioned on top of pole N95. The pole top damper was changed near the mid-point of data collection, on November 9th, 2017. With these configurations, the local effects on vibrations and damping performance can be observed. 


\subsubsection{Datasets and environmental conditions}

A total of 275 datasets were collected over a period of five months from August 29th, 2017 to January 30th, 2018. Most of the datasets were collected during moderate to strong wind events as shown in Table 8.9. A complete list of all collected datasets is available in Table D.1. As noted previously in Section 8.2.3.5, two damping configurations were considered. The damper switch occurred on November 9th, 2017.

Table 8.9: Collected datasets during wind events with corresponding environmental conditions.

\begin{tabular}{cccccccc}
\hline \multirow{2}{*}{$\begin{array}{c}\text { Damping } \\
\text { config. }\end{array}$} & $\begin{array}{c}\text { Wind } \\
\text { event } \\
\text { ID }\end{array}$ & Date & $\begin{array}{c}\text { Number } \\
\text { of } \\
\text { datasets } \\
\text { collected }\end{array}$ & $\begin{array}{c}\text { Maximum } \\
\text { sust. } \\
\text { wind } \\
(\mathrm{km} / \mathrm{h})\end{array}$ & $\begin{array}{c}\text { Maximum } \\
\text { wind } \\
\text { gust } \\
(\mathrm{km} / \mathrm{h})\end{array}$ & $\begin{array}{c}\text { Average wind } \\
\text { direction }\end{array}$ \\
\cline { 7 - 9 } & 1 & $2017-08-31$ & 36 & 49.0 & 58.6 & WSW & 231.0 \\
& 2 & $2017-09-02$ & 15 & 63.8 & 75.5 & NW & 301.2 \\
& 3 & $2017-10-16$ & 14 & 86.2 & 107.6 & WSW & 224.8 \\
No.1 & 4 & $2017-10-27$ & 19 & 91.2 & 102.0 & SSE & 148.8 \\
& 5 & $2017-10-30$ & 20 & 93.9 & 106.5 & SE & 120.1 \\
& 6 & $2017-11-04$ & 22 & 81.3 & 96.3 & NW & 297.7 \\
\hline \multirow{2}{*}{ No.2 } & 7 & $2017-11-10$ & 36 & 67.9 & 91.0 & WNW & 263.0 \\
& 8 & $2017-11-17$ & 40 & 88.5 & 104.4 & NW & 293.3 \\
& 9 & $2017-11-30$ & 18 & 58.5 & 70.9 & NNW & 311.7 \\
& 10 & $2017-12-13$ & 21 & 102.2 & 119.3 & E & 75.4 \\
& 11 & $2018-01-24$ & 13 & 74.3 & 78.0 & SW & 194.6 \\
& 12 & $2018-01-30$ & 6 & 70.8 & 83.6 & NE & 13.8 \\
\hline
\end{tabular}

*For configuration no.1 the pole top damper is positioned on pole N96. For configuration no.2 the pole top damper is on pole N95.

*Average wind direction in degrees with respect to bridge alignment.

The wind measurements were obtained from existing wind stations installed near pier 20 (see Figure 3.2 for pier numbering). The sustained winds are 10-minute mean, while gusts are based on peak measurement (one-second mean). Wind directions are averaged over the wind event and are noted in Table 8.9 in both cardinal direction 
and in degrees with respect to the bridge alignment which is on an approximate NNE alignment.

\subsubsection{Data analysis}

This section describes the data processing and the analysis operations carried out on the raw data. For this report, data in the x-direction (perpendicular to the bridge deck) and z-direction (parallel to the bridge deck) from the top sensors located on poles N95 (sensor no. 4) and N96 (sensor no. 1) were used for analysis. A resultant signal was also created from the $\mathrm{x}$ and $\mathrm{z}$-data as described in the following section.

\subsubsection{Data processing}

Data processing techniques include removal of duplicate records (if present), detrending by mean removal, scaling of data, double integration of acceleration data with filtering to obtain displacement data. The filters are high-pass sixth order Chebyshev type 2 filter $(0.1 \mathrm{~Hz}$ cut-off for filtering of acceleration data and $0.5 \mathrm{~Hz}$ cut-off for filtering of velocity and displacement data). The resultant data signals were obtained by transforming the corresponding elements of the two-dimensional Cartesian coordinate arrays $x$ and $z$ into polar coordinates $\theta$ and $\rho$; where $\rho$ is the amplitude (radius) in $\mathrm{mm}$, and $\theta$ is the corresponding angle in degrees.

\subsubsection{Time domain (RMS)}

A time history analysis was performed on the acceleration and displacement data to determine the relative power of the displacement signals as a first metric to evaluate damping performance. The root-mean-square (RMS) of a signal provides an indication of total power of the signal. The RMS of each displacement signal $(x, z$ and $\rho)$ 
was therefore calculated according to Equation (8.2) follows:

$$
x_{R M S}=\sqrt{\frac{1}{N} \sum_{n=1}^{N}\left|x_{n}\right|^{2}}
$$

where $x_{n}$ are displacement values and $N$ is the total number of displacement samples in the signal. Figure 8.18 shows a sample displacement time history with associated RMS value for a 300 seconds dataset. Section 8.2.6 summarizes the results.
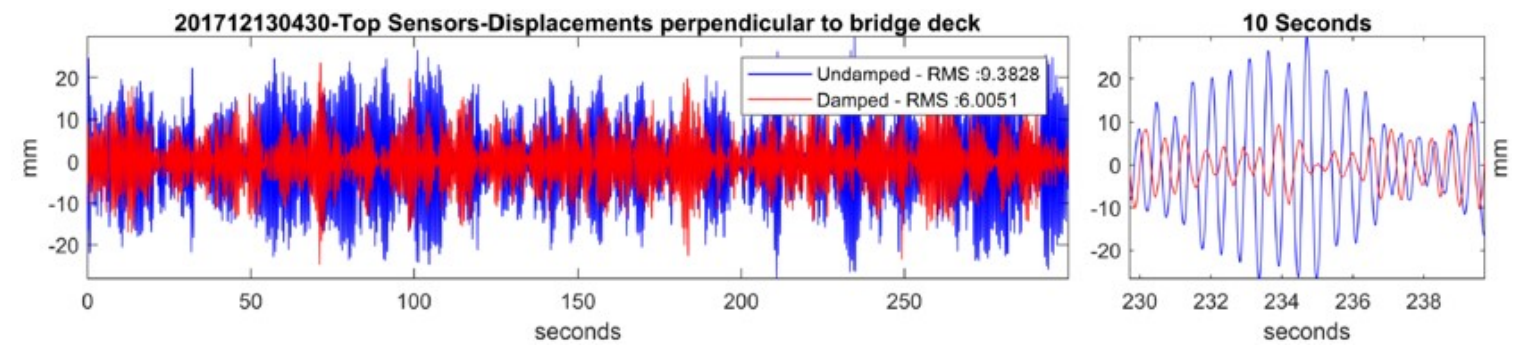

Figure 8.18: Pole top displacement time history with RMS.

\subsubsection{Frequency domain}

A frequency domain analysis was also carried out on both the acceleration and displacements signals. The power spectral density (PSD) function represents the power content of a signal in an infinitesimal frequency band. An improved estimator of the PSD by Welch is employed in the analysis of the light pole acceleration data [68]. The method consists of dividing the time series data into windowed overlapping segments, computing a modified periodogram of each segment, and then averaging the PSD estimates. These segments are subjected to a windowing operation using a Hamming window to prevent leakage in the Fast Fourier Transform (FFT) from time domain to frequency domain of the data. An n-point FFT is applied to the windowed data where $n$ is 8192 in this case. The periodogram of each windowed segment is then 
computed to obtain the segment's power spectrum. The set of periodograms is averaged to form the spectrum of all segments which is then scaled with the sampling frequency to compute the power spectral density. Results of the frequency domain analysis are shown in sample Figure 8.19 and in Section 8.2.6.

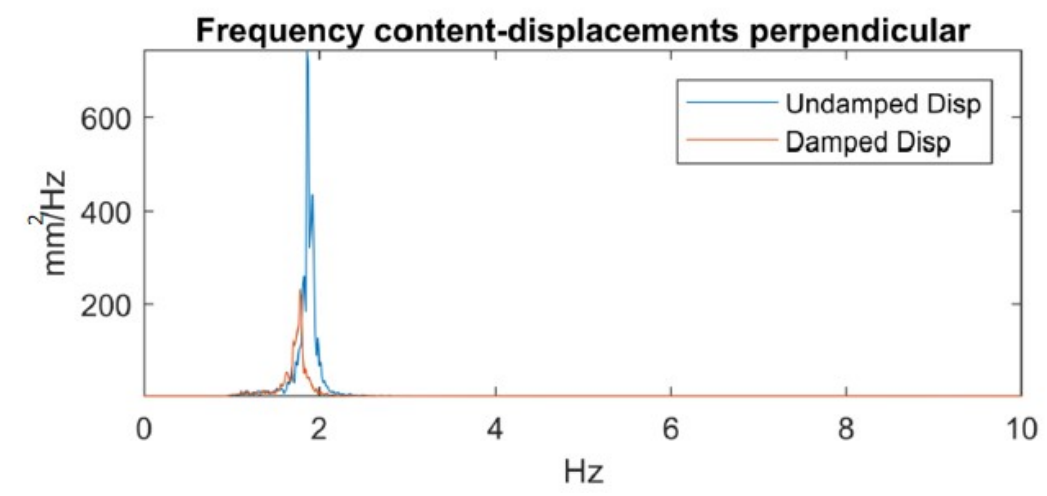

Figure 8.19: Frequency content of pole top displacement signal.

\subsubsection{Pole base stress estimation}

Pole base stresses were estimated from the displacements signals in the $\mathrm{x}$ and $\mathrm{z}$ directions based on the stress analysis results of the FEM model of the light pole detailed in the previous study (Section 8.1.4.2). An approximation of the linear relationship between the pole top displacement subjected to uniform loading and base stresses was obtained. A rigidity estimate of $0.77 \mathrm{MPa} / \mathrm{mm}$ was therefore considered and applied to the displacement signals to obtain stress time-histories. It should be noted that the rigidity thus obtained is an estimate since actual wind loading is not necessarily uniform over the length of the pole.

\subsubsection{Rainflow count for fatigue analysis}

A fatigue analysis studies how damage accumulates in an object subjected to cyclical changes in stress. The number of cycles necessary to reach a structural failure depends 
on the cycle amplitude. The wind excitation and the corresponding pole base stress time histories contain cycles of diverse amplitude, and the presence of hysteresis in the pole vibration has the effect of nesting some cycles within others, either completely or partially.

Rainflow counting estimates the number of load change cycles as a function of cycle amplitude [69]. Initially, the rainflow method turns the stress history into a sequence of reversals. Reversals are the local minima and maxima where the stress changes sign. The function counts cycles by considering a moving reference point of the sequence, and a moving ordered three-point subset with specific characteristics. At the end, the function collects the different cycles and half-cycles and tabulates their ranges, their means, and the points at which they start and end. This information can then be used to produce a histogram. Using the function available from the signal processing toolbox of Matlab [52], the histograms obtained from the light pole data in the perpendicular $(\mathrm{x})$ and parallel $(\mathrm{z})$ directions are shown in Figures 8.20a and 8.20b, while Figures 8.20c and 8.20d shows the histograms for the combined base stresses according to AASHTO using the following relations [70]:

$$
\begin{aligned}
& f_{x z}=f_{x}+0.414 f_{z} \\
& f_{z x}=f_{z}+0.414 f_{x}
\end{aligned}
$$

where $f_{x z}$ and $f_{z x}$ are the estimated combined base stresses in the general $x$ and $z$ directions respectively, while $f_{x}$ and $f_{z}$ are the estimated base stresses in the perpendicular and parallel directions. The base stress signals are obtained from the displacements time histories factored by the estimated rigidity of the steel poles as described in Section 8.2.5.4. 


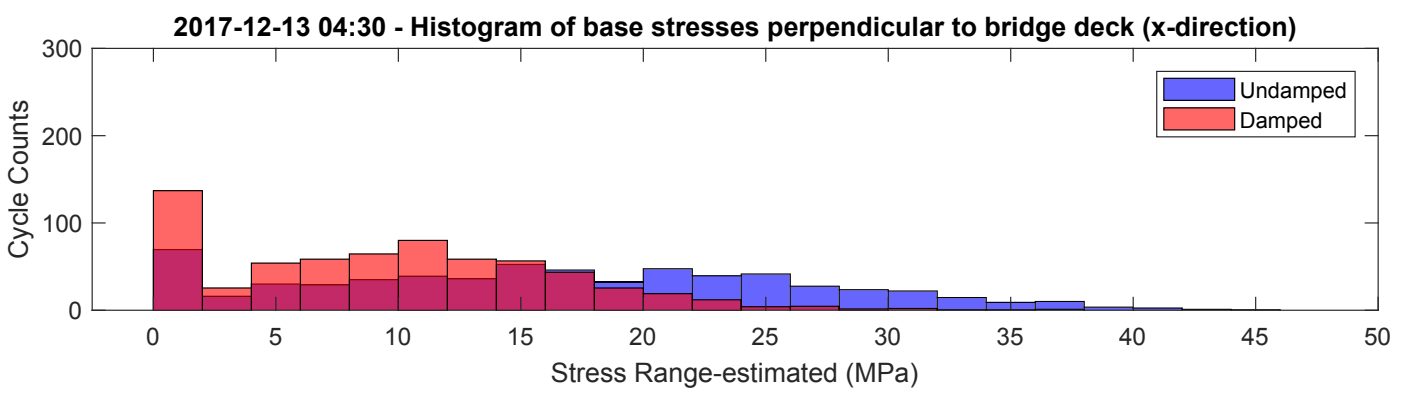

(a) Histogram of base stresses perpendicular to bridge deck (x-direction)

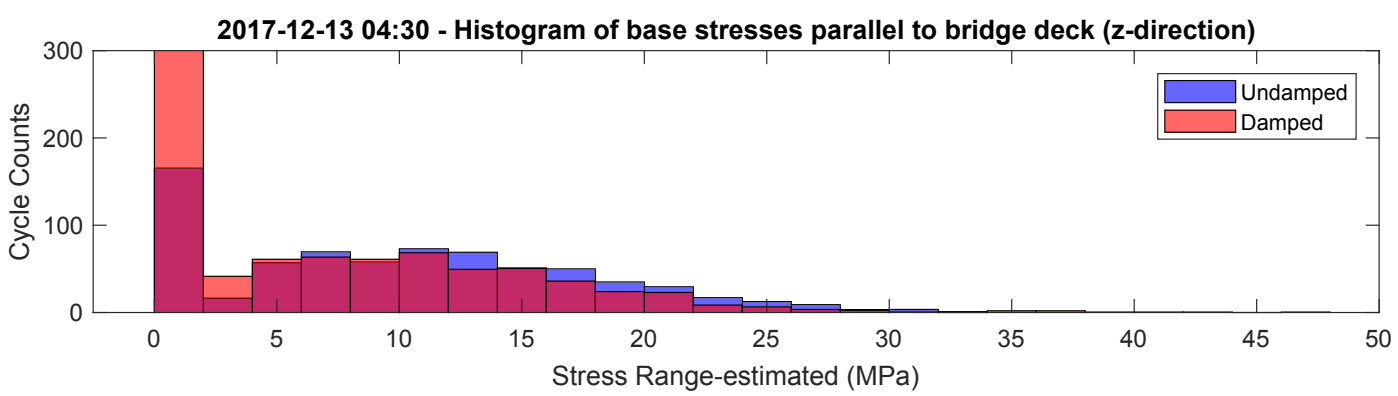

(b) Histogram of base stresses parallel to bridge deck (z-direction)

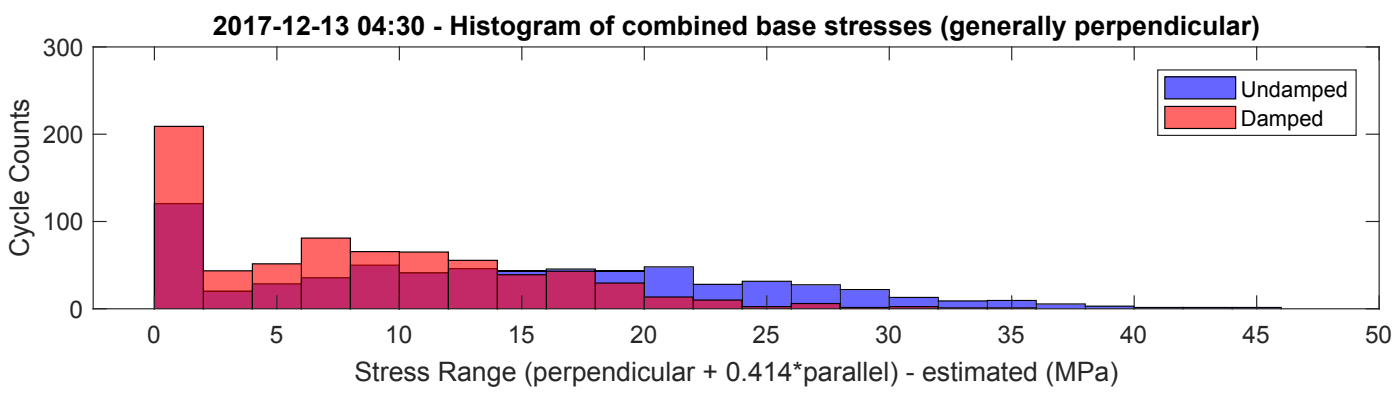

(c) Histogram of combined base stresses generally perpendicular to bridge deck $(x+0.414 z)$

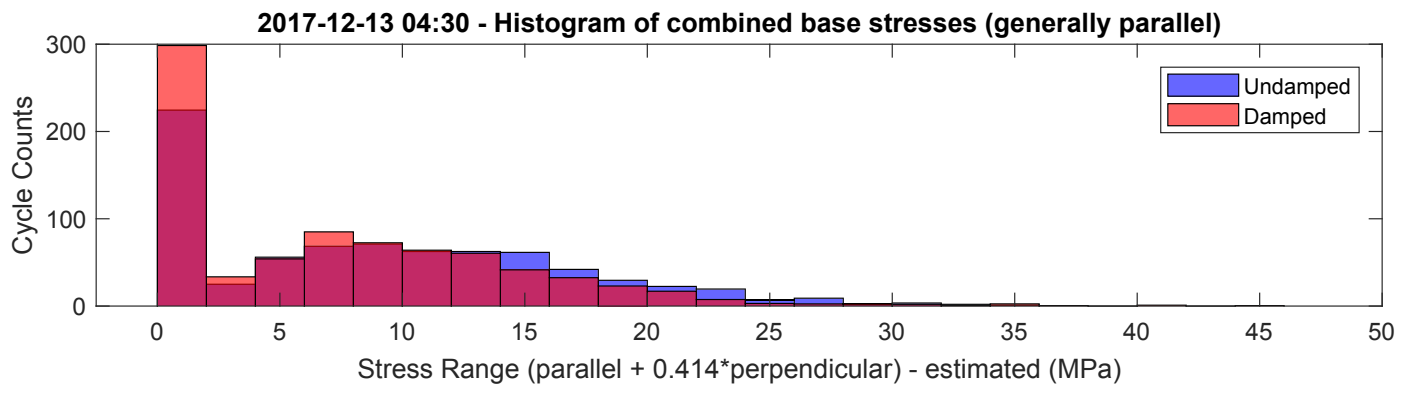

(d) Histogram of combined base stresses generally parallel to bridge deck $(z+0.414 x)$

Figure 8.20: Rainflow counts for a sample five minutes dataset. 


\subsubsection{Cumulative fatigue damage (cFD)}

According to S16-09-Design of steel structures [71], the total damage that results from variable amplitude loading shall satisfy Equation (8.4):

$$
c F D=\sum\left|\frac{(n N)_{i}}{N_{f i}}\right| \leq 1.0
$$

where $\mathrm{cFD}$ is the cumulated fatigue damage expressed in fraction of total fatigue life; $(n N)_{i}$ is the number of stress range cycles at stress range level $i, F_{s r i} . N_{f i}$ is the number of cycles that would cause failure at $F_{\text {sri }}$; it is typically obtained from the appropriate S-N curve based on the fatigue category of the steel connection detail. Alternatively, $N_{f i}$ can be calculated according to Equation (8.5):

$$
\begin{aligned}
& N_{f i}=\gamma F_{s r i}^{-3} \text { for } F_{s r i} \geq F_{s r t} \\
& N_{f i}=\gamma^{\prime} F_{s r i}^{-5} \text { for } F_{s r i} \geq F_{s r t}
\end{aligned}
$$

where $\gamma$ and $\gamma^{\prime}$ are fatigue life constants and $F_{\text {srt }}$ is the constant amplitude threshold stress range for a particular fatigue category. For the estimation of cumulative fatigue damage, all cycles of the stress history are considered, even those with amplitudes below the threshold stress range. For the original light poles, based on the shop drawings, we can assume that the pole to base plate connection is of category E1 according to S16-09 [71]. Category E1 is idealized as a fillet-welded HSS to base plate as shown in Figure 8.21. Fatigue constants are therefore taken as: $\gamma=128 \times 10^{9}$ and $\gamma^{\prime}=0.0415 \times 10^{15}$ and $F_{\text {srt }}=18 \mathrm{MPa}[71]$.

Using the rainflow counts of combined stress histories from the fatigue analysis described in Section 8.2.5.5, an estimate of cFD is calculated for each collected dataset. Complete results are shown in the last two columns of Table D.1. Averaged results are presented in Section 8.2.6. 


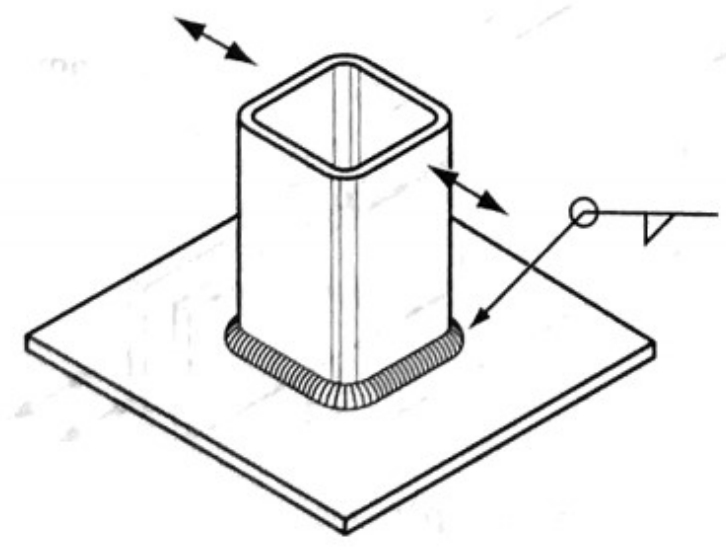

Figure 8.21: Pole to base plate connection of fatigue category E1 [71].

\subsubsection{Fatigue life estimations}

As a first estimation of fatigue life, a simple statistical fatigue model was obtained using the sustained 10-minute mean wind speed data correlated with the estimations of fatigue damage (cFD) for each of the 275 collected datasets as shown in Figure 8.22.

The expressions of Equation (8.6) describe the best fit exponential models for estimating fatigue damage for a 300 seconds sample set, complete with the corresponding coefficients of determination, for the combined stress signals primarily in the perpendicular direction.

$$
\begin{aligned}
& c F D=1.1 \times 10^{-9} e^{0.1140 v} \text { for undamped pole }\left(R^{2}=0.69\right) \\
& c F D=9.4 \times 10^{-10} e^{0.1113 v} \text { for damped pole }\left(R^{2}=0.72\right)
\end{aligned}
$$

Applying the fatigue models to historical wind data collected since November 1997 (date of first wind records) we can estimate the remaining fatigue service life of the bridge light poles. Wind data has been collected almost continually since very early in the bridge life. On average, a wind measurement is taken every 6 to 7 minutes. Since the model is fitted for fatigue damage estimates of 300 seconds 


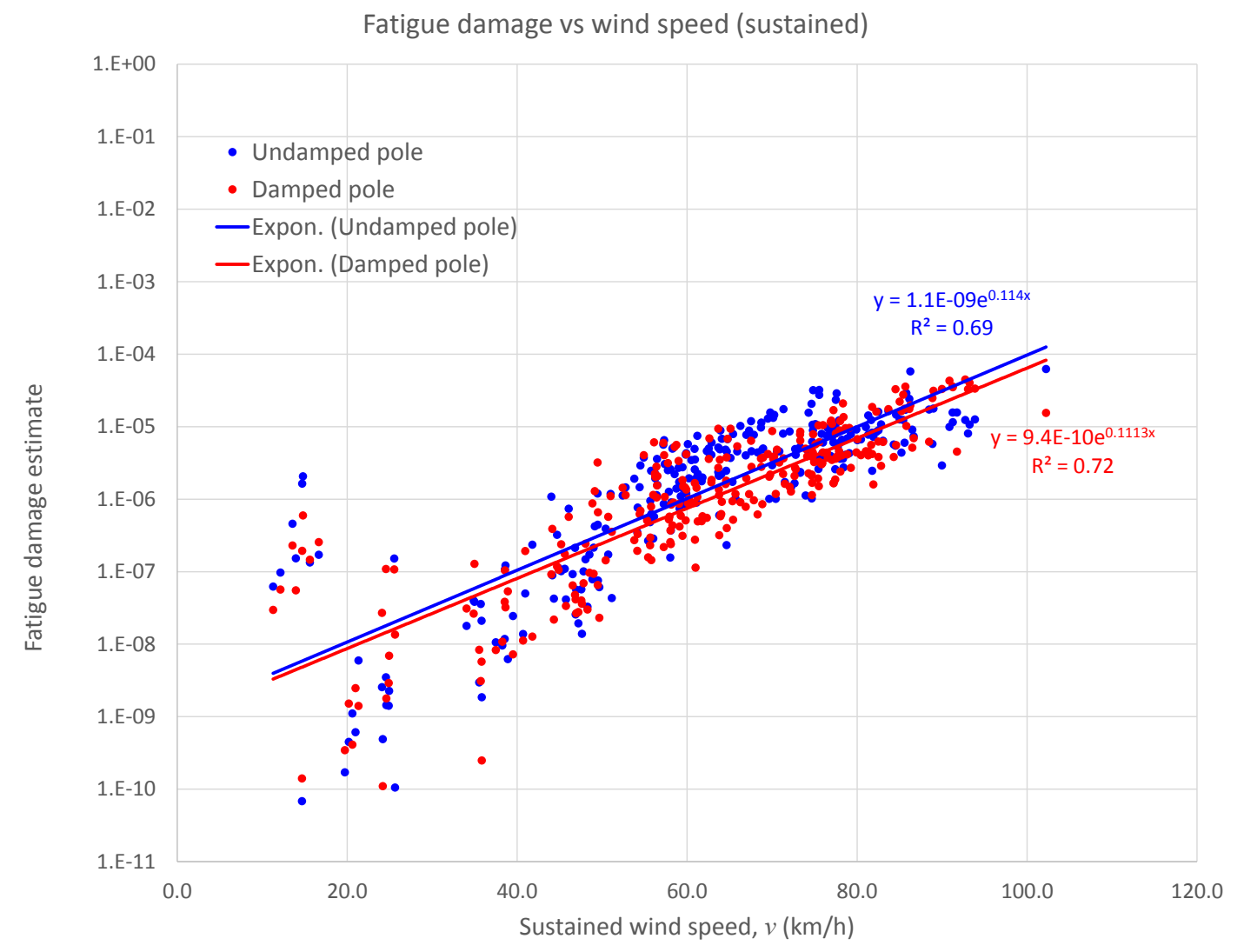

Figure 8.22: Fatigue damage versus measured ten minute mean wind speed, v, with best fit exponential model for both the undamped and the damped poles.

duration, the fatigue damage estimate from a single wind sample was prorated based on this sampling frequency (elapsed time between wind samples). Using the available 1.7 million samples, the fatigue damage for each wind sample was estimated based on the wind speed. The estimated fatigue life curves for the original light poles installed on the confederation bridge are presented in Section 8.2.6.3.

\subsubsection{Results}

The following section presents the results of all the calculated metrics described in the previous section. 


\subsubsection{Extended monitoring results}

The analysis results for each of the 275 collected dataset are included in Table D.1.

\subsubsection{Damping performance}

The analysis results averaged over all datasets are included in the last column of Table 8.10. For a more detailed perspective, the remaining columns of Table 8.10 show the analysis results averaged over each of the 12 wind events (the wind events are identified in Table 8.9) divided in two separate damping configurations. In configuration no.1 the pole-top damper is installed over pole N96 and in configuration no.2 the pole-top impact damper is installed on pole N95. Damping performance can be observed in terms of percentage of damped versus undamped for each calculated metric (in bold).

\subsubsection{Estimated fatigue service life}

With the methods described in Section 8.2.5, it is now possible to provide an estimate of cumulative fatigue damage (cFD) of the original light poles installed on the Confederation Bridge. Figure 8.23 shows the cFD curves overlaid on the sustained wind plot from 1997 to 2018. The curves are based on the exponential fatigue models of Section 8.2.5.7 using all the collected datasets for both damping configurations. The thick black curve shows the fatigue damage of the original undamped poles. The gray curves show fatigue damage of the damped pole; the thin gray line is hypothetical (assumes the presence of a pole-top damper since Nov. 1997), while the thick gray line is actual damping since installation of dampers in October 2017. Linear trend lines are then calculated and used to project the curves to 2025 . 
Table 8.10: Analysis results averaged over wind events.

\begin{tabular}{|c|c|c|c|c|c|c|c|c|c|c|c|c|c|c|}
\hline \multirow{2}{*}{\multicolumn{2}{|c|}{$\begin{array}{c}\text { Configuration } \\
\text { Wind event ID }\end{array}$}} & \multicolumn{6}{|c|}{ No.1 } & \multicolumn{6}{|c|}{ No.2 } & \multirow{7}{*}{ 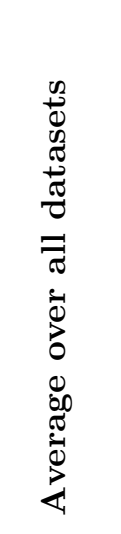 } \\
\hline & & 1 & 2 & 3 & 4 & 5 & 6 & 7 & 8 & 9 & 10 & 11 & 12 & \\
\hline \multicolumn{2}{|c|}{ Date } & 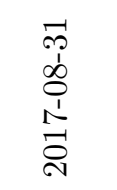 & 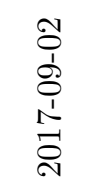 & $\begin{array}{l}0 \\
0 \\
1 \\
\stackrel{1}{1} \\
\stackrel{1}{1} \\
\stackrel{0}{0}\end{array}$ & 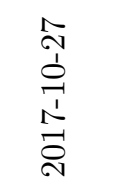 & $\begin{array}{l}0 \\
0 \\
0 \\
\stackrel{1}{1} \\
\stackrel{1}{0} \\
\stackrel{0}{N}\end{array}$ & 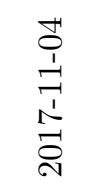 & 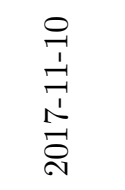 & 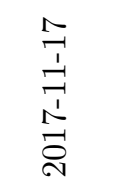 & 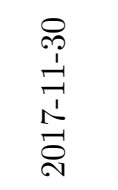 & 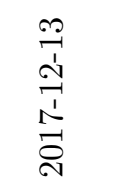 & 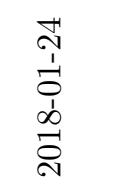 & 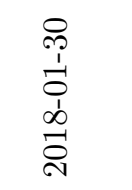 & \\
\hline \multirow{2}{*}{\multicolumn{2}{|c|}{$\begin{array}{l}\text { Max. sust. wind }(\mathrm{km} / \mathrm{h}) \\
\text { Max. wind gusts }(\mathrm{km} / \mathrm{h})\end{array}$}} & 49.0 & 63.8 & 86.2 & 91.2 & 93.9 & 81.3 & 67.9 & 88.5 & 58.5 & 102.2 & 74.3 & 70.8 & \\
\hline & & 58.6 & 75.5 & 107.6 & 102.0 & 106.5 & 96.3 & 91.0 & 104.4 & 70.9 & 119.3 & 78.0 & 83.6 & \\
\hline \multirow{2}{*}{$\begin{array}{l}\text { Avg. wind } \\
\text { direction }\end{array}$} & Cardinal & WSW & NW & WSW & SSE & $\mathrm{SE}$ & NW & WNW & NW & NNW & $\mathrm{E}$ & SW & $\mathrm{NE}$ & \\
\hline & Degrees & 231 & 301 & 225 & 149 & 120 & 298 & 263 & 293 & 312 & 75 & 195 & 14 & \\
\hline \multirow{3}{*}{ 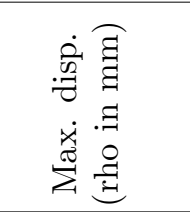 } & Undamped & 5.7 & 10.1 & 17.1 & 14.9 & 18.3 & 14.9 & 14.1 & 17.0 & 9.0 & 22.9 & 13.1 & 13.8 & 13.6 \\
\hline & Damped & 5.5 & 8.6 & 15.7 & 18.8 & 25.1 & 12.9 & 12.8 & 17.9 & 10.7 & 19.9 & 10.8 & 15.7 & 13.8 \\
\hline & $\begin{array}{c}\text { Perform.* } \\
(\%)\end{array}$ & 95.6 & 84.9 & 92.2 & 126.1 & 137.0 & 86.3 & 91.2 & 105.1 & 118.4 & 86.9 & 82.4 & 113.7 & 101.6 \\
\hline \multirow{3}{*}{ 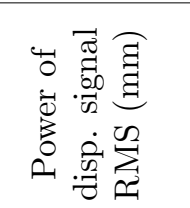 } & Undamped & 2.1 & 3.4 & 5.5 & 5.2 & 6.3 & 4.9 & 4.1 & 5.1 & 3.0 & 7.5 & 4.5 & 4.3 & 4.4 \\
\hline & Damped & 1.9 & 2.7 & 5.0 & 6.0 & 7.5 & 3.9 & 3.6 & 5.0 & 3.2 & 5.6 & 3.0 & 4.2 & 4.1 \\
\hline & $\begin{array}{l}\text { Perform. } * \\
(\%)\end{array}$ & 93.2 & 77.6 & 90.4 & 116.5 & 119.9 & 78.8 & 87.9 & 97.1 & 107.8 & 74.3 & 66.8 & 95.8 & 92.9 \\
\hline \multirow{3}{*}{ 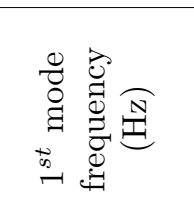 } & Undamped & 1.869 & 1.867 & 1.863 & 1.863 & 1.866 & 1.862 & 1.89 & 1.895 & 1.886 & 1.882 & 1.889 & 1.896 & 1.88 \\
\hline & Damped & 1.763 & 1.772 & 1.773 & 1.778 & 1.783 & 1.778 & 1.771 & 1.755 & 1.763 & 1.761 & 1.752 & 1.768 & 1.77 \\
\hline & $\begin{array}{c}\text { Damping } \\
\text { effect* }(\%)\end{array}$ & 94.3 & 94.9 & 95.2 & 95.4 & 95.5 & 95.5 & 93.7 & 92.6 & 93.5 & 93.6 & 92.7 & 93.3 & 94.1 \\
\hline \multirow{3}{*}{ 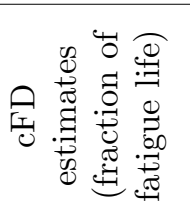 } & Undamped & $1.7 \times 10^{-}$ & $6.1 \times 10$ & $6.5 \times 10^{-}$ & $6.6 \times 10^{-}$ & $6.0 \times 10^{-}$ & $4.2 \times 10^{-}$ & $3.7 \times 10^{-}$ & $7.6 \times 10^{-}$ & $1.3 \times 10^{-}$ & $2.1 \times 10^{-}$ & $6.4 \times 10^{-}$ & $3.4 \times 10^{-6}$ & $5.3 \times 10^{-6}$ \\
\hline & Damped & $7.4 \times 10^{-}$ & $3.5 \times 10^{-}$ & $6.5 \times 10^{-}$ & $1.4 \times 10^{-}$ & $2.3 \times 10^{-}$ & $2.3 \times 10^{-}$ & $1.1 \times 10^{-}$ & $3.8 \times 10^{-}$ & $1.6 \times 10^{-}$ & $5.4 \times 10^{-}$ & $1.2 \times 10^{-}$ & $2.7 \times 10^{-6}$ & $4.6 \times 10^{-6}$ \\
\hline & $\begin{array}{c}\text { Perform.* } \\
(\%)\end{array}$ & 95.6 & 84.9 & 92.2 & 126.1 & 137.0 & 86.3 & 91.2 & 105.1 & 118.4 & 86.9 & 82.4 & 113.7 & 86.8 \\
\hline
\end{tabular}




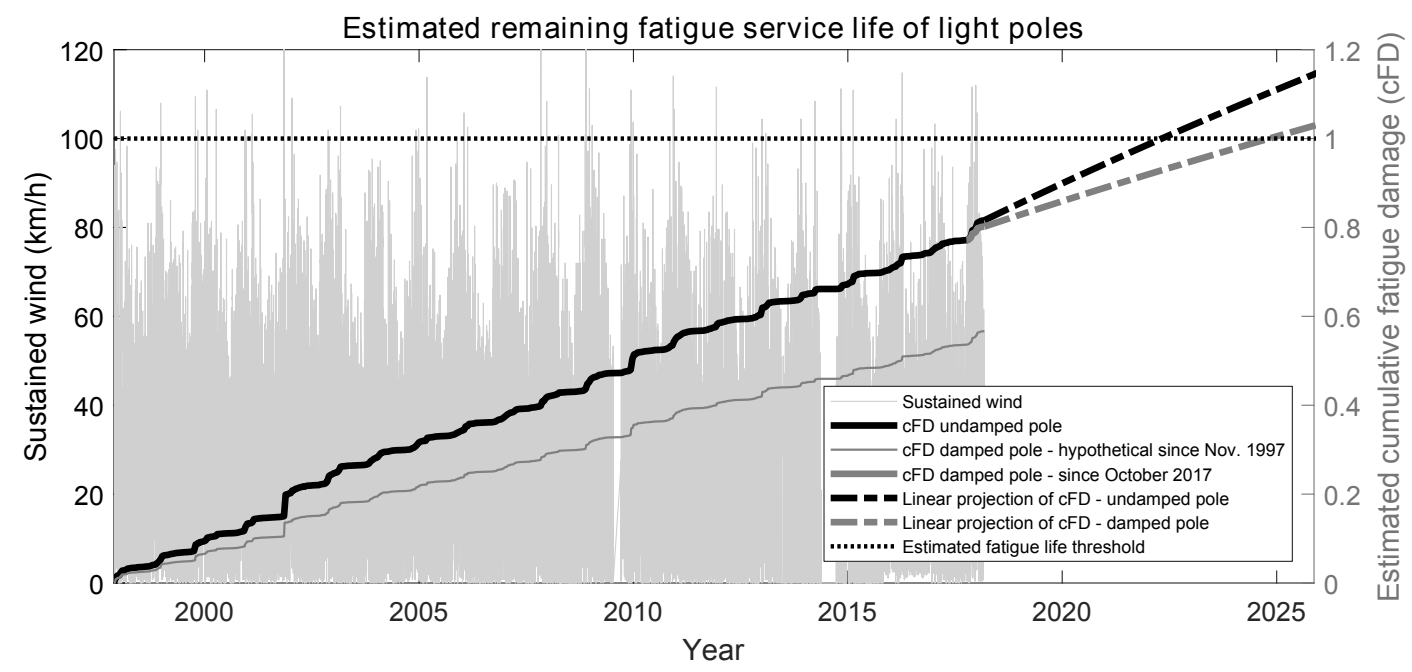

(a) November 1997 to March 2018, with projections to 2025.

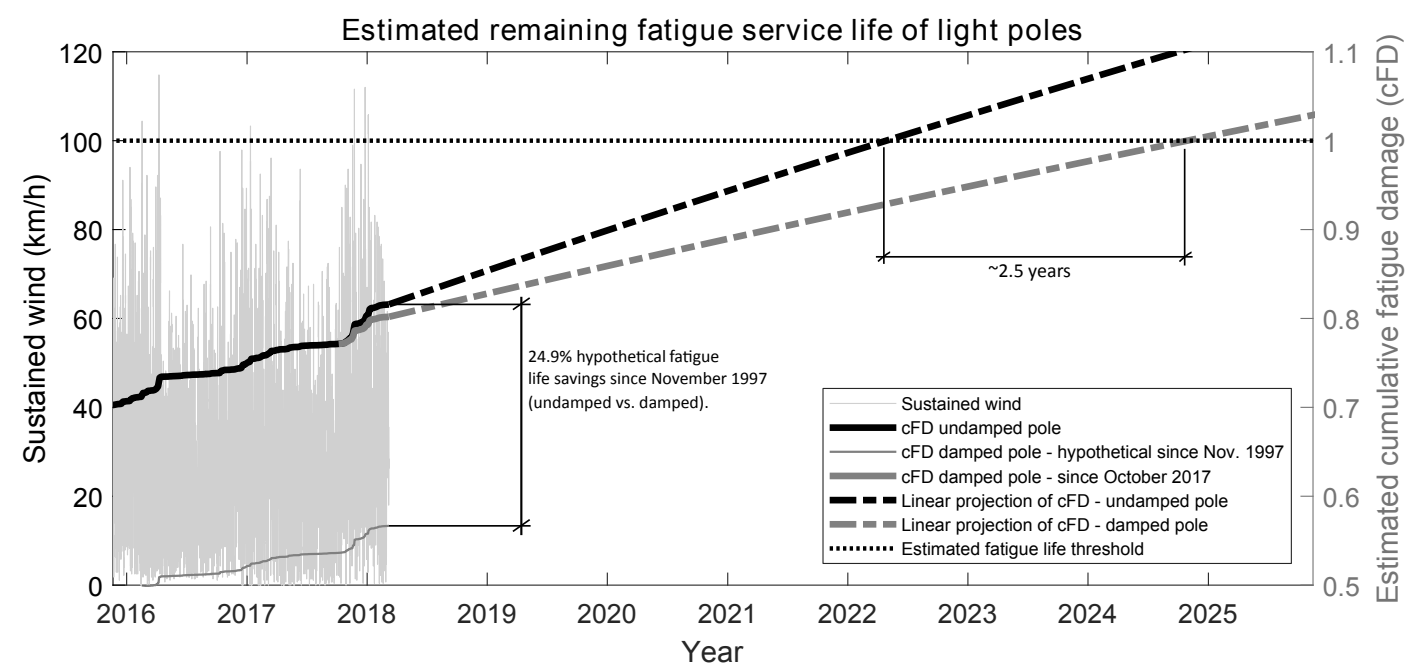

(b) November 2015 to March 2018, with projections to 2025.

Figure 8.23: Estimated cumulative fatigue damage (cFD) of Confederation Bridge light poles (damped vs undamped).

Figure 8.23b shows an expanded view recent fatigue damage (from 2015 to 2018 with projections to 2015) and provides an estimate on remaining fatigue service life for both the undamped and damped poles. The fatigue life threshold of 1.0 is clearly indicated on the figure. Also indicated on the figure is the estimated increase in the 
fatigue service life provided by the pole-top impact dampers installed in October 2017 (approximately 2.5 years). According to this figure, a $24.9 \%$ hypothetical reduction of fatigue damage could have been achieved had the dampers been installed since November 1997.

\subsubsection{Interpretation and discussions}

A relatively large amount of data has been collected from which plenty of useful metrics were calculated. The results provide a better understanding of pole vibration behavior and damping performance. In this section the analysis results presented in Section 8.2.6 will be interpreted and discussed.

Firstly, the damping performance of the pole-top impact dampers can be evaluated from several calculated metrics (maximum displacements, RMS, frequency and cFD summarized in Table 8.10). While the damping effects are not evident on the maximum observed displacements, they do have a significant impact on the most important metrics: the power of the displacement signal (RMS) and the fatigue damage (cFD) where overall reductions of $7 \%$ and $13 \%$ respectively have been obtained over all collected datasets. While the damper does not reduce the peak displacements, it effectively reduces the number of strong displacement cycles which is just as important in limiting fatigue damage.

By breaking down the results over the twelve different wind events that were measured, we can see significant variations of damping performance as shown quantitatively in Table 8.10. While a strong correlation exists across all metrics with respect to sustained wind speed, it is more difficult to correlate the actual damping performance with the wind characteristics (speed and/or direction). For example, for three of the twelve wind events (wind events 4 and 5 of configuration no. 1 and wind event 9 of configuration no.2), the results in Table 8.10 seem to show negative 
damping effects on the pole. Localized differences between the measured poles account for these results. Since poles are located $83 \mathrm{~m}$ apart, differences in vibration and damping behavior are due to a combination of several factors as described in Section 8.2.3.5.

To observe the effects of these localized differences in vibration and damping behavior between poles, the pole top damper was changed from pole N96 to pole N95 near the mid-point of data collection (on November 9th, 2017). Both poles are structurally similar and have been installed on the bridge since 1997. Six wind events were collected before and after changing the damper location. The damper's performance on the same pole is difficult to evaluate since both damped and undamped configurations cannot be measured on the same pole for the same wind event. However, Figure 8.24 shows the correlation of fatigue damage with wind speed for each undamped and damped configuration on pole N95 and N96 respectively.

We can observe from the best fit exponential trend lines that the damping effects are significant on pole N96 while minimal on pole N95. Applying these models to the historic wind record from November 1997 to March 2018, we can use the fatigue life savings (which is the difference between the undamped and the damped cumulative fatigue damage over the life span of the poles, or rather since November 1997) as a metric to evaluate damping performance. Table 8.11 summarizes these results. It should be noted that the damped fatigue damage estimate presented in this table is hypothetical since dampers were only installed in October 2017. Calculations are based on the hypothetical case where the damper was outfitted on the original poles since installation in 1997. It should also be noted that the sample size (number of datasets) used for establishing the fatigue models of the individual poles shown in Figure 8.24 was effectively reduced by half as compared with the overall fatigue models shown in Figure 8.22. 


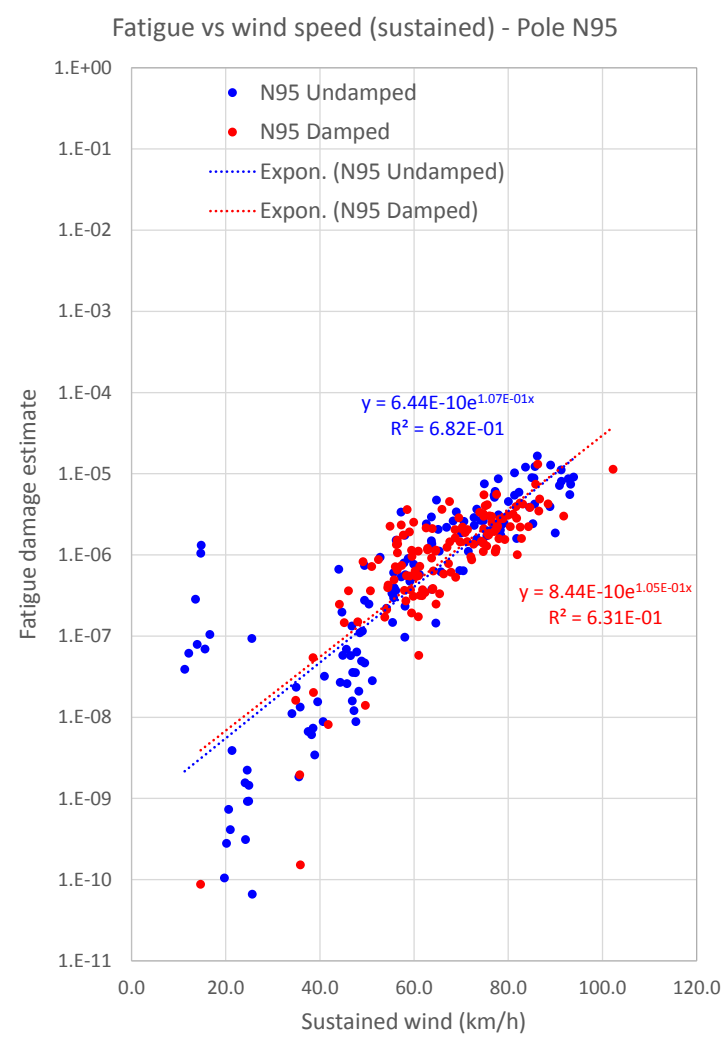

(a) Pole N95.

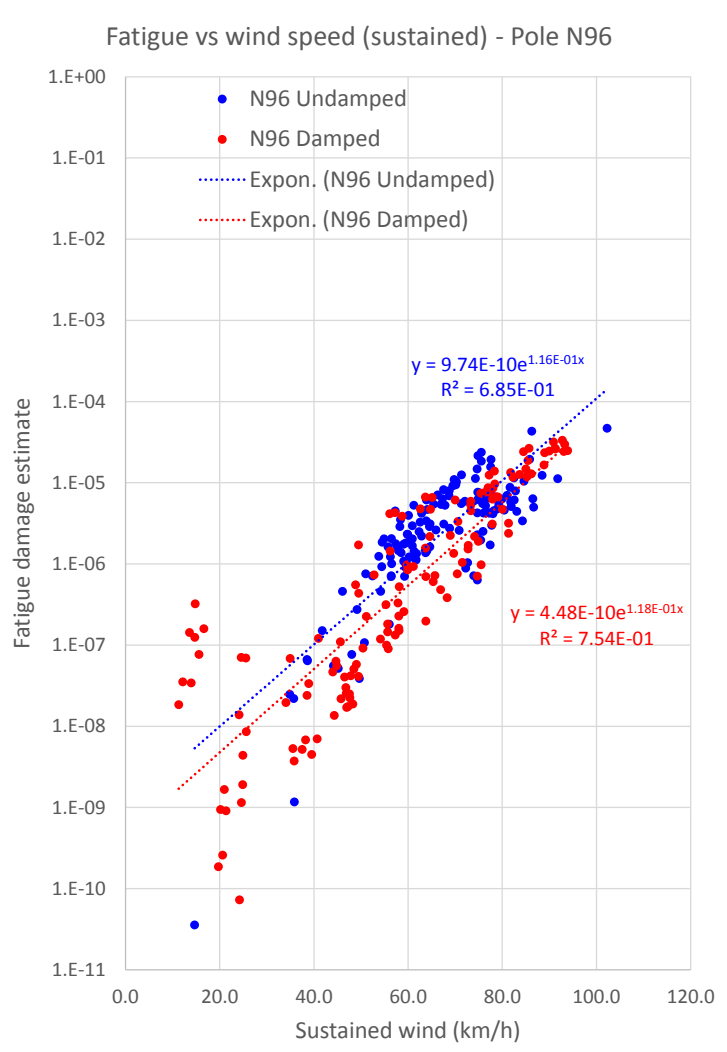

(b) Pole N96.

Figure 8.24: Fatigue damage versus wind speed with corresponding exponential fatigue model.

The most significant results from this study are visualized in Figure 8.23. This figure demonstrate the effectiveness of the methodology described in this report in providing useful engineering information to bridge operators. An estimate of historical cumulative fatigue damage is provided in these figures based on measurements taken in a span of 5 months.

While overall it would seem that poles are at $81.6 \%$ of fatigue life, by breaking down results per individual pole, we can see that fatigue damage and associated damping performance vary greatly from pole to pole due to localized effects. From this breakdown, we can see that pole N95 has relatively low fatigue damage as compared to pole N96 (28.8\% vs $86.9 \%$ of total fatigue service life). Also, we can see that 
Table 8.11: Estimate of cumulative fatigue damage (cFD) in percentage of total fatigue life (cumulated from November 1997 to March 2018).

\begin{tabular}{cccc}
\hline & Undamped pole & $\begin{array}{c}\text { Damped pole } \\
\text { (hypothetical*) }\end{array}$ & $\begin{array}{c}\text { Damper } \\
\text { performance }\end{array}$ \\
\hline Overall & $81.6 \%$ & $56.7 \%$ & $24.9 \%$ \\
Pole N95 & $28.8 \%$ & $30.9 \%$ & $-2.1 \%$ \\
Pole N96 & $86.9 \%$ & $46.6 \%$ & $40.3 \%$ \\
\hline
\end{tabular}

*Hypothetical scenario where a damper is assumed present since original pole installation in 1997.

the damping performance is significantly higher for pole N96 than pole N95 $(40.3 \%$ reduction in $\mathrm{cFD}$ vs $2.1 \%$ increase in $\mathrm{cFD}$ since 1997). Damping performance in Table 8.11 is evaluated by subtracting the historical $\mathrm{cFD}$ of the hypothetical damped pole from the $\mathrm{cFD}$ of the undamped pole.

In fact, according to the results, the installed pole-top damper has a negligible effect on Pole N95. These results seem to confirm that Pole N95 is inherently damped (see Section 8.2.3.5) and would therefore not require a pole-top impact damper, however the presence of a damper does not significantly adversely affect the pole which, in any case, has relatively low fatigue damage.

More work is required to obtain accurate estimates of cFD with associated uncertainty:

- More data needs to be collected across a broad range of wind speeds and direction and on several poles in both undamped and damped configurations.

- The current models used for estimating fatigue damage are based only on correlations with sustained wind speeds. Detailed parametric models are required and should include correlations with sustained wind speeds, wind gusts, wind direction, wind features (energy, frequency, etc.), temperature, pole location, and any other parameter susceptible of influencing vibration behavior.

- The wind record needs to be patched and completed. Some records are missing 
(particularly in 2009 and 2014) and could be filled in with data from other wind sensors. Also, wind records (and therefore cumulative fatigue damage) originate in November 1997 while the bridge opening was in May 1997 and the installation of light poles likely preceded this date by several months. The inclusion of these missing wind records would cause an increase in the estimated cumulative fatigue damage.

- Changes to light poles during their lifetime could have affected the cumulative fatigue damage curves. The current fatigue models are based on the light poles in their current configuration (with a chain damper installed in 2002 and with LED fixture changed in 2014). To account for these past changes to the light poles, measurements could be taken on poles with the historic configurations to build the missing fatigue models and to apply them to the wind records of the corresponding years. It is thought that the inclusion of the chain damper, while primarily added to reduce second mode vibrations, did have a beneficial effect on reducing the first mode vibrations. This would indicate that the fatigue damage cumulated during the first years of service without the chain damper should be greater than what is currently estimated.

Even without accurate $\mathrm{cFD}$ estimates, relative results clearly indicate that dampers are effective in reducing fatigue damage. Overall, from Figure 8.23b, we can estimate that by installing the dampers in October 2017, the fatigue service life of the light poles was increased by an average of approximately 2.5 years.

It should be noted that the fall and winter seasons of 2017-2018 were very windy. Despite collecting data over 12 wind events, several strong wind events were missed due to technical issues with the sensors and the data acquisition system which were strained under the extreme environmental conditions. Of note is the wind event of November 23rd with sustained winds well above $100 \mathrm{~km} / \mathrm{h}$ for several hours with 
gusts up to $117 \mathrm{~km} / \mathrm{h}$, and the wind event of December 26th with sustained winds above $110 \mathrm{~km} / \mathrm{h}$ for a short duration (30min). Most of these strong wind events that matched or exceeded the intensity of previous damage causing events, occurred after the installation of the pole-top dampers and interestingly, no pole damage or failures were reported.

\subsubsection{Conclusions and recommendations}

The objectives initially stated in Section 8.2.2 have been attained. Firstly, the inoperation vibrations of installed light poles were monitored by a custom wireless remote data acquisition system for a period of 5 months during the windy season on the Confederation Bridge. 275 Datasets were collected primarily during medium to strong wind events (12 events total) and under two different configurations (the poletop damper was switched from pole N96 to pole N95 mid-way through the monitoring period).

Secondly, the performance of the custom prototype damper was evaluated based on several metrics, the most significant of which are signal power expressed as RMS and the cumulative fatigue damage (cFD) expressed in fractions of total fatigue life. Global reductions of 7\% RMS and 13\% cFD were observed over all collected datasets which indicates a good overall performance of the pole-top impact damper.

A fatigue analysis method is also presented in this report as a proof of concept for calculating the cumulative fatigue damage $(\mathrm{cFD})$ from vibration data and for estimating the remaining fatigue service life of in-operation bridge light poles. Despite the current (but surmountable) limitations of the method (as noted in Section 8.2.6.3), it has proven to be a very promising analysis method of which the results were used in this study as a third metric for evaluating the damping performance of the prototype damper. It was found that the dampers installed in the fall of 2017 could increase 
the remaining fatigue service life of the light poles by 2.5 years. Hypothetically, if the dampers had originally been installed with the light poles, the cumulative fatigue damage would have been reduced by an average of $25 \%$ with a projected service life increase of $48 \%$ (which represents an additional 12 years of service above the actual estimated life of 25 years).

Lastly, a significant finding resulting from the fatigue analysis is the identification of localized effects on vibration and damping. The two subject poles of this study are adjacent, separated by 83 meters on the north side of the bridge deck and are structurally similar. Despite their physical proximity, several factors can account for the significant variability of the measured cumulative fatigue damage and the damping performance (see Sections 8.2.3.5 and 8.2.6.3). Results indicate that one pole (N96) has relatively high fatigue damage ( $87 \%$ of total fatigue life) with good damping performance provided by the installed pole-top damper (40\% cFD reduction achieved over the life of the hypothetical damped pole), while the other pole (N95) has relatively low fatigue damage (29\% of total fatigue life) with negligible damping performance provided by the damper. From this very limited sample set of two light poles, it is stated (with cautious reservations) that the dampers seem to have a positive effect on poles that have relatively high fatigue damage and no effect (or very little negative effect) on poles that have relatively low fatigue damage.

Recommendations:

- Having been already outfitted with pole-top dampers since October 2017, no changes to the current damping configurations of the light poles are recommended.

- It is recommended to reinstate a monitoring program to collect additional data over multiple poles ( 5 to 6 poles) to improve the current fatigue models and to try to identify poles that are more susceptible to fatigue damage. The selection 
of light poles to be monitored should account for localized differences susceptible of varying the vibration and damping characteristics of the poles. Data should be collected over a period of several months, ideally in windy season and across a broad range of wind conditions.

\subsection{Estimating the Remaining Service Life of Bridge Bearings Using Dynamic Measure- ments}

The second proposed study involves estimating the remaining service life of bridge bearings using vibration monitoring data.

It is proposed to use the data from accelerometers installed in the longitudinal direction of the instrumented portion of the Confederation Bridge in order to estimate the dynamic small-amplitude bearing travel. It is believed that these small amplitude displacements significantly reduce the service life of the bridge bearings due to their relatively high frequency. Estimating the cumulative displacements from these measurements would help in estimating the remaining service life of the bridge bearings.

To evaluate the feasibility of this study, some cumulative displacements were obtained from the vibrations measured at pier 31 using the existing double integration algorithm from the SPLASH application. A separate algorithm was written to obtain longitudinal pier sweep values in millimeters per minute; results are shown in Table 8.12.

It should be noted that the preliminary results shown in Table 8.12 do not actually

represent bearing travel. Data from sensors installed in the longitudinal direction on 
Table 8.12: Longitudinal pier sweep estimates $(\mathrm{mm} / \mathrm{min})$ obtained from vibration measurements of pier 30 .

\begin{tabular}{|c|c|c|c|c|c|}
\hline \multirow[b]{2}{*}{ Date } & \multirow[b]{2}{*}{ Time } & \multirow[b]{2}{*}{ Event type } & \multirow[b]{2}{*}{$\begin{array}{l}\text { Duration } \\
(\mathrm{s})\end{array}$} & \multicolumn{2}{|c|}{$\begin{array}{c}\text { Average longitudinal cumulative } \\
\text { displacements in }(\mathrm{mm} / \mathrm{min}) \text { at location } \\
\text { 18L and P31- } 4 \mathrm{~L}^{*}\end{array}$} \\
\hline & & & & Location 18L & Location P31-4L \\
\hline 2014-04-25 & $04: 22$ & traffic & 600 & 11.1 & 9.3 \\
\hline $2015-02-15$ & $18: 38$ & strong wind & 109 & 48.4 & 35.9 \\
\hline $2015-02-15$ & $20: 38$ & strong wind & 105 & 81.5 & 65.6 \\
\hline
\end{tabular}

*see Table 4.2 and Figure 3.4 for location of sensors.

both sides of an expansion joint of the bridge are required for these measurements.

The double integration algorithm currently integrated in the SPLASH applications may have some shortcomings, especially due to the filtering which effectively zeros the integration constants. The initial conditions at each calculation step are therefore lost (initial displacement and velocity). However, this may not be significant if we calculate the sweep over a certain number of displacement cycles (the more the better) since there should not be any residual displacements in the short term (realizing that temperature effects on deformations occur on longer time scales relative to the dynamic displacement oscillations occurring during traffic or wind events).

For this study, it is proposed to expand on the preliminary results of Table 8.12 with many more datasets and with measurements at the four pier locations. Since the instrumentation renewal, the sensors in all four piers were replaced and with the third version of SPLASH many of the noted issues have been resolved. These improvements will therefore permit estimates on bearing travel at two separate expansion joints. Bearing travel would be estimated from the synchronized differential sweep measurements of the two piers adjacent to an expansion joint. It should be 
noted that the third version of the SPLASH application was developed after the preliminary study presented in Table 8.12 and should greatly accelerate the processing of the data.

This work can be significant especially if real-time service life predictions of bridge bearings can be provided. A parametric model for predicting service life of sliding surface bridge bearings has been proposed in [72] based on simulated physical models. The authors of this study have stated the very real need for verification of their proposed model using real in-service data.

Possible issues to be resolved in this study include the loss of initial conditions in the current double integration algorithm. The algorithm may need to be reviewed for adequacy. The use of additional accelerometers or displacement sensors at the expansion joint may be required for measurements of initial conditions. Also, the information on installed bearings will need to be gathered (manufacturer, make, model, technical specifications, etc.).

If successful, studies of the kind described in this section would show the usefulness and the importance of vibration monitoring for practical structural health monitoring applications.

\subsection{Use of Vibration Monitoring for Estimating Prestress Loss of In-Service External Post Tensioning Cables}

The proposed study summarily described in this section concerns the vibration monitoring of the external post tensioning cables in order to easily and accurately estimate the remaining tension in the tendons. Promising work at the University of Texas at 
Austin show the feasibility of estimating tension via vibration monitoring [73, 74]. The proposed study consists of installing a single (or a small number) of accelerometers on an external post-tensioning cable and pulling in the center of the cable with a sudden release, to excite only the first mode of vibration which would simplify the analysis and perhaps lead to increased accuracy. Other references include [75-77]. 


\section{Chapter 9}

\section{Conclusions}

The ultimate goal of VBSHM applications is reliable and consistent non-destructive condition assessments of structures. This chapter will recapitulate the work carried out toward this goal and will highlight the author's original contributions towards the achievement of the objectives stated in Chapter 1.

\subsection{Discussion}

The path to reliable and consistent condition assessments from vibration response measurements is through a thorough understanding of the causes of noise and variability in the analysis results. In this regard, the objectives stated in Section 1.2 have been achieved:

- Several known sources of uncertainties inside the monitoring system have been identified and removed during the complete renewal of the monitoring system as described in Chapter 4. By choosing a single type of accelerometer (instead of multiple types as in the first generation) with better range and resolution and without the need for extra signal treatment prior to recording, the resulting measurement noise is minimized. 
- The data processing engine has been completely overhauled in order to automate and streamline all data operations as described in Chapter 5. This work was carried out with the underlying goal of removing all unneeded data operations that contributed to computational noise. The automation was required in order to batch process the large amounts of collected raw vibration responses.

- The existing stochastic subspace identification algorithm described in Section 2.1 was improved and automated to reduce the uncertainty associated with computation and human error. A new Automated Inline Full Space Identification (AI-FSI) technique is thus proposed and is presented in Section 2.2. This new technique integrates all aspects of automated modal parameter estimations (MPE) and modal tracking.

- With the new automated processing and analysis tools, an unprecedented feat was realized with the processing and analysis of all the historical data collected by the Confederation Bridge monitoring project since 1998. This represents over 250000 logger files, 40000 hours of recording and 28TB of raw and processed data collected over 20 years. The historical database of raw and processed vibration data along with the associated environmental data is presented in Chapter 6 .

- Data smoothing and filtering were carried out on the results of modal tracking as described in Sections 7.2 and 7.3. Evident correlations with environmental conditions are observed from these results.

- Several possible sources of variability were identified and quantified as described in Chapter 7. Through multiple regression analysis it has been shown that depending on the mode, these identified sources of variability can explain between $24 \%$ and $53 \%$ of the variations observed in the estimated modal frequencies. 
This is a significant finding that can further improve future condition or damage identification techniques that track modal features.

- A novel approach using the residuals from the regression models for damage detection is described in Section 7.5. A change point algorithm has been implemented with promising results in identifying shifts in residual means. Shift amplitudes in the order of the standard deviation of residuals $(0.68 \%$ to $0.95 \%$ of mean frequency) as shown in Table 7.9 are now possible.

- The sensitivity of the change detection has been evaluated and results are shown in Table 7.10. In fact, a shift in mean of approximately $0.4 \%$ was successfully detected by the change point algorithm on the residuals of the $0.47 \mathrm{~Hz}$ and $0.67 \mathrm{~Hz}$ modes; albeit with lower accuracy and longer detection lags than those obtained for higher amplitude shifts. This indicates that the use of modal features for the identification of lower levels of damage is possible.

- Finally, practical examples of the successful applications of vibration based monitoring for the evaluation of the health of structural components was provided in Chapter 8. A fatigue analysis method is presented as a proof of concept for calculating the cumulative fatigue damage (cFD) from vibration response data and for estimating the remaining fatigue service life of in-operation bridge light poles. Such successful case studies are required to further advance research in this field for eventual large scale adoption and improved infrastructure management.

\subsection{Future work}

With a complete database comprised of over 20 years of processed and analyzed vibration response data, future research work using features such as modal parameters 
are greatly facilitated. Some possible future studies are proposed here:

- The identification and gathering of additional possible sources of variability such as data on temperature variations, traffic patterns, thermocouple, humidity, concrete strength through testing, etc. These types of data could help to account for more variations in the modal parameters and provide better detection results.

- There is a strong potential in the field of machine learning and non-linear parametric modeling. The identification of more robust parametric models of a healthy state through advanced model training techniques applied to the estimated modal parameters could lead to better quality residuals for damage detection. Care must be taken when modeling to avoid over-fitting of the data.

- Parametric models (via regression or other methodologies) should be developed for all modal parameters of the structure (modal frequencies, mode shapes and damping ratios) and for each principal identified modes.

- The use of specialized Kalman filtering (Unscented Kalman Filter [58] or Outlier Resistant Extended Kalman Filters [78]) with transition functions using parametric modeling coefficients should be investigated, especially for mode shape tracking where the relations between the degrees of freedom are well established. A modification to the change detection algorithm could be implemented for use on the Kalman estimates based on slope changes instead of mean shifts.

- As a logical next step, damage simulation results with real uncertainties, such as those proposed by [79] could be used to test the change detection algorithm described in Chapter 7. Or a new method to simulate damage with real field uncertainties could be developed. Since many of the damage detection techniques rely on observing changes in the estimated modal properties, the proposed study would involve developing a new technique called Reverse SSI (R-SSI) where the 
estimated modal properties from the SSI are manually shifted to represent different levels of damage (as determined from FE modeling of damage scenarios) all the while preserving the valuable noise and uncertainties of the signal. It is believed that the inverse operations of the SSI can result in full time histories of a damaged event containing all the real world uncertainties and noise. These damage simulation techniques should lead to robust testing of the proposed change detection algorithm.

- On the practical side, it is recommended that the monitoring system continue its operation in continuous collection mode and that all equipment be monitored and fixed as soon as issues arise.

- Studies using vibration responses to evaluate the health of bridge components were presented in Chapter 8. These practical studies provide actionable engineering information to bridge operators. Two of these proposed studies (on bridge bearings and on external post tendons) are to be further advanced as part of the continued SHM of the Confederation Bridge.

The vibration monitoring program of the Confederation Bridge is a key project in the quest for smart infrastructure systems and as such should be a priority for the current and the future managers of this important infrastructure. 


\section{List of References}

[1] B. Haggart, "Canada's infrastructure debt - Part I : Assessing the infrastructure shortfall," Tech. Rep. PRB 03-55E, Library of Parliament, June 2004.

[2] A. Paterson, Setting the New Progressive Agenda, Canada 2020, vol. e-book. 2015.

[3] C. Cautillo, N. Zon, and M. Mendelsohn, Rebuilding Canada - A new framework for renewing Canada's infrastructure. Mowat Research No. 92, Mowat Centre, 2014.

[4] "The Foundations of a Competitive Canada: The Need for Strategic Infrastructure Investment," tech. rep., Canadian Chamber of Commerce, Dec. 2013.

[5] H. Mackenzie, "Canada's Infrastructure Gap, where it came from and why it will cost so much to fix it," tech. rep., Canadian Centre for Policy Alternatives, Jan. 2013.

[6] "The Canadian Infrastructure Report Card - Informing the future," tech. rep., Canadian Construction Association (CCA), the Canadian Public Works Association (CPWA), the Canadian Society for Civil Engineering (CSCE) and the Federation of Canadian Municipalities (FCM), 2016.

[7] M. Holden and C. Vander Ploeg, "At the Intersection: The Case for Sustained and Strategic Public Infrastructure Investment," Tech. Rep. 9781-927488041, Canada West Foundation, 2013.

[8] Decision Making and Investment Planning, Managing Infrastructure Assets, vol. 1.1. Federation of Canadian Municipalities and National Research Council, 2004.

[9] M. Gagnon and Statistics Canada, Age of public infrastructure: a provincial perspective. Ottawa: Statistics Canada, 2008. OCLC: 217140521. 
[10] D. J. Vanier, L. A. Newton, and S. Rahman, "A Framework for municipal infrastructure management for Canadian municipalities," tech. rep., National Research Council Canada, 2006.

[11] T. E. El-Diraby and A. Beheshti, "Re-engineering organizations for optimal deployment of smart materials and intelligent devices in civil infrastructure," in Proceedings of SPIE, vol. 4704, 2002.

[12] T. E. El-Diraby, "A Framework for Integrated Data Management in Smart Infrastructure Systems," in Construction Research Congress, pp. 1-8, American Society of Civil Engineers, 2003.

[13] C. R. Farrar and K. Worden, "An Introduction to Structural Health Monitoring," Philosophical Transactions: Mathematical, Physical and Engineering Sciences, vol. 365, no. 1851, pp. 303-315, 2007.

[14] A. Tehranian and D. T. Lau, "Pattern recognition by time series analysis of monitoring vibration responses of bridges," in Proceedings of the 5th European Conference on Structural Control, 2012.

[15] R. T. Ratay, "Structural Condition Assessment An Introduction," in Structures Congress 2006, pp. 1-2, American Society of Civil Engineers, 2006.

[16] J. M. W. Brownjohn, A. De Stefano, Y.-L. Xu, H. Wenzel, and A. E. Aktan, "Vibration-based monitoring of civil infrastructure: challenges and successes," Journal of Civil Structural Health Monitoring, vol. 1, no. 3, pp. 79-95, 2011.

[17] E. Reynders, J. Houbrechts, and G. De Roeck, "Fully automated (operational) modal analysis," Mechanical Systems and Signal Processing, vol. 29, pp. 228-250, 2012 .

[18] F. Ubertini, C. Gentile, and A. L. Materazzi, "Automated modal identification in operational conditions and its application to bridges," Engineering Structures, vol. 46, pp. 264-278, Jan. 2013.

[19] S. Marwitz and V. Zabel, "Relations between the quality of identified modal parameters and measured data obtained by structural monitoring," in ISMA International Conference on Noise and Vibration Engineering, 2018.

[20] B. Peeters, System identification and damage detection in civil engineering. PhD thesis, Katholieke Universiteit Leuven, Leuven, 2000. 
[21] M. S. Rahman, Comparison of system identification techniques with field vibration data for structural health monitoring of bridges. PhD thesis, Carleton University, Ottawa, ON Canada, 2012.

[22] N. A. Londono, Use of vibration data for structural health monitoring of bridges. PhD thesis, Carleton University, Ottawa, ON Canada, 2006.

[23] B. Peeters and C. E. Ventura, "Comparative study of modal analysis techniques for bridge dynamic characteristics," Mechanical Systems and Signal Processing, vol. 17, no. 5, pp. 965-988, 2003.

[24] S. L. Desjardins, Real-time computer platform for vibration-based structural health monitoring of the Confederation Bridge. M.A.Sc. thesis, Carleton University, Ottawa, ON Canada, 2005.

[25] J. B. Jakobsen, Fluctuating Wind Load and Response of a Line-Like Engineering Struture with Emphasis on Motion-Induced Wind Forces. PhD, Dept. of Structural Eng., University of Trondhiem, Trondheim, Norway, 1995.

[26] J.-N. Juang, Applied System Identification. Upper Saddle River, NJ, USA: Prentice-Hall, Inc., 1994.

[27] B. Peeters and G. De Roeck, "Reference based stochastic subspace identification for output-only modal analysis," Mechanical Systems and Signal Processing, vol. 13, no. 6, pp. 855-878, 1999.

[28] N. A. Londono and D. T. Lau, "Verification of the Dynamic Properties of the Confederation Bridge," in Proceedings of the annual conference of the Canadian Society for Civil Engineering, CSCE - Canadian Society for Civil Engineering, 2003.

[29] N. A. Londono, D. T. Lau, and M. Rahman, "Characteristics of dynamic monitoring data and observed behaviour of the Confederation Bridge due to operational load variations," Canadian Journal of Civil Engineering, vol. 40, no. 5, p. 393, 2013.

[30] N. A. Londono, S. L. Desjardins, and D. T. Lau, "Use of stochastic subspace identification methods for post-disaster condition assessment of highway bridges," in Proceedings of the 13th World Conference on Earthquake Engineering, 2004. 
[31] C. Rainieri and G. Fabbrocino, "Influence of model order and number of block rows on accuracy and precision of modal parameter estimates in stochastic subspace identification," International Journal of Lifecycle Performance Engineering, vol. 1, pp. 317-334, Jan. 2014.

[32] E. Reynders and G. D. Roeck, "Reference-based combined deterministic stochastic subspace identification for experimental and operational modal analysis," Mechanical Systems and Signal Processing, vol. 22, pp. 617-637, Apr. 2008.

[33] E. Neu, F. Janser, A. A. Khatibi, and A. C. Orifici, "Fully Automated Operational Modal Analysis using multi-stage clustering," Mechanical Systems and Signal Processing, vol. 84, no. Part A, pp. 308-323, 2017.

[34] R. Allemang, "The modal assurance criterion - Twenty years of use and abuse," Sound Er vibration, vol. 37, pp. 14-23, 2003.

[35] R. Brincker and C. Ventura, Introduction to Operational Modal Analysis. Chichester, West Sussex: Wiley, Sept. 2015.

[36] M. Pastor, M. Binda, and T. Hararik, "Modal Assurance Criterion," Procedia Engineering, vol. 48, pp. 543-548, 2012.

[37] M. S. Cheung, G. S. Tadros, T. Brown, W. H. Dilger, A. Ghali, and D. T. Lau, "Field monitoring and research on performance of the Confederation Bridge," Canadian Journal of Civil Engineering, vol. 24, no. 6, pp. 951-962, 1997.

[38] N. R. Canada, "Reference Maps," Dec. 2014.

[39] M. Montreuil, D. Lau, and T. Brown, "A distributed data acquisition system for monitoring the Confederation Bridge," in Proceedings of the 44 th International Instrumentation Symposium, vol. 44, (Reno, Nevada, U.S.A), pp. 318-333, May 1998.

[40] L. G. Jaeger, A. A. Mufti, G. Tadros, and P. Wong, "Seismic design for the Confederation Bridge," Canadian Journal of Civil Engineering, vol. 24, no. 6, pp. 922-933, 1997.

[41] D. T. Lau, T. Brown, M. S. Cheung, and W. C. Li, "Dynamic modeling and behavior of the Confederation Bridge," Canadian Journal of Civil Engineering, vol. 31, no. 2, pp. 379-390, 2004. 
[42] N. Naumoski, S. Foo, and M. S. Cheung, "Dynamic characteristics of the Confederation Bridge," Canadian Journal of Civil Engineering, vol. 29, no. 3, pp. 448458, 2002.

[43] M. S. Cheung, D. T. Lau, and W. C. Li, "Local vibration of girder box on Confederation Bridge, Internal report," tech. rep., 2004.

[44] M. Zhang, System identification analysis of the dynamic monitoring data of the Confederation Bridge. M.A.Sc. thesis, Carleton University, Ottawa, ON Canada, 2002.

[45] N. Naumoski, M. S. Cheung, and S. Foo, "Dynamic performance of the Confederation Bridge due to traffic and wind," Canadian Journal of Civil Engineering, vol. 31, no. 3, pp. 487-498, 2004.

[46] N. Londono and D. Lau, "Variability of dynamic properties from Confederation Bridge monitoring data," in Proceedings of the 1st International Conference on Structural Health Monitoring and Intelligent Infrastructure, vol. 2, (Tokyo, Japan), pp. 543-550, 2003.

[47] S. L. Desjardins, N. A. Londono, D. T. Lau, and H. Khoo, "Real-Time Data Processing, Analysis and Visualization for Structural Monitoring of the Confederation Bridge," Advances in Structural Engineering, vol. 9, pp. 141-157, Feb. 2006.

[48] A. Ghali, M. Elbadry, and S. Megally, "Two-year deflections of the Confederation Bridge," Canadian Journal of Civil Engineering, vol. 27, no. 6, pp. 1139-1149, 2000 .

[49] R. O'Connell, Advances in intelligent continuous structural health monitoring applications of bridges. M.A.Sc. thesis, Carleton University, Ottawa, ON Canada, 2009.

[50] A. McGregor, D. Bennett, S. Majumdar, B. Nandy, J. Melendez, M. St-Hilaire, D. Lau, and J. Liu, "A Cloud-Based Platform for Supporting Research Collaboration," in The Institute of Electrical and Electronics Engineers, Inc. (IEEE) Conference Proceedings, p. 1107, The Institute of Electrical and Electronics Engineers, Inc. (IEEE), June 2015.

[51] R. Sears, C. van Ingen, and J. Gray, "To BLOB or Not To BLOB: Large Object Storage in a Database or a Filesystem?," Technical report MSR-TR-2006-45, 
Microsoft Research Microsoft Corporation, One Microsoft Way Redmond, WA 98052, Apr. 2006.

[52] Mathworks, "Signal processing toolbox, v.7 (R2018b)," Matlab 9.5, 2018.

[53] S. W. Doebling, C. R. Farrar, M. B. Prime, and D. W. Shevitz, "Damage identification and health monitoring of structural and mechanical systems from changes in their vibration characteristics: A literature review," Tech. Rep. LA-13070-MS, Los Alamos National Lab., NM (United States), May 1996.

[54] C. R. Farrar, T. A. Duffey, S. W. Doebling, and D. A. Nix, "A Statistical Pattern Recognition Paradigm for Vibration-Based Structural Health Monitoring," in Proceedings of the 2 nd International Workshop on Structural Health Monitoring, pp. 764-773, 1999.

[55] Z. Duan and K. Zhang, "Data Mining Technology for Structural Health Monitoring," Pacific Science Review, vol. 8, pp. 27-36, 2006.

[56] T.-H. Yi, S. C. Stiros, X.-W. Ye, and J. Li, "Structural Health MonitoringOriented Data Mining, Feature Extraction, and Condition Assessment," Mathematical Problems in Engineering, vol. 2014, no. Article ID 941780, p. 3, 2014.

[57] A. H. Jazwinski, Stochastic Processes and Filtering Theory. Mineola, N.Y: Dover Publications, Nov. 2007.

[58] D. Simon, Optimal State Estimation: Kalman, H Infinity, and Nonlinear Approaches. Hoboken, N.J: Wiley-Interscience, 1 edition ed., June 2006.

[59] B. D. O. Anderson and J. B. Moore, Optimal Filtering. Mineola, NY: Dover Publications, Jan. 2005.

[60] M. Lavielle, "Using penalized contrasts for the change-point problem," Signal Processing, vol. 85, pp. 1501-1510, Aug. 2005.

[61] R. Killick, P. Fearnhead, and I. A. Eckley, "Optimal detection of changepoints with a linear computational cost," Journal of the American Statistical Association, vol. 107, pp. 1590-1598, Dec. 2012. arXiv: 1101.1438.

[62] D. T. Lau, Y.-Y. Chang, and N. A. Londono, "Experimental determination of modal properties of lamppost from Confederation Bridge," Technical report rv13, Ottawa-Carleton Bridge Research Institute, Ottawa, Apr. 2002. 
[63] R. Briden, "A guide to the selection, installation and maintenance including the cause and effects of pole vibration," White Paper WP513001EN, Eaton, Peachtree City, GA, Jan. 2016.

[64] L. Caracoglia and N. P. Jones, "Numerical and experimental study of vibration mitigation for highway light poles," Engineering Structures, vol. 29, pp. 821-831, May 2007.

[65] R. A. Cook, D. Bloomquist, D. S. Richard, and M. A. Kalajian, "Damping of Cantilevered Traffic Signal Structures," Journal of Structural Engineering, vol. 127, pp. 1476-1483, Dec. 2001.

[66] SAFI, "SAFI Structural Analysis and Design Software," 2018.

[67] D. Systemes, "SOLIDWORKS 3d CAD Design Software," 2018.

[68] P. Welch, "The use of fast Fourier transform for the estimation of power spectra: A method based on time averaging over short, modified periodograms," Audio and Electroacoustics, IEEE Transactions on, vol. 15, no. 2, pp. 70-73, 1967.

[69] ASTM, ASTM Standard E 1049, 1985 (2011). Standard Practices for Cycle Counting in Fatigue Analysis., West Conshohocken, PA: ASTM International, 2011.

[70] AASHTO, LRFD Specifications for Structural Supports for Highway Signs. Washington D, 2015.

[71] \{Canadian Standards Association\}, CAN/CSA S16-09 - Limit states design of steel structures. Mississauga, Ontario, Canada, Sept. 2009.

[72] A. Azizinamini, N. Ala, and E. H. Power, "Predicting the Service Life of Sliding Surfaces in Bridge Bearings," Journal of Bridge Engineering, vol. 21, no. 2, p. 4015035, 2016.

[73] J. K. Lee, Evaluation of external post-tensioned tendons using vibration signatures. PhD Thesis, University of Texas at Austin, Austin, Texas, U.S.A., 2007.

[74] C. A. McKinstry, Frequency response of damaged external post-tensioned tendons. M.A.Sc. thesis, University of Texas at Austin, Austin, Texas, U.S.A., Oct. 2010.

[75] R. Geier, G. De Roeck, and R. Flesch, "Accurate cable force determination using ambient vibration measurements," Structure and Infrastructure Engineering, vol. 2, no. 1, pp. 43-52, 2006. 
[76] Y. Namita, H. Zui, and T. Shinke, "Practical Formulas for Estimation of Cable Tension by Vibration Method," Journal of Structural Engineering, vol. 122, no. 6, pp. 651-656, 1996.

[77] A. A. Sagues, S. C. Kranc, and T. G. Eason, "Vibrational Tension Measurement of External Tendons in Segmental Post tensioned Bridges," Journal of Bridge Engineering, vol. 11, no. 5, pp. 582-589, 2006.

[78] H.-Q. Mu and K.-V. Yuen, "Novel Outlier-Resistant Extended Kalman Filter for Robust Online Structural Identification," Journal of Engineering Mechanics, vol. 141, no. 1, p. 4014100, 2015.

[79] N. Y. Khan, Simulation Of Damage Vibration Response Of Structures With Realistic Field Uncertainties. M.A.Sc. thesis, Carleton University, Ottawa, ON Canada, 2018. 
Appendix A

\section{Operational Mode Shapes}




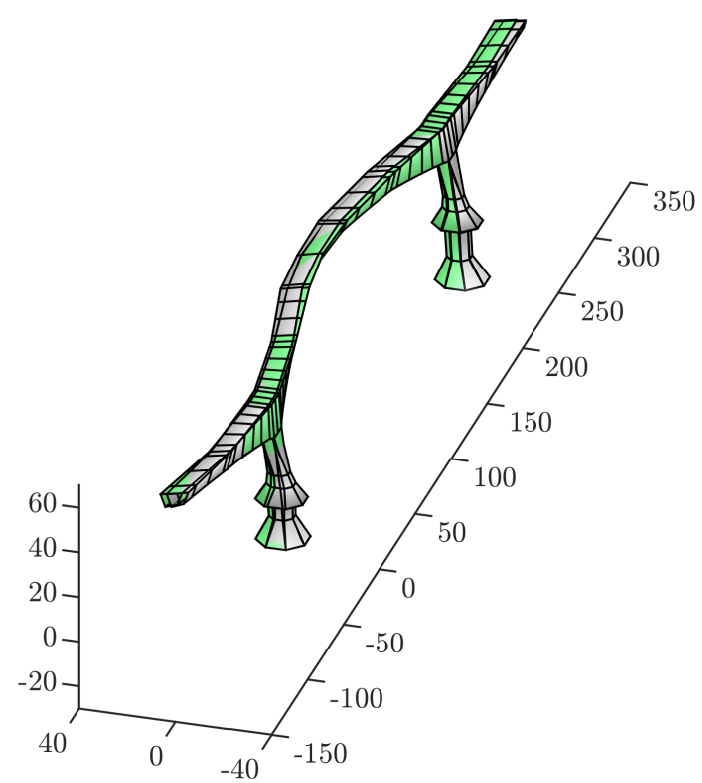

(a) Isometric view.

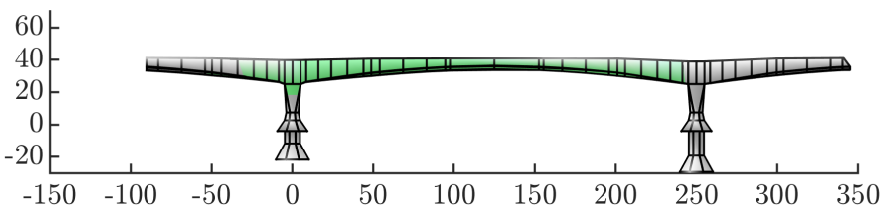

(c) Side elevation.

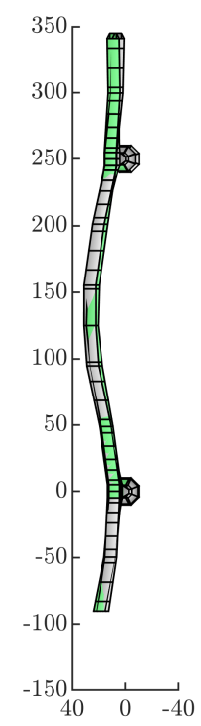

(b) Top view.

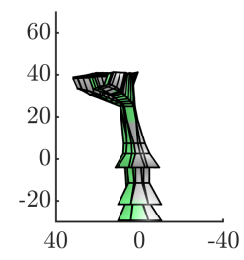

(d) Section view.

Figure A.1: Mode-shape comparison of transverse $0.47 \mathrm{~Hz}$ mode. Grey model with full lines represents extracted mode-shapes while green model with dashed lines represents the mode-shape result from the analytical $3 \mathrm{D}$ beam model (MAC $=0.972)$. 

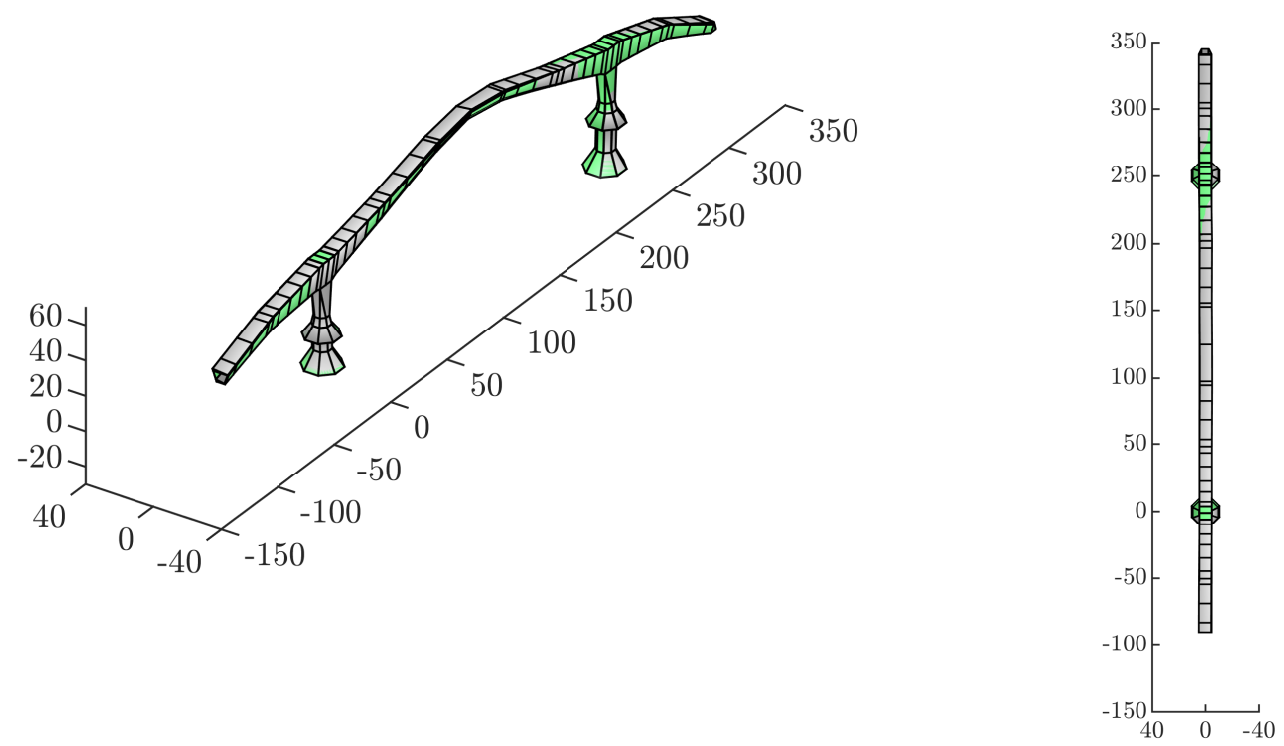

(a) Isometric view.

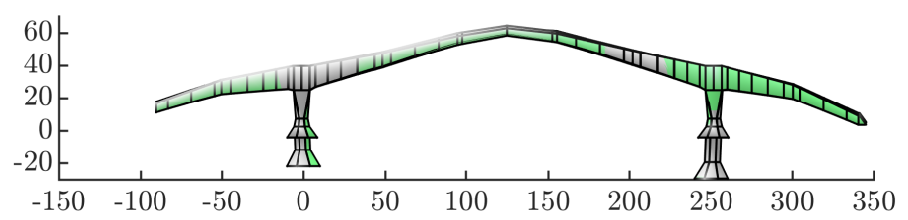

(c) Side elevation. (b) Top view.

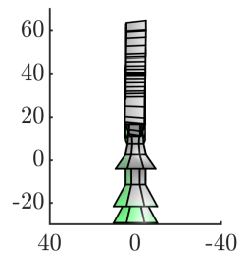

(d) Section view.

Figure A.2: Mode-shape comparison of transverse $0.67 \mathrm{~Hz}$ mode. Grey model with full lines represents extracted mode-shapes while green model with dashed lines represents the mode-shape result from the analytical $3 \mathrm{D}$ beam model (MAC $=0.995)$. 

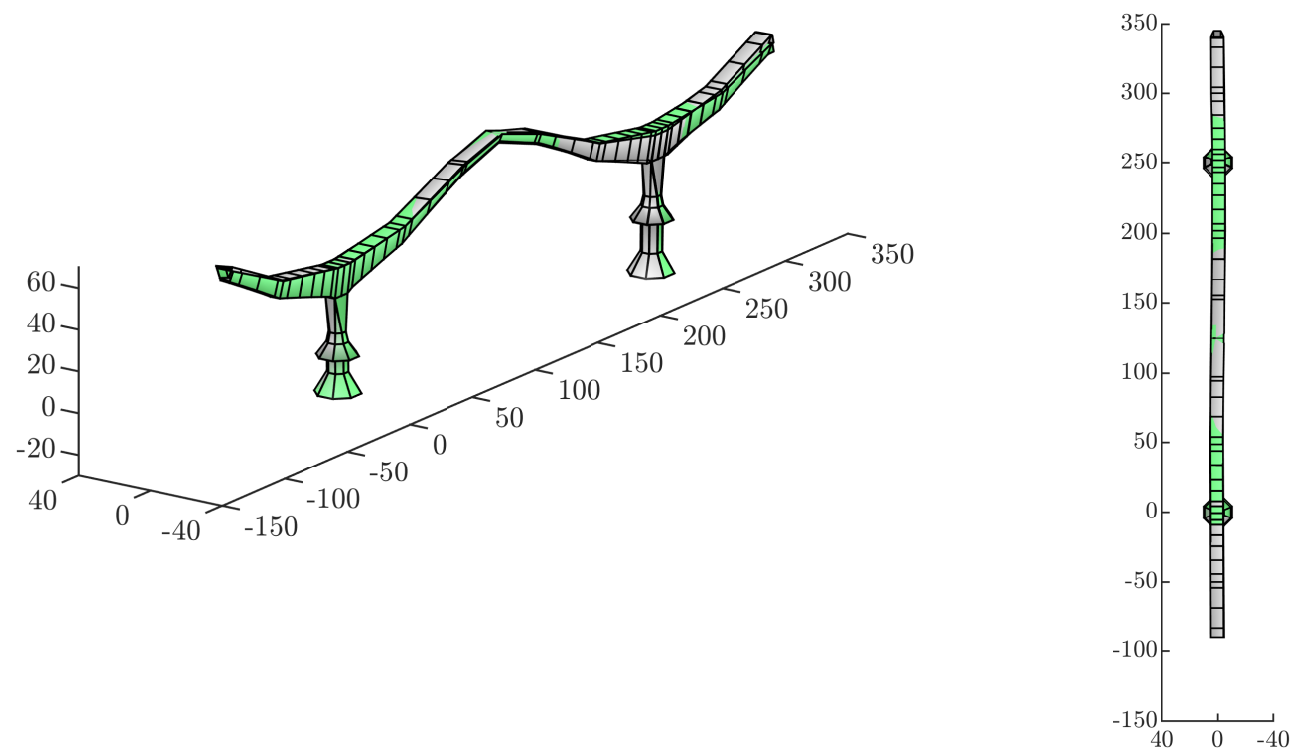

(a) Isometric view.

(b) Top view.
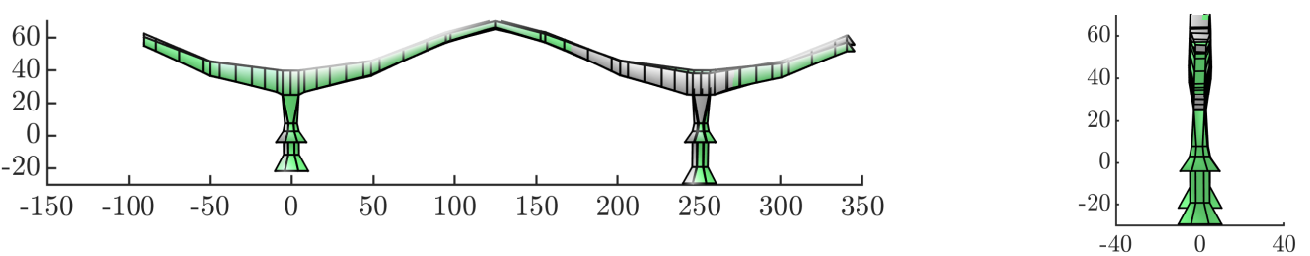

(c) Side elevation.

(d) Section view.

Figure A.3: Mode-shape comparison of transverse $0.96 \mathrm{~Hz}$ mode. Grey model with full lines represents extracted mode-shapes while green model with dashed lines represents the mode-shape result from the analytical 3D beam model (MAC $=0.993)$. 


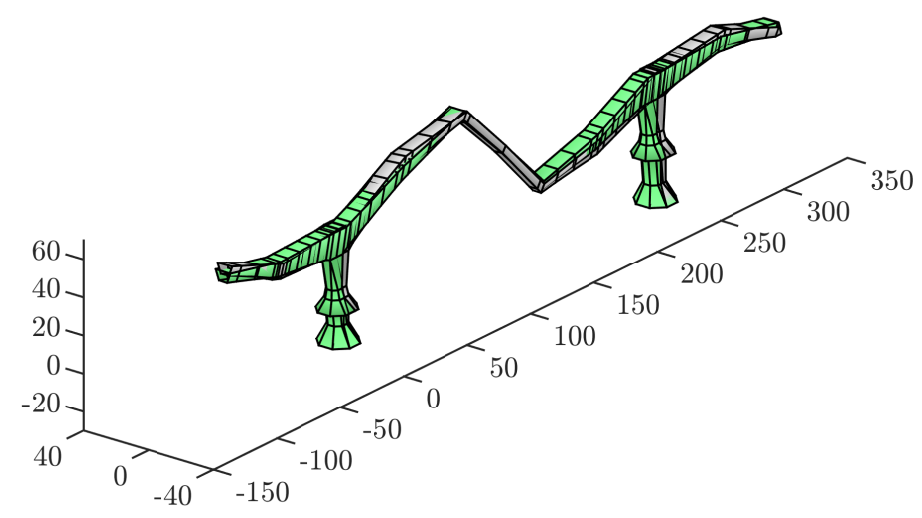

(a) Isometric view.
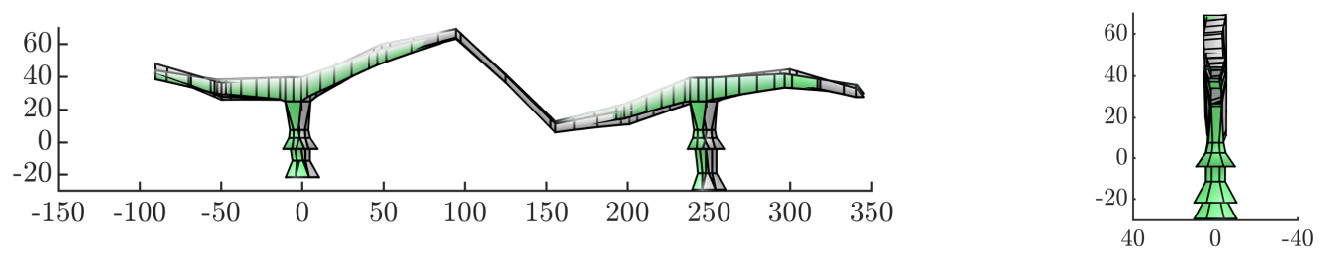

(c) Side elevation.

(d) Section view.

Figure A.4: Mode-shape comparison of transverse $1.62 \mathrm{~Hz}$ mode. Grey model with full lines represents extracted mode-shapes while green model with dashed lines represents the mode-shape result from the analytical $3 \mathrm{D}$ beam model (MAC $=0.945)$. 

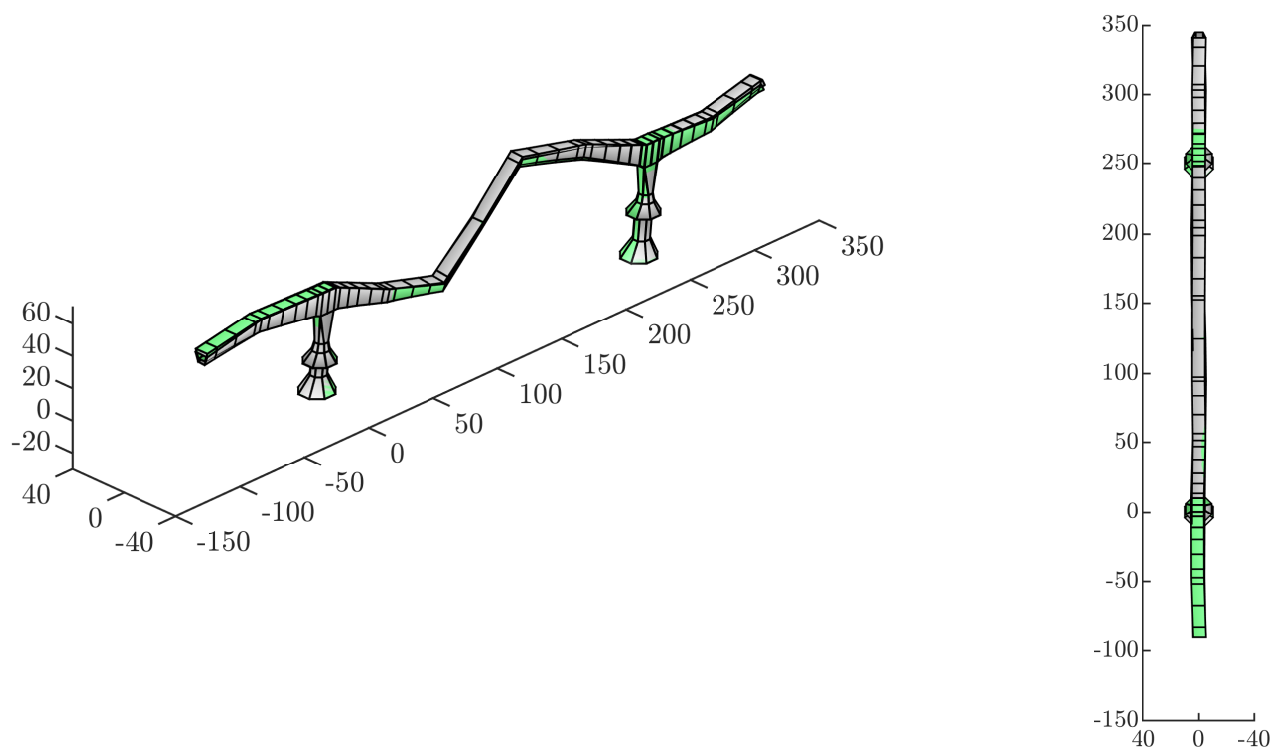

(a) Isometric view.

(b) Top view.
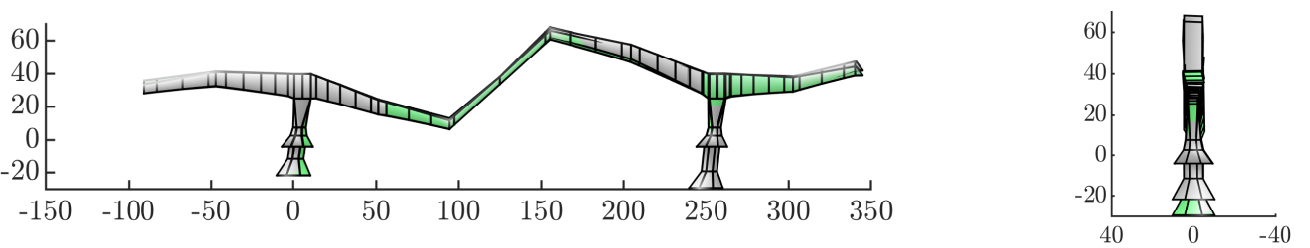

(c) Side elevation.

(d) Section view.

Figure A.5: Mode-shape comparison of transverse $1.85 \mathrm{~Hz}$ mode. Grey model with full lines represents extracted mode-shapes while green model with dashed lines represents the mode-shape result from the analytical 3D beam model (MAC $=0.976)$. 


\section{Appendix B}

\section{Linear regression parameter estimations}

Table B.1: Linear regression coefficients for modal frequencies of $0.47 \mathrm{~Hz}$ mode, ordered according to significance.

\begin{tabular}{|c|c|c|c|c|c|}
\hline Coefficient Name & $\beta$ & $\mathrm{SE}$ & tStat & pValue & $\begin{array}{r}95 \% \text { c.i. } \\
\text { for } \beta(\%)\end{array}$ \\
\hline OnesConstant & $4.314 \times 10^{-1}$ & $6.117 \times 10^{-3}$ & 70.5 & 0.000 & 2.8 \\
\hline ModeIdentifications & $8.886 \times 10^{-5}$ & $4.320 \times 10^{-6}$ & 20.6 & $1.166 \times 10^{-88}$ & 9.5 \\
\hline WindSpeed & $9.162 \times 10^{-5}$ & $7.779 \times 10^{-6}$ & 11.8 & $2.070 \times 10^{-31}$ & 16.6 \\
\hline ModalMAC & $1.993 \times 10^{-2}$ & $4.317 \times 10^{-3}$ & 4.6 & $4.060 \times 10^{-6}$ & 42.5 \\
\hline Dotw_Wed & $-1.412 \times 10^{-3}$ & $3.182 \times 10^{-4}$ & -4.4 & $9.383 \times 10^{-6}$ & 155.8 \\
\hline Dotw_Mon & $-1.072 \times 10^{-3}$ & $3.028 \times 10^{-4}$ & -3.5 & $4.033 \times 10^{-4}$ & 144.6 \\
\hline SensorsCombCat_SensorConfig_81 & $2.148 \times 10^{-2}$ & $6.502 \times 10^{-3}$ & 3.3 & $9.625 \times 10^{-4}$ & 59.3 \\
\hline SensorsCombCat_SensorConfig_52 & $1.797 \times 10^{-2}$ & $5.606 \times 10^{-3}$ & 3.2 & $1.357 \times 10^{-3}$ & 61.2 \\
\hline Dotw_Thu & $-8.785 \times 10^{-4}$ & $3.090 \times 10^{-4}$ & -2.8 & $4.490 \times 10^{-3}$ & 131 \\
\hline SensorsCombCat_SensorConfig_102 & $1.835 \times 10^{-2}$ & $6.496 \times 10^{-3}$ & 2.8 & $4.758 \times 10^{-3}$ & 69.4 \\
\hline AirTemperature & $3.878 \times 10^{-5}$ & $1.725 \times 10^{-5}$ & 2.2 & $2.464 \times 10^{-2}$ & 87.2 \\
\hline SensorsCombCat_SensorConfig_92 & $-1.368 \times 10^{-2}$ & $6.461 \times 10^{-3}$ & -2.1 & $3.429 \times 10^{-2}$ & 107.4 \\
\hline Hour_20 & $-1.129 \times 10^{-3}$ & $5.467 \times 10^{-4}$ & -2.1 & $3.898 \times 10^{-2}$ & 105.1 \\
\hline Hour_10 & $1.089 \times 10^{-3}$ & $5.543 \times 10^{-4}$ & 2.0 & $4.949 \times 10^{-2}$ & 99.8 \\
\hline
\end{tabular}


Table B.2: Analysis of variance of the linear regression model for modal frequencies of the $0.47 \mathrm{~Hz}$ mode.

\begin{tabular}{|c|c|c|c|c|c|}
\hline Coefficient:Combination & SumSq & $\mathrm{DF}$ & MeanSq & $\mathrm{F}$ test & pValue \\
\hline ModeIdentifications & $8.731 \times 10^{-3}$ & 1 & $8.731 \times 10^{-3}$ & 423.1 & $1.162 \times 10^{-88}$ \\
\hline WindSpeed & $2.863 \times 10^{-3}$ & 1 & $2.863 \times 10^{-3}$ & 138.7 & $2.069 \times 10^{-31}$ \\
\hline SensorsCombCat & $4.147 \times 10^{-3}$ & 106 & $3.912 \times 10^{-5}$ & 1.9 & $1.476 \times 10^{-7}$ \\
\hline ModalMAC & $4.397 \times 10^{-4}$ & 1 & $4.397 \times 10^{-4}$ & 21.3 & $4.060 \times 10^{-6}$ \\
\hline Dotw & $6.622 \times 10^{-4}$ & 6 & $1.104 \times 10^{-4}$ & 5.3 & $1.666 \times 10^{-5}$ \\
\hline Hour & $9.713 \times 10^{-4}$ & 23 & $4.223 \times 10^{-5}$ & 2.0 & $2.321 \times 10^{-3}$ \\
\hline AirTemperature & $1.043 \times 10^{-4}$ & 1 & $1.043 \times 10^{-4}$ & 5.1 & $2.464 \times 10^{-2}$ \\
\hline rmsTransverse & $5.796 \times 10^{-5}$ & 1 & $5.796 \times 10^{-5}$ & 2.8 & $9.388 \times 10^{-2}$ \\
\hline rmsVertical & $5.541 \times 10^{-5}$ & 1 & $5.541 \times 10^{-5}$ & 2.7 & $1.014 \times 10^{-1}$ \\
\hline Error & $6.966 \times 10^{-2}$ & 3375 & $2.064 \times 10^{-5}$ & 1.0 & $5.000 \times 10^{-1}$ \\
\hline
\end{tabular}

Table B.3: Linear regression coefficients for modal frequencies of $0.67 \mathrm{~Hz}$ mode, ordered according to significance.

\begin{tabular}{|c|c|c|c|c|c|}
\hline Coefficient Name & $\beta$ & $\mathrm{SE}$ & tStat & pValue & $\begin{array}{r}95 \% \text { c.i. } \\
\text { for } \beta(\%)\end{array}$ \\
\hline OnesConstant & $7.184 \times 10^{-1}$ & $7.506 \times 10^{-3}$ & 95.7 & 0.000 & 2.0 \\
\hline WindSpeed & $-2.195 \times 10^{-4}$ & $8.325 \times 10^{-6}$ & -26.4 & $2.828 \times 10^{-139}$ & 192.6 \\
\hline ModeIdentifications & $-3.844 \times 10^{-5}$ & $3.107 \times 10^{-6}$ & -12.4 & $2.124 \times 10^{-34}$ & 184.2 \\
\hline ModalMAC & $-4.066 \times 10^{-2}$ & $5.122 \times 10^{-3}$ & -7.9 & $2.799 \times 10^{-15}$ & 175.3 \\
\hline rmsTransverse & -1.598 & $2.175 \times 10^{-1}$ & -7.3 & $2.549 \times 10^{-13}$ & 173.3 \\
\hline Dotw_Sat & $-2.456 \times 10^{-3}$ & $3.760 \times 10^{-4}$ & -6.5 & $7.494 \times 10^{-11}$ & 170.0 \\
\hline rmsVertical & $5.031 \times 10^{-1}$ & $8.100 \times 10^{-2}$ & 6.2 & $5.925 \times 10^{-10}$ & 31.6 \\
\hline SensorsCombCat_SensorConfig_73 & $3.059 \times 10^{-2}$ & $8.091 \times 10^{-3}$ & 3.8 & $1.592 \times 10^{-4}$ & 51.9 \\
\hline Dotw_Mon & $1.398 \times 10^{-3}$ & $3.785 \times 10^{-4}$ & 3.7 & $2.256 \times 10^{-4}$ & 53.1 \\
\hline Hour_7 & $2.413 \times 10^{-3}$ & $6.924 \times 10^{-4}$ & 3.5 & $4.991 \times 10^{-4}$ & 56.3 \\
\hline SensorsCombCat_SensorConfig_8 & $2.639 \times 10^{-2}$ & $8.090 \times 10^{-3}$ & 3.3 & $1.120 \times 10^{-3}$ & 60.1 \\
\hline Dotw_Sun & $-1.146 \times 10^{-3}$ & $3.909 \times 10^{-4}$ & -2.9 & $3.399 \times 10^{-3}$ & 133.1 \\
\hline SensorsCombCat_SensorConfig_15 & $1.867 \times 10^{-2}$ & $6.678 \times 10^{-3}$ & 2.8 & $5.198 \times 10^{-3}$ & 70.1 \\
\hline Hour_8 & $1.835 \times 10^{-3}$ & $6.930 \times 10^{-4}$ & 2.6 & $8.138 \times 10^{-3}$ & 74.0 \\
\hline SensorsCombCat_SensorConfig_58 & $1.661 \times 10^{-2}$ & $6.615 \times 10^{-3}$ & 2.5 & $1.209 \times 10^{-2}$ & 78.1 \\
\hline SensorsCombCat_SensorConfig_84 & $2.019 \times 10^{-2}$ & $8.090 \times 10^{-3}$ & 2.5 & $1.263 \times 10^{-2}$ & 78.6 \\
\hline SensorsCombCat_SensorConfig_50 & $2.002 \times 10^{-2}$ & $8.092 \times 10^{-3}$ & 2.5 & $1.342 \times 10^{-2}$ & 79.3 \\
\hline SensorsCombCat_SensorConfig_61 & $1.964 \times 10^{-2}$ & $8.089 \times 10^{-3}$ & 2.4 & $1.525 \times 10^{-2}$ & 80.8 \\
\hline SensorsCombCat_SensorConfig_25 & $1.404 \times 10^{-2}$ & $5.786 \times 10^{-3}$ & 2.4 & $1.527 \times 10^{-2}$ & 80.8 \\
\hline
\end{tabular}


Table B.3 (continued). Linear regression coefficients for modal frequencies of $0.67 \mathrm{~Hz}$ mode, ordered according to significance.

\begin{tabular}{|c|c|c|c|c|c|}
\hline Coefficient Name & $\beta$ & $\mathrm{SE}$ & tStat & pValue & $\begin{array}{r}95 \% \text { c.i. } \\
\text { for } \beta(\%)\end{array}$ \\
\hline SensorsCombCat_SensorConfig_16 & $1.672 \times 10^{-2}$ & $7.028 \times 10^{-3}$ & 2.4 & $1.741 \times 10^{-2}$ & 82.4 \\
\hline SensorsCombCat_SensorConfig_80 & $1.911 \times 10^{-2}$ & $8.082 \times 10^{-3}$ & 2.4 & $1.811 \times 10^{-2}$ & 82.9 \\
\hline SensorsCombCat_SensorConfig_76 & $1.455 \times 10^{-2}$ & $6.271 \times 10^{-3}$ & 2.3 & $2.041 \times 10^{-2}$ & 84.5 \\
\hline SensorsCombCat_SensorConfig_23 & $1.346 \times 10^{-2}$ & $5.822 \times 10^{-3}$ & 2.3 & $2.084 \times 10^{-2}$ & 84.8 \\
\hline SensorsCombCat_SensorConfig_14 & $1.862 \times 10^{-2}$ & $8.091 \times 10^{-3}$ & 2.3 & $2.145 \times 10^{-2}$ & 85.2 \\
\hline SensorsCombCat_SensorConfig_34 & $1.828 \times 10^{-2}$ & $8.077 \times 10^{-3}$ & 2.3 & $2.371 \times 10^{-2}$ & 86.6 \\
\hline SensorsCombCat_SensorConfig_26 & $1.287 \times 10^{-2}$ & $5.723 \times 10^{-3}$ & 2.2 & $2.463 \times 10^{-2}$ & 87.2 \\
\hline SensorsCombCat_SensorConfig_22 & $1.707 \times 10^{-2}$ & $8.090 \times 10^{-3}$ & 2.1 & $3.490 \times 10^{-2}$ & 92.9 \\
\hline Hour_10 & $1.440 \times 10^{-3}$ & $6.958 \times 10^{-4}$ & 2.1 & $3.859 \times 10^{-2}$ & 94.8 \\
\hline SensorsCombCat_SensorConfig_52 & $1.656 \times 10^{-2}$ & $8.086 \times 10^{-3}$ & 2.0 & $4.061 \times 10^{-2}$ & 95.7 \\
\hline SensorsCombCat_SensorConfig_5 & $1.193 \times 10^{-2}$ & $5.826 \times 10^{-3}$ & 2.0 & $4.064 \times 10^{-2}$ & 95.7 \\
\hline SensorsCombCat_SensorConfig_20 & $1.249 \times 10^{-2}$ & $6.104 \times 10^{-3}$ & 2.0 & $4.075 \times 10^{-2}$ & 95.8 \\
\hline SensorsCombCat_SensorConfig_82 & $1.653 \times 10^{-2}$ & $8.080 \times 10^{-3}$ & 2.0 & $4.088 \times 10^{-2}$ & 95.9 \\
\hline SensorsCombCat_SensorConfig_67 & $1.637 \times 10^{-2}$ & $8.087 \times 10^{-3}$ & 2.0 & $4.305 \times 10^{-2}$ & 96.9 \\
\hline Dotw_Thu & $-7.771 \times 10^{-4}$ & $3.883 \times 10^{-4}$ & -2.0 & $4.544 \times 10^{-2}$ & 102.0 \\
\hline SensorsCombCat_SensorConfig_54 & $1.178 \times 10^{-2}$ & $5.937 \times 10^{-3}$ & 2.0 & $4.728 \times 10^{-2}$ & 98.8 \\
\hline
\end{tabular}

Table B.4: Analysis of variance of the linear regression model for modal frequencies of the $0.67 \mathrm{~Hz}$ mode.

\begin{tabular}{|c|c|c|c|c|c|}
\hline Coefficient:Combination & SumSq & DF & MeanSq & $F$ test & pValue \\
\hline WindSpeed & $2.245 \times 10^{-2}$ & 1 & $2.245 \times 10^{-2}$ & 695.2 & $2.804 \times 10^{-139}$ \\
\hline ModeIdentifications & $4.942 \times 10^{-3}$ & 1 & $4.942 \times 10^{-3}$ & 153.0 & $2.123 \times 10^{-34}$ \\
\hline Dotw & $4.093 \times 10^{-3}$ & 6 & $6.822 \times 10^{-4}$ & 21.1 & $1.876 \times 10^{-24}$ \\
\hline ModalMAC & $2.034 \times 10^{-3}$ & 1 & $2.034 \times 10^{-3}$ & 63.0 & $2.799 \times 10^{-15}$ \\
\hline rmsTransverse & $1.743 \times 10^{-3}$ & 1 & $1.743 \times 10^{-3}$ & 54.0 & $2.548 \times 10^{-13}$ \\
\hline SensorsCombCat & $6.797 \times 10^{-3}$ & 83 & $8.190 \times 10^{-5}$ & 2.5 & $1.612 \times 10^{-12}$ \\
\hline rmsVertical & $1.246 \times 10^{-3}$ & 1 & $1.246 \times 10^{-3}$ & 38.6 & $5.924 \times 10^{-10}$ \\
\hline Hour & $2.028 \times 10^{-3}$ & 23 & $8.816 \times 10^{-5}$ & 2.7 & $1.706 \times 10^{-5}$ \\
\hline AirTemperature & $2.289 \times 10^{-6}$ & 1 & $2.289 \times 10^{-6}$ & 0.1 & $7.901 \times 10^{-1}$ \\
\hline Error & $1.073 \times 10^{-1}$ & 3324 & $3.229 \times 10^{-5}$ & 1.0 & $5.000 \times 10^{-1}$ \\
\hline
\end{tabular}


Table B.5: Linear regression coefficients for modal frequencies of $0.96 \mathrm{~Hz}$ mode, ordered according to significance.

\begin{tabular}{|c|c|c|c|c|c|}
\hline Coefficient Name & $\beta$ & $\mathrm{SE}$ & tStat & pValue & $\begin{array}{r}95 \% \text { c.i. } \\
\text { for } \beta(\%)\end{array}$ \\
\hline OnesConstant & $9.412 \times 10^{-1}$ & $8.851 \times 10^{-3}$ & 106.3 & 0.000 & 1.8 \\
\hline ModeIdentifications & $4.833 \times 10^{-5}$ & $3.485 \times 10^{-6}$ & 13.9 & $2.312 \times 10^{-42}$ & 14.1 \\
\hline rmsTransverse & -3.394 & $3.434 \times 10^{-1}$ & -9.9 & $1.162 \times 10^{-22}$ & 180.2 \\
\hline ModalMAC & $2.414 \times 10^{-2}$ & $3.934 \times 10^{-3}$ & 6.1 & $9.576 \times 10^{-10}$ & 31.9 \\
\hline AirTemperature & $-2.032 \times 10^{-4}$ & $3.425 \times 10^{-5}$ & -5.9 & $3.331 \times 10^{-9}$ & 167.0 \\
\hline Dotw_Sun & $-3.180 \times 10^{-3}$ & $6.047 \times 10^{-4}$ & -5.3 & $1.555 \times 10^{-7}$ & 162.7 \\
\hline rmsVertical & $6.912 \times 10^{-1}$ & $1.318 \times 10^{-1}$ & 5.2 & $1.681 \times 10^{-7}$ & 37.4 \\
\hline Hour_7 & $4.455 \times 10^{-3}$ & $1.102 \times 10^{-3}$ & 4.0 & $5.464 \times 10^{-5}$ & 48.5 \\
\hline Dotw_Wed & $-2.243 \times 10^{-3}$ & $5.976 \times 10^{-4}$ & -3.8 & $1.774 \times 10^{-4}$ & 147.8 \\
\hline Hour_13 & $4.090 \times 10^{-3}$ & $1.123 \times 10^{-3}$ & 3.6 & $2.750 \times 10^{-4}$ & 53.8 \\
\hline Hour_16 & $3.928 \times 10^{-3}$ & $1.122 \times 10^{-3}$ & 3.5 & $4.691 \times 10^{-4}$ & 56.0 \\
\hline Dotw_Sat & $-2.086 \times 10^{-3}$ & $5.977 \times 10^{-4}$ & -3.5 & $4.919 \times 10^{-4}$ & 143.8 \\
\hline Hour_14 & $3.855 \times 10^{-3}$ & $1.120 \times 10^{-3}$ & 3.4 & $5.859 \times 10^{-4}$ & 57.0 \\
\hline Hour_9 & $3.711 \times 10^{-3}$ & $1.103 \times 10^{-3}$ & 3.4 & $7.774 \times 10^{-4}$ & 58.3 \\
\hline Dotw_Thu & $-1.989 \times 10^{-3}$ & $5.965 \times 10^{-4}$ & -3.3 & $8.689 \times 10^{-4}$ & 141.2 \\
\hline Hour_10 & $3.624 \times 10^{-3}$ & $1.090 \times 10^{-3}$ & 3.3 & $8.982 \times 10^{-4}$ & 59.0 \\
\hline Hour_15 & $3.501 \times 10^{-3}$ & $1.104 \times 10^{-3}$ & 3.2 & $1.529 \times 10^{-3}$ & 61.8 \\
\hline Hour_11 & $2.846 \times 10^{-3}$ & $1.099 \times 10^{-3}$ & 2.6 & $9.638 \times 10^{-3}$ & 75.7 \\
\hline Hour_12 & $2.831 \times 10^{-3}$ & $1.111 \times 10^{-3}$ & 2.5 & $1.088 \times 10^{-2}$ & 76.9 \\
\hline Hour_8 & $2.772 \times 10^{-3}$ & $1.096 \times 10^{-3}$ & 2.5 & $1.151 \times 10^{-2}$ & 77.5 \\
\hline Hour_17 & $2.396 \times 10^{-3}$ & $1.097 \times 10^{-3}$ & 2.2 & $2.899 \times 10^{-2}$ & 89.8 \\
\hline Hour_6 & $2.438 \times 10^{-3}$ & $1.122 \times 10^{-3}$ & 2.2 & $2.982 \times 10^{-2}$ & 90.2 \\
\hline Dotw_Tue & $-1.235 \times 10^{-3}$ & $5.968 \times 10^{-4}$ & -2.1 & $3.865 \times 10^{-2}$ & 105.2 \\
\hline WindSpeed & $-2.603 \times 10^{-5}$ & $1.336 \times 10^{-5}$ & -1.9 & $5.149 \times 10^{-2}$ & 99.4 \\
\hline
\end{tabular}

Table B.6: Analysis of variance of the linear regression model for modal frequencies of the $0.96 \mathrm{~Hz}$ mode.

\begin{tabular}{|c|c|c|c|c|c|}
\hline Coefficient:Combination & SumSq & $\mathrm{DF}$ & MeanSq & F test & pValue \\
\hline ModeIdentifications & $1.274 \times 10^{-2}$ & 1 & $1.274 \times 10^{-2}$ & 192.4 & $2.309 \times 10^{-42}$ \\
\hline rmsTransverse & $6.465 \times 10^{-3}$ & 1 & $6.465 \times 10^{-3}$ & 97.6 & $1.162 \times 10^{-22}$ \\
\hline ModalMAC & $2.494 \times 10^{-3}$ & 1 & $2.494 \times 10^{-3}$ & 37.7 & $9.575 \times 10^{-10}$ \\
\hline AirTemperature & $2.331 \times 10^{-3}$ & 1 & $2.331 \times 10^{-3}$ & 35.2 & $3.331 \times 10^{-9}$ \\
\hline Dotw & $3.092 \times 10^{-3}$ & 6 & $5.153 \times 10^{-4}$ & 7.8 & $2.523 \times 10^{-8}$ \\
\hline
\end{tabular}


Table B.6 (continued). Analysis of variance of the linear regression model for modal frequencies of the $0.96 \mathrm{~Hz}$ mode.

\begin{tabular}{|c|c|c|c|c|c|}
\hline Coefficient:Combination & SumSq & $\mathrm{DF}$ & MeanSq & $F$ test & pValue \\
\hline Hour & $5.090 \times 10^{-3}$ & 23 & $2.213 \times 10^{-4}$ & 3.3 & $1.328 \times 10^{-7}$ \\
\hline rmsVertical & $1.821 \times 10^{-3}$ & 1 & $1.821 \times 10^{-3}$ & 27.5 & $1.681 \times 10^{-7}$ \\
\hline SensorsCombCat & $5.263 \times 10^{-3}$ & 52 & $1.012 \times 10^{-4}$ & 1.5 & $9.101 \times 10^{-3}$ \\
\hline WindSpeed & $2.513 \times 10^{-4}$ & 1 & $2.513 \times 10^{-4}$ & 3.8 & $5.149 \times 10^{-2}$ \\
\hline Error & $1.866 \times 10^{-1}$ & 2818 & $6.620 \times 10^{-5}$ & 1.0 & $5.000 \times 10^{-1}$ \\
\hline
\end{tabular}

Table B.7: Linear regression coefficients for modal frequencies of $1.62 \mathrm{~Hz}$ mode, ordered according to significance.

\begin{tabular}{|c|c|c|c|c|c|}
\hline Coefficient Name & $\beta$ & $\mathrm{SE}$ & tStat & pValue & $\begin{array}{r}95 \% \text { c.i. } \\
\text { for } \beta(\%)\end{array}$ \\
\hline OnesConstant & 1.550 & $1.392 \times 10^{-2}$ & 111.376 & 0.000 & 1.8 \\
\hline ModeIdentifications & $1.130 \times 10^{-4}$ & $5.464 \times 10^{-6}$ & 20.681 & $2.858 \times 10^{-89}$ & 9.5 \\
\hline AirTemperature & $-5.428 \times 10^{-4}$ & $4.380 \times 10^{-5}$ & -12.393 & $1.755 \times 10^{-34}$ & 184.2 \\
\hline rmsVertical & 1.731 & $1.695 \times 10^{-1}$ & 10.212 & $4.098 \times 10^{-24}$ & 19.2 \\
\hline rmsTransverse & -3.886 & $4.423 \times 10^{-1}$ & -8.785 & $2.509 \times 10^{-18}$ & 177.7 \\
\hline ModalMAC & $6.198 \times 10^{-2}$ & $8.842 \times 10^{-3}$ & 7.010 & $2.896 \times 10^{-12}$ & 28 \\
\hline WindSpeed & $8.980 \times 10^{-5}$ & $1.742 \times 10^{-5}$ & 5.154 & $2.707 \times 10^{-7}$ & 38 \\
\hline Hour_17 & $-4.192 \times 10^{-3}$ & $1.395 \times 10^{-3}$ & -3.004 & $2.685 \times 10^{-3}$ & 134.7 \\
\hline Hour_9 & $-3.409 \times 10^{-3}$ & $1.429 \times 10^{-3}$ & -2.385 & $1.712 \times 10^{-2}$ & 117.8 \\
\hline Hour_18 & $-3.283 \times 10^{-3}$ & $1.400 \times 10^{-3}$ & -2.345 & $1.907 \times 10^{-2}$ & 116.4 \\
\hline Hour_13 & $-3.342 \times 10^{-3}$ & $1.449 \times 10^{-3}$ & -2.306 & $2.115 \times 10^{-2}$ & 115.0 \\
\hline Hour_14 & $-2.936 \times 10^{-3}$ & $1.450 \times 10^{-3}$ & -2.025 & $4.299 \times 10^{-2}$ & 103.2 \\
\hline
\end{tabular}

Table B.8: Analysis of variance of the linear regression model for modal frequencies of the $1.62 \mathrm{~Hz}$ mode.

\begin{tabular}{|c|c|c|c|c|c|}
\hline Coefficient:Combination & SumSq & DF & MeanSq & $\mathrm{F}$ test & pValue \\
\hline ModeIdentifications & $5.415 \times 10^{-2}$ & 1 & $5.415 \times 10^{-2}$ & 427.7 & $2.847 \times 10^{-89}$ \\
\hline AirTemperature & $1.944 \times 10^{-2}$ & 1 & $1.944 \times 10^{-2}$ & 153.6 & $1.754 \times 10^{-34}$ \\
\hline rmsVertical & $1.320 \times 10^{-2}$ & 1 & $1.320 \times 10^{-2}$ & 104.3 & $4.097 \times 10^{-24}$ \\
\hline
\end{tabular}


Table B.8 (continued). Analysis of variance of the linear regression model for modal frequencies of the $1.62 \mathrm{~Hz}$ mode.

\begin{tabular}{|c|c|c|c|c|c|}
\hline Coefficient:Combination & SumSq & $\mathrm{DF}$ & MeanSq & $\mathrm{F}$ test & pValue \\
\hline rmsTransverse & $9.771 \times 10^{-3}$ & 1 & $9.771 \times 10^{-3}$ & 77.2 & $2.508 \times 10^{-18}$ \\
\hline ModalMAC & $6.222 \times 10^{-3}$ & 1 & $6.222 \times 10^{-3}$ & 49.1 & $2.896 \times 10^{-12}$ \\
\hline WindSpeed & $3.363 \times 10^{-3}$ & 1 & $3.363 \times 10^{-3}$ & 26.6 & $2.707 \times 10^{-7}$ \\
\hline SensorsCombCat & $1.771 \times 10^{-2}$ & 74 & $2.393 \times 10^{-4}$ & 1.9 & $8.395 \times 10^{-6}$ \\
\hline Dotw & $1.015 \times 10^{-3}$ & 6 & $1.691 \times 10^{-4}$ & 1.3 & $2.375 \times 10^{-1}$ \\
\hline Hour & $3.367 \times 10^{-3}$ & 23 & $1.464 \times 10^{-4}$ & 1.2 & $2.747 \times 10^{-1}$ \\
\hline Error & $4.044 \times 10^{-1}$ & 3194 & $1.266 \times 10^{-4}$ & 1.0 & $5.000 \times 10^{-1}$ \\
\hline
\end{tabular}

Table B.9: Linear regression coefficients for modal frequencies of $1.85 \mathrm{~Hz}$ mode, ordered according to significance.

\begin{tabular}{|c|c|c|c|c|c|}
\hline Coefficient Name & $\beta$ & $\mathrm{SE}$ & tStat & pValue & $\begin{array}{r}95 \% \text { c.i. } \\
\text { for } \beta(\%)\end{array}$ \\
\hline OnesConstant & 1.909 & $1.380 \times 10^{-2}$ & 138.294 & 0.000 & 1.4 \\
\hline ModalMAC & $-4.818 \times 10^{-2}$ & $6.235 \times 10^{-3}$ & -7.727 & $1.541 \times 10^{-14}$ & 174.6 \\
\hline rmsTransverse & -4.510 & $5.892 \times 10^{-1}$ & -7.655 & $2.677 \times 10^{-14}$ & 174.4 \\
\hline ModeIdentifications & $-5.166 \times 10^{-5}$ & $7.036 \times 10^{-6}$ & -7.343 & $2.753 \times 10^{-13}$ & 173.3 \\
\hline AirTemperature & $-3.988 \times 10^{-4}$ & $5.453 \times 10^{-5}$ & -7.314 & $3.398 \times 10^{-13}$ & 173.2 \\
\hline Hour_13 & $7.955 \times 10^{-3}$ & $1.860 \times 10^{-3}$ & 4.278 & $1.954 \times 10^{-5}$ & 45.8 \\
\hline Hour_16 & $7.864 \times 10^{-3}$ & $1.844 \times 10^{-3}$ & 4.264 & $2.075 \times 10^{-5}$ & 46.0 \\
\hline Hour_15 & $7.247 \times 10^{-3}$ & $1.866 \times 10^{-3}$ & 3.883 & $1.055 \times 10^{-4}$ & 50.5 \\
\hline Hour_12 & $6.783 \times 10^{-3}$ & $1.856 \times 10^{-3}$ & 3.655 & $2.624 \times 10^{-4}$ & 53.7 \\
\hline Hour_9 & $6.677 \times 10^{-3}$ & $1.890 \times 10^{-3}$ & 3.533 & $4.175 \times 10^{-4}$ & 55.5 \\
\hline Hour_11 & $6.415 \times 10^{-3}$ & $1.863 \times 10^{-3}$ & 3.444 & $5.826 \times 10^{-4}$ & 56.9 \\
\hline WindSpeed & $-7.495 \times 10^{-5}$ & $2.229 \times 10^{-5}$ & -3.363 & $7.827 \times 10^{-4}$ & 141.7 \\
\hline Hour_19 & $5.719 \times 10^{-3}$ & $1.843 \times 10^{-3}$ & 3.102 & $1.939 \times 10^{-3}$ & 63.2 \\
\hline SensorsCombCat_SensorConfig_46 & $-5.401 \times 10^{-2}$ & $1.819 \times 10^{-2}$ & -2.969 & $3.009 \times 10^{-3}$ & 134.0 \\
\hline Dotw_Wed & $2.842 \times 10^{-3}$ & $9.760 \times 10^{-4}$ & 2.912 & $3.625 \times 10^{-3}$ & 67.3 \\
\hline Hour_6 & $5.283 \times 10^{-3}$ & $1.838 \times 10^{-3}$ & 2.873 & $4.091 \times 10^{-3}$ & 68.2 \\
\hline Hour_10 & $5.154 \times 10^{-3}$ & $1.855 \times 10^{-3}$ & 2.778 & $5.502 \times 10^{-3}$ & 70.6 \\
\hline Hour_17 & $5.053 \times 10^{-3}$ & $1.820 \times 10^{-3}$ & 2.776 & $5.541 \times 10^{-3}$ & 70.6 \\
\hline Hour_14 & $5.197 \times 10^{-3}$ & $1.896 \times 10^{-3}$ & 2.740 & $6.181 \times 10^{-3}$ & 71.6 \\
\hline Hour_7 & $5.048 \times 10^{-3}$ & $1.852 \times 10^{-3}$ & 2.726 & $6.443 \times 10^{-3}$ & 71.9 \\
\hline Hour_5 & $5.069 \times 10^{-3}$ & $1.881 \times 10^{-3}$ & 2.695 & $7.081 \times 10^{-3}$ & 72.8 \\
\hline Hour $\_8$ & $4.843 \times 10^{-3}$ & $1.831 \times 10^{-3}$ & 2.645 & $8.223 \times 10^{-3}$ & 74.1 \\
\hline
\end{tabular}


Table B.9 (continued). Linear regression coefficients for modal frequencies of $1.85 \mathrm{~Hz}$ mode, ordered according to significance.

\begin{tabular}{|c|c|c|c|c|c|}
\hline Coefficient Name & $\beta$ & $\mathrm{SE}$ & tStat & pValue & $\begin{array}{r}95 \% \text { c.i. } \\
\text { for } \beta(\%)\end{array}$ \\
\hline Hour_1 & $4.981 \times 10^{-3}$ & $1.922 \times 10^{-3}$ & 2.591 & $9.612 \times 10^{-3}$ & 75.7 \\
\hline Hour_4 & $4.797 \times 10^{-3}$ & $1.874 \times 10^{-3}$ & 2.560 & $1.052 \times 10^{-2}$ & 76.6 \\
\hline Dotw_Sat & $-2.520 \times 10^{-3}$ & $1.053 \times 10^{-3}$ & -2.393 & $1.678 \times 10^{-2}$ & 118.1 \\
\hline Hour_21 & $4.045 \times 10^{-3}$ & $1.833 \times 10^{-3}$ & 2.207 & $2.739 \times 10^{-2}$ & 88.8 \\
\hline SensorsCombCat_SensorConfig_40 & $-3.837 \times 10^{-2}$ & $1.821 \times 10^{-2}$ & -2.107 & $3.523 \times 10^{-2}$ & 106.9 \\
\hline Hour_18 & $3.582 \times 10^{-3}$ & $1.808 \times 10^{-3}$ & 1.981 & $4.772 \times 10^{-2}$ & 99.0 \\
\hline
\end{tabular}

Table B.10: Analysis of variance of the linear regression model for modal frequencies of the $1.85 \mathrm{~Hz}$ mode.

\begin{tabular}{|c|c|c|c|c|c|}
\hline Coefficient:Combination & SumSq & $\mathrm{DF}$ & MeanSq & $\mathrm{F}$ test & pValue \\
\hline SensorsCombCat & $3.721 \times 10^{-2}$ & 57 & $6.528 \times 10^{-4}$ & 4.0 & $3.297 \times 10^{-21}$ \\
\hline ModalMAC & $9.735 \times 10^{-3}$ & 1 & $9.735 \times 10^{-3}$ & 59.7 & $1.541 \times 10^{-14}$ \\
\hline rmsTransverse & $9.554 \times 10^{-3}$ & 1 & $9.554 \times 10^{-3}$ & 58.6 & $2.677 \times 10^{-14}$ \\
\hline ModeIdentifications & $8.791 \times 10^{-3}$ & 1 & $8.791 \times 10^{-3}$ & 53.9 & $2.753 \times 10^{-13}$ \\
\hline AirTemperature & $8.723 \times 10^{-3}$ & 1 & $8.723 \times 10^{-3}$ & 53.5 & $3.397 \times 10^{-13}$ \\
\hline Dotw & $6.945 \times 10^{-3}$ & 6 & $1.158 \times 10^{-3}$ & 7.1 & $1.609 \times 10^{-7}$ \\
\hline WindSpeed & $1.844 \times 10^{-3}$ & 1 & $1.844 \times 10^{-3}$ & 11.3 & $7.827 \times 10^{-4}$ \\
\hline Hour & $7.707 \times 10^{-3}$ & 23 & $3.351 \times 10^{-4}$ & 2.1 & $2.215 \times 10^{-3}$ \\
\hline rmsVertical & $3.646 \times 10^{-6}$ & 1 & $3.646 \times 10^{-6}$ & 0.0 & $8.811 \times 10^{-1}$ \\
\hline Error & $4.427 \times 10^{-1}$ & 2715 & $1.631 \times 10^{-4}$ & 1.0 & $5.000 \times 10^{-1}$ \\
\hline
\end{tabular}


Appendix C

Wireless sensor specification 


\section{LORD DATASHEET}

\section{G-Link2 ${ }^{\text {tm }}$-LXRS}

\section{Wireless Accelerometer Node}

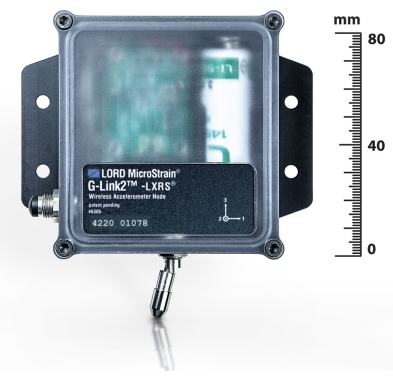

G-Link2 ${ }^{\mathrm{TM}}$-LXRS ${ }^{\circledR}$ - ruggedized node with high-speed sampling and optional integrated three-axis accelerometer or an external single-axis accelerometer

LORD MicroStrain ${ }^{\circledR}$ LXRS ${ }^{\circledR}$ Wireless Sensor Networks enable simultaneous, high-speed sensing and data aggregation from scalable sensor networks. Our wireless sensing systems are ideal for test and measurement, remote monitoring, system performance analysis, and embedded applications.

The gateways are the heart of the LORD MicroStrain wireless sensing system. They coordinate and maintain wireless transmissions across a network of distributed wireless sensor nodes. Some nodes have integrated sensors, while others are designed with multi-sensor connectivity for application flexibility. The LORD MicroStrain LXRS wireless communication protoco between LXRS nodes and gateways enable high-speed sampling, \pm 32 microseconds node-to-node synchronization, and lossless data throughput under most operating conditions.

Users can easily program nodes for data logging, continuous, and periodic burst sampling with the Node Commander ${ }^{\circledR}$ software. The web-based SensorCloud ${ }^{\mathrm{TM}}$ interface optimizes data aggregation, analysis, presentation, and alerts for gigabytes of sensor data from remote networks.
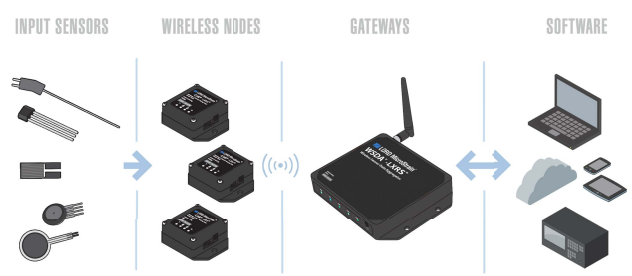

\section{Product Highlights}

- On-board triaxial, or external single axis MEMS accelerometer with up to $+/-200 \mathrm{~g}$ measurement range

- Wireless framework is ideal for measuring vibration and acceleration in remote applications.

- High resolution data with 16 -bit A/D converter

- User-programmable sample rates up to $\mathrm{KHz}$

- Small, lightweight IP67 enclosure rated for outdoor use

\section{Features and Benefits}

High Performance

- Lossless data throughput and node-to-node sampling synchronization of $\pm 32 \mu \mathrm{S}$ in $\mathrm{LXRS}^{\circledR}{ }^{\circledR}$-enabled modes

- Wireless range up to $2 \mathrm{~km}$ (800 m typical)

- User-programmable filters for optimized anti-aliasing

\section{Ease of Use}

- Scalable networks for easy expansion

- Internal or external accelerometer option for installation versatility

- Remote configuration, acquisition, and display of sensor data with SensorConnect ${ }^{\mathrm{TM}}$ or Node Commander ${ }^{\circledR}$

- Optional web-based SensorCloud ${ }^{\mathrm{TM}}$ platform optimizes data storage, viewing, alerts, and analysis.

- Easy custom integration with open-source, comprehensive communications and command library

\section{Cost Effective}

- End-to-end wireless sensing solution reduces development and deployment time

- Volume discounts

\section{Applications}

- Condition-based monitoring

- Health monitoring of rotating components, aircraft, structures, and vehicles

- Vibration monitoring

- Vehicle dynamics testing

- Product testing

Wireless Simplicity, Hardwired Reliability ${ }^{T M}$

Figure C.1: Data-sheet for the wireless sensors used in the light pole studies of Chapter 8. (1 of 2 ) 


\section{G-Link2 $^{\text {TM }}$-LXRS ${ }^{\circledR}$ Wireless Accelerometer Node}

\section{Specifications}

\begin{tabular}{|c|c|}
\hline \multicolumn{2}{|r|}{ General } \\
\hline Sensor input channels & Single-axis MEMS accelerometer (option), 1 channel \\
\hline Integrated sensors & $\begin{array}{l}\text { Triaxial MEMS accelerometer (option), } 3 \text { channels } \\
\text { Internal temperature, } 1 \text { channel }\end{array}$ \\
\hline Data storage capacity & $4 \mathrm{M}$ bytes (up to $2,000,000$ data points) \\
\hline \multicolumn{2}{|c|}{ Accelerometer Channels (integrated or external) } \\
\hline Measurement range & $\begin{array}{l} \pm 2 g \text { or } \pm 10 g \text { standard }( \pm 5 g, \pm 30 g, \pm 50 g, \pm 100 g \text {, or } \pm 200 g \\
\text { options available) }\end{array}$ \\
\hline Accelerometer bandwidth & 0 to $\leq 100 \mathrm{~Hz}$ (-3 dB cutoff), high bandwidth option available \\
\hline Accuracy and resolution & $\begin{array}{l}<0.3 \% \text { error (typical @ } 25 \mathrm{~Hz}, 1 / 2 \text { of dynamic range with } \\
\text { sinusoidal input), } 16 \text { bit resolution }\end{array}$ \\
\hline Noise & $\begin{array}{l} \pm 2 \mathrm{~g}: 130 \mu \mathrm{g} / \sqrt{\mathrm{Hz}}, \pm 10 \mathrm{~g}: 420 \mu \mathrm{g} / \sqrt{\mathrm{Hz}} \text { (typical with } 100 \mathrm{~Hz} \\
\text { anti-aliasing filter setting) }\end{array}$ \\
\hline Anti-aliasing filter bandwidth & $\begin{array}{l}\text { Fifth order low-pass Butterworth filter, user programmable } \\
\text { bandwidth from } 26 \mathrm{~Hz} \text { to } 1 \mathrm{KHz}\end{array}$ \\
\hline \multicolumn{2}{|c|}{ Integrated Temperature Channel } \\
\hline Measurement Range & $-40^{\circ} \mathrm{C}$ to $125^{\circ} \mathrm{C}$ \\
\hline Accuracy and resolution & $\pm 5^{\circ} \mathrm{C}$ (over full range), 16 bit \\
\hline \multicolumn{2}{|r|}{ Sampling } \\
\hline Sampling modes & Synchronized, low duty cycle, datalogging \\
\hline Sampling rates & $\begin{array}{l}\text { Continuous sampling: } 32 \text { to } 512 \mathrm{~Hz} \\
\text { Periodic burst sampling: } 32 \mathrm{~Hz} \text { to } 10 \mathrm{KHz} \\
\text { Datalogging: } 32 \mathrm{~Hz} \text { to } 10 \mathrm{KHz}\end{array}$ \\
\hline Sample rate stability & $\pm 3 \mathrm{ppm}$ \\
\hline Network capacity & $\begin{array}{l}\text { Up to } 125 \text { nodes per RF channel (and per gateway) depending } \\
\text { on the number of active channels and sampling settings. } \\
\text { Refer to the system bandwidth calculator: } \\
\text { http://www.microstrain.com/configure-your-system }\end{array}$ \\
\hline Synchronization between nodes & $\pm 32 \mu \mathrm{sec}$ \\
\hline \multicolumn{2}{|r|}{ Operating Parameters } \\
\hline Wireless communication range & $\begin{array}{l}\text { Outdoor/line-of-sight: } 2 \mathrm{~km} \text { (ideal)*, } 800 \mathrm{~m} \text { (typical) })^{* *} \\
\text { Indoor/obstructions: } 50 \mathrm{~m} \text { (typical)** }\end{array}$ \\
\hline $\begin{array}{l}\text { Radio frequency (RF) } \\
\text { transceiver carrier }\end{array}$ & $\begin{array}{l}2.405 \text { to } 2.470 \mathrm{GHz} \text { direct sequence spread spectrum over } 14 \\
\text { channels, license free worldwide, radiated power programmable } \\
\text { from } 0 \mathrm{dBm}(1 \mathrm{~mW}) \text { to } 16 \mathrm{dBm} \text { ( } 39 \mathrm{~mW}) \text {; low power option } \\
\text { available for use outside the U.S.A. - limited to } 10 \mathrm{dBm}(10 \mathrm{~mW})\end{array}$ \\
\hline Power source & $\begin{array}{l}\text { Internal: } 3.6 \mathrm{~V} \mathrm{dc}, 2.6 \mathrm{Ah}, \text { AA replaceable lithium battery (Saft } \\
\text { LS14500 included), External: } 2.2 \mathrm{Vdc} \text { to } 5 \mathrm{Vdc}\end{array}$ \\
\hline Power consumption & 1 channel: $20.1 \mathrm{~mA}$ (average), 3 channels: $34.9 \mathrm{~mA}$ (average) \\
\hline Operating temperature & $-40^{\circ} \mathrm{C}$ to $+85^{\circ} \mathrm{C}$ \\
\hline Acceleration limit & tested to $380 \mathrm{~g}$ \\
\hline MTBF & 378,000 hours (Telcordia method, SR332) \\
\hline \multicolumn{2}{|r|}{ Physical Specifications } \\
\hline Dimensions & $\begin{array}{l}\text { Node: } 68 \mathrm{~mm} \times 85 \mathrm{~mm} \times 33.5 \mathrm{~mm} \text { with mounting tabs, external } \\
\text { accelerometer (option): } 32 \mathrm{~mm} \times 21.5 \mathrm{~mm} \times 16 \mathrm{~mm}\end{array}$ \\
\hline Weight & $\begin{array}{l}\text { Node with internal accelerometer and battery: } 178 \text { grams } \\
\text { node with external accelerometer, cable and battery: } 252 \text { grams }\end{array}$ \\
\hline Environmental rating & IP67 \\
\hline Enclosure material & Aluminum and clear polycarbonate \\
\hline \multicolumn{2}{|r|}{ Integration } \\
\hline Compatible gateways & All WSDA ${ }^{\circledR}$ base stations and gateways \\
\hline Compatible sensors & $\begin{array}{l}\text { LORD MicroStrain }{ }^{\Theta} \text { accelerometer } \\
\text { (external accelerometer option) }\end{array}$ \\
\hline Connectors & $\begin{array}{l}\text { M5 screw-on IP67 connector } \\
\text { (external accelerometer option) }\end{array}$ \\
\hline Software & $\begin{array}{l}\text { SensorCloud }{ }^{\mathrm{TM}} \text {, SensorConnect }{ }^{\mathrm{TM}}, \text { Node Commander }^{\circledR} \text {, } \\
\text { WSDA }{ }^{\circledR} \text { Data Downloader, Live Connect }{ }^{\mathrm{M} M} \text {, Windows } \\
\text { XP/Vista/7 compatible }\end{array}$ \\
\hline Software development kit (SDK) & $\begin{array}{l}\text { Data communications protocol available with EEPROM maps } \\
\text { and sample code (OS and computing platform independent) } \\
\text { http://www.microstrain.com/software/mscl }\end{array}$ \\
\hline Regulatory compliance & FCC (U.S.), IC (Canada), CE, ROHS \\
\hline
\end{tabular}

*Measured with antennas elevated, no obstructions, and no RF interferers.

"Actual range varies with conditions such as obstructions, $\mathrm{RF}$ interference, antenna height $\&$ orientation.

Copyright $\odot 2016$ LORD Corporation
Document 8400-0072 Revision E. Subject to change without notice.

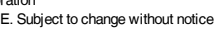

www.microstrain.com
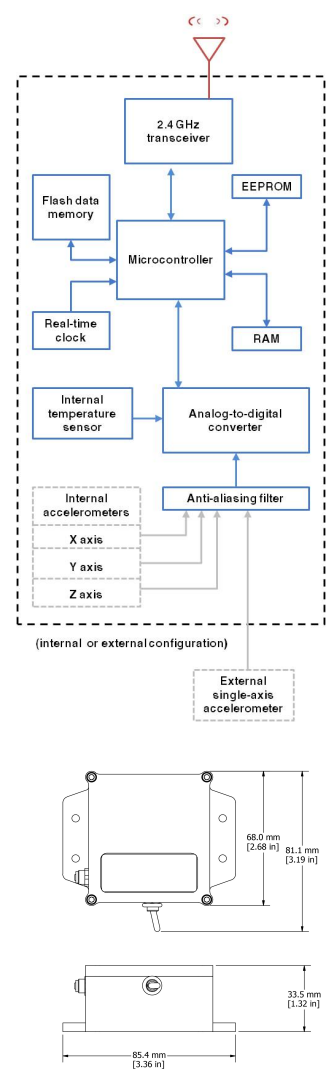

G-Link2 ${ }^{\text {TM }}$-LXRS ${ }^{\circledR}$ Node

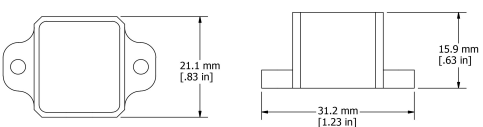

External Accelerometer

\section{LOORD SENSIIG}

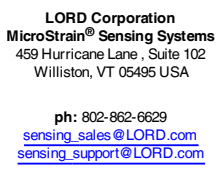

Figure C.2: Data-sheet for the wireless sensors used in the light pole studies of Chapter 8. (2 of 2) 
Appendix D

Light pole monitoring - complete list of collected datasets and associated results 
Table D.1: List of collected datasets with analysis results for the Confederation Bridge light pole study of Section 8.2.

\begin{tabular}{|c|c|c|c|c|c|c|c|c|c|c|c|c|c|c|}
\hline & \multirow[t]{2}{*}{$\begin{array}{l}\text { Data- } \\
\text { set } \\
\#\end{array}$} & \multirow[t]{2}{*}{$\begin{array}{l}\text { Time stamp } \\
\text { (local time) }\end{array}$} & \multirow[t]{2}{*}{$\begin{array}{c}\text { Dataset } \\
\text { dur. } \\
\text { (sec.) }\end{array}$} & \multirow[t]{2}{*}{$\begin{array}{l}\text { Sust. } \\
\text { wind } \\
(\mathrm{km} / \mathrm{h})\end{array}$} & \multirow[t]{2}{*}{$\begin{array}{l}\text { Wind } \\
\text { gusts } \\
(\mathrm{km} / \mathrm{h})\end{array}$} & \multirow[t]{2}{*}{$\begin{array}{l}\text { Wind } \\
\text { dir. } \\
\text { (de- } \\
\text { grees) }\end{array}$} & \multicolumn{2}{|c|}{$\begin{array}{c}\text { Maximum } \\
\text { displacement } \\
\text { (resultant, mm) }\end{array}$} & \multicolumn{2}{|c|}{$\begin{array}{l}\text { Strength of } \\
\text { signal RMS } \\
\quad(\mathrm{mm})\end{array}$} & \multicolumn{2}{|c|}{$\begin{array}{l}\text { Fundamental } \\
\text { frequency } \\
\text { (displacement) }(\mathrm{Hz})\end{array}$} & \multicolumn{2}{|c|}{$\begin{array}{c}\text { Cumulative fatigue } \\
\text { damage }(c F D) \text { estimate }\end{array}$} \\
\hline & & & & & & & Undamp. & Damped & Undamp. & Damped & Undamp. & Damped & Undamp. & Damped \\
\hline & 1 & 2017-08-29 16:32 & 90 & 19.7 & 21.2 & 132.7 & 1.9 & 1.7 & 0.9 & 0.7 & 1.859 & 1.750 & $1.70 \mathrm{E}-10$ & $3.45 \mathrm{E}-10$ \\
\hline & 2 & 2017-08-29 $16: 35$ & 90 & 20.2 & 21.9 & 133.9 & 2.5 & 2.7 & 1.1 & 1.3 & 1.859 & 1.766 & 4. $49 \mathrm{E}-10$ & $1.51 \mathrm{E}-09$ \\
\hline & 3 & 2017-08-29 16:41 & 90 & 20.6 & 22.2 & 135.6 & 2.4 & 1.9 & 1.2 & 0.9 & 1.859 & 1.750 & $1.11 \mathrm{E}-09$ & $4.11 \mathrm{E}-10$ \\
\hline & 4 & 2017-08-29 16:43 & 90 & 21.0 & 22.6 & 137.4 & 2.6 & 2.7 & 1.1 & 1.3 & 1.859 & 1.750 & $6.11 \mathrm{E}-10$ & $2.47 \mathrm{E}-09$ \\
\hline & 5 & 2017-08-31 18:01 & 90 & 25.6 & 27.2 & 125.7 & 2.4 & 4.1 & 0.9 & 1.9 & 1.844 & 1.750 & $1.05 \mathrm{E}-10$ & $1.35 \mathrm{E}-08$ \\
\hline & 6 & $2017-08-31 \quad 18: 03$ & 90 & 24.9 & 26.8 & 124.4 & 2.6 & 3.3 & 1.3 & 1.5 & 1.859 & 1.766 & 1.41E-09 & $2.90 \mathrm{E}-09$ \\
\hline & 7 & 2017-08-31 18:05 & 90 & 24.6 & 25.4 & 125.3 & 2.7 & 2.5 & 1.1 & 1.1 & 1.859 & 1.750 & $1.44 \mathrm{E}-09$ & $1.78 \mathrm{E}-09$ \\
\hline & 8 & $2017-08-31 \quad 18: 07$ & 90 & 24.2 & 25.8 & 126.2 & 2.2 & 2.9 & 0.9 & 1.2 & 1.859 & 1.781 & $4.90 \mathrm{E}-10$ & 1.10E-10 \\
\hline & 9 & $2017-08-31$ 18:30 & 90 & 24.1 & 25.4 & 125.8 & 2.5 & 4.6 & 1.2 & 1.7 & 1.859 & 1.750 & $2.55 \mathrm{E}-09$ & $2.70 \mathrm{E}-08$ \\
\hline & 10 & $2017-08-31 \quad 18: 45$ & 90 & 24.9 & 26.1 & 130.4 & 2.5 & 3.7 & 1.1 & 1.5 & 1.875 & 1.750 & $2.27 \mathrm{E}-09$ & $6.91 \mathrm{E}-09$ \\
\hline & 11 & 2017-08-31 19:00 & 90 & 25.5 & 26.8 & 134.8 & 6.8 & 5.6 & 2.6 & 2.3 & 1.891 & 1.766 & $1.52 \mathrm{E}-07$ & $1.08 \mathrm{E}-07$ \\
\hline & 12 & $2017-08-31 \quad 19: 15$ & 90 & 24.6 & 26.1 & 137.2 & 3.5 & 6.4 & 1.4 & 2.1 & 1.875 & 1.766 & $3.48 \mathrm{E}-09$ & $1.09 \mathrm{E}-07$ \\
\hline & 13 & $2017-08-31 \quad 19: 30$ & 90 & 21.3 & 24.0 & 140.0 & 3.7 & 2.4 & 1.3 & 1.1 & 1.875 & 1.750 & $5.97 \mathrm{E}-09$ & $1.41 \mathrm{E}-09$ \\
\hline & 14 & $2017-08-31 \quad 20: 14$ & 90 & 16.6 & 18.7 & 193.5 & 6.9 & 6.2 & 3.0 & 2.6 & 1.875 & 1.750 & $1.71 \mathrm{E}-07$ & $2.55 \mathrm{E}-07$ \\
\hline-1 & 15 & $2017-08-31 \quad 20: 48$ & 600 & 14.8 & 16.6 & 190.7 & 13.3 & 9.2 & 4.4 & 3.2 & 1.859 & 1.781 & $2.07 \mathrm{E}-06$ & $5.96 \mathrm{E}-07$ \\
\hline \# & 16 & $2017-08-31 \quad 21: 03$ & 300 & 14.7 & 16.9 & 223.6 & 11.6 & 7.3 & 3.4 & 2.6 & 1.844 & 1.781 & $1.64 \mathrm{E}-06$ & $1.94 \mathrm{E}-07$ \\
\hline$\vec{z}$ & 17 & 2017-08-31 21:50 & 300 & 15.6 & 19.1 & 232.4 & 6.5 & 6.3 & 2.2 & 2.1 & 1.875 & 1.750 & 1.34E-07 & $1.46 \mathrm{E}-07$ \\
\hline च & 18 & $2017-08-31 \quad 22: 15$ & 300 & 13.9 & 16.9 & 231.6 & 7.0 & 5.7 & 2.5 & 2.0 & 1.859 & 1.750 & $1.53 \mathrm{E}-07$ & $5.52 \mathrm{E}-08$ \\
\hline b & 19 & $2017-08-31 \quad 22: 40$ & 300 & 13.6 & 16.9 & 240.8 & 10.3 & 7.7 & 3.0 & 2.4 & 1.875 & 1.750 & $4.57 \mathrm{E}-07$ & $2.29 \mathrm{E}-07$ \\
\hline a & 20 & $2017-08-3123: 05$ & 300 & 11.3 & 13.8 & 234.6 & 6.8 & 5.7 & 2.3 & 1.8 & 1.891 & 1.781 & $6.24 \mathrm{E}-08$ & $2.98 \mathrm{E}-08$ \\
\hline$\Xi$ & 21 & $2017-08-31 \quad 23: 30$ & 300 & 12.1 & 16.2 & 226.2 & 8.2 & 6.8 & 2.4 & 2.1 & 1.875 & 1.734 & $9.76 \mathrm{E}-08$ & $5.66 \mathrm{E}-08$ \\
\hline 5 & 22 & $2017-08-31 \quad 23: 55$ & 300 & 47.5 & 55.0 & 303.4 & 6.0 & 4.9 & 2.2 & 2.0 & 1.859 & 1.750 & $5.72 \mathrm{E}-08$ & 4.03E-08 \\
\hline & 23 & 2017-09-01 00:20 & 300 & 44.3 & 54.3 & 302.0 & 6.9 & 4.8 & 2.7 & 1.8 & 1.875 & 1.781 & $4.26 \mathrm{E}-08$ & $2.19 \mathrm{E}-08$ \\
\hline & 24 & 2017-09-01 00:45 & 300 & 40.7 & 48.3 & 285.2 & 5.6 & 5.8 & 1.7 & 1.7 & 1.875 & 1.781 & $1.38 \mathrm{E}-08$ & $1.12 \mathrm{E}-08$ \\
\hline & 25 & 2017-09-01 01:10 & 300 & 38.5 & 45.9 & 276.3 & 4.1 & 4.8 & 1.6 & 1.9 & 1.875 & 1.75 & $1.18 \mathrm{E}-08$ & $3.84 \mathrm{E}-08$ \\
\hline & 26 & 2017-09-01 01:35 & 300 & 46.9 & 53.6 & 289 & 6 & 6.3 & 2.2 & 1.9 & 1.875 & 1.781 & $2.57 \mathrm{E}-08$ & 4.15E-08 \\
\hline & 27 & 2017-09-01 02:00 & 300 & 47.8 & 54 & 294.1 & 7.1 & 7.2 & 2.6 & 2.4 & 1.891 & 1.797 & $1.01 \mathrm{E}-07$ & $6.91 \mathrm{E}-08$ \\
\hline & 28 & 2017-09-01 $02: 25$ & 300 & 49 & 58.6 & 293.3 & 8.3 & 7.6 & 3.3 & 2.2 & 1.875 & 1.797 & $2.15 \mathrm{E}-07$ & $9.39 \mathrm{E}-08$ \\
\hline & 29 & 2017-09-01 02:50 & 300 & 46.9 & 54 & 293.5 & 7.7 & 5.6 & 2.5 & 2 & 1.859 & 1.797 & $5.63 \mathrm{E}-08$ & $2.67 \mathrm{E}-08$ \\
\hline & 30 & 2017-09-01 03:15 & 300 & 44.7 & 53.6 & 303.8 & 7.6 & 6.9 & 3.1 & 2.1 & 1.875 & 1.75 & $3.23 \mathrm{E}-07$ & $1.18 \mathrm{E}-07$ \\
\hline & 31 & 2017-09-01 03:40 & 300 & 47.6 & 55.4 & 305 & 5.6 & 5.6 & 2 & 1.8 & 1.875 & 1.734 & $1.39 \mathrm{E}-08$ & $3.60 \mathrm{E}-08$ \\
\hline & 32 & 2017-09-01 04:05 & 300 & 40.9 & 49.7 & 301.7 & 6.6 & 8.1 & 2.3 & 2.3 & 1.875 & 1.766 & $5.01 \mathrm{E}-08$ & $1.93 \mathrm{E}-07$ \\
\hline & 33 & 2017-09-01 04:30 & 300 & 47.2 & 57.5 & 295 & 5.3 & 4.8 & 1.7 & 1.9 & 1.859 & 1.781 & $1.94 \mathrm{E}-08$ & $2.78 \mathrm{E}-08$ \\
\hline & 34 & 2017-09-01 04:55 & 300 & 34 & 42.7 & 256.3 & 4.8 & 5.5 & 1.9 & 1.9 & 1.859 & 1.766 & 1.79E-08 & $3.11 \mathrm{E}-08$ \\
\hline & 35 & $2017-09-01 \quad 05: 20$ & 300 & 37.5 & 44.8 & 254.5 & 3.9 & 5 & 1.5 & 1.5 & 1.875 & 1.75 & $1.06 \mathrm{E}-08$ & $8.32 \mathrm{E}-09$ \\
\hline & 36 & 2017-09-01 05:45 & 300 & 39.5 & 46.9 & 260.3 & 4.7 & 4.6 & 1.8 & 1.5 & 1.859 & 1.75 & 2.45E-08 & 7.22E-09 \\
\hline & 37 & 2017-09-01 06:10 & 300 & 35.5 & 43.4 & 263.9 & 3.9 & 4.6 & 1.4 & 1.6 & 1.875 & 1.75 & $2.96 \mathrm{E}-09$ & 8.37E-09 \\
\hline & 38 & 2017-09-01 06:35 & 300 & 35.8 & 41.6 & 266.8 & 4.6 & 4.5 & 1.7 & 1.5 & 1.859 & 1.734 & $2.11 \mathrm{E}-08$ & $5.75 \mathrm{E}-09$ \\
\hline & 39 & 2017-09-01 07:00 & 300 & 38.2 & 45.5 & 262.2 & 3.8 & 5.4 & 1.5 & 1.7 & 1.875 & 1.766 & $9.59 \mathrm{E}-09$ & $1.08 \mathrm{E}-08$ \\
\hline & 40 & 2017-09-01 07:25 & 300 & 38.9 & 44.8 & 264.8 & 4.2 & 5.1 & 1.4 & 2 & 1.875 & 1.766 & $6.22 \mathrm{E}-09$ & $5.36 \mathrm{E}-08$ \\
\hline & 41 & 2017-09-01 17:33 & 300 & 48.5 & 56.1 & 273 & 6.5 & 6.9 & 2.3 & 2.1 & 1.875 & 1.75 & 1.72E-07 & $9.69 \mathrm{E}-08$ \\
\hline & 42 & 2017-09-01 17:47 & 300 & 44.9 & 52.9 & 267.2 & 6.5 & 6.9 & 2.1 & 2.4 & 1.859 & 1.75 & $1.13 \mathrm{E}-07$ & $1.06 \mathrm{E}-07$ \\
\hline & 43 & 2017-09-01 17:54 & 300 & 45.7 & 54.7 & 268.3 & 5.4 & 5.9 & 2.1 & 2 & 1.875 & 1.734 & $4.13 \mathrm{E}-08$ & $3.37 \mathrm{E}-08$ \\
\hline
\end{tabular}


Table D.1 (continued). List of collected datasets with analysis results for the Confederation Bridge light pole study of Section 8.2.

\begin{tabular}{|c|c|c|c|c|c|c|c|c|c|c|c|c|c|c|}
\hline & \multirow[t]{2}{*}{$\begin{array}{l}\text { Data- } \\
\text { set } \\
\#\end{array}$} & \multirow[t]{2}{*}{$\begin{array}{l}\text { Time stamp } \\
\text { (local time) }\end{array}$} & \multirow[t]{2}{*}{$\begin{array}{l}\text { Dataset } \\
\text { dur. } \\
\text { (sec.) }\end{array}$} & \multirow[t]{2}{*}{$\begin{array}{l}\text { Sust. } \\
\text { wind } \\
(\mathrm{km} / \mathrm{h})\end{array}$} & \multirow[t]{2}{*}{$\begin{array}{l}\text { Wind } \\
\text { gusts } \\
(\mathrm{km} / \mathrm{h})\end{array}$} & \multirow[t]{2}{*}{$\begin{array}{l}\text { Wind } \\
\text { dir. } \\
\text { (de- } \\
\text { grees) }\end{array}$} & \multicolumn{2}{|c|}{$\begin{array}{c}\text { Maximum } \\
\text { displacement } \\
\text { (resultant, mm) }\end{array}$} & \multicolumn{2}{|c|}{$\begin{array}{l}\text { Strength of } \\
\text { signal RMS } \\
\quad(\mathrm{mm})\end{array}$} & \multicolumn{2}{|c|}{$\begin{array}{l}\text { Fundamental } \\
\text { frequency } \\
\text { (displacement)(Hz) }\end{array}$} & \multicolumn{2}{|c|}{$\begin{array}{c}\text { Cumulative fatigue } \\
\text { damage }(\mathrm{cFD}) \text { estimate }\end{array}$} \\
\hline & & & & & & & Undamp. & $\overline{\text { Damped }}$ & Undamp. & $\overline{\text { Damped }}$ & Undamp. & $\overline{\text { Damped }}$ & Undamp. & $\overline{\text { Damped }}$ \\
\hline & 44 & 2017-09-01 18:00 & 300 & 46.5 & 55.4 & 268.5 & 7 & 5.4 & 2.1 & 2 & 1.875 & 1.75 & $9.24 \mathrm{E}-08$ & $6.46 \mathrm{E}-08$ \\
\hline 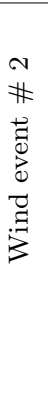 & $\begin{array}{l}45 \\
46 \\
47 \\
48 \\
49 \\
50 \\
51 \\
52 \\
53 \\
54 \\
55 \\
56 \\
57 \\
58 \\
59\end{array}$ & $\begin{array}{ll}2017-09-02 & 02: 20 \\
2017-09-02 & 02: 30 \\
2017-09-02 & 03: 00 \\
2017-09-02 & 03: 30 \\
2017-09-02 & 04: 00 \\
2017-09-02 & 04: 30 \\
2017-09-02 & 05: 00 \\
2017-09-02 & 05: 30 \\
2017-09-02 & 06: 00 \\
2017-09-02 & 06: 30 \\
2017-09-02 & 07: 00 \\
2017-09-02 & 07: 30 \\
2017-09-02 & 08: 00 \\
2017-09-02 & 08: 30 \\
2017-09-02 & 09: 00\end{array}$ & $\begin{array}{l}300 \\
300 \\
300 \\
300 \\
300 \\
300 \\
300 \\
300 \\
300 \\
300 \\
300 \\
300 \\
300 \\
300 \\
300\end{array}$ & $\begin{array}{c}55.4 \\
58.1 \\
59.8 \\
63.8 \\
58.1 \\
58 \\
55.6 \\
57.9 \\
59.1 \\
55.7 \\
54.1 \\
50.4 \\
49.5 \\
46.8 \\
44\end{array}$ & $\begin{array}{l}66.3 \\
65.6 \\
66.7 \\
75.5 \\
67 \\
70.2 \\
64.9 \\
65.3 \\
67.4 \\
67.7 \\
64.2 \\
61.4 \\
57.9 \\
56.4 \\
51.9\end{array}$ & $\begin{array}{c}298 \\
296.8 \\
291 \\
287.6 \\
289.4 \\
286.2 \\
292.1 \\
299.8 \\
305.7 \\
309.7 \\
309.6 \\
307 \\
312.8 \\
315.3 \\
317.1\end{array}$ & $\begin{array}{l}8.2 \\
12.1 \\
14.7 \\
9.7 \\
10.8 \\
9.1 \\
9.3 \\
11 \\
9.3 \\
10 \\
8.2 \\
11.6 \\
10.3 \\
7 \\
10.7\end{array}$ & $\begin{array}{l}7.1 \\
10 \\
14.3 \\
9.4 \\
9.9 \\
7.5 \\
7.8 \\
9.3 \\
11.4 \\
8.6 \\
7.7 \\
6.8 \\
6.5 \\
6.2 \\
6.9\end{array}$ & $\begin{array}{l}3.2 \\
4.1 \\
4 \\
3.5 \\
4.1 \\
3 \\
3.3 \\
4.1 \\
3.5 \\
3.2 \\
3.2 \\
3.1 \\
3.1 \\
2.7 \\
3\end{array}$ & $\begin{array}{l}2.5 \\
3.2 \\
3.7 \\
2.8 \\
2.5 \\
2.6 \\
2.5 \\
2.8 \\
2.9 \\
2.8 \\
2.4 \\
2.4 \\
2.2 \\
2.1 \\
2.2\end{array}$ & $\begin{array}{l}1.875 \\
1.844 \\
1.859 \\
1.859 \\
1.859 \\
1.891 \\
1.875 \\
1.875 \\
1.875 \\
1.859 \\
1.859 \\
1.875 \\
1.859 \\
1.875 \\
1.859\end{array}$ & $\begin{array}{l}1.766 \\
1.75 \\
1.781 \\
1.797 \\
1.766 \\
1.781 \\
1.781 \\
1.781 \\
1.75 \\
1.797 \\
1.766 \\
1.781 \\
1.75 \\
1.781 \\
1.75\end{array}$ & $\begin{array}{l}2.67 \mathrm{E}-07 \\
9.04 \mathrm{E}-07 \\
8.53 \mathrm{E}-07 \\
6.00 \mathrm{E}-07 \\
3.70 \mathrm{E}-07 \\
1.57 \mathrm{E}-07 \\
4.82 \mathrm{E}-07 \\
1.26 \mathrm{E}-06 \\
7.45 \mathrm{E}-07 \\
9.50 \mathrm{E}-07 \\
3.49 \mathrm{E}-07 \\
3.91 \mathrm{E}-07 \\
4.45 \mathrm{E}-07 \\
2.13 \mathrm{E}-07 \\
1.08 \mathrm{E}-06\end{array}$ & $\begin{array}{l}1.58 \mathrm{E}-07 \\
8.42 \mathrm{E}-07 \\
1.45 \mathrm{E}-06 \\
3.18 \mathrm{E}-07 \\
2.40 \mathrm{E}-07 \\
2.56 \mathrm{E}-07 \\
2.32 \mathrm{E}-07 \\
5.25 \mathrm{E}-07 \\
4.19 \mathrm{E}-07 \\
2.95 \mathrm{E}-07 \\
1.94 \mathrm{E}-07 \\
1.44 \mathrm{E}-07 \\
6.53 \mathrm{E}-08 \\
4.78 \mathrm{E}-08 \\
9.20 \mathrm{E}-08\end{array}$ \\
\hline & $\begin{array}{l}60 \\
61 \\
62 \\
63 \\
64\end{array}$ & $\begin{array}{ll}2017-09-04 & 15: 35 \\
2017-09-04 & 15: 42 \\
2017-09-04 & 15: 54 \\
2017-09-04 & 16: 00 \\
2017-10-12 & 10: 32\end{array}$ & $\begin{array}{l}300 \\
300 \\
300 \\
300 \\
300 \\
\end{array}$ & $\begin{array}{l}51.1 \\
49.5 \\
48.3 \\
45.6 \\
35\end{array}$ & $\begin{array}{c}61 \\
58.9 \\
55.4 \\
54 \\
42.3 \\
\end{array}$ & $\begin{array}{l}259.7 \\
261.1 \\
261.3 \\
257.6 \\
332.1 \\
\end{array}$ & $\begin{array}{l}6.9 \\
6.9 \\
5.6 \\
7.7 \\
5.5\end{array}$ & $\begin{array}{l}7.7 \\
11.7 \\
5.4 \\
6.9 \\
6.6\end{array}$ & $\begin{array}{l}2.1 \\
2.2 \\
2.2 \\
2.5 \\
1.7\end{array}$ & $\begin{array}{l}2.5 \\
2.6 \\
1.8 \\
2.2 \\
1.9\end{array}$ & $\begin{array}{l}1.859 \\
1.875 \\
1.875 \\
1.875 \\
1.859 \\
\end{array}$ & $\begin{array}{l}1.75 \\
1.75 \\
1.766 \\
1.75 \\
1.75 \\
\end{array}$ & $\begin{array}{l}4.33 \mathrm{E}-08 \\
7.56 \mathrm{E}-08 \\
3.28 \mathrm{E}-08 \\
1.10 \mathrm{E}-07 \\
3.84 \mathrm{E}-08 \\
\end{array}$ & $\begin{array}{l}3.54 \mathrm{E}-07 \\
6.58 \mathrm{E}-07 \\
3.00 \mathrm{E}-08 \\
1.76 \mathrm{E}-07 \\
1.28 \mathrm{E}-07\end{array}$ \\
\hline 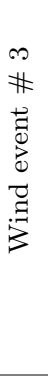 & $\begin{array}{l}65 \\
66 \\
67 \\
68 \\
69 \\
70 \\
71 \\
72 \\
73 \\
74 \\
75 \\
76 \\
77 \\
78\end{array}$ & $\begin{array}{ll}2017-10-15 & 22: 16 \\
2017-10-16 & 01: 00 \\
2017-10-16 & 01: 30 \\
2017-10-16 & 02: 00 \\
2017-10-16 & 02: 30 \\
2017-10-16 & 03: 00 \\
2017-10-16 & 03: 30 \\
2017-10-16 & 04: 00 \\
2017-10-16 & 04: 30 \\
2017-10-16 & 05: 00 \\
2017-10-16 & 05: 30 \\
2017-10-16 & 06: 00 \\
2017-10-16 & 06: 30 \\
2017-10-16 & 07: 00\end{array}$ & $\begin{array}{l}300 \\
300 \\
300 \\
300 \\
300 \\
300 \\
300 \\
300 \\
300 \\
300 \\
300 \\
300 \\
300 \\
300\end{array}$ & $\begin{array}{l}57.2 \\
73.3 \\
64.8 \\
86.2 \\
83.7 \\
79.1 \\
80 \\
71.5 \\
68.9 \\
63.7 \\
63.8 \\
62.6 \\
56.2 \\
55.8 \\
\end{array}$ & $\begin{array}{c}74.8 \\
96 \\
82.6 \\
104.4 \\
107.6 \\
103.7 \\
101.6 \\
94.2 \\
86.1 \\
86.5 \\
80.5 \\
78.7 \\
72 \\
68.8\end{array}$ & $\begin{array}{c}216.6 \\
224.6 \\
230.2 \\
228 \\
223.5 \\
226.4 \\
225.1 \\
228.5 \\
224.8 \\
224 \\
228 \\
221.6 \\
222.9 \\
222.5\end{array}$ & $\begin{array}{l}17.1 \\
15.6 \\
21.2 \\
24.4 \\
27.4 \\
16.8 \\
17.3 \\
12.3 \\
17.1 \\
15.8 \\
14.5 \\
15.9 \\
12.1 \\
11.4\end{array}$ & $\begin{array}{c}20 \\
15.3 \\
15.1 \\
22.8 \\
23.8 \\
16 \\
19.3 \\
11.6 \\
15.9 \\
12.9 \\
9.8 \\
16.3 \\
14.3 \\
7.3 \\
\end{array}$ & $\begin{array}{l}5.3 \\
5.7 \\
6.3 \\
8.5 \\
7.4 \\
6.1 \\
5.9 \\
4.4 \\
5.1 \\
5.3 \\
4.7 \\
5.2 \\
4 \\
3.1 \\
\end{array}$ & $\begin{array}{l}5 \\
5.4 \\
5.9 \\
6.9 \\
6.9 \\
5.8 \\
5.6 \\
4.4 \\
4.4 \\
4.2 \\
3.5 \\
5.1 \\
3.9 \\
2.6\end{array}$ & $\begin{array}{l}1.859 \\
1.859 \\
1.859 \\
1.859 \\
1.844 \\
1.875 \\
1.875 \\
1.859 \\
1.859 \\
1.875 \\
1.859 \\
1.859 \\
1.875 \\
1.859\end{array}$ & $\begin{array}{l}1.781 \\
1.781 \\
1.766 \\
1.781 \\
1.766 \\
1.766 \\
1.781 \\
1.797 \\
1.781 \\
1.781 \\
1.766 \\
1.781 \\
1.75 \\
1.75\end{array}$ & $\begin{array}{l}5.64 \mathrm{E}-06 \\
5.25 \mathrm{E}-06 \\
6.71 \mathrm{E}-06 \\
2.42 \mathrm{E}-05 \\
1.64 \mathrm{E}-05 \\
4.31 \mathrm{E}-06 \\
6.54 \mathrm{E}-06 \\
1.72 \mathrm{E}-06 \\
5.01 \mathrm{E}-06 \\
5.17 \mathrm{E}-06 \\
2.11 \mathrm{E}-06 \\
4.27 \mathrm{E}-06 \\
2.28 \mathrm{E}-06 \\
6.24 \mathrm{E}-07\end{array}$ & $\begin{array}{l}5.96 \mathrm{E}-06 \\
7.83 \mathrm{E}-06 \\
6.79 \mathrm{E}-06 \\
1.76 \mathrm{E}-05 \\
1.75 \mathrm{E}-05 \\
9.65 \mathrm{E}-06 \\
6.71 \mathrm{E}-06 \\
1.63 \mathrm{E}-06 \\
3.74 \mathrm{E}-06 \\
2.71 \mathrm{E}-06 \\
1.14 \mathrm{E}-06 \\
6.90 \mathrm{E}-06 \\
2.25 \mathrm{E}-06 \\
1.44 \mathrm{E}-07\end{array}$ \\
\hline $\begin{array}{l}\# \\
\#\end{array}$ & $\begin{array}{l}79 \\
80 \\
81 \\
82\end{array}$ & $\begin{array}{ll}2017-10-26 & 20: 30 \\
2017-10-26 & 21: 45 \\
2017-10-26 & 21: 54 \\
2017-10-26 & 22: 00\end{array}$ & $\begin{array}{l}300 \\
300 \\
300 \\
300\end{array}$ & $\begin{array}{l}65.1 \\
85.7 \\
89 \\
85\end{array}$ & $\begin{array}{c}75.5 \\
96 \\
97 \\
93.5\end{array}$ & $\begin{array}{c}153.4 \\
145.8 \\
143.1 \\
140\end{array}$ & $\begin{array}{c}13.1 \\
20 \\
19 \\
19.6\end{array}$ & $\begin{array}{l}15.5 \\
29.5 \\
28.8 \\
21.6\end{array}$ & $\begin{array}{c}4.3 \\
7 \\
7 \\
6.3\end{array}$ & $\begin{array}{l}5.6 \\
8.6 \\
8 \\
7.6\end{array}$ & $\begin{array}{l}1.844 \\
1.859 \\
1.859 \\
1.891\end{array}$ & $\begin{array}{l}1.797 \\
1.797 \\
1.781 \\
1.797\end{array}$ & $\begin{array}{l}3.70 \mathrm{E}-06 \\
1.71 \mathrm{E}-05 \\
1.77 \mathrm{E}-05 \\
1.26 \mathrm{E}-05\end{array}$ & $\begin{array}{l}9.40 \mathrm{E}-06 \\
3.57 \mathrm{E}-05 \\
3.12 \mathrm{E}-05 \\
2.22 \mathrm{E}-05\end{array}$ \\
\hline
\end{tabular}


Table D.1 (continued). List of collected datasets with analysis results for the Confederation Bridge light pole study of Section 8.2.

\begin{tabular}{|c|c|c|c|c|c|c|c|c|c|c|c|c|c|c|}
\hline & \multirow[t]{2}{*}{$\begin{array}{l}\text { Data- } \\
\text { set } \\
\#\end{array}$} & \multirow[t]{2}{*}{$\begin{array}{l}\text { Time stamp } \\
\text { (local time) }\end{array}$} & \multirow[t]{2}{*}{$\begin{array}{c}\text { Dataset } \\
\text { dur. } \\
\text { (sec.) }\end{array}$} & \multirow[t]{2}{*}{$\begin{array}{l}\text { Sust. } \\
\text { wind } \\
(\mathrm{km} / \mathrm{h})\end{array}$} & \multirow[t]{2}{*}{$\begin{array}{l}\text { Wind } \\
\text { gusts } \\
(\mathrm{km} / \mathrm{h})\end{array}$} & \multirow[t]{2}{*}{$\begin{array}{l}\text { Wind } \\
\text { dir. } \\
\text { (de- } \\
\text { grees) }\end{array}$} & \multicolumn{2}{|c|}{$\begin{array}{c}\text { Maximum } \\
\text { displacement } \\
\text { (resultant, } \mathrm{mm} \text { ) }\end{array}$} & \multicolumn{2}{|c|}{$\begin{array}{l}\text { Strength of } \\
\text { signal RMS } \\
\quad(\mathrm{mm})\end{array}$} & \multicolumn{2}{|c|}{$\begin{array}{l}\text { Fundamental } \\
\text { frequency } \\
\text { (displacement) }(\mathrm{Hz})\end{array}$} & \multicolumn{2}{|c|}{$\begin{array}{c}\text { Cumulative fatigue } \\
\text { damage (cFD) estimate }\end{array}$} \\
\hline & & & & & & & Undamp. & Damped & Undamp. & Damped & Undamp. & Damped & Undamp. & Damped \\
\hline $\begin{array}{l}H \\
\# \\
\# \\
\overrightarrow{0} \\
0 \\
0 \\
\overrightarrow{0} \\
\vdots \\
3\end{array}$ & $\begin{array}{l}83 \\
84 \\
85 \\
86 \\
87 \\
88 \\
89 \\
90 \\
91 \\
92 \\
93 \\
94 \\
95 \\
96 \\
97\end{array}$ & $\begin{array}{ll}2017-10-26 & 22: 30 \\
2017-10-26 & 23: 00 \\
2017-10-26 & 23: 30 \\
2017-10-27 & 00: 00 \\
2017-10-27 & 00: 30 \\
2017-10-27 & 01: 00 \\
2017-10-27 & 01: 30 \\
2017-10-27 & 02: 00 \\
2017-10-27 & 02: 30 \\
2017-10-27 & 03: 00 \\
2017-10-27 & 03: 30 \\
2017-10-27 & 04: 00 \\
2017-10-27 & 04: 30 \\
2017-10-27 & 05: 00 \\
2017-10-27 & 05: 30\end{array}$ & $\begin{array}{l}300 \\
300 \\
300 \\
300 \\
300 \\
300 \\
300 \\
300 \\
300 \\
300 \\
300 \\
300 \\
300 \\
300 \\
300\end{array}$ & $\begin{array}{c}85.4 \\
78.4 \\
64.6 \\
70 \\
77 \\
82.3 \\
91.2 \\
77.2 \\
77.1 \\
63.7 \\
58.7 \\
56.1 \\
48.9 \\
49.4 \\
52.8 \\
\end{array}$ & $\begin{array}{l}94.2 \\
86.8 \\
72.7 \\
78.7 \\
89.3 \\
90 \\
102 \\
84.7 \\
84.7 \\
67.7 \\
67.4 \\
63.5 \\
52.9 \\
57.9 \\
62.4\end{array}$ & $\begin{array}{l}137.8 \\
134.2 \\
126 \\
129.2 \\
131.2 \\
133.2 \\
134.9 \\
137.1 \\
137.6 \\
148.1 \\
167.8 \\
170.4 \\
176.7 \\
187.4 \\
192.9\end{array}$ & $\begin{array}{c}18.8 \\
12.9 \\
9.2 \\
15.8 \\
17.7 \\
19.3 \\
20.9 \\
17.2 \\
17.1 \\
11 \\
10.7 \\
10 \\
6.6 \\
11.5 \\
12.4\end{array}$ & $\begin{array}{l}26.9 \\
17 \\
13.8 \\
17.8 \\
18.7 \\
22.6 \\
28.1 \\
19.4 \\
16.7 \\
16.4 \\
15.5 \\
13.8 \\
10.8 \\
11.9 \\
11.5\end{array}$ & $\begin{array}{l}6.4 \\
5 \\
3.4 \\
6.5 \\
6.8 \\
6.5 \\
6.9 \\
5.8 \\
5.4 \\
3.8 \\
3.7 \\
3.3 \\
2.5 \\
3.6 \\
4\end{array}$ & $\begin{array}{l}7.7 \\
6.6 \\
4.3 \\
5.9 \\
6.6 \\
7.2 \\
8.5 \\
6.9 \\
6.1 \\
5.4 \\
4.8 \\
4.9 \\
2.9 \\
3.8 \\
3\end{array}$ & $\begin{array}{l}1.859 \\
1.859 \\
1.859 \\
1.859 \\
1.859 \\
1.891 \\
1.844 \\
1.859 \\
1.859 \\
1.859 \\
1.859 \\
1.875 \\
1.875 \\
1.875 \\
1.859\end{array}$ & $\begin{array}{l}1.75 \\
1.766 \\
1.781 \\
1.766 \\
1.75 \\
1.734 \\
1.828 \\
1.781 \\
1.781 \\
1.797 \\
1.781 \\
1.781 \\
1.781 \\
1.75 \\
1.781\end{array}$ & $\begin{array}{l}1.24 \mathrm{E}-05 \\
2.75 \mathrm{E}-06 \\
2.32 \mathrm{E}-07 \\
2.94 \mathrm{E}-06 \\
7.70 \mathrm{E}-06 \\
8.47 \mathrm{E}-06 \\
1.57 \mathrm{E}-05 \\
8.75 \mathrm{E}-06 \\
7.26 \mathrm{E}-06 \\
2.36 \mathrm{E}-06 \\
1.40 \mathrm{E}-06 \\
5.73 \mathrm{E}-07 \\
7.87 \mathrm{E}-08 \\
1.99 \mathrm{E}-06 \\
1.45 \mathrm{E}-06\end{array}$ & $\begin{array}{l}2.75 \mathrm{E}-05 \\
1.36 \mathrm{E}-05 \\
3.77 \mathrm{E}-06 \\
8.72 \mathrm{E}-06 \\
1.22 \mathrm{E}-05 \\
1.61 \mathrm{E}-05 \\
3.60 \mathrm{E}-05 \\
1.69 \mathrm{E}-05 \\
1.10 \mathrm{E}-05 \\
9.36 \mathrm{E}-06 \\
5.59 \mathrm{E}-06 \\
6.07 \mathrm{E}-06 \\
8.74 \mathrm{E}-07 \\
3.22 \mathrm{E}-06 \\
1.14 \mathrm{E}-06\end{array}$ \\
\hline $\begin{array}{l}10 \\
\# \\
\text { \# } \\
\overrightarrow{0} \\
\overrightarrow{0} \\
\overrightarrow{0} \\
\overrightarrow{0} \\
3\end{array}$ & $\begin{array}{l}98 \\
99 \\
100 \\
101 \\
102 \\
103 \\
104 \\
105 \\
106 \\
107 \\
108 \\
109 \\
110 \\
111 \\
112 \\
113 \\
114 \\
115 \\
116 \\
117 \\
\end{array}$ & $\begin{array}{ll}2017-10-30 & 08: 37 \\
2017-10-30 & 09: 00 \\
2017-10-30 & 10: 00 \\
2017-10-30 & 10: 30 \\
2017-10-30 & 11: 00 \\
2017-10-30 & 11: 30 \\
2017-10-30 & 12: 00 \\
2017-10-30 & 12: 30 \\
2017-10-30 & 13: 00 \\
2017-10-30 & 13: 31 \\
2017-10-30 & 13: 56 \\
2017-10-30 & 14: 02 \\
2017-10-30 & 14: 27 \\
2017-10-30 & 14: 36 \\
2017-10-30 & 14: 42 \\
2017-10-30 & 15: 00 \\
2017-10-30 & 15: 15 \\
2017-10-30 & 15: 30 \\
2017-10-30 & 15: 45 \\
2017-10-30 & 16: 00\end{array}$ & $\begin{array}{l}300 \\
300 \\
300 \\
300 \\
300 \\
300 \\
300 \\
300 \\
300 \\
300 \\
300 \\
300 \\
300 \\
300 \\
300 \\
300 \\
300 \\
300 \\
300 \\
300 \\
\end{array}$ & $\begin{array}{l}81.8 \\
78.3 \\
75.5 \\
78 \\
73.3 \\
78.6 \\
76.9 \\
76 \\
77.9 \\
85.6 \\
84.5 \\
93.1 \\
90.9 \\
93.2 \\
92.7 \\
93.9 \\
88.9 \\
91.3 \\
90 \\
85.2 \\
\end{array}$ & $\begin{array}{c}88.9 \\
85.7 \\
86.8 \\
87.5 \\
82.6 \\
89.3 \\
86.5 \\
88.6 \\
85.7 \\
94.9 \\
96 \\
106.5 \\
100.9 \\
105.5 \\
103 \\
104.8 \\
100.2 \\
100.9 \\
97 \\
94.9 \\
\end{array}$ & $\begin{array}{l}127.8 \\
121.1 \\
114.1 \\
111 \\
115.9 \\
114.8 \\
107.7 \\
109 \\
118.9 \\
124 \\
118.7 \\
120.5 \\
121.9 \\
125.4 \\
125 \\
124.7 \\
124.3 \\
123.9 \\
124.8 \\
128.7 \\
\end{array}$ & $\begin{array}{l}15.2 \\
15.6 \\
14.8 \\
15.4 \\
13.5 \\
18.6 \\
14.4 \\
16.7 \\
19 \\
18.1 \\
18.2 \\
21.7 \\
20.9 \\
29.7 \\
26.3 \\
18.1 \\
18.7 \\
19.9 \\
16.9 \\
15.1 \\
\end{array}$ & $\begin{array}{l}20.2 \\
24.2 \\
25.7 \\
22.6 \\
19.9 \\
22.8 \\
22.2 \\
22.3 \\
19.3 \\
23.8 \\
29.9 \\
26.6 \\
32.5 \\
33.2 \\
30.2 \\
26.7 \\
22.5 \\
28.9 \\
28.8 \\
20.5 \\
\end{array}$ & $\begin{array}{l}5.4 \\
5.7 \\
5 \\
5.3 \\
5 \\
5.8 \\
5.4 \\
5.7 \\
5.3 \\
6.5 \\
6.2 \\
7.2 \\
7.1 \\
8.6 \\
9.1 \\
6.9 \\
6.9 \\
7.2 \\
6.1 \\
5.5 \\
\end{array}$ & $\begin{array}{l}7.1 \\
7.1 \\
6.5 \\
6.6 \\
6.3 \\
6.3 \\
6.7 \\
6.4 \\
6 \\
7.3 \\
8.4 \\
8.7 \\
8.8 \\
9.3 \\
9.4 \\
9 \\
7.7 \\
8.4 \\
8.2 \\
6.6 \\
\end{array}$ & $\begin{array}{l}1.891 \\
1.875 \\
1.875 \\
1.875 \\
1.859 \\
1.844 \\
1.906 \\
1.859 \\
1.859 \\
1.859 \\
1.859 \\
1.859 \\
1.844 \\
1.859 \\
1.875 \\
1.859 \\
1.875 \\
1.844 \\
1.875 \\
1.875 \\
\end{array}$ & $\begin{array}{l}1.781 \\
1.75 \\
1.813 \\
1.797 \\
1.797 \\
1.797 \\
1.797 \\
1.75 \\
1.813 \\
1.766 \\
1.766 \\
1.797 \\
1.766 \\
1.766 \\
1.781 \\
1.828 \\
1.766 \\
1.781 \\
1.766 \\
1.781 \\
\end{array}$ & $\begin{array}{l}2.46 \mathrm{E}-06 \\
3.62 \mathrm{E}-06 \\
2.61 \mathrm{E}-06 \\
3.61 \mathrm{E}-06 \\
2.32 \mathrm{E}-06 \\
4.09 \mathrm{E}-06 \\
3.94 \mathrm{E}-06 \\
3.60 \mathrm{E}-06 \\
4.45 \mathrm{E}-06 \\
6.03 \mathrm{E}-06 \\
5.48 \mathrm{E}-06 \\
8.07 \mathrm{E}-06 \\
9.94 \mathrm{E}-06 \\
1.07 \mathrm{E}-05 \\
1.24 \mathrm{E}-05 \\
1.26 \mathrm{E}-05 \\
5.79 \mathrm{E}-06 \\
1.15 \mathrm{E}-05 \\
2.91 \mathrm{E}-06 \\
4.38 \mathrm{E}-06\end{array}$ & $\begin{array}{l}1.88 \mathrm{E}-05 \\
2.09 \mathrm{E}-05 \\
1.04 \mathrm{E}-05 \\
1.20 \mathrm{E}-05 \\
8.52 \mathrm{E}-06 \\
9.57 \mathrm{E}-06 \\
1.16 \mathrm{E}-05 \\
1.05 \mathrm{E}-05 \\
8.84 \mathrm{E}-06 \\
1.77 \mathrm{E}-05 \\
3.29 \mathrm{E}-05 \\
3.28 \mathrm{E}-05 \\
4.32 \mathrm{E}-05 \\
4.03 \mathrm{E}-05 \\
4.48 \mathrm{E}-05 \\
3.35 \mathrm{E}-05 \\
2.48 \mathrm{E}-05 \\
3.52 \mathrm{E}-05 \\
3.30 \mathrm{E}-05 \\
1.64 \mathrm{E}-05\end{array}$ \\
\hline $\begin{array}{l}0 \\
\#\end{array}$ & $\begin{array}{l}118 \\
119 \\
120 \\
121 \\
122 \\
123 \\
\end{array}$ & $\begin{array}{ll}2017-11-04 & 00: 00 \\
2017-11-04 & 00: 30 \\
2017-11-04 & 01: 00 \\
2017-11-04 & 01: 30 \\
2017-11-04 & 02: 00 \\
2017-11-04 & 02: 30\end{array}$ & $\begin{array}{l}300 \\
300 \\
300 \\
300 \\
300 \\
300 \\
\end{array}$ & $\begin{array}{l}69.7 \\
61.1 \\
70.6 \\
74.9 \\
72.7 \\
65.7 \\
\end{array}$ & $\begin{array}{c}85.7 \\
70.2 \\
86.1 \\
90 \\
84 \\
77.3 \\
\end{array}$ & $\begin{array}{l}283.4 \\
283.5 \\
292.5 \\
294.1 \\
297.2 \\
296.8 \\
\end{array}$ & $\begin{array}{c}11.5 \\
12 \\
14 \\
17.3 \\
13.5 \\
10.9 \\
\end{array}$ & $\begin{array}{c}16.7 \\
12.2 \\
14.2 \\
16 \\
15.8 \\
10.1 \\
\end{array}$ & $\begin{array}{l}4 \\
3.4 \\
5.2 \\
6.7 \\
5.3 \\
3.7 \\
\end{array}$ & $\begin{array}{l}4.1 \\
3.6 \\
4.4 \\
4.2 \\
4.1 \\
3.4 \\
\end{array}$ & $\begin{array}{l}1.875 \\
1.859 \\
1.844 \\
1.828 \\
1.859 \\
1.891 \\
\end{array}$ & $\begin{array}{l}1.781 \\
1.75 \\
1.797 \\
1.797 \\
1.766 \\
1.766 \\
\end{array}$ & $\begin{array}{l}1.01 \mathrm{E}-06 \\
8.99 \mathrm{E}-07 \\
4.56 \mathrm{E}-06 \\
1.06 \mathrm{E}-05 \\
4.91 \mathrm{E}-06 \\
1.03 \mathrm{E}-06 \\
\end{array}$ & $\begin{array}{l}2.04 \mathrm{E}-06 \\
1.44 \mathrm{E}-06 \\
4.83 \mathrm{E}-06 \\
3.25 \mathrm{E}-06 \\
2.70 \mathrm{E}-06 \\
1.18 \mathrm{E}-06 \\
\end{array}$ \\
\hline
\end{tabular}


Table D.1 (continued). List of collected datasets with analysis results for the Confederation Bridge light pole study of Section 8.2.

\begin{tabular}{|c|c|c|c|c|c|c|c|c|c|c|c|c|c|c|}
\hline & \multirow[t]{2}{*}{$\begin{array}{l}\text { Data- } \\
\text { set } \\
\#\end{array}$} & \multirow[t]{2}{*}{$\begin{array}{l}\text { Time stamp } \\
\text { (local time) }\end{array}$} & \multirow[t]{2}{*}{$\begin{array}{l}\text { Dataset } \\
\text { dur. } \\
\text { (sec.) }\end{array}$} & \multirow[t]{2}{*}{$\begin{array}{l}\text { Sust. } \\
\text { wind } \\
(\mathrm{km} / \mathrm{h})\end{array}$} & \multirow[t]{2}{*}{$\begin{array}{l}\text { Wind } \\
\text { gusts } \\
(\mathrm{km} / \mathrm{h})\end{array}$} & \multirow[t]{2}{*}{$\begin{array}{l}\text { Wind } \\
\text { dir. } \\
\text { (de- } \\
\text { grees) }\end{array}$} & \multicolumn{2}{|c|}{$\begin{array}{c}\text { Maximum } \\
\text { displacement } \\
\text { (resultant, } \mathrm{mm})\end{array}$} & \multicolumn{2}{|c|}{$\begin{array}{l}\text { Strength of } \\
\text { signal RMS } \\
(\mathrm{mm})\end{array}$} & \multicolumn{2}{|c|}{$\begin{array}{l}\text { Fundamental } \\
\text { frequency } \\
\text { (displacement)(Hz) }\end{array}$} & \multicolumn{2}{|c|}{$\begin{array}{c}\text { Cumulative fatigue } \\
\text { damage (cFD) estimate }\end{array}$} \\
\hline & & & & & & & Undamp. & $\overline{\text { Damped }}$ & Undamp. & $\overline{\text { Damped }}$ & Undamp. & $\overline{\text { Damped }}$ & Undamp. & Damped \\
\hline 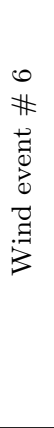 & $\begin{array}{l}124 \\
125 \\
126 \\
127 \\
128 \\
129 \\
130 \\
131 \\
132 \\
133 \\
134 \\
135 \\
136 \\
137 \\
138 \\
139\end{array}$ & $\begin{array}{ll}2017-11-04 & 03: 00 \\
2017-11-04 & 03: 30 \\
2017-11-04 & 04: 00 \\
2017-11-04 & 04: 30 \\
2017-11-04 & 05: 00 \\
2017-11-04 & 05: 30 \\
2017-11-04 & 06: 00 \\
2017-11-04 & 06: 30 \\
2017-11-04 & 07: 00 \\
2017-11-04 & 07: 30 \\
2017-11-04 & 08: 30 \\
2017-11-04 & 09: 00 \\
2017-11-04 & 09: 30 \\
2017-11-04 & 10: 00 \\
2017-11-04 & 10: 30 \\
2017-11-04 & 11: 00\end{array}$ & $\begin{array}{l}300 \\
300 \\
300 \\
300 \\
300 \\
300 \\
300 \\
300 \\
300 \\
300 \\
300 \\
300 \\
300 \\
300 \\
300 \\
300\end{array}$ & $\begin{array}{l}55.3 \\
70.4 \\
72.8 \\
73.3 \\
81.3 \\
81.3 \\
77.9 \\
75.5 \\
74.7 \\
74.1 \\
68.3 \\
65.3 \\
66.9 \\
59.9 \\
57.2 \\
57.2\end{array}$ & $\begin{array}{c}65.6 \\
78.7 \\
81.9 \\
87.9 \\
92.4 \\
96.3 \\
91.4 \\
90 \\
90 \\
86.8 \\
79.4 \\
76.2 \\
79.7 \\
68.4 \\
69.2 \\
65.6\end{array}$ & $\begin{array}{c}303 \\
286.3 \\
290.4 \\
291.3 \\
293.2 \\
299.4 \\
294.9 \\
294.6 \\
296.6 \\
295.3 \\
302.8 \\
306.5 \\
314 \\
310.6 \\
311.5 \\
311.3\end{array}$ & $\begin{array}{l}10.3 \\
13.8 \\
14.8 \\
15.1 \\
24.2 \\
23.3 \\
18.6 \\
14.7 \\
17.3 \\
15.1 \\
21.4 \\
14.3 \\
12.9 \\
11.2 \\
12.7 \\
9.4\end{array}$ & $\begin{array}{c}9.8 \\
11.1 \\
13.1 \\
16.8 \\
17.1 \\
15 \\
14.6 \\
11.9 \\
13.7 \\
14.1 \\
11.5 \\
12.4 \\
9.1 \\
11.1 \\
9.1 \\
7.8\end{array}$ & $\begin{array}{l}3.3 \\
4.6 \\
5.3 \\
4.9 \\
6.9 \\
7 \\
6.3 \\
5 \\
5.6 \\
5.3 \\
4.9 \\
4.1 \\
4.8 \\
3.7 \\
4.1 \\
3.7\end{array}$ & $\begin{array}{l}2.9 \\
3.6 \\
4.2 \\
4.7 \\
4.8 \\
4.6 \\
4.7 \\
4 \\
3.8 \\
4.3 \\
3.5 \\
3.5 \\
3.5 \\
3.2 \\
3 \\
2.7\end{array}$ & $\begin{array}{l}1.844 \\
1.875 \\
1.891 \\
1.891 \\
1.844 \\
1.844 \\
1.859 \\
1.844 \\
1.875 \\
1.859 \\
1.875 \\
1.875 \\
1.859 \\
1.844 \\
1.875 \\
1.859\end{array}$ & $\begin{array}{l}1.766 \\
1.75 \\
1.781 \\
1.781 \\
1.766 \\
1.797 \\
1.781 \\
1.766 \\
1.797 \\
1.797 \\
1.797 \\
1.781 \\
1.781 \\
1.766 \\
1.766 \\
1.781\end{array}$ & $\begin{array}{l}5.36 \mathrm{E}-07 \\
1.01 \mathrm{E}-06 \\
4.09 \mathrm{E}-06 \\
2.38 \mathrm{E}-06 \\
1.43 \mathrm{E}-05 \\
7.77 \mathrm{E}-06 \\
1.22 \mathrm{E}-05 \\
3.47 \mathrm{E}-06 \\
4.50 \mathrm{E}-06 \\
4.79 \mathrm{E}-06 \\
4.43 \mathrm{E}-06 \\
1.73 \mathrm{E}-06 \\
4.03 \mathrm{E}-06 \\
1.19 \mathrm{E}-06 \\
2.59 \mathrm{E}-06 \\
8.58 \mathrm{E}-07\end{array}$ & $\begin{array}{l}5.08 \mathrm{E}-07 \\
1.18 \mathrm{E}-06 \\
2.59 \mathrm{E}-06 \\
6.43 \mathrm{E}-06 \\
4.53 \mathrm{E}-06 \\
4.03 \mathrm{E}-06 \\
4.60 \mathrm{E}-06 \\
1.51 \mathrm{E}-06 \\
1.14 \mathrm{E}-06 \\
3.89 \mathrm{E}-06 \\
6.17 \mathrm{E}-07 \\
9.38 \mathrm{E}-07 \\
7.88 \mathrm{E}-07 \\
1.36 \mathrm{E}-06 \\
3.70 \mathrm{E}-07 \\
2.18 \mathrm{E}-07\end{array}$ \\
\hline 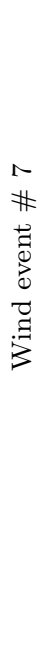 & $\begin{array}{l}140 \\
141 \\
142 \\
143 \\
144 \\
145 \\
146 \\
147 \\
148 \\
149 \\
150 \\
151 \\
152 \\
153 \\
154 \\
155 \\
156 \\
157 \\
158 \\
159 \\
160 \\
161 \\
162 \\
163 \\
164 \\
165\end{array}$ & $\begin{array}{ll}2017-11-10 & 14: 29 \\
2017-11-10 & 16: 00 \\
2017-11-10 & 16: 30 \\
2017-11-10 & 17: 00 \\
2017-11-10 & 17: 30 \\
2017-11-10 & 18: 00 \\
2017-11-10 & 18: 30 \\
2017-11-10 & 19: 00 \\
2017-11-10 & 19: 30 \\
2017-11-10 & 20: 00 \\
2017-11-10 & 20: 30 \\
2017-11-10 & 21: 00 \\
2017-11-10 & 21: 30 \\
2017-11-10 & 22: 00 \\
2017-11-10 & 22: 30 \\
2017-11-10 & 23: 00 \\
2017-11-10 & 23: 30 \\
2017-11-11 & 00000 \\
2017-11-11 & 00: 30 \\
2017-11-11 & 01: 00 \\
2017-11-11 & 01: 30 \\
2017-11-11 & 02: 00 \\
2017-11-11 & 02: 30 \\
2017-11-11 & 03: 00 \\
2017-11-11 & 03: 30 \\
2017-11-11 & 04: 00\end{array}$ & $\begin{array}{l}300 \\
300 \\
300 \\
300 \\
300 \\
300 \\
300 \\
300 \\
300 \\
300 \\
300 \\
300 \\
300 \\
300 \\
300 \\
300 \\
300 \\
300 \\
300 \\
300 \\
300 \\
300 \\
300 \\
300 \\
300 \\
300\end{array}$ & $\begin{array}{l}59.2 \\
61.7 \\
61.2 \\
57.4 \\
59.4 \\
64.1 \\
63.7 \\
67.6 \\
60.9 \\
64.7 \\
64 \\
67.5 \\
57.9 \\
62.9 \\
67.9 \\
61.8 \\
67.3 \\
64.5 \\
60.5 \\
54.2 \\
56 \\
59.1 \\
61.2 \\
56.4 \\
56.4 \\
54.4\end{array}$ & $\begin{array}{l}70.2 \\
72.7 \\
70.9 \\
74.5 \\
76.2 \\
75.9 \\
73.7 \\
79.7 \\
74.1 \\
76.6 \\
78.7 \\
79.7 \\
79 \\
91 \\
82.6 \\
79.7 \\
83.3 \\
84.7 \\
73.4 \\
69.9 \\
67.7 \\
76.6 \\
77.6 \\
70.6 \\
71.6 \\
75.2\end{array}$ & $\begin{array}{l}288.6 \\
272.1 \\
266.6 \\
267.8 \\
267.3 \\
266.6 \\
263.1 \\
269.1 \\
266.1 \\
266.5 \\
265 \\
266 \\
263.4 \\
267.7 \\
263.7 \\
264.5 \\
267.8 \\
257.1 \\
255.2 \\
251.7 \\
248.9 \\
252.4 \\
253.7 \\
250.7 \\
254.3 \\
255.3\end{array}$ & $\begin{array}{l}13.6 \\
11.5 \\
12.3 \\
13.7 \\
11.9 \\
15.6 \\
12.7 \\
13.8 \\
19 \\
15.2 \\
12.9 \\
19.3 \\
13.3 \\
21.2 \\
17.1 \\
11.4 \\
19.6 \\
15.2 \\
13.6 \\
10.3 \\
9.3 \\
10.6 \\
20.9 \\
12.7 \\
13.4 \\
11.5\end{array}$ & $\begin{array}{c}9.1 \\
10.6 \\
9.4 \\
17.5 \\
8.8 \\
14.3 \\
14 \\
12.3 \\
13.4 \\
9.7 \\
11.5 \\
21.6 \\
12.6 \\
14 \\
16.2 \\
11 \\
14.1 \\
16.4 \\
18.9 \\
9.9 \\
12.6 \\
12.6 \\
12.7 \\
14.8 \\
16.3 \\
9.7\end{array}$ & $\begin{array}{l}3.3 \\
3.7 \\
3.8 \\
3.9 \\
3.5 \\
4.6 \\
4.3 \\
4.3 \\
4.4 \\
4.4 \\
4.1 \\
5.7 \\
3.6 \\
4.6 \\
4.9 \\
3.7 \\
5 \\
4.3 \\
4.2 \\
3.4 \\
3 \\
4 \\
4.5 \\
3.3 \\
4 \\
3.5\end{array}$ & $\begin{array}{l}3.1 \\
3.1 \\
3.2 \\
3.5 \\
3.3 \\
3.3 \\
4 \\
4.4 \\
3.6 \\
3.1 \\
3.4 \\
5.2 \\
3.2 \\
4 \\
3.9 \\
3.5 \\
3.9 \\
3.7 \\
4.2 \\
3.1 \\
3.5 \\
3.6 \\
3.5 \\
3.7 \\
3.7 \\
3.4\end{array}$ & $\begin{array}{l}1.875 \\
1.891 \\
1.891 \\
1.906 \\
1.891 \\
1.891 \\
1.891 \\
1.875 \\
1.875 \\
1.906 \\
1.891 \\
1.875 \\
1.891 \\
1.891 \\
1.891 \\
1.891 \\
1.875 \\
1.906 \\
1.891 \\
1.922 \\
1.922 \\
1.875 \\
1.891 \\
1.906 \\
1.875\end{array}$ & $\begin{array}{l}1.75 \\
1.75 \\
1.75 \\
1.75 \\
1.766 \\
1.766 \\
1.734 \\
1.766 \\
1.766 \\
1.781 \\
1.766 \\
1.75 \\
1.781 \\
1.797 \\
1.781 \\
1.766 \\
1.781 \\
1.797 \\
1.781 \\
1.813 \\
1.781 \\
1.781 \\
1.766 \\
1.766 \\
1.781 \\
1.766\end{array}$ & $\begin{array}{l}1.09 \mathrm{E}-06 \\
1.76 \mathrm{E}-06 \\
2.25 \mathrm{E}-06 \\
3.06 \mathrm{E}-06 \\
1.55 \mathrm{E}-06 \\
6.79 \mathrm{E}-06 \\
2.11 \mathrm{E}-06 \\
4.60 \mathrm{E}-06 \\
4.95 \mathrm{E}-06 \\
4.55 \mathrm{E}-06 \\
2.20 \mathrm{E}-06 \\
1.19 \mathrm{E}-05 \\
2.58 \mathrm{E}-06 \\
6.03 \mathrm{E}-06 \\
7.71 \mathrm{E}-06 \\
1.99 \mathrm{E}-06 \\
8.82 \mathrm{E}-06 \\
4.84 \mathrm{E}-06 \\
3.46 \mathrm{E}-06 \\
7.69 \mathrm{E}-07 \\
2.87 \mathrm{E}-07 \\
1.73 \mathrm{E}-06 \\
7.50 \mathrm{E}-06 \\
1.14 \mathrm{E}-06 \\
3.58 \mathrm{E}-06 \\
1.46 \mathrm{E}-06\end{array}$ & $\begin{array}{l}5.91 \mathrm{E}-07 \\
4.98 \mathrm{E}-07 \\
4.93 \mathrm{E}-07 \\
1.07 \mathrm{E}-06 \\
3.12 \mathrm{E}-07 \\
9.18 \mathrm{E}-07 \\
1.36 \mathrm{E}-06 \\
2.80 \mathrm{E}-06 \\
8.86 \mathrm{E}-07 \\
3.99 \mathrm{E}-07 \\
6.23 \mathrm{E}-07 \\
6.39 \mathrm{E}-06 \\
5.74 \mathrm{E}-07 \\
1.85 \mathrm{E}-06 \\
9.70 \mathrm{E}-07 \\
5.82 \mathrm{E}-07 \\
1.17 \mathrm{E}-06 \\
1.61 \mathrm{E}-06 \\
8.71 \mathrm{E}-07 \\
3.31 \mathrm{E}-07 \\
1.15 \mathrm{E}-06 \\
8.64 \mathrm{E}-07 \\
1.08 \mathrm{E}-06 \\
1.06 \mathrm{E}-06 \\
1.55 \mathrm{E}-06 \\
6.22 \mathrm{E}-07\end{array}$ \\
\hline
\end{tabular}


Table D.1 (continued). List of collected datasets with analysis results for the Confederation Bridge light pole study of Section 8.2 .

\begin{tabular}{|c|c|c|c|c|c|c|c|c|c|c|c|c|c|c|}
\hline & \multirow[t]{2}{*}{$\begin{array}{l}\text { Data- } \\
\text { set } \\
\#\end{array}$} & \multirow[t]{2}{*}{$\begin{array}{l}\text { Time stamp } \\
\text { (local time) }\end{array}$} & \multirow[t]{2}{*}{$\begin{array}{l}\text { Dataset } \\
\text { dur. } \\
\text { (sec.) }\end{array}$} & \multirow[t]{2}{*}{$\begin{array}{l}\text { Sust. } \\
\text { wind } \\
(\mathrm{km} / \mathrm{h})\end{array}$} & \multirow[t]{2}{*}{$\begin{array}{l}\text { Wind } \\
\text { gusts } \\
(\mathrm{km} / \mathrm{h})\end{array}$} & \multirow[t]{2}{*}{$\begin{array}{l}\text { Wind } \\
\text { dir. } \\
\text { (de- } \\
\text { grees) }\end{array}$} & \multicolumn{2}{|c|}{$\begin{array}{c}\text { Maximum } \\
\text { displacement } \\
\text { (resultant, } \mathrm{mm})\end{array}$} & \multicolumn{2}{|c|}{$\begin{array}{l}\text { Strength of } \\
\text { signal RMS } \\
\quad(\mathrm{mm})\end{array}$} & \multicolumn{2}{|c|}{$\begin{array}{l}\text { Fundamental } \\
\text { frequency } \\
\text { (displacement)(Hz) }\end{array}$} & \multicolumn{2}{|c|}{$\begin{array}{c}\text { Cumulative fatigue } \\
\text { damage (cFD) estimate }\end{array}$} \\
\hline & & & & & & & Undamp. & $\overline{\text { Damped }}$ & Undamp. & $\overline{\text { Damped }}$ & Undamp. & $\overline{\text { Damped }}$ & Undamp. & Damped \\
\hline $\begin{array}{l}\sim \\
\# \\
\# \\
\overrightarrow{0} \\
\overrightarrow{0} \\
\overrightarrow{0} \\
\vec{\Xi} \\
\vec{j}\end{array}$ & $\begin{array}{l}166 \\
167 \\
168 \\
169 \\
170 \\
171 \\
172 \\
173 \\
174 \\
175\end{array}$ & $\begin{array}{lll}2017-11-11 & 04: 30 \\
2017-11-11 & 05: 00 \\
2017-11-11 & 05: 30 \\
2017-11-11 & 06: 00 \\
2017-11-11 & 06: 30 \\
2017-11-11 & 07: 00 \\
2017-11-11 & 07: 30 \\
2017-11-11 & 08: 00 \\
2017-11-11 & 08: 30 \\
2017-11-11 & 09: 00\end{array}$ & $\begin{array}{l}300 \\
300 \\
300 \\
300 \\
300 \\
300 \\
300 \\
300 \\
300 \\
300\end{array}$ & $\begin{array}{l}58.4 \\
59.8 \\
60.2 \\
60.9 \\
58.3 \\
53.8 \\
55.8 \\
60.9 \\
62.9 \\
62.3\end{array}$ & $\begin{array}{l}76.6 \\
74.8 \\
74.8 \\
74.1 \\
70.9 \\
68.4 \\
75.9 \\
76.2 \\
77.6 \\
76.6\end{array}$ & $\begin{array}{l}255.5 \\
260.8 \\
260 \\
259.4 \\
260.9 \\
261.8 \\
266.6 \\
269.5 \\
271.7 \\
271.9\end{array}$ & $\begin{array}{l}16.8 \\
13.1 \\
11.8 \\
14.6 \\
14.2 \\
12.2 \\
13 \\
14.1 \\
14.3 \\
11.3\end{array}$ & $\begin{array}{l}12.3 \\
11.9 \\
11.6 \\
10.4 \\
9.5 \\
10.5 \\
10.8 \\
14.6 \\
14.6 \\
11.7\end{array}$ & $\begin{array}{l}4.3 \\
4.1 \\
3.7 \\
4 \\
4.2 \\
3.4 \\
4.1 \\
4 \\
4.5 \\
4.3\end{array}$ & $\begin{array}{l}3.8 \\
3.2 \\
3.5 \\
3 \\
3.1 \\
2.9 \\
3.6 \\
4 \\
4.2 \\
3.3\end{array}$ & $\begin{array}{l}1.875 \\
1.891 \\
1.891 \\
1.875 \\
1.875 \\
1.891 \\
1.875 \\
1.891 \\
1.891 \\
1.891\end{array}$ & $\begin{array}{l}1.781 \\
1.766 \\
1.781 \\
1.766 \\
1.75 \\
1.766 \\
1.797 \\
1.797 \\
1.766 \\
1.75\end{array}$ & $\begin{array}{l}5.14 \mathrm{E}-06 \\
4.12 \mathrm{E}-06 \\
1.95 \mathrm{E}-06 \\
3.52 \mathrm{E}-06 \\
4.99 \mathrm{E}-06 \\
1.92 \mathrm{E}-06 \\
2.47 \mathrm{E}-06 \\
2.58 \mathrm{E}-06 \\
4.11 \mathrm{E}-06 \\
4.61 \mathrm{E}-06\end{array}$ & $\begin{array}{l}8.98 \mathrm{E}-07 \\
5.10 \mathrm{E}-07 \\
1.01 \mathrm{E}-06 \\
2.76 \mathrm{E}-07 \\
4.35 \mathrm{E}-07 \\
2.73 \mathrm{E}-07 \\
7.90 \mathrm{E}-07 \\
1.67 \mathrm{E}-06 \\
1.70 \mathrm{E}-06 \\
5.53 \mathrm{E}-07\end{array}$ \\
\hline $\begin{array}{l}\infty \\
\#\end{array}$ & $\begin{array}{l}176 \\
177 \\
178 \\
179 \\
180 \\
181 \\
182 \\
183 \\
184 \\
185 \\
186 \\
187 \\
188 \\
189 \\
190 \\
191 \\
192 \\
193 \\
194 \\
195 \\
196 \\
197 \\
198 \\
199 \\
200 \\
201 \\
202 \\
203 \\
204 \\
205 \\
206 \\
207\end{array}$ & $\begin{array}{ll}2017-11-17 & 14: 53 \\
2017-11-17 & 15: 20 \\
2017-11-17 & 15: 40 \\
2017-11-17 & 16: 00 \\
2017-11-17 & 16: 20 \\
2017-11-17 & 16: 40 \\
2017-11-17 & 17: 00 \\
2017-11-17 & 17: 20 \\
2017-11-17 & 17: 40 \\
2017-11-17 & 18: 00 \\
2017-11-17 & 18: 20 \\
2017-11-17 & 18: 40 \\
2017-11-17 & 1900 \\
2017-11-17 & 19: 20 \\
2017-11-17 & 19: 40 \\
2017-11-17 & 2000 \\
2017-11-17 & 20: 20 \\
2017-11-17 & 20: 40 \\
2017-11-17 & 21: 00 \\
2017-11-17 & 21: 20 \\
2017-11-17 & 21: 40 \\
2017-11-17 & 22: 00 \\
2017-11-17 & 22: 20 \\
2017-11-17 & 22: 40 \\
2017-11-17 & 23: 00 \\
2017-11-17 & 23: 20 \\
2017-11-17 & 23: 40 \\
2017-11-18 & 00: 00 \\
2017-11-18 & 00: 20 \\
2017-11-18 & 00: 40 \\
2017-11-18 & 01: 00 \\
2017-11-18 & 01: 20\end{array}$ & $\begin{array}{l}300 \\
300 \\
300 \\
300 \\
300 \\
300 \\
300 \\
300 \\
300 \\
300 \\
300 \\
300 \\
300 \\
300 \\
300 \\
300 \\
300 \\
300 \\
300 \\
300 \\
300 \\
300 \\
300 \\
300 \\
300 \\
300 \\
300 \\
300 \\
300 \\
300 \\
300 \\
300\end{array}$ & $\begin{array}{c}76 \\
75.8 \\
77.2 \\
74.7 \\
74.7 \\
77.5 \\
82.5 \\
83.1 \\
82.5 \\
86.5 \\
88.5 \\
82.8 \\
84.6 \\
81 \\
80.5 \\
81.9 \\
81.7 \\
79.3 \\
75.9 \\
78.2 \\
77.4 \\
77 \\
76.5 \\
78.9 \\
77.7 \\
79.9 \\
81.8 \\
86.7 \\
84.3 \\
75.1 \\
77.2 \\
74.8\end{array}$ & $\begin{array}{l}86.5 \\
86.5 \\
90 \\
88.2 \\
92.1 \\
90.3 \\
91.4 \\
93.5 \\
95.3 \\
100.9 \\
104.4 \\
98.4 \\
95.6 \\
95.6 \\
92.1 \\
95.3 \\
91.4 \\
92.1 \\
88.2 \\
87.5 \\
90.3 \\
87.2 \\
87.5 \\
90 \\
90.7 \\
92.1 \\
96 \\
103.7 \\
98.4 \\
89.6 \\
91 \\
91.7\end{array}$ & $\begin{array}{l}282.3 \\
288.7 \\
290.3 \\
291.8 \\
289.4 \\
288.7 \\
287.8 \\
287.7 \\
289.3 \\
293.8 \\
295.4 \\
293.8 \\
289.8 \\
289.6 \\
289.3 \\
290.4 \\
291.7 \\
293.7 \\
298.4 \\
300.4 \\
298.6 \\
299.1 \\
300.3 \\
302 \\
300.9 \\
295.9 \\
294.9 \\
296.5 \\
292.2 \\
292.2 \\
290.5 \\
289.7\end{array}$ & $\begin{array}{l}21.6 \\
19.3 \\
18.2 \\
15.5 \\
15.3 \\
18.1 \\
20.3 \\
16.3 \\
17.9 \\
21.7 \\
19.7 \\
17 \\
19.8 \\
18.9 \\
16.5 \\
16 \\
17.4 \\
16.5 \\
15.7 \\
14.5 \\
12.9 \\
19.4 \\
20.3 \\
15.1 \\
14.4 \\
18 \\
19.5 \\
19.4 \\
15.7 \\
17 \\
17.1 \\
16.2\end{array}$ & $\begin{array}{c}17.6 \\
18 \\
16.3 \\
20.2 \\
19.3 \\
15.3 \\
18.3 \\
26 \\
25 \\
19.2 \\
19.7 \\
15.8 \\
16.5 \\
21.3 \\
20.3 \\
19.9 \\
17.6 \\
19.4 \\
16.2 \\
15.3 \\
16.3 \\
15 \\
17 \\
20 \\
18 \\
16.5 \\
16 \\
17.1 \\
15.6 \\
17.6 \\
15.3 \\
18.5\end{array}$ & $\begin{array}{l}5.1 \\
5.3 \\
4.9 \\
5.1 \\
4.7 \\
5.6 \\
6.1 \\
4.9 \\
5.3 \\
5.6 \\
6.6 \\
5.7 \\
6.3 \\
5.7 \\
5.1 \\
5.3 \\
5.9 \\
5.5 \\
4.6 \\
5.1 \\
4.3 \\
5.3 \\
5.7 \\
5.2 \\
4.9 \\
5.3 \\
5.6 \\
5.4 \\
4.9 \\
5.6 \\
4.6 \\
5.2\end{array}$ & $\begin{array}{l}4.9 \\
4.8 \\
4.5 \\
4.4 \\
4.8 \\
5.1 \\
5.2 \\
5.7 \\
5.7 \\
5.7 \\
6.2 \\
4.8 \\
5.4 \\
5.2 \\
5.4 \\
4.6 \\
5.5 \\
4.9 \\
4.8 \\
4.8 \\
4.3 \\
5 \\
5 \\
4.8 \\
5 \\
4.9 \\
5.5 \\
5.8 \\
5 \\
5.1 \\
4 \\
4.6\end{array}$ & $\begin{array}{l}1.906 \\
1.891 \\
1.906 \\
1.938 \\
1.875 \\
1.906 \\
1.891 \\
1.891 \\
1.875 \\
1.891 \\
1.891 \\
1.891 \\
1.922 \\
1.875 \\
1.859 \\
1.891 \\
1.891 \\
1.906 \\
1.906 \\
1.891 \\
1.906 \\
1.906 \\
1.922 \\
1.875 \\
1.922 \\
1.875 \\
1.906 \\
1.891 \\
1.906 \\
1.891 \\
1.891 \\
1.891\end{array}$ & $\begin{array}{l}1.75 \\
1.766 \\
1.734 \\
1.797 \\
1.781 \\
1.75 \\
1.766 \\
1.75 \\
1.75 \\
1.781 \\
1.734 \\
1.734 \\
1.766 \\
1.75 \\
1.719 \\
1.734 \\
1.719 \\
1.734 \\
1.734 \\
1.734 \\
1.766 \\
1.734 \\
1.781 \\
1.781 \\
1.734 \\
1.781 \\
1.766 \\
1.766 \\
1.766 \\
1.75 \\
1.75 \\
1.766\end{array}$ & $\begin{array}{l}6.03 \mathrm{E}-06 \\
7.97 \mathrm{E}-06 \\
6.11 \mathrm{E}-06 \\
6.21 \mathrm{E}-06 \\
4.14 \mathrm{E}-06 \\
1.08 \mathrm{E}-05 \\
1.61 \mathrm{E}-05 \\
6.41 \mathrm{E}-06 \\
8.90 \mathrm{E}-06 \\
9.07 \mathrm{E}-06 \\
1.72 \mathrm{E}-05 \\
1.08 \mathrm{E}-05 \\
1.47 \mathrm{E}-05 \\
9.83 \mathrm{E}-06 \\
6.67 \mathrm{E}-06 \\
7.24 \mathrm{E}-06 \\
1.24 \mathrm{E}-05 \\
7.87 \mathrm{E}-06 \\
4.33 \mathrm{E}-06 \\
6.62 \mathrm{E}-06 \\
2.59 \mathrm{E}-06 \\
9.15 \mathrm{E}-06 \\
9.56 \mathrm{E}-06 \\
6.88 \mathrm{E}-06 \\
5.31 \mathrm{E}-06 \\
9.15 \mathrm{E}-06 \\
8.27 \mathrm{E}-06 \\
7.41 \mathrm{E}-06 \\
5.66 \mathrm{E}-06 \\
1.08 \mathrm{E}-05 \\
6.04 \mathrm{E}-06 \\
8.92 \mathrm{E}-06\end{array}$ & $\begin{array}{l}4.41 \mathrm{E}-06 \\
3.03 \mathrm{E}-06 \\
1.66 \mathrm{E}-06 \\
1.68 \mathrm{E}-06 \\
2.19 \mathrm{E}-06 \\
3.22 \mathrm{E}-06 \\
6.24 \mathrm{E}-06 \\
6.17 \mathrm{E}-06 \\
3.83 \mathrm{E}-06 \\
5.13 \mathrm{E}-06 \\
6.19 \mathrm{E}-06 \\
2.89 \mathrm{E}-06 \\
5.56 \mathrm{E}-06 \\
4.59 \mathrm{E}-06 \\
4.04 \mathrm{E}-06 \\
1.60 \mathrm{E}-06 \\
5.62 \mathrm{E}-06 \\
2.70 \mathrm{E}-06 \\
3.14 \mathrm{E}-06 \\
4.01 \mathrm{E}-06 \\
1.87 \mathrm{E}-06 \\
3.48 \mathrm{E}-06 \\
3.74 \mathrm{E}-06 \\
4.44 \mathrm{E}-06 \\
3.81 \mathrm{E}-06 \\
4.42 \mathrm{E}-06 \\
4.20 \mathrm{E}-06 \\
6.95 \mathrm{E}-06 \\
3.82 \mathrm{E}-06 \\
5.72 \mathrm{E}-06 \\
1.67 \mathrm{E}-06 \\
4.35 \mathrm{E}-06\end{array}$ \\
\hline
\end{tabular}


Table D.1 (continued). List of collected datasets with analysis results for the Confederation Bridge light pole study of Section 8.2 .

\begin{tabular}{|c|c|c|c|c|c|c|c|c|c|c|c|c|c|c|}
\hline & \multirow[t]{2}{*}{$\begin{array}{l}\text { Data- } \\
\text { set } \\
\#\end{array}$} & \multirow[t]{2}{*}{$\begin{array}{l}\text { Time stamp } \\
\text { (local time) }\end{array}$} & \multirow[t]{2}{*}{$\begin{array}{l}\text { Dataset } \\
\text { dur. } \\
\text { (sec.) }\end{array}$} & \multirow[t]{2}{*}{$\begin{array}{l}\text { Sust. } \\
\text { wind } \\
(\mathrm{km} / \mathrm{h})\end{array}$} & \multirow[t]{2}{*}{$\begin{array}{l}\text { Wind } \\
\text { gusts } \\
(\mathrm{km} / \mathrm{h})\end{array}$} & \multirow[t]{2}{*}{$\begin{array}{l}\text { Wind } \\
\text { dir. } \\
\text { (de- } \\
\text { grees) }\end{array}$} & \multicolumn{2}{|c|}{$\begin{array}{c}\text { Maximum } \\
\text { displacement } \\
\text { (resultant, mm) }\end{array}$} & \multicolumn{2}{|c|}{$\begin{array}{l}\text { Strength of } \\
\text { signal RMS } \\
\quad(\mathrm{mm})\end{array}$} & \multicolumn{2}{|c|}{$\begin{array}{c}\text { Fundamental } \\
\text { frequency } \\
\text { (displacement) }(\mathrm{Hz})\end{array}$} & \multicolumn{2}{|c|}{$\begin{array}{c}\text { Cumulative fatigue } \\
\text { damage (cFD) estimate }\end{array}$} \\
\hline & & & & & & & Undamp. & $\overline{\text { Damped }}$ & Undamp. & $\overline{\text { Damped }}$ & Undamp. & $\overline{\text { Damped }}$ & Undamp. & $\overline{\text { Damped }}$ \\
\hline $\begin{array}{l}\infty \\
\#\end{array}$ & $\begin{array}{l}208 \\
209 \\
210 \\
211 \\
212 \\
213 \\
214 \\
215 \\
\end{array}$ & 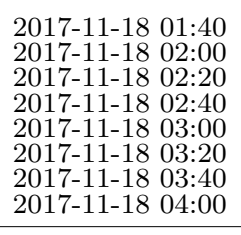 & $\begin{array}{l}300 \\
300 \\
300 \\
300 \\
300 \\
300 \\
300 \\
300 \\
\end{array}$ & $\begin{array}{c}76.5 \\
78 \\
75.4 \\
74 \\
74.7 \\
74.8 \\
72.6 \\
72.2 \\
\end{array}$ & $\begin{array}{l}88.9 \\
86.5 \\
95.3 \\
87.2 \\
88.9 \\
88.2 \\
85.4 \\
84 \\
\end{array}$ & $\begin{array}{l}289.7 \\
290.5 \\
295.7 \\
295.4 \\
293.5 \\
296.1 \\
300.3 \\
297.5 \\
\end{array}$ & $\begin{array}{c}15.9 \\
18.7 \\
14 \\
13.4 \\
10.6 \\
20.6 \\
12.9 \\
13.2 \\
\end{array}$ & $\begin{array}{c}23 \\
19.8 \\
12.8 \\
19.1 \\
15.4 \\
17.8 \\
14.7 \\
12.5 \\
\end{array}$ & $\begin{array}{l}5.1 \\
4.8 \\
4.4 \\
3.9 \\
3.6 \\
5.2 \\
4.2 \\
4.4 \\
\end{array}$ & $\begin{array}{l}4.9 \\
5.1 \\
4.1 \\
5.4 \\
5 \\
5.2 \\
4.4 \\
4.2 \\
\end{array}$ & $\begin{array}{l}1.906 \\
1.891 \\
1.906 \\
1.891 \\
1.891 \\
1.891 \\
1.875 \\
1.891 \\
\end{array}$ & $\begin{array}{l}1.75 \\
1.781 \\
1.766 \\
1.734 \\
1.75 \\
1.781 \\
1.75 \\
1.75 \\
\end{array}$ & $\begin{array}{l}7.55 \mathrm{E}-06 \\
6.14 \mathrm{E}-06 \\
3.53 \mathrm{E}-06 \\
1.12 \mathrm{E}-06 \\
1.02 \mathrm{E}-06 \\
1.07 \mathrm{E}-05 \\
1.66 \mathrm{E}-06 \\
1.45 \mathrm{E}-06 \\
\end{array}$ & $\begin{array}{l}4.39 \mathrm{E}-06 \\
2.40 \mathrm{E}-06 \\
1.93 \mathrm{E}-06 \\
4.97 \mathrm{E}-06 \\
4.79 \mathrm{E}-06 \\
3.79 \mathrm{E}-06 \\
2.10 \mathrm{E}-06 \\
1.27 \mathrm{E}-06 \\
\end{array}$ \\
\hline & 216 & 2017-11-28 15:00 & 300 & 14.7 & 19.4 & 280 & 1.5 & 4.9 & 0.5 & 1 & 1.906 & 1.781 & $6.83 \mathrm{E}-11$ & $1.40 \mathrm{E}-10$ \\
\hline $\begin{array}{l}\infty \\
\# \\
\# \\
\overrightarrow{0} \\
\overrightarrow{0} \\
\overrightarrow{0} \\
\overrightarrow{0} \\
\vec{z}\end{array}$ & $\begin{array}{l}217 \\
218 \\
219 \\
220 \\
221 \\
222 \\
223 \\
224 \\
225 \\
226 \\
227 \\
228 \\
229 \\
230 \\
231 \\
232 \\
233 \\
234\end{array}$ & $\begin{array}{ll}2017-11-29 & 22: 30 \\
2017-11-29 & 23: 00 \\
2017-11-29 & 23: 30 \\
2017-11-30 & 00: 00 \\
2017-11-30 & 00: 30 \\
2017-11-30 & 01: 00 \\
2017-11-30 & 01: 30 \\
2017-11-30 & 02: 00 \\
2017-11-30 & 02: 30 \\
2017-11-30 & 03: 00 \\
2017-11-30 & 03: 30 \\
2017-11-30 & 04: 00 \\
2017-11-30 & 04: 30 \\
2017-11-30 & 05: 00 \\
2017-11-30 & 05: 30 \\
2017-11-30 & 06: 00 \\
2017-11-30 & 06: 30 \\
2017-11-30 & 07: 00\end{array}$ & $\begin{array}{l}300 \\
300 \\
300 \\
300 \\
300 \\
300 \\
300 \\
300 \\
300 \\
300 \\
300 \\
300 \\
300 \\
300 \\
300 \\
300 \\
300 \\
300 \\
\end{array}$ & $\begin{array}{l}54.9 \\
58.5 \\
57.7 \\
57.3 \\
56.4 \\
56.5 \\
56.2 \\
52.4 \\
51 \\
49.1 \\
50.7 \\
48 \\
45.2 \\
44.1 \\
46.1 \\
38.6 \\
38.6 \\
34.9\end{array}$ & $\begin{array}{l}66.7 \\
70.9 \\
70.6 \\
69.2 \\
69.2 \\
66.3 \\
63.5 \\
62.1 \\
58.9 \\
58.2 \\
57.9 \\
55.4 \\
52.2 \\
50.4 \\
52.2 \\
45.2 \\
44.4 \\
41.6\end{array}$ & $\begin{array}{c}325 \\
325 \\
310 \\
310 \\
310 \\
310 \\
310 \\
310 \\
310 \\
317.5 \\
302.5 \\
302.5 \\
310 \\
310 \\
310 \\
317.5 \\
310 \\
310\end{array}$ & $\begin{array}{l}11.5 \\
11.1 \\
11.3 \\
13.6 \\
9.3 \\
10.9 \\
10.6 \\
9.4 \\
11.3 \\
10.3 \\
6.8 \\
7.3 \\
5.7 \\
6.1 \\
9.4 \\
6.4 \\
6.3 \\
5\end{array}$ & $\begin{array}{c}14 \\
15.3 \\
13.1 \\
15.2 \\
12.3 \\
13.5 \\
13.7 \\
12.1 \\
11.3 \\
11.4 \\
9.8 \\
7 \\
7.5 \\
9.4 \\
9.1 \\
6.9 \\
5.1 \\
5.4\end{array}$ & $\begin{array}{l}4 \\
3.8 \\
4 \\
4.4 \\
3.5 \\
3.4 \\
3.8 \\
3.2 \\
3.3 \\
2.6 \\
2.5 \\
2.2 \\
2.1 \\
2.2 \\
2.8 \\
1.8 \\
2 \\
1.7\end{array}$ & $\begin{array}{l}4.3 \\
4.7 \\
4.1 \\
4 \\
4.1 \\
3.8 \\
3.9 \\
3.6 \\
3.4 \\
3.1 \\
2.9 \\
2.6 \\
2.2 \\
2.6 \\
2.7 \\
1.9 \\
1.7 \\
1.7\end{array}$ & $\begin{array}{l}1.891 \\
1.891 \\
1.891 \\
1.906 \\
1.891 \\
1.875 \\
1.891 \\
1.875 \\
1.875 \\
1.875 \\
1.875 \\
1.875 \\
1.906 \\
1.922 \\
1.875 \\
1.891 \\
1.875 \\
1.875\end{array}$ & $\begin{array}{l}1.766 \\
1.766 \\
1.781 \\
1.766 \\
1.766 \\
1.75 \\
1.766 \\
1.75 \\
1.75 \\
1.781 \\
1.75 \\
1.781 \\
1.781 \\
1.766 \\
1.766 \\
1.75 \\
1.75 \\
1.75\end{array}$ & $\begin{array}{l}3.74 \mathrm{E}-06 \\
2.22 \mathrm{E}-06 \\
2.51 \mathrm{E}-06 \\
6.53 \mathrm{E}-06 \\
1.09 \mathrm{E}-06 \\
1.57 \mathrm{E}-06 \\
1.94 \mathrm{E}-06 \\
1.12 \mathrm{E}-06 \\
1.18 \mathrm{E}-06 \\
4.23 \mathrm{E}-07 \\
1.72 \mathrm{E}-07 \\
1.48 \mathrm{E}-07 \\
1.02 \mathrm{E}-07 \\
8.92 \mathrm{E}-08 \\
7.38 \mathrm{E}-07 \\
1.08 \mathrm{E}-07 \\
1.22 \mathrm{E}-07 \\
3.98 \mathrm{E}-08\end{array}$ & $\begin{array}{l}4.05 \mathrm{E}-06 \\
5.26 \mathrm{E}-06 \\
3.15 \mathrm{E}-06 \\
4.03 \mathrm{E}-06 \\
2.81 \mathrm{E}-06 \\
2.08 \mathrm{E}-06 \\
2.04 \mathrm{E}-06 \\
1.43 \mathrm{E}-06 \\
1.10 \mathrm{E}-06 \\
1.29 \mathrm{E}-06 \\
5.70 \mathrm{E}-07 \\
2.43 \mathrm{E}-07 \\
2.38 \mathrm{E}-07 \\
3.89 \mathrm{E}-07 \\
5.69 \mathrm{E}-07 \\
1.04 \mathrm{E}-07 \\
3.23 \mathrm{E}-08 \\
2.63 \mathrm{E}-08\end{array}$ \\
\hline 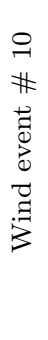 & $\begin{array}{l}235 \\
236 \\
237 \\
238 \\
239 \\
240 \\
241 \\
242 \\
243 \\
244 \\
245 \\
246 \\
247\end{array}$ & $\begin{array}{ll}2017-12-12 & 21: 00 \\
2017-12-12 & 21: 30 \\
2017-12-12 & 22: 00 \\
2017-12-12 & 22: 30 \\
2017-12-12 & 23: 00 \\
2017-12-12 & 23: 30 \\
2017-12-13 & 00: 00 \\
2017-12-13 & 00: 30 \\
2017-12-13 & 01: 00 \\
2017-12-13 & 01: 30 \\
2017-12-13 & 0200 \\
2017-12-13 & 02: 30 \\
2017-12-13 & 03: 00\end{array}$ & $\begin{array}{l}300 \\
300 \\
300 \\
300 \\
300 \\
300 \\
300 \\
300 \\
300 \\
300 \\
300 \\
300 \\
300\end{array}$ & $\begin{array}{l}62.5 \\
67 \\
69.5 \\
70.1 \\
68.7 \\
65.9 \\
70.2 \\
75.5 \\
74.8 \\
75.5 \\
74.6 \\
77.6 \\
77.5\end{array}$ & $\begin{array}{c}73 \\
78.3 \\
79.4 \\
79.7 \\
79 \\
78.7 \\
84.3 \\
88.2 \\
90.3 \\
85 \\
83.6 \\
89.6 \\
87.9\end{array}$ & $\begin{array}{l}62.5 \\
77.5 \\
70 \\
70 \\
70 \\
70 \\
70 \\
77.5 \\
77.5 \\
77.5 \\
77.5 \\
77.5 \\
77.5\end{array}$ & $\begin{array}{c}15 \\
19.5 \\
22.2 \\
22.1 \\
20.5 \\
18.5 \\
22.5 \\
23.7 \\
28.2 \\
25.3 \\
24.6 \\
23.3 \\
25.6\end{array}$ & $\begin{array}{l}15.8 \\
14.7 \\
22 \\
20.1 \\
16.1 \\
15.8 \\
24.1 \\
28.9 \\
21.8 \\
18.1 \\
15.7 \\
19.9 \\
22.5\end{array}$ & $\begin{array}{l}5.3 \\
6.3 \\
7 \\
6.8 \\
6.7 \\
5.5 \\
7 \\
8.4 \\
9 \\
8.2 \\
7.8 \\
8.5 \\
8\end{array}$ & $\begin{array}{l}4.9 \\
4.8 \\
5.3 \\
5.1 \\
4.6 \\
5.7 \\
5.5 \\
5.9 \\
6.2 \\
5.3 \\
5.1 \\
5.6 \\
6.4\end{array}$ & $\begin{array}{l}1.859 \\
1.875 \\
1.859 \\
1.875 \\
1.875 \\
1.891 \\
1.906 \\
1.859 \\
1.859 \\
1.875 \\
1.875 \\
1.906 \\
1.875\end{array}$ & $\begin{array}{l}1.781 \\
1.734 \\
1.75 \\
1.766 \\
1.766 \\
1.766 \\
1.781 \\
1.781 \\
1.766 \\
1.75 \\
1.75 \\
1.75 \\
1.75\end{array}$ & $\begin{array}{l}5.01 \mathrm{E}-06 \\
7.74 \mathrm{E}-06 \\
1.29 \mathrm{E}-05 \\
1.32 \mathrm{E}-05 \\
1.14 \mathrm{E}-05 \\
4.56 \mathrm{E}-06 \\
1.45 \mathrm{E}-05 \\
2.75 \mathrm{E}-05 \\
3.19 \mathrm{E}-05 \\
3.21 \mathrm{E}-05 \\
2.06 \mathrm{E}-05 \\
2.88 \mathrm{E}-05 \\
2.35 \mathrm{E}-05\end{array}$ & $\begin{array}{l}3.59 \mathrm{E}-06 \\
1.95 \mathrm{E}-06 \\
4.21 \mathrm{E}-06 \\
3.62 \mathrm{E}-06 \\
2.80 \mathrm{E}-06 \\
5.36 \mathrm{E}-06 \\
3.89 \mathrm{E}-06 \\
6.13 \mathrm{E}-06 \\
7.88 \mathrm{E}-06 \\
3.26 \mathrm{E}-06 \\
2.13 \mathrm{E}-06 \\
3.86 \mathrm{E}-06 \\
8.05 \mathrm{E}-06\end{array}$ \\
\hline
\end{tabular}


Table D.1 (continued). List of collected datasets with analysis results for the Confederation Bridge light pole study of Section 8.2.

\begin{tabular}{|c|c|c|c|c|c|c|c|c|c|c|c|c|c|c|}
\hline & \multirow[t]{2}{*}{$\begin{array}{l}\text { Data- } \\
\text { set } \\
\#\end{array}$} & \multirow[t]{2}{*}{$\begin{array}{l}\text { Time stamp } \\
\text { (local time) }\end{array}$} & \multirow[t]{2}{*}{$\begin{array}{l}\text { Dataset } \\
\text { dur. } \\
\text { (sec.) }\end{array}$} & \multirow[t]{2}{*}{$\begin{array}{l}\text { Sust. } \\
\text { wind } \\
(\mathrm{km} / \mathrm{h})\end{array}$} & \multirow[t]{2}{*}{$\begin{array}{l}\text { Wind } \\
\text { gusts } \\
(\mathrm{km} / \mathrm{h})\end{array}$} & \multirow[t]{2}{*}{$\begin{array}{l}\text { Wind } \\
\text { dir. } \\
\text { (de- } \\
\text { grees) }\end{array}$} & \multicolumn{2}{|c|}{$\begin{array}{c}\text { Maximum } \\
\text { displacement } \\
\text { (resultant, } \mathrm{mm} \text { ) }\end{array}$} & \multicolumn{2}{|c|}{$\begin{array}{l}\text { Strength of } \\
\text { signal RMS } \\
\quad(\mathrm{mm})\end{array}$} & \multicolumn{2}{|c|}{$\begin{array}{l}\text { Fundamental } \\
\text { frequency } \\
\text { (displacement)(Hz) }\end{array}$} & \multicolumn{2}{|c|}{$\begin{array}{c}\text { Cumulative fatigue } \\
\text { damage (cFD) estimate }\end{array}$} \\
\hline & & & & & & & Undamp. & $\overline{\text { Damped }}$ & Undamp. & $\overline{\text { Damped }}$ & Undamp. & $\overline{\text { Damped }}$ & Undamp. & $\overline{\text { Damped }}$ \\
\hline 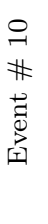 & $\begin{array}{l}248 \\
249 \\
250 \\
251 \\
252 \\
253 \\
254 \\
255\end{array}$ & $\begin{array}{ll}2017-12-13 & 03: 30 \\
2017-12-13 & 04: 00 \\
2017-12-13 & 04: 30 \\
2017-12-13 & 05: 00 \\
2017-12-13 & 05: 30 \\
2017-12-13 & 06: 00 \\
2017-12-13 & 06: 30 \\
2017-12-13 & 07: 00\end{array}$ & $\begin{array}{l}300 \\
300 \\
300 \\
300 \\
300 \\
300 \\
300 \\
300\end{array}$ & $\begin{array}{l}85.8 \\
86.3 \\
102.2 \\
91.7 \\
68.7 \\
71.3 \\
63.9 \\
66.3\end{array}$ & $\begin{array}{c}98.1 \\
100.6 \\
119.3 \\
108.3 \\
78.3 \\
85.7 \\
69.5 \\
73.7\end{array}$ & $\begin{array}{l}77.5 \\
77.5 \\
777.5 \\
77.5 \\
77.5 \\
77.5 \\
85 \\
77.5\end{array}$ & $\begin{array}{c}28.2 \\
38.2 \\
30 \\
21 \\
19.5 \\
20.9 \\
16 \\
17\end{array}$ & $\begin{array}{l}25.1 \\
30.5 \\
33.1 \\
19.5 \\
17.8 \\
13.9 \\
11.6 \\
11.4\end{array}$ & $\begin{array}{c}9 \\
10.8 \\
11.4 \\
7.4 \\
6.3 \\
6.4 \\
6 \\
6\end{array}$ & $\begin{array}{l}7.4 \\
8.6 \\
8.5 \\
5.2 \\
4.9 \\
4.6 \\
3.5 \\
4\end{array}$ & $\begin{array}{l}1.906 \\
1.906 \\
1.859 \\
1.891 \\
1.891 \\
1.922 \\
1.859 \\
1.891\end{array}$ & $\begin{array}{l}1.781 \\
1.766 \\
1.781 \\
1.766 \\
1.75 \\
1.781 \\
1.75 \\
1.719\end{array}$ & $\begin{array}{l}2.89 \mathrm{E}-05 \\
5.80 \mathrm{E}-05 \\
6.26 \mathrm{E}-05 \\
1.57 \mathrm{E}-05 \\
9.72 \mathrm{E}-06 \\
8.03 \mathrm{E}-06 \\
8.88 \mathrm{E}-06 \\
1.03 \mathrm{E}-05\end{array}$ & $\begin{array}{l}1.02 \mathrm{E}-05 \\
1.97 \mathrm{E}-05 \\
1.55 \mathrm{E}-05 \\
4.54 \mathrm{E}-06 \\
3.63 \mathrm{E}-06 \\
2.23 \mathrm{E}-06 \\
5.83 \mathrm{E}-07 \\
9.08 \mathrm{E}-07\end{array}$ \\
\hline & 256 & 2018-01-21 14:37 & 300 & 35.8 & 40.9 & 295 & 3.1 & 3 & 1 & 0.9 & 1.906 & 1.781 & $1.85 \mathrm{E}-09$ & $2.49 \mathrm{E}-10$ \\
\hline $\begin{array}{l}\vec{J} \\
\# \\
\# \\
\overrightarrow{0} \\
0 \\
0 \\
\overrightarrow{3} \\
\overrightarrow{3} \\
\overrightarrow{3}\end{array}$ & $\begin{array}{l}257 \\
258 \\
259 \\
260 \\
261 \\
262 \\
263 \\
264 \\
265 \\
266 \\
267 \\
268 \\
269\end{array}$ & 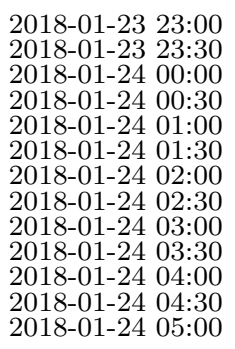 & $\begin{array}{l}300 \\
300 \\
300 \\
300 \\
300 \\
300 \\
300 \\
300 \\
300 \\
300 \\
300 \\
300 \\
300\end{array}$ & $\begin{array}{l}54.5 \\
59 \\
60.1 \\
71.3 \\
74.3 \\
72.1 \\
65.4 \\
69.8 \\
68.8 \\
61 \\
49.7 \\
41.8 \\
35.7\end{array}$ & $\begin{array}{c}59.6 \\
66 \\
65.3 \\
75.9 \\
78 \\
77.3 \\
71.6 \\
78 \\
72.3 \\
64.9 \\
54 \\
45.9 \\
48\end{array}$ & $\begin{array}{c}122.5 \\
92.5 \\
92.5 \\
212.5 \\
220 \\
220 \\
227.5 \\
220 \\
220 \\
220 \\
227.5 \\
227.5 \\
227.5\end{array}$ & $\begin{array}{c}12.1 \\
13.1 \\
15.1 \\
17.5 \\
19.7 \\
15.4 \\
17.2 \\
17.5 \\
13.6 \\
11.4 \\
6 \\
6.2 \\
5.4\end{array}$ & $\begin{array}{l}9.1 \\
14.3 \\
11.2 \\
16.2 \\
14.5 \\
14.9 \\
13.8 \\
12.8 \\
9.6 \\
7.6 \\
7.1 \\
4.8 \\
4.5\end{array}$ & $\begin{array}{l}4.4 \\
4.8 \\
5 \\
6.5 \\
6 \\
5.3 \\
5.4 \\
6.1 \\
4.3 \\
4 \\
2.4 \\
2.4 \\
1.7\end{array}$ & $\begin{array}{l}3 \\
4 \\
3.3 \\
4.3 \\
4.2 \\
3.5 \\
3.3 \\
4 \\
3.1 \\
2.1 \\
1.4 \\
1.5 \\
1.1\end{array}$ & $\begin{array}{l}1.875 \\
1.906 \\
1.875 \\
1.891 \\
1.906 \\
1.875 \\
1.891 \\
1.875 \\
1.891 \\
1.906 \\
1.906 \\
1.891 \\
1.875\end{array}$ & $\begin{array}{l}1.766 \\
1.734 \\
1.75 \\
1.75 \\
1.766 \\
1.75 \\
1.766 \\
1.734 \\
1.75 \\
1.75 \\
1.75 \\
1.766 \\
1.75\end{array}$ & $\begin{array}{l}2.92 \mathrm{E}-06 \\
2.70 \mathrm{E}-06 \\
5.74 \mathrm{E}-06 \\
1.74 \mathrm{E}-05 \\
1.56 \mathrm{E}-05 \\
8.61 \mathrm{E}-06 \\
7.93 \mathrm{E}-06 \\
1.57 \mathrm{E}-05 \\
4.84 \mathrm{E}-06 \\
1.92 \mathrm{E}-06 \\
6.11 \mathrm{E}-08 \\
2.36 \mathrm{E}-07 \\
3.58 \mathrm{E}-08\end{array}$ & $\begin{array}{l}6.94 \mathrm{E}-07 \\
3.37 \mathrm{E}-06 \\
8.88 \mathrm{E}-07 \\
3.67 \mathrm{E}-06 \\
2.27 \mathrm{E}-06 \\
1.49 \mathrm{E}-06 \\
5.21 \mathrm{E}-07 \\
2.19 \mathrm{E}-06 \\
8.56 \mathrm{E}-07 \\
1.14 \mathrm{E}-07 \\
2.30 \mathrm{E}-08 \\
1.27 \mathrm{E}-08 \\
3.10 \mathrm{E}-09\end{array}$ \\
\hline 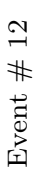 & $\begin{array}{l}270 \\
271 \\
272 \\
273 \\
274 \\
275\end{array}$ & $\begin{array}{l}2018-01-3011: 30 \\
2018-01-3012: 00 \\
2018-01-3012: 30 \\
2018-01-3013: 00 \\
2018-01-3013: 30 \\
2018-01-3014: 00\end{array}$ & $\begin{array}{l}300 \\
300 \\
300 \\
300 \\
300 \\
300\end{array}$ & $\begin{array}{l}59.5 \\
59.9 \\
59.5 \\
70.8 \\
63.9 \\
64.6\end{array}$ & $\begin{array}{l}72.7 \\
72.3 \\
76.9 \\
83.6 \\
79.4 \\
82.5\end{array}$ & $\begin{array}{l}17.5 \\
17.5 \\
17.5 \\
10 \\
10 \\
10\end{array}$ & $\begin{array}{l}11.5 \\
12.3 \\
12 \\
14.7 \\
13.5 \\
18.7\end{array}$ & $\begin{array}{l}14.4 \\
16.4 \\
10 \\
19.7 \\
20.3 \\
13.3\end{array}$ & $\begin{array}{l}3.7 \\
4.5 \\
4 \\
4.6 \\
5 \\
4.2\end{array}$ & $\begin{array}{l}3.8 \\
4.5 \\
3.8 \\
4.3 \\
4.4 \\
4.1\end{array}$ & $\begin{array}{l}1.906 \\
1.891 \\
1.906 \\
1.891 \\
1.891 \\
1.891\end{array}$ & $\begin{array}{l}1.766 \\
1.781 \\
1.75 \\
1.766 \\
1.766 \\
1.781\end{array}$ & $\begin{array}{l}1.39 \mathrm{E}-06 \\
4.30 \mathrm{E}-06 \\
2.75 \mathrm{E}-06 \\
4.55 \mathrm{E}-06 \\
4.89 \mathrm{E}-06 \\
2.47 \mathrm{E}-06\end{array}$ & $\begin{array}{l}1.50 \mathrm{E}-06 \\
4.19 \mathrm{E}-06 \\
1.83 \mathrm{E}-06 \\
3.22 \mathrm{E}-06 \\
3.49 \mathrm{E}-06 \\
1.80 \mathrm{E}-06\end{array}$ \\
\hline
\end{tabular}

* For configuration no.1 the pole top damper is positioned on pole N96. For configuration no.2 the pole top damper is on pole N95.

** Average wind direction in degrees with respect to bridge alignment. 